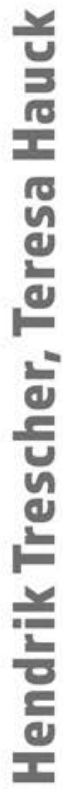
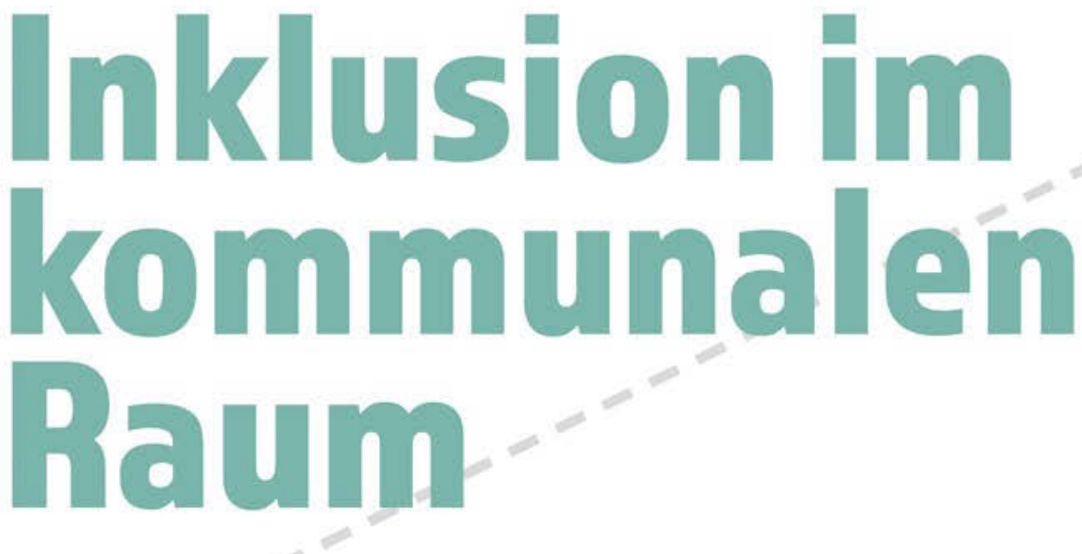

\title{
Sozialraumentwicklung
} im Kontext von Behinderung, Flucht und Demenz 
Hendrik Trescher, Teresa Hauck

Inklusion im kommunalen Raum

Gesellschaft der Unterschiede | Band 65 
Hendrik Trescher (Prof. Dr. phil. habil.) ist Soziologe und Pädagoge und hat die Professur für Erziehungswissenschaft mit dem Schwerpunkt Inklusion und Exklusion an der Philipps-Universität Marburg inne. Seine Forschungsgebiete sind Inklusion und Raum, politische Partizipation von Menschen mit Behinderung, Pädagogik bei kognitiven Beeinträchtigungen, Methoden qualitativer Sozialforschung, Disability Studies sowie Subjekt- und Diskursforschung im Kontext von Teilhabe und Ausschluss.

Teresa Hauck ist wissenschaftliche Mitarbeiterin im Projekt »Kommune Inklusiv« an der Philipps-Universität Marburg. In ihrer Dissertation untersucht sie Inklusionsverständnisse von pädagogisch Handelnden im Kontext der Betreuung von Menschen mit geistiger Behinderung. 
Hendrik Trescher, Teresa Hauck

\section{Inklusion im kommunalen Raum}

Sozialraumentwicklung im Kontext von Behinderung, Flucht und Demenz 


\section{Bibliografische Information der Deutschen Nationalbibliothek}

Die Deutsche Nationalbibliothek verzeichnet diese Publikation in der Deutschen Nationalbibliografie; detaillierte bibliografische Daten sind im Internet über http://dnb.d-nb.de abrufbar.

\section{(9) $(1) \Theta \Theta$}

Dieses Werk ist lizenziert unter der Creative Commons Attribution-NonCommercial-NoDerivs 4.0 Lizenz (BY-NC-ND). Diese Lizenz erlaubt die private Nutzung, gestattet aber keine Bearbeitung und keine kommerzielle Nutzung. Weitere Informationen finden Sie unter https://creativecommons.org/licenses/by-nc-nd/4.o/deed.de

Um Genehmigungen für Adaptionen, Übersetzungen, Derivate oder Wiederverwendung zu kommerziellen Zwecken einzuholen, wenden Sie sich bitte an rights@transcriptpublishing.com

Die Bedingungen der Creative-Commons-Lizenz gelten nur für Originalmaterial. Die Wiederverwendung von Material aus anderen Quellen (gekennzeichnet mit Quellenangabe) wie z.B. Schaubilder, Abbildungen, Fotos und Textauszüge erfordert ggf. weitere Nutzungsgenehmigungen durch den jeweiligen Rechteinhaber.

\section{(c) 2020 transcript Verlag, Bielefeld}

Umschlaggestaltung: Kordula Röckenhaus, Bielefeld

Satz: Mark-Sebastian Schneider, Bielefeld

Druck: Majuskel Medienproduktion GmbH, Wetzlar

Print-ISBN 978-3-8376-5267-3

PDF-ISBN 978-3-8394-5267-7

https://doi.org/10.14361/9783839452677

Gedruckt auf alterungsbeständigem Papier mit chlorfrei gebleichtem Zellstoff. Besuchen Sie uns im Internet: https://www.transcript-verlag.de

Unsere aktuelle Vorschau finden Sie unter www.transcript-verlag.de/vorschau-download 


\section{Inhalt}

I. Hinführung, Aufbau und AdressatInnen ...................................11

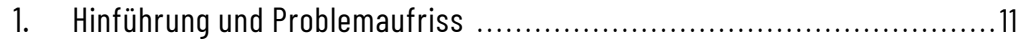

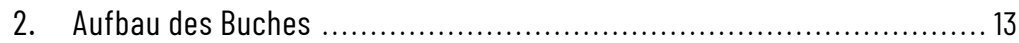

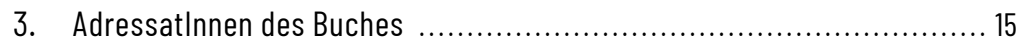

II. Behinderung, Inklusion und Sozialraum ................................... 17

4. Behinderung als Praxis, Inklusion als Kritik ................................... 17

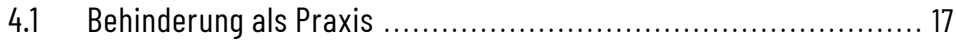

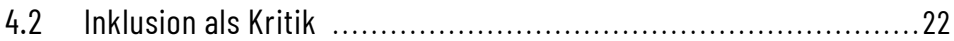

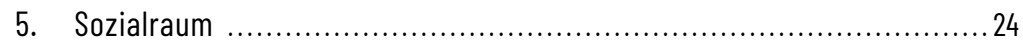

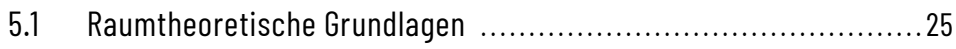

5.2 Raum und Subjekt als Praxen

der wechselseitigen Hervorbringung .................................. 26

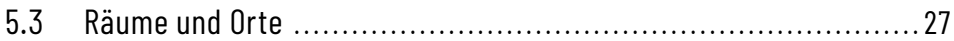

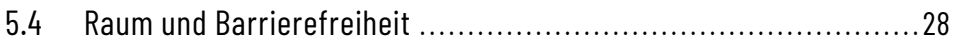

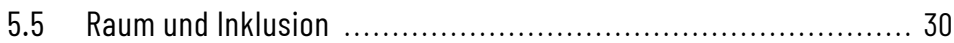

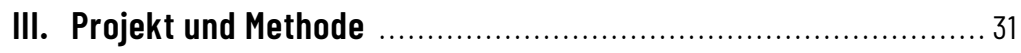

6. Sozialraumanalysen als Forschungsmethode ……......................... 31

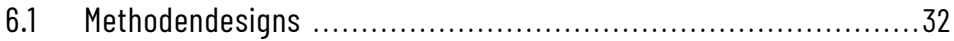

6.2 Betrachtung einer Studie: Sozialraumanalysen

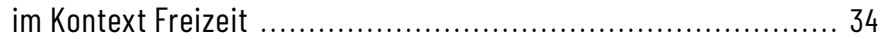

7. Beschreibung des Projekts »Kommune Inklusiv« .......................... 36

8. Beschreibung der Gesamtstudie .............................................. 38

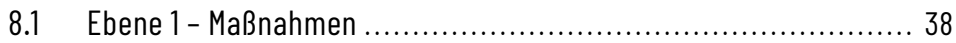

8.2 Ebene 2 - Sozialräume …............................................... 39

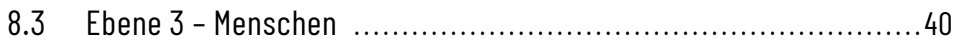


9. Beschreibung der hier dargelegten Teilstudie -

Sozialräume beforschen

9.1 Sozialräume beschreiben und erfahren ............................... 42

9.2 Erfahrungen im Miteinander erforschen ............................. 43

9.3 Sozialraumbefragung und Sozialraummonitoring ................... 43

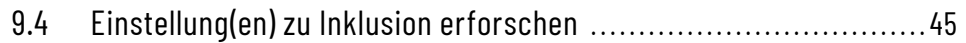

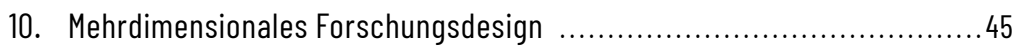

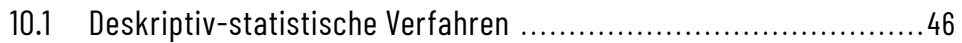

10.2 Qualitativ-inhaltsanalytische Verfahren ................................. 47

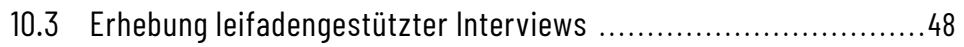

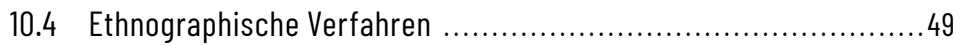

10.5 Zum Verhältnis quantitativer und qualitativer Forschungsverfahren ................................... 51

IV. Sozialräume beschreiben und erfahren ................................. 53

11. Öffentliche Orte und Barrierefreiheit ..........................................54

11.1 Annäherungen an ein operationalisierbares Verständnis

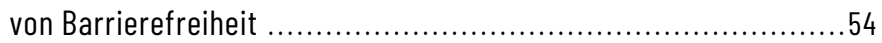

11.2 Barrierefreiheit öffentlicher Orte

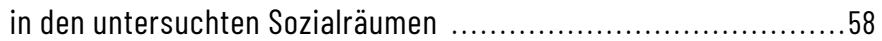

11.3 Gesamtbetrachtung und Diskussion .................................... 72

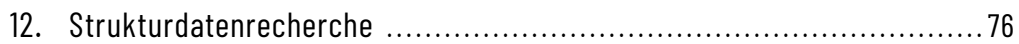

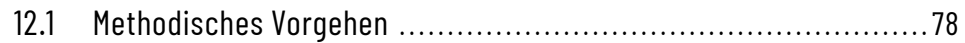

12.2 Herausforderungen der Strukturdatenrecherche ......................79

12.3 Beschreibung der Sozialräume ........................................... 81

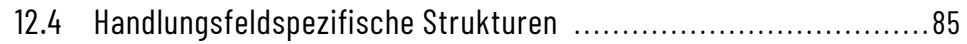

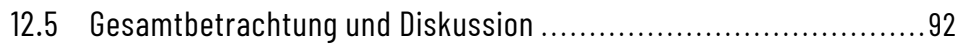

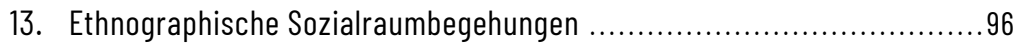

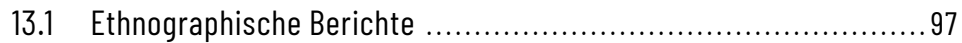

13.2 Gesamtbetrachtung und Diskussion .................................. 143

V. Erfahrungen im Miteinander erforschen ............................... 149

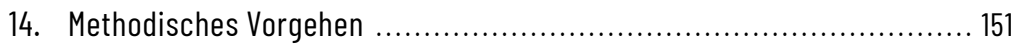

14.1 Forschungsleitende Fragestellungen ............................................ 151

14.2 Behinderung, Fluchtmigrationshintergrund und Demenz als Differenzkategorien ................................. 152

14.3 Erhebung: Leitfadengestützte Interviews ............................. 152 
14.4 Pre-Tests: Leitfadengestützte Telefoninterviews .156

14.5 Auswertung: Deskriptiv-statistische und qualitativ-

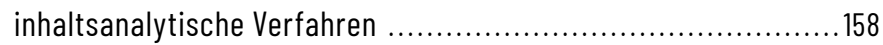

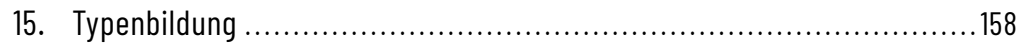

16. Sozialraumanalysen im Handlungsfeld Arbeit ................................ 162

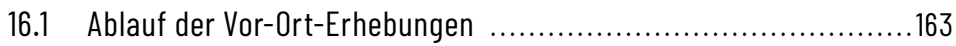

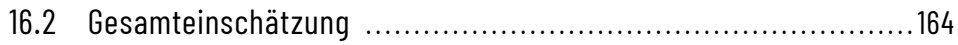

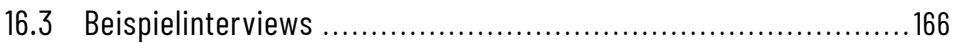

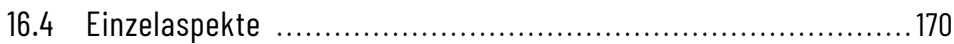

16.5 Aktuelle Teilhabe von Menschen mit Behinderung und Menschen mit Fluchtmigrationshintergrund ......................173

16.6 Erfahrungen im Miteinander .................................................. 174

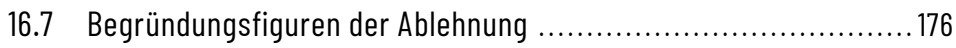

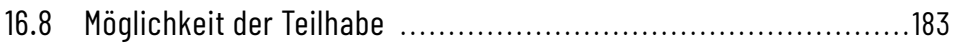

16.9 Schwierigkeiten und Handlungsperspektiven im Kontext

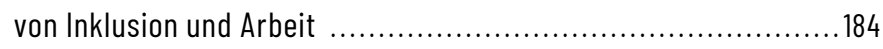

17. Sozialraumanalysen im Handlungsfeld Freizeit ............................. 196

17.1 Ablauf der leitfadengestützten Telefoninterviews .....................196

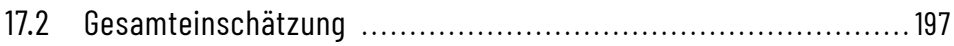

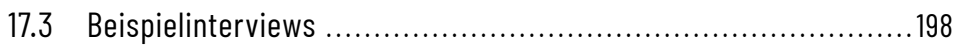

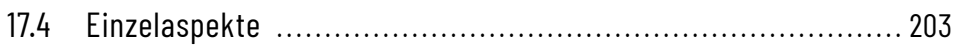

17.5 Aktuelle Teilhabe von Menschen mit Behinderung, Menschen mit Fluchtmigrationshintergrund und Menschen mit Demenz ..... 208

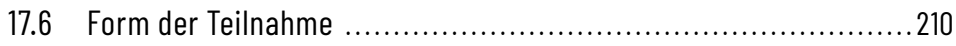

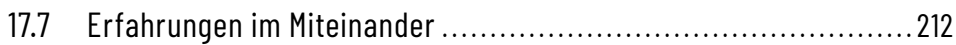

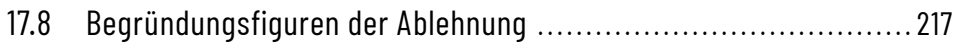

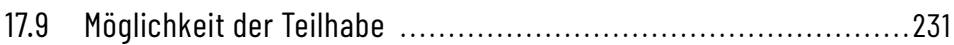

17.10 Möglichkeit, eine Begleitperson mitzubringen ....................... 232

17.11 Unterstützungsmöglichkeiten durch die anderen Teilnehmenden . 233

17.12 Schwierigkeiten und Handlungsperspektiven im Kontext von Inklusion und Freizeit ............................................. 235

18 Erfahrungen im Miteinander erforschen: Gesamtbetrachtung und Diskussion ............................................ 245

VI. Einstellung(en) zu Inklusion erforschen ............................. 257

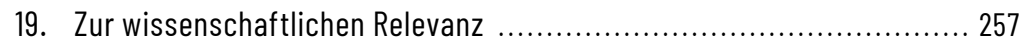

20. Konstruktion des Surveys, Pre-Test, Erhebung ............................ 259 
20.1 Geistige Behinderung als Differenzkategorie ........................ 259

20.2 Zur Konstruktion und Testung des Fragebogens ..................... 260

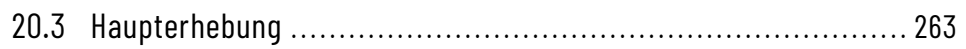

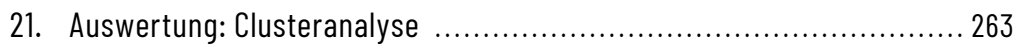

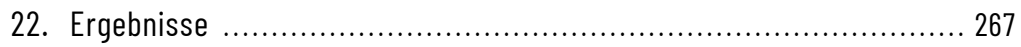

22.1 Zusammenfassung und Charakterisierung der Cluster ............. 267

22.2 Deskriptive Statistik der Cluster ....................................... 274

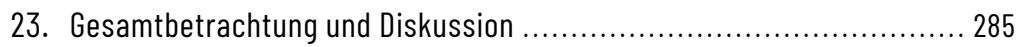

24. Methodische Gegenüberstellung des Surveys

»Einstellung(en) zu Inklusion « und der Sozialraumanalysen

in den Handlungsfeldern Arbeit und Freizeit ................................ 290

24.1 Interpretationsebene: Einstellung vs. Typisierung .................. 290

24.2 Legitimation: Testung und Forschungstradition

vs. Komplexität und Würdigung des Einzelfalls ........................291

24.3 Gegenstand: Hypothetische vs. tatsächliche Lebenspraxis ......... 292

24.4 Übertragbarkeit: Deutschlandweit, bevölkerungsrepräsentativ vs. sozialraumbezogen, modellhaft ... 292

24.5 Datengenerierung: Online-Fragebogenerhebung

vs. persönliches Interview 293

24.6 Differenzkategorie: Geistige Behinderung vs. Behinderung, Fluchtmigration und Demenz ......................................... 294

24.7 Gemeinsamkeit: Ergebnisse zeigen ähnliche Problematiken auf .. 294

24.8 Desiderat: Latente Sinnzusammenhänge erforschen .............. 295

\section{Zusammenfassung - Inklusion, Aneignung,}

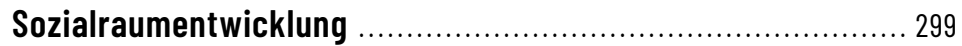

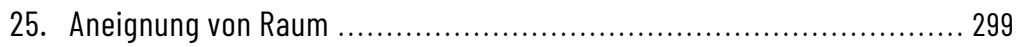

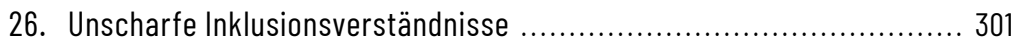

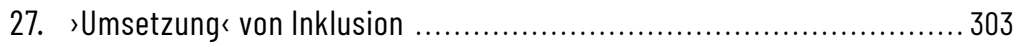

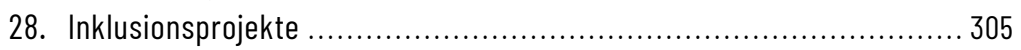

29. Akteurlnnen und kommunale Netzwerke im Kontext

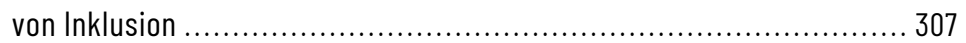

30. Inklusion zwischen Recht und Gerechtigkeit .............................. 309

31. Inklusion, Gerechtigkeit und Politik .............................................. 311

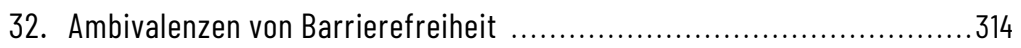

33. Bewusstseinsbildung und Sensibilisierung ..................................... 317

34. Frage nach öffentlichen Mitteln ............................................... 319 


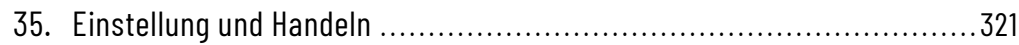

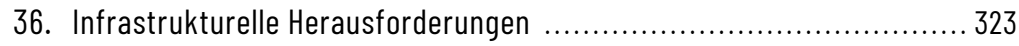

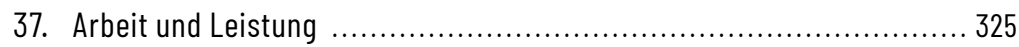

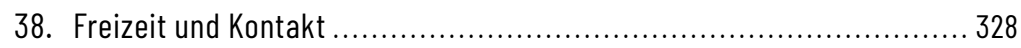

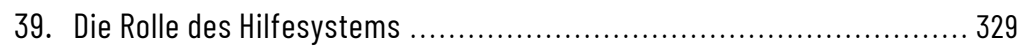

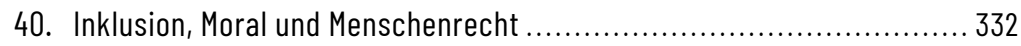

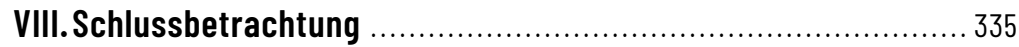

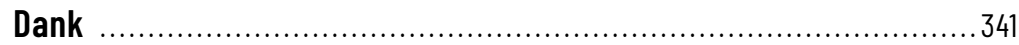

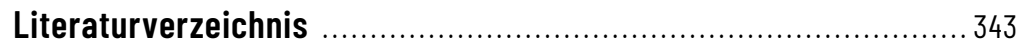





\section{Hinführung, Aufbau und AdressatInnen}

\section{Hinführung und Problemaufriss}

Sozialraum wird im Kontext Inklusion immer wieder als bedeutsam benannt, beispielsweise bezüglich sogenannter Sozialraumorientierung. Thematisiert wird diese unter anderem im Kontext Pflege und Alter (Maurer 2015), in Bezug auf Empowerment und Behinderung (Theunissen und Kulig 2011), im Bereich Schule (Schroeder 2016) oder auch im Kontext Migration (Nuss 2019) beziehungsweise Migration und Behinderung (Seifert 2014) $)^{1}$. Zumeist wird dabei unter Sozialraum ein durch kommunale Grenzen definierter Ort verstanden, während einige andere einen relationalen Zugang wählen und Sozialraum eher als etwas fassen, das in der interaktionalen Aushandlung hervorgebracht wird. Daraus folgen unterschiedliche Forschungszugänge, von denen einige oftmals als Sozialraumanalysen bezeichnet werden und eine (vollumfängliche) Untersuchung des jeweiligen Ortes und seiner ihn konstituierenden Praxen unter je bestimmten Fragestellungen vornehmen. Das jeweilige Forschungsinteresse und Sozialraumverständnis korrespondieren zumeist mit den methodischen Herangehensweisen. Im Kontext von Inklusion stehen dabei vor allem die Fragen im Vordergrund, wo und inwiefern sich in einem Sozialraum inklusive Praxen vollziehen und in welcher Hinsicht Handlungsbedarfe ausgemacht werden können. Eine Möglichkeit, sich diesen und weiteren Fragen anzunähern, ist, konkrete Projekte $\mathrm{zu}$ untersuchen, die in einem Sozialraum initiiert werden, um Inklusion im jeweiligen Ort zu ermöglichen. Forschungszugänge können hier beispielsweise begleitend installiert werden, um Prozesse abbilden und Veränderun-

1 Eine problematisierende Einordnung des Phänomens sogenannter Sozialraumorientierung in Bezug auf den Rückbau sozialstaatlicher Verantwortlichkeit geben beispielsweise Dahme und Wohlfahrt 2011. 
gen nachzeichnen zu können. Begleitforschung kann dadurch Ergebnisse sukzessive an die Verantwortlichen im Sozialraum rückkoppeln, damit diese die Möglichkeit haben, Projekte und Angebote entsprechend zu steuern und gegebenenfalls entlang der Erkenntnisse aus der Begleitforschung $\mathrm{zu}$ adaptieren. Ein Beispiel für eine solche Begleitforschung ist die Studie zum Projekt »Kommune Inklusiv«, die am Gegenstand des Projekts Grundlagenforschung zum Thema Inklusion und Sozialraum betreibt und deren Ergebnisse in diesem Band vorgestellt und diskutiert werden. Im Projekt »Kommune Inklusiv« werden fünf sogenannte Sozialräume in Deutschland - Erlangen, Rostock, Schneverdingen, Schwäbisch Gmünd und die Verbandsgemeinde Nieder-Olm - über einen Zeitraum von fünf Jahren von der Aktion Mensch e.V. finanziell und inhaltlich dabei unterstützt, Maßnahmen zu entwickeln und durchzuführen, die an >Inklusion< orientiert sind und dazu beitragen sollen, die Teilhabemöglichkeiten von Menschen zu erweitern, die von Ausschluss bedroht oder betroffen sind. Die breit und auf mehreren Ebenen multiperspektivisch verfahrende Begleitforschung wird durch die übergeordnete Forschungsfrage geleitet: Inwiefern verändern sich Sozialräume, wenn inklusionsorientierte Maßnahmen gefördert werden? Anhand dieser wird der Prozess in den jeweiligen Sozialräumen untersucht, um im Ergebnis darstellen zu können, inwiefern sich diese über den Verlauf des Projekts (gegebenenfalls) verändert haben. In diesem Band liegt der Fokus auf einer umfänglichen Untersuchung der fünf projektrelevanten Sozialräume, die anhand eines mehrdimensionalen Forschungsdesigns erfolgt. Die Studie ist, ebenso wie das hier vorgelegte Buch, im Dreiklang von Theorie, Empirie und Praxis verortet und folgt dem Anspruch, diese sinnhaft miteinander zu verbinden und wechselseitig zu konstituieren. In diesem Sinne korrespondiert beispielsweise die Wahl der Forschungsmethoden mit den theoretischen Grundannahmen. Schließlich ist ein weiterer Anspruch der Begleitforschung, Ergebnisse praxisnah zu diskutieren und handlungspraktische Hinweise dafür zu geben, wie mit Herausforderungen im Kontext von Sozialraum und Inklusion umgegangen werden kann. Jene Ansprüche spiegeln sich im Aufbau des Buches wider, der im nachfolgenden Kapitel erläutert wird. 


\section{Aufbau des Buches}

Das Buch ist in acht übergeordnete Teile gegliedert. Im ersten Teil »I - Hinführung, Aufbau und AdressatInnen« wird das Thema Sozialraum und Inklusion einleitend behandelt und es wird beschrieben, an wen sich das Buch richtet. Daraufhin wird im zweiten Teil »II - Behinderung, Inklusion und Sozialraum « das theoretische Fundament der Studie entfaltet. In Kapitel "4 - Behinderung als Praxis, Inklusion als Kritik« wird gezeigt, inwiefern in der Studie Behinderung und Inklusion entlang einer dekonstruktivistischen Logik gedacht werden. Das bedeutet, dass Behinderung als Subjektstatus infrage gestellt und schließlich abgebaut werden kann, denn Behinderung vollzieht sich je situativ und kann insofern alle Personen betreffen, die an Barrieren stoßen und von Ausschluss bedroht oder betroffen sind. Dieses kritische Infragestellen bedeutet schließlich Inklusion (Trescher 2018b). Die theoretischen Grundannahmen zu Sozialraum und Barrierefreiheit werden in Kapitel »5-Sozialraum « dargelegt, wobei von einem relationalen Verständnis von Raum ausgegangen wird, in dem sich Raum und Subjekt wechselseitig konstituieren. Dem folgt der dritte Teil "III - Projekt und Methode«, in dem die methodischen Grundannahmen (Kapitel »6 - Sozialraumanalysen als Forschungsmethode«) ebenso dargelegt werden wie eine »Beschreibung des Projekts »Kommune Inklusiv« (Kapitel 7). Die Begleitforschung zum Projekt ist auf mehreren Ebenen verortet und überaus komplex, weshalb in Kapitel »8 - Beschreibung der Gesamtstudie« ein Überblick über diese gegeben wird, woraufhin in Kapitel $» 9-$ Beschreibung der hier dargelegten Teilstudie - Sozialräume beforschen « ausführlich auf die diesem Buch zugrundeliegende Teilstudie eingegangen wird. Schließlich wird in Kapitel $» 10-$ Mehrdimensionales Forschungsdesign « das für die Teilstudie entwickelte methodische Setting detailliert dargelegt, anhand dessen die untersuchten Sozialräume umfassend analysiert werden können. Im vierten Teil »IV - Sozialräume beschreiben und erfahren « werden Ergebnisse der ersten, felderöffnenden Forschungsverfahren vorgestellt und diskutiert. In Kapitel »11 - Öffentliche Orte und Barrierefreiheit « werden die Ergebnisse von ExpertInneninterviews mit vier Personen dargelegt, die sich als SelbstvertreterInnen in repräsentativer Funktion im Bereich Barrierefreiheit engagieren. Untersucht wurden dabei vier Dimensionen von Barrierefreiheit: Mobilität, Sehen, Hören und Lesen/ Verstehen. Daran anschließend wird gezeigt, inwiefern öffentliche Orte in den untersuchten Sozialräumen als barrierefrei in den jeweiligen Dimensio- 
nen eingeschätzt werden können, wobei zusätzlich die Dimension Fremdsprache (Englisch) untersucht wurde. In Kapitel »12-Strukturdatenrecherche« werden die fünf untersuchten Sozialräume anhand der Recherche struktureller Daten untereinander vergleichbar gemacht. Unter anderem wurden dazu die EinwohnerInnenzahl, das durchschnittliche Einkommen, die Anzahl behindertenspezifischer Wohn- und Beschäftigungsmöglichkeiten sowie die Anzahl der Kindertagesstätten und Schulen erhoben. Diesem eher beschreibenden Blick werden in Kapitel »13-Ethnographische Sozialraumbegehungen « die Ergebnisse eines Forschungsverfahrens an die Seite gestellt, das auf die Erfahrung und das Erleben von Barrieren in den fünf untersuchten Sozialräumen ausgerichtet ist. Hierbei stand vor allem die Frage im Vordergrund, an welche Barrieren der Teilhabe die EinwohnerInnen jeweils stoßen und welche Möglichkeiten ihrer Überwindung es gibt. Jedes Kapitel schließt mit einer Gesamtbetrachtung und Diskussion der Ergebnisse, die sowohl in theoretischer, methodischer als auch handlungspraktischer Hinsicht erfolgt. Dem folgt der zentrale fünfte Teil »V - Erfahrungen im Miteinander erforschen«, in dem die Ergebnisse ebenjener Sozialraumanalysen ausführlich dargelegt und diskutiert werden. In Kapitel»14-Methodisches Vorgehen « wird gezeigt, wie die zugrundeliegenden Fragestellungen forschungspraktisch untersucht wurden. Zentrales Forschungsverfahren und zentrales Ergebnis ist die sogenannte Typenbildung, die im gleichnamigen Kapitel »15 - Typenbildung « beschrieben wird. Im Ergebnis können bezüglich Arbeit und Inklusion respektive Freizeit und Inklusion vier Typen unterschieden werden - voll ablehnende, teilweise ablehnende, (noch) zurückhaltende und offene Interviewpersonen - deren Beschreibung in diesem Kapitel erfolgt. Wie sich diese in den beiden untersuchten Handlungsfeldern Arbeit und Freizeit verteilen, wird in den jeweiligen nachfolgenden Kapiteln dargestellt. In Kapitel "16 - Sozialraumanalysen im Handlungsfeld Arbeit« werden die Ergebnisse der Sozialraumanalysen zum Thema Arbeit veranschaulicht. Neben einiger forschungspraktischer Hinweise wird exemplarisch Einblick in zwei Interviews gegeben, woraufhin zentrale Ergebnisse bezüglich der aktuellen Teilhabe von Menschen mit Behinderung und Menschen mit Fluchtmigrationshintergrund im Kontext Arbeit dargelegt werden. Abgeschlossen wird das Kapitel durch eine Diskussion von Schwierigkeiten und Handlungsperspektiven im Kontext von Inklusion und Arbeit. Das korrespondierende Kapitel »17-Sozialraumanalysen im Handlungsfeld Freizeit« ist ähnlich aufgebaut. Methodischen Hinweisen folgen zwei Beispielinterviews und es werden daraufhin Einzel- 
aspekte und die aktuelle Teilhabe dargelegt von Menschen mit Behinderung, Menschen mit Fluchtmigrationshintergrund und - hier wurde der Bereich Freizeit gegenüber dem Bereich Arbeit erweitert - Menschen mit Demenz. Ebenfalls schließt das Kapitel damit, dass Schwierigkeiten und Handlungsperspektiven im Kontext Inklusion und Freizeit diskutiert werden. Dieser Teil des Buches, in dem die breiten Ergebnisse der Sozialraumanalysen dargestellt werden, schließt mit Kapitel $\$ 18$ - Erfahrungen im Miteinander erforschen: Gesamtbetrachtung und Diskussion «. Hier werden die Ergebnisse einander gegenüberstellend diskutiert und theoretische, methodische und handlungspraktische Anschlussfragen abgewogen. Im sechsten Teil "VI - Einstellung(en) zu Inklusion erforschen« werden Ergebnisse des gleichnamigen Surveys dargestellt. Hier wird in Kapitel $\$ 19-Z$ Zur wissenschaftlichen Relevanz«, Kapitel »20 - Konstruktion des Surveys, Pre-Test, Erhebung « und Kapitel »21 - Auswertung: Clusteranalyse« das methodische und forschungspraktische Vorgehen erläutert, woraufhin in Kapitel „22 - Ergebnisse« zusammengefasst wird, welche Einstellungen zu Inklusion differenziert und wie diese jeweils beschrieben werden können. Auch dieser Teil schließt vorerst mit dem Kapitel »23-Gesamtbetrachtung und Diskussion«, in dem theoretische, methodische und handlungspraktische Anschlussmöglichkeiten erläutert werden, wobei besonderes Augenmerk auf die sogenannte Kontakthypothese gelegt wird. Daraufhin wird in Kapitel »24-Methodische Gegenüberstellung des Surveys "Einstellung(en) zu Inklusion « und der Sozialraumanalysen in den Handlungsfeldern Arbeit und Freizeit «as Forschungsdesign des Einstellungssurveys mit dem der Sozialraumanalysen kontrastiert. Schließlich werden zentrale Erkenntnisse im siebten Teil „VII - Zusammenfassung Inklusion, Aneignung, Sozialraumentwicklung « noch einmal zusammengetragen und weiterführende Fragen diskutiert. Das Buch endet mit dem achten Teil „VIII - Schlussbetrachtung«.

\section{Adressatlnnen des Buches}

Aus der Trias Theorie, Empirie und Praxis ergeben sich die Personenkreise, an die sich als potenzielle LeserInnen gerichtet wird. Adressiert werden WissenschaftlerInnen aus unterschiedlichen sozialwissenschaftlichen Disziplinen, die Interesse an der Verknüpfung von sozialraumorientierter Forschung und Inklusion haben. Die Studie erhebt den Anspruch, einen 
Beitrag zum Diskurs um Sozialraum und Inklusion zu leisten und darüber hinaus eine Antwort auf die Frage zu geben, wie Sozialräume umfänglich und mehrdimensional beforscht werden können. Zudem richtet sich das Buch ausdrücklich an Personen, die in der Praxis tätig sind und dabei vor der Herausforderung stehen, Inklusionsansprüche handlungspraktisch $\mathrm{zu}$ wenden, die von unterschiedlicher Seite an sie herangetragen werden (Interessenverbände, (Sozial-)Politik, Wissenschaft etc.). Insofern will die Studie dabei unterstützen, Handlungs- und Reflexionsanstöße auf die Frage zu geben, was Verantwortliche und Engagierte in Städten und Kommunen tun können, um Teilhabemöglichkeiten für jene zu eröffnen, die bislang (je situativ) Ausschluss erfahren. 


\section{Behinderung, Inklusion und Sozialraum}

Die Studie bewegt sich in ihrer theoretischen Rahmung primär in der wechselseitigen Verflechtung von Behinderung, Inklusion und Sozialraum. Inwiefern diese im Kontext der Studie konstituiert werden und miteinander in Verbindung stehen, wird im Folgenden entfaltet.

\section{Behinderung als Praxis, Inklusion als Kritik}

Behinderung wird im Kontext dieser Studie als Praxis des Behindert-werdens verstanden, die sich immer dann vollzieht, wenn Personen von (allgemeinen) Diskursen ausgeschlossen werden. Inklusion ist in diesem Verständnis folglich der Prozess, der jenen Behinderungspraxen gegenläufig ist. Da Inklusion bestehende Strukturen und Praxen infrage stellt, wird diese als Kritik verstanden. Dieses Theorem wird im Folgenden erläutert ${ }^{1}$.

\subsection{Behinderung als Praxis}

Behinderungspraxen vollziehen sich diskursiv, wobei auf ein foucaultsches Diskursverständnis referiert wird. Foucault versteht Diskurse als »Praktiken [...], die systematisch die Gegenstände bilden, von denen sie sprechen« (Foucault 1981, S. 74), was bedeutet, dass Diskurs Vollzugskraft innewohnt. Diskurs ist insofern nicht unbeweglich und manifest, sondern wird immer wieder neu hervorgebracht durch Subjekte (siehe Reckwitz 2003, S. 298) die wiederum in diesem Prozess ebenfalls in je bestimmter Weise hervorgebracht werden beziehungsweise sich selbst hervorbringen (Foucault 2005,

1 Eine ausführliche Herleitung findet sich unter anderem in Trescher (2018a). 
S. 275; Saar 2013, S. 27). Es wird von einer Art >Gesamtdiskurs` ausgegangen, der jede erfahrbare(n) Wirklichkeit(en) umfasst, während sich je bestimmte Diskurse auf einen konkreten Gegenstand beziehen. Diskurse haben (auch) dadurch unscharfe Grenzen, anhand derer sie ein Stück weit voneinander abgegrenzt werden können (Reckwitz 2008b, S. 11) und gleichzeitig Überschneidungen und Gemeinsamkeiten offensichtlich werden (Trescher 2018a, S. 22). Diskurse werden durch innere und äußere Ausschließungssysteme reguliert, die kontrollieren beziehungsweise steuern, inwiefern ein Diskurs verbreitet wird (Foucault 2003, S. 11; siehe auch Trescher 2015b, S. 261ff). Auf diese Weise wird sichergestellt, »dass das, was gesagt werden kann und was als legitimes Wissen angenommen wird, limitiert bleibt und nur innerhalb deutlich eingeschränkter und sichtbarer Grenzen erscheint« (Mills 2007, S. 80). Innere und äußere Ausschließungsprozeduren von Diskurs können entlang dreier Fragen zusammengeführt werden, nämlich der Frage nach Exklusion, der Frage nach dem Normalen und der Frage nach den Sprechenden (Foucault 2003; siehe auch Trescher 2018b). Bei der Frage nach Exklusion geht es um Zugänge und Zugangsmöglichkeiten zu bestimmten Diskursen, also darum: Wer wird zu Diskursen zugelassen und wer nicht? Verhandelt werden bei der Frage nach dem Zugang sowohl etwaige (Diskurs-)Grenzen, die immer unscharf sind, als auch unter welchen Umständen eine Person zu einem bestimmten Diskurs zugelassen wird (beispielsweise aufgrund einer Qualifikation). Exklusionspraxen vollziehen sich unter anderem durch das, was Foucault als »Ausgrenzung des Wahnsinns« (Foucault 2003, S. 16) bezeichnet, anhand dessen geregelt wird, wer im je bestimmten Diskurs >sprechen darf und wer nicht (wobei ssprechen und alle Ausdrucks- beziehungsweise Gestaltungsformen umfasst; Foucault 2003, S. 27ff). So werden einige Personen systematisch von bestimmten Diskursen ausgeschlossen, da sie als (kognitiv, sozial, physisch etc.) andersartig und/oder nicht zurechnungsfähig konstruiert werden. Ein Beispiel für eine solche Statuszuweisung und den damit verbundenen Ausschluss ist, dass einigen Menschen mit kognitiven Beeinträchtigungen bis vor kurzem noch das Wahlrecht pauschal abgesprochen wurde ${ }^{2}$. Dieses Gesetz wurde jedoch im Juli 2019 aufgehoben (BGBl. I). An dieser Gesetzesänderung zeigt

2 Menschen, die eine Betreuung in allen Angelegenheiten haben, waren durch $\S 13$ des Bundeswahlgesetzes von der Teilnahme an Wahlen ausgeschlossen, was auf viele Menschen mit kognitiven Beeinträchtigungen zutraf. 
sich, inwiefern Diskursgrenzen (und somit Exklusionspraxen) flexibel sind und sich verändern können und es wird deutlich, dass die Konstruktion von ’kognitiver Beeinträchtigung kontingent ist. Ein weiteres Beispiel für Exklusionspraxen ist, dass ein Ort bestimmte Voraussetzungen erfüllen muss, damit er uneingeschränkt zugänglich ist. Zudem ist zu prüfen, ob die betreffende Person die Erlaubnis hat, jenen Ort aufzusuchen oder ob es dazu ausgewählter Zugangsberechtigungen bedarf (beispielsweise, MitarbeiterIn einer konkreten Firma zu sein). Neben diesen manifesten Barrieren können latente Barrieren den Zugang zu Orten (und Diskursen) erschweren. Dies vollzieht sich beispielsweise dann, wenn eine Person sich den Eintritt zu einem bestimmten Ort nicht leisten kann. Ist also für eine Person der Kinobesuch finanziell nicht erschwinglich, so wird sie ausgeschlossen vom Diskurs um kulturelles Erleben und allem, was damit zusammenhängt (Freizeit verbringen, FreundInnen treffen, Sozialkontakte knüpfen und pflegen etc.). Wenn ein Ort in diesem Sinne zugänglich ist, dann ist damit eine Voraussetzung von teilhabender Aneignung geschaffen - teilhabende Aneignung selbst aber eben noch nicht. Neben der Frage nach Zugängen stellt sich die Frage nach dem Normalen, also nach der inneren Ausgestaltung von Diskursen: Was ist in Diskursen zugelassen zu sagen (und was nicht)? Diese Frage betrifft bestimmte (auch implizite) Regeln, Normen oder Verhaltenskodizes, die regulieren, was >das Normale des Diskurses ist. Dies können Sittlichkeitsnormen, wie zum Beispiel (unausgesprochene) Dresscodes, dem jeweiligen Diskurs inhärente Abläufe, eine bestimmte Art des (verbalsprachlichen) Ausdrucksvermögens oder auch Tabus sein, die in der Ordnung des jeweiligen Diskurses nicht angesprochen werden dürfen (siehe Foucault 2003, S. 11). Wer diese Vorgaben nicht kennt oder nicht einhalten kann beziehungsweise will, dessen Teilhabemöglichkeiten am Diskurs sind deutlich eingeschränkt. Eine Normalisierungspraxis ist beispielsweise »das verbotene Wort (Foucault 2003, S. 16), anhand dessen reguliert wird, was in einem Diskurs zulässig ist zu >sagen und was nicht. Auch »der Wille zur Wahrheit« (Foucault 2003, S. 16) reguliert >das Normale< des Diskurs, da durch ihn bestimmt wird, welches Wissen im Diskurs als wahr gilt. In diesem Sinne sind beispielsweise wissenschaftliche Entdeckungen oder Erkenntnisse nicht als solche aufzufassen, sondern sie stellen das Zulassen neuer Wahrheiten im Diskurs dar (siehe Foucault 2003, S. 14f), wodurch die (unscharfen) Grenzen des Diskurses verschoben wurden. Auch hier kann das Beispiel des Wahlrechts für Menschen mit kognitiven Beeinträchtigungen beziehungsweise 
Menschen, die unter vollumfänglicher Betreuung stehen, herangezogen werden - wurde doch die sneue Wahrheit als gültig befunden, dass jene Personen durchaus wahlfähig sind. Ein weiteres Beispiel für Normalisierungspraxen ist, dass gerade im Wissenschaftsdiskurs eine gewisse elaborierte Sprache üblich ist und Personen, die sich nicht derart ausdrücken (können), häufig ausgeschlossen werden oder gar nicht erst die Möglichkeit zum Sprechen bekommt. Dies verweist unmittelbar auf die dritte Frage nach den Sprechenden: Wer darf sich im Diskurs äußern und wird gehört? Im Zusammenhang damit steht die Frage, wie viel Aufmerksamkeit und Gewicht dem Wort der jeweiligen Person beigemessen wird. Hierbei können ebenfalls latente Mechanismen wirksam werden, die den Ausschluss vom je bestimmten Diskurs befördern und eine "Selektion unter den sprechenden Subjekten« (Foucault 2003, S. 26) vornehmen. Ein Beispiel für eine solche Regulierung der Vergabe von Sprechrollen ist, dass eine Person zwar an einem Diskurs teilhaben kann (und ihn dadurch mithervorbringt), ihrem Wort allerdings nicht die gleiche Bedeutung beigemessen wird, wie dem von anderen. In Bezug auf das Wahlrecht von Menschen mit kognitiven Beeinträchtigungen kann ausgeführt werden, dass jene Personen zwar inzwischen wählen dürfen, auch die, die bislang ausgeschlossen waren, es allerdings trotzdem kaum PolitikerInnen mit kognitiven Beeinträchtigungen in Deutschland gibt (Trescher 2016c). Dies liegt unter anderem daran, dass Menschen mit geistiger Behinderung im Gros mehrheitsgesellschaftlich nicht als politische Person anerkannt werden oder sich nicht trauen, Ansprüche geltend zu machen und auf einer Sprechrolle zu beharren. Ausschluss vollzieht sich also nicht nur durch eingeschränkte Zugangsmöglichkeiten, sondern auch aufgrund von Vorurteilen, Ängsten oder anderen latenten Barrieren.

Jene ausschließenden Diskursteilhabebarrieren werden in und durch Dispositive(n) (re-)produziert. Dispositive sind dabei als »Netz« (Foucault 1978, S. 120) zu verstehen, das Diskurse miteinander verbindet und anhand derer das Subjekt an bestimmte Positionen im Diskurs gebunden und auf diese beschränkt wird. Durch ein sogenanntes Behinderungsdispositiv werden Personen, die sbehindert genannt werden, in ihren Teilhabemöglichkeiten beschränkt. Dies vollzieht sich auch dann, wenn diesen Personen vordergründig dieselben Teilhabemöglichkeiten zugesprochen werden (wie eben in Bezug auf das Wahlrecht). Zum Tragen kommen dabei insbesondere bestimmte Einstellungen und Vorbehalte, die gegenüber sogenannten Menschen mit Behinderung vorgebracht und in denen diese oftmals als 
>defizitär konstruiert werden, was als Begründung für Ausschlusspraxen herangezogen wird. »Diese Einstellungen etc. werden durch das Dispositiv zusammengehalten, welches als eine Art Hinterbühne fungiert, auf der die Diskurse und diskursiven Praxen reguliert werden, die auf das Subjekt wirken und es auf bestimmte Subjektpositionen beschränken" (Trescher 2018c, S. 14). Dispositive werden dabei als kontingent angenommen. Sie sind also - ebenso wie Diskurs - wandelbar. An dieses Verständnis von Diskurs und Dispositiv knüpft das hier zugrunde gelegte Verständnis von Behinderung als Praxis an, das diese als je situative Praxis des Behindert-werdens konstituiert. Es wird davon ausgegangen, dass sich Behinderung immer dann vollzieht, wenn Subjekte je situativ an Diskursteilhabebarrieren sto$ß^{3}{ }^{3}$. Dadurch kann der Status sbehindert, der in gängigen Behinderungsverständnissen nach wie vor untrennbar an das Subjekt geknüpft ist, von diesem entkoppelt werden. Dies hat zur Folge, dass Subjekte, die bislang nicht als >behindert $\star$ adressiert werden, dennoch je situativ >behindert werden können. Beispielsweise stoßen auch Personen, die einen Kinderwagen schieben immer dann an Diskursteilhabebarrieren, wenn kein ebenerdiger beziehungsweise stufenloser Zugang möglich ist, Menschen mit Fluchtmigrationshintergrund erfahren oftmals dadurch Ausschluss, dass sie die deutsche Sprache (noch) nicht gut verstehen und Menschen mit Demenz werden teilweise durch hospitalisierende Unterbringungsstrukturen in ihrer Teilhabe an Praxen der Mehrheitsgesellschaft behindert. Behinderungspraxen sind insofern machtvolle Prozesse, die sich immer dann vollziehen, wenn einem Subjekt oder einer Gruppe von Subjekten der Zugang zu (je konkret bezugsrelevanten) Diskursen sowie die Möglichkeit, im Diskurs zu ssprechen<, erschwert oder verschlossen wird. Das bedeutet schließlich, dass sich von gängigen Behinderungsbegriffen gelöst werden kann, die nie ganz ihren essentialistischen Kern aufgeben konnten (Trescher 2018a, S. 37), und es möglich ist, auch Personen und Ausschlusspraxen darunter zu fassen, die in gängigen Verständnissen nicht als >behindert bezeichnet werden. Das Verständnis von Behinderung als Praxis folgt dabei der Frage: Wer wird in welcher Art und Weise (nicht) als im Diskurs sprechendes Subjekt hervorgebracht (und bringt sich wechselseitig selbst hervor)? Mit diesem Verständnis von Behinderung als Praxis, die sich immer dann vollzieht, wenn Personen

3 Dies ist ausführlich dargelegt in Trescher (2015b, S. 333f, 2017a, 2017c, 2017d, 2017e, 2018b, 2019). 
je situativ an Barrieren stoßen, geht einher, dass Behinderung theoretisch auflösbar ist, indem Diskursteilhabe ermöglicht wird. Jene Diskursteilhabemöglichkeiten beziehen sich dabei zunächst auf allgemeine Diskurse (im Gegensatz zu besonderen Diskursen). Diskurse werden dann als allgemein verstanden, wenn sie prinzipiell allen Personen offenstehen oder offenstehen sollten, woran schließlich das normative Moment deutlich wird, das diesem Verständnis von Behinderung als Praxis inhärent ist. Besondere Diskurse sind durch bestimmte Qualifikationen und/oder bestimmtes Wissen begrenzt; zu ihnen haben nicht alle Personen Zugang (und sollten auch nicht unbedingt Zugang haben). »Die Entscheidung darüber, welche Diskurse allgemein und welche besonders sind und wer diese Entscheidung trifft beziehungsweise treffen darf, ist normativ und kumuliert in Fragen der Gerechtigkeit, in denen ausgehandelt werden muss, welche Diskurse grundsätzlich allen Subjekten zugänglich sein sollen« (Trescher 2018c, S. 15; siehe auch Forst 1994, S. 215ff, 2005, S. 24ff; Herzog 2013). Zugang zu allgemeinen Diskursen wird ermöglicht, indem Diskursteilhabebarrieren dekonstruiert werden. Behinderung als je subjektive Praxis des Behindert-werdens ist dabei nicht ausschließlich als analytische Kategorie zu verstehen, sondern vollzieht sich auch als Erfahrung von Ausgrenzung, Teilhabebarrieren oder Diskriminierung. Behinderung ist also auch das, "was sich als Erfahrung von und mit Behinderung niederschlägt, sich in Biografien und Karrieren verfestigt, was Gegenstand und Bedingung von Anpassungen, Bewältigungsstrategien, Leiden oder Widerstand [...] darstellt« (Groenemeyer 2014, S. 154; siehe diesbezüglich auch Shakespeare 2010, S. 270). Behinderung als emotionale Erfahrung kann sozialräumlich (re-)produziert werden (Weisser 2010, S. 6).

\subsection{Inklusion als Kritik}

Inklusion ist, wie oben bereits angerissen, im Verständnis des hier dargelegten Theorems als Praxis zu verstehen, die Behinderung gegenläufig ist. Dennoch vollziehen sich Teilhabe und Ausschluss nicht als Antinomien, sondern sind eingebettet in ein diskursives Netz, in dem unterschiedliche Subjektpositionen möglich sind. »[D]ie Welt des Diskurses ist nicht zweigeteilt zwischen dem zugelassenen und dem ausgeschlossenen oder dem herrschenden und dem beherrschten Diskurs. Sie ist als eine Vielfältigkeit von diskursiven Elementen, die in verschiedenartigen Strategien ihre Rolle spie- 
len können, zu rekonstruieren « (Foucault 2014, S. 100). Inklusion als Praxis, die Behinderungspraxen gegenläufig ist, ist entlang dieses Theorems nicht als moralische Idee zu verstehen, die beispielsweise menschenrechtlichen Zugängen entspringt, sondern als eine Praxis der Kritik, anhand derer Diskursteilhabebarrieren dekonstruiert werden. Dabei wird Bezug genommen auf dekonstruktionslogische Arbeiten Butlers (u.a. 1991), die das Verständnis von Dekonstruktion, wie Derrida (u.a. 2016) es entwarf, als Begriff und Praxis weiterentwickelt hat (Reckwitz 2008a, S. 89ff). Dekonstruktion wird als Praxis des Infragestellens verstanden, die Widersprüche, Zusammenhänge und Ambivalenzen offenlegt und so der Analyse zugänglich macht (Zima 2016, S. 1; Zirfas 2001, S. 50; siehe auch Saar 2007, S. 167). Es handelt sich dabei also um eine Art kritisches Lesen<, das (unter anderem) das Ziel verfolgt, »das Vorherrschende [...] in seiner gesellschaftlichen Funktion zur Aufrechterhaltung der herrschenden Norm(alität) sowie in seinen Konstitutions- und Konstruktionsprozessen zu untersuchen, dabei Ausgeschlossenes zu erkennen und Hierarchisierungen aufzuweichen " (Hartmann 2001, S. 80f; siehe auch Engelmann 1990, S. 27). Inklusion geht also immer mit einem kritischen Infragestellen von Strukturen und Praxen einher, die Behinderung als Praxis (re-)produzieren. Inklusion ist kritisch, »da sie nicht nur die Handlungspraxis, sondern auch (Herrschafts-)Systeme sowie deren Funktionen in Frage stellt" (Trescher 2017a, S. 51; siehe auch Schäper 2015, S. 82ff). Inklusion als Kritik hat »die Funktion der Entunterwerfung "(Foucault 1992, S. 15). Insofern ist es nachvollziehbar, dass Inklusion krisenhaft für alle an diesem Prozess beteiligten Subjekte und Diskurse ist, da mit ihr bestehende Ordnungen aufgebrochen und schließlich Diskurse verschoben werden (Trescher 2018b). Inklusion als kritische Praxis ist also nicht »die Realisierung eines positiv definierbaren gesellschaftlichen (Ideal-)Zustands« (Dannenbeck 2013, S. 461), sondern vollzieht sich prozesshaft - Inklusion wird im Vollzug konstituiert. 


\section{Sozialraum}

In der (sozial-/sonder-)pädagogischen Handlungspraxis wird die sogenannte Sozialraumorientierung in den letzten Jahren in gewisser Weise zu einem neuen Leitbild erhoben (Wansing 2016, S. 258ff; Kessl et al. 2005, S. 191ff) ${ }^{4}$. Wansing (2016) erkennt darin einen gewissen Pragmatismus, »die komplexen Umweltfaktoren, die zur Konstitution von Behinderung beitragen, innerhalb eines festgelegten territorialen Rahmens buchstäblich zu verorten und in dieser räumlichen Dimension handlungspraktisch zu bearbeiten « (Wansing 2016, S. 258f). Ein möglicher Umkehrschluss dessen wäre, Sozialräume primär als »Verwaltungseinheiten« (Kessl et al. 2005, S. 194) zu verstehen. In Bezug auf die AdressatInnen sozialraumorientierten (pädagogischen) Handelns merkt Boettner (2009) problematisierend an, dass »die Orientierung auf den lokalen Lebenszusammenhang durchaus zwiespältig $\mathrm{zu}$ sehen [ist]. Wer sich in der abweichenden Normalität eines marginalisierten Sozialraumes einrichtet, sitzt darin auch fest. Der sozialräumliche Rückzug mildert die Erfahrung der gesellschaftlichen Randlage und verstetigt sie dadurch zugleich« (Boettner 2009, S. 261). Die zentrale Frage, die hierdurch aufgeworfen wird, ist, inwiefern Sozialräume marginalisiert sind und ob es nicht eher Menschen sind, die durch (unter anderem sozialräumliche) Praxen marginalisiert werden. Sozialraum wäre hierbei also nicht ausschließlich als lokaler Ort, so wie es Wansing (2016) und Boettner (2009)

4 Dies zeigt sich nicht zuletzt in der Ausgestaltung und Ausrichtung von Projekten, die anhand dieses Leitbilds zur Veränderung von Sozialräumen beitragen wollen. Neben dem Projekt »Kommune Inklusiv«, das in dieser Studie im Vordergrund steht, kann hier beispielsweise das Projekt »Inklusive Gemeinwesen planen« (Rohrmann und Schädler 2014) genannt werden, in dem die Forschenden kommunale Strukturen untersuchten, die entlang der UN-Konvention über die Rechte von Menschen mit Behinderungen Teilhabe ermöglichen sollen. Im Gros haben so gut wie alle Städte und Kommunen in Deutschland kleinere und größere Projekte angestoßen, anhand derer sie >Inklusion < in ihrem Sozialraum ermöglichen wollen. Stellvertretend für die Vielzahl an Projekten können die »Aktionspläne für Inklusion« hervorgehoben werden, die in allen Bundesländern formuliert wurden (eine Übersicht findet sich beispielsweise hier: einfachmachen 2019). Auch auf Bundesebene werden Projekte angestoßen, wobei beispielhaft die Konferenzreihe »Inklusiver Sozialraum« des Bundesministeriums für Arbeit und Soziales (2019) angeführt werden kann. Im Zusammenhang damit ist die Bundesfachstelle Barrierefreiheit zu nennen, die das dazugehörige Projekt »Initiative Sozialraum Inklusiv«seit 2018 leitet (Bundesfachstelle Barrierefreiheit o.J.). 
mehr oder weniger explizit zugrunde legen, sondern als sich vollziehende Praxis zu verstehen, die Menschen in je bestimmter Art und Weise hervorbringt - zum Beispiel als (nicht) marginalisiert. Raum ist immer schon Sozialraum, da er diskursiv hervorgebracht wird durch handelnde Subjekte und ihre Aneignungspraxen (siehe Kapitel 5; Kessl et al. 2005, S. 193). Wenn hier über Sozialraum gesprochen wird, dann immer ausgehend eines raumsoziologischen Verständnisses, das Raum als relational versteht. Forschung zu Sozialräumen, die von einem relationalen Raumverständnis ausgeht, bewegt sich immer ein Stück weit in der Ambivalenz, zwar ein theoretisches Verständnis von Sozialraum als sich diskursiv vollziehende Praxis zugrunde zu legen, dieses allerdings auf lokale Orte zu beziehen, die sich vor allem über ihre Grenzen definieren (beispielsweise über Stadtgrenzen eines 'Sozialraums). Besonders herausgefordert wird dieses Verhältnis dann, wenn Städte und Orte durch beispielsweise Eingemeindungen in gewisser Weise künstlich zusammengeführt werden, sich das soziale Leben allerdings (noch) nicht auf dieser Ebene vollzieht. Es stellt sich die Frage, inwiefern solche Gebilde sowohl aus praxistheoretischer als auch aus geographischer Sicht noch als >Sozialraum < bezeichnet werden können.

\subsection{Raumtheoretische Grundlagen}

Einer Annäherung an das hier zugrunde gelegte Verständnis von Sozialraum gehen theoretische Vorarbeiten voraus, in denen raumsoziologische Grundlagen entfaltet werden. Hierbei wird sich an einem Raumverständnis von Löw (2001) orientiert, die unter Raum »eine relationale (An)Ordnung sozialer Güter und Menschen (Lebewesen) an Orten « (Löw 2001, S. 224; einen Überblick gibt Trescher 2017f, S. 17ff) versteht. Der Terminus (An)Ordnung verweist dabei darauf, dass Raum sowohl strukturierende (>ordnendes) als auch handlungsorientierte (ranordnendes) Dimensionen hat (Löw 2001, S. 166), woraus resultiert, dass sich Raum »in der Wechselwirkung zwischen Handeln und Strukturen « (Löw 2001, S. 191) konstituiert. Hiermit wiederum geht eine "Dualität von Raum « (Löw 2001, S. 226) einher. Das bedeutet, dass Raum zwar je bestimmte Handlungen ermöglicht, gleichzeitig jedoch Handlungsmöglichkeiten begrenzt (Löw 2001, S. 226). Raum ist in diesem Verständnis weder absoluter (Behälter-)Raum noch relativer (Zeit-)Raum, sondern relational. »[W]ir leben nicht in einer Leere, die wir mit Menschen und Dingen füllen könnten. [...] Wir leben vielmehr innerhalb einer Menge von 
Relationen, die Orte definieren, welche sich nicht aufeinander reduzieren und einander absolut nicht überlagern lassen « (Foucault 2018, S. 319f; siehe auch Schroer 2008, S. 146ff; Füller und Michel 2012, S. 9ff; Schreiber 2009). Relationaler Raum wird in Aushandlungsdiskursen hervorgebracht und konstituiert seinerseits Diskurs ${ }^{5}$. Die konstituierenden Raumpraxen vollziehen sich innerhalb unscharfer Grenzen, die als diskursive Ordnungen Raum hervorbringen und seine fortwährende Re-Konstitution tragen. Raum ist demnach eine Praxis, die der Ordnung des Diskurses unterworfen ist, anhand derer über Praxen des Ein- und Ausschlusses bestimmt wird, wer wie am Diskurs teilhat und wer nicht (Foucault 2003). Jene Praxen »sind immer auch räumlich organisierte Ausschlüsse« (Schroer 2008, S. 150; siehe auch Weisser 2010, S. 7). Die Relationalität von Raum, also das Hervorbringen von Raum in Aushandlungsdiskursen, vollzieht sich in Prozessen des Spacings und der Syntheseleistung (Löw 2001, S. 158ff). Spacing meint dabei vor allem das Positionieren von Subjekten und Artefakten sowie die Bewegungen zwischen den je bestimmten Platzierungen (Löw 2001, S. 158f). Die Syntheseleistung dagegen umfasst die Fähigkeit des Subjekts, (einzelne) Menschen und Orte miteinander zu verknüpfen (Löw 2001, S. 159). »Welt« wird insofern »als ein Netz, dessen Stränge sich kreuzen und Punkte verbinden« (Foucault 2018, S. 317), verstanden. Durch die Synthese des handelnden Subjekts wird jenes Netz geknüpft und Raum wird objektiviert, das heißt, »daß er - ein Produkt menschlicher Tätigkeit - als gegenständlich erlebt wird« (Löw 2001, S. 164). Daraus folgt die Frage nach dem Verhältnis von Subjekt und Raum.

\subsection{Raum und Subjekt als Praxen der wechselseitigen Hervorbringung}

»Menschen machen Räume« (Bilstein 2018, S. 29), Räume smachen aber ebenso Menschen und insofern kann gesagt werden, dass sich Raum und Subjekt wechselseitig hervorbringen (Trescher 2018a, S. 45ff). Raum wird durch Subjekte in Aneignungspraxen konstituiert (Hüllemann et al. 2016, S. 11), wobei die Frage im Vordergrund steht, als was Raum angeeignet - und in diesem Prozess hervorgebracht - wird. Aneignung kann hierbei als Praxis des Subjekts verstanden werden, sich zu etwas ins Verhältnis zu setzen (Trescher 2018a, S. 46). Aneignung vollzieht sich in sozialen Handlungen

5 Zur Diskursivität von Raum siehe auch Nugel 2017, S. 273. 
und ist zwischen (aus-)handelnden Subjekten situiert, denn erst »in Aushandlungsprozessen mit anderen Handelnden« (Löw 2001, S. 228; siehe auch Deinet 2010, S. 38) kann sich das Subjekt Raum (in oben genannten Prozessen des Spacings und der Syntheseleistung) aneignen. Das bedeutet also auch, dass sich Raumkonstitution in Praxen der »diskursiven Selbstverständigung einer Kultur« (Bilstein 2018, S. 33) vollzieht. Dabei kann Raum je unterschiedlich angeeignet werden, abhängig davon, welche Ideen, Handlungen und Emotionen das konstituierende Subjekt damit verknüpft beziehungsweise synthetisiert (siehe dazu Trescher 2018a, S. 46). Entgegen einiger, insbesondere routinemäßiger, Verständnisse von Aneignung ist es nicht möglich, sich Raum nicht anzueignen. Selbst eine Aneignung, die aus einigen Verstehenszugängen heraus als gescheitert bezeichnet würde, ist eine sich vollziehende Aneignungspraxis, die Raum und Subjekt wechselseitig in je bestimmter Art und Weise hervorbringt. Beispielsweise ist es möglich, dass sich viele Menschen mit Behinderung Raum primär als »Territorium der Anderen « (Trescher und Hauck 2017) aneignen, was nicht bedeutet, dass sie sich Raum nicht aneignen können, sondern dass sie sich Raum vor allem als etwas aneignen, das für sie Teilhabebarrieren errichtet. Ausgeschlossen von der Aneignung von Raum sind sie allerdings nicht. Das Subjekt wird in diesen Raumaneignungspraxen ebenfalls in je bestimmter Art und Weise hervorgebracht (und bringt sich selbst hervor) - zum Beispiel als ausgeschlossen von routinemäßigen Praxen und insofern je situativ sbehindert Subjekt stehen also, das wird hier deutlich, in einem gegenseitigen Hervorbringungsverhältnis, in dem Raum einerseits durch Subjekte konstituiert wird und andererseits selbst Subjekte als je bestimmte hervorbringt (siehe auch Berdelmann und Reh 2015, S. 190f). Einer Veränderung des Raumverständnisses folgt in diesem Sinne also auch eine veränderte Sichtweise auf die Konstitution des Subjekts ${ }^{6}$. Raum und Subjekt sind folglich kontingente diskursive Praxen.

\subsection{Räume und Orte}

Bei der Betrachtung von Raum stellt sich die Frage nach dessen Verhältnis zu Orten. Orte sind »meist geographisch markiert « (Löw 2001, S. 199), können jedoch auch virtuell oder symbolisch sein (Löw 2001, S. 198ff; Stoetzer 2014,

\footnotetext{
6 In Bezug auf das Private stellen dies Beyvers et al. (2017, S. 5) fest.
} 
S. 21). Raum wird, wie oben bereits ausgeführt, in Prozessen des Spacings und der Syntheseleistung hervorgebracht. Für jenes Spacing, das auch als Platzierung beschrieben wird, sind Orte unerlässlich, denn sie bereiten die Positionen, »an denen plaziert werden kann« (Löw 2001, S. 198). Dabei ist es wichtig hervorzuheben, dass Orte auch unabhängig der jeweiligen Platzierung existieren und dass sich an Orten je unterschiedliche Platzierungen vollziehen können (Löw 2001, S. 91). Das bedeutet, dass es zu einer »Überlagerung unterschiedlicher Räume an einem Ort « (Stoetzer 2014, S. 16) kommen kann. Jene Orte sind »konkret benennbar und einzigartig« (Löw 2001, S. 199). Sie geben darüber hinaus dem raumkonstituierenden Subjekt eine Position vor, an dem es >Körper ist<, sich also als "Synthetisierende[s] an einem Ort befindet« (Löw 2001, S. 202). Das Verhältnis von Raum und Ort ist ebenso wie das Verhältnis von Raum und Subjekt wechselseitig: »Die Konstitution von Raum bringt [...] systematisch auch Orte hervor, so wie Orte die Entstehung von Raum erst möglich machen« (Löw 2001, S. 198). Für die hier erfolgte, auch diskurstheoretische Annäherung an ein Verständnis von Raum spielt das Verhältnis von Raum und Ort insofern eine bedeutsame Rolle, dass die Materialität von Raum nicht negiert, sondern vielmehr ebenfalls als diskursiv hervorgebracht verstanden wird. Hierbei wird sich an einem Diskursverständnis orientiert, das davon ausgeht, dass alles von Diskurs ist - es gibt nichts Außerdiskursives (siehe Laclau und Mouffe 2006, S. 143ff).

\subsection{Raum und Barrierefreiheit}

Gegenwärtige Verständnisse von Barrieren respektive Barrierefreiheit sind zumeist an ein Verständnis von Raum als absoluten Behälterraum geknüpft. Barrieren werden infolgedessen oft primär physisch gedacht, ausschließlich auf dieser Ebene problematisiert und schließlich zumeist lediglich in physischer Hinsicht abgebaut. Diese Engführung von Barrierefreiheit findet sich nicht nur in der Handlungspraxis, sondern auch und insbesondere in Forschungszusammenhängen (Trescher 2018c, S. 34ff). Beispielsweise problematisiert Wansing (2016) zwar zutreffend: »Die Existenz von physikalischen Barrieren in erheblichem Maße kann [...] als materialisierte Symbolik von Unterdrückung, Diskriminierung und sozialer Ungleichheit von Bürgerinnen und Bürgern mit Beeinträchtigungen gedeutet werden « (Wansing 2016, S. 260). Dennoch greift sie damit etwas zu kurz, denn Barrieren sind weit über physikalische Hindernisse (im absoluten Raum) hinaus errichtet und 
führen zu Ausschluss an Praxen der Mehrheitsgesellschaft. Barrieren entstehen je situativ und sind sozial, emotional, physisch etc. erlebbar (Trescher 2020a). Infolgedessen stoßen nicht nur Personen an Barrieren, die bislang als >behindert bezeichnet werden, sondern alle Personen, deren Teilhabe je situativ eingeschränkt wird (siehe Kapitel 4). Barrieren stellen eine Aushandlungspraxis dar - ebenso wie eine (häufig moralisch aufgeladene) sogenannte Barrierefreiheit. Problematisiert werden kann zudem, dass das weitläufig bekannte Symbol, das oft zur Kennzeichnung von >barrierefreien Zugängen verwendet wird - ein sogenanntes Rollstuhlpiktogramm - zu einer Trennung beiträgt zwischen »those who are deemed `normak (needing no access accommodations) from those who are not (Ben-Moshe und Powell 2007, S. 503). Jenes Piktogramm wird dadurch zu einem Symbol und einer Praxis der Unterdrückung anhand der eine dichotome Unterteilung in zwei Gruppen (>beeinträchtigt « und `nicht beeinträchtigt) reproduziert und verfestigt wird. Behinderung als Status (negativer) Andersartigkeit wird auf diese Weise manifestiert und es kann beispielsweise »die Nutzung eines Behindertenparkplatzes buchstäblich zu einem Stigma werden « (Dederich 2012, S. 111; siehe auch Goffman 1975). Auf der anderen Seite wird anhand der Praxen, die sich rund um das Thema Barrierefreiheit vollziehen, bestimmten Personen Zugang ermöglicht, wo er ihnen zuvor gegebenenfalls verschlossen war. Es zeigt sich also, dass Barrierefreiheit unbedingt mehrdimensional abwägend betrachtet werden muss. Dazu gehört, sich mit den rechtlichen und begrifflichen Ambivalenzen von Barrierefreiheit zu beschäftigen. So ist Barrierefreiheit zwar in einigen Gesetzen und Vorgaben (mehr oder weniger bindend) rechtlich festgeschrieben (unter anderem im Grundgesetz, im Allgemeinen Gleichbehandlungsgesetz oder in der UN-Konvention über die Rechte von Menschen mit Behinderungen). Dennoch kann eingeworfen werden, dass Gesetze und Vorgaben zwar, gegebenenfalls sogar einklagbare, Grundlagen für eine Teilhabe an der Mehrheitsgesellschaft schaffen, allerdings selbst keine Praxen verändern und damit höchstens transitiv zu einem Abbau von Barrieren beitragen (Trescher 2018c, S. 20; siehe Kapitel 30). "Das Gesetz ist das Ergebnis von Auseinandersetzungen innerhalb eines Kräfteverhältnisses, das Gewinner und Verlierer schafft. Es garantiert nichts, solange sich Menschen nicht dafür einsetzen« (Weisser 2010, S. 8). Insbesondere bezüglich der UN-Konvention über die Rechte von Menschen mit Behinderungen muss problematisiert werden, dass diese nur bedingt einklagbar ist, was ihre Reichweite weitergehend einschränkt. Ganz grund- 
sätzlich wird schließlich klar, dass Barrieren »bevor sie abgebaut werden können, wahrgenommen und verstanden werden [müssen]« (Tervooren und Weber 2012a, S. 11). Es bedarf also einer empirischen Erhebung von Barrieren und durch sie entstehende Ausschlusspraxen. Auch auf begrifflicher Ebene kann >Barrierefreiheit` problematisiert werden, ist der Begriff doch untrennbar mit Barrieren verbunden und ohne das Vorhandensein dieser nicht denk- und handelbar. Barrierefreiheit bezeichnet in diesem Sinne weniger die Abwesenheit von Barrieren als den Willen, Barrieren abzubauen »[d]ie Freiheit von Barrieren setzt also ihre Existenz voraus« (Trescher 2018c, S. 16). Obwohl jene Ambivalenz nicht außer Acht gelassen werden darf, war es dennoch notwendig, in der forschungspraktischen Ausgestaltung dieser Studie ein operationalisierbares Verständnis von Barrierefreiheit zugrunde zu legen. Dieses ist in Kapitel 11.1 entfaltet.

\subsection{Raum und Inklusion}

Vor dem Hintergrund einer Idee von Inklusion stellt sich die Frage, wie manifeste ebenso wie latente Diskursteilhabebarrieren abgebaut werden können, die sich in und durch Raum vollziehen und als Behinderungspraxen wirksam werden, indem sie Teilhabemöglichkeiten einschränken. Inklusion bedarf folglich einer Idee, Diskursteilhabemöglichkeiten zu eröffnen. Eine Herangehensweise ist dabei, wie oben beschrieben (siehe Kapitel 4.2), Inklusion als dekonstruktive Praxis zu verstehen, die sich in Prozessen der (An)Ordnung durch handlungsmächtige Subjekte vollzieht (Trescher und Hauck 2017). Inklusion heißt dabei nicht nur, Zugänge zu Diskursen zu eröffnen, indem Teilhabebarrieren abgebaut werden, sondern auch Personen als handlungsmächtig zu subjektivieren, Diskurs aktiv zu gestalten (Trescher 2018b). Dazu ist es nicht ausreichend, sicherzustellen, dass Personen, die je situativ als behindert hervorgebracht werden, einen Ort physisch erreichen können. Mindestens ebenso wichtig ist, welche räumlichen Aneignungsmöglichkeiten der Ort potenziell eröffnet und inwiefern die jeweilige Person sich diesen als teilhabend aneignen kann. Dazu braucht es, über den Zugang und die Subjektivierung als handlungsmächtig hinaus, Gefühle von Verbundenheit und Zugehörigkeit, was deutlich macht, dass Teilhabe nicht ausschließlich technisch generiert werden kann, sondern insbesondere im sozialen Miteinander entsteht (Trescher und Hauck 2020). 


\section{Projekt und Methode}

\section{Sozialraumanalysen als Forschungsmethode}

Kongruent $\mathrm{zu}$ differierenden Verständnissen des Begriffs 'Sozialraum finden sich einige unterschiedliche inhaltliche und method(olog)ische Herangehensweisen zur empirischen Erfassung und Beschreibung von Sozialräumen. Dies liegt insbesondere darin begründet, dass das jeweilige Raumverständnis und die forschungspraktische Herangehensweise an den Gegenstand miteinander verknüpft und voneinander abhängig sind (Riege 2007, S. 380). Sozialraumanalysen beziehungsweise umfassende Untersuchungen von Sozialräumen werden oftmals mit dem Ziel durchgeführt, »Belege für [...] Probleme vvor Ort‘, in Städten und Gemeinden aufzuzeigen - und Anreize für ein Nachdenken über den Zustand der Mentalitäten der eigenen Gemeinde oder der Stadtgesellschaft zu setzen« (Heitmeyer 2013, S. 9). Demgegenüber geht es sozialraumanalytischen Verfahren, die eher sozialarbeiterisch ausgerichtet sind, zumeist darum, Aneignungspraxen im jeweiligen Sozialraum zu untersuchen, um »das Erleben von Menschen in ihrem Lebensraum zu verstehen und (hierauf aufbauend) Schlußfolgerungen für die Art und Weise der Lebensbewältigung ziehen zu können« (Wendt 2017, S. 146). Dabei werden Sozialraumanalysen oftmals von der Annahme getragen, »dass bestimmte Merkmale des unmittelbaren Lebensraums einen nachweisbaren Einfluss auf die individuellen Sichtweisen, Wahrnehmungen und Erfahrungen von Personen haben « (Heitmeyer und Grau 2013, S. 12). Im Folgenden wird herausgearbeitet, wie unterschiedliche methodische Herangehensweisen an die Analyse von Sozialräumen begründet werden. Im Anschluss wird auf die Studie »Inklusion. Zur Dekonstruktion von Diskursteilhabebarrieren im Kontext von Freizeit und Behinderung« (Trescher 2015b) Bezug genommen, deren sozialraumanalytisches Forschungsdesign Vorbild für die hiesige Studie ist. 


\subsection{Methodendesigns}

Die methodischen Herangehensweisen an die Untersuchung von Sozialräumen sind, wie oben bereits beschrieben, zahlreich und je unterschiedlich ausgestaltet. Mit Boettner (2009) können dabei zwei Ausrichtungen sozialraumanalytischer Forschungsmethoden unterschieden werden: Sozialraumanalysen als sozialstatistische Verfahren, die vor allem in der soziologischen Stadtforschung zu finden sind, und eher sozialarbeiterisch geprägte Herangehensweisen, in denen Sozialraumanalysen der handlungspraktischen Erfassung der Lebenswelt der AdressatInnen dienen (Boettner 2009, S. 262f). Riege (2007) differenziert demgegenüber drei unterschiedliche Forschungszugänge, nämlich sozioökonomische Studien, Untersuchungen zur Sozialraumnutzung und Untersuchungen zum Sozialraumerleben (Riege 2007, S. 380ff) ${ }^{1}$. Unabhängig ihrer vorgängigen Einteilung finden sich in der Forschungslandschaft zahlreiche Studien, die sich als Sozialraumanalysen bezeichnen und die methodisch je unterschiedlich verfahren. Quantitativ verfahrende Sozialraumanalysen untersuchen beispielsweise die Einstellung der Menschen vor Ort zu einem bestimmten Gegenstand (u.a. Heitmeyer und Grau 2013, S. 14f) oder erfolgen als sozialstatistische Erhebungen der strukturellen Gegebenheiten im Sozialraum (Eifler et al. 2017, S. 5f; Landeshauptstadt Mainz 2018, S. If; Meier 2013, S. 9f), die insbesondere darauf abzielen, Typologien $\mathrm{zu}$ generieren und Sozialräume untereinander vergleichbar zu machen (Riege 2007, S. 381). Ein Beispiel für quantitativ ausgerichtete Sozialraumanalysen, die primär nach sozialstatistischen Methoden verfahren, ist die Studie zur »Fortschreibung der Analyse der sozialräumlichen Struktur der Landeshauptstadt Mainz« (2018). In dieser wurden Sozialraumanalysen mit der Begründung durchgeführt, sie seien »wichtige Hilfsmittel für die kommunale Sozialplanung und zielgerichtete Entwicklung der sozialen Infrastruktur« (Ebling und Lensch 2018). Indikatorenbereiche, die mit Hilfe quantitativer Methoden untersucht wurden, waren unter anderem: die Flächennutzung, demographische Angaben, die Haushalts- und Familienstrukturen oder auch strukturelle Überblicke über Angebote und Nutzungsverhalten der Kinder- und Jugendhilfe. Ziel dabei war, dass »[k]ommunale Handlungsfelder [...] sichtbar und räumliche

1 Einen Überblick zum Repertoire forschungsmethodischer Herangehensweisen an sozialraumanalytische Untersuchungen findet sich in Riege und Schubert (2005, S. 43ff). 
Handlungsschwerpunkte identifiziert und überprüft« (Landeshauptstadt Mainz 2018, S. 1) werden. Ebenso zahlreich und vielfältig sind eher qualitativ ausgerichtete Verfahren, die sich unter anderem ethnographischer Methoden bedienen, wie beispielsweise Begehungen oder teilnehmenden Beobachtungen (Wendt 2017, S. 146f; Kurtenbach 2017, S. 120ff). Qualitative Studien ermöglichen eine "Gewinnung der sozialräumlichen Perspektiven« (Riege 2007, S. 382), anhand der subjektive "Erlebnisse, Erfahrungen und Wahrnehmungen « (Riege 2007, S. 383) der Analyse zugänglich gemacht werden können. Ein Beispiel dafür ist eine Studie zur Stadtteilanalyse, die von Geiling (2005) vorgelegt wurde und in der »die sozialen Beziehungen und positionsbedingten Probleme von sozialen Gruppen" (Geiling 2005, S. 223) im Vordergrund stehen. Methodisch operieren die Forschenden anhand von Stadtteilbegehungen, Rekonstruktionen der Siedlungsgeschichte, der Analyse sozialstatistischer Daten sowie Interviews mit ExpertInnen und EinwohnerInnen (Geiling 2005, S. 225) ${ }^{2}$. Ein anderes Beispiel für eine Stadtteilanalyse ist die Studie Kurtenbachs (2017), die die Frage verfolgt, wie eine »umweltvermittelte[...] Normanpassung innerhalb eines Wohngebiets" (Kurtenbach 2017, S. 23) entsteht. Methodisch wurde diese Frage anhand der Analyse kleinteiliger Daten amtlicher Statistiken, ExpertInneninterviews und einer intensiven Feldforschungsphase untersucht (Kurtenbach 2017, S. 23f). Auch wenn die hiesige Darstellung nahelegt, Sozialraumanalysen würden entweder quantitativ oder qualitativ durchgeführt, so handelt es sich doch zumeist um mehrdimensionale Forschungsdesigns (Riege 2007, S. 382). Über einen mehrdimensionalen Forschungszugang können unterschiedliche Fragestellungen am sinnhaftesten miteinander verknüpft werden. Ein sehr bekanntes Beispiel für eine mehrdimensional verfahrende Sozialraumanalyse ist die Studie zur Lebenssituation der »Arbeitslosen von Marienthal (Jahoda et al. 2018) aus den 1930er Jahren, in der sich die Forschenden zum Ziel setzten, »die Verwendung exakten Zahlenmaterials' mit dem Sich Einleben in die Situation« (Lazarsfeld 2005, S. 73) zu verknüpfen. Dabei überwiegt dennoch eine quantifizierende Herangehensweise (Lazarsfeld 2005, S. 73f). Unabhängig ihrer methodischen Ausrichtung zielen sozialraumanalytische Untersuchungen zumeist darauf ab, Veränderungen im Sozialraum empiriegeleitet anzustoßen. Insofern gibt es beispielsweise sogenannte interventionistische Ansätze (Heitmeyer und Grau 2013, S. 15;

2 Ähnlich verfahren Karhoff und Riege (2005) sowie von Seggern und Tessin (2005). 
Landeshauptstadt Mainz 2018, S. If; 313ff; Meier 2013, S. 103ff), die sich teils als »[a]ktivierende Sozialforschung" (Heitmeyer und Grau 2013, S. 31) verstehen. Andere Forschende entwickeln demgegenüber Handlungsleitlinien dafür, wie Sozialräume so verändert werden können, dass bestimmte Personen mehr Teilhabemöglichkeiten haben (Rohrmann et al. 2010).

\subsection{Betrachtung einer Studie: Sozialraumanalysen im Kontext Freizeit}

Beispielhaft für eine mehrdimensional verfahrende Sozialraumanalyse kann die Studie "Inklusion. Zur Dekonstruktion von Diskursteilhabebarrieren im Kontext von Freizeit und Behinderung« (Trescher 2015b) genannt werden. In der Studie wurde der Frage auf den Grund gegangen, welche Differenzen es zwischen der Ausgestaltung routinemäßiger Freizeitgestaltung von Menschen ohne Behinderung und institutionalisiert lebenden Menschen mit geistiger Behinderung gibt, welche Bedürfnisse vorhanden sind und inwiefern diese gegenwärtig realisiert werden können. Zentral war zudem die Frage, welche Möglichkeiten für Menschen mit geistiger Behinderung zur Teilhabe an Freizeitangeboten der Mehrheitsgesellschaft bestehen. Es wurde ein mehrdimensionales Forschungssetting entwickelt, das auf drei verschiedenen Ebenen operationalisiert wurde: auf Ebene der Lebenswelt, auf der Subjektebene und der Institutionsebene. Die Wahl des Untersuchungsortes fiel auf die Stadt Frankfurt a.M., da davon ausgegangen wurde, dass dort eine ausreichend breite Materialgrundlage erhoben werden konnte. Um auch den Aspekt der eher ländlichen Umgebung zu berücksichtigen, wurde eine Vergleichsstudie in einer Kleinstadt mit circa 25.000 EinwohnerInnen durchgeführt. Auf Ebene der Lebenswelt war es das Ziel, die Fragen zu beantworten, wie stark Menschen mit (geistiger) Behinderung gegenwärtig in Freizeitaktivitäten der routinemäßigen Lebenspraxis eingebunden sind und inwiefern Freizeitvereine und -gruppen sich als aufgeschlossen erweisen gegenüber einer Teilnahme von Menschen mit (geistiger) Behinderung beziehungsweise welche Teilhabebarrieren vorhanden oder zu erwarten sind. Im Rahmen einer Voruntersuchung (Empirische Phase I - Felderöffnung) wurde dazu felderöffnend gesammelt, welche Freizeitaktivitäten es in der Mehrheitsgesellschaft überhaupt gibt (Trescher 2015b, S. 49ff, 2015c, S. 39f, 2016a, S. 100f). Daraufhin wurden 324 Leitfadeninterviews mit VertreterInnen von Freizeitvereinen und -gruppen telefonisch erhoben, die wiederum 
mittels Verfahren Qualitativer Inhaltsanalyse ausgewertet wurden (Empirische Phase II - Subsumtion) (Trescher 2015b, S. 55ff). Auf der Subjektebene wurden sechs Interviews mit Menschen mit geistiger Behinderung aus je verschiedenen Wohnkontexten geführt und anhand der rekonstruktiven Verfahren der Objektiven Hermeneutik ausgewertet (u.a. Oevermann 2002; siehe zur Anwendung Trescher 2015b, S. 145ff), um latente Barrieren, wie Vorbehalte und Ängste, offenlegen zu können (Trescher 2015b, S. 189ff). Auf der Institutionsebene wurden vier gesprächsförmige Interviews mit MitarbeiterInnen von denjenigen Einrichtungen geführt, in denen die zuvor interviewten Menschen mit geistiger Behinderung wohnen (Trescher 2015b, S. 215ff). Die interviewten MitarbeiterInnen sind in den Einrichtungen unter anderem auch für die Freizeitgestaltung zuständig (Empirische Phase III Rekonstruktion). Insgesamt betrachtet kann auf Grundlage der erarbeiteten Ergebnisse festgehalten werden, dass aufseiten der Mehrheitsgesellschaft zwar Ängste und Vorbehalte bezüglich der Teilhabe von Menschen mit geistiger Behinderung an routinemäßigen Freizeitaktivitäten identifiziert werden konnten, demgegenüber aber auch eine große Bereitschaft ausgemacht wurde, sich auf deren Teilnahme einzulassen und Teilhabemöglichkeiten zu eröffnen. Weiterhin stellte sich heraus, dass der Ausschluss von mehrheitsgesellschaftlichen Freizeitaktivitäten primär über die institutionalisierten Strukturen erfolgt, in denen Menschen mit geistiger Behinderung zumeist leben. Deutlich wurde auch, dass die Ablehnung respektive Akzeptanz gegenüber der Teilhabe von Menschen mit geistiger Behinderung in Relation zu einem lebensgeschichtlichen Kontakt der Interviewpersonen mit der Bezugsgruppe steht (Trescher 2015b, S. 118f, 2016a, S. 107ff, 2016b, S. 37f). Nehmen also Menschen mit geistiger Behinderung an Freizeitaktivitäten teil, wirkt sich dies tendenziell positiv auf die Bereitschaft der Freizeitvereine und -gruppen aus, jenen Personen Teilhabe zu ermöglichen. »In diesem Sinne kann begründet davon ausgegangenen werden, dass ein längerfristiger zwischenmenschlicher Kontakt zu Menschen mit geistiger Behinderung auch die je subjektive Konstruktion von 'geistiger Behinderung، verändert und die Bereitschaft erhöht, diese an den eigenen (in diesem Fall freizeitlichen) Lebenspraxen teilhaben zu lassen« (Trescher 2015b, S. 119). Es kann also davon ausgegangen werden, dass sich die sogenannte Kontakthypothese bestätigt, die, eingeführt von Allport (1954), besagt, dass Vorurteile gegenüber je bestimmten Personen oder Gruppen durch regelmäßigen und 
qualitativ wertvollen lebenspraktischen Kontakt abgebaut werden können (Allport 1954, S. 281; siehe auch Cloerkes 2007, S. 145ff; Dollase 2001) ${ }^{3}$.

\section{Beschreibung des Projekts "Kommune Inklusiv"}

Das Projekt »Kommune Inklusiv« wurde im Jahr 2017 von der Aktion Mensch e.V. initiiert. Über den Zeitraum von fünf Jahren werden fünf Städte und Gemeinden in Deutschland - Erlangen, Rostock, Schneverdingen, Schwäbisch Gmünd und die Verbandsgemeinde Nieder-Olm - finanziell unterstützt und inhaltlich begleitet auf dem »Weg zu einer inklusiven Gesellschaft« (Zibell 2018, S. 93). Diese fünf sogenannten Sozialräume wurden unter zahlreichen Bewerbungen anderer Städte und Gemeinden in einem mehrschrittigen Verfahren ausgewählt und sollen nun in einer Art Pilotprojekt Vorreiter dafür werden, einen Sozialraum im Hinblick auf >Inklusion` zu verändern. Im Fokus stehen dabei sogenannte Inklusionsnetzwerke, in denen unterschiedliche Institutionen zusammenkommen und am gemeinsamen Gegenstand der sinklusiven Weiterentwicklung arbeiten und die von sogenannten Netzwerk- beziehungsweise SozialraumkoordinatorInnen organisiert und geleitet werden (Zibell 2018, S. 93). Die KoordinatorInnen des Projekts entwickeln, organisieren und/oder begleiten die Maßnahmen in den Sozialräumen. Sie werden unmittelbar von Aktion Mensch e.V. adressiert, unter anderem in regelmäßig stattfindenden Treffen, in denen der Prozess reflektiert und Einblick in das Vorgehen in den anderen Sozialräumen genommen werden soll. Die KoordinatorInnen sind für die wissenschaftliche Begleitforschung zudem die Schnittstellen zu den Sozialräumen. Die Forschenden stehen im stetigen Austausch mit den KoordinatorInnen, um wechselseitig Informationen und Erkenntnisse weiterzugeben. Von den Mitteln, die den Sozialräumen respektive dem Netzwerk zur Verfügung gestellt werden, werden Maßnahmen finanziert, die dazu beitragen sollen, das Ziel eines sinklusiven Sozialraums zu erreichen. Beispiele für Maßnahmen sind unter anderem

3 Allport (1954) stellt fest: »Prejudice (unless deeply rooted in the character structure of the individual) may be reduced by equal status contact between majority and minority groups in the pursuit of common goals. The effect is greatly enhanced if this contact is sanctioned by institutional supports (i.e., by law, custom or local atmosphere), and provided it is of a sort that leads to the perception of common interests and common humanity between members of the two groups« (Allport 1954, S. 281). 
die Erstellung eines Leitfadens, in dem Informationen für Arbeitgebende bezüglich der Beschäftigung von Menschen mit Behinderung und/oder Menschen mit Fluchtmigrationshintergrund zusammengefasst sind, oder auch ein regelmäßiges Treffen für Arbeitgebende, auf dem sie sich untereinander und mit geladenen ExpertInnen zum Thema Arbeit und Inklusion austauschen können. In einem anderen Sozialraum werden Menschen mit bestimmten Unterstützungsbedarfen vorübergehend Freizeitassistenzen bereitgestellt, die sie beim Erproben neuer Freizeitaktivitäten begleiten. Ein weiteres Beispiel ist ein sogenannter inklusiver Stadtlauf, in dem Menschen mit und ohne Beeinträchtigungen miteinander wetteifern und dadurch zu mehr Sichtbarkeit für diverse Bedarfe im Sozialraum beitragen. Die Sozialräume haben jeweils unterschiedliche AdressatInnengruppen definiert, an die sich die Maßnahmen primär richten und die als besonders von Ausschluss gefährdet wahrgenommen werden. Jene Gruppen wurden durch die KoordinatorInnen gemeinsam mit Aktion Mensch e.V. sowie im Hinblick auf die vor Projektstart bereits vorhandenen Strukturen sorgfältig ausgewählt. AdressatInnengruppen sind dabei beispielsweise Menschen mit (geistiger) Behinderung, SeniorInnen (mit Behinderung), Menschen mit (Flucht-)Migrationshintergrund oder auch Kinder und Jugendliche (mit Behinderung). Das Projekt hat Modellcharakter und zielt darauf ab, »eine Schablone für Projekte und Netzwerkvorbilder rund um Inklusion « (Zibell 2018, S. 94) zu entwerfen, anhand derer andere Sozialräume Veränderungen vorantreiben können. Bereits während der Laufzeit des Projekts wird deshalb immer wieder zu Symposien und ähnlichen Veranstaltungen geladen, an denen nicht nur die KoordinatorInnen der fünf derzeit geförderten Sozialräume teilnehmen, sondern auch andere VertreterInnen von Städten und Kommunen, die an einer inklusiven Weiterentwicklung des von ihnen repräsentierten Sozialraums interessiert sind und sich vom Modellprojekt wegweisende Ideen und Handlungsanstöße erhoffen. Eine wichtige Stütze des Modellprojekts ist dabei die wissenschaftliche Begleitforschung, die den Prozess im Gesamten untersucht und immer wieder Rückmeldung zu aktuellen Forschungsergebnissen gibt, anhand derer Verantwortliche in Städten und Kommunen die Prozesse vor Ort steuern und gegebenenfalls adaptieren können. Beispielsweise ergaben unter anderem die ethnographischen Sozialraumbegehungen, dass bewusstseinsbildende Maßnahmen dabei unterstützen könnten, die EinwohnerInnen der jeweiligen Sozialräume für diverse Bedarfe zu sensibilisieren, die bislang oftmals wenig Be- 
achtung finden (siehe Kapitel 13). Verantwortliche und Handelnde vor Ort können auf ein solches Forschungsergebnis reagieren und entsprechende Angebote konzipieren, die, im hiesigen Fall, zu mehr Bewusstsein bei der Mehrheitsbevölkerung beitragen. Die Sozialräume, die das Projekt »Kommune Inklusiv« umsetzen, respektive die KoordinatorInnen und Beteiligten werden so zu MultiplikatorInnen von sowohl Praxiserfahrungen als auch Forschungsergebnissen. Darüber hinaus wird abgebildet, ob und inwiefern sich die untersuchten Sozialräume über den Projektzeitraum verändern und mit ihnen die Teilhabemöglichkeiten von Menschen, die von Ausschluss bedroht oder betroffen sind. Die Ausgestaltung der Studie wird im Folgenden beschrieben.

\section{Beschreibung der Gesamtstudie}

Das Projekt »Kommune Inklusiv« wird durch die Philipps-Universität Marburg (Prof. Dr. Hendrik Trescher) und die Goethe-Universität Frankfurt (Prof. Dr. Dieter Katzenbach) auf drei Ebenen beforscht ${ }^{4}$.

\subsection{Ebene 1- Maßnahmen}

Auf Ebene 1 werden die Maßnahmen evaluiert, die die Verantwortlichen in den Sozialräumen entwickeln und umsetzen und die zum Ziel haben, die Lebenssituation der Menschen, die als AdressatInnen des Projekts definiert wurden (aber auch anderer Personen, die von Ausschluss bedroht oder betroffen sind) zu verbessern. Die Evaluation der Maßnahmen erfolgt entlang der übergeordneten Forschungsfrage:

Tabelle 1: Forschungsleitende Fragestellung der Maßnahmenevaluation

Ebene 1- Maßnahmen

Wie nehmen die Teilnehmenden die Maßnahmen wahr?

4 Leitung Ebenen 1 und 2: Prof. Dr. Hendrik Trescher, Leitung Ebene 3: Prof. Dr. Dieter Katzenbach 
Forschungspraktisch werden an die Teilnehmenden nach Besuch oder Abschluss einer Maßnahme eine Einladung zu einem Onlinesurvey gesendet, in dem in geschlossenen und teiloffenen Elementen nach der je individuellen Bewertung der Maßnahme, dem persönlichen Nutzen und Weiterentwicklungspotenzialen gefragt wird. Sollte eine Onlineerhebung nicht möglich sein, werden die Surveys in ausgedruckter Form zur Verfügung gestellt. Dies erfolgt beispielsweise dann, wenn den Teilnehmenden kein Internetanschluss zur Verfügung steht, wie es nach wie vor auf viele institutionalisiert lebende Menschen mit geistiger Behinderung zutrifft (Reichstein 2016, S. 82; Trescher 2017f, S. 133ff), oder wenn die Sozialform der Maßnahme eine andere Form der Erhebung begünstigt. Damit die Surveys so barrierearm wie möglich sind, wurden diese an die Bedarfe von unterschiedlichen AdressatInnen angepasst, wozu unter anderem eine Übersetzung in sogenannte Leichte Sprache gehört. Ein Jahr nach der ersten Befragung findet eine Follow-Up-Erhebung statt, um so die nachhaltige Wirksamkeit der Maßnahme $\mathrm{zu}$ untersuchen, insofern die jeweilige Person in die erneute Befragung (und damit verbundene Kontaktierung) eingewilligt hat. Die Auswertung der Surveys erfolgt anhand deskriptiv-statistischer und qualitativ-inhaltsanalytischer Verfahren.

\subsection{Ebene 2 - Sozialräume}

Auf Ebene 2 stehen die fünf geförderten Sozialräume in ihrer jeweiligen Ausgestaltung im Vordergrund. Fokussiert wird insbesondere, inwiefern sich dort Praxen der Teilhabe ebenso wie Praxen des Ausschlusses vollziehen. Ziel der Begleitforschung auf Ebene 2 ist, $\mathrm{zu}$ untersuchen, ob und inwiefern sich die initiierten Prozesse und Maßnahmen des Projekts auf die Strukturen der Sozialräume und das Leben im jeweiligen Sozialraum auswirken und ob Teilhabemöglichkeiten für Menschen geschaffen werden, die bislang in vielerlei Hinsicht von Ausschluss bedroht oder betroffen sind. Die übergeordnete Frage ist, ob und inwiefern sich eine Region über einen längeren Zeitraum hinweg entwickelt, wenn sinklusive < beziehungsweise auf das Eröffnen von Teilhabemöglichkeiten ausgerichtete Maßnahmen durchgeführt werden. Die Beforschung der Sozialräume auf Ebene 2 erfolgt sehr breit und verfolgt dabei die folgenden Fragestellungen: 
Tabelle 2: Forschungsleitende Fragestellungen der Untersuchung der Sozialräume

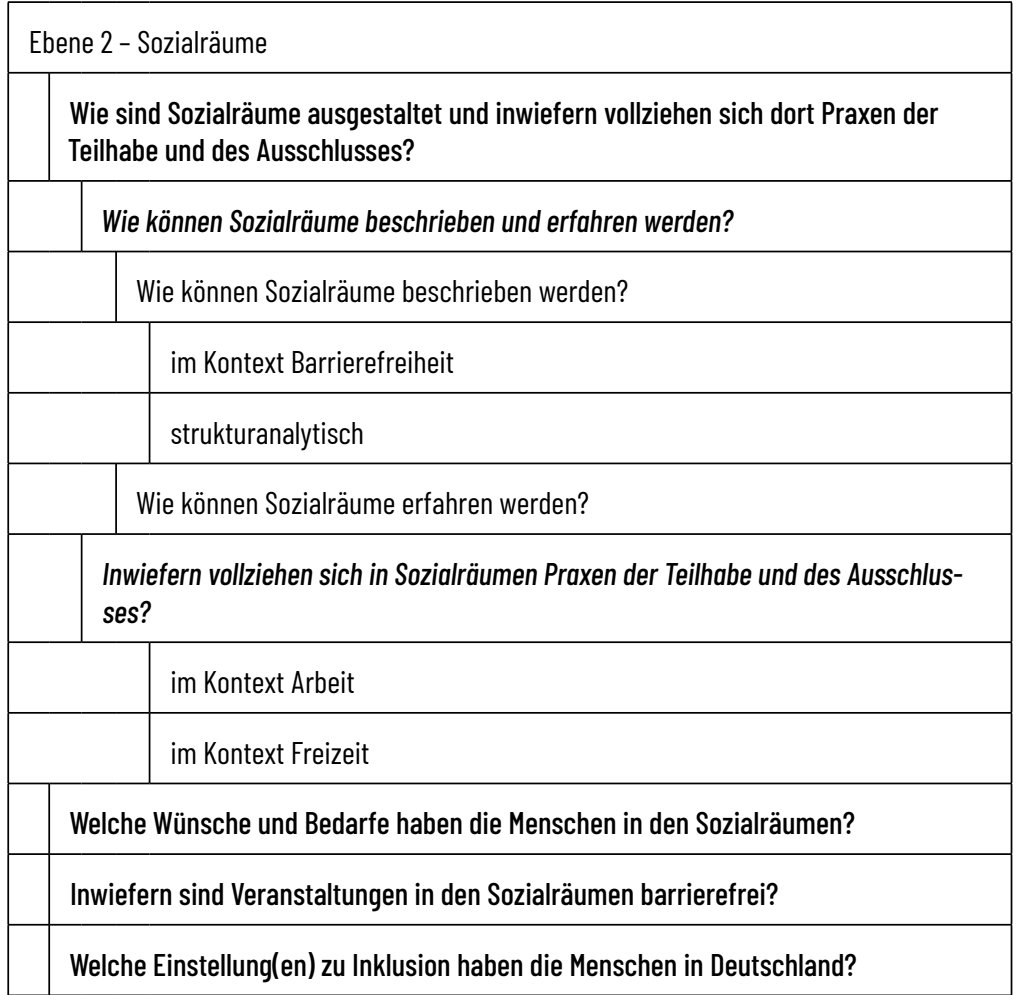

Die Forschungsfragen werden in einzelnen Teilstudien operationalisiert, auf die in Kapitel IV und Kapitel V detaillierter eingegangen wird. Die Ergebnisse der Begleitforschung auf Ebene 2 sind Gegenstand dieser Veröffentlichung.

\subsection{Ebene 3 - Menschen}

Auf der dritten Ebene stehen individuumszentrierte Fallstudien im Mittelpunkt, die die subjektive Wahrnehmung der Personen untersuchen, die in den Sozialräumen leben und die in besonderer Weise von Ausschluss bedroht oder betroffen sind. Die Untersuchungen erfolgen entlang der übergeordneten Fragestellung: 
Tabelle 3: Forschungsleitende Fragestellung der Einzelfallstudien

Ebene 3 - Menschen

Wie erleben Menschen den Sozialraum, die von Ausschluss bedroht oder betroffen sind?

Eines der Ziele ist, zu analysieren, ob und inwiefern die Maßnahmen in den Sozialräumen von den AdressatInnen wahrgenommen werden und sich gegebenenfalls ihre Lebenssituation verändert. Die Ergebnisse der Einzelfallstudien werden hier nicht abgebildet, da sie in der Verantwortung des Forschungsteams der Goethe-Universität Frankfurt liegen.

\section{Beschreibung der hier dargelegten Teilstudie - Sozialräume beforschen}

Was im vorangegangenen Kapitel bereits überblicksartig dargestellt wurde, wird hier noch einmal detaillierter dargelegt, liegt der Fokus des Buches doch darauf, Ergebnisse der Teilstudie auf Ebene 2 - Sozialräume vorzustellen und zu diskutieren. Auf jener zweiten Ebene steht eine Analyse der Sozialräume selbst im Vordergrund, die sich entlang der übergeordneten Forschungsfrage vollzieht:

Ebene 2 - Sozialräume

Wie sind Sozialräume ausgestaltet und inwiefern vollziehen sich dort Praxen der Teilhabe und des Ausschlusses?

Im Folgenden werden die untergeordneten Forschungsfragen und das jeweils daran geknüpfte forschungspraktische Vorgehen skizziert, zudem wird auf die jeweiligen nachfolgenden Kapitel verwiesen, in denen Vorgehen, Ergebnisse und Diskussion dargelegt sind. 


\subsection{Sozialräume beschreiben und erfahren}

Um sich den Sozialräumen initial anzunähern, werden sowohl deskriptivabstrakte als auch ethnographische Verfahren herangezogen. Diese erfolgen entlang der nachstehenden Forschungsfragen:

\begin{tabular}{|l|l|}
\hline \multicolumn{2}{|l|}{ Wie können Sozialräume beschrieben und erfahren werden? } \\
\hline & Wie können Sozialräume beschrieben werden? \\
\hline & im Kontext Barrierefreiheit \\
\hline & strukturanalytisch \\
\hline & Wie können Sozialräume erfahren werden? \\
\hline
\end{tabular}

Anhand von vier Interviews mit SelbstvertreterInnen in repräsentativer Funktion, die selbst unterschiedlicher Unterstützung bedürfen (Mobilität, Sehen, Hören oder Lesen/Verstehen), wurde ein Verständnis von Barrierefreiheit entwickelt und untersucht, welche Rolle diese im Alltag spielt sowie inwiefern eine Praxis ausgestaltet sein muss, um barrierefrei nutzbar zu werden (siehe Kapitel 11.1). Daran anschließend wurde in jedem Sozialraum untersucht, inwiefern öffentliche Einrichtungen des sozialen Lebens barrierefrei zugänglich sind (unter anderem Museen, Ämter und Behörden, Theater, Kinos, Schwimmbäder usw.). Dabei wurden sowohl der Internetauftritt, der Ort selbst und die dort auszuübende Tätigkeit oder Veranstaltung geprüft. Ein zentrales Ergebnis dessen ist die Identifizierung von Merkmalen, inwiefern Barrierefreiheit in je unterschiedlichen Dimensionen (Mobilität, Sehen, Hören, Lesen/Verstehen und Fremdsprache/Englisch) gestaltet werden kann (siehe Kapitel 11.2). Eine Beschreibung von Metadaten der fünf untersuchten Sozialräume eröffnet diese weitergehend als Forschungsgegenstand und es können auf dieser deskriptiven Ebene erste Vergleiche zwischen den Sozialräumen gezogen werden (siehe Kapitel 12). In ethnographischen Sozialraumbegehungen wurden die fünf Sozialräume hinsichtlich behindernder Barrieren und Möglichkeiten ihrer Überwindung erfahren. Im Vordergrund steht dabei das Erleben von und die Interaktion mit sowohl dem Sozialraum als auch seinen BewohnerInnen (siehe Kapitel 13). 


\subsection{Erfahrungen im Miteinander erforschen}

Kern der Ebene 2 sind sogenannte Sozialraumanalysen, anhand derer die Erfahrungen im Miteinander von Menschen mit und Menschen ohne je bestimmte Unterstützungsbedarfe untersucht werden. Diese werden zu drei Zeitpunkten (am Anfang, in der Mitte und am Ende des Projekts) in den Handlungsfeldern Arbeit und Freizeit durchgeführt ${ }^{5}$. Das Handlungsfeld Barrierefreiheit/Mobilität ist dabei als Querschnittsthema für beide Handlungsfelder relevant.

\begin{tabular}{|l|l|}
\hline \multicolumn{2}{|l|}{$\begin{array}{l}\text { Inwiefern vollziehen sich in Sozialräumen Praxen der Teilhabe und des Ausschlus- } \\
\text { ses? }\end{array}$} \\
\hline & Im Kontext Arbeit \\
\hline & Im Kontext Freizeit \\
\hline
\end{tabular}

Die Sozialraumanalysen erfolgen anhand eines mehrdimensionalen Forschungsinstrumentes, das eigens für diese entwickelt wurde (siehe dazu Kapitel 10 und Kapitel 14). Anhand leitfadengestützter Telefon- und faceto-face-Interviews wird untersucht, inwiefern Menschen an Arbeits- und Freizeitpraxen der Mehrheitsgesellschaft teilhaben sowie inwiefern sie dort möglicherweise an Barrieren stoßen. Die der Untersuchung zugrunde gelegten Differenzkategorien sind >Behinderung, >Fluchtmigrationshintergrund und `Demenz , anhand derer Personen, die Ausschluss erfahren, in der Breite untersucht werden können.

\subsection{Sozialraumbefragung und Sozialraummonitoring}

Teil der Sozialraumanalysen ist darüber hinaus der Onlinesurvey "Sozialraumbefragung", der sich an die Menschen richtet, die in den fünf untersuchten Sozialräumen leben. Forschungsleitende Fragestellung ist hierbei:

5 Zusätzlich wurden Sozialraumanalysen im Handlungsfeld Bildung durchgeführt, aufgrund der geringen Repräsentativität der Ergebnisse (die Rückmeldungen auf den Survey zum Thema Bildung waren sehr gering und somit kaum aussagekräftig) werden diese allerdings hier nicht abgebildet. Problematisiert werden kann hierbei, inwiefern Forschung auf die Kooperationsbereitschaft der Personen und Institutionen vor Ort angewiesen ist. 
Welche Wünsche und Bedarfe haben die Menschen in den Sozialräumen?

Es wird untersucht, wie sich die EinwohnerInnen der fünf Sozialräume zu Inklusion verhalten und inwiefern sie der Teilhabe von Menschen mit Behinderung, Menschen mit Fluchtmigrationshintergrund und Menschen mit Demenz gegenüberstehen. Zudem haben die AdressatInnen des Onlinesurveys die Möglichkeit, in offenen Frageteilen unmittelbare Veränderungsvorschläge zu machen, die ungefiltert an die KoordinatorInnen in den jeweiligen Sozialräumen weitergegeben werden können. Die Ergebnisse dieses Onlinesurveys sind nicht Teil der hiesigen Ausführungen. Dies liegt darin begründet, und dabei handelt es sich um eine wichtige Erkenntnis im Forschungsprozess, dass (a) die untersuchte Stichprobengröße zu gering ist, um repräsentative Aussagen treffen zu können, und dass (b) insbesondere Personen, die Inklusion eher ablehnend gegenüber eingestellt sind, nicht durch den Survey erreicht wurden. Insofern konnten lediglich die qualitativen Daten, die durch den Survey generiert wurden, genutzt werden. In der Konsequenz bedeutet das, dass die KoordinatorInnen, die den Onlinesurvey in den jeweiligen Sozialräumen gestreut haben, noch nicht die Breite der Bevölkerung erreichen können, sondern primär Personen, die sich ohnehin im Bereich Inklusion engagieren oder diesbezüglich Interesse haben. Es zeigt sich also, dass sich Netzwerke erst bilden müssen. Unter großen Anstrengungen und unterstützt durch Aktion Mensch e.V. wird der Survey derzeit überarbeitet und es wird ein Design entwickelt, das eine repräsentative Befragung der EinwohnerInnen ermöglicht. Entsprechende Ergebnisse werden nach Abschluss des Erhebungs- und Auswertungsprozesses an anderer Stelle veröffentlicht.

Des Weiteren werden auf Ebene 2 im Rahmen des sogenannten Sozialraummonitorings monatlich Veranstaltungen in den Sozialräumen auf ihre barrierefreie Zugänglichkeit untersucht. Dies erfolgt entlang der Fragestellung:

Inwiefern sind Veranstaltungen in den Sozialräumen barrierefrei?

Ziel des Sozialraummonitorings ist es, über den gesamten Projektzeitraum hinweg zu untersuchen, inwiefern Personen, die von Ausschluss bedroht oder betroffen sind, bei je bestimmten Veranstaltungen in den untersuchten Sozialräumen an Teilhabebarrieren stoßen. Kern dabei sind monatli- 
che Analysen je aktueller Veranstaltungen hinsichtlich ihrer barrierefreien Zugänglichkeit. Untersucht werden beispielsweise jahreszeitenspezifische Märkte, Ausstellungen, Musikveranstaltungen, Sportveranstaltungen oder auch naturbezogene Tätigkeiten (Wanderungen usw.). Im Ergebnis wird es so möglich, darzustellen, ob und inwiefern sich die Ausgestaltung von Veranstaltungen und/oder der Zugang zu diesen verändert, sodass Menschen daran teilhaben können, ohne an Barrieren zu stoßen. Da die Ergebnisse des Sozialraummonitorings ihre Validität primär aus der langen Erhebungsdauer schöpfen, ist die Untersuchung noch nicht abgeschlossen, weshalb hier keine entsprechenden Ergebnisse dargestellt werden.

\subsection{Einstellung(en) zu Inklusion erforschen}

Um zu untersuchen, wie Menschen in Deutschland zu Inklusion eingestellt sind, wurde der Onlinesurvey »Einstellung(en) zu Inklusion« durchgeführt.

Welche Einstellung(en) zu Inklusion haben die Menschen in Deutschland?

Operationalisiert wurde jene Forschungsfrage, indem die Einstellungen zu (einer Teilhabe von) Menschen mit geistiger Behinderung untersucht wurden. Dieser Fokus wurde gewählt, da Menschen mit geistiger Behinderung nach wie vor oftmals massive Teilhabebarrieren erfahren und so in besonderer Weise von Ausschluss betroffen sind (u.a. Trescher 2018b, 2020b). Die Ergebnisse des Surveys, der in dieser Form in Deutschland das erste Mal durchgeführt wurde, sind in Kapitel VI dargestellt (siehe auch Trescher et al. 2020a, 2020b).

\section{Mehrdimensionales Forschungsdesign}

Das Forschungsdesign der Gesamtstudie wurde als mehrdimensionale Methodik entwickelt, anhand derer unterschiedliche Perspektiven auf den Forschungsgegenstand eingenommen werden können, »die eine umfassendere Erfassung, Beschreibung und Erklärung (Kelle und Erzberger 2010, S. 304) desselben möglich machen. In diesem Verständnis ergänzen sich qualitative und quantitative Forschungsmethoden insofern, dass mehrdimensionale Erklärungs- und Verstehenszugänge auf den jeweiligen Gegenstand er- 
öffnet werden können (Kelle und Erzberger 2010, S. 306; siehe auch Promberger 2011). Mehrdimensionale Verfahren bedürfen dabei eines gemeinsamen theoretischen Rahmens, anhand dessen die Ergebnisse zueinander in Relation gesetzt und diskutiert werden können (Kelle und Erzberger 2010, S. 306). Diese theoretische Rahmung wurde in den einleitenden Kapiteln dargelegt (siehe Kapitel II). Im Folgenden werden nun methodische Voraussetzungen und Vorgehensweisen deskriptiv-statistischer Verfahren (Kapitel 10.1) und qualitativ-inhaltsanalytischer Verfahren (Kapitel 10.2) erläutert. Daraufhin wird Bezug genommen auf die Erhebung leitfadengestützter Interviews (Kapitel 10.3). Als weiterer qualitativer Forschungszugang, der im Rahmen der Studie Anwendung fand, werden Verfahren ethnographischer Feldforschung erläutert (Kapitel 10.4). Das vorliegende Kapitel zum mehrdimensionalen Forschungsdesign der Studie wird mit einer Diskussion des Verhältnisses quantitativer und qualitativer Forschungsverfahren geschlossen (Kapitel 10.5).

\subsection{Deskriptiv-statistische Verfahren}

Quantitative Forschungsverfahren folgen dem Paradigma des Erklärens, anhand dessen Gegenstände beschrieben und zueinander in Relation gesetzt werden können, während qualitative Verfahren eher auf das Verstehen abzielen und folglich »die sinnhafte Erschließung der sozialen Praxis« (Silkenbeumer 2010, S. 262) fokussieren. Quantifizierende, deskriptiv-statistisch verfahrende Forschungsmethoden haben eine $\gg$ Exploration und Beschreibung eines komplexen sozialen Sachverhalts oder Problemfelds" (Silkenbeumer 2010, S. 268) zum Gegenstand und verfolgen das Ziel, »die Informationen großer Datenmengen zu verdichten, um Strukturen sichtbar zu machen « (Völkl und Korb 2018, S. 1). Deskriptiv-statistische Verfahren können entlang der Anzahl der Variablen, die Gegenstand ihrer Analyse sind, in univariate, bivariate und multivariate Analyseverfahren differenziert werden (Völkl und Korb 2018, S. 2f). Während im Kontext der hier dargelegten Studie bei den sogenannten Sozialraumanalysen (siehe Kapitel V) anhand bivariater Analyseverfahren Zusammenhänge zwischen jeweils zwei Variablen untersucht und diskutiert werden, werden im Survey »Einstellung(en) zu Inklusion « (siehe Kapitel VI) multivariate Datenanalysen durchgeführt, die mehrdimensionale Zusammenhänge offenlegen (Raithel 2008, S. 120). Bivariate Datenanalysen erfolgen zumeist anhand sogenannter Kreuztabellen 
und können zudem anhand dieser veranschaulicht werden, wie in den entsprechenden Kapiteln noch einmal erläutert und dargestellt wird.

\subsection{Qualitativ-inhaltsanalytische Verfahren}

Qualitativ-inhaltsanalytische Verfahren entsprechen dem Paradigma des Verstehens (Mayring 2010b, S. 19) und folgen einer subsumierenden Logik. Der zentrale Vertreter qualitativ-inhaltsanalytischer Verfahren ist Philipp Mayring, auf dessen Ausführungen zur Methodik sich hier primär gestützt wird (Mayring 2010b; zur Anwendungspraxis siehe auch Trescher 2015b, S. 74ff). Der Gegenstand qualitativer Inhaltsanalysen ist sprachlich vermittelt und muss in protokollierter Form vorliegen, er beschränkt sich allerdings nicht auf Verbalsprache, sondern »es kann sich auch um musikalisches, bildliches, plastisches o.ä. Material handeln« (Mayring 2010a, S. 468). Jenes Material wird »immer in seinem Kommunikationszusammenhang verstanden « (Mayring 2010b, S. 48), was zudem bedeutet, dass es nicht losgelöst von seinem Entstehungskontext analysiert werden soll (Mayring 2010b, S. 48). Die qualitative Inhaltsanalyse folgt je bestimmten Regeln, die allerdings an den jeweiligen Gegenstand angepasst werden (Mayring 2010b, S. 48f). Sie ist also »kein Standardinstrument (Mayring 2010b, S. 49), das in immer gleicher Form angewendet werden kann. Dennoch ist es unbedingt notwendig, das jeweilige forschungspraktische Vorgehen detailliert darzulegen, um so eine intersubjektive Nachvollziehbarkeit und Überprüfung der Ergebnisse sicherzustellen (Mayring 2010b, S. 49). Hierzu soll ein sogenanntes Ablaufmodell erstellt werden (Mayring 2010a, S. 471). Um intersubjektive Geltungsüberprüfung zu ermöglichen, ist es ebenso wichtig, theoriegeleitet zu arbeiten, das heißt, »dass der Stand der Forschung zum Gegenstand und vergleichbaren Gegenstandsbereichen systematisch bei allen Verfahrensentscheidungen herangezogen wird« (Mayring 2010b, S. 51). Kern qualitativ-inhaltsanalytischer Verfahren ist das Erarbeiten eines Kategoriensystems, das in gewisser Weise das zentrale »Ergebnis der Analyse darstellt" (Mayring 2010b, S. 50). Es wird »in einem Wechselverhältnis zwischen der Theorie (der Fragestellung) und dem konkreten Material entwickelt, durch Konstruktions- und Zuordnungsregeln definiert und während der Analyse überarbeitet und rücküberprüft« (Mayring 2010b, S. 59). Im Zuge der Analyse gilt es, sogenannte Analyseeinheiten zu definieren, anhand derer die Größe des Materials festgelegt wird, das in einer Kategorie zusammen- 
geführt werden darf (Kodiereinheit und Kontexteinheit), sowie in welcher Reihenfolge das Material ausgewertet wird (Auswertungseinheit) (Mayring 2010 b, S. 59). In der qualitativen Inhaltsanalyse werden drei unterschiedliche Interpretationsformen unterschieden, die aufeinander folgend oder jede für sich einzeln angewendet werden können. Insofern stellen die $\mathrm{Zu}$ sammenfassung, die Explikation und die Strukturierung jeweils eigenständige qualitativ-inhaltsanalytische Verfahrensweisen dar, Mischformen sind jedoch denkbar (Mayring 2010b, S. 64f). Während in der zusammenfassenden Inhaltsanalyse die zentralen Inhalte des Materials herausgearbeitet werden, zieht die explizierende Inhaltsanalyse zusätzliche Informationen heran, um unverständliche Teile zu interpretieren (Mayring 2010b, S. 65). In der strukturierenden Inhaltsanalyse werden schließlich vorab festgelegte Kriterien an das Material herangetragen, um »bestimmte Aspekte aus dem Material herauszufiltern« (Mayring 2010b, S. 65). Bei der zusammenfassenden Inhaltsanalyse ist es naheliegend, die Kategorien aus dem Material zu erschließen (induktive Kategorienbildung), wogegen in der explizierenden und strukturierenden Analyse Kategorien zumeist im Vorhinein festgelegt und an das Material angelegt werden (deduktive Kategorienanwendung) (Mayring 2010b, S. 66). Jenes induktive Vorgehen bei der Kategorienbildung ist im Rahmen dieser Studie zentral (siehe Kapitel 15). Die qualitative Inhaltsanalyse erfordert, dass mehrere Forschende unabhängig voneinander dasselbe Material analysieren und daraufhin ihre Ergebnisse vergleichen und diskutieren. Die auf diese Weise hergestellte Intercoderreliabilität dient ebenfalls dazu, valide Ergebnisse zu erzielen (Mayring 2010b, S. 51). Auch wenn bei der Qualitativen Inhaltsanalyse im Vorhinein festgelegt wird, wie das Material bearbeitet werden soll, liegt es doch an den Forschenden selbst, das Vorgehen so $\mathrm{zu}$ adaptieren, dass die forschungsleitenden Fragen sinnhaft beantwortet werden können. Es besteht also durchaus Gestaltungsspielraum im Auswertungsprozess, was die Qualitative Inhaltsanalyse als Hermeneutik kennzeichnet, die in der Interpretation als Kunstlehre Anwendung findet (Trescher 2015b, S. 75).

\subsection{Erhebung leifadengestützter Interviews}

$\mathrm{Zu}$ sozialwissenschaftlichen Interviews kann ganz allgemein gesagt werden, dass es übergeordnete Formen gibt, die sich bezüglich des Grades der Strukturierung des Interviewverlaufs und der daraus generierten Antwor- 
ten durch den/die ForscherIn unterscheiden (Friebertshäuser und Langer 2010, S. 439). Im Allgemeinen werden hierbei sogenannte erzählgenerierende Interviews und Leitfadeninterviews unterschieden (Friebertshäuser und Langer 2010, S. 439). In sogenannten erzählgenerierenden Interviews, die eine eher offene Erhebungsform darstellen, werden der Gesprächsverlauf und der Inhalt überwiegend durch die interviewte Person bestimmt (Friebertshäuser und Langer 2010, S. 439). In sogenannten Leitfadeninterviews hingegen erfolgt die Strukturierung durch den Interviewleitfaden, der zuvor durch den/die ForscherIn entwickelt wurde (Trescher 2015b, S. 59; siehe auch Friebertshäuser 1997, S. 375f) (Lamnek 2005, S. 367; Helfferich 2014, S. 560). Beim Leitfadeninterview kann zwischen einer standardisierten und einer halbstandardisierten Form unterschieden werden. Während Leitfadeninterviews, die der standardisierten Form folgen, stärker strukturiert sind (unter anderem vollständig ausformulierte Fragen und vorgegebene Antwortmöglichkeiten), werden in der halbstandardisierten Form offenere Themenfelder angesprochen, die im Laufe des Interviews verändert, ergänzt oder ausgelassen werden können (Friebertshäuser und Langer 2010, S. 439; Hopf 2010, S. 351). Wie von Friebertshäuser und Langer (2010) betont, kann allerdings diese "grobe Kategorisierung (Friebertshäuser und Langer 2010, S. 439) in standardisierte und halb-standardisierte Formen des Leitfadeninterviews nicht uneingeschränkt vorgenommen werden, »da es auch Mischformen gibt« (Friebertshäuser und Langer 2010, S. 439), in denen beispielsweise im Verlauf eines vorstrukturierten, standardisierten Leitfadeninterviews offene Erzählpassagen bewusst herbeigeführt werden können (Helfferich 2014, S. 565f; siehe auch Trescher 2015b, S. 60). Die je konkrete Ausgestaltung der Leitfadeninterviews und das forschungspraktische Vorgehen bei der Erhebung sind in den jeweiligen Kapiteln eingehen erläutert (siehe Kapitel 11.1.1 und Kapitel 14.3).

\subsection{Ethnographische Verfahren}

Für die Vor-Ort-Begehungen der Sozialräume wurde ein ethnographisches Forschungsdesign entwickelt, das vom Anspruch getragen ist, den Sozialraum und die ihm eigenen Strukturen, Praxen, Interaktionen etc. zu erfahren. Dadurch wird es möglich, gegebenenfalls vorhandene Barrieren lebenspraktisch zu erschließen. Ethnographie heißt in diesem Zusammenhang, sich je bestimmten Lebenspraxen anzunähern und sozusagen Welt 
mit den Augen der darin lebenden Menschen zu sehen (Honer 2010, S. 195; siehe auch Riege 2007, S. 383). "Es geht darum, >die Fremde (Honer 2010, S. 197) und diese zu verstehen. Grundlegend ist dabei »eine sozialwissenschaftliche Haltung der Neugier, die davon ausgeht, dass soziale Wirklichkeiten Überraschendes und Verwunderliches bereithalten, sowie die Bereitschaft, sich der Dynamik und Logik eines Feldes auszusetzen« (Breidenstein et al. 2013, S. 7). Da sich mit der ethnographischen Begehung der Sozialräume in gewisser Weise der eigenen Lebenswelt verstehend angenähert wird, kann dieses Vorgehen auch als ein "Medium der gesellschaftlichen Selbstbeobachtung (Lüders 2010, S. 390) aufgefasst werden. Das konkrete methodische Vorgehen ist an Seifert (2010, S. 301f) und Honer (2010, S. 197) angelehnt. Der große und vielgestaltige Materialkorpus, der in aufwendigen und kleinteiligen Besuchen der Sozialräume generiert wurde, setzt sich aus zahlreichen Beobachtungsprotokollen und gesprächsförmigen Interviews mit Menschen im Sozialraum zusammen, die immer wieder angesprochen wurden. Ein weiterer Schwerpunkt sind Analysen von Dokumenten und Artefakten, beispielsweise Busfahrpläne, Flyer aus dem Touristenbüro, Hinweis- und Verkehrsschilder etc. Bei den Begehungen wurden Wohnquartiere, Innenstadtbereiche, öffentliche Verkehrsmittel, kulturelle Einrichtungen, Behörden/städtische Verwaltungen und weitere den Sozialraum auszeichnende Orte miteinbezogen. In zahlreichen und kleinteiligen Beobachtungsprotokollen wird der Sozialraum beschrieben und es werden konkrete Interaktionen dokumentiert, an denen teilgenommen wurde oder die eher saus der Ferne wahrgenommen wurden. Grundsätzlich wird zudem immer der öffentliche Personennahverkehr genutzt, um zu entdecken, wo dort potenzielle Barrieren liegen (Trescher et al. 2017). Immer wieder wird das Gespräch mit den Menschen vor Ort gesucht, um Einblick in deren Erfahrungen - insbesondere bezüglich erlebter Teilhabebarrieren - nehmen zu können. Teils werden auch kurze gesprächsförmige Interviews geführt, um Wissen über bestimmte Praxen zu generieren (beispielsweise ein kurzes Interview mit einer Mitarbeiterin eines Schwimmbades, um über die Möglichkeiten aufgeklärt zu werden, dieses als Person mit je bestimmten Unterstützungsbedarfen zu besuchen). In weiteren Analysen wird sich mit den Sozialräumen eigenen Dokumenten und Artefakten befasst, die gesammelt oder fotografiert und so dem Materialkorpus hinzugefügt werden. Diese Dokumente und Artefakte sind besonders zahlreich und vermitteln einen ganz eigenen Eindruck vom Sozialraum. Aus diesen unterschiedlichen $\mathrm{Zu}$ - 
gängen zu den Sozialräumen entsteht schließlich ein sehr großer und vielgestaltiger Materialkorpus, dessen Analyse in ethnographischen Berichten resultiert (siehe dazu u.a. Lüders 2010, S. 396). In diesen ethnographischen Berichten (siehe Kapitel 13.1) wird das mehrdimensional Erfahrene und Protokollierte eingeordnet und verdichtet (Lüders 2010, S. 397).

\subsection{Zum Verhältnis quantitativer und qualitativer Forschungsverfahren}

In mehrdimensional oder auch Mixed Methods genannten Forschungsdesigns werden, wie oben dargelegt, quantitative und qualitative Forschungsverfahren miteinander verknüpft, was die Frage aufwirft, wie sich die jeweiligen Ausrichtungen respektive Ansätze zueinander verhalten. Zentrale Voraussetzung dafür, quantitative und qualitative Forschungsverfahren sinnhaft miteinander zu verbinden, ist die Einsicht, dass jene Forschungsmethoden »keineswegs einen einheitlichen Block darstellen, sondern ein großes Spektrum teilweise auch heterogener Ansätze umfassen « (Kuckartz 2014, S. 28). Quantitative und qualitative Forschungsmethoden werden insofern nicht einander gegenübergestellt, sondern können sich überlappen und ergänzen. Dabei wird von der beziehungsweise den Forschungsfrage(n) ausgegangen, die in der jeweiligen Studie untersucht werden sollen und es wird entlang der Annahme verfahren: »Welche Methoden sind bei der Beantwortung der Forschungsfrage nützlich?« (Kuckartz 2014, S. 35). Folglich kann anhand mehrdimensionaler Forschungsdesigns ein breites Spektrum an Forschungsfragen bearbeitet werden, was wiederum breite Einblicke in den jeweiligen Forschungsgegenstand ermöglicht (Engler 1997, S. 126). Unterschiedliche Verstehenszugänge zum selben Gegenstand können ergänzend sein und das Verständnis der untersuchten Lebenspraxis erweitern, wobei beachtet werden sollte, dass die gewählten Verfahren »tatsächlich miteinander vereinbar sind, sich ergänzen und unterschiedliche Perspektiven liefern" (Kuckartz 2014, S. 35; siehe auch Engler 1997, S. 129). Bei sogenannten mehrdimensionalen Forschungsdesigns wie dem hiesigen ist es zudem notwendig, die Ergebnisse der quantitativen und qualitativen Untersuchungen nicht beziehungslos nebeneinander bestehen zu lassen, sondern miteinander zu verknüpfen (Kuckartz 2014, S. 157) - erst daraus ergibt sich der Gewinn ebenjener Verfahren (siehe auch Kapitel 24). 



\section{Sozialräume beschreiben und erfahren}

Ein Schwerpunkt der Untersuchung der Sozialräume liegt auf der Beschreibung und Erfahrung dieser. Während beschreibende Zugänge einen deskriptiven, strukturanalytischen Blickwinkel einnehmen, zielen erfahrende Ausrichtungen darauf ab, dass sich Behinderung als Praxis auch und insbesondere in je subjektiven Erfahrungen des Behindert-werdens niederschlägt (siehe Kapitel 4). Diese Erfahrungen werden teils sozialräumlich (re-)produziert (Weisser 2010, S. 6). Bei der Analyse sind die folgenden Fragen, wie oben bereits dargestellt, forschungsleitend.

Tabelle 4: Forschungsleitende Fragestellung der Beschreibung und Erfahrung von Sozialräumen

\begin{tabular}{|l|l|}
\hline \multicolumn{2}{|l|}{ Wie können Sozialräume beschrieben und erfahren werden? } \\
\hline & Wie können Sozialräume beschrieben werden? \\
\hline & im Kontext Barrierefreiheit \\
\hline & strukturanalytisch \\
\hline & Wie können Sozialräume erfahren werden? \\
\hline
\end{tabular}

Die initiale Beschreibung und Erfahrung der Sozialräume ist felderöffnend und erfolgt im Rahmen einer Recherche zu öffentlichen Orten und Barrierefreiheit (Kapitel 11), einer Strukturdatenrecherche (Kapitel 12) sowie ethnographischer Sozialraumbegehungen (Kapitel 13), wobei primär bei Letzteren ein erfahrender Verstehenszugang angewendet wird. 


\section{1. Öffentliche Orte und Barrierefreiheit}

Teil der beschreibenden Erschließung und Analyse der fünf untersuchten Sozialräume ist eine Recherche zu öffentlichen Orten im Kontext Barrierefreiheit. Diese erfolgt in zwei Schritten, nämlich zum einen anhand einer Analyse von Interviews mit SelbstvertreterInnen in repräsentativer Funktion aus den Bereichen Mobilität, Sehen, Hören und Lesen/Verstehen und zum anderen anhand der Analyse von öffentlichen Orten in den fünf Sozialräumen. Dadurch soll sich dem Verständnis von Barrierefreiheit angenähert werden wie es (a) bei selbst Betroffenen sowie (b) im gesamtgesellschaftlichen Diskurs gebräuchlich ist. Übergeordnetes Ziel ist, Kriterien zur Einschätzung der barrierefreien Zugänglichkeit von Orten und Tätigkeiten herauszuarbeiten. Wie eingangs bereits dargelegt (Kapitel 5.4), wird die Frage nach Barrierefreiheit auch davon geleitet, dass diese nicht nur für Menschen mit je unterschiedlichen Unterstützungsbedarfen von Bedeutung ist, sondern (je situativ) jede Person betreffen kann. Insbesondere mit fortschreitendem Alter können beispielsweise eine eingeschränkte Mobilität und/oder nachlassendes Seh- und Hörvermögen dazu führen, dass Personen an Barrieren stoßen. Dadurch wird Barrierefreiheit zu einer Thematik, mit der sich jede Person im Laufe ihres Lebens früher oder später auseinandersetzen wird. Diese Perspektive macht deutlich, dass Barrierefreiheit ein gesamtgesellschaftliches Thema ist und als solches auch der gesamtgesellschaftlichen Aufmerksamkeit bedarf - nicht zuletzt deshalb wird der Frage nach Barrierefreiheit im Rahmen der Studie eine solche Bedeutung zuerkannt.

\subsection{Annäherungen an ein operationalisierbares Verständnis von Barrierefreiheit}

Um sich einem Verständnis von Barrierefreiheit anzunähern, wurden in einem ersten Schritt Menschen mit unterschiedlichen Unterstützungsbedarfen (Mobilität, Sehen, Hören oder Lesen/Verstehen) als ExpertInnen in eigener Sache (Hermes und Rohrmann 2006, S. 7) und als SelbstvertreterInnen in repräsentativer Funktion interviewt ${ }^{1}$. Ziel dessen war, ein Verständ-

1 Es sei angemerkt, dass das Interview mit dem/der Selbstvertreterln aus dem Bereich Hören durch eine/n anwesende/n Gebärdensprachdolmetscherln übersetzt wurde. Auf ein Interview mit einem/einer Selbstvertreterln aus dem Bereich Fremdsprache (Englisch) 
nis dafür zu entwickeln, auf welche Barrieren Menschen mit Beeinträchtigungen im Alltag treffen und welche Strategien bereits vorliegen, Barrieren zu überwinden. Es sei angemerkt, dass die vielgestaltigen Ergebnisse hier lediglich zusammenfassend dargestellt werden können, um die Anonymität der Interviewpersonen zu wahren.

\subsubsection{Methodisches Vorgehen}

\section{Entlang der Forschungsfrage "Was bedeutet Barrierefreiheit für mich und} für den Personenkreis, den ich vertrete?« wurden Leitfadeninterviews mit SelbstvertreterInnen aus den Bereichen Mobilität, Sehen, Hören und Lesen/ Verstehen geführt, die qualitativ-inhaltsanalytisch ausgewertet wurden ${ }^{2}$. Jene Beeinträchtigungsdimensionen wurden nach eingehender Recherche als besonders von Barrieren (und infolgedessen Ausschluss) betroffen ausgemacht. Die Interviews wurden entlang eines vorab entworfenen Leitfadens erhoben, in den die für die Interviews zentralen Interessen eingingen. Im Folgenden ist der Leitfaden schematisch dargestellt, der bei den Interviews Anwendung fand. Im Gros wurde danach gefragt, worin Barrieren bei der Nutzung von Internetseiten, öffentlichen Orten und Veranstaltungen liegen und wie diese überwunden werden können. Die Interviews dienten also in erster Hinsicht einer Wissenssammlung.

Tabelle 5: Leitfaden für die Interviews mit SelbstvertreterInnen im Kontext Barrierefreiheit

\begin{tabular}{|l|l|}
\hline Internet & \\
\cline { 1 - 1 } Öffentliche Orte & $\begin{array}{l}\text { - Worin liegen Barrieren? } \\
\text { - Wie können diese abgebaut beziehungs- } \\
\text { weise überwunden werden? }\end{array}$ \\
\cline { 1 - 2 } Veranstaltungen & \\
\hline
\end{tabular}

wurde verzichtet, da sich die Forschenden diesbezüglich selbst dazu geeignet sahen, zu antizipieren, worin Barrieren und Möglichkeiten ihrer Überwindung liegen, wenn eine Person auf Unterstützung im Bereich Fremdsprache (Englisch) angewiesen ist.

2 Methodische Erläuterung zur Erhebungsmethode des Leitfadeninterviews und zum Auswertungsverfahren der Qualitativen Inhaltsanalyse finden sich in Kapitel 10.3 und Kapitel 10.2 . 


\subsubsection{Barrieren und Möglichkeiten ihrer Überwindung}

In Bezug auf Internetseiten wird deutlich, dass Barrieren immer dann entstehen, wenn nur eine bestimmte Möglichkeit besteht, die Inhalte zu erfassen - zum Beispiel (schwere) Schriftsprache. Die SelbstvertreterInnen stimmen darin überein, dass gerade durch unterschiedliche Darstellungsformen Internetseiten zugänglich gemacht werden können (Inhalte in Leichter Sprache und Deutscher Gebärdensprache, Größe und Kontrast veränderbar, Vorlesefunktion etc.). Teilweise verweisen die SelbstvertreterInnen auf die Inhalte der sogenannten BITV (Verordnung zur Schaffung barrierefreier Informationstechnik nach dem Behindertengleichstellungsgesetz, Barrierefreie-Informationstechnik-Verordnung - BITV 2.0), die zur Orientierung sinnvoll seien. Der/die SelbstvertreterIn mit Lernschwierigkeiten macht darauf aufmerksam, dass für viele Menschen mit Unterstützungsbedarfen im Bereich Lesen/Verstehen Barrieren vor allem aus ihrer oftmals sehr geschlossenen Wohnsituation resultieren, in der kein Internetanschluss zur Verfügung steht (siehe dazu auch Reichstein 2016, S. 82; Trescher 2017f, S. 133ff). Zudem können sich einige Personen aufgrund des sehr geringen Gehalts, das sie in einer Werkstatt für Menschen mit Behinderung (WfbM) ausgezahlt bekommen, keinen Internetanschluss und/oder ein internetfähiges Endgerät leisten (siehe dazu auch Trescher 2018c, S. 138). In Bezug auf Orte entstehen Barrieren immer dann, wenn diese auf unterschiedlichen Ebenen angeordnet und unübersichtlich gestaltet sind. Problematisch ist zudem, wenn stufenlose Wege fehlen, keine Führungslinien vorhanden sind oder keine Symbole den Ort und seine Nutzung erläutern. Folgerichtig fordern die interviewten SelbstvertreterInnen, öffentliche Orte besser auszubauen, indem ebenjene stufenlose Zugänglichkeit und Führungslinien sowie Symbole gewährleistet werden. Gleichzeitig gibt der/die blinde SelbstvertreterIn zu bedenken, dass Führungslinien in gewisser Weise einen begrenzten Nutzen haben, denn gerade in Fußgängerzonen seien diese kaum sinnvoll. Er/sie sagt: »Wo sollen die denn dann hinführen?« Übergreifend machen die SelbstvertreterInnen darauf aufmerksam, dass es zu wenig Expertise für die Bedarfe von Menschen mit Beeinträchtigungen gibt und, auch deshalb, $\mathrm{zu}$ wenig Berücksichtigung im mehrheitsgesellschaftlichen Diskurs. Sie fordern teilweise zentrale Anlaufstellen, die sowohl informieren als auch sich dafür einsetzen, dass Teilhabe barrierearm ermöglicht wird. Bei Veranstaltungen schließlich entstehen Barrieren nach Maßgabe der SelbstvertreterInnen primär dadurch, dass ein Bewusstsein für diverse Bedarfe fehlt. 
Ein weiteres Problem ist mangelnde Flexibilität und fehlende Bereitschaft, Abläufe zu verändern. Bei Großveranstaltungen bestehen Barrieren vor allem darin, sich in großen Menschenmengen zu bewegen beziehungsweise zurechtzufinden. In Bezug auf Mobilität werden insbesondere Kabelkanäle zur Herausforderung. Teilhabe an Veranstaltungen kann laut der SelbstvertreterInnen insbesondere dadurch ermöglicht werden, dass Menschen ohne Beeinträchtigung für die Bedarfe von Menschen mit bestimmten Beeinträchtigungen sensibilisiert werden. Notwendig sei dazu auch, Begegnungsund Austauschräume zu schaffen. Darüber hinaus sollen bauliche und/oder technische Lösungen, die bereits vorhanden sind, auch genutzt werden. Dies betrifft insbesondere technische und digitale Anwendungen, die zur Orientierung in Gebäuden oder Ähnlichem genutzt werden können (zum Beispiel iBeacons, Greta und Starks etc.). Generell sei es sinnvoll, ein Mehr-SinnePrinzip bei der Gestaltung von Orten und der Planung von Veranstaltungen zu verfolgen, um unterschiedliche Zugänge zu bereiten. Dabei dürften jedoch nicht Barrieren für die einen errichtet werden, indem Barrieren für andere (scheinbar) abgebaut werden. Das konkrete Beispiel, das dabei genannt wird, betrifft Bordsteine, die für Menschen mit Sehbeeinträchtigungen/ Blindheit Orientierungslinie sind, für Menschen mit Mobilitätsbeeinträchtigungen dagegen ein manifestes Hindernis darstellen (siehe dazu auch Leidner 2007, S. 31; Trescher 2018c, S. 19f). Ein/e SelbstvertreterIn merkt demgegenüber problematisierend an, dass technische oder bauliche Hilfsmittel nicht immer die beste Unterstützung bieten. Er/sie sagt: »Manchmal ist es am einfachsten, jemanden anzusprechen und sich weiterhelfen zu lassen«. Bei anderen SelbstvertreterInnen wiederum ist eine gewisse Resignation zu erkennen und sie gehen eher nicht davon aus, dass es so etwas wie eine barrierefreie Stadt jemals geben wird. Eine Person sagt: »Es wird auch künftig so sein, dass ich Probleme mit dem ÖPNV habe. Das ist dann halt einfach so und das ist dann eben Pech«. Eine solche Haltung resultiert möglicherweise aus Ausgrenzungserfahrungen, von denen alle SelbstvertreterInnen berichten. Beispielsweise sagt eine Interviewperson: »Die KollegInnen haben mir nicht das Gefühl gegeben, dass ich herzlich willkommen bin im Team. Manchmal sind die Menschen selbst die Barrieren«. Hieran zeigt sich erneut, wie wichtig es ist, Berührungspunkte und Begegnungsmöglichkeiten für Menschen mit und Menschen ohne Beeinträchtigungen zu schaffen, sodass eine gewisse Sensibilität und ein Bewusstsein dafür gebildet werden, dass Barrieren nicht nur baulich, sondern auch im Miteinander entstehen können. 


\subsection{Barrierefreiheit öffentlicher Orte in den untersuchten Sozialräumen}

Im Rahmen der Recherchen zu öffentlichen Orten und Barrierefreiheit in den fünf untersuchten Sozialräumen stand die Frage danach im Vordergrund, inwiefern öffentliche Orte beziehungsweise dort ausgeübte Tätigkeiten sowie die jeweiligen Internetauftritte des betreffenden Ortes/der betreffenden Aktivität als barrierefrei bezeichnet werden können. Zur Beantwortung dieser Fragen wurden in allen fünf Sozialräumen öffentliche Orte (Theater, Kinos, Fußballstadien, Schwimmbäder, Kongresszentren, Bibliotheken, Bürgerämter und viele mehr) hinsichtlich ihrer barrierefreien Nutzungsmöglichkeiten analysiert. Insgesamt wurden auf diese Art und Weise exemplarisch 50 Orte untersucht. Die Recherchen dienen (a) dem weitergehenden Kennenlernen der Sozialräume und waren darüber hinaus (b) ein wichtiger Schritt in der Vorbereitung der ethnographischen Sozialraumbegehungen, denn durch die Recherchen wurden sozialraumspezifisches Wissen generiert und weiterführende Fragen aufgeworfen, die bei der Entwicklung der Forschungsinstrumente zentral bedeutsam waren (siehe Kapitel 10). Im Folgenden wird das methodische Vorgehen der Recherchen zu öffentlichen Orten und Barrierefreiheit in den fünf Sozialräumen erläutert (Kapitel 11.2.1), woraufhin die Kriterien zur Kategorisierung der Orte als (nicht) barrierefrei erläutert (Kapitel 11.2.2) und die Ergebnisse dessen dargelegt werden (Kapitel 11.2.3). Abschließend werden die Ergebnisse zusammengeführt und diskutiert (Kapitel 11.3).

\subsubsection{Forschungsfrage und methodisches Vorgehen}

Die zugrundliegende Forschungsfrage der Recherchen zu öffentlichen Orten und Barrierefreiheit ist: Kann eine Person mit Unterstützungsbedarf das Angebot beziehungsweise die Tätigkeit nutzen? Dieser Unterstützungsbedarf wurde hinsichtlich der Dimensionen Mobilität, Sehen, Hören, Lesen/ Verstehen und Fremdsprache (Englisch) ausdifferenziert und sowohl Internetauftritte als auch Orte des öffentlichen Lebens sowie darin ausgeübte Tätigkeiten dahingehend untersucht. Diese Untersuchung entlang unterschiedlicher Ausprägungen von Unterstützungsbedarfen erlaubt eine Analyse unabhängig von gängigen Behinderungskategorien und ist Zeichen des Versuchs, Behinderung nicht als individuelle Größe, sondern als Praxis des Behindert-werdens zu verstehen, die sich unabhängig von dem, was bislang 
mehrheitlich als >Behinderung bezeichnet wird, je situativ vollzieht (siehe Kapitel 4.1). Ganz konkret heißt das beispielsweise, dass Personen, die einen Rollator nutzen, oder Personen, die einen Kinderwagen befördern und die gegenwärtig nicht unter der Kategorie >behindert « gefasst werden, dennoch Unterstützungsbedarfe im Bereich Mobilität haben können. Im Folgenden ist ausdifferenziert, was im Einzelnen unter Unterstützungsbedarf in den jeweiligen Ausprägungen verstanden wird und welche möglichen Unterstützungsbedarfe dabei antizipiert wurden ${ }^{3}$.

\section{Unterstützungsbedarf im Bereich Mobilität}

Im Bereich Mobilität bezieht sich der Unterstützungsbedarf auf all jene Personen, die aufgrund etwaiger Mobilitätseinschränkungen an Barrieren stoßen, infolge derer sie nicht beziehungsweise nur eingeschränkt an routinemäßigen Praxen des öffentlichen Lebens teilhaben können. Dies kann zum Beispiel Personen betreffen, die einen Rollstuhl oder Rollator nutzen, oder auch Personen, die einen Kinderwagen befördern. Auch Personen, die (temporär oder längerfristig) auf Gehhilfen wie zum Beispiel Krücken angewiesen sind, können Unterstützungsbedarfe im Bereich Mobilität haben. Folglich entstehen Barrieren immer dann, wenn Stufen, Treppen, steile Hänge usw. nicht umgangen werden können (beispielsweise durch Rampen, Aufzüge oder absenkbare Verkehrsmittel).

3 Das Verständnis von Unterstützungsbedarf, das den Recherchen zu öffentlichen Orten und Barrierefreiheit zugrunde liegt, kann unabhängig von dem, was bislang mehrheitlich unter der Differenzkategorie >Behinderung verstanden wird, gedacht werden und erstreckt sich in der Folge auf alle Personen, die je situativ bestimmter Unterstützung bedürfen. Wichtig ist in dieser Hinsicht auch zu erwähnen, dass viele Menschen mit unterschiedlichen Beeinträchtigungen beziehungsweise Unterstützungsbedarfen im Laufe ihres Lebens zum Teil vielfältige Strategien dahingehend entwickelt haben, mit alltäglichen Barrieren umzugehen und die, trotz einer Umwelt, die im hiesigen Forschungszusammenhang als snicht barrierefrei< kategorisiert würde, auf so gut wie keine Unterstützung angewiesen sind. Nichtsdestotrotz sind Fragen von Barrierefreiheit von Bedeutung, da die barrierefreie Zugänglichkeit beziehungsweise Nutzung die prinzipielle Möglichkeit der Teilhabe an Praxen des öffentlichen Lebens bereiten kann und dadurch Chancen auch für diejenigen eröffnet, die bislang (aus welchen Gründen auch immer) im Alltag an Teilhabebarrieren stoßen und infolgedessen von Ausschluss bedroht beziehungsweise betroffen sind. 


\section{Unterstützungsbedarf im Bereich Sehen}

Personen, die auf Unterstützungsbedarf im Bereich Sehen angewiesen sind, stoßen immer dann an Barrieren, wenn beispielsweise eine Orientierung in Gebäuden ausschließlich aufgrund optischer Markierungen möglich ist (zum Beispiel Wegweiser ohne Braillezeichen, mit sehr kleiner Schrift oder sehr kontrastarm). Dies betrifft vor allem Personen, die eine (mehr oder minder ausgeprägte) Sehbeeinträchtigung haben sowie blinde Personen.

\section{Unterstützungsbedarf im Bereich Hören}

Im Bereich Hören zielt Unterstützungsbedarf teils auf die Verstärkung beziehungsweise technische Abnahme akustischer Signale sowie teils auf schrift- oder gebärdensprachgestützte Übersetzungen von Lautsprache. Diese Unterstützungsmöglichkeiten richten sich an all diejenigen Personen, die in ihrem Hörvermögen eingeschränkt sind, wie beispielsweise (altersbegleitend) schwerhörige oder gehörlose Menschen.

\section{Unterstützungsbedarf im Bereich Lesen/Verstehen}

Im Bereich Lesen/Verstehen ist Unterstützungsbedarf immer dann gegeben, wenn Personen (deutscher) Schriftsprache beziehungsweise komplexen sprachlichen Inhalten nur eingeschränkt folgen können. Dies betrifft Personen, die von (funktionalem) Analphabetismus betroffen sind ebenso wie einige Menschen mit Lernschwierigkeiten, Menschen mit Demenz oder ganz allgemein Menschen, für die Deutsch eine Fremdsprache ist, die sie (noch) nicht gut beherrschen. Unterstützungsmöglichkeiten liegen hier primär in der Verwendung von Leichter beziehungsweise Einfacher Sprache (siehe dazu Netzwerk Leichte Sprache e.V. o.J.; Stefanowitsch 2014; Oomen-Welke $2015^{4}$ ) und auch in der entsprechenden grafischen Aufbereitung von Inhalten, zum Beispiel anhand von Piktogrammen, die das Geschriebene noch einmal auf anschauliche Art und Weise verdeutlichen.

\section{Unterstützungsbedarf im Bereich Fremdsprache (Englisch)}

Unterstützungsbedarf im Bereich Fremdsprache (Englisch) besteht dann, wenn Inhalte ausschließlich in deutscher Sprache zur Verfügung stehen und damit Personen, die nicht oder nur kaum Deutsch sprechen, verschlossen

4 Mit Leichter Sprache und Barrierefreiheit setzt sich beispielsweise Trescher (2018c, S. 146ff) problematisierend auseinander. 
bleiben. Für diese Personen ist es notwendig, dass Inhalte auch in anderen Sprachen zur Verfügung gestellt werden. Hier liegt ein besonderer Fokus auf der englischen Sprache, da diese, wie sich gezeigt hat, in großen Teilen der Welt verbreitet ist und viele Menschen diese (zumindest rudimentär) verstehen und sich anhand dieser verständigen können - Englisch ist »die Verkehrssprache schlechthin« (Leitner 2009, S. 8) ${ }^{5}$.

Forschungspraktisch wurden in jedem Sozialraum exemplarisch jeweils zehn Einrichtungen des öffentlichen Lebens (zum Beispiel Veranstaltungsorte, Einkaufszentren, Schwimmbäder, Bibliotheken etc. sowie das Bürgerbüro beziehungsweise die Stadtverwaltung) hinsichtlich ihrer (barrierefreien) Ausgestaltung untersucht. Bei der Beurteilung der Einrichtungen hinsichtlich ihrer Barrierefreiheit werden fünf Kategorien unterschieden, die im Forschungsprozess sukzessive herausgearbeitet wurden. Diese sind im Folgenden übersichtlich abgebildet.

\section{Tabelle 6: Kategorien der Einschätzung von Barrierefreiheit}

\begin{tabular}{|l|l|}
\hline$\checkmark$ & $\begin{array}{l}\text { barrierefrei } \\
\text { Diese Einschätzung wurde gewählt, wenn alle Angebote beziehungs- } \\
\text { weise Orte einschränkungslos nutzbar/erreichbar waren. }\end{array}$ \\
\hline$\times$ & $\begin{array}{l}\text { nicht barrierefrei } \\
\text { Internetseiten, Orte und Tätigkeiten werden dann als >nicht barriere- } \\
\text { frei< kategorisiert, wenn sie in keiner Hinsicht nutzbar/erreichbar } \\
\text { waren. }\end{array}$ \\
\hline \multirow{6}{*}{$\checkmark$} & $\begin{array}{l}\text { eingeschränkt barrierefrei } \\
\text { Die Kategorie >eingeschränkt barrierefrei< kommt immer dann zum } \\
\text { Tragen, wenn nur einzelne Angebote beziehungsweise Bereiche bar- } \\
\text { rierefrei nutzbar sind oder Barrierefreiheit ausschließlich durch die } \\
\text { Nutzung eines Sonderweges oder durch eine Assistenz/Begleitperson } \\
\text { gewährleistet wird. }\end{array}$ \\
\hline
\end{tabular}

5 Dass dadurch dennoch zahlreiche Personen ausgeschlossen werden, die Englisch nicht verstehen und sprechen können, ist klar. Es bedurfte allerdings einer forschungspraktischen Idee davon, wie die Dimension >Fremdsprache s sinnhaft operationalisiert werden konnte, weshalb sich nach eingehender Recherche darauf verständigt wurde, diese auf die englische Sprache engzuführen. 


\begin{tabular}{|l|l|}
\hline \multirow{5}{*}{} & $\begin{array}{l}\text { Frage nicht sinnhaft } \\
\text { Diese Kategorisierung wurde beispielsweise in Bezug auf die Nutzung } \\
\text { von Internetseiten im Kontext von Mobilitätsbeeinträchtigungen } \\
\text { gewählt, da Personen mit Mobilitätsbeeinträchtigungen laut des } \\
\text { interviewten Selbstvertreters/der interviewten Selbstvertreterin bei } \\
\text { der Nutzung des Internets nicht an Barrieren stoßen. }\end{array}$ \\
\hline & $\begin{array}{l}\text { keine Angabe } \\
\text { Mit dieser Kennzeichnung wurden Orte versehen, bei denen, trotz } \\
\text { mehrfacher Kontaktversuche, keine entsprechenden Informationen } \\
\text { erfragt werden konnten. }\end{array}$ \\
\hline
\end{tabular}

\subsubsection{Kriterien zur Kategorisierung öffentlicher Orte als (nicht) barrierefrei}

Im Folgenden ist dargelegt, wie bei der Kategorisierung von Internetseiten, Orten und dort stattfindenden Aktivitäten hinsichtlich der barrierefreien Zugänglichkeit vorgegangen und aufgrund welcher Parameter entschieden wurde, ob ein Ort (eingeschränkt) barrierefrei ist oder nicht. Dies war nicht immer ohne Weiteres festzulegen, weshalb hier exemplarisch entsprechende Grenzfälle diskutiert werden. Dadurch wird zudem der Kategorisierungsprozess transparent und nachvollziehbar gemacht.

\section{Barrierefreiheit der Internetseite}

Bei der Überprüfung des Internetauftritts des jeweiligen Ortes beziehungsweise Angebots hinsichtlich der barrierefreien Zugänglichkeit wurde sich primär an den Richtlinien orientiert, die in der aktualisierten BarrierefreieInformationstechnik-Verordnung (BITV 2.0) festgehalten sind. Grundlegend muss dem vorausgeschickt werden, dass, unabhängig der vorgängig gebildeten Unterstützungsbedarfe, Barrieren dadurch entstehen können, dass ein Zugang zum Internet zur Verfügung stehen muss, was beispielsweise in Bezug auf stationäres Wohnen von Menschen mit geistiger Behinderung nicht immer gegeben ist (Trescher 2017f, S. 133ff, 2018c, S. 142ff) und dass die betreffende Person über das kulturelle Wissen verfügen muss, das Internet für sich zu nutzen und sich auf entsprechenden Seiten zurechtzufinden. Gerade viele ältere und alte Menschen sind davon also bereits ausgeschlossen, da sie häufig nicht über einen Internetanschluss beziehungsweise das entsprechende ExpertInnenwissen verfügen (Tesch-Römer et al. 2016; Seifert 2016). 
- Mobilität: Da die Frage nach barrierefreien Internetseiten im Bereich Mobilität nicht sinnhaft ist, findet sich in der Recherchedokumentation an diesen Stellen ein »-«.

- Sehen: Im Bereich Sehen wurde eine Internetseite dann als barrierefrei kategorisiert, wenn es Möglichkeiten gab, die Schriftgröße oder den Kontrast $\mathrm{zu}$ ändern, eine Vorlesefunktion vorhanden war und wenn Bilder einen sogenannten Alternativtext eingebettet hatten, der diese beschreibt (vgl. BITV 2.0). Nach Aussage des Selbstvertreters/der Selbstvertreterin benötigen viele blinde oder hochgradig sehbehinderte Menschen nicht zwingend eine Sprachausgabe, da »die überwiegenden blinden, hochgradig sehbehinderten Menschen, die mit PC und Internet arbeiten, sowieso mit Sprachausgaben arbeiten«. Darüber hinaus profitieren auch andere Personengruppen wie beispielsweise (funktionale) AnalphabetInnen oder Menschen mit (mehr oder minder ausgeprägten) Seheinschränkungen, wie es auf viele Menschen im Alter zutrifft, von einer Sprachausgabe. War nur einer der genannten Aspekte gegeben, so wurde die Internetseite als seingeschränkt barrierefrei< kategorisiert.

- Hören: Im Bereich Hören wurde eine Internetseite dann als barrierefrei bezeichnet, wenn es eingebettete Videos gab, die den Text in die Deutsche Gebärdensprache übersetzen. Nach Aussage des Selbstvertreters/ der Selbstvertreterin »brauchen 80 Prozent der Gehörlosen diese Videos tatsächlich«. Dies war, wie hier problematisiert werden kann, in keinem der untersuchten Fälle gegeben. An dieser Stelle besteht also noch deutliches Weiterentwicklungspotenzial. Waren nur einzelne Bereiche der Internetseite (zum Beispiel die Hauptseite) in Deutscher Gebärdensprache verfügbar, so wurde die Seite als reingeschränkt barrierefreiく kategorisiert.

- Lesen/Verstehen: Im Bereich Lesen/Verstehen wurde eine Internetseite immer dann als barrierefrei bezeichnet, wenn sie in Leichter Sprache verfasst ist beziehungsweise alle Inhalte zusätzlich in Leichter Sprache zur Verfügung stehen. Als reingeschränkt barrierefrei wurde eine Internetseite dann kategorisiert, wenn nur einzelne Seiten in Leichter Sprache zur Verfügung stehen (zum Beispiel die Hauptseite), weiterführende Informationen jedoch nicht.

- Fremdsprache (Englisch): Im Bereich Fremdsprache (Englisch) wurde eine Internetseite dann als barrierefrei verzeichnet, wenn die Sprache der Seite von Deutsch auf Englisch umgestellt werden kann. Auch hier galt 
die Kategorie seingeschränkt barrierefrei<, wenn nur einzelne Teile der Internetseite in englischer Sprache verfügbar sind.

In der Forschungspraxis selbst war es nicht immer einfach zu entscheiden, ob eine Internetseite barrierefrei ist oder nicht, insbesondere da (trotz der Interviews mit den SelbstvertreterInnen) teilweise die handlungspraktische Expertise als NutzerIn mit Unterstützungsbedarf fehlte ${ }^{6}$. Im Hinblick auf die Verfügbarkeit der Informationen in sogenannter Leichter Sprache war es beispielsweise häufiger so, dass es zwar Informationen auch in Leichter Sprache gab, diese jedoch auf der Internetseite gleichsam >versteckt waren, sodass gezielt danach gesucht werden musste. Dies stellte die ForscherInnen vor die Herausforderung, $\mathrm{zu}$ entscheiden, ob eine solche Internetseite noch als barrierefrei oder bereits als eingeschränkt barrierefrei zu kategorisieren ist. Denn gerade für Personen, die Unterstützungsbedarfe im Bereich Lesen/Verstehen haben, ist die Navigation auf einer Internetseite potenziell schwieriger und mit Mühen verbunden. Des Weiteren kam es immer wieder vor, dass Internetseiten, die als »barrierefrei« betitelt sind, nicht beziehungsweise nur in einzelnen Bereichen als (eingeschränkt) barrierefrei kategorisiert werden konnten, da unter anderem auf keiner der untersuchten Internetseiten Videos in Deutscher Gebärdensprache vorhanden waren.

\section{Barrierefreiheit des Ortes}

Der jeweilige Ort, an dem die Tätigkeit/Veranstaltung etc. stattfindet, wurde ebenfalls hinsichtlich seiner barrierefreien Ausgestaltung analysiert. Bei der Erhebung wurden Informationen herangezogen, die auf der Internetseite zur Verfügung stehen, sowie, sollte anhand dieser Informationen die Frage nicht beantwortet werden können, per E-Mail beziehungsweise telefonisch Kontakt mit Verantwortlichen vor Ort aufgenommen, um die Information zu erfragen. Dies hatte zur Folge, dass sich bei der anschließenden Kategorisierung auf die Einschätzung der kontaktierten Person verlassen werden musste. Diese forschungspraktische Einschränkung wurde nach Abwägen alternativer Erhebungsmöglichkeiten hingenommen, da sich der Frage so am sinnvollsten und forschungsökonomischsten genähert werden konnte.

6 Hier sind deutliche Weiterentwicklungspotenziale hinsichtlich partizipativer Forschungsmethoden auszumachen. 
- Mobilität: Im Bereich Mobilität wurde ein Ort dann als barrierefrei kategorisiert, wenn alle Bereiche des Ortes von Menschen mit Mobilitätseinschränkungen aufgesucht werden können - gegebenenfalls mit Hilfsmitteln wie zum Beispiel Aufzug, Lifter, Rampe etc. Orte sind auch dann barrierefrei hinsichtlich Mobilität, wenn sie ebenerdig beziehungsweise ohne Stufen zugänglich sind. >Eingeschränkt barrierefrei< sind dagegen alle Orte, in denen nicht alle Bereiche für Menschen mit Mobilitätseinschränkungen erreichbar sind (beispielsweise, wenn ein Aufzug nicht in allen Stockwerken hält oder Ähnliches).

- Sehen: Barrierefreiheit im Bereich Sehen ist immer dann gegeben, wenn für Menschen mit Sehbeeinträchtigungen alle Bereiche zugänglich sind und zwar ohne fremde Hilfe. Dazu ist beispielsweise ein haptischer Orientierungsstreifen auf dem Fußboden (sogenanntes Blindenleitsystem) notwendig. Die Kategorisierung als reingeschränkt barrierefrei< erfolgte immer dann, wenn es einer sehbeeinträchtigten beziehungsweise blinden Person nicht möglich ist, alle Bereiche ohne die Unterstützung einer Begleitperson zu erreichen. Im Forschungsprozess erwies es sich teils, trotz des Interviews mit einem/einer blinden SelbstvertreterIn, als schwierig, diese Unterscheidung zu treffen, da von den ortsfremden und nicht sehbeeinträchtigten ForscherInnen nicht immer optimal eingeschätzt werden konnte, ob alle Bereiche des Ortes hinsichtlich des Unterstützungsbedarfs Sehen zugänglich sind. Infolgedessen musste sich in diesen Fällen auf eine Einschätzung der befragten Person verlassen werden, die allerdings diesbezüglich zusätzlich auf Erfahrungswerte mit sehbeeinträchtigten beziehungsweise blinden BesucherInnen des Ortes zurückgreifen konnte, was als ein Gewinn angesehen wird. Deutlich wird nichtsdestotrotz, dass eine Generalisierung schwierig ist.

- Hören: Im Bereich Hören wurde sich, nach Rücksprache mit dem/der gehörlosen SelbstvertreterIn darauf verständigt, dass die Frage nach der Barrierefreiheit des (physischen) Ortes in Bezug auf Unterstützungsbedarf im Bereich Hören sehr vom Einzelfall abhängig ist und dass, auch wenn es Möglichkeiten der Unterstützung bei der Nutzung von Orten gibt (beispielsweise Lichtsignale bei Gefahr, akustische Durchsagen zusätzlich als visuelle Anzeigen etc.), im Allgemeinen die Frage nach der Barrierefreiheit des Ortes nicht sinnhaft ist, da Menschen mit Hörbeeinträchtigungen zumeist vor keinen besonderen Herausforderungen bei der Nutzung eines Ortes stehen. Barrieren können bei der Rezeption 
von Angeboten (zum Beispiel im Kino, Theater) und bei der Auseinandersetzung mit nicht gebärdensprachkompetenten Personen (zum Beispiel Verkaufspersonal) entstehen, jedoch weniger bei der Aneignung des Ortes an sich. Infolgedessen wurde bei der überblicksartigen Darstellung der Ergebnisse an dieser Stelle ein Strich ( $>-<)$ eingefügt, der anzeigt, dass die Frage nicht sinnhaft ist.

- Lesen/Verstehen: Im Bereich Lesen/Verstehen wurde ein Ort als barrierefrei kategorisiert, wenn Beschilderungen ohne besondere Lesekompetenzen nachvollziehbar waren beziehungsweise mit Piktogrammen unterstützt wurden. `Eingeschränkt barrierefrei< war ein Ort, wenn nur einzelne Bereiche dementsprechend ausgeschildert waren.

- Fremdsprache (Englisch): Im Bereich Fremdsprache (Englisch) wurde die Kategorie sbarrierefrei< gewählt, sobald Beschilderungen nicht nur in Deutsch, sondern auch in Englisch vorhanden waren. Die Kategorisierung seingeschränkt barrierefrei< wurde dementsprechend immer dann vorgenommen, wenn nur einzelne Bereiche (zusätzlich) in Englisch ausgeschildert waren.

In Bezug auf Orte war die Kategorisierung insbesondere in den Fällen problematisch, in denen die Informationen auf der Internetseite im Gegensatz zu dem standen, was zusätzlich per E-Mail oder telefonisch bei den Verantwortlichen vor Ort erfragt wurde. Beispielsweise zeigte sich bei näherem Nachfragen sehr häufig, dass unter ১barrierefreier Zugänglichkeit< ausschließlich der uneingeschränkte Zugang für RollstuhlnutzerInnen verstanden wird. Hieran wird deutlich, dass das routinemäßig vorherrschende Verständnis von Barrierefreiheit (noch immer) einseitig auf die Definition zzugänglich für RollstuhlnutzerInnen< beschränkt ist. Hier eröffnet sich also eine Perspektive für unter anderem Öffentlichkeitsarbeit, die hinsichtlich Fragen von Barrierefreiheit sensibilisiert und einen differenzierteren $\mathrm{Zu}$ gang dazu etabliert.

\section{Barrierefreiheit des Angebots/der Tätigkeit}

In einem dritten Schritt wurde die konkrete Tätigkeit, die am jeweils untersuchten Ort ausgeübt wird, in den Fokus der Untersuchung genommen. Hier stand die Frage im Vordergrund, ob und inwiefern Personen mit unterschiedlichen Unterstützungsbedarfen am jeweiligen Angebot beziehungsweise der jeweiligen Tätigkeit teilnehmen können (zum Beispiel ein 
Fußballspiel im Stadion anschauen, ein Theaterstück ansehen, ein Konzert besuchen oder Ähnliches). Ebenso wie bei der Frage nach der Barrierefreiheit des Ortes wurde hier, sollte es notwendig sein, nach einer Recherche auf der Internetseite, Kontakt zu Verantwortlichen vor Ort aufgenommen (per E-Mail oder telefonisch), um die Frage zu klären, ob und inwiefern die Tätigkeit barrierefrei in den hier zugrunde gelegten Dimensionen ist.

- Mobilität: Im Bereich Mobilität wurde eine Tätigkeit als barrierefrei kategorisiert, wenn der Ort barrierefrei zugänglich war. Ebenso wurde im eventuell vorliegenden Fall einer >eingeschränkten Barrierefreiheit verfahren, denn die Nutzung der Tätigkeit war gegeben, sobald der Ort der Tätigkeit aufgesucht werden konnte.

- Sehen: Im Bereich Sehen wurde eine Tätigkeit als barrierefrei kategorisiert, wenn eine akustische Begleitung beziehungsweise Beschreibung gegeben war (zum Beispiel Hörfassungen im Kino). ।Eingeschränkt barrierefrei< war eine Tätigkeit dann, wenn nur einzelne Angebote barrierefrei waren (zum Beispiel Hörfassungen nur ausgewählter Filme).

- Hören: Im Bereich Hören wurde die Kategorie barrierefrei gewählt, sobald entsprechende Unterstützungssysteme routinemäßig vorhanden waren (zum Beispiel eine Induktionsanlage) beziehungsweise nach entsprechender Anmeldung zur Verfügung gestellt wurden (zum Beispiel DolmetscherInnen für Deutsche Gebärdensprache). Konnten diese Unterstützungsangebote nur in einzelnen Bereichen beziehungsweise nur bei ausgewählten Veranstaltungen angeboten werden, so wurde die Tätigkeit als reingeschränkt barrierefrei< kategorisiert.

- Lesen/Verstehen und Fremdsprache (Englisch): In den Bereichen Lesen/Verstehen und Fremdsprache (Englisch) wurde eine Tätigkeit als barrierefrei kategorisiert, sobald sie (auch) in Leichter Sprache beziehungsweise in Englisch angeboten wurde. Wurden nur einzelne Veranstaltungen in Leichter Sprache beziehungsweise in Englisch abgehalten, so wurde die Tätigkeit als >eingeschränkt barrierefrei< kategorisiert. Auch in diesem Zusammenhang wurde klar, dass die Frage nicht in Bezug auf alle Tätigkeiten sinnvoll ist, beispielsweise bei einem Fußballspiel oder einem 
Konzert, bei denen im Allgemeinen kein Lesen oder Verstehen auf einem hohen Niveau notwendig sind?

Die Einschätzung, ob und inwiefern ein Angebot beziehungsweise eine konkrete Tätigkeit barrierefrei ist, war mit am schwierigsten zu treffen, denn zumeist bestand das Angebot aus mehreren möglichen Aktivitäten (zum Beispiel im Schwimmbad: Schwimmen in Kursen, Schwimmen ohne Kurse, Sauna etc.) und es musste festgelegt werden, auf welche der Aktivitäten sich die Kategorisierung bezieht. Zudem besteht eine generelle Schwierigkeit der Kategorisierung darin, dass sich Fragen von Teilnahme und Teilhabe primär in Praxen des Aushandelns in der konkreten Situation vollziehen und eine vorgängige Kategorisierung deshalb nur eingeschränkt möglich ist. Trotz dieser forschungspraktischen Einschränkungen konnten in vielen Fällen potenzielle Teilhabebarrieren aufgedeckt werden, an die Menschen mit unterschiedlichen Unterstützungsbedarfen stoßen (können). Zur Veranschaulichung von Herausforderungen bei der Kategorisierung soll hier exemplarisch der Fall eines Kletterwaldes in einem der untersuchten Sozialräume herangezogen werden. Der Kletterwald verfügt über einen Rollstuhlparcours, in dem Menschen, die einen Rollstuhl nutzen, das Angebot des Kletterwaldes, zumindest in einem gesonderten Parcours, in Anspruch nehmen können. Problematisiert werden kann dabei, dass der besagte Parcours am Ein- und Ausgang mit einer Stufe ausgestattet ist, damit, wie der O-Ton des auf der Internetseite eingestellten Videos über den Rollstuhlparcours verdeutlicht, RollstuhlfahrerInnen »nicht ganz ohne Aufsicht auf Tour gehen«. Dies schränkt die scheinbare Barrierefreiheit massiv ein und macht Menschen, die einen Rollstuhl nutzen, abhängig von einer Begleitperson. Gleichzeitig werden dadurch überhaupt erst Teilhabemöglichkeiten für Menschen mit Unterstützungsbedarfen im Bereich Mobilität eröffnet - wenn auch mit Einschränkungen. Barrierefreiheit ist also eine durchaus ambivalente Praxis, die der Aushandlung bedarf, wobei wiederum Barrieren entstehen können. Dabei kommt zudem zum Tragen, dass in Überlegungen zu Barrierefreiheit »immer erst Barrieren manifestiert werden, bevor ausge-

7 Barrieren liegen hier eher darin, Informationen über Veranstaltungen zu beschaffen. Dieser Aspekt war nicht Teil der Untersuchung, könnte jedoch für eine weitergehende, zukünftige Studie herangezogen werden. 
hend davon darüber nachgedacht wird, wie diese abgebaut werden können « (Trescher 2018c, S. 16; siehe Kapitel 5.4).

\subsubsection{Kategorisierung öffentlicher Orte als (nicht) barrierefrei}

Die Ergebnisse der Kategorisierung von Internetseiten, Orten und Tätigkeiten hinsichtlich ihrer barrierefreien Zugänglichkeit zeigen schließlich, dass in allen fünf Sozialräumen bereits ein gewisses Bewusstsein für diverse $\mathrm{Zu}$ gangsbedarfe besteht und auch einiges dafür unternommen wurde, barrierefreie Teilhabe zu ermöglichen. Dennoch zeigt sich immer wieder und an vielen Stellen, inwiefern nach wie vor Barrieren manifest sind und somit die Teilhabemöglichkeiten von Menschen mit je bestimmten Unterstützungsbedarfen einschränken. Da sich die Ergebnisse der Kategorisierung quer über die Sozialräume kaum unterscheiden, wird im Folgenden exemplarisch ein Einblick in die Ergebnisse der Untersuchung eines der fünf Sozialräume gegeben. Ein Blick in die Ergebnistabelle zeigt, dass ein Großteil der untersuchten Einrichtungen nicht barrierefrei zugänglich ist sowie die jeweils darin stattfindenden Aktivitäten nicht barrierefrei rezipiert werden können (entlang der oben explizierten Kriterien). Als Ort, an dem Menschen mit Unterstützungsbedarfen an besonders umfassende Barrieren stoßen, stellt sich das untersuchte Theater (2) heraus. Dies ist in keinerlei Hinsicht barrierefrei (außer der Ort selbst in Bezug auf die Kategorie Lesen/Verstehen). Deutlich wurde zudem, dass auf so gut wie keiner Internetseite die Informationen derart aufbereitet wurden, dass diverse NutzerInnen sie barrierefrei abrufen können. Hier besteht also das Potenzial zu Veränderung - nicht nur in den untersuchten Sozialräumen, sondern auch darüber hinaus (siehe dazu Makarova et al. 2016; Bernasconi 2007). 
Tabelle 7: Öffentliche Orte und Barrierefreiheit in einem untersuchten Sozialraum

\begin{tabular}{|c|c|c|c|c|c|c|c|c|c|c|c|}
\hline \multicolumn{2}{|c|}{$\begin{array}{l}\text { Barrierefrei- } \\
\text { heit }\end{array}$} & \multirow{2}{*}{$\begin{array}{l}\text { 올 } \\
\text { 言 } \\
\text { 言 }\end{array}$} & \multirow{2}{*}{ 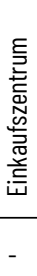 } & \multirow{2}{*}{ 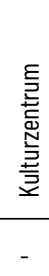 } & \multirow{2}{*}{ 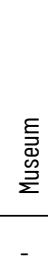 } & \multirow{2}{*}{ 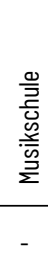 } & \multirow{2}{*}{ 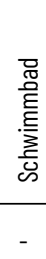 } & \multirow{2}{*}{ 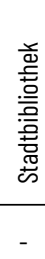 } & \multirow{2}{*}{ 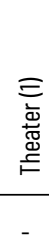 } & \multirow{2}{*}{ 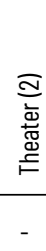 } & \multirow{2}{*}{ 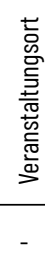 } \\
\hline \multirow{5}{*}{ 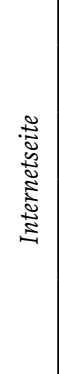 } & Mobilität & & & & & & & & & & \\
\hline & Sehen & $(\checkmark)$ & $x$ & $x$ & $(\checkmark)$ & $x$ & $x$ & $(\checkmark)$ & $x$ & $x$ & $x$ \\
\hline & Hören & $x$ & $x$ & $x$ & $x$ & $x$ & $x$ & $x$ & $x$ & $x$ & $x$ \\
\hline & $\begin{array}{l}\text { Lesen/ } \\
\text { Verstehen }\end{array}$ & $x$ & $x$ & $x$ & $x$ & $x$ & $x$ & $(\checkmark)$ & $(\checkmark)$ & $x$ & $x$ \\
\hline & $\begin{array}{l}\text { Fremd- } \\
\text { sprachen }\end{array}$ & $\checkmark$ & $x$ & $x$ & $\checkmark$ & $x$ & $x$ & $\checkmark$ & $x$ & $x$ & $x$ \\
\hline \multirow{5}{*}{$\overrightarrow{0}$} & Mobilität & $\checkmark$ & - & $\checkmark$ & $\checkmark$ & $(\checkmark)$ & $\checkmark$ & $\checkmark$ & $(\checkmark)$ & $x$ & $\checkmark$ \\
\hline & Sehen & $x$ & $x$ & $x$ & $x$ & k.A. & $\checkmark$ & $(\checkmark)$ & $x$ & $x$ & $\checkmark$ \\
\hline & Hören & - & $x$ & - & - & - & - & - & - & - & - \\
\hline & $\begin{array}{l}\text { Lesen/ } \\
\text { Verstehen }\end{array}$ & $x$ & $x$ & $\checkmark$ & $x$ & k.A. & $\checkmark$ & $\checkmark$ & $\checkmark$ & $\checkmark$ & $\checkmark$ \\
\hline & $\begin{array}{l}\text { Fremd- } \\
\text { sprachen }\end{array}$ & $\checkmark$ & $x$ & $x$ & $x$ & k.A. & $(\checkmark)$ & $x$ & $x$ & $x$ & $x$ \\
\hline \multirow{5}{*}{ 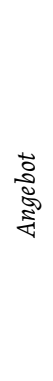 } & Mobilität & $\checkmark$ & - & $\checkmark$ & $\checkmark$ & $(\checkmark)$ & - & $\checkmark$ & $(\checkmark)$ & $x$ & $\checkmark$ \\
\hline & Sehen & $\checkmark$ & $x$ & $(\checkmark)$ & $x$ & - & - & $x$ & $x$ & $x$ & $\checkmark$ \\
\hline & Hören & $x$ & $x$ & $(v)$ & $x$ & - & - & $\checkmark$ & $(\checkmark)$ & $x$ & $\checkmark$ \\
\hline & $\begin{array}{l}\text { Lesen/ } \\
\text { Verstehen }\end{array}$ & $x$ & $x$ & k.A. & $x$ & $(\checkmark)$ & - & $\checkmark$ & $x$ & $x$ & $(\checkmark)$ \\
\hline & $\begin{array}{l}\text { Fremd- } \\
\text { sprachen }\end{array}$ & $\checkmark$ & $x$ & $(\checkmark)$ & $x$ & $(\checkmark)$ & - & $\checkmark$ & $x$ & $x$ & $(\checkmark)$ \\
\hline
\end{tabular}


Bezüglich der Gesamtergebnisse wird deutlich, dass die untersuchten Sozialräume sich kaum darin unterscheiden, welche Personen mit welchen Unterstützungsbedarfen am ehesten an Barrieren stoßen beziehungsweise sich in einer nicht barrierefrei gestalteten Umwelt zurechtfinden müssen. Es kann also nicht gesagt werden, dass in einem bestimmten Sozialraum mehr barrierefreie Zugänge für Personen mit einem je bestimmten Unterstützungsbedarf bereitstehen. Hervorgehoben werden muss zudem, dass kein öffentlicher Ort verzeichnet wurde, der in allen untersuchten Dimensionen einschränkungslos barrierefrei genutzt beziehungsweise begangen werden kann (dies betrifft sowohl Internetseite, Ort und Angebot als auch die fünf untersuchten Ausprägungen von Barrierefreiheit). Barrieren liegen also vielfach und in zahlreichen Dimensionen vor. Dabei kann im Vergleich auch kein Typ von Orten ausgemacht werden, der in allen untersuchten Sozialräumen besonders zahlreiche Barrieren aufweist oder im Gegenteil barrierefrei in vielerlei Hinsicht ist. Vielmehr ist es so, dass sich die Orte mit der geringsten Barrierefreiheit in allen untersuchten Sozialräumen unterscheiden - ein Muster ist nicht zu erkennen. Interessant ist zudem, dass in keinem der Sozialräume der Internetauftritt des jeweiligen Bürgerbüros in allen untersuchten Dimensionen barrierefrei war. Zumeist war es vielmehr so, dass keine barrierefreien Inhalte zur Verfügung standen. Hier besteht in allen untersuchten Sozialräumen Entwicklungspotenzial und es wird die Frage aufgeworfen, wie sich die Stadt ihren BürgerInnen präsentiert und Informationen zur Verfügung stellt, insbesondere jenen, die oftmals an Barrieren stoßen und deshalb von der Lebenspraxis der Mehrheitsgesellschaft ein Stück weit abgeschnitten sind. Als ein positives Beispiel kann das Rathaus der Verbandsgemeinde Nieder-Olm herausgegriffen werden, in dem sich ganz offensichtlich bereits umfänglicher mit Fragen von Barrierefreiheit beschäftigt wurde, was nicht zuletzt die ethnographischen Sozialraumbegehungen vor Ort bestätigen (siehe Kapitel 13). Gleichzeitig muss problematisiert werden, dass es sich bei diesem Positivbeispiel um einen Einzelfall handelt. Übergreifend zeigt sich, dass insbesondere Menschen mit Sehbeeinträchtigungen und Menschen mit Hörbeeinträchtigungen besonders von Ausschluss bedroht oder betroffen sind, da in diesen Dimensionen kaum barrierefreie Orte gefunden wurden. Menschen mit Unterstützungsbedarfen im Bereich Lesen/Verstehen folgen dicht darauf und stoßen insofern ebenfalls sehr häufig an Barrieren bei der Nutzung öffentlicher Orte 
in ihrem Sozialraum ${ }^{8}$. Ein Problem, das dadurch annehmbar reproduziert wird, ist die Abhängigkeit von Begleitpersonen und Assistenzen, was nicht nur soziale, sondern auch finanzielle Auswirkungen hat. Denn die jeweiligen Personen werden dadurch (a) zusätzlich ๖besondert und es ist für sie möglicherweise schwieriger, in statusgleichen Kontakt mit Personen aus der Mehrheitsgesellschaft zu treten. Zudem ist die Abhängigkeit von Assistenz (b) zumeist eine deutlich merkbare finanzielle Belastung, sollte die Assistenz nicht aus dem privaten Umfeld akquiriert werden können, sondern in persona Beschäftigter der Behindertenhilfe, die für ihre Dienste entlohnt werden. Möglicherweise geht damit zudem ein hoher bürokratischer Aufwand einher, entsprechende (finanzielle) Unterstützungsleistungen zu beantragen. Flexibilität und Spontaneität, beispielsweise ohne längere Vorbereitung und Organisation ein Museum zu besuchen, werden dadurch merklich eingeschränkt, was wiederum eine Teilhabe an Praxen der Mehrheitsgesellschaft erschwert.

\subsection{Gesamtbetrachtung und Diskussion}

Nachdem das methodische Vorgehen erläutert und die Ergebnisse dargelegt wurden, werden hier die zentralen Ergebnisse noch einmal zusammengetragen und diskutiert. Die Gesamtbetrachtung und Diskussion erfolgt dabei jeweils auf theoretischer, methodischer und handlungspraktischer Ebene. In allen danach folgenden Kapitel wird ebenso verfahren.

Theoretische Gesamtbetrachtung und Diskussion Auf theoretischer Ebene eröffnen die Ergebnisse der Analyse öffentlicher Orte im Kontext Barrierefreiheit insbesondere raumtheoretische Anschlüsse, wobei die Frage im Vordergrund steht, wer sich welchen Ort auf welche Art und Weise aneignet beziehungsweise aneignen kann. Indem Personen mit bestimmten Unterstützungsbedarfen der Zugang zu Orten im Sozialraum erschwert wird, können sie sich diese primär als »Territorium der Anderen " (Trescher und Hauck 2017) aneignen (siehe auch Kapitel 5). Jene Personen werden dadurch behindert und in ihrer Teilhabe an Praxen der Mehrheitsgesellschaft eingeschränkt, was anschaulich verdeutlicht, inwie-

8 Dies ist ein Ergebnis, das in den Sozialraumanalysen in den Handlungsfeldern Arbeit und Freizeit bestätigt wird (siehe Kapitel 16 und Kapitel 17). 
fern sich Behinderung, wie einleitend dargelegt (siehe Kapitel 4.1), als Praxis vollzieht, die Ausschluss und >Besonderung` produziert und Menschen folglich als ausgeschlossen und besonders< hervorbringt. Problematisiert werden kann in dieser Hinsicht und mit Blick auf die Ergebnisse, dass sogenannte Barrierefreiheit weitestgehend als etwas postuliert wird, das Teilhabe ermöglicht oder sogar mit IInklusion gleichgesetzt wird (u.a. Behrendt 2017, S. 51f; Moser 2010, S. 83f) und insofern behindernde Praxen abbauen soll. Bis zu einem gewissen Grad ist das sicherlich zutreffend, können doch durch sogenannte barrierefreie Gestaltung von beispielsweise Orten $\mathrm{Zu}$ gangsmöglichkeiten für Personen geschaffen werden, die bislang ausgeschlossen waren. Völlig frei von Barrieren, wie der Begriff suggeriert, werden Orte dadurch jedoch nicht. Zudem stimmt dies nicht mit dem überein, was (zumindest im hier zugrunde gelegten Theorem) unter Inklusion verstanden wird. Inklusion ist eine Praxis, die sich vollziehen muss und kein Zustand, der hergestellt werden kann. Ungeklärt ist dabei auch, wie mit Orten und Praxen umzugehen ist, die auch nach entsprechender sogenannter barrierefreier Gestaltung nicht uneingeschränkt durch alle Personen genutzt werden können. Häufig bleibt hier lediglich die Möglichkeit zur Sondernutzung, was allerdings wiederum eine behindernde Praxis darstellt, die die jeweilige Person als >besonders und unterstützungsbedürftig hervorbringt. Barrierefreiheit respektive die damit verbundenen und darin begründeten Praxen sind also durchaus ambivalent. Ein weiterer Aspekt, der problematisierend aufgegriffen wird, betrifft sogenannte Leichte Sprache und die Verknüpfung dieser mit dem Postulat barrierefreier Teilhabe für Menschen mit Unterstützungsbedarfen Lesen/Verstehen. Dabei wird zumeist nicht bedacht, dass Leichte Sprache, ebenso wie Barrierefreiheit, als sehr ambivalent zu betrachten ist. Leichte Sprache kann zwar Zugänge ermöglichen, wird allerdings aufgrund ihrer Reduktion teilweise als infantilisierend oder beleidigend wahrgenommen (Trescher 2018c, S. 146f) und kann zudem komplexe Inhalte nicht immer umfassend und abstrichslos transportieren (Kupke und Schlummer 2010, S. 70ff). Kritisiert wird teilweise auch, dass Leichte Sprache aus sprachwissenschaftlicher Sicht nicht ausreichend fundiert ist (Zurstrassen 2015, S. 128f). Gleichzeitig existieren zahlreiche sogenannte Übersetzungsbüros für Leichte Sprache, was verdeutlicht, inwiefern es sich dabei in gewisser Weise um ein für einzelne Personen lukratives Geschäftsmodell handelt (Zurstrassen 2015, S. 131). Es kann gesagt werden, dass Leichte Sprache primär auf die Legitimation des Gegenstandes ab- 
zielt und aus einem äußeren >Inklusionsdruck heraus entstand (Reichstein 2016, S. 81). Dabei »entbehrt [es] nicht einer gewissen Ironie, dass im Zuge der Inklusionsdebatte eine eigene Sprache für Menschen mit Lernschwierigkeiten entwickelt wird« (Zurstrassen 2015, S. 130). Zentral ist jedoch die Problematik, dass Leichte Sprache nicht allen Personen, die durch sie adressiert werden, automatisch Teilhabe ermöglicht. Vielmehr werden bestimmte Personen (erneut) ausgeschlossen, wodurch Leichter Sprache eine gewisse exkludierende Wirkmächtigkeit zukommt (Dannenbeck 2012, S. 59; Zurstrassen 2015, S. 129). Denn Personen, die nicht lesen können, erfahren auch durch Leichte (Schrift-)Sprache keinen Abbau von Barrieren (Kurzenberger et al. 2012, S. 122). Zudem wird durch das Schaffen einer Art Zweitsprache eine Dichotomie manifestiert, die der von >behindert und >nicht behindert sehr ähnlich ist. Es kann also gesagt werden: "Leichte Sprache überwindet nicht nur Grenzen und schafft individuelle Teilhabeoptionen - Leichte Sprache erzeugt gleichzeitig auch Differenz und reproduziert die binäre Logik von inklusiven/exklusiven Ordnungen « (Dannenbeck 2012, S. 59). Die Konsequenz ist, dass in gewisser Weise einige Personen als >Ausgeschlossene der Ausgeschlossenen hervorgebracht werden, die in ihren Teilhabemöglichkeiten unverändert massiv eingeschränkt werden. Demgegenüber darf nicht unbeachtet bleiben, dass Leichte Sprache von SelbstvertreterInnen in repräsentativer Funktion ebenso gefordert wird wie von einigen Menschen mit Lernschwierigkeiten oder kognitiven Beeinträchtigungen, die in unterschiedlichen Studien interviewt wurden (u.a. Trescher 2018c). Leichte Sprache stellt insofern eine Art Ermächtigungspraxis jenes Personenkreises dar, insbesondere deshalb, da diese eng verknüpft ist mit Bewegungen wie beispielsweise »Mensch zuerst - Netzwerk People First Deutschland e.V.« In einer Studie zum Thema Barrierefreiheit und kognitiver Beeinträchtigung zeigte sich darüber hinaus, dass die interviewten Menschen mit kognitiven Beeinträchtigungen teils sogenannte Leichte Sprache einfordern, da sie davon ausgehen, dadurch Teilhabebarrieren überwinden zu können (Trescher 2018c, S. 132). Es wird also deutlich, dass Leichte Sprache respektive ihre Ausgestaltungspraxis sehr ambivalent ist und dringend einer weitergehenden Untersuchung bedarf.

9 www.menschzuerst.de/pages/startseite/leichte-sprache.php (zuletzt am 26.11.2019). 


\section{Methodische Gesamtbetrachtung und Diskussion}

Mit Blick auf die Ergebnisse kann in methodischer Hinsicht festgehalten werden, dass, trotz des teils differenzierten Einblicks, die Aussagen, die über die Barrierefreiheit einzelner Internetseiten, Orte und Aktivitäten getroffen werden können, ein Stück weit holzschnittartig sind. Es zeigt sich immer wieder, dass durch diese Form der Untersuchung die konkrete Lebenspraxis, in der Barrieren beziehungsweise ihre Überwindung vielfach eine Aushandlungspraxis mit der Umwelt sind, nur eingeschränkt in den Blick genommen werden kann. Dennoch handelt es sich beim letztlich hier Anwendung gefundenen forschungspraktischen Vorgehen um die bestmögliche Näherung der Kategorisierung von öffentlichen Orten als (nicht) barrierefrei. Auch ausgehend von diesem Ergebnis wurde das Verfahren der ethnographischen Sozialraumbegehungen geplant und operationalisiert, das ebenjene subjektive Auseinandersetzung mit Barrieren und Praktiken ihrer Überwindung zum Gegenstand macht (siehe Kapitel 13).

\section{Handlungspraktische Gesamtbetrachtung und Diskussion}

Eine handlungspraktische Reflexion der Ergebnisse macht klar, dass die Ausgestaltung sogenannter Barrierefreiheit oftmals mangelhaft ist und die Teilhabemöglichkeiten zahlreicher Personen im Sozialraum behindert. Es zeigt sich dabei auch, dass Barrierefreiheit als Begriff und Praxis zu statisch ist und oftmals nicht die diversen Bedürfnisse der unterschiedlichen RezipientInnen erfüllen kann. Daraus folgt, dass es flexiblerer Unterstützungssysteme bedarf, die je situativ Anwendung finden können. Wie diese im Konkreten aussehen können, ist eine Frage, die Gestaltung, Verwaltung, Architektur, Selbstvertretung etc. gemeinsam verfolgen sollten, um in kooperativer Zusammenarbeit Ideen zu entwickeln, wie Zugänge eröffnet werden können. Klar ist dabei auch, dass Barrierefreiheit eine Aushandlungspraxis ist, weshalb es insbesondere ein Bewusstsein dafür braucht, durch welche Mechanismen bestimmte Personen von öffentlichen Orten ausgeschlossen werden. Wichtige Fragen sind dabei, was einerseits eingefordert werden kann und welche Veränderungen und Praxen andererseits zumutbar sind. Teil dieser Bewusstseinsbildung ist die Reflexion dessen, sicherzustellen, dass die zur Verfügung gestellte Information, der öffentliche Ort und die darin auszuübende Aktivität nicht losgelöst voneinander betrachtet werden. So ist es beispielsweise im Hinblick auf die Dimensionen Mobilität nicht ausreichend, zwar eine Rampe zum Eingang des örtlichen Schwimm- 
bads zu bauen, jedoch im Inneren keine Umkleidemöglichkeiten für Rollstuhlnutzende und/oder keinen Lifter ins Wasser zur Verfügung zu stellen. Es bedarf also einer Auseinandersetzung mit Barrieren und Möglichkeiten, diese abzubauen, die die Gesamtheit von Information, Ort und Aktivität bedenkt und nicht ausschließlich Einzelaspekte in den Blick nimmt. Eine Ambivalenz von Barrierefreiheit, die auf handlungspraktischer Ebene ebenso reflektiert werden muss wie in theoretischer Hinsicht, besteht darin, dass Unterstützungssysteme zwar Zugänge eröffnen können, demgegenüber allerdings zur >Besonderung der AdressatInnen beitragen, wodurch diese in gewisser Hinsicht als unterstützungsbedürftig etikettiert werden, was wiederum dazu beitragen kann, Zugänge eher zu verschließen als sie zu eröffnen. Was die Recherche ebenfalls offensichtlich macht, ist, dass ein gewisser je individueller Unterstützungsbedarf bedeuten kann, sich intensiv damit auseinandersetzen zu müssen, inwiefern welche Orte barrierefrei sind und welche Art von Unterstützung organisiert werden muss, um den jeweiligen Ort zu besuchen. Handlungspraktisch bedarf es also möglicherweise einer besseren Kennzeichnung und Information über die Barrierefreiheit öffentlicher Orte - insbesondere jenseits der barrierefreien Zugänglichkeit für Rollstuhlnutzende. Darüber hinaus gilt es, zu untersuchen, wie sich je bestimmte, sbarrierefreie Strukturen auf Nutzende auswirken, ob diese Auswirkungen zumutbar sind oder ob Handlungsbedarf besteht, diese zu verändern. $\mathrm{Zu}$ diskutieren wäre hierbei beispielsweise, ob und inwiefern es einer Person zuzumuten ist, einen gesonderten Eingang verwenden zu müssen, da der reguläre Eingang nicht barrierefrei genutzt werden kann. Ein weiterer und schließlich letzter Aspekt, der angesprochen werden soll, betrifft die Niedrigschwelligkeit von Hilfen und Unterstützungsstrukturen. Ist der Aufwand sehr groß, Zugänge zu bestimmten Orten (sowie Informationen und Aktivitäten) zu schaffen, so sinkt womöglich die Bereitschaft, überhaupt teilnehmen zu wollen. Barrierefreiheit sollte auch in dieser Hinsicht nicht weitergehend behindernd wirksam werden.

\section{Strukturdatenrecherche}

Im Rahmen einer Strukturdatenrecherche werden die fünf Sozialräume abstrakt beschrieben, die Gegenstand der hiesigen Studie sind (beispielsweise EinwohnerInnenzahl, Arbeitslosenquote, durchschnittliches Ein- 
kommen etc.). Die Strukturdatenrecherche folgt dabei dem Gedanken, dass "Wirtschaftsstrukturen, Bildungsangebote, räumliche Dichte und Mobilitätsbedingungen, Angebote sozialer Dienstleistungen, lokale Sozialpolitik und vieles mehr [...] einen entscheidenden Einfluss auf das [hat], was im Inklusionsdiskurs als Teilhabe am gesellschaftlichen Leben bezeichnet wird« (Kratz et al. 2016, S. 16). Ziel ist, die untersuchten Sozialräume in ausgesuchten Themenbereichen abstrakt beziehungsweise deskriptiv-statistisch zu beschreiben. Darüber hinaus sind die folgenden drei Punkte relevant:

1. Felderöffnung: Die Felderöffnung ist der erste Schritt dabei, die Sozialräume näher kennenzulernen und (vergleichend) zugänglich zu machen. Folglich soll anhand der Strukturdatenrecherche untersucht werden, wie sich die fünf untersuchten Sozialräume zueinander verhalten und zwar unter anderem in Bezug auf die EinwohnerInnengröße, das durchschnittliche Einkommen, den sogenannten Mietspiegel etc. Darüber hinaus stehen zielgruppenspezifische Fragen im Vordergrund:Wie viele Menschen mit Behinderung, Fluchtmigrationshintergrund oder Demenz leben im Sozialraum? Wie viele Einrichtungen der Behindertenhilfe und Altenhilfe gibt es? Wie viele Förder-, integrative und Regelbildungseinrichtungen gibt es?

2. Einordnung der Ergebnisse: Die Strukturdatenrecherche bildet zweitens eine Art Referenzrahmen, der herangezogen werden kann, um (insbesondere) die Ergebnisse der Sozialraumanalysen (siehe Kapitel V) weitergehend einzuordnen. Insofern bilden die Strukturdaten einen Status quo ab, ausgehend von dem Veränderungen abgelesen werden können, die möglicherweise im Zusammenhang mit Maßnahmen stehen, die im Rahmen des untersuchten Projekts durchgeführt werden.

3. Strukturdaten als Ergebnis: Schließlich sind die recherchierten Strukturdaten selbst Ergebnis, die erste Rückschlüsse darauf zulassen, wie die Sozialräume je ausgestaltet sind. Beispielsweise kann anhand der Höhe der Arbeitslosenquote auf eine gewisse Prekarität im Bereich Arbeit geschlossen werden oder eine geringe Dichte an Angeboten der Behindertenhilfe weist auf nur geringe Wahlmöglichkeiten der AdressatInnen hin. Ausgehend davon können im Rahmen der Sozialraumanalysen vertiefende Fragen gestellt und verfolgt werden. 
Das methodische Vorgehen wird in diesem Kapitel ebenso dargelegt (Kapitel 12.2) wie Herausforderungen der Recherche (Kapitel 12.3), woraufhin eine strukturelle Beschreibung der fünf Sozialräume (Kapitel 12.4) sowie ein Überblick über handlungsfeldspezifische Strukturdaten (Kapitel 12.5) folgen. Es sei darauf hingewiesen, dass die sehr umfangreiche Strukturdatenrecherche hier nicht vollumfänglich abgebildet wird, sondern lediglich ausgewählt und exemplarisch. Aus Gründen der Übersichtlichkeit (und da einige dieser Strukturdaten weniger relevant und/oder aussagekräftig sind) wird auf eine Darstellung der Bevölkerungsdichte, des durchschnittlichen Kaufpreises von Wohnimmobilien in $€ / \mathrm{m}^{2}$, der Entfernungen der jeweiligen Wohneinrichtungen der Behinderten- und Altenhilfe zum Stadtzentrum sowie der Freizeitangebote im Kontext Behinderung, Fluchtmigration und Alter verzichtet. Sollte es für die weiteren Ausführungen relevant sein, werden diese zusätzlichen Ergebnisse der Strukturdatenrecherche ergänzend herangezogen.

\subsection{Methodisches Vorgehen}

Das methodische Vorgehen bei der Strukturdatenrecherche sieht vor, eine Übersicht mit relevanten Daten und Größen zu erstellen, die in unterschiedlichen Themen- und Handlungsfeldern angeordnet sind. Die Recherche wurde in vier aufeinander aufbauenden Rechercheschritten vorgenommen, die im Folgenden beschrieben werden.

1. Recherche durch die KoordinatorInnen des untersuchten Projekts: Die Strukturdatenrecherche wurde von den KoordinatorInnen des untersuchten Projekts in den Sozialräumen begonnen, nachdem ihnen eine Liste über die erforderlichen Daten zugestellt wurde. Rasch stellte sich heraus, dass (a) der zeitliche Aufwand für die KoordinatorInnen sehr hoch war, die erforderlichen Daten zu recherchieren, und diese zudem belastet waren durch andere zu dem Zeitpunkt notwendigen Aufgaben. Darüber hinaus zeigte sich, dass (b) die Daten teilweise unveröffentlicht waren und in einigen Fällen sehr deutlich von offiziell veröffentlichten Daten abwichen. Um einen vergleichbaren, für das Projekt validen Datenkorpus zu generieren, wurden die Rechercheschritte (2), (3) und (4) durchgeführt. 
2. Desktoprecherche: Die Recherchen anhand einer Internetsuchmaschine und unter anderem in den Internetauftritten der Statistischen Ämter des Bundes und der Länder konnten einen Teil der erforderlichen Strukturdaten offenlegen.

3. Kontaktaufnahme per E-Mail: Strukturdaten, die nicht ohne Weiteres anhand der Desktoprecherche gefunden werden konnten, wurden in vielen Fällen per E-Mail-Kontaktaufnahme angefragt. Im Gros war der Rücklauf diesbezüglich sehr positiv, wobei insbesondere die Kooperationsbereitschaft der MitarbeiterInnen der kontaktierten Behindertenhilfeträger hervorgehoben werden muss.

4. Telefonrecherche: Schließlich nahm eine Telefonrecherche einen beträchtlichen Anteil der Strukturdatenrecherche ein, anhand derer diese entscheidend um noch fehlende Angaben ergänzt wurde. Kontaktiert wurden dabei vor allem Personen, die in der Verwaltung der jeweiligen Sozialräume tätig sind.

\subsection{Herausforderungen der Strukturdatenrecherche}

Eine der größten Herausforderungen der Recherche bestand darin, vergleichbare Daten über die fünf untersuchten Sozialräume zu generieren. Diesem Anspruch kann zwar größtenteils entsprochen werden, allerdings kommt es dennoch immer wieder vor, dass die dargelegten Daten nur eingeschränkt vergleichbar sind. Dafür sind vor allem zwei Aspekte verantwortlich, die im Folgenden ausgeführt und problematisiert werden.

\section{Aktualität vs. Vergleichbarkeit}

Im Rahmen der Recherchen mussten immer wieder Spannungsverhältnisse zwischen unterschiedlichen Ansprüchen abgewogen werden. Die größten Spannungen bestehen im Anspruch, möglichst aktuelle Daten zu generieren, die gleichzeitig einschränkungslos miteinander verglichen werden können. Die Konsequenz daraus war, dass teilweise weniger aktuelle Daten zugrunde gelegt werden mussten, um dem Anspruch der Vergleichbarkeit $\mathrm{zu}$ genügen. Dies erfolgte unter anderem dann, wenn die Daten anhand unterschiedlicher Definitionen generiert wurden. Hiervon sind insbesondere die Angaben zur Anzahl der im herkömmlichen Sinne als >behindert< geltenden Menschen in den Sozialräumen betroffen, erwies es sich doch bei dieser Recherche als äußerst schwierig, zuverlässige Angaben für die einzel- 
nen Sozialräume zu finden. Während teils Zahlen überhaupt nicht verfügbar waren, differierte in den Fällen, in denen Angaben gefunden wurden, die Definition von `Behinderung . Beispielsweise wurden teils Menschen mit einem Grad der Behinderung ab 20 als sbehindert kategorisiert, wogegen in anderen Angaben von einem Grad der Behinderung von 50 und mehr ausgegangen wurde. Dies hat zur Folge, dass die Zahlen nicht vergleichbar sind. Es musste nun also ein Weg gefunden werden, vergleichbare Daten zu recherchieren. Schlussendlich wurde dazu auf die Angaben der Bundesländer zurückgegriffen, da für diese - vergleichbare - Angaben zur Anzahl der Menschen mit Schwerbehinderung (ab einem Grad der Behinderung von 50) im jeweiligen Bundesland vorliegen. Diese wurden anhand der EinwohnerInnenzahl näherungsweise für die einzelnen Sozialräume berechnet, woraus sich vergleichbare Werte ergeben. Dass es sich hierbei um eine forschungspraktische Einschränkung handelt, ist klar und es muss betont werden, dass die in Kapitel 12.4 jeweils dargestellten Zahlen und Anteile lediglich näherungsweise Angaben sind. Die gegebenenfalls geringere Genauigkeit wird allerdings zugunsten der Vergleichbarkeit der Daten in Kauf genommen. Ähnliche Herausforderungen stellten sich in Bezug auf die Anzahl der Menschen mit Fluchtmigrationshintergrund, wobei ebenfalls die Definition des Personenkreises schwierig und oftmals nicht übereinstimmend war und zudem die Fluktuation in den Sozialräumen so hoch ist, dass die Anzahl der Menschen mit Fluchtmigrationshintergrund durch permanente $\mathrm{Zu}$ - und Wegzüge sehr stark variiert, wodurch letztlich keine validen Daten diesbezüglich erhoben werden konnten. Auch die Anzahl der Menschen mit Demenz war nicht zu recherchieren, da diese weder von den Sozialräumen noch von den Ländern erhoben wird. Hier wurde auf eine Recherche demographischer Angaben ausgewichen, um zumindest Informationen über die Altersverteilung der Menschen in den Sozialräumen zur Verfügung zu haben. Problematisch war darüber hinaus, dass die Strukturdaten - entgegen der Planung im Forschungsdesign - über einen vergleichsweise langen Zeitraum erhoben werden mussten, was insbesondere daran lag, dass den Verantwortlichen in den Sozialräumen die angefragten Daten nicht ohne Weiteres zur Verfügung standen. Dies wiederum kann dahingehend problematisiert werden, dass im Sozialraum selbst offenbar Informationen zur deskriptiven Beschreibung dessen fehlen, was potenziell die Planung und/oder Ausführung von inklusionsorientierten Maßnahmen beeinflusst, da beispielsweise unklar ist, wie viele Personen zum AdressatInnenkreis 
der jeweiligen Maßnahme gezählt werden. Durch diese Hürden im Rechercheprozess, die zur Verlängerung des anvisierten Erhebungszeitraums führten, wurde es notwendig, einzelne Daten immer wieder zu aktualisieren, um auch hier Vergleichbarkeit zu gewährleisten. Auf diese Weise sollte beispielsweise vermieden werden, dass die aktuelle Anzahl der Vereine mit einer veralteten EinwohnerInnenzahl verglichen wird (siehe Kapitel 12.5.4).

\section{Leerstellen im Datenkorpus}

Als problematisch stellte sich heraus, dass einzelne Daten teils nicht für alle Sozialräume verfügbar waren, weshalb bezüglich einzelner Themen auf Daten ausgewichen werden musste, die sich auf die Region und/oder den Kreis beziehen. Dass dadurch einzelne Angaben weniger genau - und damit auch weniger aussagekräftig - sind, wurde vor dem Hintergrund in Kauf genommen, dass so zumindest eine näherungsweise Aussage für den jeweiligen Sozialraum möglich ist. Dies betrifft unter anderem die Daten zu den drei jeweils stärksten Wirtschaftsbranchen in den Sozialräumen (siehe Kapitel 12.4). Einige Daten jedoch konnten auch mit größtem Rechercheaufwand nicht in der gewünschten Art und Weise offengelegt werden, weshalb es einige Leerstellen im Datenkorpus gibt, die auch nicht mit näherungsweisen Angaben geschlossen werden können. Dies betrifft beispielsweise, wie oben bereits problematisiert, die Anzahl der Menschen mit Demenz in den Sozialräumen.

\subsection{Beschreibung der Sozialräume}

Die fünf untersuchten Sozialräume sind heterogen und unterscheiden sich bezüglich einiger Strukturdaten teils deutlich voneinander. Demgegenüber können jedoch immer wieder Gemeinsamkeiten ausgemacht werden, aus denen annehmbar ähnliche Herausforderungen erwachsen, bei deren Bewältigung sich die Sozialräume gegenseitig unterstützen können. Im Folgenden werden die einzelnen Sozialräume entlang deskriptiv-statistischer Daten beschrieben, woraufhin Einblick in handlungsfeldspezifische Strukturen ${ }^{10}$ gegeben wird. Eine tiefergehende qualitative Beschreibung

10 In Bezug auf die handlungsfeldspezifischen Strukturen in den einzelnen Sozialräumen sei darauf hingewiesen, dass die Zahlen zumeist aus unterschiedlichen Quellen recherchiert wurden. Während ein Teil der Daten per Desktoprecherche zusammengetragen 
der Sozialräume, insbesondere hinsichtlich der qualitativen Erfahrung von Barrieren, findet sich in den Ergebnissen der ethnographischen Sozialraumbegehungen (siehe Kapitel 13).

\subsubsection{Erlangen}

Die bayerische Großstadt Erlangen hat 113.164 EinwohnerInnen. Ihre drei stärksten Wirtschaftsbranchen sind (1) Herstellung von elektrischen Erzeugnissen, (2) Gesundheitswesen sowie (3) Einzelhandel (ohne Handel mit Kraftfahrzeugen). ArbeitnehmerInnen verdienen in Erlangen durchschnittlich $24.139 €$ pro Jahr. Circa 3,7 \% der potenziell Erwerbstätigen sind als sarbeitslos $<$ registriert. Die durchschnittliche Miete beträgt $12,25 € \mathrm{pro} \mathrm{m}^{2}$. In Erlangen leben ungefähr 10.003 Menschen mit Schwerbehinderung (entspricht circa 8,8 \% der Bevölkerung), circa 17,9 \% der Bevölkerung sind über 65 Jahre alt ${ }^{11}$.

\subsubsection{Rostock}

Rostock (Mecklenburg-Vorpommern) ist mit 208.299 EinwohnerInnen der größte Sozialraum, der in dieser Studie untersucht wird. Im Stadtteil südstadt, auf dem im Rahmen des Projekts ein besonderer Fokus liegt, wohnen 15.035 Personen. Für die Strukturdatenrecherche hatte diese Fokussierung zur Folge, dass parallel zu den Daten bezüglich der Stadt Rostock ebenfalls Daten erhoben werden sollten, die sich ausschließlich auf den Stadtteil Südstadt beziehen. Dies war mit größeren Einschränkungen verbunden, da häufig keine stadtteilbezogenen Daten vorlagen. Infolgedessen können nur in Einzelfällen zusätzliche Angaben zum Stadtteil Südstadt gemacht werden. Die drei stärksten Wirtschaftsbranchen in Rostock sind (1) Gesundheitswesen, (2) Einzelhandel (ohne Handel mit Kraftfahrzeugen), (3) Sozialwesen (ohne Heime). Die EinwohnerInnen Rostocks verdienen

wurde, wurden einige Angaben bei den Trägern und Institutionen angefragt und per EMail oder telefonisch übermittelt. Aufgrund der Vielfalt der verwendeten Quellen, die den hier dargelegten Zahlen zugrunde liegen, wird auf eine detaillierte Angabe aller Verweise verzichtet.

11 Stadt Erlangen, Bürgermeister- und Presseamt, Sachgebiet Statistik und Stadtforschung 2016, S. 24; Bundesagentur für Arbeit 2018c; Statistische Ämter des Bundes und der Länder, Deutschland 2018; Statistik der Bundesagentur für Arbeit 2020; PWIB WohnungsInfobörse $\mathrm{CmbH}$ 2018a; Cesundheitsberichterstattung des Bundes 2018; Bayerisches Landesamt für Statistik 2018, S. 6. 
im Jahr durchschnittlich $18.488 €$, wobei es sich im Vergleich mit den anderen Sozialräumen um den geringsten jährlichen Verdienst handelt. Zudem ist in Rostock die Arbeitslosenquote mit 7,4 \% am höchsten. Der durchschnittliche Mietpreis beträgt 7,72€ pro $\mathrm{m}^{2}$. In Rostock leben circa 24.267 Menschen mit Schwerbehinderung, was einem Bevölkerungsanteil von circa 11,7\% entspricht. Durchschnittlich 23,3\% der EinwohnerInnen Rostocks sind über 65 Jahre alt ${ }^{12}$.

\subsubsection{Schneverdingen}

Schneverdingen (Nieder-Sachsen) hat 18.795 EinwohnerInnen und ist damit der kleinste untersuchte Sozialraum. Die drei stärksten Wirtschaftsbranchen sind (1) Öffentliche Verwaltung, Verteidigung, Sozialversicherung, (2) Einzelhandel (ohne Handel mit Kraftfahrzeugen), (3) Gesundheitswesen, wobei anzumerken ist, dass sich hierbei auf die Angaben des Kreises beziehungsweise des zuständigen Arbeitsamtes (Celle) gestützt wurde, da für Schneverdingen keine unmittelbaren Daten zur Verfügung stehen. Durchschnittlich verdienen die ArbeitnehmerInnen in Schneverdingen 20.093€ pro Jahr (den Angaben zugrunde liegen hierbei die Daten des Landkreises Heidekreis). Die Arbeitslosenquote liegt bei 5,1\%. Durchschnittlich müssen die EinwohnerInnen Schneverdingens 5,69€ Miete pro $\mathrm{m}^{2}$ aufbringen, was im Vergleich zu den anderen Sozialräumen des Projekts der niedrigste Wert ist. In Schneverdingen leben im Durchschnitt 1.760 Menschen mit Schwerbehinderung. Das entspricht einem Bevölkerungsanteil von 9,4\%. Die Menschen in Schneverdingen sind im Vergleich zu den anderen Sozialräumen durchschnittlich am ältesten, circa 24,7\% der EinwohnerInnen sind über 65 Jahre alt ${ }^{13}$.

12 Rathaus Rostock 2018; Bundesagentur für Arbeit 2018e; Statistische Ämter des Bundes und der Länder, Deutschland 2018; Statistik der Bundesagentur für Arbeit 2018d; PWIB Wohnungs-Infobörse $\mathrm{CmbH}$ 2018b; Statistisches Amt Mecklenburg-Vorpommern 2017, S. 24; Hansestadt Rostock. Der Oberbürgermeister. Presse- und Informationsstelle 2016, S. 34 .

13 Stadt Schneverdingen 2018; Bundesagentur für Arbeit 2018b; Statistische Ämter des Bundes und der Länder, Deutschland 2018; Statistik der Bundesagentur für Arbeit 2018a; iib Institut Innovatives Bauen Dr. Hettenbach CmbH 2018b; Landkreis Emsland 2018, S. 1; Landesamt für Statistik Niedersachsen 2018. 


\subsubsection{Schwäbisch Gmünd}

Schwäbisch Gmünd liegt in Baden-Württemberg und hat 60.743 EinwohnerInnen. Dort sind die drei stärksten Wirtschaftsbranchen (1) Herstellung von elektronischen Erzeugnissen, (2) Metallerzeugung und -bearbeitung, (3) Maschinenbau (Angaben des Arbeitsamtes Aalen). Durchschnittlich verdienen ArbeitnehmerInnen in Schwäbisch Gmünd 23.226€ pro Jahr, 2,5 \% der potenziellen ArbeitnehmerInnen sind arbeitslos (im Vergleich zu den anderen untersuchten Sozialräumen entspricht dies dem niedrigsten Wert). Der durchschnittliche Mietpreis liegt bei $8,24 €$ pro $\mathrm{m}^{2}$. In Schwäbisch Gmünd leben durchschnittlich 5.205 Menschen mit Schwerbehinderung, was einem Anteil an der Bevölkerung von 8,6 \% entspricht. Circa $20 \%$ der EinwohnerInnen sind über 65 Jahre alt ${ }^{14}$.

\subsubsection{Verbandsgemeinde Nieder-Olm}

Die Verbandsgemeinde Nieder-Olm liegt in Rheinland-Pfalz und setzt sich aus acht eigenständigen Gemeinden zusammen. Insgesamt hat die Verbandsgemeinde Nieder-Olm 34.888 EinwohnerInnen. Die drei stärksten Wirtschaftsbranchen in diesem Sozialraum sind (1) Gesundheitswesen, (2) Einzelhandel (ohne Handel mit Kraftfahrzeugen), (3) Öffentliche Verwaltung, Verteidigung, Sozialversicherung. ArbeitnehmerInnen verdienen im Durchschnitt 24.639€ pro Jahr (Angaben des Arbeitsamtes Mainz-Bingen), wobei es sich um den höchsten durchschnittlichen Verdienst im Vergleich zu den anderen untersuchten Sozialräumen handelt. Durchschnittlich 3,4 \% der potenziellen ArbeitnehmerInnen sind arbeitslos. Für die Miete von Wohnraum müssen in der Stadt Nieder-Olm, die stellvertretend und exemplarisch für alle Gemeinden der Verbandsgemeinde ausgewählt wurde, durchschnittlich 9,49€ pro $\mathrm{m}^{2}$ aufgewendet werden. In der Verbandsgemeinde leben durchschnittlich 2.711 Menschen mit Schwerbehinderung, was einem Bevölkerungsanteil von 7,8 \% entspricht. Ungefähr 19,8 \% der EinwohnerInnen sind über 65 Jahre alt ${ }^{15}$.

14 Statistisches Landesamt Baden-Württemberg 2018c; Bundesagentur für Arbeit 2018a; Statistische Ämter des Bundes und der Länder, Deutschland 2018; Statistik der Bundesagentur für Arbeit 2018c; PWIB Wohnungs-Infobörse GmbH 2018c; Statistisches Landesamt Baden-Württemberg 2018b; Statistisches Landesamt Baden-Württemberg 2018a.

15 Statistisches Landesamt Rheinland-Pfalz 2018, S. 2; Bundesagentur für Arbeit 2018d; Statistische Ämter des Bundes und der Länder, Deutschland 2018; Statistik der Bundes- 


\subsection{Handlungsfeldspezifische Strukturen}

\subsubsection{Werkstätten für Menschen mit Behinderung}

Im Folgenden ist die Anzahl an Plätzen in Werkstätten für Menschen mit Behinderung (WfbM) dargestellt, die in den untersuchten Sozialräumen jeweils vorhanden sind. Weitere Daten aus dem Bereich Arbeit und Behinderung liegen vor (beispielsweise Anzahl der Werkstätten in den untersuchten Sozialräumen), jedoch wird hier der Fokus auf die Anzahl der Plätze gelegt und ausschließlich diese Daten abgebildet und diskutiert.

Tabelle 8: Werkstätten für Menschen mit Behinderung

\begin{tabular}{|l|c|c|c|c|c|}
\hline & $\begin{array}{l}\text { Erlan- } \\
\text { gen }\end{array}$ & Rostock & $\begin{array}{l}\text { Schnever- } \\
\text { dingen }\end{array}$ & $\begin{array}{l}\text { Schwä- } \\
\text { bisch } \\
\text { Gmünd }\end{array}$ & $\begin{array}{l}\text { VG Nie- } \\
\text { der-Olm }\end{array}$ \\
\hline $\begin{array}{l}\text { Plätze in } \\
\text { den WfbM }\end{array}$ & 325 & 1200 & 0 & 520 & $46^{16}$ \\
\hline
\end{tabular}

Die Tabelle zeigt, dass es in Schneverdingen keine Plätze in einer Werkstatt für Menschen mit Behinderung gibt, was daran liegt, dass eine solche Einrichtung dort nicht vorhanden ist. Diesbezüglich muss angemerkt werden, dass EinwohnerInnen Schneverdingens die Angebote der Lebenshilfe Soltau mitnutzen, wo es unter anderem eine Werkstatt für Menschen mit Behinderung gibt. Auch wenn ganz grundsätzlich davon ausgegangen wird, dass die EinwohnerInnen der jeweiligen Sozialräume auch Angebote der Behindertenhilfe aus anderen Gemeinden nutzen (können), ist dennoch zu problematisieren, inwiefern dies ein relevanter Faktor in der Bewertung des Sozialraums beziehungsweise im Hinblick auf (potenzielle) strukturelle Veränderungen im Kontext Inklusion ist. Menschen mit Behinderung, die Angebote außerhalb ihres direkten Wohnortes nutzen, stellen sich unter Umständen zusätzliche Herausforderungen im Kontext Mobilität. Darüber hinaus sind generelle Fragen des Aus- und Abbaus von Werkstattplätzen zu

agentur für Arbeit 2018b; iib Institut Innovatives Bauen Dr. Hettenbach GmbH 2018a; Reh 2018; Statistisches Landesamt Rheinland-Pfalz 2018, S. 4.

16 Hierbei handelt es sich um die Außenstelle einer Werkstatt für Menschen mit Behinderung, deren Hauptsitz in Mainz ist. 
diskutieren, insbesondere im Zusammenhang damit, dass diese zwar als Zeichen von Ausschluss verstanden werden können, da Menschen mit Behinderung dort exklusiv und fernab des sogenannten ersten Arbeitsmarktes einer Beschäftigung nachgehen, demgegenüber allerdings auch als 'geschützte< Arbeitsstätten dazu beitragen können, Menschen mit Behinderung so zu qualifizieren, dass sie zukünftig auf dem ersten Arbeitsmarkt eine Beschäftigung finden. Perspektivisch ist es interessant zu beobachten, ob und inwiefern sich die Anzahl der Werkstätten und jeweiligen Plätze über den Projektverlauf verändert und so gegebenenfalls Aufschluss darüber geben kann, dass Menschen mit Behinderung inklusive Teilhabemöglichkeiten im Bereich Arbeit nutzen.

\subsubsection{Stationär und ambulant betreutes Wohnen der Behindertenhilfe}

Im Folgenden sind die Plätze in Angeboten stationär betreuten Wohnens der Behindertenhilfe in den fünf untersuchten Sozialräumen abgebildet. Korrespondierend zur Untersuchung des Angebots im Handlungsfeld Arbeit und Behinderung wird hier ebenfalls die Anzahl der Plätze beziehungsweise der betreuten Personen fokussiert.

Tabelle 9: Einrichtungen stationär betreuten Wohnens der Behindertenhilfe

\begin{tabular}{|c|c|c|c|c|c|}
\hline & Erlangen & Rostock & $\begin{array}{l}\text { Schnever- } \\
\text { dingen }\end{array}$ & $\begin{array}{l}\text { Schwä- } \\
\text { bisch } \\
\text { Gmünd }\end{array}$ & $\begin{array}{l}\text { VG Nie- } \\
\text { der-Olm }\end{array}$ \\
\hline $\begin{array}{l}\text { Plätze/betreu- } \\
\text { te Personen in } \\
\text { Einrichtungen } \\
\text { des stationär } \\
\text { betreuten } \\
\text { Wohnens der } \\
\text { Behinderten- } \\
\text { hilfe }\end{array}$ & 136 & 344 & 0 & 383 & 91 \\
\hline
\end{tabular}

In Schneverdingen sind keine Einrichtungen stationär betreuten Wohnens vorhanden, allerdings können EinwohnerInnen Wohneinrichtungen im Umkreis nutzen - ebenso wie in Bezug auf Werkstätten für Menschen mit Behinderung. Zusätzlich zu den stationär betreuten Wohneinrichtungen 
wurden Anbieter des ambulant betreuten Wohnens der Behindertenhilfe recherchiert. Dies ist im Folgenden dargestellt.

Tabelle 10: Anbieter ambulant betreuten Wohnens der Behindertenhilfe

\begin{tabular}{|l|c|c|c|c|c|}
\hline & Erlangen & Rostock & $\begin{array}{l}\text { Schnever- } \\
\text { dingen }\end{array}$ & $\begin{array}{l}\text { Schwä- } \\
\text { bisch } \\
\text { Gmünd }\end{array}$ & $\begin{array}{l}\text { VG Nieder- } \\
\text { Olm }\end{array}$ \\
\hline $\begin{array}{l}\text { Anbieter des } \\
\text { ambulant } \\
\text { betreuten } \\
\text { Wohnens der } \\
\text { Behinderten- } \\
\text { hilfe }\end{array}$ & 2 & 12 & 0 & 2 & 0 \\
\hline
\end{tabular}

In Erlangen gibt es zwei Anbieter für ambulant betreutes Wohnen für Menschen mit Behinderung. In Rostock sind es dagegen zwölf Anbieter. Dies kann in der Konsequenz bedeuten, dass Menschen mit Behinderung in Rostock möglicherweise größere Wahlmöglichkeiten haben, an welchen Träger sie sich wenden wollen, als dies in Erlangen der Fall ist. In Schwäbisch Gmünd gibt es zwei Anbieter für ambulant betreutes Wohnen, wogegen es in Schneverdingen und der Verbandsgemeinde Nieder-Olm keine derartigen Anbieter gibt ${ }^{17}$.

\subsubsection{Stationär betreutes Wohnen der Altenhilfe}

Um Aussagen über die Wohn- und Betreuungssituation von älteren und alten Menschen treffen zu können, wurde untersucht, wie viele (Plätze in) Einrichtungen des stationär betreuten Wohnens der Altenhilfe es jeweils in den Sozialräumen gibt.

17 Die Anzahl der Plätze im ambulant betreuten Wohnen der Behindertenhilfe richtet sich nach dem je gegebenen Bedarf und ist deshalb eine variable Größe. Die Anzahl der jeweiligen Klientlnnen liegt zwar vor, ist an dieser Stelle jedoch wenig sinnhaft, weshalb darauf verzichtet wird. 
Tabelle 11: Einrichtungen stationär betreuten Wohnens der Altenhilfe

\begin{tabular}{|l|c|c|c|c|c|}
\hline & Erlangen & Rostock & $\begin{array}{l}\text { Schnever- } \\
\text { dingen }\end{array}$ & $\begin{array}{l}\text { Schwä- } \\
\text { bisch } \\
\text { Gmünd }\end{array}$ & $\begin{array}{l}\text { VG Nie- } \\
\text { der-OIm }\end{array}$ \\
\hline $\begin{array}{l}\text { Einrichtungen } \\
\text { des stationär } \\
\text { betreuten } \\
\begin{array}{l}\text { Wohnens der } \\
\text { Altenhilfe }\end{array}\end{array}$ & 12 & 24 & 2 & 10 & 3 \\
\hline $\begin{array}{l}\text { Plätze/ } \\
\text { betreute } \\
\text { Personen }\end{array}$ & 1.167 & 2.301 & 150 & 669 & 456 \\
\hline
\end{tabular}

Die Daten zeigen, dass die stationär betreuten Wohneinrichtungen der Altenhilfe im Gros sehr viele BewohnerInnen haben (beispielsweise bis $\mathrm{zu}$ durchschnittlich 152 BewohnerInnen pro Altenheim in der Verbandsgemeinde Nieder-Olm), was darauf aufmerksam macht, inwiefern die Deinstitutionalisierung von Alten- und Pflegeheimen nach wie vor eine wichtige Aufgabe ist (Trescher 2013, S. 310ff).

\subsubsection{Vereine}

Im Folgenden ist die näherungsweise Anzahl der Vereine in den einzelnen Sozialräumen dargelegt. Um einen Eindruck davon zu bekommen, wie die Anzahl der Vereine jeweils im Verhältnis zur Anzahl der EinwohnerInnen steht und die Sozialräume so untereinander vergleichbar zu machen, wurde zusätzlich die Anzahl der Vereine pro 10.000 EinwohnerInnen im jeweiligen Sozialraum angegeben. 
Tabelle 12: Vereine

\begin{tabular}{|l|c|c|c|c|c|}
\hline & Erlangen & Rostock & $\begin{array}{l}\text { Schnever- } \\
\text { dingen }\end{array}$ & $\begin{array}{l}\text { Schwä- } \\
\text { bisch } \\
\text { Gmünd }\end{array}$ & $\begin{array}{l}\text { VG Nieder- } \\
\text { Olm }\end{array}$ \\
\hline $\begin{array}{l}\text { Anzahl } \\
\text { der Ver- } \\
\text { eine }\end{array}$ & 116 & 447 & 130 & 513 & 268 \\
\hline $\begin{array}{l}\text { Anzahl } \\
\text { der Ver- } \\
\text { eine pro } \\
10.000 \\
\begin{array}{l}\text { Einwoh- } \\
\text { nerlnnen }\end{array}\end{array}$ & 10,25 & 21,46 & 69,17 & 84,45 & 76,82 \\
\hline
\end{tabular}

Vereine finden sich oftmals im Bereich Freizeit, der sich prinzipiell eher wertrational definiert, also über gemeinsame Interessen, und tendenziell herrschaftsfrei ist (Trescher 2015b, S. 32ff). Über das Mitwirken in Vereinen können folglich Berührungspunkte zwischen der Mehrheitsgesellschaft und von Ausschluss bedrohten oder betroffenen Personen entstehen, die in einem gemeinsamen Interesse gründen und so Möglichkeiten der Vergemeinschaftung (und damit schließlich Inklusion) eröffnen. Schwäbisch Gmünd hat, absolut als auch im Verhältnis zu den EinwohnerInnen, die meisten Vereine ${ }^{18}$.

\subsubsection{Kindertagesstätten}

In den Sozialräumen gibt es sowohl Sonder-/Förderkindertagesstätten, integrative Kindertagesstätten als auch sogenannte Regelkindertagesstätten, in denen nicht ausdrücklich Kinder mit und Kinder ohne Behinderung gemeinsam betreut werden. Dies ist allerdings dennoch möglich, beispielsweise im Rahmen einer Einzelintegration der Eingliederungshilfe ${ }^{19}$. Im Folgenden ist

18 Erlangen: Stadt Erlangen, Oberbürgermeister Dr. Florian Janik 2018; Rostock: Adeos Media $\mathrm{CmbH}$ 2018; Schneverdingen: Die Angabe wurde per E-Mail übermittelt; Schwäbisch Gmünd: Stadt Schwäbisch Gmünd 2018; VG Nieder-Olm: Verbandsgemeindeverwaltung Nieder-Olm, Bürgermeister Ralph Spiegler 2018.

19 Bundessozialhilfegesetz §39; Speck 2001, S. 376ff. 
dargelegt, wie viele Kindertagesstätten es in den Sozialräumen jeweils gibt und welchem Schwerpunkt diese zugeordnet werden können.

Tabelle 13: Kindertagesstätten

\begin{tabular}{|l|c|c|c|c|c|}
\hline & Erlangen & Rostock & $\begin{array}{l}\text { Schnever- } \\
\text { dingen }\end{array}$ & $\begin{array}{l}\text { Schwä- } \\
\text { bisch } \\
\text { Gmünd }\end{array}$ & $\begin{array}{l}\text { VG Nie- } \\
\text { der-0lm }\end{array}$ \\
\hline $\begin{array}{l}\text { Sonder-/För- } \\
\text { derkinderta- } \\
\text { gesstätten }\end{array}$ & 3 & 1 & 0 & 1 & 0 \\
\hline $\begin{array}{l}\text { Integrative } \\
\text { Kindertages- } \\
\text { tätten }\end{array}$ & 3 & 27 & 3 & 2 & 1 \\
\hline $\begin{array}{l}\text { Regelkinder- } \\
\text { tagesstätten }\end{array}$ & 133 & 40 & 7 & 54 & 22 \\
\hline gesamt & 139 & 68 & 10 & 57 & 23 \\
\hline
\end{tabular}

Die hohe Anzahl an Kindertagesstätten in der Verbandsgemeinde NiederOlm mag zunächst verwunderlich erscheinen, begründet sich jedoch annehmbar darin, dass alle Gemeinden der Verbandsgemeinde auch eigene Kindertagesstätten haben.

\subsubsection{Schulen}

In den Sozialräumen gibt es unterschiedliche Schulformen, die hier zwecks Darstellung in den Rubriken Grundschulen, Förderschulen und weiterführende Schulen zusammengeführt sind. Im Folgenden ist dargelegt, wie viele Schulen es in den Sozialräumen jeweils gibt und welcher Rubrik diese zugeordnet werden können. 
Tabelle 14: Schulen

\begin{tabular}{|l|c|c|c|c|c|}
\hline & Erlangen & Rostock & $\begin{array}{l}\text { Schne- } \\
\text { verdingen }\end{array}$ & $\begin{array}{l}\text { Schwä- } \\
\text { bisch } \\
\text { Gmünd }\end{array}$ & $\begin{array}{l}\text { VG Nie- } \\
\text { der-0Im }\end{array}$ \\
\hline Grundschulen & 16 & 18 & 4 & 18 & 6 \\
\hline Förderschulen & 3 & 7 & 0 & 4 & 2 \\
\hline $\begin{array}{l}\text { Weiterführende } \\
\text { Schulen }\end{array}$ & 41 & 23 & 1 & 12 & 2 \\
\hline
\end{tabular}

Schneverdingen unterscheidet sich insofern von den anderen Sozialräumen, dass dort keine Förderschule vorhanden ist. Bei der Recherche stellte sich heraus, dass es dort zwar bis zum Ende des Schuljahres 2016/17 eine Förderschule gab, diese jedoch geschlossen wurde. In Bezug auf Fragen inklusiver Beschulung von SchülerInnen mit und SchülerInnen ohne Behinderung bleibt abzuwarten, ob und inwiefern sich die Anzahlen der jeweiligen Schulformen verändern und ob beispielsweise Förderschulen perspektivisch abgeschafft werden. Denkbar ist auch, dass andere und erweiterte Schulformen geschaffen werden. Diese Veränderungen werden annehmbar im Rahmen der Sozialraumanalysen sichtbar und können entsprechend herausgearbeitet und diskutiert werden.

\subsubsection{Weiterbildungseinrichtungen und (Fach-)Hochschulen}

Neben Kindertagesstätten und Schulen wurde im Handlungsfeld Bildung der Weiter-/Erwachsenenbildungs- und (Fach-)Hochschulbildungsbereich untersucht. Die Ergebnisse dessen sind im Folgenden dargestellt. 
Tabelle 15: Weiter-/Erwachsenenbildungseinrichtungen und (Fach-)Hochschulen

\begin{tabular}{|l|c|c|c|c|c|}
\hline & Erlangen & Rostock & $\begin{array}{l}\text { Schnever- } \\
\text { dingen }\end{array}$ & $\begin{array}{l}\text { Schwä- } \\
\text { bisch } \\
\text { Gmünd }\end{array}$ & $\begin{array}{l}\text { VG Nieder- } \\
\text { Olm }\end{array}$ \\
\hline $\begin{array}{l}\text { Weiter-/Er- } \\
\text { wachsenen- } \\
\text { bildungsein- } \\
\text { richtungen }\end{array}$ & 6 & 8 & 1 & 10 & 2 \\
\hline $\begin{array}{l}\text { (Fach-)Hoch- } \\
\text { schulen }\end{array}$ & 1 & 2 & 0 & 3 & 0 \\
\hline
\end{tabular}

Trotz seiner vergleichsweise geringeren Größe hat Schwäbisch Gmünd die meisten Weiter-/Erwachsenenbildungseinrichtungen ebenso wie (Fach-) Hochschulen. Hervorgehoben werden kann zudem, dass in den beiden kleineren Sozialräumen (Schneverdingen und Verbandsgemeinde Nieder-Olm) Weiter-/Erwachsenenbildungseinrichtungen vorhanden sind, was Erwachsenen die Möglichkeit bietet, Interessen nachzugehen und/oder neue Interessen zu entwickeln.

\subsection{Gesamtbetrachtung und Diskussion}

Nachdem zentrale Ergebnisse der Strukturdatenrecherche dargelegt wurden, werden daraus resultierende Fragestellungen diskutiert. Dies erfolgt in theoretischer, methodischer und handlungspraktischer Hinsicht.

\section{Theoretische Gesamtbetrachtung und Diskussion}

Die Ergebnisse der Recherche zeigen, dass ein großer handlungspraktischer Bedarf besteht, Informationen über die strukturelle Ausgestaltung von Sozialräumen zur Verfügung zu stellen, um ausgehend davon organisationales, pädagogisches und/oder verwaltungslogisches Handeln planen zu können, das (beispielsweise) auf die Erweiterung von Teilhabemöglichkeiten für von Ausschluss bedrohte oder betroffene Menschen abzielt. Diesem dringenden Bedarf an Wissen über Strukturen, Barrieren und je individuelle (Teilhabe-)Bedarfe steht gegenüber, dass diesem Streben nach Wissen in gewisser Weise immer ein Moment der Überwachung innewohnt. Es ist also eine Abwägungsfrage, zu entscheiden, ob über bestimmte Strukturdaten ein zentrales Register geführt oder ob dies grundsätzlich unterlassen werden 
sollte, um die Privatheit und schließlich auch Privatsphäre der betreffenden Personen zu schützen ${ }^{20}$. Diese Frage stellt sich insbesondere vor dem Hintergrund, dass Überwachungspraxen sowohl das Private verletzen als auch in der Konsequenz - zumindest potenziell - regulierend sind, eröffnen sie doch implizit Handlungsbedarfe und -optionen. Eine enge Überwachung von Personen kann darüber hinaus eine Verletzung ihrer Würde bedeuten, denn, um die Würde des Menschen zu wahren, ist es notwendig, diesem einen privaten Bereich der Lebensgestaltung zur Verfügung zu stellen, der vor jeglicher Einflussnahme geschützt ist (Wetz 2002, S. 86) ${ }^{21}$. Ein weiterer Aspekt, der mit Blick auf die Strukturdatenrecherche diskutiert werden kann, ist die Frage, welche Expertise es dafür braucht, Teilhabebarrieren zu erkennen und dazu beizutragen, sie abzubauen. Oder plakativer formuliert: Welche Kompetenzen braucht es für Inklusion? Bedacht werden muss dabei, ob es überhaupt einer bestimmten Expertise für Inklusion bedarf. Gerade im Zusammenhang mit der vorangegangenen Diskussion bezüglich Wissenssammlung, Überwachung und Würdeverletzung stellt sich die Frage, ob, und wenn ja, inwiefern, es notwendig sein kann, ein Wissen über die Strukturen und EinwohnerInnen des Sozialraums zu sammeln, um auf Missstände aufmerksam zu machen und schließlich Möglichkeiten für jene Personen zu eröffnen, die in besonderer Weise von Ausschluss betroffen sind. Braucht es überhaupt personenspezifisches Wissen oder widerspricht dies nicht vielmehr dem Gedanken, dichotome Statuszuweisungen im Kontext von Inklusion aufzuweichen? Hinsichtlich der Diskussion von Expertise und was diese begründet, ist das Sammeln struktureller Daten also ebenfalls durchaus ambivalent. Als eine Anschlussmöglichkeit weiterführender theoretischer Auseinandersetzungen bleibt nun die Frage, wie das Verhältnis von Wissensbedarf, Überwachung und möglicher würdeverletzender Regulierung ausgehandelt und diskutiert werden kann.

20 Zur Unterscheidung von Privatsphäre, Privatheit und Privatangelegenheit siehe Trescher (2013, S. 307ff; weiterführend Rössler 2001).

21 Zum Zusammenhang von Würde und der Wahrung des Privaten siehe Trescher (2013, S. 307ff, 2015a). 


\section{Methodische Gesamtbetrachtung und Diskussion}

Insgesamt lässt sich sagen, dass die Ziele der Strukturdatenrecherche erreicht wurden. Das Feld wurde deskriptiv erschlossen und es wurde eine Grundlage dafür geschaffen, Ergebnisse der Sozialraumanalysen einzuordnen und weitergehend zu diskutieren. Methodisch herausfordernd war, wie eingangs bereits diskutiert (siehe Kapitel 12.3), dass nicht immer vergleichbare Strukturdaten der fünf untersuchten Sozialräume generiert werden konnten. Hier mussten forschungspraktische Einschränkungen vorgenommen werden (wie beispielsweise Daten zugrunde legen, die sich auf größere Kreise und nicht alleinig auf den jeweils interessierenden Sozialraum beziehen), die allerdings den näherungsweisen Vergleich der Sozialräume nicht einschränken, sondern vielmehr erst ermöglichen ${ }^{22}$. Forschungspraktisch sind methodische Erweiterungen denkbar. Insbesondere Verfahren des sogenannten Dataminings können hierbei eine Rolle spielen, anhand derer große Datenmengen auf Querverbindungen und bislang unbekannte $\mathrm{Zu}$ sammenhänge untersucht werden. Problematisiert werden kann darüber hinaus, dass die generierten Daten zwar für die weitergehende Einordnung der nachfolgenden Untersuchungen herangezogen werden können, allerdings würden Sozialräume, die sich um den Abbau von Teilhabebarrieren bemühen, von jenen Daten eher profitieren, wenn diese ihnen zu Beginn des Prozesses zur Verfügung gestellt würden. Dadurch könnten sie ausgehend von jenen Strukturdaten Netzwerke bilden und potenzielle NetzwerkpartnerInnen suchen sowie schließlich je konkrete Maßnahmen planen. Städte und Kommunen, die sich entsprechende Projekte vornehmen, bedürfen also umfassenden Wissens über die strukturelle Ausgestaltung des jeweiligen Ortes, um ein solches Projekt bedürfnisnah und bestmöglich planen zu können. Dieses Wissen muss erhoben und/oder von wissenschaftlicher Seite zur Verfügung gestellt werden.

\section{Handlungspraktische Gesamtbetrachtung und Diskussion}

Die Recherchen legen offen, dass die Strukturdaten selbst bereits als Ergebnis herangezogen werden können und Rückschlüsse auf die Ausgestaltung der Sozialräume zulassen, mit der wiederum handlungspraktische Herausforderungen einhergehen können. Beispielsweise kann problemati-

22 Eine problematisierende Perspektive auf strukturanalytische, quantitative Raumstudien eröffnet Bauriedl (2008). 
siert werden, dass durch permanente $\mathrm{Zu}$ - und Wegzüge von Menschen mit Fluchtmigrationshintergrund wenig Konstanz gegeben ist, was aufseiten der geflüchteten Personen zu Schwierigkeiten führen kann, Anschluss im Sozialraum zu finden. Aufseiten derjenigen Personen, die Maßnahmen planen und anbieten, bedeutet dies, dass jene Angebote oftmals nur punktuell möglich sind und es eher schwierig ist, längerfristige Aktivitäten durchzuführen. In Bezug auf Menschen mit Demenz wird die Problematik offensichtlich, dass von den Kommunen keine Daten darüber erhoben werden, wie viele Menschen mit Demenz im jeweiligen Sozialraum leben. Das bedeutet im Umkehrschluss, dass diese kaum je durch Maßnahmen adressiert werden - und auch gar nicht adressiert werden können, da die Kontaktaufnahme kaum geling ${ }^{23}$. Die Strukturdaten machen zudem darauf aufmerksam, dass gerade in Rostock teilweise Lebensbedingungen vorherrschen, mit denen ein hoher Arbeitslosigkeitsanteil und damit verbundene Armutsrisiken einhergehen. Dies muss bei der weiteren Analyse mitgedacht werden (insbesondere bei der Sozialraumanalyse im Kontext Arbeit) und es bedarf einer Reflexion dessen, inwieweit sich den Verantwortlichen in diesem Sozialraum gegebenenfalls andere Herausforderungen stellen als in den swohlhabenderen` Sozialräumen. In Ansätzen deutet sich darüber hinaus eine gewisse Differenz zwischen großstädtischen und kleinstädtischen Sozialräumen an, insbesondere was die Wahlmöglichkeiten für bestimmte Dienstleistungen angeht. So haben Menschen mit Behinderung in größeren Städten gegebenenfalls mehr Wahlmöglichkeiten, welche Wohn- und/ oder Betreuungsangebote sie nutzen wollen. Demgegenüber sind in der Kleinstadt unter Umständen bürokratische Hürden geringer, da Anliegen möglicherweise rauf dem kleinen Dienstweg، bearbeitet werden können. Gleichzeitig kann problematisiert werden, dass spezialisierte Versorgungsinstitutionen häufig zentral organisiert sind (zum Beispiel sogenannte Komplexeinrichtungen, aber auch im kleineren Rahmen integrative Kindertagesstätten oder behinderungsspezifische Förderschulen), was mit längeren Anfahrten und einem Ausschluss aus dem persönlichen sozialen Nahraum (Nachbarschaft) verbunden ist (Kratz et al. 2016, S. 11). »Je spezieller sie [Versorgungsinstitutionen; $\mathrm{HT} / \mathrm{TH}]$ ausgelegt sind, umso geringer ist die räum-

23 Dies spiegeln die Ergebnisse der Sozialraumanalyse im Kontext Freizeit wider, die zeigen, dass Menschen mit Demenz kaum an routinemäßigen Freizeitpraxen in den Sozialräumen teilhaben (siehe Kapitel 17.2). 
liche Versorgungsdichte, weil im Verhältnis zur Gesamtbevölkerung weniger Personen als Zielgruppe infrage kommen. Der Einzugsbereich erweitert sich entsprechend und wird süberregionak, was häufig verbunden ist mit langen Fahrtzeiten, einer Trennung der betroffenen Menschen vom gewohnten Wohnumfeld, der Nachbarschaft« (Kratz et al. 2016, S. 11). In Bezug auf Projekte, die Inklusion im Sozialraum voranbringen wollen, kann problematisiert werden, wie wichtig ein Wissen darüber ist, welche EinwohnerInnen des Sozialraums potenzielle AdressatInnen von sogenannten inklusionsorientierten Maßnahmen sind, wie zahlreich diese vertreten sind und wie diese erreicht werden können. Fehlt dieses Wissen, dann planen Personen, die ein solches Projekt steuern, ein Stück weit ins Leere beziehungsweise entwickeln Maßnahmen, bei denen im Vorhinein Rahmenbedingungen und potenzielle AdressatInnen offen sind. Es wäre also gegebenenfalls sinnvoll, vor der Planung von Maßnahmen genau zu untersuchen, wie der Sozialraum strukturell ausgestaltet ist, wo Menschen an Barrieren stoßen und welche Personen(-Gruppen) besonders stark von Ausschluss bedroht oder betroffen sind. Wird dies nicht getan, kann berechtigterweise die Frage gestellt werden, ob und inwiefern noch von einem sinnhaften Gebrauch der zur Verfügung gestellten Mittel gesprochen werden kann, wenn Projektverantwortliche ein eher geringes Wissen über die Menschen in den Sozialräumen haben, in denen sie das Projekt leiten. Mit diesem Wissen ist nämlich eine deutlich effektivere Maßnahmen- und Netzwerkplanung möglich - denn Netzwerke sollen ja das sein, was nach Abschluss der unmittelbaren Projektphase bestehen bleibt. Inwiefern dabei Ambivalenzen zwischen Wissensgenerierung und Überwachungspraxis ausgehandelt werden müssen, wurde oben diskutiert. Eine weitere Erkenntnis ist, dass an entsprechenden Schnittstellen der kommunalen Verwaltungsapparate Wissen, Ressourcen und Zugangsmöglichkeiten $\mathrm{zu}$ fehlen scheinen, anhand derer unkompliziert Strukturdaten zur Verfügung gestellt werden könnten. Möglicherweise deutet dies darauf hin, dass Projekte, wie das hier untersuchte, eher geringen Rückhalt in sowohl Stadtverwaltung als auch Bürgerschaft haben, was wiederum die Reichweite und Nachhaltigkeit eines solchen Projekts von vorneherein behindern kann. 


\section{Ethnographische Sozialraumbegehungen}

Anhand ethnographischer Sozialraumbegehungen wurden die Sozialräume qualitativ daraufhin untersucht, wo und inwiefern Barrieren bestehen, die Personen in ihren Teilhabemöglichkeiten behindern. Ebensolche qualitativen Verfahren werden der Relationalität von Raum gerecht, wogegen quantitative Untersuchungen problematisch sind, da sie »Raum zu einer prädiskursiven Einheit« (Bauriedl 2008, S. 282), also einer Art Behälterraum, machen und somit diametral zur Idee einer relationalen Hervorbringung von Raum stehen. Bei den ethnographischen Sozialraumbegehungen handelt es sich um eine exemplarische Untersuchung, die keine allgemein gültigen Aussagen über die Sozialräume trifft. Primäres Anliegen ist vielmehr, Barrieren und etwaige Versuche, diese zu überwinden, problemzentriert zu diskutieren, um so Problematiken und eventuelle Ambivalenzen zu reflektieren ${ }^{24}$. Das methodische Vorgehen wurde bereits in Kapitel 10.4 skizziert, weshalb im Folgenden die aus den Begehungen entstandenen ethnographischen Berichte dargestellt werden. Dabei werden teils Querverweise zwischen den einzelnen Sozialräumen vorgenommen, um Redundanzen zu vermeiden.

\subsection{Ethnographische Berichte}

Ein zentrales Ergebnis der ethnographischen Sozialraumbegehungen ist, dass in allen Sozialräumen in gewisser Hinsicht ein Bewusstsein für Teilhabebarrieren und daraus hervorgehende Ausschlusspraxen besteht und bereits Veränderungen vorgenommen wurden, um Menschen mit unterschiedlichen Unterstützungsbedarfen Teilhabemöglichkeiten zu eröffnen. Trotz dieser zahlreichen Bemühungen werden immer wieder Barrieren offenbar, die Teilhabe einschränken können, sei es durch Gestaltungen, Kommunikationsbarrieren, fehlende Beschilderungen/Übersetzungen usw. (Trescher und Hauck 2017, 2018; Trescher et al. 2017). Immer wieder wird deutlich, dass der Umgang mit Barrieren und ihr Abbau oftmals primär technisch er-

24 Es sei darauf hingewiesen, dass aus Gründen der Anonymisierung einige Beispiele, insbesondere, wenn es sich um konkrete Personen handelt, hier nicht dargestellt werden können. Zusätzlich werden alle Personen in der weiblichen Form benannt, um auch dadurch Rückschlüsse auf Einzelpersonen und daran geknüpfte Aussagen beziehungsweise Beobachtungen zu verhindern. Zudem wurden Aussagen teils paraphrasiert, um mögliche Rückschlüsse auf Sprechende weitergehend zu vermeiden. 
folgen, womit eine gewisse Ferne zur tatsächlichen Lebenswirklichkeit einhergeht. Dies zeigt sich beispielsweise in extrem steilen Rampen, die keine Zugangsmöglichkeiten für Menschen eröffnen, die einen Rollstuhl nutzen oder einen Kinderwagen schieben, oder auch in einem Kino, das in einem Informationsflyer als barrierefrei im Bereich Hören aufgeführt wird, bei dem bei näherer Betrachtung allerdings klar wird, dass sich dies lediglich auf den Aufzug (Anzeige der Stockwerke) bezieht und nicht auf das Filmprogramm des Kinos (beispielsweise Filme mit Untertiteln). Auf diese Art und Weise bleiben Teilhabebarrieren bestehen - trotz entgegengesetzter Bemühungen. Ein weiterer wichtiger Aspekt betrifft Einstellungen und Haltungen der Menschen zu Barrierefreiheit und Teilhabe von Menschen mit unterschiedlichen Unterstützungsbedarfen. Beispielsweise wurde mit einer Frau gesprochen, die kritisiert, dass eine Schule mit Förderschwerpunkt »wegen der Inklusion« geschlossen wurde. Inklusion wird hier als verantwortlich für eine Entscheidung gesehen, die diese Dame als wenig nachvollziehbar erachtet $^{25}$. Aufgrund der Auswahl der Beispiele, anhand derer die Ergebnisse der ethnographischen Sozialraumbegehungen verdeutlicht werden, könnte mitunter der Eindruck entstehen, dass Teilhabebarrieren primär in Bezug auf Unterstützungsbedarfe im Bereich Mobilität manifest werden, dies ist jedoch nicht so. In Bezug auf andere Unterstützungsbedarfe (Sehen, Hören, Lesen/Verstehen etc.) sind vielmehr die Barrieren so zahlreich, dass die Problematisierung dieser sehr redundant wäre - was allerdings ihre Brisanz in keiner Weise schmälert, ganz im Gegenteil. Es ist eine große Problematik bei der Diskussion um Barrierefreiheit, dass gerade die Dimensionen Hören und Lesen/Verstehen kaum bedacht werden, wodurch Menschen mit entsprechenden Unterstützungsbedarfen ausgeblendet werden (siehe umfassend Trescher 2018c). Sie werden dadurch im Sozialraum ein Stück weit unsichtbar. Im Folgenden sind die ethnographischen Berichte für die einzelnen Sozialräume handlungsfeldbezogen und problemfokussiert dargelegt.

25 Inwiefern die Einstellung(en) zu Inklusion bei der Wahrnehmung und Bewertung von Barrieren wirksam werden, wurde im gleichnamigen deutschlandweiten Survey untersucht, dessen Ergebnisse in Kapitel 22 dargestellt sind. 


\subsubsection{Erlangen}

\section{Öffentlicher Personennahverkehr}

In Erlangen ist, wie in den übrigen Sozialräumen auch, der öffentliche Personennahverkehr in Ansätzen auf verschiedene Unterstützungsbedarfe eingerichtet. Die Busse verfügen teils über Rampen, haben Anzeigen und Durchsagen. Die Fahrpläne sind, und auch dies trifft im Gros auf alle Sozialräume $\mathrm{zu}$, teils nicht ohne Weiteres $\mathrm{zu}$ verstehen und gerade für Ortsfremde kann es schwierig sein, sich $\mathrm{zu}$ orientieren. Stellvertretend für die Erfahrungen und Beobachtungen, die gesammelt werden konnten, soll die folgende Szene herangezogen werden.

An einer innerstädtischen Bushaltestelle, an der der Bordstein erhöht ist, hält ein Niederflurbus. Dieser fährt nicht nahe genug an den Bordstein heran, sodass eine Frau, die mit einem Kinderwagen unterwegs ist, Schwierigkeiten hat, einzusteigen. Einen kurzen Augenblick später hält der nächste Bus an dieser Haltestelle. Auch dieser fährt nicht nahe genug heran, sodass eine Frau mit zwei Gehstöcken es kaum schafft, die Distanz zwischen Bordstein und Bus zu überwinden.

An diesem Beispiel zeigt sich eindrücklich, inwiefern >barrierefreie< Gestaltung (erhöhter Bordstein, Niederflurbus) in der Lebenspraxis doch zur Barriere werden kann. Barrierefreiheit ist hier also eher als eine Aushandlungspraxis und weniger als die Bereitstellung technischer Ausstattungen zu verstehen.

Der zentrale Omnibusbahnhof in der Stadtmitte wird wie folgt wahrgenommen:

Es gibt an der Haltestelle circa sechs bis acht Haltepunkte (wie viele es tatsächlich sind, erschließt sich nicht ganz), die an schmalen Inseln angeordnet sind. Diese sind an den seitlichen Enden abgeflacht und an den Längsseiten mit einem Bordstein versehen. Zwischen den Inseln besteht der Bodenbelag aus Kopfsteinpflaster. Unterstände oder Warteplätze mit Sitzgelegenheiten gibt es aufden Inseln nicht. Das Ein- und Aussteigen aus den Bussen und darauffolgende Überqueren der Straße, um von der Haltestelle wegzukommen, ist mit Rollstuhl, Rollator, Kinderwagen oder Fahrrad sicherlich beschwerlich. Zudem fällt die Orientierung an den vielen Haltepunkten schwer. Fahrgäste müssen 
im Vorhinein wissen, an welchem der Haltepunkte der Bus abfährt, den sie nutzen wollen. Dies wird möglicherweise dadurch erschwert, dass es keine großflächigen Symbole beziehungsweise Piktogramme oder groß gedruckte Zahlen gibt, die die Busse und ihre Fahrtziele anzeigen. Durchsagen oder dynamische Anzeigen, auf denen die Dauer angezeigt wird, bis der nächste Bus einfährt, gibt es ebenfalls nicht.

Die Nutzung dieser zentralen Haltestelle ist nicht einfach, wodurch Fahrgäste an ganz unterschiedliche Barrieren stoßen können - Orientierung, Information, Überqueren der Inseln (gegebenenfalls beim Umsteigen auch schnell) usw. Die selbstständige Nutzung des öffentlichen Personennahverkehrs wird dadurch möglicherweise erschwert. Erlangen ist an das Streckennetz der Deutschen Bahn angebunden, sodass von dort auch weiter entfernt liegende Städte erreicht werden können. Der Bahnhof wurde wie folgt erlebt:

Am Bahnhofsgebäude ist nur die mittlere der fünf Eingangstüren eine automatische Schiebetür, alle anderen müssen per Hand geäffnet werden. Um ohne Treppen zum Gleis zu gelangen, muss die Bahnhofshalle durchquert, eine Tür nach draußen per Hand geöffnet und dort mit dem Aufzug zur Unterführung gefahren werden, von der aus wiederum per Aufzug die Gleise erreicht werden können.

Eine Reise mit der Bahn von Menschen, die auf die Nutzung von Aufzügen angewiesen sind, gestaltet sich bereits vor dem eigentlichen Antritt der Reise als komplex und mit erhöhtem Zeitaufwand verbunden. Weitere Beobachtungen zeigen, dass es auf dem gesamten Bahnhofsvorplatz keine sogenannten Blindenleitsysteme gibt. Einzig an der gegenüberliegenden Haltestelle ist eine Stelle als Gefahrenstelle (Noppen auf dem Boden) gekennzeichnet, anhand derer wohl verhindert werden soll, dass blinde oder sehbeeinträchtigte Personen unvermittelt auf der (sehr stark befahrenen) Straße laufen, da der Bordstein dort auf Straßenniveau abgesenkt ist. Hieran kann außerdem eindrücklich verdeutlicht werden, inwiefern unterschiedliche Bedarfe - abgesenkte Bordsteine für einfachere Mobilität vs. vorhandene Bordsteine zur Orientierung - miteinander konkurrieren können. Eine Bahnfahrt nach Erlangen und von dort wieder zurück zum Ausgangsort wurde wie folgt erlebt: 
Aufder Hinfahrt mit einer Niederflurbahn kommt diese auffreier Strecke zum Stehen. Die Durchsage, dass dies ein außerplanmäßiger Halt ist und die Türen geschlossen gehalten werden sollen, erfolgte erst nach einigen Minuten, was bei einigen Fahrgästen sichtlich zu Nervosität und Unruhe führte. Durchsagen der Haltestellen oder eine Anzeige dieser gab es nicht, was uns ebenfalls unruhig werden ließ. Wir sicherten uns mit einer Smartphone-App ab, nach welcher Haltestelle die für uns relevante kommen würde. Aufder Rückfahrt sind in der Niederflurbahn, die wir nutzen, Durchsagen und Anzeigen der Haltestellen. Wir beobachten, wie eine Person, die einen elektrisch angetriebenen Rollstuhl nutzt, die Bahn ohne (für uns erkennbare) Schwierigkeiten verlässt.

Insgesamt wird bei der Reflexion des öffentlichen Personennahverkehrs in Erlangen deutlich, dass gerade Menschen, die Unterstützungsbedarfe im Bereich Lesen/Verstehen haben, wie es auf viele Menschen mit kognitiven Beeinträchtigungen, aber auch auf viele gehörlose Menschen zutrifft, an Barrieren bei der Nutzung von Bus und Bahn stoßen.

\section{Verwaltung}

Im Bereich der Verwaltung wird sich hier auf eine Reflexion der Begehung des Rathauses beschränkt.

Zum Eingang des Rathauses in Erlangen führt eine Rampe. Der Eingang selbst ist eine sich automatisch drehende Drehtür. Am seitlichen Eingang neben der Drehtür gibt es einen Eingang, der als »barrierefrei« benannt ist. Dort findet sich das Hinweisschild, dass dieser "nur bei Bedarf« verwendet werden darf. Unter dieser Vorschrift sind die Piktogramme eines Rollstuhls und eines Kinderwagens. Im Gebäude selbst sind drei Aufzüge vorhanden, von denen allerdings nur einer breit genug ist, dass er mit einem Rollstuhl befahren werden kann, wie auch aus der entsprechenden Bezeichnung dieses Aufzugs hervorgeht. In den Aufzügen werden die Stockwerke nicht angesagt, sondern ausschließlich angezeigt.

Am Beispiel dieser kurzen Beobachtungen können ganz grundlegende Problematiken und Herausforderungen diskutiert werden, die mit Barrieren und einer Forderung nach deren Abbau einhergehen. Der Zusatz, der seitliche Eingang dürfe nur von bestimmten Personen verwendet werden, zeigt, wie eng nach wie vor die Idee von Barrierefreiheit an bestimmte Vorstellungen 
von Beeinträchtigung geknüpft wird, wobei dies hier bereits dadurch abgeschwächt wird, dass neben einem Rollstuhl auch ein Kinderwagen symbolhaft abgebildet wurde. Es stellt sich die Frage, weshalb nicht alle Personen, die gerne die Drehtür - aus welchen Gründen auch immer - umgehen wollen, diesen seitlichen Eingang benutzen dürfen. Zudem wird hier deutlich, dass Personen, die einen Rollstuhl nutzen, als bedürftig konstruiert werden. Mit diesem Labeling gehen (potenziell) immer auch Ausschlusspraxen einher. Dadurch, dass nur einer der drei Aufzüge breit genug für einen Rollstuhl ist, werden Personen, die einen solchen nutzen, von vorneherein auf einen bestimmten Weg festgelegt. Diese sind davon abhängig, dass der Aufzug verfügbar ist, und können nicht einfach auf einen der anderen ausweichen. Personen, die einen Rollstuhl nutzen, stoßen dadurch potenziell an Teilhabebarrieren. Zudem wird einmal mehr deutlich, inwiefern Barrierefreiheit oftmals ausschließlich über die Nutzung eines Sonderweges erreicht wird. Auch hier zeigt sich, dass ein Umbau hin zu Barrierefreiheit im technischen Sinne oftmals eine Kosten-/Nutzenabwägung ist und dies deshalb auch nicht immer perfekt gelingt. So ist es beispielsweise, nicht zuletzt auch aus Kostengründen, nicht möglich, öffentliche Gebäude so umzubauen, dass Menschen, die einen Rollstuhl nutzen, immer und überall dieselben Wege nutzen, wie Menschen, die keinen Rollstuhl nutzen.

Als wir zum Mittagessen mit dem Aufzug in eines der oberen Stockwerke fahren, ist dieser sehr voll und eine mit uns fahrende Rathausmitarbeiterin sagt: "Ja, das ist gerade zur Mittagszeit häufiger so, dass der Aufzug sehr voll ist. Da ist es manchmal einfacher, die Treppe zu nehmen, anstatt ewig aufden Aufzug zu warten und sich dann auch noch reinquetschen zu müssen. Wenn man Pech hat, ist das Essen leer, bis man oben ankommt«, ergänzt sie schmunzelnd.

An diesem kurzen Gespräch kristallisiert sich noch einmal sehr deutlich heraus, inwiefern die Abhängigkeit vom Aufzug zur Barriere werden kann, denn Personen, die auf diesen angewiesen sind, können nicht auf die Treppe ausweichen. Verschärft wird die Situation für Personen, die einen Rollstuhl nutzen, dadurch, dass, wie bereits beschrieben, ihnen nur ein Aufzug zur Verfügung steht. 


\section{Einkaufen}

In der Fußgängerzone ist ein Großteil der Geschäfte ebenerdig zu erreichen. Der Bodenbelag ist circa alle zwei Meter durch einen schmalen Streifen Kopfsteinpflaster unterbrochen, was einerseits Orientierung geben, andererseits jedoch auch als störend empfunden werden kann, da der Boden an diesen Stellen eher uneben ist. Es stehen in der Fußgängerzone Sitzbänke zur Verfügung. Besondere Orientierungssysteme gibt es hier nicht (Leitstreifen, Piktogramme). Exemplarisch für alle untersuchten Geschäfte, soll ein zentral gelegener Supermarkt beschrieben werden:

Der Supermarkt kann stufenlos erreicht werden und verfügt über eine automatisch öffnende Schiebetür. Die Durchgänge sind teils zu schmal, um sie mit einem Rollstuhl, Rollator oder Kinderwagen zu durchqueren. Auch das Nutzen eines Einkaufwagens ist an diesen Stellen erschwert. Hinzu kommt, dass der Pfandautomat an einer eher unzugänglichen Stelle steht, sodass dieser nur eingeschränkt oder in Einzelfällen gar nicht erreicht werden kann. Das Eingabefach des Pfandautomaten ist in Brusthöhe (stehend) und gegebenenfalls zu hoch, um es aus einer sitzenden Position heraus zu erreichen. Orientierungsmöglichkeiten, beispielsweise anhand von Leitstreifen, Piktogrammen, Braille oder Ähnlichem, gibt es nicht.

Situationen wie diese finden sich in allen untersuchten Sozialräumen (und auch darüber hinaus) und stellen eine zentrale Problematik von Einkaufsmärkten dar. Der Einkauf des täglichen Bedarfs kann dadurch sehr schnell zur Belastung werden, die, insofern möglich, an andere abgeben wird. Dadurch entstehen Barrieren bei lebenspraktisch grundlegenden Tätigkeiten, die so gesehen in gewisser Weise zum Ausschluss aus Praxen der Mehrheitsgesellschaft führen. In einem anderen Geschäft dagegen ist es möglich, auch als Person, die einen Rollstuhl nutzt, größtenteils barrierefrei einzukaufen:

In diesem Supermarkt sind die Gänge sehr breit und können, in unserer Wahrnehmung, einschränkungslos genutzt werden. Barrieren entstehen hier in anderer Hinsicht, wie uns diese Dame, mit der wir ins Gespräch kommen, schildert: "Eigentlich kann ich hier ganz gut einkaufen, deshalb komme ich auch immer hierher. Ein Problem ist nur, dass die Türen der Kühlschränke nach außen öffnen und ich dort nicht so einfach an die Sachen rankomme. Außerdem sind die Regale so hoch, dass ich, wenn ich etwas Bestimmtes brauche, im- 
mer nachfragen oder mit jemanden gemeinsam einkaufen muss. Das stört mich ein bisschen. Aber ich kenne mich inzwischen so gut aus, dass ich weiß, welche Produkte eher in der Regalmitte stehen und dann kaufe ich einfach die«.

Es zeigt sich also, dass Barrieren auch dann entstehen können, wenn Wege einschränkungslos zugänglich sind. Barrierefreiheit umfasst also deutlich mehr als das Verbreitern von Eingängen und Wegen. Barrierefreiheit betrifft alltägliche Praxen und konstituiert sich in dieser Hinsicht vor allem in der Aushandlung (Trescher 2018c, S. 20).

\section{Ausgehen}

Gerade in der Altstadt ist ein Großteil der Restaurants und Kneipen nur über Stufen zu erreichen, was primär an der historischen Bausubstanz liegt, bei der Umbauten und Veränderungen oftmals nicht ohne Weiteres möglich sind. Unterstützungsmöglichkeiten für blinde oder gehörlose Menschen finden sich in keinen der Restaurants, die dahingehend stichprobenartig untersucht wurden. Teilhabemöglichkeiten für Personen, die diesbezüglich auf Unterstützung angewiesen sind, werden dadurch erheblich eingeschränkt. Weder im Theater noch in einem der untersuchten Kinos finden sich Informationen über die barrierefreie Zugänglichkeit der Veranstaltungen. Diese müssen online oder telefonisch erfragt werden.

\section{Freizeit und Naherholung}

Unter der Überschrift Freizeit und Naherholung wird die ethnographische Begehung von unter anderem Parks und Grünflächen, Stadtbibliothek, Volkshochschule, Hallenbad, Stadtmuseum und weiteren zusammengeführt. Die Beschreibung des Schlossgartens ist wie folgt:

Der Schlossgarten kostet keinen Eintritt und ist täglich von 6:30 Uhr bis 20:00 Uhr geöffnet, allerdings ist der Eintritt für Fahrräder verboten. Der Wegbelag besteht aus festgetretener, lehmiger Erde und fühlt sich verhältnismäßig eben an - zumindest als FußgängerIn. Die zahlreichen Sitzbänke laden zum Verweilen ein. Sie haben eine normale Sitzhöhe und sind nicht etwa erhöht (um beispielsweise das Aufstehen zu erleichtern). Die Orientierung im Park ist durch kein Leitsystem oder Ähnliches unterstützt. Aufder Überblickskarte ist kein Standort eingezeichnet. 
Die Möglichkeit, mitten in der Stadt einen Park aufsuchen zu können, bietet für die BürgerInnen Erlangens zügig und unkompliziert erreichbare Naherholung - insofern diese in Innenstadtnähe wohnen. Dadurch, dass der Park kostenlos ist, steht dieser grundsätzlich allen Personen offen. Die Orientierung im Park selbst kann unter Umständen dadurch erschwert sein, dass es keine unterstützenden Orientierungssysteme gibt, was gerade bei Personen, die diesbezüglich auf Unterstützung angewiesen sind, zu gewissen Einschränkungen führen kann. Am Beispiel der Stadtbibliothek kann erneut die Problematik verdeutlicht werden, dass barrierefreie Eingänge sehr häufig mit der Inkaufnahme eines Sonderwegs verknüpft sind:

Zum vorderen Eingang der Stadtbibliothek führen vier Stufen. An der Eingangstür findet sich ein Schild, das den Weg zum »barrierefreien Zugang" ausweist. Versehen ist diese Information zusätzlich mit einem Rollstuhlpiktogramm. Der Weg des barrierefreien Zugangs führt rechts um die Ecke des Hauses, sodass dieses sozusagen von der Seite beziehungsweise von hinten betreten wird. Am vorderen Eingang ist ein Schild, das den ersten Fahrradständer als Parkplatz für »Behindertenfahrräder« ausweist. Auch hier ist ein Rollstuhlpiktogramm abgebildet.

So positiv es ist, dass auch für Fahrräder ein entsprechend markierter Parkplatz bereitgestellt wird, stellt sich die Frage, wie festgelegt wird, welche Personen ein Recht darauf haben, hier ihr Fahrrad abzustellen. Für den sogenannten barrierefreien Zugang, muss ein Sonderweg genutzt werden. Es erfolgt so eine Trennung zwischen Personen, die den Vordereingang nehmen können, und Personen, die den Seiten-/Hintereingang nehmen müssen, an der erkennbar wird, dass Barrierefreiheit auch an das vermeintliche Fehlen bestimmter Fähigkeiten geknüpft ist. Klar ist, dass es lebenspraktisch teils nicht anders als über Seiten-/Hintereingänge möglich ist, Zugänge zu eröffnen, beispielsweise aufgrund bestehender Bausubstanz und nur eingeschränkten Möglichkeiten, diese zu verändern. Positiv hervorgehoben werden kann, dass überhaupt für Menschen, die beispielsweise einen Rollstuhl nutzen, der Zugang zur Stadtbibliothek möglich ist. Gleichzeitig ist es allerdings auch wichtig, zu reflektieren, dass dadurch eine Einteilung von Personen in >fähig « und `nicht fähig ‘ weiter fortgeschrieben wird. Barrierefreiheit beziehungsweise entsprechende Vorkehrungen werden dadurch in gewisser 
Weise selbst zur Behinderungspraxis. Eine weitere Beobachtung wurde im Rahmen der Begehung einer Zweigstelle der Volkshochschule dokumentiert:

Die Zweigstelle liegt in einem historischen Gebäude, das über einen ebenerdigen Eingang erreicht werden kann, in den allerdings eine Metallfuge eingelassen ist, die die Eintretenden zum Stolpern bringen kann. Die Tür muss mit der Hand geöffnet werden. Über eine weitere Tür gelangen wir in den Innenhof. Der Bodenbelag dessen ist geteert, aber trotzdem sehr uneben. Nach dem Durchqueren des Innenhofs sehen wir eine weitere Tür, die dieses Mal über die Betätigung eines Schalters automatisch geöffnet werden kann. Im Innenhofliegen die Toiletten; keine davon ist >barrierefreik.

Für Menschen, die auf die automatische Öffnung von Türen angewiesen sind, ist der Zugang zu den Räumlichkeiten der Zweigstelle nicht ohne Unterstützung zu bewältigen. Es irritiert, dass eine der innenliegenden Türen mit einem Öffnungsautomatismus versehen ist, kann dort doch nur hingelangt werden, wenn zwei weitere Türen mit der Hand geöffnet werden. Dies ist erneut ein Beispiel dafür, dass Barrierefreiheit mitunter zwar mitgedacht, allerdings häufig nur punktuell umgesetzt wird, sodass die Reichweite dieser infrage gestellt werden kann. Schließlich sei noch ein kurzes Gespräch, das mit einer Mitarbeiterin des Hallenbads geführt wurde, wiedergegeben:

Ich frage, ob und inwiefern Menschen, die aufeinen Rollstuhl angewiesen sind, hier schwimmen gehen können. Die Mitarbeiterin antwortet mir freundlich, dass das alles gar kein Problem sei. "Die Rollstuhlfahrer dürfen mit dem Rollstuhl bis ans Becken fahren und da ist dann so eine Art Aufzug, mit dem sie ins Becken hinuntergelassen werden können. Das Wasser beginnt nämlich erst einige Zentimeter nach der Beckenkante«. Sie weist mich noch daraufhin, dass Menschen, die einen Schwerbehindertenausweis haben, einen reduzierten Eintrittspreis zahlen.

Hier zeigt sich, dass das Hallenbad auf Menschen, die Unterstützungsbedarfe im Bereich Mobilität haben, ausgerichtet ist. Allerdings ist es unter Umständen nicht ganz einfach, in das Gebäude, in dem das Schwimmbad liegt, zu gelangen. Es gibt zwar eine Rampe, aber die Tür öffnet nicht automatisch, weshalb einige Personen hier wiederum von der Unterstützung anderer abhängig sind. 


\subsubsection{Rostock}

\section{Öffentlicher Personennahverkehr}

Die Straßenbahnhalte sind an vielen Stellen in Rostock gut ausgebaut. Es kann stufenlos ein- und ausgestiegen werden, an den Haltestellen ist ein sogenanntes Blindenleitsystem und eine Durchsage benennt die einfahrende Bahn mit Nummer und Richtung, was den Wartenden erleichtert, sich zu orientieren. In den Bahnen selbst sind mehrere Plätze für Rollstühle respektive Personen, die einen solchen nutzen, reserviert. Zudem werden die angefahrenen Haltestellen durchgesagt und sind auch auf einer Anzeige nachlesbar. Dies erleichtert die Orientierung ungemein - gerade auch für die ortsfremden Forschenden. Diese positive Ausgangsposition wird dann hinfällig, wenn die Teilhabemöglichkeiten einzelner Personen an der Nutzung der Straßenbahnen massiv eingeschränkt werden:

In der Innenstadt lernen wir eine Dame kennen, die mit ihrem elektrisch angetriebenen Rollstuhl jeden Tag mit der Straßenbahn zu ihrer Arbeitsstelle fährt. Seit kurzem darf sie jedoch aus, wie ihr gesagt wurde, "versicherungstechnischen Gründen «nicht mehr mit ihrem Rollstuhl in der Straßenbahn mitfahren. Sie sagt: »Ich finde es diskriminierend, dass ich jetzt nicht mehr mit öffentlichen Verkehrsmitteln zur Arbeit fahren kann."

Dieser Dame - und im Übrigen allen Personen in Rostock, die einen Elektrorollstuhl nutzen - wird die Teilhabe an routinemäßigen Praxen deutlich erschwert. Sie und alle anderen können den öffentlichen Personennahverkehr nicht einschränkungslos und somit nicht im selben Maße nutzen wie Personen ohne einen entsprechenden Unterstützungsbedarf. Dadurch verschärft sich die Abhängigkeit von privat organisierten Fahrdiensten, was wiederum die Eigenständigkeit und die Möglichkeit, spontan irgendwohin zu fahren, massiv einschränkt. Eine weitere Folge ist, dass an sozialen Praxen innerhalb der Stadt nur eingeschränkt teilgenommen werden kann, da diese schlicht nicht erreicht werden - zumindest nicht mit der Straßenbahn. Dadurch werden mögliche Berührungspunkte zwischen Menschen mit und Menschen ohne entsprechende Unterstützungsbedarfe verringert, was wiederum Inklusion im Sinne des Abbaus von Teilhabebarrieren erschwert. Weitere Barrieren können dadurch entstehen, dass Fahrpläne nicht immer auf Anhieb nachvollzogen werden können oder die Kästen, in denen diese 
aushängen, derart überladen sind mit Informationen und Werbung, dass es unter Umständen schwierig ist, die für den Moment relevanten Informationen auf Anhieb zu finden. Erleichtert wird dies an einigen Stellen dadurch, dass dynamische Anzeigen die kommenden Straßenbahnen und ihre Richtung anzeigen, sowie, dass, wie oben bereits beschrieben, bei Einfahrt der Bahnen die Nummer und Richtung dieser genannt werden. Der Fahrkartenkauf gestaltete sich aus Perspektive der Forschenden als verhältnismäßig einfach, wobei zu bedenken gegeben werden muss, dass das Display des Automaten im Sitzen nicht einsehbar ist und auch der Münzeinwurf sowie das Feld zur Eingabe der PIN bei Kartenzahlung nicht erreicht werden. Auch Braille, eine Sprachausgabe oder eine Version mit Piktogrammen und/ oder Leichter Sprache wurden nicht vorgefunden. Der Fahrkartenkauf kann somit für einige Personen zur Barriere werden, die unter Umständen dazu führt, den öffentlichen Personennahverkehr ganz zu meiden, wodurch wiederum Teilhabemöglichkeiten am sozialen Leben der Stadt eingeschränkt werden. In Bezug auf den Regional- und Fernverkehr wurden mehrere Beobachtungen und Erfahrungen gesammelt, von denen einige ausgewählte im Folgenden geschildert werden:

Wir fahren mit der S-Bahn aus Rostock hinaus. An der Haltestelle, an der wir einsteigen, hält die Bahn sehr weit vom Gleis entfernt, sodass wir uns fragen, wie hier unproblematisch eingestiegen werden kann, wenn Fahrgäste beispielsweise mit Rollstuhl, Kinderwagen, Koffer usw. unterwegs sind. Als sich die Türen öffnen, wird eine Art Brücke ausgefahren, die den Abstand zwischen Gleis und Bahn deutlich verringert, wodurch allerdings eine Stufe entsteht, da der Eingang in die Bahn höher liegt als das Gleis. Wir fragen uns, wie hier sbarrierefreik eingestiegen werden kann.

An dieser Stelle zeigt sich erneut, inwieweit Veränderungen, die im Sinne von Barrierefreiheit vorgenommen werden, oftmals nur punktuell sinnvoll sind und anderenorts eher Barrieren erzeugen als diese abbauen. Es ist eine große Herausforderung, Umbaumaßnahmen so vorzunehmen, dass tatsächlich Barrieren verringert und nicht eher verschoben oder sogar (re-)produziert werden. Am Hauptbahnhof können die Gleise per Aufzug erreicht werden, wofür allerdings mehr Zeit benötigt wird, als wenn die Treppe verwendet wird. Reisende, die auf den Aufzug angewiesen sind, müssen also unter Umständen mehr Zeit einplanen - gerade auch beim Umsteigen -, wodurch sich 
unter Umständen die Reisezeit deutlich verlängert. Rolltreppen gibt es keine. Die Orientierung im Bahnhof wird durch sogenannte Blindenleitsysteme an den Gleisen und Anzeigen der abfahrenden Züge unterstützt. Leitsysteme im Gebäude oder auf dem Vorplatz gibt es nicht. Die Fahrkartenautomaten sind alle in Stehhöhe, wodurch die oben bereits geschilderte Problematik (in Bezug auf die Fahrkartenautomaten des Stadtverkehrs), potenziell reproduziert wird. An der Haltestelle Hauptbahnhof Nord dagegen gab es zum Zeitpunkt der Untersuchung weder eine Durchsage noch eine Anzeige der einfahrenden Züge, was die Orientierung deutlich erschweren kann.

\section{Verwaltung}

Beispielhaft für Verwaltungseinrichtungen in Rostock wird sich hier mit dem Rathaus auseinandergesetzt.

Das Rathaus ist an allen Eingängen über Stufen zu erreichen. An einem seitlichen Eingang ist eine Klingel angebracht, die bei Bedarfeines barrierefreien Zugangs betätigt werden soll - zumindest schließen wir dies aus dem Rollstuhlpiktogramm, das unter der Klingel angebracht ist. Über der Klingel ist ein Schild mit der Aufschrift "Ausgezeichnet im Wettbewerb Denkmalschutz barrierefrei 2008 des Bund Heimat und Umwelt«.

Das Rathaus ist ein Beispiel dafür, inwiefern es bei der Umgestaltung historischer Gebäude entlang einer Idee von Barrierefreiheit zur Abwägung von Interessen und Möglichkeiten kommt und wie dennoch Zugänge eröffnet werden können. Problematisiert werden kann allerdings, dass auch hier der Sonderweg in Kauf genommen werden muss, um in das Gebäude zu gelangen. Ähnlich wie bereits in Bezug auf die Stadtbibliothek in Erlangen diskutiert, wird auch hier >Beeinträchtigung oder >Unterstützungsbedarf als Differenzkategorie hervorgebracht, die in Praxen des Ausschlusses resultiert. Menschen, die das Gebäude nicht via Stufen betreten können, werden - auch physisch - getrennt von jenen, die dazu in der Lage sind. Dass der Eintritt in das Gebäude nur nach vorherigem Klingeln möglich ist, hebt die Andersartigkeit dieses Zugangs noch schärfer hervor, da das Gebäude nicht eigenständig betreten werden kann. Die Abhängigkeit der Eintretenden wird dadurch manifestiert. Dies verhält sich ein Stück weit konträr zu der Auszeichnung des `barrierefreien Zugangs`, der so prominent über der Klingel angebracht ist. Sicherlich ist es wichtig und wertvoll, dass für Men- 
schen, die Unterstützungsbedarfe im Bereich Mobilität haben, ein Zugang zum Rathaus ermöglicht wurde. Gleichzeitig muss reflektiert werden, inwiefern dadurch fähigkeitsbezogene Dichotomien fortgeschrieben werden. Darüber hinaus zeigt sich auch hier eine Fokussierung auf ein Verständnis von Barrierefreiheit als >rollstuhlgeeignet, wodurch andere Unterstützungsdimensionen (Sehen, Hören, Lesen/Verstehen oder Fremdsprache) ausgeblendet werden.

\section{Einkaufen und Besorgungen}

Einkaufsmöglichkeiten sind in Rostock zahlreich, weshalb hier nur ein kurzer Ausschnitt wiedergegeben wird. Zusätzlich werden eine besuchte Postfiliale sowie ausgewählte Bankfilialen beschrieben. Ein Großteil der Geschäfte, die in einer zentralen Einkaufsstraße gelegen sind, kann ausschließlich über Stufen erreicht werden, wodurch die in dieser Straße gelegenen Läden kaum durch Personen frequentiert werden, die auf Unterstützung beim Treppensteigen angewiesen sind. Es kann gefragt werden, ob es nicht auch im Sinne des Einzelhandels ist, die eigenen Geschäfte zugänglicher zu gestalten, um die Anzahl möglicher KundInnen zu erhöhen. Hier liegt ein großes Potenzial, das bislang vom Einzelhandel wenig genutzt wird. Vielmehr ist es so, dass die Initiative für Veränderungen häufig primär von behindertenspezifischen Interessensverbänden ausgeht. In der Innenstadt sind viele Geschäfte ebenerdig zu erreichen (zumindest auf der Haupteinkaufsstraße), weitere Orientierungsmöglichkeiten fehlen allerdings auch hier. Dies betrifft teils auch Fußgängerübergänge, die durch eine Ampel reguliert werden.

An einer Kreuzung gibt es eine Ampel mit Akustiksignal. Wir betätigen den Schalter, der das Akustiksignal anfordert. Als die Ampel auf»grün« umschaltet, können wir jedoch kein akustisches Signal vernehmen.

Hieran wird deutlich, dass sbarrierefreie Anpassungen nicht nur eingebaut, sondern auch regelmäßig überprüft und gewartet werden müssen, sodass sie störungsfrei genutzt werden können. Dies wiederum ist oftmals auch eine Kostenfrage.

An einer weiteren Ampel, die FußgängerInnen über eine stark mit Autos befahrene Straße leitet, ist die Grünphase derart kurz, dass wir es während dieser nicht aufdie andere Straßenseite schaffen. Wir fragen uns, wie Menschen, die 
unter Umständen länger benötigen, um die Straße zu überqueren, sicher auf der anderen Seite ankommen können .

Hier wird eine Barriere offenbar, die sich immer wieder findet und die durchaus zur Gefahr werden kann - unabhängig irgendeines Unterstützungsbedarfes. Im Zusammenhang mit der Gestaltung des Innenstadtbereichs kann zudem einer der Aufgänge vom Fluss in die Altstadt problematisiert werden:

Dieser Aufgang verfügt ausschließlich über Treppen (keine Rampen, kein Aufzug) und der Bodenbelag ist gepflastert. Auf der rechten und linken Seite des Aufgangs befinden sich Geländer, weitere Unterstützungen zur Fortbewegung gibt es nicht, beispielsweise auch kein Leitsystem, das Orientierung gibt.

Menschen, die in dieser Situation auf entsprechende Unterstützungen angewiesen sind, können diesen Aufgang nicht uneingeschränkt und selbstständig nutzen und müssen infolgedessen möglicherweise Umwege in Kauf nehmen. Die direkte Verbindung zwischen Fluss und Altstadt bleibt ihnen verschlossen. Auf diese Art und Weise werden Ausschlusspraxen (re-)produziert. Eine Postfiliale, die im Rahmen der Begehungen aufgesucht wurde, wurde wie folgt erlebt:

Wir betreten das Gebäude durch eine automatische Schiebetür. Dahinter befinden sich Geldautomaten, Paketboxen und Briefkästen. Alle sind so angebracht, dass sie im Stehen bedient werden müssen. In diesem Raum befindet sich ebenfalls ein Aufzug, neben dem auf einem Schild vermerkt ist, dass weitere Informationen (beispielsweise bezüglich Dienstleistungen und AnsprechpartnerInnen) im ersten Obergeschoss zu finden sind. Dieses kann ausschließlich über den Aufzug erreicht werden. Die Treppe, die außen am Gebäude verläuft, führt - entgegen unserer ersten Annahmen - nicht zur Post, sondern zur philosophischen Fakultät der Universität. Etwas verwirrt verlassen wir die Post wieder.

Als zentral problematisch wurde hier wahrgenommen, dass der Aufbau des Gebäudes wenig routinemäßig ist und deshalb möglicherweise nicht auf Anhieb verstanden wird. Ortsfremde und/oder Menschen, die Unterstützung bei der Orientierung benötigen, sind hier (zumindest im ersten Moment) unter Umständen ein wenig hilflos. Eine eindeutigere Beschilderung würde unterstützen. In Bezug auf die untersuchten Bankfilialen wurden in 
erster Linie die dort vorhandenen Geldautomaten in den Blick genommen. Es fanden sich in keiner der unterschiedlichen Filialen Geldautomaten, die sitzend bedient werden können. Sprachausgaben gab es ebenfalls keine und eine Beschriftung der Tasten mit Braille war zwar überwiegend, allerdings nicht in allen Fällen vorhanden. Grundlegende Tätigkeiten wie Geldabheben können somit zur Barriere für Menschen mit ganz unterschiedlichen Unterstützungsbedarfen werden, woraus wiederum ein erhöhter Aufwand bei der Auszahlung von Bargeld entsteht - unter anderem Abhängigkeit von den Schalteröffnungszeiten der Filialen oder Abhängigkeit von anderen Personen, die beim Geldabheben unterstützen. Eine der Bankfilialen wurde wie folgt wahrgenommen:

Wir stehen vor der verschlossenen Tür der Bankfiliale. Mit der EC-Karte öffnen wir die Tür. Diese summt, öffnet sich allerdings nicht. Erst nachdem ich kraftvoll dagegen drücke, geht die Tür auf.

An Beispielen wie diesen kann diskutiert werden, dass Zugänge, die zwar barrierefrei genutzt werden könnten, oftmals aber aufgrund von Defekten nicht oder nur eingeschränkt zur Verfügung stehen. Barrierefreiheit heißt also auch, regelmäßige Wartungen durchzuführen und gegebenenfalls im Bedarfsfall unterstützendes Personal zur Verfügung zu stellen. Zu problematisieren ist, dass viele Menschen diesen >Defekt Menschen mit gewissen Beeinträchtigungen aber womöglich nicht. Das heißt, dieser >Defekt<, der womöglich als >nicht so schlimm < eingestuft wird, kann dennoch für einige Personen zur manifesten Barriere werden.

\section{Ausgehen}

Ein Aspekt der Sozialraumbegehungen in Rostock war die Untersuchung von Restaurants und anderen Ausgehlokalitäten, insbesondere jener, die bei einem Onlinereiseführer als besonders gut eingestuft worden waren. Eines dieser Restaurants stellte sich als besonders unzugänglich in der Dimension Mobilität heraus, wie im Folgenden deutlich wird:

Die Tür des Restaurants öffnet nach außen, nach Überqueren der Schwelle folgt eine Stufe beziehungsweise Kante, an die sich eine weitere Tür anschließt, die nach außen öffnet. Hier ist an der Stufe eine Rampe angebracht. Das Res- 
taurant erstreckt sich über mehrere Ebenen, die über Treppen miteinander verbunden sind. Einen Aufzug gibtes nicht.

Für Menschen, die Unterstützung im Bereich Mobilität bedürfen, ist ein Besuch in diesem Restaurant mit einigen Barrieren verbunden. Diese Personen können nur eingeschränkt an dem teilhaben, was das Restaurant bietet. Es vollziehen sich so Ausschlusspraxen bestimmter Personen von der Mehrheitsgesellschaft und darin verorteten kulturellen Praxen, wie die des gemeinsamen Essens und Trinkens. Auch in weiteren untersuchten Restaurants manifestieren sich Treppen als Barrieren. Es wird teils aber auch deutlich, inwiefern Rampen, die eigentlich Zugänge ermöglichen sollen, dieser Funktion kaum nachkommen:

Zum Restaurant führt eine lange, steile Rampe, die allerdings abgesperrt ist. Selbst wenn sie zur Benutzung freigegeben wäre, würde der Weg durch einen großen Blumenkübel, der vor der Eingangstür abgestellt ist, teilweise versperrt. Die Tür öffnet nach außen und muss per Hand aufgezogen werden.

Diese Fokussierung von Barrieren, die im Bereich Mobilität manifest werden, darf, wie eingangs bereits problematisiert wurde, nicht den Eindruck erwecken, dass in anderen Dimensionen keine Barrieren entstünden. Vielmehr ist das Gegenteil oft der Fall, dass nämlich in anderen Dimensionen nach wie vor viele Barrieren vorhanden sind. Beispielsweise finden sich auch in Gebäuden, die als barrierefrei bezeichnet werden, immer wieder potenzielle Barrieren für Menschen mit bestimmten Unterstützungsbedarfen (insbesondere im Bereich Sehen oder Hören). Diese ganz grundlegende Problematik zieht sich durch alle untersuchten Sozialräume.

\section{Freizeit und Naherholung}

Im Bereich Freizeit kann unter anderem die Tourist-Information im Stadtzentrum problematisiert werden, wie aus der folgenden Beschreibung hervorgeht.

Wir stehen vor der Tourist-Information und fragen uns, wie das Gebäude betreten werden kann, würden wir einen Rollstuhl nutzen, mit einem Kinderwagen unterwegs sein oder Ähnliches, da Treppenstufen zur Eingangstür führen. Nachdem wir die Treppe hinaufgestiegen sind, sehen wir in einem Fenster den folgenden Hinweis »Barrierefreier Eingang hinter dem Gebäude«, der mit 
einem Pfeil sowie einem Kinderwagen- und einem Rollstuhlpiktogramm versehen ist. Irritiert gehen wir die Treppe wieder hinunter und überlegen, wie diese Information von Menschen gesehen werden kann, die tatsächlich den alternativen Eingang nutzen wollen.

Hieran zeigt sich erneut sehr eindrücklich, dass eine gewisse Idee von Barrierefreiheit bei den Planenden vorhanden ist und auch die Notwendigkeit anerkannt wird, alternative Zugänge zur Verfügung zu stellen. Gleichzeitig kann die Ausführung dahingehend problematisiert werden, dass sie manchmal wenig praktikabel ist und unter Umständen nur eingeschränkt zum Abbau von Barrieren beiträgt. Die Problematik des Sonderweges wurde oben bereits diskutiert, weshalb an dieser Stelle lediglich darauf verwiesen werden soll. Von den zahlreichen untersuchten Veranstaltungsorten wird an dieser Stelle exemplarisch die Stadthalle herausgegriffen.

Zum Eingang, an dem auch der Vorverkauf von Eintrittskarten zu finden ist, führen circa zehn Stufen. An der Seite des Gebäudes gibt es einen Außenaufzug, der wie eine Art Hebebühne zu funktionieren scheint. Dieser darfnur von autorisierten Personen genutzt werden. Am Seiteneingang befindet sich eine Rampe, an die sich allerdings Treppenstufen anschließen, weshalb auch hier kein stufenloser Zutritt zum Gebäude möglich ist. Andere Orientierungssysteme fehlen.

Auch an dieser Stelle wird deutlich, inwiefern >Wege zur Kultur (Tervooren und Weber 2012b) teils nur eingeschränkt zugänglich sind, wodurch Teilhabebarrieren errichtet werden. Aus Warnemünde, was für viele Rostocker das Naherholungsgebiet erster Wahl zu sein scheint, soll hier exemplarisch nur ein Kuriosum hervorgehoben werden:

Der Weg zu einer barrierefreien Toilette ist mit einem streppabwärts«-Piktogramm ausgeschildert.

Hier zeigt sich erneut, inwiefern Anpassungen, die sbarrierefrei< genannt werden, teils in der Lebenspraxis scheitern oder es an Möglichkeiten fehlt, Symbole verständlicher beziehungsweise zutreffender abzubilden. Schließlich soll das Erleben bei der Begehung der Volkshochschule zur Diskussion gestellt werden. 
An der Tür zum Eingang der Volkshochschule sind Informationen ausgehängt, unter anderem auch in arabischer Sprache. Die Tür muss mit der Hand geöffnet werden und öffnet nach innen. Aufder Schwelle ist eine Kante, über die ich prompt stolpere. Im Erdgeschoss gibt es einen Empfang, der mit einer Mitarbeiterin besetzt ist, die adressiert werden kann, um sich beispielsweise im Gebäude zurechtzufinden. Im Inneren gibt es einen Aufzug, mit dem wir in den vierten Stock zur Aula fahren. Im Aufzug gibt es keine Braille auf den Tasten und die Stockwerke werden nicht angesagt. Die Aula kann stufenlos erreicht werden. Die Tür öffnet nicht automatisch und nach außen.

Am Eingang zur Volkshochschule finden wir zum ersten Mal Informationen in einer anderen Sprache als Deutsch, was von Sensibilität zeugt, sich auf unterschiedliche Sprachen einzustellen und Menschen, die diese sprechen, ganz unmittelbar zu adressieren. Dadurch können Teilhabebarrieren abgebaut werden. Insbesondere die Gestaltung des Aufzugs kann eine Orientierung im Gebäude erschweren, da die Stockwerke nicht angesagt werden. Menschen, die in dieser Hinsicht Unterstützungsbedarfe haben, erfahren so unter Umständen Ausschluss. Türen, die nach außen und zudem nicht automatisch öffnen, können problematisch sein für Menschen, die mit einem Rollstuhl, Kinderwagen oder Ähnlichem unterwegs sind. Gleichzeitig erfüllen solche Türen Sicherheitsvorgaben, da diese im Notfall (beispielsweise bei einem Brand) schnell nach außen geöffnet werden müssen. Solche sogenannten Fluchttüren verhalten sich ambivalent zu den Bedarfen, die Menschen mit Unterstützungsbedarfen im Bereich Mobilität haben. Hieran zeigt sich erneut, inwiefern Barrierefreiheit immer auch eine Abwägung von Bedürfnissen ist.

\section{Südstadt}

In Rostock haben die Projektverantwortlichen einen Schwerpunkt auf den Stadtteil Südstadt gelegt.

In der Südstadt wohnen die Menschen vor allem in Hochhäusern, die dort zahlreich vorhanden sind. Es gibt einen Supermarkt und nach Aussage einer Einwohnerin, mit der wir uns unterhalten, gibt es hier "auch sonst nichts«. Sie berichtet, dass sie »in die Stadt « fährt, wenn sie Cafés, Restaurants oder Einkaufsmärkte besuchen will. In der Südstadt sei »nichts los«. 
Es stellt sich die Frage, wie Menschen, die weniger mobil und flexibel sind als obige Dame, ihren Alltag in der Südstadt gestalten. Das soziale und kulturelle Angebot scheint dort eher eingeschränkt, was die Dame im Gespräch noch einmal bestätigt. Dies zeigt eine Form von Teilhabebarrieren auf, die in dieser Form bislang nicht thematisiert wurden, nämlich die des sozialen Ausschlusses, der aus einer bestimmten Wohnsituation resultiert. Im Fokus des Projekts steht das Stadtteilbegegnungszentrum in der Südstadt.

Das Stadtteilbegegnungszentrum liegt im Erdgeschoss eines Hochhauses. Es verfügt über einen »Behindertenparkplatz«. Zum Eingang führt eine kleine Stufe, an der Seite gibt es eine zusätzliche Rampe. Die Eingangstür öffnet nach außen und das Gebäude kann daraufhin ebenerdig betreten werden. Das Programm, das in einem Glaskasten aushängt, richtet sich an alle Altersgruppen und fokussiert dabei SeniorInnen sowie Kinder und Jugendliche. Informationen in einer anderen Sprache als Deutsch oder in Leichter Sprache und/oder mit Piktogrammen versehen gibt es nicht.

Es ist positiv, dass in einem Stadtteil, in dem es verhältnismäßig wenige Orte der Begegnung sowie Freizeitaktivitäten und kulturelle Angebote gibt, ein Begegnungszentrum etabliert wird, das dem möglicherweise entgegenwirken kann. Handlungsperspektiven ergeben sich daraus, weitere und andere Personen anzusprechen - wie beispielsweise obige Dame, mit der sich unterhalten wurde - um auch diesen Teilhabemöglichkeiten und Zugänge zum sozialen und kulturellen Leben zu eröffnen. Ein Einkaufsmarkt wurde in der Südstadt untersucht. In diesem gibt es eine >barrierefreie< Toilette:

Die Tür der >barrierefreien Toilette, die durch ein Rollstuhlpiktogramm als solche gekennzeichnet wird, öffnet nach außen. Es gibt keine Möglichkeit zur automatischen Türöffnung. Die Toilette ist nicht abschließbar. Im Raum befindet sich außerdem ein Wickeltisch. Auch dies ist an der Tür durch ein entsprechendes Piktogramm verdeutlicht.

So sinnvoll und wichtig eine >barrierefreie< Toilette ist, so problematisch ist es, dass diese nach außen öffnet und zudem mit der Hand aufgezogen werden muss. Besonders problematisch ist, dass jene Toilette, auf die einzelne Personen in ganz besonderer Weise angewiesen sind, nicht abgeschlossen werden kann. 


\subsubsection{Schneverdingen}

\section{Öffentlicher Personennahverkehr}

Der öffentliche Personennahverkehr ist in Schneverdingen nicht sehr gut ausgebaut. Die Menschen, die dort leben, sind sich dieser Problematik bewusst und selbst aktiv geworden, um Abhilfe zu schaffen. Mit dem Aufbau des »Bürgerbusses«, bei dem BewohnerInnen der Stadt den Bus ehrenamtlich fahren, ist es möglich, auch in Schneverdingen (und den angrenzenden Orten) mobil zu sein. Bezüglich der Haltestellen und Busse des regulären Busverkehrs, den es ebenfalls gibt, wurde die folgende Beobachtung gemacht:

An der Bushaltestelle gibt es vier Haltepunkte aufjeweils sehr schmalen Inseln, bei denen teils nur auf einer Seite der Bordstein abgesenkt ist. Aufjeder Insel ist ein Unterstand, der Wartenden Sitzgelegenheiten bietet. Die Inseln sind so schmal, dass mit Rollstuhl, Rollator oder Kinderwagen nicht an diesem Unterstand vorbeigefahren werden kann. Es ist unklar, wie Personen, die einen Rollstuhl oder Rollator nutzen oder mit Kinderwagen unterwegs sind, hier in den Bus einsteigen können - der zudem kein Niederflurbus ist.

Hier wird es also Menschen mit Unterstützungsbedarfen im Bereich Mobilität deutlich erschwert, eigenständig unterwegs zu sein, was die Möglichkeiten zur Aneignung von Raum als subjektiv bedeutsamen Handlungsraum einschränken kann (Trescher und Hauck 2017, 2019). Eine weitere Haltestelle wurde wie folgt wahrgenommen:

Die Haltestelle liegt direkt an einem Schulkomplex (Grund-und Förderschule mit Schwerpunkt Lernen) und wird folglich annehmbar primär von SchülerInnen genutzt. Die Haltestelle ist auf einer Insel angeordnet und es gibt mehrere Halteplätze. Die Mitte der Insel ist von den Haltepunkten abgezäunt, der Boden besteht hier aus Sand und Gras. Wir gehen davon aus, dass sich in diesem Bereich die wartenden SchülerInnen aufhalten sollen. Für Personen, die einen Rollstuhl oder Gehhilfen nutzen, ist dieser Bereich kaum zugänglich. Die Haltepunkte sind nicht mit zusätzlichen Wegweisern oder Piktogrammen versehen, die beispielsweise die Haltestelle erklären. Anzeigen oder Durchsagen der Abfahrtszeiten und ankommenden Busse gibt es ebenfalls nicht. Wir können uns vorstellen, wie durcheinander, voll und laut es sein muss, wenn die SchülerInnen nach Schulschluss in großen Gruppen hier aufden Bus warten. 
Es ist bekannt, dass gerade schulnahe Haltestellen zu bestimmten Zeiten stark frequentiert werden. Dies wird möglicherweise dann problematisch, wenn Haltestellen wenig übersichtlich gestaltet und Warteplätze nicht uneingeschränkt zugänglich sind. Gerade SchülerInnen, die Unterstützungsbedarfe im Bereich Mobilität oder bei der Orientierung haben, können dadurch unter Umständen nicht gleichermaßen teilhaben, woraus wiederum folgt, dass ein zentrales Moment dessen, was es heißt, `SchülerIn`zu sein, nicht uneingeschränkt erlebt werden kann. Denkbar ist, dass jene SchülerInnen auf alternative Beförderungswege zurückgreifen müssen und dadurch sowohl in räumlicher als auch sozialer Hinsicht von ihren MitschülerInnen getrennt werden. Diese SchülerInnen werden somit als sanders hervorgebracht, woraus häufig Ausschluss resultiert. Eine zusätzliche Problematik des Busverkehrs ist, dass dieser in teils sehr geringen Frequenzen verkehrt. Beispielsweise fahren einige Busse außerhalb der Schulzeit im Drei-StundenTakt und samstags gibt es teils lediglich zwei Verbindungen. Hinzu kommt, dass die Fahrpläne nicht sehr übersichtlich gestaltet und gerade für Ortsfremde schwierig nachzuvollziehen sind, insbesondere da in den Plänen einige Ausnahmen und Änderungen verzeichnet sind, auf die anhand von Fußnoten und unterschiedlichen Symbolen hingewiesen wird. Dies ist eine generelle Problematik, die alle untersuchten Sozialräume betrifft. Die Fahrpläne des Bürgerbusses sind dagegen einfacher verständlich, was vor allem daran liegt, dass keine zusätzlichen Informationen abgedruckt sind, die es erschweren, die eigentlich interessierende Abfahrtszeit herauszufinden. Einzig problematisch ist hier, dass am Wochenende keine Fahrten ermöglicht werden können, wodurch gerade im Bereich Freizeit, die sich oftmals am Wochenende vollzieht, die Mobilität eingeschränkt ist. Schneverdingen ist an den regionalen Bahnverkehr angebunden. Im Bahnhof Schneverdingen ist die Orientierung für Ortsfremde und Menschen mit unterschiedlichen Unterstützungsbedarfen unter Umständen dadurch erschwert, dass es keine Durchsagen der ankommenden Züge und Anschlussverbindungen gibt. Zudem zeigt die, zwar vorhandene, Anzeige lediglich die Uhrzeit an. Weitere Einschränkungen können dadurch entstehen, dass ein sogenannter Blindenleitstreifen ausschließlich direkt am Gleis vorhanden ist und im Gebäude selbst oder aus diesem heraus keine speziellen Orientierungsmöglichkeiten gegeben werden. Über den Bahnhof finden sich folgende Informationen in der Broschüre »barrierefrei erleben«, die in der Touristeninformation ausliegt: 
»Fahrkartenschalter und Fahrscheinautomaten befinden sich innerhalb des Bahnhofgebäudes, der Zugang erfolgt über eine Treppe an der Straßenseite und auf der Bahnsteigseite über eine Stufe. Rollstuhlfahrer werden deshalb gebeten, sich am Fenster an der Bahnsteigseite bemerkbar zu machen, Hilfestellung erfolgt. Der Zugang zum Bahnsteig selbst ist ebenerdig. Anreisende wie Abreisende, die beim Ein-, beziehungsweise Ausstieg auf Hilfe angewiesen sind, werden gebeten, sich rechtzeitig anzumelden, damit Hilfestellung garantiert werden kann« (Schneverdingen Touristik 2012, S. 8).

Der Zugang zum Bahnhof ist also nicht uneingeschränkt möglich und Menschen, die einen Rollstuhl nutzen, erfahren hier massiven Ausschluss. Die Angewiesenheit auf andere wird hier ganz deutlich manifestiert, wodurch jene Personen als grundsätzlich hilfebedürftig hervorgebracht werden. Wären die Fahrscheinautomaten stufenlos erreichbar, würden Personen, die einen Rollstuhl nutzen, nicht behindert. In Bezug auf die Broschüre kann zudem problematisiert werden, dass diese zwar einer Leerstelle entgegenwirkt und wichtige Informationen bereithält - es wurde sich ganz offensichtlich mit Barrierefreiheit beschäftigt, was nicht unbedingt selbstverständlich ist. Demgegenüber bleiben die Informationen allerdings auf ein Verständnis von Barrierefreiheit im Bereich Mobilität beschränkt, wodurch Informationen zu anderen Bereichen (beispielsweise Sehen, Hören, Lesen/Verstehen und weitere) nach wie vor fehlen. Hierin kann ganz konkretes Handlungspotenzial ausgemacht werden. Im Bahnhof Schneverdingen hält der sogenannte »Heide-Sprinter«. In diese Niederflurbahn kann stufenlos ein- und ausgestiegen werden und im Zug selbst gibt es Durchsagen und Anzeigen der jeweils nächsten Haltestellen. Die Anbindung an den Fernverkehr erfolgt über den Bahnhof Buchholz, der von Schneverdingen aus direkt erreicht werden kann. Dies ist nicht möglich, ohne eine Barriere zu überwinden:

Als wir am Bahnhof in Buchholz ankommen, müssen wir beim Aussteigen eine hohe Stufe hinaufsteigen. Es scheint, als sei der Bahnsteig nicht für Niederflurbahnen ausgelegt. In den am gegenüberliegenden Bahnsteig abfahrenden Zug »Metronom«kann stufenlos eingestiegen werden.

Menschen, die Unterstützungsbedarf im Bereich Mobilität haben, können nicht einschränkungsfrei von Schneverdingen aus in weiter entfernt liegende Städte reisen, wodurch ihnen ein Stück weit der Anschluss an >die Welt 
außerhalb verwehrt wird und sie sich in gewisser Weise »mit einer halben Welt abfinden« (Goffman 1975, S. 32) müssen. Auf diese Weise vollzieht sich Ausschluss von routinemäßigen Lebenspraxen - wie beispielsweise am Wochenende nach Hamburg zu fahren oder Ähnliches.

\section{Verwaltung}

In Bezug auf das Rathaus in Schneverdingen zeigt sich ebenfalls, was in Bezug auf Erlangen und Rostock bereits diskutiert wurde - zum Eingang in das Rathaus muss ein seitlicher Eingang genutzt werden:

Der stufenlose Zugang zum Rathaus ist als "Behindertengerechter Eingang" ausgeschildert. Der Hinweis ist zusätzlich mit einem Piktogramm einer Person versehen, die einen Rollstuhl nutzt. Der Ratssaal und weitere Sitzungszimmer können nicht unmittelbar aufgesucht werden, sondern der Zugang erfolgt seitlich in das Nebengebäude, von dort mit dem Aufzug nach oben und über einen verbindenden Flur hin zum Ratssaal.

Hier kann unter anderem problematisiert werden, dass Personen, die den stufenlosen Eingang nutzen, als sbehindert adressiert und infolgedessen auch hervorgebracht werden. Eine mögliche (und naheliegende) Handlungsperspektive diesbezüglich wäre beispielsweise, zumindest die Benennung dieses Eingangs zu ändern. Durch die Abhängigkeit vom `Sonderweg` wird die Adressierung bestimmter Personen als >behindert< reproduziert. Dadurch, dass der Zugang zum Ratssaal nur über Umwege zu erreichen ist, wird für bestimmte Personen eine Barriere manifest, die deren Teilhabemöglichkeiten am politischen Geschehen einschränken. Politische Partizipation ist somit in gewisser Weise davon abhängig, Stufen laufen zu können. Auch hieran wird deutlich, inwiefern nachträglich eingebaute Zugänge nicht immer optimale Lösungen darstellen, sondern eher Kompromisse, die aber nichtsdestotrotz dort Zugänge eröffnen, wo sich zuvor Ausschluss vollzog.

\section{Ausgehen}

Auch in Schneverdingen wurde ein Restaurant besucht, das in einem Internetreiseführer als besonders gut empfohlen wurde. Dieses kann wie folgt beschrieben werden: 
Zum Eingang des Restaurants führt eine Rampe. Allerdings öffnet die Eingangstür nach außen, sodass beim Eintritt zwar die Tür erreicht werden kann, um sie zu öffnen muss jedoch die Rampe wieder rückwärts hinuntergegangen/-gefahren werden. Personen, die einen Rollstuhl oder Rollator nutzen oder beispielsweise mit einem Kinderwagen unterwegs sind, sind dadurch immer davon abhängig, dass eine andere Person sie beim Öffnen der Tür unterstützt. Im Restaurant selbst ist es eher eng, sodass gerade für RollstuhlnutzerInnen das Bewegen im Raum erschwert ist. Eine >barrierefreie Toilette gibt es nicht. In den Speisekarten gibt es keine Braille und/oder Abbildungen, die Personen mit entsprechenden Unterstützungsbedarfen die Auswahl des Essens ohne fremde Hilfe ermöglichen.

Auch in Bezug auf dieses Restaurant zeigt sich, dass eine Idee von Barrierefreiheit vorhanden ist, welche in der lebenspraktischen Umsetzung jedoch nicht unproblematisch ist. Es stellt sich nun die Frage, inwiefern Handlungsoptionen gedacht werden können, anhand derer Menschen mit unterschiedlichen Unterstützungsbedarfen Teilhabemöglichkeiten eröffnet werden können.

\section{Freizeit}

Schneverdingen hat ein reges Vereinsleben, das die möglichen Freizeitaktivitäten im Sozialraum maßgeblich prägt. Eindrücke einiger Freizeiteinrichtungen werden im Folgenden dargelegt:

Das Schwimmbad ist auch über eine Rampe zugänglich. Neben der nichtautomatischen Drehtür befindet sich seitlich eine weitere Tür, die mit einem Schalter automatisch geöffnet werden kann. Weitere Unterstützungen bei der Orientierung (Leitstreifen, Symbole und Abbildungen etc.) können wir nicht erkennen. Personen, die einen Schwerbehindertenausweis haben, zahlen einen vergünstigten Eintrittspreis.

Der Zugang zum Schwimmbad in Schneverdingen ist stufenlos möglich. Andere Unterstützungsdimensionen außer der dadurch bedachten Mobilität finden keine Berücksichtigung - wie es so gut wie überall der Fall ist. In Bezug auf die Eintrittsvergünstigungen bei vorliegendem Schwerbehindertenausweis kann ganz grundlegend problematisiert werden, dass damit zwar eine finanzielle Entlastung von Personen vorgenommen wird, die unter 
Umständen nur über geringe finanzielle Mittel verfügen. Demgegenüber ist es allerdings so, dass dem Preisnachlass zwingend vorausgehen muss, sich selbst als sschwerbehindert zu erkennen zu geben. In dieser Ambivalenz wird >Behinderungくals Differenzkategorie reproduziert. Die Zugänge zu Sport- und Turnhalle erfolgen ebenerdig beziehungsweise über eine Rampe. Automatische Türöffnungen gibt es bei beiden nicht. Die Geschäftsstelle des Kulturvereins Schneverdingen e.V. ist ausschließlich über eine Stufe zu erreichen. Selbiges trifft auch auf die Geschäftsstelle der Diakonie Schneverdingen zu. Das Mehrgenerationenhaus ist über einen seitlichen Eingang zu erreichen, an den eine Rampe angelegt wurde. An dieser Stelle kann erneut auf die obigen Diskussionen bezüglich Eingängen verwiesen werden, bei denen ein Umweg in Kauf genommen werden muss und inwiefern dadurch Personen als randersartig، hervorgebracht werden. In Bezug auf das Kino wurde folgende Beobachtung gemacht:

Das Kino "Lichtspiel« wird durch eine schmale Eingangstür erreicht, bei der uns unklar ist, ob ein Rollstuhl ohne Weiteres hindurchpasst. Problematisch ist unter Umständen auch, dass der Bürgersteig vor dem Kino leicht abfallend ist, sodass Rollstühle, Rollatoren, Kinderwagen und Ähnliches daran gehindert werden müssen, ungebremst in Richtung Straße zu rollen.

Sollte die Tür des Kinos tatsächlich zu schmal dafür sein, mit einem Rollstuhl durchquert zu werden, wird so für Menschen, die darauf angewiesen sind, einen Rollstuhl zu nutzen, der Zugang zum Kino massiv eingeschränkt. In Schneverdingen gibt es eine Stadtbücherei, die über eine Rampe erreicht werden kann. Die Eingangstür kann nicht automatisch geöffnet werden. Barrieren können in Bezug auf die Stadtbücherei vor allem darin liegen, dass sie nicht sehr häufig geöffnet hat. Die Stadtbücherei hat an Wochentagen jeweils 3,5 Stunden am Nachmittag geöffnet; dienstags und donnerstags kommen weitere 2,5 Stunden Öffnung am Vormittag hinzu. Samstags ist die Stadtbibliothek vormittags für 2,5 Stunden geöffnet. Diese changierenden Öffnungszeiten erschweren es möglicherweise, den Überblick zu behalten und die richtigen Öffnungszeiten abzupassen. Hinzu kommt, dass am Wochenende, wenn im Allgemeinen viele Menschen Zeit haben, in die Bücherei zu gehen, nur eine kurze Zeitspanne zur Verfügung steht, um Medien $\mathrm{zu}$ entleihen/zurückzubringen. Gleichzeitig ist dies erneut Beispiel für eine Kosten-/Nutzenabwägung, die kulturelle Angebote im Allgemeinen betrifft. 
Diese Problematik wird dadurch verschärft, dass Menschen mit bestimmten Unterstützungsbedarfen nur auf bestimmte Angebote zurückgreifen können. Deren Möglichkeiten, kulturelle und/oder Freizeitangebote zu nutzen, werden dadurch unter Umständen deutlich eingeschränkt.

\section{Alltag}

Im gesamten Stadtgebiet finden sich größtenteils abgesenkte Bordsteine. Sogenannte Blindenleitsysteme oder Ampeln mit akustischen Signalen gibt es dagegen so gut wie keine. Lediglich an einer größeren Kreuzung wurde eine Ampel mit zusätzlicher akustischer Signalfunktion festgestellt. Diese änderte jedoch das Signal nicht, als die Ampel auf sgrün umsprang, wodurch sie ihrer unterstützenden Funktion nicht nachkommt. Schneverdingen ist auf TouristInnen eingestellt, was sich auch an den vielen Wegweisern zeigt. Diese sind zumeist ohne Piktogramme oder andere Ergänzungen, um das Verstehen zu erleichtern und die Abhängigkeit von Schriftsprache zu reduzieren. Auch sind die Wegweiser ausschließlich in deutscher Sprache beschriftet. Zwei Bankfilialen wurden begangen. Die eine kann durch eine seitliche Rampe erreicht werden, die zu einer nicht-automatischen Drehtür führt. Daneben findet sich eine weitere Tür, die allerdings nicht per Schalter geöffnet werden kann, sondern mit der Hand aufgezogen werden muss. Die Geldautomaten befinden sich alle in einer Höhe, in der sie ausschließlich stehend bedient werden können. Dies trifft auch auf die Geldautomaten der anderen Bankfiliale zu. Zudem ist dort kein stufenloser Eintritt möglich.

\section{Bildung}

In Schneverdingen teilen sich eine Grundschule und eine Förderschule mit dem Schwerpunkt Lernen ein gemeinsames Gelände:

Der Schulhof und, so weit wir das beurteilen können, auch das Gebäude, werden von Grund- und Förderschule gleichermaßen genutzt. Der Bodenbelag des Schulhofs besteht teils aus Asphalt (dort befinden sich Tischtennisplatten) und teils aus Sand (dort stehen Klettergerüste). Vor dem Nebeneingang befindet sich ein umzäunter Bereich, der für SkaterInnen vorgesehen ist, befindet sich hier doch beispielsweise eine 'Halfpipes. Der Haupteingang ist ebenerdig zu erreichen. Die Türen können nicht automatisch per Schalter geöffnet werden. Dies ist auch am Seiteneingang der Fall, zu dem zusätzlich Treppenstufen führen. Besondere Leitsysteme oder Orientierungshilfen erkennen wir keine. 
Die gemeinsame Nutzung von Gebäude und Schulhof legt nahe, dass die, häufig starren, Grenzen zwischen Regel- und Förderbeschulung aufgeweicht werden können. Inwiefern dies in der Lebenspraxis tatsächlich der Fall ist, kann nicht beurteilt werden, da zum Begehungszeitraum schulfrei war. Dies ist aber prinzipiell eine spannende Frage, die zukünftig aufgegriffen werden könnte. Im Rahmen der Begehung wurde folgendes Gespräch geführt:

Als wir an der Schule stehen, kommen wir ins Gespräch mit einer Frau, die annehmbar in der Nähe wohnt und gerade mit ihrem Hund spazieren geht. Sie erzählt, dass die Förderschule jetzt geschlossen würde »wegen der Inklusion«. Wir haben den Eindruck, dass sie diese Entscheidung für wenig nachvollziehbarhält.

Mit dieser Aussage trifft die Dame mitten ins Herz der Debatte um schulische Inklusion, die oftmals auf die Annahme beschränkt wird: Alle Förderschulen müssen geschlossen werden. Für den hiesigen Zusammenhang ist wichtig, dass `Inklusion< dadurch oftmals Abneigung entgegengebracht wird, da sie als einseitig verantwortlich für Entscheidungen gesehen wird, die nicht allen Personen nachvollziehbar scheinen. Insofern sollte also auch darüber nachgedacht werden, wie Inklusion als Begriff und Praxis in den Sozialraum getragen wird und welches Verständnis davon in der Bevölkerung überhaupt vorherrscht. Interessanterweise zeigte eine kurze Recherche im Rahmen der Ausarbeitung der ethnographischen Berichte, dass die Förderschule tatsächlich geschlossen wurde und sich auch auf der Internetseite der Schule die Begründung findet: »Die Förderschule wurde im Rahmen der Inklusion am 31. Juli 2017 geschlossen! «².

\subsubsection{Schwäbisch Gmünd}

\section{Öffentlicher Personennahverkehr}

Der Bahnhof in Schwäbisch Gmünd verfügt über ein gut ausgebautes sogenanntes Blindenleitsystem, das allerdings abrupt endet, sobald das Bahnhofgelände verlassen wird. Hieran zeigt sich, dass unterschiedliche Zuständigkeiten im öffentlichen Raum (Deutsche Bahn innerhalb des Bahnhofgeländes, Stadt beziehungsweise Kommune außerhalb) nicht immer

26 www.osterwaldschule-schneverdingen.de/ (zuletzt am 15.07.2018). 
sinnhaft und barrierefrei ineinandergreifen. Zudem kann das Gebäude stufenlos betreten werden und die Eingangstüren öffnen automatisch. In dieser Hinsicht stoßen Menschen mit entsprechenden Unterstützungsbedarfen also an so gut wie keine Barrieren. Problematisch kann es dagegen sein, tatsächlich mit der Bahn fahren zu wollen, wie uns diese Dame berichtet:

Die Frau, die einen Rollstuhl nutzt, berichtet uns in leicht lakonischem Ton, dass man sich bei der Bahn im Regionalverkehr mittlerweile »nur noch einen Tag im Voraus (und nicht wie zuvor drei Tage im Voraus) anmelden muss, um als Rollstuhlfahrerin Unterstützung beim Einsteigen in den Zug zu bekommen.

Dadurch ist ein spontanes Verreisen nicht möglich. Menschen mit Unterstützungsbedarfen im Bereich Mobilität werden so in ihrer Flexibilität eingeschränkt und die Abhängigkeit von Hilfestellungen und bürokratischen Vorgängen (wie beispielsweise der verbindlichen Voranmeldung einer Bahnreise) wird manifestiert. Im Bahnhofsgebäude selbst wird die Orientierung unter Umständen dadurch erschwert, dass es keine dynamische Fahrgastinformation gibt, die die kommenden Verbindungen, Abfahrtsgleise beziehungsweise Gleiswechsel oder Verspätungen anzeigt. Der zentrale Busbahnhof jenseits des Bahnhofs kann wie folgt beschrieben werden:

Es gibt am Busbahnhof insgesamt sechs Haltestellen, die wiederum in jeweils 4 Haltepunkte (A, B, C, D) unterteilt sind. Die anfahrenden Busse haben also 24 mögliche Haltepunkte, was die Orientierung - gerade für Ortsfremde - erschweren kann. Hinzu kommt, dass es keine Anzeigen oder Ansagen bezüglich der einfahrenden Busse gibt, sondern lediglich gedruckte Aushangfahrpläne. Diese sind alle an einer großen Fläche und zusätzlich an den jeweiligen Haltepunkten angebracht. Auf uns wirkt dies auf den ersten Blick sehr unübersichtlich und wir sind froh, dass wir zu Fuß weitergehen können und nicht davon abhängig sind, einen Bus zu nutzen. Zusätzlich erschwert wird die Orientierung dadurch, dass es keine sogenannten Blindenleitsysteme gibt. Es ist uns unklar, wie sich Menschen mit starken Sehbeeinträchtigungen hier selbstständig zurechtfinden sollen.

Die Orientierung an diesem Busbahnhof ist durch die vielen und (zumindest für Ortsfremde) unübersichtlichen Haltepunkte deutlich erschwert. Ortsfremde Personen und/oder Personen, die Unterstützung bei der Orien- 
tierung benötigen, sind dadurch möglicherweise abgeschreckt und meiden unter Umständen diese Haltestelle. Dadurch wird der persönliche Fortbewegungsradius enger und es werden schließlich Teilhabemöglichkeiten an Praxen der Mehrheitsgesellschaft eingeschränkt. Eine weitere, etwas außerhalb des Stadtzentrums liegende Bushaltestelle wurde wie folgt wahrgenommen:

Der Bus der Linie, die wirnehmen sollen, um in die Fußgängerzone zu gelangen, fährt je nach Abfahrtszeit zwei unterschiedliche Haltestellen an, die wenige hundert Meter voneinander entfernt liegen. Da wir das nicht aufAnhieb verstehen und es auch nicht ausgeschildert ist, verpassen wir den Bus, den wir eigentlich nehmen sollten, und müssen auf den nächsten warten. An der Haltestelle, an der wir nun warten, gibt es keine elektronische Anzeige und auch keinen Fahrkartenautomaten, was dann problematisch werden kann, wenn Personen, die einen Rollstuhl nutzen und über die hintere Tür einsteigen, beim Busfahrer/bei der Busfahrerin einen Fahrschein kaufen müssen, allerdings durch den engen Gang nicht bis nach vorne durchfahren können. Die Haltestelle ist mit einem hohen Bordstein versehen, auf den stufenlos aufgefahren werden kann, um dann - ebenfalls stufenlos - in den Bus einzusteigen. Zudem gibt es ein sogenanntes Blindenleitsystem, das vor und nach der Haltestelle allerdings abrupt endet. Beides ist bei vielen Haltestellen, an denen wir aufdem Weg in die Stadt vorbeifahren, nicht der Fall. Der Bus, den wir dann schließlich nehmen, verfügt über eine Rampe, sodass stufenlos eingestiegen werden kann. An Haltestellen, die keinen erhöhten Bordstein haben, ist dies nicht möglich. Da es im Bus weder eine Anzeige noch eine Durchsage der kommenden Haltestellen gibt, verpassen wir unsere Zielhaltestelle - was für uns unproblematisch ist, da wir den Weg zu Fuß zurückgehen können, für Menschen mit entsprechenden Unterstützungsbedarfen ist dies unter Umständen nicht so einfach zu bewältigen.

Die Schilderung dieser Busfahrt zeigt sehr deutlich mehrere Problematiken, an die gerade ortsfremde BesucherInnen der Stadt Schwäbisch Gmünd stoßen, die allerdings zu ganz alltäglichen Barrieren für die EinwohnerInnen der Stadt werden können. Der Busverkehr in Schwäbisch Gmünd kann nicht barrierefrei genutzt werden, was darin begründet ist, dass es zu wenige (auch zuverlässige) Anzeigen und Ansagen sowohl an der Haltestelle als auch im Fahrzeug selbst gibt und dass Haltestellen nur teilweise sbarrierefrei< ausgebaut sind. So kann es zum Problem werden, wenn von der zuständigen Verkehrsgesellschaft zwar Niederflurbusse angeschafft werden, allerdings 
nicht alle Haltestellen passend dazu ausgebaut sind. Eine Busfahrerin teilt uns im Gespräch mit:

"Die Haltestellen sind nicht alle behindertengerecht, das ist aber Aufgabe der Stadt, sich darum zu kümmern. Aber bisher konnten wir noch jeden mitnehmen. Meistens geht es ja eh nur um Kinderwagen. Rollstuhlfahrer haben wir eher selten."

Hieran wird deutlich, dass Barrierefreiheit auch und vor allem ein bürokratischer Akt ist, im Zuge dessen unterschiedliche Zuständigkeiten verhandelt werden müssen. Erkennbar ist auch die Zuversicht der Busfahrerin, im Bedarfsfall Unterstützungsmöglichkeiten zu finden, sodass alle Personen unabhängig etwaiger Beeinträchtigungen am Busverkehr teilhaben können. Barrierefreiheit wird hier in der intersubjektiven Aushandlung konstituiert, die Teilhabemöglichkeiten eröffnet. An Beispielen wie diesen zeigt sich, dass viele Personen sehr engagiert sind und sich bemühen, Barrieren abzubauen, wodurch schließlich Menschen mit Unterstützungsbedarfen Teilhabemöglichkeiten eröffnet werden können. Ganz besonders im Gedächtnis geblieben ist uns die Schilderung einer Mitarbeiterin in einem öffentlichen Gebäude, die immer wieder die Rollatoren von älteren BesucherInnen die Treppe hoch- und runterträgt und einmal sogar zusammen mit einer Kollegin eine Person samt Rollstuhl auf diese Art in das nächste Stockwerk befördert hat ${ }^{27}$. In gewisser Weise ist daran jedoch problematisch, dass Menschen mit entsprechenden Unterstützungsbedarfen von anderen Personen abhängig sind und Angebote, in diesem Falle den Busverkehr, nicht selbstständig nutzen können. Ebenfalls kann reflektiert werden, dass Personen, die einen Rollstuhl nutzen, kaum als Fahrgäste wahrgenommen werden, wobei nicht bedacht wird, dass dies womöglich primär daran liegt, dass der Busverkehr nicht einschränkungslos genutzt werden kann. Auf die Frage, ob und inwiefern der Busverkehr auch von blinden Personen genutzt wird, antwortet eine andere Busfahrerin:

27 Aus Gründen der Anonymisierung soll hier nicht weiter ausgeführt werden, um welche Einrichtung in welchem Sozialraum es sich handelt. 
"Es gab mal einen Blinden, der ist immer mit dem Bus zur Arbeit gefahren, aber da wussten ja eh alle, wo der aussteigen muss. Und jetzt ist der in Rente, also fährt eh keiner mehr mit.«

Auf die Fragen, ob es teils Rückfragen zu den Aushangfahrplänen gibt oder ob sie Erfahrungen mit Verständnisproblemen gemacht hat, antwortet ebenjene Busfahrerin:

"Den Aushangfahrplan müsste der Dümmste kapieren, da hatten wir noch nie Probleme mit. Leute, die total neben der Spur sind, haben ja eh nen Betreuer dabei, die kann man ja auch nicht alleine rumlaufen lassen."

In diesen Ausschnitten aus dem Gesprächsprotokoll wird ersichtlich, inwiefern Beeinträchtigung oder Unterstützungsbedarf negativ konstruiert wird - insbesondere im Bereich Lesen/Verstehen. Hier eröffnen sich Handlungsoptionen, um über bewusstseinsbildende Maßnahmen zur Sensibilisierung der breiten Öffentlichkeit für diverse Unterstützungsbedarfe beizutragen.

\section{Verwaltung}

Am Rathaus in Schwäbisch Gmünd ist ein Schild mit der folgenden Inschrift angebracht:

"Barrierefreier Zugang über Milchgässle«. Neben der Schrift ist das Piktogramm von einer Person, die in einem Rollstuhl sitzt, und einem Kind, das sich in einem Kinderwagen befindet. Neben den Piktogrammen ist ein Pfeil, der nach rechts zeigt.

Auch hier zeigt sich, dass es schwierig ist, Bestandsbauten so zu verändern, dass sie stufenlos betreten werden können. Insofern ist es positiv, dass der Bedarf eines barrierefreien Eingangs bedacht und umgesetzt wird. Dennoch kann problematisiert werden, dass auf diese Art und Weise Personen, die auf einen stufenlosen Eingang angewiesen sind, ein Stück weit von der Mehrheitsgesellschaft getrennt werden - auch wenn dies die einzige Möglichkeit zu sein scheint, überhaupt Zugänge zu eröffnen. Dieselbe Problematik wurde bereits in Bezug auf das Rathaus in Rostock diskutiert, worauf an dieser Stelle verwiesen werden soll. Andere Dimensionen von Barrierefreiheit wer- 
den hier nicht beachtet, woran erneut deutlich wird, inwiefern ein Verständnis von Barrierefreiheit als barrierefrei im Bereich Mobilität überwiegt.

\section{Einkaufen}

In der Fußgängerzone von Schwäbisch Gmünd sind einige Geschäfte nicht ebenerdig zu erreichen. Inwiefern dies zum Problem werden kann, zeigt die folgende Beobachtung:

Eine Frau, die einen Rollstuhl nutzt, befindet sich vor dem Geschäft eines Mobilfunkanbieters, wo sie von der dort angestellten Mitarbeiterin beraten wird. Der Eingang zum Geschäft hat eine Stufe, die von Personen, die einen Rollstuhl nutzen, nicht überwunden werden kann, weshalb das Beratungsgespräch vor der Tür stattfinden muss. Die Verkäuferin läuft immer wieder ins Geschäft und holt verschiedene Gegenstände, die sie vor der Tür dann der Frau zeigt. Nach einer Weile kommt sie uns sehr genervt vor.

Die betreffende Dame muss aufgrund mangelnder Zugänglichkeit des Geschäfts ein Beratungsgespräch auf der Straße in Kauf nehmen. Nun kann eingewandt werden, dies sei doch ein Beispiel dafür, wie Barrieren lebenspraktisch überwunden werden können. Zu bedenken gegeben werden muss allerdings, dass auf diese Art und Weise die Privatsphäre der Dame massiv verletzt wird, könnte doch potenziell jede/r PassantIn das Gespräch mithören. Die originär privaten Angelegenheiten der Dame werden öffentlich gemacht - sie wird in ihrer Privatsphäre beeinträchtigt. Im Gespräch mit einer Passantin erfahren wir das Folgende:

"Ich erinnere mich, dass es mal eine Initiative gab, alle Geschäfte in der Innenstadt mit einer automatischen Türöffnung zu versehen. Die ist dann letzten Endes aber gescheitert. Ich weiß gar nicht so genau, warum."

Demgegenüber konnte auch ein Beispiel dafür dokumentiert werden, wie Menschen mit Unterstützungsbedarfen im Bereich Mobilität auf dem ersten Arbeitsmarkt in einem Geschäft arbeiten:

In einem Geschäft der deutschlandweiten Einkaufsmarktkette »REWE « gibt es einen sogenannten "Rollstuhl-Kurier«, der von einer Person, die einen Rollstuhl nutzt, angeboten wird und ähnliche Dienstleistungen wie ein sogenann- 
ter Fahrradkurier bietet. Der Rollstuhl-Kurier bringt die Waren, die von den KundInnen telefonisch vorbestellt werden, innerhalb der Innenstadt zu diesen nach Hause beziehungsweise zur Mittagspause ins Büro/zur Arbeitsstelle, sodass diese nicht selbst den Weg in den Einkaufsmarkt antreten müssen.

Auf diese Art und Weise werden Menschen, die einen Rollstuhl nutzen, sichtbar im Handlungsraum und eignen sich Raum, im hiesigen Fall als Arbeitsplatz, an. Insofern übernimmt der Rollstuhl-Kurier aus Schwäbisch Gmünd also eine Art Vorreiterrolle und seine Arbeit trägt dazu bei, dass sich die öffentliche Wahrnehmung von Menschen mit Unterstützungsbedarfen verändert. Praxen wie diese tragen zu oben genannter Bewusstseinsbildung bei.

\section{Ausgehen}

Auch in Schwäbisch Gmünd wurde ein von einem Internetreiseführer empfohlenes Restaurant besucht. Dieses kann wie folgt beschrieben werden:

Über einige Stufen erreichen wir die Eingangstür des Restaurants. Nachdem wir diese durchquert haben, sehen wir, dass das eigentliche Restaurant im ersten Obergeschoss liegt und über eine breite Treppe erreicht werden kann. Einen Aufzug gibt es nicht.

Dieses Restaurant ist für Menschen mit Unterstützungsbedarfen im Bereich Mobilität nicht ohne umfängliche Hilfestellungen von anderen Personen zu erreichen. Dadurch können diese nicht an routinemäßigen Praxen teilhaben, wie im konkreten Fall, in einem sehr gut bewerteten Restaurant einzukehren, und erfahren somit sozialen Ausschluss. In Bezug auf Restaurantbesuche stehen gerade gehörlose Menschen häufig vor Barrieren, wie die folgende gehörlose Dame im Gespräch berichtet:

"Viele Restaurants bestehen darauf, dass Tische telefonisch reserviert werden. Gehörlose Menschen nutzen dafür jedoch Faxgeräte. Es kam schon vor, dass ich ein Fax an ein Restaurant geschickt habe und dann kam zurück: ,Wir können Tischreservierungen leider nur telefonisch entgegennehmen. Ich musste dann jemanden bitten, für mich dort anzurufen.»

Eine weitere anwesende gehörlose Dame berichtet von ganz ähnlichen Erfahrungen: 
"Einmal bekam ich vom Restaurant die Rückmeldung, dass ich ja einen Nachbarn bitten könnte, für mich anzurufen. Aber ich will doch auch nicht, dass die Nachbarn darüber Bescheid wissen, wo ich gerne Essen gehe!«

In Bezug auf das Beauftragen von Handwerksdienstleistungen berichtet sie von ganz ähnlichen Schwierigkeiten:

"Einmal wollte ich Handwerker beauftragen und habe ihnen dazu ein Fax geschickt. Als lange keine Antwort daraufkam, habe ich meine Schwiegertochter gebeten, für mich dort anzurufen und nachzufragen. Da haben sich die Handwerker entschuldigt und meinten, sie bekommen so selten Faxe und haben sich deshalb nicht darum gekümmert. Ich habe die Erfahrung gemacht, dass, wenn ich auf das Fax schreibe, dass ich gehörlos bin und deswegen nicht telefonieren kann, eher auf mein Anliegen eingegangen wird. Aber eigentlich will ich das ja auch nicht, dass ich immer und überall drauf schreiben muss: >Bitte beachten Sie, ich bin gehörlos‘.«

Die lebenspraktische Abhängigkeit vom Telefon zieht weitere Abhängigkeiten nach sich, nämlich beispielsweise Verwandte oder Bekannte bitten zu müssen, stellvertretend für einen selbst zu telefonieren. Hieran zeigt sich, inwiefern eine gewisse Inflexibilität und Unwissen darüber, dass nicht alle Personen ohne Weiteres auf das Telefon zurückgreifen können, zur Barriere werden. Im Gespräch berichten die gehörlosen Personen, dass es in vielen Fällen schon hilfreich wäre, wenn ihre Gegenüber langsam sprechen und mehr Mimik und Gestik benutzen würden, da es dann einfacher sei, dem Gesprächsverlauf zu folgen. Kritisiert wird auch, dass es kaum Interesse, aber auch zu wenige Angebote gebe, Deutsche Gebärdensprache zu erlernen. Dadurch gebe es zu wenige GebärdensprachdolmetscherInnen, die infolgedessen frühzeitig angefragt werden müssen, da sie sonst ausgebucht sind. Handlungspotenziale bestehen also darin, auf diverse Kommunikationsbedürfnisse aufmerksam zu machen und gegebenenfalls Deutsche Gebärdensprache als mögliche zu erlernende Fremdsprache mehr in den Fokus der breiten Öffentlichkeit zu rücken. 


\section{Freizeit}

Im Bereich Freizeit wurde sich unter anderem ausführlich mit der Stadtbibliothek in Schwäbisch Gmünd beschäftigt. Deren Begehung wurde wie folgt protokolliert:

Die Eingangstüren zur Stadtbibliothek sind per Hand zu öffnen, eine automatische Türöffnung gibt es nicht. Die Bibliothek ist auf mehreren Ebenen angeordnet, die durch einen Aufzug erreicht werden können, der allerdings bereits seit drei Monaten defekt ist. Von den circa 100.000 verfügbaren Medien werden 267 unter dem Schlagwort »Leichte beziehungsweise Einfache Sprache" geführt. In einer gesonderten Abteilung sind diese Medien zugänglich, die sich an Jugendliche und Erwachsene richten. Einmal im Monat findet dort auch ein Lesekreis statt. Die in der Stadtbibliothek verfügbaren Medien decken circa 25 verschiedene Sprachen ab. Für geflüchtete Personen gibt es gesonderte Angebote, wie beispielsweise die Aufnahme weiterer Sprachen beziehungsweise Anschaffung entsprechender Medien.

Personen, die keine Unterstützungsbedarfe im Bereich Mobilität haben, können die Angebote der Stadtbibliothek, die durchaus divers und auf unterschiedliche NutzerInnen zugeschnitten sind, gut nutzen. Insbesondere das Angebot von Medien in Leichter beziehungsweise Einfacher Sprache eröffnet Menschen, die Unterstützungsbedarfe im Bereich Lesen/Verstehen haben, die Möglichkeit, am kulturellen Leben in der Stadt teilzuhaben. Personen allerdings, die in irgendeiner Weise auf einen Aufzug angewiesen sind, bleibt die Stadtbibliothek als Ort und als soziale Praxis schon seit Monaten verschlossen. Dies ist hoch problematisch, denn dadurch vollzieht sich Ausschluss. Weitere strukturelle Teilhabebarrieren Im Bereich Freizeit schildert die folgende gehörlose Dame, mit der wir ins Gespräch gekommen sind:

»Mein Sohn (8 Jahre) möchte so gerne mit mir zusammen ins Kino gehen. Aber für mich ist das zwecklos, ich kann nichts davon verstehen, was auf der Leinwand passiert. Ich habe den Kinobetreiber schon öfter mal darauf angesprochen, warum es nicht auch Filme mit Untertiteln gibt, aber er meinte, er könne da nichts machen. Und dann bekomme ich noch nicht einmal Rabatt im Kino, obwohl ich die Dialoge ja gar nicht verstehe." 
Durch diese strukturelle Barriere werden sowohl die betreffende Dame als auch ihr Sohn in ihren Teilhabemöglichkeiten am sozialen Leben eingeschränkt. Freizeitaktivitäten, wie ein Kinobesuch, können so nicht gemeinsam erlebt werden, woraus sowohl für die Mutter als auch für den Sohn Ausschluss resultiert. Hieran zeigt sich, dass es nicht zwingend einer Kategorisierung als beeinträchtigt bedarf, um an Teilhabebarrieren zu stoßen, sondern, dass es sich dabei um sehr komplexe Zusammenhänge handeln kann, von denen letztlich auch andere sbetroffen< sind, wie im hiesigen Beispiel der Sohn der Dame. Weiterhin zeigt sich, wie wichtig es ist, mit Menschen ins Gespräch zu kommen, sie ernst zu nehmen und ihre Vorschläge lösungsorientiert zu diskutieren. Menschen mit bestimmten Unterstützungsbedarfen sind ExpertInnen in eigener Sache und wissen selbst am besten, worin Barrieren liegen und wie diese unter Umständen abgebaut werden könnten. Die Innenstadt von Schwäbisch Gmünd ist touristisch erschlossen, es gibt Wegweiser und Hinweistafeln. Diese sind möglicherweise für Menschen mit Unterstützungsbedarfen im Bereich Lesen/Verstehen schwierig nachzuvollziehen, da es keine Piktogramme oder andere Darstellungsarten gibt, die auf Schriftsprache verzichten. Für Menschen mit Sehbeeinträchtigungen oder blinde Menschen gibt es keine Informationen in Braille.

\section{Bildung}

Eine der gehörlosen Damen, mit denen wir ins Gespräch kamen, berichtet von Schwierigkeiten, die für sie in der Schule ihres Kindes entstehen:

»Ein Problem ist, dass Elternabende häufig sehr kurzfristig anberaumt werden. Das macht es für mich sehr schwierig, einen Dolmetscher zu organisieren. Deshalb konnte ich manchmal schon nicht am Elternabend teilnehmen. Es hat auch nichts geholfen, dass ich mit den Lehrern gesprochen habe und gesagt habe, dass gehörlose Eltern frühzeitig informiert werden müssen. Ich habe gesagt, dass das sehr wichtig ist, aber es hat nichts geändert."

Eine danebenstehende Dame ergänzt:

"Ja, das war früher schon ganz genauso. Da hat sich auch nie etwas dran geändert. Tja, aber jetzt sind die Kinder ja schon groß.» 
Aus Perspektive der Personen, die diese Problematik schildern, scheint es kaum nachvollziehbar, warum Termine nicht frühzeitig angekündigt werden können. Auch wenn die Perspektive der Lehrkräfte unbekannt ist, kann problematisiert werden, dass hier offenbar das Bewusstsein dafür fehlt, wie schwierig es ist, GebärdensprachdolmetscherInnen zu beauftragen. Dieses Wissen in der breiten Öffentlichkeit zu verankern und so zu mehr Sensibilität beizutragen, wie auch dazu, dass flexibler auf unterschiedliche Bedürfnisse reagiert wird, ist eine mögliche Handlungsperspektive. Davon profitieren nicht nur gehörlose Personen, sondern alle, die kurzfristige Termine nicht wahrnehmen können, beispielsweise arbeitsbedingt (vor allem Schichtdienst), wegen fehlender Betreuungskräfte für jüngere Kinder oder pflegebedürftige Familienangehörige, fehlender DolmetscherInnen (nicht nur für Deutsche Gebärdensprache, sondern auch darüber hinaus) etc.

\subsubsection{Verbandsgemeinde Nieder-Olm}

\section{Öffentlicher Personennahverkehr}

In der Stadt Nieder-Olm verkehrt der öffentliche Personennahverkehr in größeren zeitlichen Intervallen, weshalb eine genaue Planung der Verbindungen wichtig ist. Spontanes und wenig geplantes Reisen beziehungsweise Unternehmungen mit Bus und Bahn sind nur eingeschränkt möglich. Der Bahnhof Nieder-Olm wurde wie folgt erlebt:

Innerhalb des Bahnhofgeländes gibt es Rampen und abgeflachte Bordsteine. Teils muss ein (weiterer) Umweg in Kaufgenommen werden, um das gewünschte Gleis zu erreichen. Uns fällt auf, dass der Bodenbelag teils sehr uneben ist. Zudem ist im Bahnhof ein sogenanntes Blindenleitsystem vorhanden, das jedoch endet, sobald dieser verlassen wird. Weitere Vorkehrungen, die bei der Orientierung unterstützen könnten, erkennen wir nicht. In die Regionalbahn kann stufenlos ein-und wieder ausgestiegen werden. Auf dem Bahnhofsgelände liegen viele Glasscherben aufdem Boden. Die Warteplätze sind teils dreckig und zerstört.

Wie auch in den anderen Sozialräumen teils beobachtet wurde, beschränken sich unterstützende Orientierungs- und Mobilitätsangebote auf das Bahnhofsgelände. Sobald dieses verlassen wird, enden sogenannte Blindenleitsysteme und können Wege nicht mehr stufenlos begangen werden. Dies 
liegt primär daran, wie oben bereits problematisiert, dass unterschiedliche Zuständigkeiten vorliegen (innerhalb des Bahnhofs die Deutsche Bahn und außerhalb die Kommune) und deshalb der Ausbau von dem, was sbarrierefrei genannt werden kann, in unterschiedlichem Tempo und mit unterschiedlichen Schwerpunktsetzungen vorangetrieben wird. Der offensichtliche Vandalismus kann dann zum Problem werden, wenn beispielsweise Glasscherben auf dem Boden liegen und Menschen, die einen Rollstuhl nutzen, oder Menschen mit starken Sehbeeinträchtigungen diesen nicht ausweichen können beziehungsweise auf die Wege angewiesen sind, die verschmutzt sind. Bezüglich der Anzeige am Bahnhof kann problematisiert werden, dass diese lediglich die aktuelle Uhrzeit und nicht die kommenden Verbindungen anzeigt. Zur Diskussion dessen sei auf die entsprechenden Ausführungen zum Bahnhof in Schneverdingen verwiesen, bei dem dies ebenfalls so ist. In Bezug auf die Fahrkartenautomaten wurde folgende Beobachtung gemacht:

Es gibt zwei Fahrkartenautomaten, wobei sich einer bei einem Unterstand befindet, an dem sich Wartende aufhalten können. Der Weg von diesem Warteplatz zum Gleis kommt uns eher weit vor und wir fragen uns, ob es möglich ist, sich schnell ein Ticket zu kaufen und dann sofort in die Bahn einzusteigen. Die Fahrkartenautomaten sind in einer Höhe angebracht, in der sie auch im Sitzen bedient werden können. In Braille sind Informationen angegeben. Allerdings übersetzen diese das Folgende: "Schwerbehinderte Menschen können die Züge des Nahverkehrs mit einem entsprechend gekennzeichneten Ausweis unentgeltlich nutzen«.

Der weite Weg vom Fahrkartenautomaten zum Gleis kann nicht nur für Menschen mit bestimmten Unterstützungsbedarfen zur Barriere werden, sondern ist unter Umständen für alle Personen problematisch. Der hiesige Fahrkartenautomat ist der einzige, der in allen Sozialräumen ausgemacht werden konnte, der auch im Sitzen bedient werden kann. Dadurch eröffnen sich Teilhabemöglichkeiten für alle Personen, die auf die niedrige Höhe angewiesen sind. Es ist positiv, dass auch Informationen in Braille an diesem Automaten verfügbar sind. Dass sich diese allerdings auf eine Erklärung des Schwerbehindertenausweises beschränken, ist schade. Wichtig wäre es, für die Bahnfahrt relevante Informationen in Braille wiederzugeben (beispielsweise Abfahrtszeiten). Auf einer Bahnfahrt von Nieder-Olm Richtung Mainz wurde das Folgende beobachtet: 
In die Bahn kann stufenlos eingestiegen werden. In einem kleinen Vorraum sind einige Sitzplätze und auch ein Platz für einen Rollstuhl. Rechts und links dieses Vorraums führen Stufen in die eigentlichen Abteile. Im Vorraum befindet sich eine barrierefreie Toilette, die aber gerade defekt ist.

Hier zeigt sich, dass Personen, die einen Rollstuhl nutzen, keine Möglichkeit haben, stufenlos das Abteil zu wechseln und aufgrund dessen in diesem Fall auch nicht die Toilette aufsuchen können. Sie müssten dazu theoretisch die Bahn verlassen und an einem anderen Eingang wieder einsteigen. Während der kurzen Halte der Bahn ist dies sicherlich nicht möglich. Beim Aussteigen stellen wir das Folgende fest:

Als wir in Mainz ankommen, können wir nicht mehr stufenlos aus der Bahn aussteigen.

Von Nieder-Olm aus ist also der Anschluss an den Fernverkehr nicht ohne Weiteres möglich. Dieselbe Problematik wurde oben bereits in Bezug auf Schneverdingen diskutiert, weshalb für eine weiterführende Diskussion darauf verwiesen werden soll. An zwei Bushaltestellen werden unterschiedliche Barrieren manifest:

Um die Haltestelle ist es insgesamt eher eng und es erscheint uns schwierig, sich dort mit Rollstuhl, Rollator oder Kinderwagen zu bewegen. Die Bordsteine sind nicht abgeflacht, was das Erreichen der Haltestelle unter Umständen zusätzlich erschwert. Es gibt weder eine Ansage noch eine Anzeige der kommenden Verbindungen. Ein sogenanntes Blindenleitsystem ist ebenfalls nicht vorhanden. Auf dem Fahrplan sind einige Verbindungen mit einem Rollstuhl-Piktogramm versehen. In der Legende ist dieses wie folgt beschrieben: »In der Regel - Niederflurbus - keine Gewähr«.

Für Ortsfremde ist die Orientierung an dieser Haltestelle schwierig, vor allem, da es keine dynamischen Anzeigen der kommenden Verbindungen gibt. Für Menschen, die Unterstützungsbedarfe im Bereich Lesen/Verstehen haben, kann dies unter Umständen zur Barriere werden, wodurch die Fortbewegung per öffentlichem Personennahverkehr im Sozialraum eingeschränkt wird. Wenig zuverlässig ist wohl auch die Art des Busses, der die Haltestelle anfährt, können sich die Fahrgäste doch nicht darauf verlassen, 
dass dies ein Niederflurfahrzeug ist, in das stufenlos eingestiegen werden kann. So ist es möglich, dass beispielsweise Menschen, die einen Rollstuhl nutzen, die Fahrt nicht antreten können, da sie nicht in den Bus gelangen. Dies erzeugt Ausschluss, auch dahingehend, dass dann unter Umständen gar nicht mehr auf den öffentlichen Personennahverkehr zurückgegriffen wird. Die andere Haltestelle kann wie folgt beschrieben werden:

Auch an dieser Haltestelle ist kein sogenanntes Blindenleitsystem. Dafür gibt es an einem Pfahl einen Schalter, mit dem die akustische Ansage der Abfahrtszeiten aktiviert werden kann. Diese Ansage ist allerdings defekt. Zudem ist der Pfahl, an dem der Schalter angebracht ist, mit Baustellenmaterial zugestellt (ein Zaunstück und ein Betonpflock, in den Schilder gestellt werden können), sodass dieser, selbst wenn er nicht defekt wäre, gar nicht ersterreicht werden kann. Zusätzlich begrenzt wird der Pfahl durch einen davorstehenden Mülleimer. Auf der Bank, die Wartenden als Sitzgelegenheit dient, liegt ein Signalschild, das wohl auf die Baustelle aufmerksam machen soll, aber nun die Sitzplätze versperrt. An einer dynamischen Verbindungsanzeige sind die kommenden Busse und ihre Ziele angezeigt. Teils ist die Abfahrtszeit als Uhrzeit und teils als »in $x$ Minuten« angegeben, was unübersichtlich wirkt.

An dieser Haltestelle werden Teilhabebarrieren nicht (primär) durch die Haltestelle selbst manifest, sondern dadurch, dass diese durch zusätzliche physische Barrieren versperrt wird. Selbstredend ist es immer möglich, dass bei elektronischen Anzeigen und Ansagen Ausfälle und Defekte entstehen, allerdings muss auch bedacht werden, dass diese Ausschluss nach sich ziehen. Es bedarf also Überlegungen, wie Defekte verhindert oder alternative Informationsmöglichkeiten bereitgestellt werden können. Ersteres kann gegebenenfalls durch regelmäßige Wartungen erzielt werden, Letzteres möglicherweise durch eine/n ServicemitarbeiterIn vor Ort, der/die im Bedarfsfall die Informationen gibt, die im Regelfall die Ansage geben würde. Es erscheint positiv, dass eine dynamische Verbindungsanzeige vorhanden ist, denn diese kann die Orientierung ungemein erleichtern. Problematisiert werden kann, dass die uneinheitliche Anzeige von Zeitpunkten und Zeiträumen unübersichtlich ist und insofern die Orientierung eher erschwert. Zusätzlich muss auch in Nieder-Olm problematisiert werden, dass Aushangfahrpläne nicht einfach nachzuvollziehen sind, wodurch gerade für Menschen mit Unterstützungsbedarfen in den Bereichen Lesen/Verstehen Barrieren manifest 
werden. Darüber hinaus zeigt sich hier ganz deutlich, inwiefern Barrierefreiheit als Aushandlungspraxis konstituiert wird, die sich zwischen unterschiedlichen Strukturen und Subjekten vollzieht. Die Person, die den Pfahl zugestellt und ein Signalschild auf die Bank gelegt hat, tat dies sicherlich nicht in der Absicht, bestimmte Personen zu behindern. Vielmehr ist anzunehmen, dass dadurch der Weg freigeräumt werden sollte, sodass Menschen mit Rollstuhl, Rollator oder Kinderwagen ungehindert an der Haltestelle vorbeilaufen oder in den Bus einsteigen können. Barrierefreiheit heißt also auch, Interessen abzuwägen. Eine Busfahrt, die an oben beschriebener Haltestelle angetreten wurde, kann wie folgt wiedergegeben werden:

Der Niederflurbus verfügt über eine Rampe zum Ausklappen. Im Businneren ist das Symbol für Barrierefreiheit ein Piktogramm, das einen Menschen mit einem Gehstock zeigt. Während der Fahrt werden die Haltestellen sowohl angesagt als auch angezeigt. Zudem ertönt ein Signalton, wenn der Halteknopf gedrückt wird und auf der Anzeige erscheint das Blinksignal »Wagen hält«. Wir bemerken, dass die Namen der Haltestellen teils stark verkürzt sind (teilweise bis zu drei Abkürzungen in einem Namen), was dazu führt, dass aufder Anzeige die Haltestellennamen teils unkenntlich beziehungsweise nicht nachzuvollziehen sind.

Die prinzipiell gute Ausstattung des Busses wird ein Stück weit dadurch beeinträchtigt, dass auf der Anzeige die Haltestellennamen stark verkürzt werden. Dies ist annehmbar ein technisches Problem, das ohne größere Mühen lösbar erscheint. Es stellt sich die Frage, weshalb bislang keine Lösung dafür gefunden wurde, denn es ist klar, dass eine solche Anzeige die Orientierung entlang der Strecke deutlich erschweren kann, wodurch möglicherweise Teilhabebarrieren errichtet werden. In Nieder-Olm stellt sich die zentrale Frage, wie die anderen Orte der Verbandsgemeinde erreicht werden können. Verbindungen mit dem öffentlichen Personennahverkehr gibt es nur zu bestimmten Zeiten, zudem besteht eine teils große Abhängigkeit davon, diesen auch nutzen zu können. Dies resultiert darin, dass für die Mobilität innerhalb der Verbandsgemeinde ein PKW unerlässlich ist. Menschen, die nicht auf diesen zurückgreifen können, werden so ein Stück weit immobil und dadurch auch in ihren Teilhabemöglichkeiten am sozialen und kulturellen Leben außerhalb ihres unmittelbaren Wohnorts eingeschränkt. 


\section{Verwaltung}

Das Rathaus der Verbandsgemeinde Nieder-Olm wurde begangen und dabei wie folgt wahrgenommen:

Das Rathaus kann über eine Rampe erreicht werden, die zu automatischen Schiebetüren führt. Mit dem Aufzug können die oberen Stockwerke angefahren werden. Der Aufzug selbst kommt uns sehr schmal vor und wir fragen uns, ob auch größere Rollstühle hier hineinpassen. Das Gebäude verfügt über ein sogenanntes Blindenleitsystem. Besonders hervorzuheben ist ein mit Braille versehener Übersichtsplan, auf dem die Stockwerke sowie die dort zu findenden Abteilungen beschrieben sind. Piktogramme, die die Orientierung vereinfachen, sehen wirkeine.

Das Rathaus der Verbandsgemeinde Nieder-Olm ist das einzige öffentliche Gebäude (zumindest insofern die Gebäude begangen werden konnten), das über ein solches Orientierungssystem für blinde Personen verfügt. Dadurch wird betreffenden Personen die selbstständige(re) Orientierung im Gebäude möglich. Dieses Bestreben, ein barrierefreies Bewegen im Gebäude zu ermöglichen, kann dahingehend erweitert werden, beispielsweise Piktogramme anzubringen, die Personen unterstützen, die (deutsche) Schriftsprache nur eingeschränkt lesen und verstehen können.

\section{Einkaufen}

In Nieder-Olm ist ein Großteil der Geschäfte ausschließlich über Stufen zu erreichen, Rampen finden sich nur selten. Dies kann zur Barriere werden, wie in der folgenden Beobachtung deutlich wird:

Eine Frau, die einen Rollstuhl nutzt, und ihre Begleiterin stehen vor einer Bäckerei, deren Eingang ausschließlich über Stufen zu erreichen ist. Die Begleiterin bespricht mit der Frau, was sie gerne haben möchte und geht dann ohne sie in die Bäckerei, um den Einkauffür sie zu erledigen. Die Frau selbst bleibt vor dem Geschäft stehen und wartet.

Dieses Beispiel, das exemplarisch für eine Vielzahl solcher Situationen steht, zeigt deutlich, inwiefern Stufen zur Barriere werden können. Die Dame, die einen Rollstuhl nutzt, wird so von der lebenspraktisch zentralen Tätigkeit, 
selbstständig und unabhängig einkaufen gehen zu können, ausgeschlossen. Darüber hinaus werden Hürden auch bei Bankfilialen deutlich:

Die Bankfiliale wird über fünf Treppenstufen erreicht, die zu einer Tür führen, die mit der Hand geöffnet werden muss. Ein sogenanntes Blindenleitsystem, das beispielsweise zu den Geldautomaten führt, ist nicht vorhanden. An den Geldautomaten sind dagegen einige der Tasten mit Braille versehen.

Eine Filiale der Deutschen Post dagegen kann über eine Rampe erreicht werden. Hier befindet sich auch eine Toilette, die mit einem Rollstuhlsymbol versehen ist. Diese Toilette ist verschlossen. Ein weiterer Aspekt, der die Mobilität in Nieder-Olm unter Umständen einschränkt, ist, dass die Straßen teils eine deutliche Steigung aufweisen. Die Fortbewegung per Rollstuhl, Rollator oder Fahrrad ist dadurch möglicherweise beschwerlich und verhindert, dass sich bestimmte Personen ungehindert im Sozialraum fortbewegen können. Klar ist allerdings auch, dass Straßenzüge nicht umfassend abgeflacht werden können. Dennoch ist die Reflexion dessen wichtig, dass nicht alle Personen sich uneingeschränkt auf steilen Straßen bewegen können, um so zu mehr Sensibilität im Umgang mit Menschen mit je bestimmten Unterstützungsbedarfen beizutragen. Für die Verbandsgemeinde Nieder-Olm liegen zwei Broschüren mit dem Titel »Barrierefreie Gastronomie- und Hotelleriebetriebe sowie Arztpraxen im Landkreis Mainz-Bingen" vor (eine als tabellarische Übersicht mit Piktogrammen und eine in Textform), in denen aufgeführt ist, welche gastronomischen Betriebe und welche Arztpraxen in welcher Hinsicht bbarrierefrei< sind. Aufgenommen sind hierbei die Dimensionen Mobilität und Sehen. Über etwaige Angebote in Leichter Sprache oder Deutsche Gebärdensprache wird per zusätzlicher Fußnote informiert. Darüber hinaus sollen gastronomische Betriebe gekennzeichnet sein, die ihre Speisekarte auch in Braille anbieten wollen. Dies will ganz offensichtlich keiner der aufgeführten Betriebe, da keiner entsprechend hervorgehoben wurde. Bei den diesbezüglich überprüften Gaststätten lag auch keine Speisekarte in Braille vor, weshalb für blinde Menschen, die Braille zur Information bedürfen, Barrieren nach wie vor bestehen bleiben. Grundsätzlich kann gesagt werden, dass für Personen mit entsprechenden Unterstützungsbedarfen eine solche Broschüre wertvoll ist, um sich im Sozialraum zu orientieren. Perspektivisch wäre es hier möglicherweise sinnvoll, weitere 
Dimensionen (Hören, Lesen/Verstehen, Fremdsprache) mit in die Übersicht aufzunehmen und darüber hinaus in der Handlungspraxis zu etablieren.

\section{Ausgehen}

Auch in der Verbandsgemeinde Nieder-Olm wurde ein Restaurant besucht, das von einem Onlinereiseführer empfohlen wurde:

Um in das Restaurant zu gelangen, müssen erst drei Stufen nach unten gegangen und der Innenhof durchquert werden, um dann mehrere Stufen zum Eingang hinaufzugehen. Von der Seite führt ein Pfad in den Innenhof, der stufenlos begangen werden kann. Der Innenraum des Restaurants ist in zwei Ebenen unterteilt, die wiederum über eine Stufe erreicht werden können. Zum Obergeschoss führt ausschließlich eine Treppe. Die Speisekarten sind auch hier ohne Abbildungen und/oder Braille.

Dieses Restaurant, das im oben genannten Onlinereiseführer als sehr gut bewertet ist, ist für Personen, die auf einen stufenlosen Zugang angewiesen sind, nicht zu erreichen. Dadurch werden bestimmte Personen von gemeinsamen Praxen ausgeschlossen, was unter Umständen sozialen Ausschluss nach sich zieht. Ein weiteres empfohlenes Restaurant gestaltet sich wie folgt:

Auch zu diesem Restaurant führen mehrere Stufen. Der Innenraum ist eher eng und wir stellen uns vor, dass es schwierig ist, dort mit Rollstuhl, Rollator oder Kinderwagen unterwegs zu sein.

Die Problematik ist hier dieselbe wie oben, sie wird allerdings dadurch verschärft, dass kein stufenlos zugänglicher Außenbereich verfügbar ist und durch die Enge der Tische und Stühle das Fortbewegen zusätzlich erschwert wird.

\section{Freizeit}

Im Bereich Freizeit scheint in der Verbandsgemeinde Nieder-Olm die Festhalle eine zentrale Rolle zu spielen.

Das Gelände, auf dem die Festhalle liegt, ist sehr weitläufig und verfügt über einen großen Parkplatz. Die Festhalle selbst kann stufenlos erreicht werden. Beim Eintritt müssen zwei Türen durchquert werden, die nicht automatisch 
öffnen beziehungsweise per Schalter geöffnet werden können. Sogenannte Blindenleitsysteme gibt es keine.

Für Menschen mit Unterstützungsbedarfen im Bereich Mobilität ist die Festhalle stufenlos zugänglich. Lediglich die Türöffnung könnte potenziell zur Barriere werden beziehungsweise hier könnte sich eine Abhängigkeit von anderen manifestieren. Für Menschen mit diversen Unterstützungsbedarfen, die über den Bereich Mobilität hinausgehen, könnte der Eintritt in die Festhalle mit Schwierigkeiten verbunden sein. Dies ist insofern problematisch, dass dadurch der Zugang zu Kultur eingeschränkt wird, finden doch ein Großteil der kulturellen Veranstaltungen in der Verbandsgemeinde Nieder-Olm hier statt, wie uns diese Dame im Gespräch berichtet:

"Ich bin häufiger hier, weil hier immer wieder schöne und interessante Veranstaltungen sind, zu denen ich hingehe. Im Sommer gehe ich auch gerne in den Biergarten dort oben.»

Dieser Biergarten ist auf der Fläche oberhalb der Festhalle gelegen und sowohl über eine Treppe als auch einen stufenlosen Weg zu erreichen. Letzterer ist allerdings verhältnismäßig steil. Im Untergeschoss sind zudem die Stadtbücherei gelegen sowie die Räumlichkeiten des sogenannten Jugendhauses und des sogenannten Spielkreises. Zu der Stadtbücherei kann folgendes gesagt werden:

Die Bücherei kann über einen längeren Pfad stufenlos erreicht werden, der vom Parkplatz aus um eine große Bepflanzung herum zum Untergeschoss führt. Der direkte Zugang erfolgt über eine Treppe. Die beiden Eingangstüren können stufenlos durchquert werden. Eine Möglichkeit zur automatischen Türöffnung gibt es nicht. Innerhalb der Stadtbücherei gibt es einen Aufzug, mit dem die oberen Etagen erreicht werden können, wie uns berichtet wird. Bücher in Braille oder in Leichter Sprache gibt es nicht. Die Stadtbücherei hat an drei Tagen in der Woche für jeweils zwei bis vier Stunden geöffnet.

Diskutiert werden sollen an dieser Stelle die Öffnungszeiten der Stadtbücherei, bei denen potenziell ähnliche Problematiken auftreten wie bei der Stadtbücherei in Schneverdingen. Auch hier ist es letztlich annehmbar eine Frage der Verfügbarkeit ausreichender finanzieller Mittel, die über die Dauer der 
Öffnung entscheidet. Allerdings haben so alle Personen nur eingeschränkt die Möglichkeit, die Angebote der Stadtbücherei zu nutzen. Auf dem Weg zur Stadtbücherei wurde folgende Beobachtung gemacht:

Am Rande des kleinen Spielplatzes vor der Stadtbücherei, der aufgrund seiner Ausstattung eher für kleinere Kinder gedacht ist, halten sich einige Jugendliche auf. Sie haben Getränke dabei und hören über ihre Mobiltelefone Musik.

Szenen wie diese finden sich in allen Sozialräumen immer wieder. Diese zeugen sehr deutlich davon, wie wichtig es ist, Angebote und Aufenthaltsorte für Jugendliche zu schaffen, an denen sie sich treffen können und wodurch nicht andere Freizeitangebote, wie der oben beschriebene Spielplatz, in ihren Nutzungsmöglichkeiten eingeschränkt werden.

\subsection{Gesamtbetrachtung und Diskussion}

In diesem Kapitel werden die zentralen Ergebnisse der ethnographischen Sozialraumbegehungen noch einmal zusammengefasst sowie in theoretischer, methodischer und handlungspraktischer Hinsicht mögliche Anschlussfragen diskutiert.

\section{Theoretische Gesamtbetrachtung und Diskussion}

Am Ende der problemzentrierten ethnographischen Berichte angelangt, kann diskutiert werden, dass Barrierefreiheit als ein sehr komplexes Gebilde $\mathrm{zu}$ begreifen ist. Es zeigt sich, dass nicht immer von vorneherein offensichtlich ist, was Barrierefreiheit im Konkreten heißt und wann etwas als sbarrierefrei< bezeichnet werden kann. Gleichzeitig wird Barrierefreiheit als Label genutzt und ist etwas, an dem sich Menschen orientieren. Dies kann dann zu Problemen führen, wenn Zugänge und/oder Tätigkeiten, die als barrierefrei deklariert sind, doch nicht uneingeschränkt genutzt werden können. Im Zusammenhang damit steht, dass Barrierefreiheit als Begriff zwar verwendet wird, sich als derart bezeichnete Praxis vollzieht und insofern durchaus eine handlungspraktisch begründete Berechtigung hat, aber dennoch ein Stück weit inhaltsleer bleibt. Es fehlt eine tatsächliche Arbeit am Begriff, eine theoretische Auseinandersetzung damit, wie Barrierefreiheit hergeleitet und fundiert werden kann sowie welche gegebenenfalls widerstreitenden Momente darin vereint sind (Trescher 2018c, S. 137f). Insbesondere 
das darin enthaltene Freiheitsparadigma bedarf einer näheren Betrachtung. Eine zentrale Problematik, die sich im Rahmen der ethnographischen Sozialraumbegehungen immer wieder zeigt, besteht darin, dass barrierefreie Zugänge oftmals nur über Sonderwege erreicht werden können. Dadurch werden zwar Teilhabemöglichkeiten an Praxen der Mehrheitsgesellschaft eröffnet, demgegenüber werden allerdings Personen, die auf diese Zugänge angewiesen sind, in gewisser Weise als sbesonders und abhängig hervorgebracht. Behinderung wird so als Status reproduziert und Teilhabe zum Preis der Besonderung erkauft. Die Frage, die sich dabei stellt, ist, was als höheres Gut zu bewerten ist beziehungsweise, was eher zumutbar ist - Teilhabemöglichkeiten, die Behinderung reproduzieren, oder Ausschluss von mehrheitsgesellschaftlichen Praxen. Zudem manifestiert die Praxis des Sonderwegs eine Dichotomie zwischen ,fähigく und snicht fähig` beziehungsweise anders gesagt zwischen >ohne weiteres zu Teilhabe in der Lage und sunterstützungsbedürftig؛. Die handlungspraktische Umsetzung von sogenannter Barrierefreiheit kann sich also als Behinderungspraxis vollziehen. Darüber hinaus stellt sich die Frage, wie diese Ambivalenz begrifflich gefasst und ausgehandelt werden kann. Die Ergebnisse zeigen zudem, dass Barrierefreiheit nach wie vor im mehrheitsgesellschaftlichen Diskurs (allerdings auch jenseits davon; Trescher 2018c, S. 137f) primär aus einer Mobilitätsperspektive verstanden wird. Insbesondere Unterstützungsbedarfe im Bereich Lesen/Verstehen, aber auch in den Bereichen Sehen und Hören, werden kaum bedacht, was sehr prekär ist für Personen, die auf entsprechende Zugänge angewiesen sind. Diese Leerstelle ist auch in theoretischer sowie empirischer Hinsicht festzustellen, wie in anderen Zusammenhängen bereits problematisiert wurde (Trescher 2018c, S. 137ff). Weiterführend ist die Frage zu stellen, inwiefern gegebenenfalls die mangelnde handlungspraktische Berücksichtigung von Barrierefreiheit jenseits von Mobilität auch auf eine unzureichende theoretische Durchdringung zurückzuführen ist. Möglicherweise fehlt der Handlungspraxis eine theoretische Grundlegung von Barrierefreiheit als Begriff, die sie als Reflexionsfolie heranziehen könnte. Denn einer solchen "theoretisch fundierte[n] Reflexionsbereitschaft" (Dannenbeck 2013, S. 463) muss eine dezidierte theoretische Begriffsbildung vorangehen. Ein weiteres wichtiges Ergebnis der ethnographischen Sozialraumbegehungen ist, dass sich Ausschluss nicht nur für die jeweilige Person mit Unterstützungsbedarf vollzieht, sondern auch für ihr nahestehende Personen wie zum Beispiel Familienmitglieder oder FreundInnen. Hier 
vollziehen sich behindernde Praxen auch mittelbar, was die Wirkmächtigkeit von Ausschluss noch einmal aus einer anderen Perspektive verdeutlicht. Schließlich kann gesagt werden, dass Barrierefreiheit respektive das, was darunter verstanden und in diesem Kontext verhandelt wird, durchaus ambivalent ist und dringend der theoretischen Auseinandersetzung bedarf.

\section{Methodische Gesamtbetrachtung und Diskussion}

Methodisch betrachtet zeigt sich, dass das Instrument der ethnographischen Sozialraumbegehungen sehr gut dazu geeignet ist, Sozialräume als solche zu erfahren. Die dichten Beschreibungen zeichnen ein vielgestaltiges Bild und geben einen guten Einblick in Strukturen und Praxen, die sich im Sozialraum vollziehen. Dadurch werden Sozialräume niedrigschwellig erschlossen und zum Gegenstand der Reflexion. Im Anschluss an die Ergebnisse ist es denkbar, den Fokus der ethnographischen Sozialraumbegehungen in der zweiten Erhebung ${ }^{28}$ insofern zu verschieben, dass die Lebens- und Teilhabegeschichten der EinwohnerInnen der untersuchten Sozialräume mehr im Vordergrund stehen. Es soll mit Menschen ins Gespräch gekommen werden, um Einblick in ihren Alltag, ihre Biographien, das Erleben von Barrieren, das Leben im Sozialraum oder auch Zukunftswünsche zu bekommen. Zur Erhebung solcher persönlicher Geschichten bedarf es einer Weiterentwicklung des methodischen Vorgehens.

\section{Handlungspraktische Gesamtbetrachtung und Diskussion}

In handlungspraktischer Hinsicht eröffnen sich zahlreiche mögliche Fragen und Herausforderungen, die die Ergebnisse der ethnographischen Sozialraumbegehungen aufwerfen. Menschen haben teils sehr unterschiedliche Bedürfnisse, wenn es um Zugangsmöglichkeiten geht, weshalb an eine Gestaltung, die barrierefrei genannt wird, sehr vielfältige Anforderungen gestellt werden. Es ist zu klären, wie den unterschiedlichen Bedürfnissen gerecht werden kann, wo gegebenenfalls Abstriche gemacht werden müssen und wie dies je zu begründen wäre. Dass Standards zur Umsetzung von Barrierefreiheit dabei nicht nur hilfreich sind, wird dann klar, wenn sich

28 Wie eingangs dargelegt wurde, werden die ethnographischen Sozialraumbegehungen sowie die Sozialraumanalysen im Projektzeitraum insgesamt drei Mal durchgeführt - zu Beginn, in der Mitte und am Ende. Dadurch sollen Veränderungen herausgearbeitet werden, die sich über den Zeitraum des Projekts möglicherweise ergeben. 
vor Augen geführt wird, dass niemals alle denkbaren Unterstützungsbedarfe erfüllt werden können. Problematisch ist dabei zudem, dass Standards von Barrierefreiheit immer eine Form der Grenzziehung darstellen, die der Komplexität von Barrieren nicht gerecht wird respektive vollumfänglich gerecht werden kann (Trescher 2018c, S. 140). Weiterhin zeigt sich, dass ein eher technisches Verständnis von Barrierefreiheit und die damit zumeist verbundene technische Ausgestaltung dieser oftmals nicht flexibel genug für individuelle Nutzende ist. In der Praxis braucht es deshalb ein erweitertes Verständnis davon, dass Barrierefreiheit vor allem als Aushandlungspraxis zu verstehen ist. Inwiefern ein solches Verständnis etabliert werden kann, bleibt als Aufgabe und Herausforderung bestehen. In diesem Kontext kann auch die Ambivalenz des Sonderwegs betrachtet werden, die oben bereits diskutiert wurde und bei der sich in handlungspraktischer Hinsicht die Frage stellt, wie damit umgegangen werden kann, dass Zugänge oftmals nur auf gesonderte Weise erfolgen können, wodurch zwar Teilhabemöglichkeiten eröffnet werden, sich gleichzeitig jedoch Behinderungspraxen vollziehen. Wie kann diese Ambivalenz ausgehandelt werden beziehungsweise ist dies überhaupt möglich? Eine problematisierende Nachfrage wäre zudem, ob nicht gegebenenfalls auf das sogenannte Rollstuhlpiktogramm verzichtet werden könnte, anhand dessen zumeist sogenannte Barrierefreiheit kenntlich gemacht wird. Dadurch wird ein »Mythos physischer oder funktionaler Normalität« (Dederich 2012, S. 111) etabliert, der Dichotomien manifestiert. Demgegenüber kann allerdings argumentiert werden, dass jenes Symbol gesamtgesellschaftlich bekannt ist und eine durchaus wichtige Funktion zuverlässig erfüllt. Auch wenn keine Alternativen zu diesem Symbol gefunden werden, so sollte doch zumindest eine Diskussion angestoßen werden, in der dieses in vielerlei Hinsicht stigmatisierende Zeichen reflektiert wird. Handlungspraktisch herausfordernd ist des Weiteren, dass Barrierefreiheit oftmals nur abschnittsweise mitgedacht wird, beispielsweise, wenn auf einen Zugang mit Rampe ein weiterer Zugang mit Stufe folgt oder wenn sogenannte Blindenleitstreifen abrupt enden und somit ins Leere führen. Unterstützungsmaßnahmen wie die genannten schränken das, was Barrierefreiheit genannt wird, in ihrer Reichweite deutlich ein und eröffnen insofern nicht unbedingt Teilhabemöglichkeiten. Demgegenüber kann argumentiert werden, dass es wohl kaum möglich ist, immer und überall jeden möglichen Bedarf zu antizipieren und (beispielsweise baulich) barrierefrei zu gestalten. Daran zeigt sich erneut, wie ambivalent barrierefreie Gestal- 
tung und wie wichtig deshalb eine reflexive Beschäftigung damit ist. Weitere Herausforderungen entstehen darüber hinaus beispielsweise im Kontext Barrierefreiheit und Denkmalschutz ebenso wie bezüglich Barrierefreiheit und Sicherheitsvorgaben. Beide drehen sich um die Fragen, an welcher Maxime sich orientiert werden soll und wie möglicherweise beide Anforderungen miteinander verbunden werden können. Bedacht werden muss auch, wie sichergestellt werden kann, dass das, was als barrierefrei geplant wurde, nicht durch Defekte außer Funktion gesetzt wird. Bauliche Veränderungen müssen gewartet werden, damit es möglichst zu keinen Ausfällen kommt, wie es bei den Begehungen teilweise festgestellt wurde. Eine weitere Problematik betrifft eher strukturschwache Regionen, in der Barrieren für alle, die dort wohnen, manifest werden, beispielsweise durch eine hohe Abhängigkeit von automobilem Verkehr. Dies gestaltet sich für Menschen mit bestimmten Unterstützungsbedarfen noch prekärer und wird bislang kaum aufgefangen. Auch hier braucht es Ideen für beispielsweise barrierefreie und flexibel nutzbare Fortbewegungsmittel. Viele Menschen mit je bestimmten Unterstützungsbedarfen erfahren Teilhabebarrieren, da sie kaum flexibel und unabhängig von anderen an Kulturveranstaltungen teilnehmen, verreisen oder alltägliche Besorgungen erledigen können. Weiterer Ausschluss vollzieht sich dadurch, dass aufgrund dessen die ohnehin oftmals sehr geringen Berührungspunkte zwischen Menschen mit Beeinträchtigungen und Menschen ohne Beeinträchtigungen geschmälert werden. Es braucht Ideen, wie Kontaktmöglichkeiten zwischen den EinwohnerInnen von Sozialräumen geschaffen werden können, dies auch vor dem Hintergrund, dass dadurch Vorurteile und Berührungsängste abgebaut werden können (siehe dazu unter anderem die Ergebnisse des Surveys »Einstellung(en) zu Inklusion« in Kapitel VI). Im Zusammenhang damit ist das Thema Bewusstseinsbildung anzusprechen, das sich im Kern um zwei Fragen dreht: Wie können Personen der Mehrheitsgesellschaft für diverse Bedarfe sensibilisiert werden? Und: Wer soll durch ebenjene bewusstseinsbildenden Maßnahmen überhaupt adressiert werden? Ideen zu entwickeln, wie zu mehr Sensibilität und Bewusstsein beigetragen werden kann, ist eine zentrale Herausforderung für alle, die sich aus handlungspraktischer Perspektive mit Fragen von Teilhabe und Ausschluss beschäftigen. 



\section{Erfahrungen im Miteinander erforschen}

Ein zentraler Teil der Studie sind, wie einführend bereits dargelegt, drei Sozialraumanalysen, die zu drei Zeitpunkten durchgeführt werden (initial, nach zwei und nach vier Jahren ${ }^{1}$ ). Gegenstand dieser Veröffentlichung sind die Ergebnisse der ersten, initialen Sozialraumanalyse, die insofern einen Ist-Stand abbildet. Anhand der sich daran anschließenden Untersuchungen sollen Veränderungen offengelegt werden, die möglicherweise über den Untersuchungszeitraum erfolgen. Die Sozialraumanalysen erfolgen entlang der Forschungsfragen:

Tabelle 16: Forschungsleitende Fragestellungen der Sozialraumanalysen

\begin{tabular}{|l|l|}
\hline \multicolumn{2}{|l|}{$\begin{array}{l}\text { Inwiefern vollziehen sich in Sozialräumen Praxen der Teilhabe und des Ausschlus- } \\
\text { ses? }\end{array}$} \\
\hline & im Kontext Arbeit \\
\hline & im Kontext Freizeit \\
\hline
\end{tabular}

Die Sozialraumanalysen verfolgen das Ziel, gegenwärtige Teilhabebarrieren und -möglichkeiten zu erschließen und, über den Vergleich der Analysen, etwaige Veränderungen nachzuzeichnen. Zudem ermöglichen die Sozialraumanalysen einen Vergleich zwischen unterschiedlichen marginalisierten beziehungsweise von Marginalisierung bedrohten Personengruppen, denn es wird die Teilhabesituation von Menschen mit Behinderung (wobei unterschiedliche Beeinträchtigungsdimensionen abgebildet werden), Menschen

1 Die Zeiträume orientieren sich am Projekt, das untersucht wird, und liegen zu Beginn, in der Mitte und am Ende des Projekts. 
mit Fluchtmigrationshintergrund und Menschen mit Demenz zum Gegenstand gemacht. Die Ergebnisse geben also einen sehr breiten Einblick in die Lebenssituation von Menschen, die oftmals von Ausschluss bedroht oder betroffen sind. Forschungspraktisch werden die Handlungsfelder Arbeit, Freizeit und Barrierefreiheit/Mobilität untersucht, wobei Letzteres als Querschnittsthema zu verstehen ist, das für beide Handlungsfelder relevant ist. Im Handlungsfeld Arbeit werden in den jeweiligen Sozialräumen vor Ort Interviews mit ArbeitnehmerInnen geführt, um zu untersuchen, inwiefern Menschen mit unterschiedlichen Unterstützungsbedarfen an Arbeit teilhaben und ob beziehungsweise inwiefern Teilhabemöglichkeiten bestehen (siehe Kapitel 16). Im Handlungsfeld Freizeit wird die Frage verfolgt, inwiefern Menschen mit unterschiedlichen Unterstützungsbedarfen an Freizeitaktivitäten der Mehrheitsgesellschaft teilhaben beziehungsweise inwiefern Möglichkeiten zur Teilhabe bestehen. Dazu werden Verantwortliche und Ansprechpersonen von Freizeitaktivitäten (sogenannte PrimärvertreterInnen $^{2}$ ) in den Sozialräumen telefonisch kontaktiert und interviewt (siehe Kapitel 17). Schlussendlich können, mit Blick auf die Gesamtergebnisse der Studie, strukturelle und inhaltliche Faktoren abgeleitet werden, anhand derer Teilhabe ermöglicht werden kann oder die zumindest die Erweiterung von Teilhabemöglichkeiten begünstigen. Da die fünf untersuchten Sozialräume zueinander heterogen sind, kann davon ausgegangen werden, dass vielschichtige Ergebnisse generiert werden, die potenziell auf andere Orte übertragen werden können. Ein zentrales Ergebnis kann hier bereits vorweggenommen werden: Im Handlungsfeld Arbeit spielt der Standortfaktor, hier verstanden als der Faktor Wohn- und Arbeitsort, eine deutlich größere Rolle als im Handlungsfeld Freizeit, denn es zeigt sich, dass im Bereich Arbeit signifikante Unterschiede zwischen Großstadt und Kleinstadt/ Gemeinde bestehen, die die Teilhabemöglichkeiten von Menschen mit Behinderung oder Menschen mit Fluchtmigrationshintergrund beeinflussen. Im Handlungsfeld Freizeit dagegen ist dies nicht so, dort spielt die Größe und Lage des jeweiligen Ortes eine untergeordnete Rolle. Die Darstellung

2 Mit dem Begriff PrimärvertreterInnen werden all jene Personen umfasst, die eine Freizeitaktivität verantworten oder dieser in irgendeiner Weise vorstehen (sei es als Trainerln, ÜbungsleiterIn, Vorstand oder Ähnliches). Als PrimärvertreterInnen werden also »ausgewiesene AnsprechpartnerInnen betrachtet, die qualitativ hinreichende Aussagen über Struktur und Abläufe der Aktivitäten bereitstellen« (Trescher 2015b, S. 44) können. 
der Ergebnisse folgt dieser Erkenntnis, weshalb diese nicht immer sozialraumspezifisch dargelegt werden, sondern teilweise entlang der Unterscheidung >Großstadt< und >Kleinstadt/Gemeinde`. Weiterhin wird immer dann auf eine sozialraumspezifische Aufbereitung verzichtet, wenn zwischen den fünf untersuchten Sozialräumen kaum Unterschiede bestehen. In diesen Fällen wird lediglich die Gesamtzahl betrachtet. Dies erhöht zudem die Anonymität der mittelbar durch Interviews mit ArbeitnehmerInnen und PrimärvertreterInnen untersuchten Unternehmen/Betriebe und Freizeitaktivitäten und trägt zum zusätzlichen Schutz dieser und der Sozialräume insgesamt bei.

\section{Methodisches Vorgehen}

Für die Sozialraumanalysen wurde ein mehrdimensional verfahrendes Forschungsinstrument entwickelt, das quantitative und qualitative Methoden sinnhaft verknüpft. Grundlage dessen sind leitfadengestützte Erhebungen, die deskriptiv-statistisch beziehungsweise qualitativ-inhaltsanalytisch ausgewertet werden (siehe Kapitel 10).

\subsection{Forschungsleitende Fragestellungen}

Die übergeordnete Forschungsfrage wurde im Zuge ihrer Operationalisierung weiter konkretisiert, sodass die folgenden Fragestellungen forschungsleitend waren:

1. Inwiefern haben Menschen in den fünf untersuchten Sozialräumen, die von Ausschluss bedroht oder betroffen sind, an Arbeit und Freizeit teil?

2. Wie ist eine etwaige Teilhabe ausgestaltet?

3. Welche Erfahrungen im Miteinander gibt es?

4. Worin werden Gründe dafür gesehen, dass keine Menschen, die von Ausschluss bedroht oder betroffen sind, an Arbeit und Freizeit teilhaben?

5. Was sind Schwierigkeiten und Handlungsperspektiven im Kontext Inklusion und Arbeit beziehungsweise Freizeit? 


\subsection{Behinderung, Fluchtmigrationshintergrund und Demenz als Differenzkategorien}

Menschen sind aus ganz unterschiedlichen Gründen von der Erfahrung bedroht oder betroffen, von in der Mehrheitsgesellschaft verorteten und sich dort vollziehenden Praxen ausgeschlossen zu werden. Um die Frage nach dem Erleben ebenjener Ausschlusserfahrungen operationalisieren $\mathrm{zu}$ können, wurden drei exemplarische Differenzkategorien gewählt - Behinderung, Fluchtmigrationshintergrund und Demenz. Personen, die unter einem oder mehreren dieser Label gefasst werden, haben häufig (zumindest potenziell) eingeschränkte Teilhabemöglichkeiten (siehe u.a. Trescher 2013, 2015b, 2017a, 2017b; Trescher und Hauck 2015). Wie eingangs bereits problematisiert wurde (siehe Kapitel 4), läuft eine solche Manifestation von Behinderungs- respektive Differenzkategorien zwar dem hier zugrunde gelegten Verständnis von Behinderung als Praxis zuwider, dennoch bedarf es, um Ausschlusspraxen zu untersuchen, gewisser vorgängiger Kategorisierungen, damit AdressatInnen der Erhebung festgelegt werden können. Deshalb wurde sich, nach entsprechender eingängiger Recherche nach Personenkreisen, die von Ausschluss bedroht oder betroffen sind, auf die drei Differenzkategorien Behinderung, Fluchtmigrationshintergrund und Demenz verständigt. Im Rahmen der Erhebungen wurde es den interviewten Personen überlassen, ob sie eine Person als behindert, fluchtmigriert oder dement bezeichnen. Dass dadurch die Zuschreibung an das jeweilige Label unterschiedlich sein kann, wurde mit dieser Annäherung an die sSprache des Feldes in Kauf genommen.

\subsection{Erhebung: Leitfadengestützte Interviews}

Die Erhebung erfolgte anhand leitfadengestützter Interviews (siehe Kapitel 10.3) und in Anlehnung an die Studie »Inklusion. Zur Dekonstruktion von Diskursteilhabebarrieren im Kontext von Freizeit und Behinderung« (Trescher 2015b).

\subsubsection{Leitfadeninterviews und Sozialraumanalysen}

Bei der Konstruktion des Leitfadens zur Erhebung im Rahmen der Sozialraumanalysen wurde mit Blick auf die ausgewählten Auswertungsverfahren (deskriptiv-statistisch und qualitativ-inhaltsanalytisch) sowohl der Einsatz 
der standardisierten Interviewform (mit konkreten Fragen) als auch die offenere halbstandardisierte Form (mit Themenfeldern) als sinnvoll erachtet. Für die erste Form sprachen, neben einem im Vergleich einfacheren Feldzugang und einer damit einhergehend größeren Anzahl an Interviews, die vor allem aufgrund der geringeren Interviewdauer möglich werden, die gute Vergleichbarkeit der erhobenen Daten und somit leichter auswertbare Ergebnisse (Lamnek 2005, S. 341f). Im Hinblick auf den Umfang des zu untersuchenden Feldes war die standardisierte Form des Interviews allerdings zu unflexibel, nicht zuletzt auch deswegen, weil der Leitfaden universell eingesetzt werden sollte und deshalb entsprechend konstruiert werden musste. Zudem lassen sich in halbstandardisierten Leitfadeninterviews vergleichsweise umfassendere und detailliertere Informationen aus den gegebenen Antworten erzeugen als in standardisierten Interviewformen (Lamnek 2005, S. 341f). Aufgrund dessen fiel im Rahmen der hiesigen Erhebungen die Wahl auf die Konstruktion eines halbstandardisierten Leitfadeninterviews, was primär in der größeren Flexibilität in der Interviewdurchführung sowie in der Vorbeugung einer "Leitfadenbürokratie« (Hopf 2010, S. 358; siehe auch Przyborski und Wohlrab-Sahr 2010, S. 142) begründet ist. Ausschlaggebend waren darüber hinaus die Vorerfahrungen aus der Studie »Inklusion. Zur Dekonstruktion von Diskursteilhabebarrieren im Kontext von Freizeit und Behinderung« (Trescher 2015b), in der ebenjene halbstandardisierten Leitfadeninterviews erfolgreich zur Datengenerierung genutzt wurden. Die Wahl des halbstandardisierten Interviews ermöglicht die Nutzung der Vorteile beider Interviewformen (in vergleichsweise kurzer Zeit mehr Interviews und nichtsdestotrotz den Erhalt der gewünschten ,Tiefe der Antworten) und damit einhergehend sowohl die zielgerichtete Erzeugung von offenen, beschreibenden Informationen als auch die Gewinnung von universellen und vergleichbaren Daten. Dies bietet zudem die Möglichkeit, flexibler auf den Gesprächsverlauf und zum Teil auch individuell auf Einzelfälle eingehen zu können.

\subsubsection{Zur Konstruktion des Leitfadens}

Zur Beantwortung der forschungsleitenden Fragestellungen wurde ein Leitfaden entworfen, anhand dessen die Interviewpersonen (ArbeitnehmerInnen und PrimärvertreterInnen von institutionalisierten Freizeitaktivitäten) befragt werden sollten. Hierbei wurde sich ebenfalls an Trescher (2015b) orientiert. Neben allgemeinen Rahmenbedingungen, beispielsweise bezüg- 
lich der barrierefreien Zugänglichkeit der interessierenden Aktivität, wurde gefragt, ob bereits Menschen mit >Behinderung, >Fluchtmigrationshintergrund oder `Demenz teilhaben und wie diese Teilhabe je ausgestaltet ist. Hatten bislang noch keine von Ausschluss bedrohten oder betroffenen Personen teil, so wurde nach den prinzipiellen Möglichkeiten ihrer Teilhabe gefragt und die Einschätzungen der Interviewpersonen dokumentiert. Die abschließenden Fragen waren offen formuliert und zielten im Kern darauf ab, Haltungen der Interviewpersonen zu Inklusion und Teilhabemöglichkeiten von Menschen zu erfahren, die von Ausschluss bedroht oder betroffen sind.

Tabelle 17: Überblick über den Interviewleitfaden in den Handlungsfeldern Arbeit und Freizeit ${ }^{3}$

\begin{tabular}{|c|c|c|}
\hline 1. Intervieweinstieg & \multicolumn{2}{|l|}{$\begin{array}{l}\text { 1.1 Begrüßung } \\
\text { 1.2 Erklären des Anliegens } \\
\text { 1.3 Vorstellen des Projekts } \\
1.4 \text { Zusichern von Anonymität }\end{array}$} \\
\hline $\begin{array}{l}\text { 2. Fokus: Unternehmen/ } \\
\text { Freizeitaktivität }\end{array}$ & $\begin{array}{l}\text { Arbeit: } \\
\text { 2.1 Tätigkeitsbereich/Bran- } \\
\text { che des Unternehmens } \\
\text { 2.2 Größe des Unterneh- } \\
\text { mens am Standort } \\
\text { 2.3 Alterspanne der Be- } \\
\text { schäftigten }\end{array}$ & $\begin{array}{l}\text { Freizeit: } \\
\text { 2.1 Beschreibung der } \\
\text { Aktivität } \\
\text { 2.2 Dauerangebot oder } \\
\text { Kurs } \\
\text { 2.3 Höhe der Kosten } \\
\text { 2.4 Altersspanne der Teil- } \\
\text { nehmenden }\end{array}$ \\
\hline $\begin{array}{l}\text { 3. Fokus: Barrierefreiheit/ } \\
\text { Erreichbarkeit }\end{array}$ & \multicolumn{2}{|c|}{$\begin{array}{l}\text { 3.1 ÖPNV-Anbindung in Gehminuten } \\
\text { 3.2 Vorhandensein eines Behindertenparkplatzes } \\
\text { 3.3 Barrierefreiheit/Zugänglichkeit des Ortes hinsichtlich } \\
\text { • Mobilitätsbeeinträchtigung } \\
\text { - Sehbeeinträchtigung } \\
\text { - Hörbeeinträchtigung } \\
\text { - kognitiver Beeinträchtigung }\end{array}$} \\
\hline
\end{tabular}

3 Es sei darauf hingewiesen, dass zwar alle erhobenen Fragen ausgewertet wurden, hier jedoch aus Cründen der Stringenz des Buches nicht vollumfänglich dargestellt werden. 


\begin{tabular}{|c|c|}
\hline 4. Fokus: Behinderung & 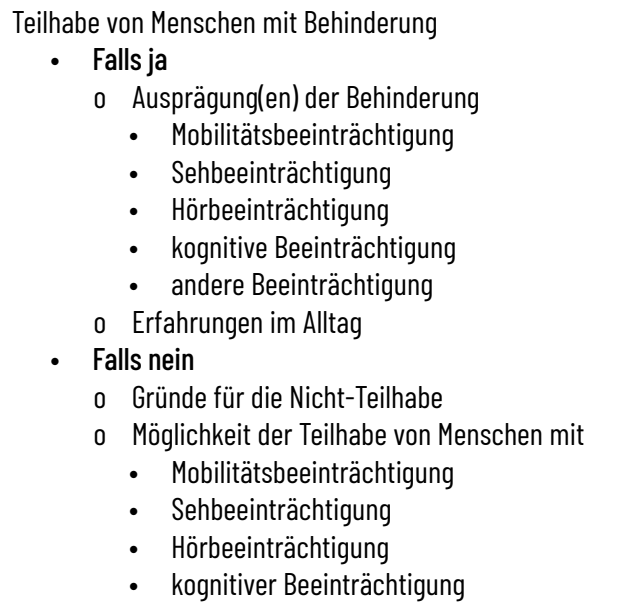 \\
\hline 5. Fokus: Fluchtmigration & $\begin{array}{l}\text { Teilhabe von Menschen mit Fluchtmigrationshintergrund } \\
\text { - Falls ja } \\
0 \text { Erfahrungen im Alltag } \\
\text { - Falls nein } \\
0 \text { Gründe für die Nicht-Teilhabe } \\
0 \text { Möglichkeit der Teilhabe von Menschen mit } \\
\quad \text { Fluchtmigrationshintergrund }\end{array}$ \\
\hline $\begin{array}{l}\text { 6. Fokus : Demenz (nur im } \\
\text { Handlungsfeld Freizeit) }\end{array}$ & $\begin{array}{l}\text { Teilhabe von Menschen mit Demenz } \\
\text { - Falls ja } \\
0 \text { Erfahrungen im Alltag } \\
\text { - Falls nein } \\
0 \text { Gründe für die Nicht-Teilhabe } \\
0 \text { Möglichkeit der Teilhabe von Menschen mit } \\
\text { Demenz }\end{array}$ \\
\hline $\begin{array}{l}\text { 7. Fokus: Inklusion/Ab- } \\
\text { schluss des Interviews }\end{array}$ & $\begin{array}{l}\text { 7.1 Ist Inklusion im Unternehmen/in der Freizeitaktivität } \\
\text { möglich? } \\
\text { 7.2 Worin liegen (potenzielle) Schwierigkeiten? } \\
\text { 7.3 Sind Maßnahmen/Vorhaben in Bezug auf Inklusion in } \\
\text { Planung? } \\
\text { 7.4 Gibt es (Veränderungs-)Wünsche an Politik und Gesell- } \\
\text { schaft in Bezug auf Inklusion? Welche? }\end{array}$ \\
\hline $\begin{array}{l}\text { 8. Einschätzung durch } \\
\text { Interviewerln }\end{array}$ & $\begin{array}{l}\text { 8.1 Besonderes/Erwähnenswertes } \\
8.2 \text { Gesamteinschätzung }\end{array}$ \\
\hline
\end{tabular}




\subsection{Pre-Tests: Leitfadengestützte Telefoninterviews}

Bei der Testung der leitfadengestützten Telefoninterviews wurde auf Erkenntnisse aus der bereits mehrfach genannten Studie zur Freizeitsituation von Menschen mit (geistiger) Behinderung zurückgegriffen und darauf aufgebaut (Trescher 2015b, S. 56ff). Darüber hinaus wurden die Interviewleitfäden in einem Sozialraum getestet, der nicht Teil der in der Studie untersuchten Sozialräume ist und der in Bezug auf Größe und Infrastruktur Strukturähnlichkeiten mit den beiden größten Sozialräumen Erlangen und Rostock aufweist und dahingehend ausgewählt wurde ${ }^{4}$. Die Pre-Tests hatten zwei Ziele, nämlich (1) ein leitfadengestütztes Telefoninterview zu erheben und (2) den ausgearbeiteten Leitfaden auf Durchführbarkeit und Sinnhaftigkeit zu testen. Die Entscheidung fiel aus arbeitspraktischen/ operativen Gründen auf die telefonische Erhebung, da einer persönlichen Befragung (face-to-face Kommunikation) keine besonderen Vorteile zugeschrieben werden konnten und diese Vorgehensweise nicht nur den Vorteil der spontaneren und schnelleren Durchführung bietet, sondern auch die Möglichkeit, eine große Anzahl an Interviews zu erheben. Die Option, im Einzelfall auch auf eine persönliche Ansprache zurückzugreifen, wurde im Zuge dessen nicht gänzlich ausgeschlossen. Hierauf sollte jedoch lediglich in jenen Fällen zurückgegriffen werden, in denen dies vonseiten der Interviewperson explizit gewünscht wurde. Des Weiteren wurde in der entstehenden Sozialsituation während eines Telefonates ein Gewinn bezüglich der Offenheit der Interviewpersonen sowie der damit einhergehenden Qualität des Datenmaterials gesehen. Aufgrund der räumlichen Distanz zwischen dem/der InterviewerIn und der Interviewperson bietet sich für Letztere/n die Möglichkeit zu größerer Anonymität während des Gespräches, da eine direkte Begegnung mit dem/der ForscherIn ausbleibt und so die Problematik einer Musterung beziehungsweise Einordnung des Gegenübers sowie eine hieraus resultierende potenzielle Beeinflussung der getroffenen Aussagen entfällt (Lueger 1989, S. 26). Zudem war es der Interviewperson (folgenlos und unkompliziert) möglich, das Interview durch Beenden des Telefonats abzubrechen, was annehmbar weitergehend zu ihrer Offenheit beiträgt, da der Eingriff in die Privatsphäre der Interviewperson aufgrund der anonymeren Gesprächssituation geringer ist (Bänziger 2009, S. 19). In Anbetracht

4 Aus Gründen der Anonymisierung wird der Name der Stadt nicht genannt. 
der im Vergleich zum persönlichen Interview größeren Spontaneität des Telefongesprächs war es nicht immer möglich, potenzielle Störfaktoren zu umgehen (beispielsweise Zeitdruck der Interviewperson oder die mögliche Anwesenheit Dritter zum Zeitpunkt des Anrufs), worin eine forschungspraktische Einschränkung liegt.

\subsubsection{Handlungsfeld Arbeit}

Im Handlungsfeld Arbeit wurden im Sozialraum, in dem die Pre-Tests durchgeführt wurden, ArbeitgeberInnen telefonisch kontaktiert, da diese bei der Konzeption der Erhebung (noch) im Fokus des Feldzugangs standen. In Bezug auf das erste Ziel der Pre-Tests, ein Leitfadeninterview mittels Telefonkontakt zu erheben, wurde sehr schnell klar, dass der Feldzugang so nur schwerlich gelingt und es forschungspraktisch gegebenenfalls sinnvoller wäre, die Erhebungsform noch einmal zu justieren. Dies lag primär darin begründet, dass (a) kaum Personen erreicht werden konnten, die leitend tätig sind, da diese in den meisten Fällen nicht telefonisch zu erreichen beziehungsweise zu Telefonterminen nicht bereit waren, und dass (b) nur circa jeder siebte Anruf zu einer erfolgreichen Erhebung des Leifadeninterviews führte. Der Pre-Test ergab also, dass die Erhebung anhand von Telefoninterviews, letztlich auch aus forschungsökonomischen Gründen, zu überdenken war. In Bezug auf das zweite Ziel des Pre-Tests, einer Testung des Leitfadens selbst, zeigte sich im Rahmen der Interviews, die erhoben werden konnten, dass der erarbeitete Leitfaden mit geringen Änderungen in der Studie Anwendung finden kann. Auch an dieser Stelle wurde noch einmal deutlich, wie sehr das methodische Setting von den Erfahrungen aus vorangegangenen Studien profitiert (v.a. Trescher 2015b).

Adaption: Ausgehend von diesen Erfahrungen wurde sich dafür entschieden, ArbeitnehmerInnen als Interviewpersonen auszuwählen und diese vor Ort in den Sozialräumen zu befragen ${ }^{5}$. Das konkrete Vorgehen bei der Erhebung ist in Kapitel 16.1 näher dargelegt.

5 Solche Befragungen vor Ort haben sich in diesem Kontext bewährt und sind lohnend (Riege 2007, S. 382). 


\subsubsection{Handlungsfeld Freizeit}

Die Pre-Tests verliefen im Handlungsfeld Freizeit unproblematisch, was insbesondere darin begründet ist, dass im Kontext der Studie »Inklusion. Zur Dekonstruktion von Diskursteilhabebarrieren im Kontext von Freizeit und Behinderung« (Trescher 2015b) bereits Erfahrungen mit der Erhebungsmethode und einem ähnlichen Leitfaden im Kontext Freizeit gesammelt wurden. Die leitfadengestützten Telefoninterviews konnten im Sozialraum, in dem die Pre-Tests durchgeführt wurden, ohne größere Einschränkungen erhoben werden. Der Leitfaden konnte ohne große Veränderungen beibehalten werden.

Adaption: Es waren keine Adaptionen des Vorgehens und keine größeren Adaptionen des Leitfadens notwendig.

\subsection{Auswertung: Deskriptiv-statistische und qualitativ- inhaltsanalytische Verfahren}

Wie im einleitenden Methodenkapitel bereits dargelegt wurde, erfolgte die Auswertung der Leitfadeninterviews mehrdimensional (siehe Kapitel 10). Anhand deskriptiv-statistischer Verfahren wurden Häufigkeiten und Verhältnisse dargestellt (siehe u.a. Benninghaus 2007; Kuckartz et al. 2013; Kuckartz et al. 2009), wogegen die Antworten, die auf offene Fragen gegeben wurden, anhand der Verfahren der Qualitativen Inhaltsanalyse untersucht wurden, wobei eine induktive Typisierung ein zentrales Ergebnis produziert (u.a. Mayring 2010b; siehe zur Anwendung Trescher 2015b, S. 74ff).

\section{Typenbildung}

Die Typenbildung erfolgte induktiv anhand der Verfahren der Qualitativen Inhaltsanalyse (Mayring 2010b; siehe zur Anwendung Trescher 2015b, S. 74ff; siehe Kapitel 10.2) und mit dem Ziel, das Datenmaterial zu typisieren. Jene Typenbildung verfolgt den Anspruch, Ähnlichkeiten und Unterschiede im Datenkorpus aufzufinden, zu strukturieren und darzulegen. Folglich werden mit dem Begriff >Typ « meinsame Eigenschaften aufweisen und anhand der spezifischen Konstellation dieser Eigenschaften beschrieben und charakterisiert werden können« (Kelle und Kluge 2010, S. 85; siehe auch Kuckartz 2016, S. 146). Daten, die 
einem bestimmten Typ zugehörig sind, zeichnen sich durch eine »interne Homogenität« (Kelle und Kluge 2010, S. 85) aus. Das bedeutet, dass die Daten jenes Typs einander größtmöglich ähneln. Demgegenüber sollen jene Daten von denen anderer Typen durch eine »externe Heterogenität« (Kelle und Kluge 2010, S. 85) gekennzeichnet sein, sich also größtmöglich von Daten anderen Typs unterscheiden. In der untenstehenden Übersicht ist dargelegt, inwiefern diese beiden Kriterien - interne Homogenität und externe Heterogenität - auf die gebildeten Typen zutreffen. Die induktive Analyse des Datenkorpus ergab vier Typen, anhand derer die Interviews respektive Interviewpersonen kategorisiert werden können. Bei Kategorisierung und Typenbildung konnte auf die Erfahrungen aus vorangegangenen Studien zurückgegriffen werden (Trescher 2015b, S. 82ff). Im Folgenden werden die vier Typen prototypisch charakterisiert. Die Beschreibung der Typen wird durch exemplarische Ausschnitte aus den Interviews in den Handlungsfeldern Arbeit und Freizeit ergänzt.

Tabelle 18: Charakterisierung der vier Typen: voll ablehnend, teilweise ablehnend, (noch) zurückhaltend, offen

Typ 1 - " voll ablehnend "

Typ 1 »voll ablehnend « ist davon gekennzeichnet, dass die Interviewpersonen eine Teilhabe von Menschen mit Behinderung, Menschen mit Fluchtmigrationshintergrund und Menschen mit Demenz kategorial ablehnen. Dies erfolgt häufig mit der Begründung, jene Personen seien nicht geeignet, die erforderlichen Tätigkeiten im je konkreten Handlungsfeld adäquat auszuführen. Dies steht in Verbindung damit, dass sich die Interviewpersonen im Gros negativ über Menschen mit Behinderung, Menschen mit Fluchtmigrationshintergrund und Menschen mit Demenz äußern. Hierbei überwiegen Konstruktionen negativer Andersartigkeit, die in einem defizitären Bild jener Personen resultieren. Interviewpersonen des Typs 1 »voll ablehnend « hatten bislang (so gut wie) keinen lebensweltlichen Kontakt zu Menschen mit Behinderung, Menschen mit Fluchtmigrationshintergrund und/ oder Menschen mit Demenz und/oder berichten von sehr negativen Erfahrungen, sollte doch schon einmal Kontakt bestanden haben. 


\section{Exemplarische Interviewpassagen im Kontext des Handlungsfeldes Arbeit}

Behinderung: »Die Beschäftigung von Menschen mit Behinderung ist nicht wirtschaftlich, denn der Betrieb wird aufgehalten. Es gibt zu wenig Angestellte und viel Arbeit, sodass keine extra Unterstützung geleistet werden kann. «(7191) ${ }^{6}$

Fluchtmigrationshintergrund: »Die Flüchtlinge sind doch alle IS-Kämpfer und Kriminelle. Gerade letzte Woche habe ich in der Zeitung gelesen, dass die Kriminalität sich gesteigert hat, seit die hier sind. Wir wollen nix mit denen zu tun haben. « (3578)

\section{Exemplarische Interviewpassagen im Kontext des Handlungsfeldes Freizeit}

Behinderung: "Das Niveau unseres Angebotes verunmöglicht das. Die Teilnahme von Menschen mit Behinderung schließt sich einfach grundsätzlich aus. « (6841) Fluchtmigrationshintergrund: "Der Chor ist nicht für Menschen auf der Durchreise.» (5200)

Demenz: »Menschen mit Demenz können nicht teilnehmen, weil die Leistungsanforderungen zu hoch sind. « (6766)

\section{Typ 2 - "teilweise ablehnend"}

Interviewpersonen werden dann zu Typ 2 »teilweise ablehnend « zugeordnet, wenn sie bestimmte Personengruppen kategorial ausschließen. Beispielsweise gibt es Personen dieses Typs, die einer Teilhabe von Menschen mit Behinderung grundsätzlich eher offen gegenüber eingestellt sind, allerdings eine Teilhabe von Menschen mit Fluchtmigrationshintergrund verneinen. Hinzu kommt, dass jene Personen bislang kaum Kontakt zu Menschen mit Behinderung, Menschen mit Fluchtmigrationshintergrund und/oder Menschen mit Demenz hatten. Insofern besteht eine deutliche Unsicherheit im Umgang mit Menschen jener Personengruppen, was oftmals darin resultiert, dass der Wunsch geäußert wird, die betreffende Person solle eine Assistenz oder Begleitperson mitbringen. Kleinere Unterstützungsleistungen zu erbringen, können sich Interviewpersonen des Typs 2 »teilweise ablehnend " ausschließlich in Bezug auf Personen vorstellen, die sie nicht von vorneherein pauschal ausschließen.

6 Es sei darauf hingewiesen, dass die Interviews ebenso wie die Schilderungen der Erhebungserfahrungen nach dem Zufallsprinzip kodiert wurden, sodass aus der vergebenen Zahl keine Rückschlüsse auf den Sozialraum möglich sind. Dadurch wird die Anonymität der Sozialräume und der interviewten Personen weitergehend gewährleistet. 


\section{Exemplarische Interviewpassagen im Kontext des Handlungsfeldes Arbeit}

Behinderung: »Menschen mit körperlicher Behinderung können hier arbeiten, kein Problem. Menschen mit geistiger Behinderung aber nicht.« (2830)

Fluchtmigrationshintergrund: »Behinderung wird schon gehen, aber Flüchtlinge finden hier keinen Platz. « (3053)

\section{Exemplarische Interviewpassagen im Kontext des Handlungsfeldes Freizeit}

Behinderung: »Generell sehe ich keine Schwierigkeiten, außer, die Menschen sind körperlich zu stark eingeschränkt und haben eine starke geistige Behinderung, sodass die Sicherheit nicht mehr gewährleistet ist." (8311)

Fluchtmigrationshintergrund: »|ch wüsste nicht, wie Flüchtlinge hier teilnehmen sollen. Behinderte haben wir manchmal, aber mehr geht nicht. « (3002)

Demenz: »Menschen mit Demenz kommen nicht auf die Idee, zu uns zu kommen, da die Tauglichkeit von einem Arzt attestiert werden muss. Es besteht die Gefahr der Eigen- und der Fremdgefährdung. « (5149)

\section{Typ 3 - "(noch) zurückhaltend "}

Interviewpersonen werden dann zu Typ 3 »(noch) zurückhaltend « zugeordnet, wenn sie zwar Vorbehalte gegenüber der Teilhabe von Menschen mit Behinderung, Menschen mit Fluchtmigrationshintergrund und/oder Menschen mit Demenz äußern und eine Teilhabe jener Personen als eher schwierig einschätzen, diese allerdings nicht grundsätzlich ausschließen. Interviewpersonen zeigen sich darüber hinaus teils dazu bereit, kleinere Hilfeleistungen für Menschen mit unterschiedlichen Unterstützungsbedarfen zu erbringen (zum Beispiel einen Fahrdienst rufen oder Ähnliches). Interviewpersonen, die Typ 3 »(noch) zurückhaltend « zugeordnet werden, hatten bislang kaum lebensweltlichen Kontakt zu Menschen mit Behinderung, Menschen mit Fluchtmigrationshintergrund und/ oder Menschen mit Demenz. In Bezug auf die von innen repräsentierte Tätigkeit verdeutlichen sie, dass bestehende Strukturen (beispielsweise Ort, Zeitpunkt und/oder Ablauf der jeweiligen Tätigkeit) eher nicht verändert werden sollen, sie schließen dies allerdings nicht vollständig aus, worin ein gewisses Potenzial zu erkennen ist, Teilhabemöglichkeiten für Personen zu eröffnen, die bislang ausgeschlossen sind.

\section{Exemplarische Interviewpassagen im Kontext des Handlungsfeldes Arbeit}

Behinderung: „Unser Laden ist offen für alles, das Problem ist die Intoleranz der Kunden.» (9406)

Fluchtmigrationshintergrund: »Bisher gab es noch keine Bewerber, da oft fachliche Voraussetzungen fehlen. ( $(8698)$ 


\section{Exemplarische Interviewpassagen im Kontext des Handlungsfeldes Freizeit}

Behinderung: »Eine Teilnahme ist möglich, aber nur, wenn die jemanden als Begleitung mitbringen. «(3268)

Fluchtmigrationshintergrund: »Die Teilnahme ist nur möglich, wenn ein Instrument auf einem bestimmten Niveau beherrscht wird. « (4302)

Demenz: »Es kommt auf die Ausprägung der Demenz an. Im Anfangsstadium ist das kein Problem. Wenn sie schon fortgeschritten ist, kann es schwieriger werden. (8343)

\section{Typ 4 - "offen «}

Typ 4 »offen « zeichnet sich dadurch aus, dass Interviewpersonen keine Vorbehalte bezüglich der Teilhabe von Menschen mit Behinderung, Menschen mit Fluchtmigrationshintergrund und/oder Menschen mit Demenz äußern. Inklusion wird häufig als gesamtgesellschaftliche Aufgabe verstanden, die auch die Interviewperson betrifft und zu der sie ihren Teil beitragen will. Jene Interviewpersonen haben beziehungsweise hatten in der Vergangenheit oftmals bereits Kontakt zu Menschen mit Behinderung, Menschen mit Fluchtmigrationshintergrund und/oder Menschen mit Demenz. Ihre Offenheit wird auch in dieser Erfahrung begründet. Darüber hinaus sehen es Interviewpersonen als unkompliziert an, die Strukturen der von ihnen vertretenen Tätigkeit zu verändern, sollten dadurch Teilhabemöglichkeiten für bestimmte Personen eröffnet werden (zum Beispiel Ort, Zeitpunkt, Ablauf etc.).

Exemplarische Interviewpassagen im Kontext des Handlungsfeldes Arbeit

Behinderung: »|ch habe gute Erfahrungen gemacht. Die Zusammenarbeit ist nicht anders, als mit anderen KollegInnen auch. " (1662)

Fluchtmigrationshintergrund: »Die KollegInnen mit Fluchtmigrationshintergrund sind hilfsbereit und engagiert, sprachliche Probleme sind nicht so erheblich. «(1899)

\section{Exemplarische Interviewpassagen im Kontext des Handlungsfeldes Freizeit}

Behinderung: »Unsere Erfahrungen sind positiv, bei uns wird kein Unterschied zwischen Menschen mit und ohne Behinderung gemacht.« (1470)

Fluchtmigrationshintergrund: »Das klappt super, höchstens entstehen mal Sprachbarrieren, aber dann spricht man langsam und verständigt sich mit Händen und Füßen. « (5331) Demenz: »Das klappt wunderbar, wir gehen offen mit den Menschen um. «(3105)

\section{Sozialraumanalysen im Handlungsfeld Arbeit}

Aufgrund der Schwierigkeiten bei der Erhebung von Telefoninterviews, die der Pre-Test offenbarte, musste im Handlungsfeld Arbeit das methodische Erhebungssetting angepasst werden (siehe Kapitel 14.4.1). Nachdem auch die 
Möglichkeit einer Onlinebefragung in Betracht gezogen wurde, wurde sich letztlich für eine Vor-Ort-Erhebung von Leitfadeninterviews entschieden. Dies hatte zur Folge, dass sich der Fokus möglicher InterviewpartnerInnen von ArbeitgeberInnen zu ArbeitnehmerInnen verschob. Damit geht einher, dass einige Angaben weniger verlässlich und infolgedessen weniger vergleichbar sind, da ArbeitnehmerInnen - gerade in größeren Unternehmen - eher nicht wissen, inwiefern beispielsweise alle Bereiche des Arbeitsplatzes barrierefrei sind und wie viele Menschen mit Beeinträchtigungen und/oder Fluchtmigrationshintergrund im Unternehmen arbeiten. Vergleichbare Aussagen bezüglich dieser Angaben wären primär über die ArbeitgeberInnen $\mathrm{zu}$ erheben gewesen, die sich allerdings als sehr unkooperativ herausstellten beziehungsweise, bei gelingendem Kontakt, ausweichend antworteten, woraus zudem eine gewisse soziale Erwünschtheit des Antwortverhaltens abgeleitet werden konnte und oftmals deutlich wurde, dass die ArbeitgeberInnen das Image ihres Unternehmens als besonders offen darstellen wollen. Ein positiver Nebeneffekt dessen, dass der Interviewfokus auf ArbeitnehmerInnen verschoben wurde, ist dagegen, dass der Einblick in Erfahrungen des alltäglichen Miteinanders dadurch annehmbar vielfältiger ist, als dies ArbeitgeberInnen schildern könnten, die zumeist ein Stück weit abgeschnitten von alltäglichen Praxen sind (zumindest in großen Betrieben).

\subsection{Ablauf der Vor-Ort-Erhebungen}

Die ForscherInnen fuhren jeweils in Zweier-Teams in die fünf Sozialräume und sprachen an öffentlichen Orten (Fußgängerzonen, Einkaufszentren, Parkplätze etc.) PassantInnen an, mit der Absicht, ein Interview mit diesen zu führen. Dabei wurde auf eine größtmögliche Heterogenität der Interviewpersonen geachtet hinsichtlich Alter, Geschlecht und Branche, in der diese Person arbeitstätig ist. Die Erfahrungen bei der Interviewerhebung waren im Gros positiv, wie beispielsweise die folgende Schilderung zeigt: In der Fußgängerzone war ab vormittags sehr viel los. Fast jede zweite oder dritte Person war dazu bereit, die Fragen zu beantworten. Die Menschen begegneten mir meist freundlich und aufgeschlossen, sobald ich ihnen versichert habe, dass ich weder etwas verkaufen noch irgendwelche Unterschriften sammeln will (I-5775). Hin und wieder reagierten die angesprochenen Personen aber auch ablehnend. Ein Beispiel hierfür ist die folgende Erfahrung: Die zwei Mitarbeitenden in dem Geschäft waren sehr gesprächig, jedoch wollten sie mit mir über alles sprechen, nur nicht über 
Inklusion, denn »die Politiker sollen sich erst mal um die Leute auf dem Land kümmern und an die Bürger im Dorf denken, bevor die sich um Flüchtlinge und Behinderte kümmern«. Die zwei Personen waren gegenüber Menschen mit Behinderung und Menschen mit Fluchtmigrationshintergrund abweisend und haben mit, mir unverständlich erscheinenden, Begründungen erläutert, warum ihre Arbeitsstelle für diese Menschen nicht geeignet sei. Sie sagten: "Die alten Leute kommen nicht mehr ins Geschäft, wenn da eine mit einem Kopftuch sitzt«. Und: »Menschen mit Behinderung schmeißen da nur alles um und können mit den Kunden nicht kommunizieren«. Zum Schluss des Gesprächs wurde mir noch gesagt, dass sie sowieso mit jemandem aus der Stadt, wie ich es bin, nicht sprechen werden (I-2259). Negative Reaktionen wie die obige waren eher die Ausnahme, zumeist reagierten die angesprochenen Personen sehr freundlich und offen. Demgegenüber zeigen solche offen ablehnenden Reaktionen gegenüber Menschen mit Behinderung und/oder Menschen mit Fluchtmigrationshintergrund, dass Inklusion weiter angestoßen und in vielerlei Hinsicht darauf abzielen muss, die breite Bevölkerung für Fragen von Teilhabe und Diversität zu sensibilisieren.

\subsection{Gesamteinschätzung}

Die Typisierung der Fälle im Handlungsfeld Arbeit ergab, dass durchschnittlich mehr als zwei Drittel der Interviewpersonen einer Teilhabe von Menschen mit Behinderung und/oder Menschen mit Fluchtmigrationshintergrund an ihrem Arbeitsplatz voll ablehnend (16,8\%) beziehungsweise teilweise ablehnend $(52,7 \%)$ gegenüberstehen. Diese Interviewpersonen sehen keine Möglichkeit, jenen Personen beziehungsweise bestimmten Personen Zugangs- und Handlungsmöglichkeiten in dem Unternehmen zu schaffen, in dem sie selbst tätig sind. Hinzu kommt, dass Menschen mit Behinderung und/oder Menschen mit Fluchtmigrationshintergrund eher als defizitär konstruiert werden. Damit verbunden sind oftmals negative und pauschalisierende Aussagen. Demgegenüber sind durchschnittlich 18,8 \% der Interviewpersonen (noch) zurückhaltend, ob und inwiefern Teilhabemöglichkeiten im Bereich Arbeit für Menschen mit Behinderung und/oder Menschen mit Fluchtmigrationshintergrund bestehen. Diese Interviewpersonen haben oftmals noch keine Erfahrungen in der Zusammenarbeit mit jenen Personen gesammelt und begegnen der Idee, diese als KollegInnen zu haben, teils mit Vorbehalten. Dennoch zeigen sie sich offen für Veränderungen. Schließlich wurden durchschnittlich 11,6\% der Interviewpersonen als offen typisiert. 
Diese Interviewpersonen zeigen eine große Bereitschaft, Teilhabemöglichkeiten für Menschen mit Behinderung und Menschen mit Fluchtmigrationshintergrund $\mathrm{zu}$ bereiten. Sie haben häufig bereits mit jenen Personen zusammengearbeitet und berichten von größtenteils positiven Erfahrungen. Darüber hinaus engagieren sie sich oftmals zusätzlich, indem sie beispielsweise geflüchtete Menschen beim Deutschlernen unterstützen. Schließlich soll noch ein Blick auf die Ergebnisse der Typisierung in den einzelnen Sozialräumen geworfen werden, welche zeigt, dass sich diese teils erheblich in ihrem jeweiligen Anteil an als (voll/teilweise) ablehnend, (noch) zurückhaltend und offen typisierten Antworten unterscheiden. Insgesamt wird klar, dass die interviewten ArbeitnehmerInnen der beiden kleineren Sozialräume, Schneverdingen und die Verbandsgemeinde Nieder-Olm, deutlich ablehnender gegenüber einer Beschäftigung von Menschen mit Behinderung und/oder Fluchtmigrationshintergrund sind, als dies bei den Interviewten aus den anderen drei Sozialräumen der Fall ist. Die Unterschiede zwischen den Sozialräumen Erlangen, Rostock und Schwäbisch Gmünd bewegen sich in einem Rahmen, der zu erwarten war. Es soll abschließend hervorgehoben werden, dass die interviewten ArbeitnehmerInnen in Schwäbisch Gmünd einer möglichen Beschäftigung von Menschen mit Behinderung und/oder Fluchtmigrationshintergrund am offensten gegenüberstehen.

Tabelle 19: Kategorisierung entlang der vier Typen im Handlungsfeld Arbeit

\begin{tabular}{|l|c|c|c|c|}
\hline \multirow{2}{*}{} & \multicolumn{4}{|c|}{ Gesamteinschätzung } \\
\cline { 2 - 5 } & $\begin{array}{c}\text { voll ab- } \\
\text { lehnend }\end{array}$ & $\begin{array}{c}\text { teilweise } \\
\text { ableh- } \\
\text { nend }\end{array}$ & $\begin{array}{c}\text { (noch) } \\
\text { zurück- } \\
\text { haltend }\end{array}$ & offen \\
\hline Erlangen & $16,2 \%$ & $48,6 \%$ & $24,3 \%$ & $10,8 \%$ \\
\hline Rostock & $8,2 \%$ & $49 \%$ & $34,7 \%$ & $8,2 \%$ \\
\hline Schneverdingen & $17,6 \%$ & $76,5 \%$ & $5,9 \%$ & $0 \%$ \\
\hline Schwäbisch Gmünd & $19 \%$ & $35,7 \%$ & $21,4 \%$ & $23,8 \%$ \\
\hline VG Nieder-0Im & $23,1 \%$ & $53,8 \%$ & $7,7 \%$ & $15,4 \%$ \\
\hline$\varnothing$ & $16,8 \%$ & $52,7 \%$ & $18,8 \%$ & $11,6 \%$ \\
\hline
\end{tabular}




\subsection{Beispielinterviews}

Anhand zweier Interviews, die im Folgenden exemplarisch abgebildet sind, wird dargelegt, wie sich der Typisierungsprozess vollzieht. Darüber hinaus werden einzelne Aussagen und Zusammenhänge diskutiert (siehe dazu unter anderem auch Kapitel 16.9). Das erste Interview, das beispielhaft herangezogen wird, wurde mit einer Person geführt, die in einem Dienstleistungsbetrieb im Bereich Gastronomie arbeitet. Der Verlauf des Interviews ist im Folgenden zusammengefasst.

Tabelle 20: Beispielinterview im Handlungsfeld Arbeit (1)

\begin{tabular}{|c|c|}
\hline Arbeitsstelle der Interviewperson & Dienstleistung im Bereich Gastronomie \\
\hline Code & 7191 \\
\hline Größe des Unternehmens & mehr als 100 MitarbeiterInnen \\
\hline Behindertenparkplätze & nein \\
\hline Nähe zum ÖPNV & weniger als 5 Gehminuten \\
\hline Barrierefreiheit des Arbeitsortes & $\begin{array}{l}\text { barrierefrei für Menschen mit Unterstüt- } \\
\text { zungsbedarf im Bereich Mobilität; nicht } \\
\text { barrierefrei für Menschen mit Unterstüt- } \\
\text { zungsbedarfen in den Bereichen Sehen, } \\
\text { Hören oder Lesen/Verstehen }\end{array}$ \\
\hline MitarbeiterInnen mit Behinderung & nein \\
\hline $\begin{array}{l}\text { Gründe dafür, dass keine Menschen mit } \\
\text { Behinderung dort arbeiten }\end{array}$ & $\begin{array}{l}\text { - »Es ist nicht wirtschaftlich.» } \\
\text { - »Der Betrieb wird aufgehalten.» } \\
\text { - »Es gibt zu wenig Angestellte.» } \\
\text { - »Es gibt viel Arbeit, deshalb können wir } \\
\text { keine extra Unterstützung leisten.» }\end{array}$ \\
\hline $\begin{array}{l}\text { Arbeitsmöglichkeiten für Menschen mit } \\
\text { Behinderung }\end{array}$ & nein \\
\hline $\begin{array}{l}\text { Mitarbeiterlnnen mit Fluchtmigrations- } \\
\text { hintergrund }\end{array}$ & nein \\
\hline
\end{tabular}




\begin{tabular}{|c|c|}
\hline $\begin{array}{l}\text { Gründe dafür, dass keine Menschen mit } \\
\text { Fluchtmigrationshintergrund dort arbeiten }\end{array}$ & $\begin{array}{l}\text { - »Es ist nicht wirtschaftlich.» } \\
\text { - »Es fehlt das Vertrauen." } \\
\text { - »Die Einstellung von MitarbeiterInnen ist } \\
\text { eine Entscheidung der Chefetage.» }\end{array}$ \\
\hline $\begin{array}{l}\text { Arbeitsmöglichkeiten für Menschen mit } \\
\text { Fluchtmigrationshintergrund }\end{array}$ & nein \\
\hline $\begin{array}{l}\text { Halten Sie Inklusion im Unternehmen für } \\
\text { umsetzbar? }\end{array}$ & nein \\
\hline $\begin{array}{l}\text { mögliche Schwierigkeiten bei der Um- } \\
\text { setzung von Inklusion }\end{array}$ & $\begin{array}{l}\text { - Fehlende Sprachkenntnisse (englisch) } \\
\text { - »Um in der Essensausgabe arbeiten zu } \\
\text { können, muss eine Hygieneprüfung auf } \\
\text { Deutsch erfolgreich abgeschlossen werden } \\
\text { und ein Gesundheitspass vorliegen. Das } \\
\text { schaffen viele nicht. « }\end{array}$ \\
\hline $\begin{array}{l}\text { Maßnahmen/Vorhaben in Bezug auf } \\
\text { Inklusion }\end{array}$ & nein \\
\hline $\begin{array}{l}\text { (Veränderungs-)Wünsche an Politik und } \\
\text { Gesellschaft }\end{array}$ & »momentan nicht« \\
\hline Gesamteinschätzung & voll ablehnend \\
\hline
\end{tabular}

Die Interviewperson wurde gegenüber einer Teilhabe von KollegInnen mit Behinderung oder Fluchtmigrationshintergrund als voll ablehnend typisiert, was insbesondere aus den ablehnenden Aussagen resultiert, die in Bezug auf die Gründe dafür formuliert wurden, dass bislang keine Personen, die entsprechend adressiert werden, an der eigenen Arbeitsstelle arbeiten. Problematisiert werden kann in Bezug auf die Angaben der Interviewperson darüber hinaus, dass, obwohl es sich um ein großes Unternehmen mit mehr als 100 MitarbeiterInnen handelt, Barrierefreiheit ausschließlich im Bereich Mobilität gegeben ist und keine weiteren Dimensionen berücksichtigt wurden. Zudem stehen keine sogenannten Behindertenparkplätze zur Verfügung. Hier zeichnen sich Handlungsperspektiven ab, die wiederum die Teilhabemöglichkeiten von Menschen, die auf barrierefreie Arbeitsorte angewiesen sind, erhöhen können. Aus den Gründen, die als ursächlich dafür benannt werden, dass keine Menschen mit Behinderung oder Menschen mit Fluchtmigrationshintergrund an der eigenen Arbeitsstelle arbeiten, resultiert eine gewisse negative Defizitorientierung, die jene Personen pauschal 
als Belastung für den Betrieb sowie die KollegInnen konstruiert. Hierbei wird insbesondere das Argument vermeintlicher wirtschaftlicher Belastung herangezogen, womit eine Konstruktion prinzipieller Minderleistung jener Personen einhergeht. Deutlich wird zudem, dass die Interviewperson sich selbst nicht als primär dafür verantwortlich sieht, die Teilhabemöglichkeiten von Menschen mit Behinderung oder Menschen mit Demenz zu verbessern, da solche Entscheidungen auf der »Chefetage« getroffen würden. Diese Haltung ist signifikant für Interviewpersonen des Typs voll ablehnend, ist sie doch Ausdruck davon, selbst so wenig wie möglich aktiv werden zu wollen, um das Verhältnis von Teilhabe und Ausschluss zu verändern. Inwiefern die Erfahrung in der Zusammenarbeit mit KollegInnen mit Behinderung oder KollegInnen mit Fluchtmigrationshintergrund bei der Beurteilung von Inklusion im Kontext Arbeit wirksam werden kann, zeigt das nachfolgend skizzierte Interview mit einer Person, die in einem Logistikdienstleistungsunternehmen angestellt ist, in dem sowohl Menschen mit Behinderung als auch Menschen mit Fluchtmigrationshintergrund arbeiten.

Tabelle 21: Beispielinterview im Handlungsfeld Arbeit (2)

\begin{tabular}{|l|l|}
\hline Arbeitsstelle der Interviewperson & Logistikdienstleistungsunternehmen \\
\hline Code & 9335 \\
\hline Größe des Unternehmens & mehr als 100 Mitarbeiterlnnen \\
\hline Behindertenparkplätze & nein \\
\hline Nähe zum ÖPNV & weniger als 5 Gehminuten \\
\hline Barrierefreiheit des Arbeitsortes & $\begin{array}{l}\text { barrierefrei für Menschen mit Unterstüt- } \\
\text { zungsbedarf im Bereich Mobilität; nicht } \\
\text { barrierefrei für Menschen mit Unterstüt- } \\
\text { zungsbedarfen in den Bereichen Sehen, } \\
\text { Hören oder Lesen/Verstehen }\end{array}$ \\
\hline Mitarbeiterlnnen mit Behinderung & $\begin{array}{l}\text { ja, mit Unterstützungsbedarf im Bereich } \\
\text { Mobilität }\end{array}$ \\
\hline $\begin{array}{l}\text { Erfahrungen in der täglichen Zusammen- } \\
\text { arbeit }\end{array}$ & "keine Unterschiede« \\
\hline
\end{tabular}




\begin{tabular}{|l|l|}
\hline $\begin{array}{l}\text { MitarbeiterInnen mit Fluchtmigrations- } \\
\text { hintergrund }\end{array}$ & ja \\
\hline $\begin{array}{l}\text { Erfahrungen in der täglichen Zusammen- } \\
\text { arbeit }\end{array}$ & "keine Unterschiede» \\
\hline $\begin{array}{l}\text { Halten Sie Inklusion im Unternehmen für } \\
\text { umsetzbar? }\end{array}$ & weiß nicht \\
\hline $\begin{array}{l}\text { mögliche Schwierigkeiten bei der Um- } \\
\text { setzung von Inklusion }\end{array}$ & $\begin{array}{l}\text { "Es kommt auf den Grad der Behinderung } \\
\text { an. « }\end{array}$ \\
\hline $\begin{array}{l}\text { Maßnahmen/Vorhaben in Bezug auf } \\
\text { Inklusion }\end{array}$ & nicht bekannt \\
\hline $\begin{array}{l}\text { (Veränderungs-)Wünsche an Politik und } \\
\text { Gesellschaft }\end{array}$ & $\begin{array}{l}\text { "Das Amt schickt die falschen Leute zu } \\
\text { den falschen Stellen. " }\end{array}$ \\
\hline Gesamteinschätzung & (noch) zurückhaltend \\
\hline
\end{tabular}

Auffällig ist, dass sowohl Menschen mit Behinderung als auch Menschen mit Fluchtmigrationshintergrund im Unternehmen arbeiten, in dem die Interviewperson beschäftigt ist. Ihre Erfahrungen in der Zusammenarbeit sind weder positiv noch negativ, sondern in gewisser Weise gleichmütig - es macht keinen Unterschied, ob der/die KollegIn als >behindert oder >fluchtmigriert bezeichnet wird. Die Personen mit Behinderung, die im Unternehmen arbeiten, haben Unterstützungsbedarfe im Bereich Mobilität und können sich annehmbar aufgrund der barrierefreien Ausgestaltung des Arbeitsortes selbstständig im Gebäude/auf dem Gelände bewegen. Eine deutlich eingeschränkte Barrierefreiheit in den Bereichen Sehen, Hören und Lesen/Verstehen hat möglicherweise zur Folge, dass Personen mit Unterstützungsbedarfen in diesen Dimensionen eher nicht im Unternehmen arbeitstätig werden (können). In gewisser Hinsicht zeigen sich auch bei dieser Interviewperson Vorbehalte gegenüber Menschen mit Behinderung, denn sie macht Inklusion vom »Grad der Behinderung« einer Person abhängig, wodurch sozusagen Inklusionsgrenzen definiert werden, die bestimmte Personen ausschließen, die als 'zu stark behindert ' gelten. Auch die Analyse dieser Aussage hat mit zu einer Typisierung jener Interviewperson als (noch) zurückhaltend beigetragen. Schließlich problematisiert die Interviewperson bürokratische Hürden, die sich in ihrer Sicht vor allem darin zeigen, dass Verwaltung und potenzielle Arbeitsplätze nicht miteinander korres- 
pondieren. Dies zeugt davon, dass sich die Interviewperson Gedanken über Möglichkeiten der Teilhabe unterschiedlicher Personen macht und ist zusätzlich Indiz für eine Typisierung der Person als (noch) zurückhaltend, da bei ihr ein gewisses Potenzial erkannt werden kann, für Fragen von Teilhabe und Ausschluss weitergehend sensibilisiert zu werden.

\subsection{Einzelaspekte}

Im Folgenden werden einzelne Ergebnisse der Analyse herausgegriffen und im Kontext der Kategorisierung entlang der vier Typen dargestellt und diskutiert.

\subsubsection{Größe des Unternehmens}

Das Verhältnis der Anzahl der MitarbeiterInnen des Unternehmens am Standort und dem jeweiligen Typ verdeutlicht, dass größere Unternehmen (ab 50 MitarbeiterInnen) einer Teilhabe von Menschen mit Behinderung und/ oder Menschen mit Fluchtmigrationshintergrund offener gegenüberstehen. So wurden Unternehmen mit mehr als 100 MitarbeiterInnen zu 48,6\% als (noch) zurückhaltend und zu $55 \%$ als offen kategorisiert. Unternehmen, die weniger als zehn MitarbeiterInnen haben, sind dagegen in 50 \% der Fälle voll beziehungsweise in $43,4 \%$ der Fälle teilweise ablehnend. Perspektivisch wäre also denkbar, vor allem kleinere Unternehmen dabei zu begleiten, Arbeitsmöglichkeiten für Menschen mit unterschiedlichen Unterstützungsbedarfen zu schaffen. Hierbei stehen vor allem bewusstseinsbildende und sensibilisierende Maßnahmen im Vordergrund, wie auch ein Blick in die qualitativen Ergebnisse zeigt (siehe Kapitel 16.7).

Tabelle 22: Größe des Unternehmens

\begin{tabular}{|l|c|c|c|c|}
\hline & \multicolumn{4}{|c|}{ Anzahl der Mitarbeiterlnnen des Unternehmens am Standort } \\
\hline & $<10$ & $10-49$ & $50-100$ & $>100$ \\
\hline voll ablehnend & $50 \%$ & $16,7 \%$ & $4,2 \%$ & $29,2 \%$ \\
\hline teilweise ablehnend & $43,4 \%$ & $35,5 \%$ & $6,6 \%$ & $14,5 \%$ \\
\hline
\end{tabular}




\begin{tabular}{|l|c|c|c|c|}
\hline $\begin{array}{l}\text { (noch) zurück- } \\
\text { haltend }\end{array}$ & $18,9 \%$ & $18,9 \%$ & $13,5 \%$ & $48,6 \%$ \\
\hline offen & $10 \%$ & $20 \%$ & $15 \%$ & $55 \%$ \\
\hline
\end{tabular}

\subsubsection{Entfernung zum öffentlichen Personennahverkehr}

Die überwiegende Mehrheit der Unternehmen, in denen die Interviewpersonen beschäftigt sind, liegen weniger als fünf Minuten Fußweg von der nächsten Haltestelle des öffentlichen Personennahverkehrs entfernt (durchschnittlich 87 \%). Einen Fußweg von fünf bis zehn Minuten legen durchschnittlich 11,3\% der Interviewpersonen zur nächsten Haltestelle des öffentlichen Personennahverkehrs zurück. Ausschließlich in Erlangen $(2,7 \%)$ und Schneverdingen (5,9 \%) wurden ArbeitnehmerInnen befragt, die in Unternehmen arbeiten, die mehr als zehn Minuten Fußweg von der nächsten Haltestelle des öffentlichen Personennahverkehrs entfernt liegen. Die Abhängigkeit davon, Auto zu fahren, ist also eher gering, da potenziell öffentliche Verkehrsmittel genutzt werden können, wobei einschränkend festgehalten werden muss, dass keine Aussagen über die Quantität der Verbindungen sowie die Ausgestaltung der Fahrzeuge getroffen werden können, da dies nicht erhoben wurde. Zudem können keine Aussagen über das Verhältnis des Typs der jeweiligen Arbeitsstelle und ihrer Entfernung zu Haltestellen des öffentlichen Personennahverkehrs getroffen werden, da hier keine $\mathrm{Zu}$ sammenhänge vorgefunden wurden.

\subsubsection{Vorhandensein von Behindertenparkplätzen}

Während bei den Unternehmen, die als voll oder teilweise ablehnend kategorisiert wurden, kein Zusammenhang zwischen dem Typ und dem Vorhandensein eines sogenannten Behindertenparkplatzes festgestellt wurde, zeigt sich, dass (noch) zurückhaltende und offene Unternehmen deutlich häufiger über einen sogenannten Behindertenparkplatz verfügen. Es liegt also der Schluss nahe, dass, sobald ein Unternehmen über Behindertenparkplätze verfügt, es einer Teilhabe von Menschen mit Behinderung offener gegenübersteht. Dies kann sicherlich auf eine gewisse Sensibilität gegenüber je bestimmten Bedarfen zurückgeführt werden. 
Tabelle 23: Vorhandensein von Behindertenparkplätzen

\begin{tabular}{|l|c|}
\hline \multicolumn{2}{|c|}{ Ja, es ist ein sogenannter Behindertenparkplatz vorhanden } \\
\hline voll ablehnend & $43,5 \%$ \\
\hline teilweise ablehnend & $56,8 \%$ \\
\hline (noch) zurückhaltend & $70,3 \%$ \\
\hline offen & $80 \%$ \\
\hline
\end{tabular}

\subsubsection{Barrierefreiheit des Arbeitsortes}

Im Gros zeigt sich, dass die Kategorisierung des Unternehmens als voll/teilweise ablehnend, (noch) zurückhaltend oder offen oftmals im Zusammenhang mit der barrierefreien Zugänglichkeit dessen steht. Sobald ein Unternehmen als barrierefrei in Bezug auf Mobilität, Sehen, Hören oder Lesen/ Verstehen eingeschätzt wird, ist es wahrscheinlicher, dass es als (noch) zurückhaltend oder offen kategorisiert wird. Es zeigt sich also auch hier, dass strukturelle Gegebenheiten die Teilhabemöglichkeiten von Menschen mit unterschiedlichen Unterstützungsbedarfen beeinflussen. Es bedarf folglich einer gewissen Sensibilisierung für Barrieren und Möglichkeiten ihres Abbaus ${ }^{7}$.

Tabelle 24: Barrierefreiheit des Arbeitsortes

\begin{tabular}{|l|c|c|c|c|}
\hline & \multicolumn{4}{|c|}{ Der Arbeitsort ist barrierefrei in Bezug auf... } \\
\hline & Mobilität & Sehen & Hören & $\begin{array}{l}\text { Lesen/ } \\
\text { Verstehen }\end{array}$ \\
\hline voll ablehnend & $50 \%$ & $25 \%$ & $13 \%$ & $26,1 \%$ \\
\hline
\end{tabular}

7 Bezüglich der Betrachtung des Zusammenhangs von Typ des Unternehmens und barrierefreier Ausgestaltung des Unternehmensortes sei angemerkt, dass die Ergebnisse aufgrund ihrer subsumtionslogischen Schließung nur eingeschränkt herangezogen werden können. Da die Barrierefreiheit des Arbeitsortes ein Faktor bei der Kategorisierung des Unternehmens ist und bei der Beschreibung der vier Typen als ausschlaggebend herangezogen wird, ist es ein Stück weit tautologisch, Barrierefreiheit und Typ miteinander ins Verhältnis zu setzen. 


\begin{tabular}{|l|c|c|c|c|}
\hline teilweise ablehnend & $51,9 \%$ & $18,4 \%$ & $10,8 \%$ & $13,3 \%$ \\
\hline (noch) zurückhaltend & $70,3 \%$ & $24,3 \%$ & $8,8 \%$ & $32,4 \%$ \\
\hline offen & $80 \%$ & $30 \%$ & $40 \%$ & $50 \%$ \\
\hline
\end{tabular}

\subsection{Aktuelle Teilhabe von Menschen mit Behinderung und Menschen mit Fluchtmigrationshintergrund}

Die Interviewpersonen wurden gefragt, ob sie KollegInnen mit Behinderung und/oder KollegInnen mit Fluchtmigrationshintergrund haben. Bei der Konstruktion dessen, wie Behinderung und Fluchtmigrationshintergrund gefasst werden, wird, wie eingangs bereits dargelegt (Kapitel 14.2), die >Sprache des Feldes`zugrunde gelegt, woraus folgt, dass es im Ermessen der Interviewpersonen lag, eine/n KollegIn als behindert oder fluchtmigriert zu bezeichnen. Dass damit forschungspraktische Einschränkungen verbunden sind, da Interviewpersonen hierbei gegebenenfalls zu je unterschiedlichen Einschätzungen kommen, scheint klar. Allerdings handelt es sich sowohl beim Status >Fluchtmigration als auch beim Status >Behinderung um kontingente Kategorien, die vor allem in der interpersonalen Zuschreibung wirkmächtig werden, beispielsweise, wenn Personen als >fluchtmigriert oder >behindert adressiert werden und daraus bestimmte Handlungspraxen hervorgehen, die (potenziell) in Ausschluss resultieren. Deshalb hat sich dieses Vorgehen als zielführend erwiesen. Im Folgenden wird dargelegt, wie groß der Anteil der interviewten ArbeitnehmerInnen ist, die KollegInnen mit Behinderung und/oder KollegInnen mit Fluchtmigrationshintergrund haben. Im Kontext Behinderung wird dies darüber hinaus bezüglich der jeweiligen Ausprägung der >Beeinträchtigung` aufgeschlüsselt, wobei hier die Bereiche Mobilität, Sehen, Hören und kognitive Beeinträchtigung abgedeckt werden. Für Beeinträchtigungen, die unter keiner dieser vorgegebenen Kategorien gefasst werden können, wurde die zusätzliche Kategorie sandere Beeinträchtigungen gebildet. Es sei erneut darauf hingewiesen, dass größtenteils keine sozialraumbezogenen Einzelergebnisse dargelegt werden (wie unter anderem im Folgenden). Davon wird abgewichen, wenn die Ergebnisse sich in den Sozialräumen deutlich voneinander unterscheiden oder Trends festzustellen sind. 
Tabelle 25: KollegInnen mit (unterschiedlichen) Beeinträchtigungen oder Fluchtmigrationshintergrund

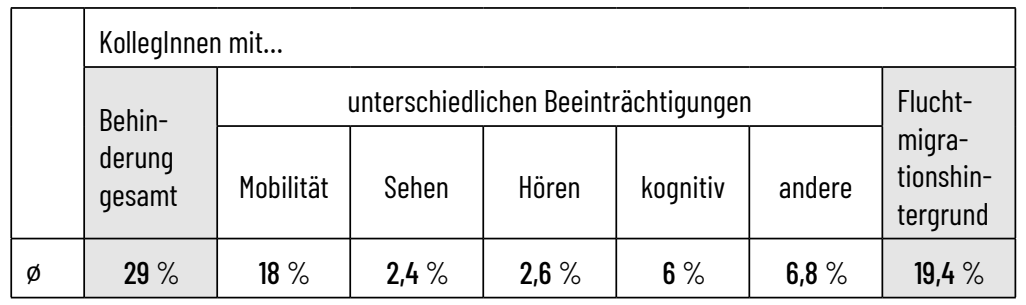

Durchschnittlich gaben $29 \%$ aller Interviewpersonen an, mit Menschen mit Behinderung zusammenzuarbeiten. Mit Blick auf die fünf untersuchten Dimensionen von Beeinträchtigung lassen sich teils deutliche Unterschiede erkennen. Während durchschnittlich 18 \% der Interviewten angaben, KollegInnen mit Mobilitätsbeeinträchtigung zu haben, fielen die Anteile in den Dimensionen Sehbeeinträchtigung $(2,4 \%)$, Hörbeeinträchtigung $(2,6 \%)$ und kognitive Beeinträchtigung (6\%) deutlich geringer aus. Durchschnittlich gaben $6,8 \%$ der Interviewten an, mit Menschen mit anderen Beeinträchtigungen zusammenzuarbeiten. $\mathrm{Zu}$ berücksichtigen ist hier, dass Mehrfachnennungen möglich waren, sodass das Ergebnis bezüglich der Frage nach der Zusammenarbeit mit KollegInnen mit Behinderung nicht mit den Ergebnissen der einzelnen Beeinträchtigungsdimensionen aufgerechnet werden kann. Es zeigt sich, dass vor allem Menschen mit Mobilitätsbeeinträchtigungen als KollegInnen mit den interviewten Personen zusammenarbeiten. Deutlich seltener sind dies Menschen mit kognitiven Beeinträchtigungen. Menschen mit Sehbeeinträchtigung und Menschen mit Hörbeeinträchtigung arbeiten am seltensten mit den Interviewten zusammen. Demgegenüber gaben durchschnittlich 19,4 \% der Interviewten an, mit KollegInnen mit Fluchtmigrationshintergrund zusammenzuarbeiten.

\subsection{Erfahrungen im Miteinander}

\subsubsection{Erfahrungen im Miteinander mit KollegInnen mit Behinderung}

Die Erfahrungen derjenigen Interviewpersonen, die mit KollegInnen mit Behinderung zusammenarbeiten, sind primär positiv, wie sie im Interview berichten. Eine Interviewperson sagt: »Die Zusammenarbeit ist nicht anders als mit anderen Kollegen auch« (1662). Diese Einschätzung wird von 
einigen Interviewpersonen geteilt, die die Zusammenarbeit mit ihren KollegInnen mit Behinderung als »normal« (5309) oder »gut« (3541) bezeichnen und "positive Erfahrungen« (6225) gemacht haben. Teilweise kann aus den Antworten der Interviewpersonen abgeleitet werden, dass sie Vorbehalte gegenüber ihren KollegInnen mit Behinderung haben, was sich unter anderem in Aussagen zeigt, die in gewisser Weise paternalistisch gefärbt sind. Ähnliches kann bezüglich Aussagen der Art festgestellt werden, die Zusammenarbeit sei »besser als erwartet (1899) und es gebe nur »selten Komplikationen« (1899), woran zwar gewisse Vorbehalte gegenüber Menschen mit Behinderung festgestellt werden können, die jedoch teilweise überwunden wurden, was einmal mehr klar macht, inwiefern Inklusion ein krisenhafter Prozess ist. Deutlich negativer beurteilen einige andere Interviewpersonen die Zusammenarbeit mit ihren KollegInnen mit Behinderung. Eine Interviewperson sagt:»Die Arbeit ist mit [Vorname Kollege/Kollegin] nicht immer einfach, aber möglich« (4334). Ein eher despektierlicher Blick auf Menschen mit Behinderung kann aus dieser Aussage einer Interviewperson abgeleitet werden: »Ich arbeite nicht mit denen zusammen« (1239). Hier werden Menschen mit Behinderung als eigene Gruppe adressiert, die sich von >den anderen $<$ - annehmbar Menschen ohne Behinderung - ganz grundsätzlich unterscheidet. Die Interviewperson distanziert sich von seinen/ihren KollegInnen mit Behinderung. Einige Interviewpersonen wiederum haben keine Erfahrungen in der Zusammenarbeit mit ihren KollegInnen mit Behinderung, da diese beispielsweise in einer anderen Abteilung arbeiten und so kein alltäglicher Kontakt besteht.

\subsubsection{Erfahrungen im Miteinander mit KollegInnen mit Fluchtmigrationshintergrund}

Während in Bezug auf die Zusammenarbeit mit Menschen mit Behinderung die Interviewpersonen zu größtenteils eher positiven Einschätzungen kamen, sind die Schilderungen in Bezug auf KollegInnen mit Fluchtmigrationshintergrund etwas mehr von Vorbehalten und teils negativen Erfahrungen geprägt, wobei die Abweichungen gering sind. Einige Interviewpersonen berichten von "guten« (5431) und "positiven Erfahrungen« (3793), andere stellen sogar fest: »Es läuft voll gut, die Leute sind meganett« (5510), oder schlicht: "alles ist super« (3875). Demgegenüber gibt es einige Interviewpersonen, die von deutlich schlechteren Erfahrungen berichten beziehungsweise in deren Antworten Vorbehalte gegenüber der Zusammenarbeit 
mit ihren geflüchteten KollegInnen zu erkennen sind. Eine Interviewperson sagt: »Ich habe da ganz gemischte Erfahrungen« (3586), während eine andere berichtet, die Zusammenarbeit sei »so naja« (9208). Eine wiederum andere Person kommt zu der Einschätzung, die Zusammenarbeit sei »schon ok« (5844). Laut einer weiteren Interviewperson seien die KollegInnen mit Fluchtmigrationshintergrund »häufig echt unzuverlässig. Das kann richtig nerven « (9956). Eine Problematik, auf die viele der Interviewpersonen in ihrem beruflichen Miteinander mit ihren geflüchteten KollegInnen treffen, ist die Sprachbarriere, da, nach Angaben dieser Interviewperson, viele dieser KollegInnen nur wenig Deutsch sprechen können. Eine Interviewperson sagt: »Sprache ist echt wichtig und das ist bei den meisten einfach noch nicht gut« (4244). Ähnlich wie in Bezug auf KollegInnen mit Behinderung haben auch einige der Interviewpersonen, die KollegInnen mit Fluchtmigrationshintergrund haben, (bislang) keinen Kontakt zu diesen gehabt, da sie beispielsweise in einer anderen Abteilung arbeiten, und können deshalb auch keine Erfahrungen im Miteinander berichten.

\subsection{Begründungsfiguren der Ablehnung}

\subsubsection{Ablehnung von Menschen mit Behinderung}

Die Interviewpersonen nennen einige unterschiedliche Gründe dafür, dass sie keine KollegInnen mit Behinderung haben. Diese sind im Folgenden zusammengefasst, wobei immer wieder Einblick in Aussagen der Interviewpersonen gegeben wird.

Menschen mit Behinderung sind nicht im gleichen Maße leistungsfähig Die Interviewpersonen sehen Gründe dafür, dass sie keine KollegInnen mit Behinderung haben, vor allem in einer vermeintlichen Minderleistung von Menschen mit Behinderung. Diese werden oftmals pauschal als sunfähig dazu angenommen, die jeweilige Tätigkeit auszuüben. Mangelnde Fähigkeiten werden Menschen mit Behinderung dabei teils auf körperlicher und teils auf intellektueller Ebene zugeschrieben. Eine Interviewperson sagt:»Für die Arbeit bei uns muss man körperlich fit sein und vor allem auch Stress aushalten können« (6348). Eine andere wiederum merkt an: »Schauspieler müssen geistig fit sein« (6655). Beide sehen in diesen Anforderungen Gründe, dass keine Menschen mit Behinderung an ihrer Arbeitsstelle arbeiten. Dies wird auch in der Aussage dieser Interviewperson deutlich:»Bei uns arbeiten keine 
Menschen mit Behinderung, weil man für den Job alle seine Sinne braucht. Man muss mit dem PC arbeiten können, die Kunden abkassieren und vor allem im Kundenkontakt immer seriös und aufmerksam sein« (3578). Weitere defizitäre Zuschreibungspraxen zeigen sich unter anderem in der pauschalen Annahme, Menschen mit Behinderung könnten nicht Auto fahren. Eine Interviewperson sagt: »Für die Arbeit braucht man einen Führerschein, denn es ist notwendig, dass man auch auswärts tätig sein kann. Menschen mit Behinderung sind also nicht geeignet« (8040). Andere stehen dagegen einer Beschäftigung von Menschen mit Behinderung ganz grundsätzlich eher negativ gegenüber, wie die Aussage dieser Interviewperson verdeutlicht: „Dass Menschen mit Behinderung hier bei uns arbeiten, ist nicht wirtschaftlich. Der Betrieb wird aufgehalten und außerdem gibt es eh schon zu wenig Angestellte für die ganze Arbeit, die hier anfällt. Da ist einfach keine Zeit, jemanden nochmal extra zu unterstützen« (7191).

\section{Strukturelle Rahmenbedingungen}

Vereinzelt werden strukturelle Rahmenbedingen dafür verantwortlich gemacht, dass keine Menschen mit Behinderung KollegInnen sind. Diese Interviewperson problematisiert: »Bei uns gibt es keinen Aufzug und deshalb kommen Rollstuhlfahrer nicht in die zweite Etage« (1760). Deutlich wird hieran zudem, dass bei der Frage nach \Menschen mit Behinderung primär von Menschen mit Mobilitätsbeeinträchtigungen ausgegangen wird. Andere Beeinträchtigungsformen werden nur sehr selten bedacht. Denkbar ist, dass es an einer gewissen Sensibilität hinsichtlich diverser Unterstützungsbedarfe fehlt sowie an zu geringen Berührungspunkten zwischen Menschen ohne und Menschen mit Behinderung. Darüber hinaus spielt der Abbau struktureller Barrieren eine wichtige Rolle, der in erster Linie durch eine Bewusstseinsmachung und Sensibilisierung angestoßen werden muss (siehe in Bezug auf Barrieren im Kontext kognitiver Beeinträchtigung Trescher 2018c).

\section{Keine Bewerbungen von Menschen mit Behinderung}

Ein weiteres Ergebnis ist demgegenüber, dass einige Interviewpersonen den Grund dafür, dass keine Menschen mit Behinderung an ihrer Arbeitsstelle beschäftigt sind, in ausbleibenden Bewerbungen sehen. Menschen mit Behinderung hätten sich bislang noch auf keine der offenen Stellen beworben, weshalb sie auch keine KollegInnen mit Behinderung haben (6890). Dies kann im Zusammenhang mit der sogenannten Kontakthypothese diskutiert 
werden (siehe Kapitel 6.2 und Kapitel 23), wonach beiderseitige Vorbehalte gegenüber Menschen mit und ohne Behinderung auch daraus resultieren, dass der lebenspraktische Kontakt zu gering oder sogar gar nicht vorhanden ist. Es braucht also Bedingungen und vor allem Handlungspraxen, in denen Menschen mit und ohne Behinderung ganz selbstverständlich miteinander arbeiten (oder wohnen, lernen, ihre Freizeit verbringen etc.), um so Vorbehalte und Ängste abzubauen. Daran ist ein gewisses Potenzial zu erkennen, mehr Menschen mit Behinderung Arbeitstätigkeiten auf dem ersten Arbeitsmarkt zu ermöglichen. Handlungspraktisch können beispielsweise Menschen mit Behinderung (noch) ausdrücklicher in Stellenausschreibungen angesprochen werden. Darüber hinaus stellt sich die Frage, inwiefern MitarbeiterInnen in (insbesondere größeren) Unternehmen überhaupt Einblick in die Bewerbungsverfahren haben und ob es tatsächlich so ist, dass keine Bewerbungen von Menschen mit Behinderung eingehen. Unabhängig davon bleibt festzuhalten, dass Interviewpersonen dies als Begründung für die geringe Beschäftigung von Menschen mit Behinderung an ihrer Arbeitsstelle heranziehen, weshalb gegebenenfalls eine größere Transparenz in Bewerbungsverfahren eine sinnhafte Veränderung sein kann, die die MitarbeiterInnen enger in diese Prozesse einbindet und so zu Bewusstseinsbildung beiträgt.

\section{Kaum Stellenausschreibungen kleiner und/oder familiengeführter Unternehmen}

Im Kontrast zu ausbleibenden Bewerbungen von Menschen mit Behinderung werden mangelnde Ausschreibungen seitens der ArbeitgeberInnen benannt, neue MitarbeiterInnen einzustellen, da es sich teils um sehr kleine und/oder familiengeführte Unternehmen handele, die kaum neue MitarbeiterInnen einstellen. Eine Interviewperson sagt: »Wir haben ein kleines Familienunternehmen, in dem alle Mitarbeiter langjährig beschäftigt sind. $\mathrm{Da}$ ist die Fluktuation einfach nicht sehr groß « (3053). Eine andere Interviewperson betont: »Hier am Standort arbeiten nur zwei Personen. Deren Einstellung war keine bewusste Entscheidung gegen Menschen mit Behinderung «(3972), was eine prinzipielle Offenheit gegenüber der Beschäftigung von Menschen mit Behinderung nahelegt, die allerdings, das darf nicht aus dem Blick verloren werden, dennoch nicht erfolgte. In Bezug auf die familiengeführten oder neu gegründeten Unternehmen, die langjährige und/ oder (bislang) nur wenige MitarbeiterInnen haben und darin begründen, 
keine Menschen mit Behinderung zu beschäftigen, stellt sich die Frage, weshalb keine Menschen mit Behinderung als FirmengründerInnen in Erscheinung treten. Ausschluss vom Arbeitsleben vollzieht sich also nicht nur in der Rolle als Angestellte/r, sondern auch und insbesondere in der Rolle als Vorgesetzte/r, GründerIn usw. Offen ist bislang, wie die Menschen im Sozialraum - sowohl Menschen mit Behinderung als auch Menschen ohne Behinderung - empowert werden können, um im Handlungsfeld Arbeit mehr Teilhabemöglichkeiten zu schaffen und zu nutzen, sodass sich diverse Arbeitspraxen im Sozialraum vollziehen können. Dadurch können perspektivisch gesamtgesellschaftliche Arbeitspraxen, die sich kontinuierlich im Wandel befinden (Voß 2010, S. 23ff), verändert werden.

\subsubsection{Ablehnung von Menschen mit Fluchtmigrationshintergrund}

Aus Perspektive der Interviewpersonen sind die Gründe dafür vielfältig, dass (bislang) keine Menschen mit Fluchtmigrationshintergrund ihre KollegInnen sind.

\section{Geringere oder schlechtere Leistungsfähigkeit}

Einige Interviewpersonen sehen in der vergleichsweise geringeren oder schlechteren Leistungsfähigkeit von Menschen mit Fluchtmigrationshintergrund die Gründe dafür, dass diese nicht an ihrer Arbeitsstelle arbeiten. Bei diesen Begründungen überwiegt oftmals eine negative Konstruktion von Menschen mit Fluchtmigrationshintergrund, in der diese als weniger $\mathrm{zu}$ Leistung bereit dargestellt werden. Eine Interviewperson sagt: »Die paar Flüchtlinge, die da waren, die haben kaum gearbeitet und dadurch haben wir echt viel Zeit verloren« (9115). Wieder andere gehen davon aus, dass Menschen mit Fluchtmigrationshintergrund schlechtere Leistung erbringen. Eine Interviewperson sagt: »Das ist dann schwer mit der Eingliederung. Bei uns auf der Arbeit wird Kundenkontakt vorausgesetzt, da muss man die Sprache perfekt beherrschen, sonst springen uns die Kunden $a b$ « (3387). Eine andere Person ist der Meinung: »Es hat sich immer wieder gezeigt, dass einfach unterschiedliche Erwartungen auf den Beruf bezogen bestehen « (5274). Diese Interviewpersonen schreiben den geflüchteten Personen, mit denen sie zusammengearbeitet haben (beziehungsweise teils sogar geflüchteten Personen im Allgemeinen), eine bestimmte Mentalität zu, die sich in einer für sie nicht nachvollziehbaren Auffassung von Arbeit und Beruf niederschlägt. Diese Zuschreibungen sind Pauschalisierungen aus denen nicht zu- 
letzt negative Einstellungen gegenüber Menschen mit Fluchtmigrationshintergrund resultieren können, wie dies teils bei Interviewpersonen der Fall ist. Eine Interviewperson sagt: »Die Flüchtlinge sind doch alle IS-Kämpfer und Kriminelle. Gerade letzte Woche habe ich in der Zeitung gelesen, dass die Kriminalität sich gesteigert hat, seit die hier sind. Wir wollen nix mit denen zu tun haben.« (3578). Diese Aussage ist ein Beispiel dafür, inwiefern sich Interviewpersonen gerade in Bezug auf Menschen mit Fluchtmigrationshintergrund teilweise rassistisch äußern. Auch wenn dies nur vereinzelt dokumentiert wurde, zeichnet sich hierbei doch ab, inwiefern solche Einstellungen in der Gesamtgesellschaft vorhanden sind und vertreten werden. Auch bei der Interviewerhebung wurden die InterviewerInnen teils mit entsprechenden Aussagen konfrontiert (siehe Kapitel 16.1).

\section{Geringe Sprachkenntnisse}

Häufig werden zu geringe oder fehlende Sprachkenntnisse als Grund dafür genannt, dass keine geflüchteten Menschen an der eigenen Arbeitsstelle arbeiten. Sprache wird als manifeste Barriere dargestellt, die Menschen ohne (sehr gute) Deutschkenntnisse ausschließt. Dies führt teils dazu, dass jene Personen, insofern sie denn Arbeit finden, eher in un- und angelernten Berufen arbeiten, wie diese Interviewperson schildert: »In medizinischen und sozialen Berufen ist Sprache einfach total wichtig. Deshalb arbeiten viele Flüchtlinge in diesem Bereich eher als Hilfs- oder Putzkräfte« (1899). Geringe Sprachkenntnisse werden auch dann als Problematik adressiert, wenn Menschen mit Fluchtmigrationshintergrund als KollegInnen im eigenen Betrieb arbeiten, wie im Vorangegangenen dargestellt wurde (Kapitel 16.6).

\section{Fehlende beziehungsweise nicht anerkannte Ausbildung}

Besonders häufig problematisieren die Interviewpersonen, geflüchtete Menschen würden nicht über die geeignete Ausbildung respektive Qualifizierung verfügen, um für die Arbeitstätigkeit geeignet zu sein, der die Interviewpersonen nachgehen. Auch in Bezug darauf überwiegen defizitäre Zuschreibungspraxen, ausgehend von denen primär der vermeintliche Tatbestand einer mangelhaften Ausbildung adressiert wird. Die Aussage einer Interviewperson steht exemplarisch für die große Zahl sehr ähnlicher Aussagen: »Der Ausbildungsstand ist einfach zu niedrig. Und dazu kommt oft auch noch, dass die Ausbildung gar nicht abgeschlossen wurde. Da ist es echt schwierig, bei uns zu arbeiten« (7723). Nur sehr wenige Interviewperso- 
nen sprechen konkret die Problematik fehlender Anerkennung von im Ausland erbrachten Abschlüssen an. Eine Interviewperson sagt: »Das ist eben die zentrale Hürde, wenn man als Flüchtling nach Deutschland kommt. Die Ausbildung wird ganz oft einfach nicht anerkannt« (6169). Dies ist ein strukturelles Problem, das viele Menschen mit Fluchtmigrationshintergrund betrifft. Veränderung ist hier insbesondere auf politischer Ebene denkbar, indem Zugangsregelungen und Arbeitsgesetze infrage gestellt und schließlich neu verhandelt werden.

\section{Fehlende Arbeitserlaubnis}

Eine weitere strukturelle Barriere wird von einer Interviewperson angesprochen, die darauf hinweist, dass viele Menschen, die nach Deutschland geflüchtet sind, gar keine Erlaubnis haben, einer beruflichen Tätigkeit nachzugehen. Sie sagt: \#in großes Problem ist, dass viele Menschen, zwar gerne arbeiten wollen, aber nicht arbeiten dürfen« (1406). Es muss bedacht werden, inwiefern dadurch Teilhabemöglichkeiten eher eingeschränkt als eröffnet werden. Mit dem Verbot, einer beruflichen Tätigkeit nachzugehen, geht einher, dass dadurch eine Abhängigkeit von finanzieller Unterstützung (re-) produziert wird, wodurch Personen, die dies erfahren, oftmals in Armut leben und infolgedessen an alltäglichen Praxen, wie Wohnen, Einkaufen, Freizeitaktivitäten nachgehen etc., nicht im gleichen Maße teilhaben können. Der Ausschluss von Arbeit zieht also zahlreiche weitere Ausschlusspraxen nach sich, wie sich an diesem Beispiel ganz deutlich zeigt.

\section{Eher wenige Arbeitsplätze in kleineren Kollegien}

Interviewpersonen argumentieren teilweise, Menschen mit Fluchtmigrationshintergrund arbeiteten nicht an der eigenen Arbeitsstelle, da das Unternehmen eher klein sei und die MitarbeiterInnen auch nur selten wechselten. Diese Begründungsfigur findet sich, wie oben dargestellt (siehe Kapitel 16.7.1), auch oftmals in Bezug auf Menschen mit Behinderung. Eine Interviewperson sagt: »Also, bei uns sind die meisten Mitarbeiter wirklich schon jahrelang da und haben oft auch ihre Ausbildung schon bei uns gemacht. Da gibt es einfach wenig Neue« (3014). Neben der Kontinuität der MitarbeiterInnen wird häufig angesprochen, dass es sich um kleine und/oder familiengeführte Unternehmen handelt, die keine Kapazität für und keinen Bedarf an weitere(n) MitarbeiterInnen haben (diese Argumentation findet sich ebenfalls in Bezug auf Menschen mit Behinderung). Eine Interviewperson sagt: »Bei 
uns ist das ein Familienunternehmen und da ist klar, die Familie reicht aus" (8645). Deutlich wird bei diesen Aussagen, die exemplarisch für zahlreiche weitere stehen, dass es eher nicht als Aufgabe des eigenen Unternehmens respektive deren Führungsebene angesehen wird, überhaupt erst Arbeitsmöglichkeiten für (beispielsweise) Menschen mit Fluchtmigrationshintergrund zu schaffen. Inklusion wird größtenteils also nicht als gesamtgesellschaftliche Aufgabe verstanden, was mit dem hohen Wert (voll/teilweise) ablehnender und (noch) zurückhaltender Typen bei der Gesamteinschätzung im Handlungsfeld Arbeit korrespondiert (siehe Kapitel 16.2).

\section{Keine Bewerbungen von Menschen mit Fluchtmigrationshintergrund}

In Bezug auf Menschen mit Fluchtmigrationshintergrund, ebenso wie in Bezug auf Menschen mit Behinderung, argumentieren die Interviewpersonen, dass Bewerbungen dieser Personen ausbleiben und dass (auch) deshalb bislang keine Einstellung vorgenommen werden konnte. Zusätzlich wird dies teils mit mangelnden Qualifikationen begründet, wie diese Interviewperson schildert: »Die bewerben sich halt nicht bei uns, weil die fachlichen Voraussetzungen fehlen oder weil sie keine Ausbildung haben« (8698). In dieser Argumentation zeichnet sich ein Gegensatz zu der ab, wie sie in Bezug auf Menschen mit Behinderung oftmals zu finden ist. Dort wird größtenteils nicht weiter ausgeführt, warum sich keine Menschen mit Behinderung bewerben, was darauf schließen lässt, dass Informationsdefizite bestehen - annehmbar aufseiten der potenziellen BewerberInnen ebenso wie aufseiten der Personen, die die Stelle ausschreiben (siehe dazu auch Kapitel 16.7.1). Im weitesten Sinne adressiert die Aussage der folgenden Interviewperson dieses Problem: »Es gibt keine Bewerbungen, da denen ganz einfach die Deutschkenntnisse fehlen« (7898). Diese Aussage kann auf zweierlei Weise gelesen werden. Einerseits ist denkbar, dass die Interviewperson auf zu geringe Deutschkenntnisse als mangelnde Qualifikation für ausgeschriebene Stellen abzielt und darin die Gründe sieht, dass sich keine Menschen mit Fluchtmigrationshintergrund bewerben. Andererseits kann diese Aussage auch so gelesen werden, dass Menschen, die die deutsche Sprache kaum oder nicht verstehen, keinen Zugang zu deutschsprachigen Ausschreibungen finden können. Diesbezüglich kann darüber nachgedacht werden, ob gegebenenfalls Stellenausschreibungen sinnhafterweise mehrsprachig veröffentlicht werden können, wie es in internationalen Unternehmen ja bereits geschieht, in kleineren Betrieben dagegen allerdings meist eher nicht. 


\subsection{Möglichkeit der Teilhabe}

Interviewpersonen, die bislang keine KollegInnen mit Behinderung und/ oder KollegInnen mit Fluchtmigrationshintergrund haben, wurden gefragt, inwiefern sie es als möglich einschätzen, dass Menschen mit unterschiedlichen Beeinträchtigungen oder fluchtmigrierte Personen an ihrer Arbeitsstelle beschäftigt werden können ${ }^{8}$. Die Ergebnisse sind in der nachfolgenden Tabelle übersichtlich dargelegt und werden daran anschließend eingehend betrachtet.

Tabelle 26: Prinzipielle Möglichkeit der Beschäftigung von Menschen mit (unterschiedlichen) Beeinträchtigungen oder Fluchtmigrationshintergrund

\begin{tabular}{|l|c|c|c|}
\hline $\begin{array}{l}\text { Ja, es besteht die prinzipielle } \\
\text { Möglichkeit der Beschäfti- } \\
\text { gung von Menschen mit }\end{array}$ & Großstadt & $\begin{array}{l}\text { Kleinstadt/Ge- } \\
\text { meinde }\end{array}$ & $\varnothing$ \\
\hline Mobilitätsbeeinträchtigung & $76,8 \%$ & $50,7 \%$ & $63,8 \%$ \\
\hline Sehbeeinträchtigung & $34,6 \%$ & $21,8 \%$ & $28,2 \%$ \\
\hline Hörbeeinträchtigung & $61,3 \%$ & $44,3 \%$ & $52,8 \%$ \\
\hline kognitiver Beeinträchtigung & $32 \%$ & $15,6 \%$ & $23,8 \%$ \\
\hline Fluchtmigrationshintergrund & $87,8 \%$ & $71,6 \%$ & $79,7 \%$ \\
\hline
\end{tabular}

Ein Blick auf die Gesamtergebnisse zeigt, dass es hier naheliegend war, die untersuchten Sozialräume in die beiden übergeordneten Kategorien >Großstadt< (Erlangen und Rostock) und >Kleinstadt/Gemeinde` (Schneverdingen, Schwäbisch Gmünd, Verbandsgemeinde Nieder-Olm) zu unterteilen, da sich diesbezüglich signifikante Unterschiede feststellen ließen, auch wenn dies in Bezug auf die jeweiligen Beeinträchtigungsdimensionen unterschiedlich stark ausgeprägt ist. Die Ergebnisse zeigen, dass Interviewpersonen, die in

8 Es sei darauf hingewiesen, dass hier die Kategorie sandere Beeinträchtigungen $<$ nicht abgefragt wurde, da im Zusammenhang der prinzipiellen Möglichkeit der Beschäftigung von Menschen mit Beeinträchtigungen nicht pauschal nach `anderen « gefragt werden kann. Es ist nicht sinnhaft, gewissermaßen eine Restkategorie zu schaffen, in der sehr heterogene Personengruppen abgebildet werden (beispielsweise Menschen mit Asperger-Autismus ebenso wie Menschen mit schwerst-mehrfachen Behinderungen). 
Großstädten befragt wurden, die Beschäftigung von Menschen mit unterschiedlichen Beeinträchtigungen oder Menschen mit Fluchtmigrationshintergrund eher als möglich einschätzen als Menschen in der Kleinstadt/Gemeinde. Werden Teilhabemöglichkeiten von Menschen betrachtet, die von Ausschluss bedroht oder betroffen sind, so scheint die Größe des Wohnortes eine bedeutsame Rolle zu spielen. Als Mensch mit bestimmten Unterstützungsbedarfen außerhalb von der Großstadt zu leben, geht also potenziell damit einher, geringere Teilhabemöglichkeiten zu haben - zumindest im Bereich Arbeit. Schließlich kann hervorgehoben werden, dass die Bereitschaft, mit Menschen mit Mobilitätsbeeinträchtigungen zusammenzuarbeiten, deutlich höher ist (durchschnittlich 63,8 \%) als beispielsweise in Bezug auf Menschen mit kognitiven Beeinträchtigungen (durchschnittlich 23,8 \%). Die geringsten Vorbehalte haben die interviewten Personen gegenüber einer Beschäftigung von Menschen mit Fluchtmigrationshintergrund (durchschnittlich 79,7 \%), was möglicherweise darin begründet ist, dass jenen Personen weniger Beeinträchtigungen attribuiert werden als Menschen mit Behinderung (siehe dazu auch Kapitel 16.7).

\subsection{Schwierigkeiten und Handlungsperspektiven im Kontext von Inklusion und Arbeit}

Im Zuge der Analyse der Interviews mit ArbeitnehmerInnen in den fünf untersuchten Sozialräumen konnten zahlreiche Schwierigkeiten ebenso wie Handlungsperspektiven herausgearbeitet werden, die die Interviewpersonen im Kontext von Inklusion und Arbeit beschreiben. Um einen qualitativen Einblick in diese Ergebnisse zu ermöglichen, wird im Folgenden problemfokussiert zusammengefasst und diskutiert, inwiefern Interviewpersonen sich zu Inklusion im Handlungsfeld Arbeit verhalten, welche Schwierigkeiten sie ausmachen und welche Handlungsperspektiven sie formulieren. Dabei wurde sich für eine sozialraumübergreifende Darstellung entschieden, da die Auswertung zeigte, dass die Aussagen quer über die Sozialräume sehr ähnlich sind. Interviewpersonen berichten von ähnlichen Barrieren und Möglichkeiten ihrer Überwindung, haben ähnliche Vorbehalte und Ängste sowie ähnliche Ideen, welche Veränderungen für eine inklusive Teilhabe am Arbeitsleben notwendig sind. Um Redundanz zu vermeiden, wurden deshalb exemplarisch einzelne Interviewpassagen herausgegriffen, an denen zentrale, wiederkehrende Problematiken diskutiert werden. 


\subsubsection{Schwierigkeiten im Kontext von Inklusion und Arbeit}

Während viele Interviewpersonen die Frage danach, ob Inklusion in ihrem eigenen Unternehmen möglich ist, einschränkungslos bejahen, beantwortet ein großer Teil der Interviewpersonen diese Frage eher zurückhaltend und sieht (teils erhebliche) Schwierigkeiten, die die Teilhabemöglichkeiten von Menschen mit Behinderung und/oder Fluchtmigrationshintergrund erschweren. Diese Schwierigkeiten sind qualitativ-inhaltsanalytisch zusammengefasst und im Folgenden problemzentriert dargestellt.

\section{Rassismus und Behindertenfeindlichkeit}

Einige Interviewpersonen äußern teils stark defizitäre bis hin zu rassistischen beziehungsweise behindertenfeindlichen Aussagen. Fast alle dieser Interviewpersonen haben starke Vorbehalten gegenüber einer Beschäftigung von Menschen mit Behinderung und/oder Fluchtmigrationshintergrund und sind (auch) infolgedessen größtenteils als »(voll oder teilweise) ablehnend« typisiert worden. Einige Interviewpersonen konstruieren Menschen mit Fluchtmigrationshintergrund als eine Gefahr, wie beispielsweise die Aussage dieser Interviewperson zeigt: "Politiker sind nicht am Ort des Geschehens. Gehen Sie zum Beispiel mal nachts in München als Frau alleine auf die Straße, da haben Sie keine Chance gegenüber Gruppen. Politiker beachten einfach nicht das Gefährdungspotenzial, das von denen ausgeht. Die lassen einfach alle nach Deutschland rein« (3583). Eine andere Interviewperson sagt: »Die Flüchtlinge sind doch alle IS-Kämpfer und Kriminelle« (3578). Neben diesen pauschalisierenden Vorurteilen gibt es Vorbehalte, die auf das Thema Arbeit im Allgemeinen oder konkret auf die jeweilige Arbeitstätigkeit bezogen werden. Einige Interviewpersonen sehen kulturell unterschiedliche Einstellungen zum Thema Arbeit und leiten daraus ab, dass einige Personen weniger geeignet seien für eine Tätigkeit in ihrem Unternehmen. Eine Interviewperson sagt: "Flüchtlinge haben manchmal andere Vorstellungen und Arbeitsauffassungen. Da heißt es nicht immer deutsche Tugenden, sondern die haben andere Prägungen " (9176). Ein weiteres wiederkehrendes Thema ist, dass einige Interviewpersonen pauschal eine Minderleistung von Menschen mit Fluchtmigrationshintergrund annehmen. Eine Interviewperson sagt beispielsweise: "Die arbeiten halt einfach langsamer « (2262), wogegen eine andere sich wie folgt äußert: "Inklusion ist im Gastrobereich schwierig, gerade für Flüchtlinge, wegen dem Kundenkontakt. Die machen einfach mehr Fehler« (4279). Andere Interviewpersonen gehen davon aus, dass 
Menschen mit Fluchtmigrationshintergrund für bestimmte Arbeitstätigkeiten nicht geeignet seien, da ihnen notwendige Kenntnisse fehlen. Ein Beispiel dafür ist die Aussage dieser Interviewperson: „Bei uns müssen die Blumen eher deutsch hergerichtet werden. Leute aus dem Süden wissen gar nicht, wie das geht« (4489). Neben der Problematik offenkundiger Vorurteile kann hier diskutiert werden, dass Interviewpersonen, wie die oben zitierten, Menschen mit Fluchtmigrationshintergrund keine Möglichkeiten einräumen, sich weiterzubilden oder bestimmte Fähigkeiten anzueignen. Das ist im Kontext Fluchtmigration dahingehend bedeutsam, dass hier Sprach- und Kommunikationsbarrieren sehr häufig vorkommen, die allerdings überwunden werden können, wenn entsprechende Unterstützungsmöglichkeiten geschaffen werden (zum Beispiel Sprachkurse, auch bezogen auf bestimmte Berufs- und Tätigkeitsfelder). Teils werden Menschen mit Fluchtmigrationshintergrund besondere Bedürfnisse zugeschrieben, die das Unternehmen, so die entsprechenden Interviewpersonen, nicht leisten kann. Ein Beispiel dafür ist die folgende Aussage: »Das Problem ist die Religion. Die [gemeint sind Menschen mit Fluchtmigrationshintergrund] sind ja extrem konservativ. Und dann ist es für das Unternehmen zusätzlicher Aufwand, zum Beispiel einen Raum mit Ausrichtung nach Osten zur Verfügung zu stellen. In Deutschland ist Religion Privatsache und beeinflusst die Arbeit nicht« (3583). Ganz anders sieht dies dagegen die nachfolgende Interviewperson, die, wie sie sagt, vor allem für einen kulturellen Paradigmenwechsel im Bereich Arbeit plädiert. Sie sagt: »Insgesamt müsste sich die Arbeitskultur ändern, denn, wenn wir ehrlich sind, stehen Inklusion doch nur Kleinigkeiten im Weg, die schnell beseitigt werden können. Zum Beispiel könnte man ohne Weiteres einen Gebetsraum oder Möglichkeiten zur Fußwaschung einrichten. Notwendig dazu ist vor allem, dass die Arbeitgeber informiert sind « (2027). Es zeigt sich also, inwiefern Interviewpersonen ganz unterschiedliche Haltungen zum selben Gegenstand haben können. Diese Personen in Dialog zu bringen, sodass Erfahrungen ausgetauscht und schließlich Ängste und Vorbehalte abgebaut werden können, kann ein Ziel von Maßnahmen sein, die Inklusion im Sozialraum fokussieren.

Behindertenfeindliche und/oder defizitorientierte Haltungen zeigen sich insbesondere in Aussagen, in denen Menschen mit Behinderung pauschal als $>$ Minderleister konstruiert werden, die der Betreuung bedürfen. Eine Interviewperson sagt: »Behinderte brauchen besondere Hilfe und das können wir hier nicht leisten. Außerdem muss man für die Arbeit klar im 
Kopf sein« (5514). Als besonders benachteiligte Personengruppe stellen sich Menschen mit kognitiven Beeinträchtigungen heraus, da Interviewpersonen in Bezug auf diese häufig eine Teilhabe am Arbeitsleben in ihrem Unternehmen kategorial ausschließen. Ein Beispiel dafür ist die Aussage dieser Interviewperson: »Bei Flüchtlingen geht Inklusion vielleicht noch, aber bei Menschen mit geistiger Behinderung auf keinen Fall« (6189). , Geistige Behinderung ‘ wird hier als eine Art Grenze konstruiert, die nicht durch Inklusion überwunden werden kann. Menschen, die als geistig behindert bezeichnet werden, werden so pauschal von der Möglichkeit der Teilhabe an der Mehrheitsgesellschaft ausgeschlossen, woraus wiederum die Frage folgt, was jene Interviewpersonen überhaupt unter >Inklusion verstehen. Interviewpersonen, die sich so deutlich defizitär äußern, können annehmbar kaum unmittelbar durch inklusionsorientierte Maßnahmen erreicht werden, da ihre ablehnende Haltung so deutlich ist. Hier kann darüber nachgedacht werden, inwiefern Ablehnung gegebenenfalls dadurch abgebaut werden könnte, dass mehr Kontakt(-möglichkeiten) zu Menschen mit Behinderung und/oder Menschen mit Fluchtmigrationshintergrund geschaffen werden, sodass Personen wie die oben zitierten Interviewten eine größere lebenspraktische Nähe zu unterschiedlichen Personen erfahren. Auf diese Weise würde Diversität mehr zum Alltag für die betreffenden Personen und so möglicherweise ihre ablehnende Haltung sukzessive verändern.

Fluchtmigrationshintergrund: Sprachbarrieren und schwierige Anerkennung von Berufsabschlüssen

Einige Interviewpersonen gehen davon aus, dass Menschen mit Fluchtmigrationshintergrund keine ausreichenden Fähigkeiten und Fertigkeiten vorweisen können, um eine Arbeitstätigkeit in ihrem Unternehmen aufzunehmen. Ein sehr großer Teil dieser Interviewpersonen benennt dabei eingeschränkte Sprach- und Kommunikationsfähigkeiten als Hürden. Eine Interviewperson konkretisiert: »Für Flüchtlinge bestehen Schwierigkeiten, da bei uns das Amtsdeutsch gut beherrscht werden muss " (4755). Eine andere Interviewperson sagt: »Die Verkäufer haben bei uns viel Kundenkontakt, da muss man die Artikel gut kennen und auch gut auf Deutsch kommunizieren können« (3081). Interviewpersonen, die sich derart äußern, sehen Barrieren eher in äußeren Strukturen und weniger in der Person selbst, denn gerade Sprach- und Kommunikationsbarrieren sind überwindbar - zwar bedarf es dazu einiger Arbeit und Unterstützung, aber es ist möglich. Von- 
seiten der Politik und entsprechender Anbieter braucht es dazu Sprachkurse und eine ausreichende Finanzierung. Weiterhin werden Problematiken in Ausbildungen gesehen, die nicht den für die Tätigkeit notwendigen Voraussetzungen entsprechen, oder darin, dass Berufsabschlüsse in Deutschland nicht anerkannt werden, was bei Menschen mit Fluchtmigrationshintergrund oftmals der Fall ist (diskutiert wird dies ebenfalls in Kapitel 16.7.2).

\section{Behinderung: Zu geringe Fähigkeiten und mangelnde Qualifizierung}

Bei Menschen mit Behinderung werden oftmals mangelnde körperliche und/ oder kognitive Fähigkeiten als Grund dafür genannt, dass diese keiner Tätigkeit im Unternehmen, in dem die Interviewperson beschäftigt ist, nachgehen können. Eine Interviewperson sagt: »Bei uns sind alle Sinne, Hände und Füße notwendig. Wir arbeiten im Stehen, außerdem muss man sich gut verständigen können, weil wir auch häufig Kundenkontakt haben. Vielleicht ist für behinderte Menschen eine Arbeit mit Maschinen besser geeignet« (8701). Demgegenüber kommt es allerdings auch vor, dass Interviewpersonen Menschen mit Behinderung als besonders geeignet für die eigene berufliche Tätigkeit sehen, wie zum Beispiel diese Interviewperson: „Es wäre sehr hilfreich, wenn jemand mit Gehörschädigung bei uns arbeiten würde« (5739). Dies liegt in diesem Fall in der Tätigkeit der Interviewperson begründet, denn sie arbeitet in einem Laden für Hörgeräteakustik. Weiterhin problematisiert eine Interviewperson, sie sehe bei Menschen mit Behinderung vor allem das Problem, »dass fachliche Anforderungen nicht erfüllt werden« (4755). Dies mag unter Umständen in mancher Hinsicht richtig sein, kann aber (a) nicht so pauschal gesagt werden und löst (b) nicht die dahinterliegenden Problematiken, dass viele Menschen mit Behinderung bereits während der Ausbildung vom allgemeinen Arbeitsmarkt ausgeschlossen werden und dass zudem der Arbeitsmarkt oftmals zu unflexibel ist für die diversen Fähigkeiten und Bedürfnisse möglicher ArbeitnehmerInnen.

\section{Tätigkeitsbezogene Schwierigkeiten}

Demgegenüber gibt es einige Interviewpersonen, die Inklusion ausschließlich in bestimmten Arbeitsbereichen ihres Unternehmens für möglich halten. Hieran zeigt sich, dass Arbeitsabläufe teils eher als manifest angesehen werden und diesen nur geringe Flexibilität eingeräumt wird. Ein solches Verhaften am Status quo zeigt sich auch an der Aussage dieser Interviewpersonen, die Inklusion zwar für möglich hält, allerdings sei eine Umsetzung schwie- 
rig. Sie sagt: "Inklusion ist eine schöne Illusion « (3203). Interviewpersonen, die sich dementsprechend äußern, stehen Inklusion zwar nicht grundlegend ablehnend gegenüber, verstehen sich selbst aber nicht als handlungsmächtig, Teilhabemöglichkeiten für bestimmte Personen zu eröffnen. Hieran gilt es anzusetzen und beispielsweise Personen, wie die genannte, zu empowern, sodass sie Möglichkeiten findet, sich für Teilhabe und Inklusion einzusetzen.

\section{Ablehnung und mangelnde Akzeptanz}

Einige Interviewpersonen sehen Schwierigkeiten im Kontext Inklusion und Arbeit in der mangelnden Akzeptanz von Menschen mit Behinderung und/ oder Menschen mit Fluchtmigrationshintergrund sowohl auf Ebene der unmittelbaren KundInnen und KlientInnen ihres Unternehmens, ihrer KollegInnen als auch auf gesamtgesellschaftlicher Ebene, in der diesen Personen mit Vorbehalten begegnet würde. In Bezug auf die Vorbehalte von KundInnen und KlientInnen des Unternehmens sagt eine Interviewperson: »Unsere breit gefächerte Klientel könnte Probleme mit Angestellten mit Behinderung oder Fluchtmigrationshintergrund haben « (1472). Eine andere Interviewperson befürchtet, »dass die Menschen von den Kunden nicht akzeptiert werden« (6924). Demgegenüber differenziert eine Interviewperson, indem sie sagt: »Unsere Kunden haben Berührungsängste gegenüber Menschen mit geistiger Behinderung. Bei Menschen mit körperlichen Beeinträchtigungen sehe ich aber keine Schwierigkeiten « (6189). Hieran wird erneut deutlich, inwiefern geistige Behinderung nach wie vor als eine Art Ausschlusskategorie konstruiert wird, mit der manifeste Teilhabegrenzen einhergehen, was wiederum zeigt, dass viele Personen ein pauschales, defizitäres Bild von Menschen mit geistiger Behinderung haben. Wieder andere Interviewpersonen sehen Vorbehalte vor allem aufseiten ihrer KollegInnen, wie eine Interviewperson verdeutlicht: „Schwierig ist vor allem die Offenheit der Kollegen. Bei denen fehlt es einfach an Akzeptanz« (6398). Eine weitere Interviewperson sagt: »Inklusion ist bei uns nicht denkbar. Die Leute im Unternehmen wollen das nicht« (7748). Dies ist eine Problematik, die andere Interviewpersonen auf gesamtgesellschaftlicher Ebene erkennen. Eine Interviewperson sagt: »Die Gesellschaft hat Vorbehalte gegenüber Behinderten und Flüchtlingen. Das ist das zentrale Problem bei Inklusion« (2843). In Bezug auf alle Antworttypen kann festgestellt werden, dass die Interviewpersonen Skepsis und Ablehnung ausschließlich außerhalb ihrer eigenen Person konstruieren. Dies wirkt fast entschuldigend, so, als befürworteten die Interviewperso- 
nen zwar eine Teilhabe von Menschen mit Behinderung und/oder Menschen mit Fluchtmigrationshintergrund (beziehungsweise wollen sich zumindest so darstellen), kommen aber nicht gegen die massiven Vorbehalte an, die in ihrem direkten und mittelbaren Umfeld bestehen. Nichtsdestotrotz können hier bewusstseinsbildende Maßnahmen sinnvoll ansetzen und gegebenenfalls sogar dazu führen, dass sich jene Personen, durch Unterstützung und Empowerment, für inklusive Teilhabemöglichkeiten (im Bereich Arbeit und darüber hinaus) engagieren.

\section{Mangelnde Barrierefreiheit}

Ein letztes Problemfeld kann schließlich in Bezug auf äußere Strukturen herausgearbeitet werden, die von den Interviewpersonen als Teilhabebarrieren dargestellt werden. Dazu gehören vor allem eine mangelnde Barrierefreiheit des Arbeitsortes und zu geringe Möglichkeiten, Umbauten vorzunehmen, wie eine Interviewperson problematisiert: »Unser Geschäft befindet sich in einem alten Haus. Da sind bauliche Maßnahmen teilweise nicht machbar und vor allem auch nicht finanzierbar (2183). Eine andere Interviewperson sieht ähnliche Schwierigkeiten, formuliert diese aber lösungsorientierter, indem sie sagt: "Wir müssten dann eben umbauen, damit der Arbeitsort barrierefrei wird. Und auch die Arbeitsweisen könnte man sicher umstrukturieren « (4432). Hieran zeigt sich großes Potenzial dazu, Menschen mit Behinderung und/oder Menschen mit Fluchtmigrationshintergrund Teilhabemöglichkeiten zu eröffnen. Daneben finden sich jedoch auch vereinzelt Interviewperson, die den Fokus ihrer Antwort weniger auf Schwierigkeiten der Teilhabe von Menschen mit Behinderung und/oder Menschen mit Fluchtmigrationshintergrund legen, sondern eher kommentieren, wie Schwierigkeiten überwunden werden könnten. Dementsprechend sagt eine Interviewperson: »Die Menschen müssen in den Arbeitsablauf miteinbezogen werden, damit man herausfinden kann, was die Person für machbar hält« (6169), woraus Forderungen nach einer gewissen Flexibilität am Arbeitsplatz abgeleitet werden können.

\subsubsection{Handlungsperspektiven im Kontext von Inklusion und Arbeit}

Einige Interviewpersonen benennen (mehr oder weniger konkrete) Maßnahmen und Handlungsideen, die Menschen mit Behinderung und/oder Menschen mit Fluchtmigrationshintergrund darin unterstützen können, im 
eigenen Unternehmen beschäftigt zu werden. Diese sind im Folgenden thematisch geordnet wiedergegeben und werden problemzentriert diskutiert.

\section{Bürokratieabbau}

Eine Handlungsperspektive, die von vielen Interviewpersonen genannt wird, ist, bürokratische und strukturelle Barrieren abzubauen, die eine Teilhabe von Menschen mit Behinderung und/oder Menschen mit Fluchtmigrationshintergrund erschweren. Eine Interviewperson problematisiert: »Von den Menschen wird eine hohe Flexibilität erwartet, während das System starr ist. Meiner Ansicht nach, müsste Flexibilität viel mehr gefördert werden« (5565). Inwiefern vorgefestigte, unbewegliche Strukturen die Biographien und Lebensentwürfe von Menschen mit Behinderung und/oder Menschen mit Fluchtmigrationshintergrund beeinflussen, wurde an anderer Stelle detailliert herausgearbeitet (Trescher 2017a), sodass hier lediglich noch einmal darauf aufmerksam gemacht werden soll, dass Bürokratie oftmals Ausschluss (re-)produziert. Auch Prozesse und Strukturen aufseiten der Unternehmen werden problematisiert, wofür beispielhaft die Aussage dieser Interviewperson steht: "Man muss die Auswahlprozesse prüfen und erreichen, dass Menschen mit Behinderung oder Geflüchtete nicht von vorneherein negativ besetzt und dann ausgeschlossen werden" (1239). Die hier skizzierte Handlungsperspektive ist also, Strukturen zu verändern und althergebrachte Praxen aufzubrechen - beispielsweise in Bezug auf Bewerbungsverfahren.

\section{Gesetzesänderungen}

Einige Interviewpersonen regen rechtliche Änderungen an, um Teilhabemöglichkeiten von Menschen mit Behinderung und/oder Menschen mit Fluchtmigrationshintergrund am Arbeitsleben zu erleichtern. Eine Interviewperson kritisiert gängige Rechtspraxis in Bezug auf die Arbeitsmöglichkeiten von Menschen, die nach Deutschland geflüchtet sind und (vorerst) keine Arbeitserlaubnis haben. Sie fordert: »Das deutsche Arbeitsrecht muss geändert werden. Denn so wie das jetzt gerade ist, haben Ausländer keine Chance auf dem Arbeitsmarkt" (9731). Kritik an der Gesetzeslage kann auch aus der Antwort einer Interviewperson abgeleitet werden, die die rechtliche Regelung der sogenannten Ausgleichsabgabe (\$160 SGB IX) infrage stellt, nach der Unternehmen Zahlungen leisten müssen, wenn sie die vorgeschriebene Anzahl an Beschäftigten mit dem Status sSchwerbehinderung nicht erfüllen. Die Interviewperson sagt: »Unternehmen müs- 
sen Inklusion umsetzen und sich nicht mehr freikaufen können« (8698). Die sogenannte Ausgleichsabgabe verfolgt zweierlei Ziele, »neben ihrem Anreiz zur Beschäftigung [ist sie] insbesondere eine Finanzierungsgrundlage für die verschiedenen Maßnahmen« (Klinkhammer und Niehaus 2015, S. 208), womit primär Maßnahmen zur Qualifikation von Menschen mit Behinderung und deren Vermittlung auf den ersten Arbeitsmarkt adressiert werden. Dabei muss hinterfragt werden, inwiefern die Ausgleichsabgabe überhaupt einen Anreiz für Firmen darstellt, ist die finanzielle Belastung doch eher gering - wenngleich das Gesamtvolumen der dadurch eingenommenen Gelder im Jahr 2018 immerhin etwa 673 Millionen Euro betrug (BIH Bundesarbeitsgemeinschaft der Integrationsämter und Hauptfürsorgestellen 2019, S. 20). Deutlich problematischer ist dagegen, dass Personen, die in der Qualifikation und Vermittlung von Menschen mit Behinderung arbeiten, gegebenenfalls widerstreitende Interessen verfolgen. Denn dadurch, dass die Einnahmen durch die Ausgleichsabgabe jene Maßnahmen refinanzieren, könnte den darin arbeitenden Personen unterstellt werden, sie beeinflussten die Maßnahme zu ihrem eigenen Vorteil: Wenn sie mehr Menschen mit Behinderung auf den ersten Arbeitsmarkt vermitteln, sinken die Einnahmen durch die Ausgleichsabgabe, wodurch wiederum die Finanzierung jener Maßnahmen verringert wird, in denen die betreffende Person arbeitet. Auch wenn den in diesem Bereich arbeitenden Personen selbstredend keine Beeinflussung unterstellt werden soll, so muss die Ambivalenz diskutiert werden, dass sie streng genommen wider sich selbst beziehungsweise ihre eigene Beschäftigung arbeiten.

\section{Barrierefreiheit und Unterstützung bei der Finanzierung}

Weiteren Handlungsbedarf sehen einige Interviewpersonen in Bezug auf Barrierefreiheit respektive die Erweiterung entsprechend ausgebauter $\mathrm{Zu}$ gänge. Eine Interviewperson kritisiert beispielsweise: »Barrierefreiheit wird nicht richtig umgesetzt, zum Beispiel im öffentlichen Verkehr« (7090). Andere Interviewpersonen berichteten dagegen, dass gerade in Bezug auf Barrierefreiheit bereits einige Maßnahmen umgesetzt wurden oder in Planung sind. Eine Interviewperson sagt: »Bei der Übernahme des Geschäfts vor einigen Jahren wurde eine Rampe gebaut, die einen barrierefreien Zugang für mobilitätseingeschränkte Kunden zur Buchhandlung ermöglicht« (2183). Im Zusammenhang mit dem Wunsch, mehr barrierefreie Zugänge zu schaffen, werden häufig die dadurch entstehenden Kosten problematisiert und 
übereinstimmend fordern die Interviewpersonen, die Finanzierung solcher Maßnahmen müsse durch den Staat geregelt sein. Eine Interviewperson sagt diesbezüglich: »Eine Maßnahme wäre ja, unser Geschäft barrierefrei zu machen. Aber da ist halt unklar, wer das am Ende bezahlt« (4466). Das Thema Kosten und Finanzierung beschäftigt mehrere Interviewpersonen hinsichtlich weiterer Bereiche. Eine Interviewperson sieht finanzielle Unterstützungsbedarfe vor allem im Bereich Schule und sagt: „Schulen müssen besser ausgestattet werden « (5739). Eine andere Interviewperson konkretisiert dies, indem sie sagt: »Die I-Kräfte in den Schulen müssen finanziert werden " (4004). Die Forderung einer anderen Interviewperson ist, wenngleich weniger konkret, deutlich umfassender, sie sagt: »Es muss vom Staat mehr Geld für Inklusion aufgewendet werden« (4432).

\section{Fluchtmigrationshintergrund: Erleichterter Zugang}

\section{zum ersten Arbeitsmarkt}

Ein nicht unerheblicher Anteil der Interviewpersonen problematisiert, dass viele Menschen mit Fluchtmigrationshintergrund einen erschwerten $\mathrm{Zu}$ gang zum Arbeitsmarkt haben, und fordert, ebenjenen Zugang durch den Abbau von Hürden zu erleichtern. Eine Interviewperson sagt: "Man muss den Menschen mehr Möglichkeiten geben, Voraussetzungen überhaupt erfüllen zu können. Zum Beispiel durch Sprachkurse, Anreize oder eine Ausbildung, die in der Zusammenarbeit mit der IHK ${ }^{9}$ erfolgt« (9242). Was von den Interviewpersonen ebenfalls mehrfach angesprochen wird, ist, dass Menschen mit Fluchtmigrationshintergrund und begrenztem Aufenthaltstitel nicht abgeschoben werden sollen, wenn sie einer Beschäftigung nachgehen. In dem Zusammenhang können auch die oben diskutierten rechtlichen Schwierigkeiten wiederaufgegriffen werden, die einer Eingliederung von Menschen mit Fluchtmigrationshintergrund in den ersten Arbeitsmarkt entgegenstehen. Eine Interviewperson fordert diesbezüglich: »Es sollte ermöglicht werden, dass Menschen mit Fluchtmigrationshintergrund schneller in den Arbeitsmarkt aufgenommen werden« (2659).

9 Annehmbar werden damit die Industrie- und Handelskammern adressiert, in denen in Deutschland Ausbildungsberufe organisiert und reguliert sind. 


\section{Behinderung: Reproduktion bestehender Strukturen}

Während in Bezug auf Menschen mit Fluchtmigrationshintergrund oftmals der Zugang zum ersten Arbeitsmarkt gefordert wird, werden in Bezug auf Menschen mit Behinderung fast immer bereits bestehende Sonderstrukturen als sinnvoll und unterstützend angenommen und bestätigt. Eine Interviewperson sagt: »Ein guter Ansatz sind diese Werkstätten für Behinderte « (6752). Dass durch diese protektiven Strukturen Teilhabemöglichkeiten eher eingeschränkt als eröffnet werden, wird dabei nicht reflektiert. Zudem wird dadurch ein Bild von Menschen mit Behinderung als besonders schutz- und unterstützungsbedürftig (re-)produziert, das ebenfalls Ausschluss vom mehrheitsgesellschaftlichen Arbeitsleben manifestiert. Auch in Bezug auf Interviewpersonen, die bereits KollegInnen mit Behinderung haben, können ausschließende Prozesse festgestellt werden. Eine Interviewperson sagt beispielsweise: »Bei uns in der Filiale werden extra Arbeitsplätze für Menschen mit Behinderung angeboten« (3697). Dies ist zwar für Menschen mit Behinderung eine Möglichkeit, auf dem ersten Arbeitsmarkt beschäftigt zu werden. Problematisiert werden kann in Bezug auf diese Aussage allerdings, dass jene gesonderten Arbeitsplätze, die explizit für Menschen mit Behinderung geschaffen werden, eine Art Sonderstatus bedeuten, wodurch die $\mathrm{Zu}$ schreibung >Behinderung< nicht abgebaut, sondern vielmehr reproduziert wird. Im Lichte eines Verständnisses von Inklusion, das stigmatisierende Zuschreibungen dekonstruieren will, ist diese Praxis infrage zu stellen.

\section{Einstellungsänderung, Offenheit und Sensibilität}

Eine weitere Perspektive, die einige Interviewpersonen formulieren, betrifft eine Veränderung der Einstellungen gegenüber Menschen mit Behinderung und/oder Menschen mit Fluchtmigrationshintergrund. Einige Interviewpersonen fordern, man müsse »Vorurteile abbauen und Chancen schaffen" (5006), »Rassismus kontrollieren« (3644) oder »die Gesellschaft« (9176) müsse "mehr open-minded werden" (9176), damit kein Mensch ausgeschlossen wird. Die Verantwortung dafür sehen sie insbesondere auf politischer Seite. Veränderungen auf politischer Ebene beziehungsweise bezüglich des politischen Systems adressiert auch eine Interviewperson, die sagt: »Im europäischen Ausland sind Menschen mit Behinderung freie Menschen und normal. Wieso funktioniert Inklusion nicht in einem so reichen Land wie Deutschland?« (6144). Hervorzuheben ist hier zweierlei: Zum einen werden Menschen mit Behinderung in Deutschland von dieser Interviewperson ganz 
offensichtlich als unfrei und unnormal wahrgenommen und zum anderen verknüpft sie Inklusion unmittelbar mit der Wirtschaftskraft eines Landes. Inklusion wird also auch hier im Zusammenhang mit Geld gesehen und, zumindest latent, als finanzielles Problem konstruiert, das im Umkehrschluss durch entsprechende Zuwendungen sgelöst‘ werden kann. Auch wenn Geld sicherlich eine Rolle dabei spielt, Teilhabemöglichkeiten für Menschen zu schaffen, die von Ausschluss bedroht oder betroffen sind, ist doch die Frage zu stellen, ob dies nicht ein wenig zu kurz gegriffen ist. Im Zusammenhang mit Einstellungen der Mehrheitsgesellschaft kann darüber hinaus die Aussage der nachfolgenden Interviewperson gesehen werden: »Inklusion sollte eine Selbstverständlichkeit werden und nicht nur in speziellen Cafés, Einrichtungen oder Bereichen stattfinden « (1899). Demgegenüber gibt es einige Interviewpersonen, die Menschen mit Behinderung als besonders schutzbedürftig und weniger zu Leistung fähig konstruieren, was oben in Bezug auf protektive Beschäftigungsstrukturen bereits diskutiert wurde. Ein Beispiel dafür ist die Aussage dieser Interviewperson: »Wenn es Jobs gibt, dann sollten auch Menschen mit Behinderung eingestellt werden, damit sie was zu tun haben und ihr Selbstwertgefühl gesteigert wird« (3364). Hier werden Menschen mit Behinderung pauschal als bemitleidenswerte Personen mit geringem Selbstwertgefühl dargestellt, die beschäftigt werden müssen, damit sie sfroh sind. Zusätzlich sollen Menschen mit Behinderung lediglich auf vorhandene Stellen eingestellt werden, was bedeutet, dass keine alternativen Möglichkeiten ihrer Beschäftigung geschaffen werden sollen, wodurch jene Personen in gewisser Weise als eine Art `Restkategorie k konstruiert werden, die aus Mitleid oder Güte am Leben der Mehrheitsgesellschaft beteiligt werden sollen - insofern sich dieses dafür nicht verändern muss. Eine letzte Handlungsperspektive soll hier abschließend genannt werden, in der die betreffende Interviewperson den Mehrwert von inklusionsorientierten Projekten und insbesondere sozialwissenschaftlicher Inklusionsforschung hervorhebt: "Also, eine Maßnahme muss ja sein, die Leute besser miteinzubeziehen. Und da sind wir ganz offensichtlich auf dem richtigen Weg, weil Sie sich ja damit beschäftigen [adressiert wird der/die InterviewerIn]« (1292). 


\section{Sozialraumanalysen im Handlungsfeld Freizeit}

Aufgrund der unproblematischen Pre-Tests im Test-Sozialraum und der Erfahrungen, die bereits aus dem Projekt »Inklusion. Zur Dekonstruktion von Diskursteilhabebarrieren im Kontext von Freizeit und Behinderung« (Trescher 2015b) gewonnen wurden, verlief die Erhebung im Handlungsfeld Freizeit in allen untersuchten Sozialräumen größtenteils einschränkungslos.

\subsection{Ablauf der leitfadengestützten Telefoninterviews}

Zum Ablauf der leitfadengestützten Telefoninterviews im Handlungsfeld Freizeit kann gesagt werden, dass zunächst per Desktoprecherche Freizeitvereine und -gruppen in den Sozialräumen und entsprechende Kontaktdaten herausgesucht wurden. Hierbei wurde auf eine sinnvolle Verteilung der Freizeitaktivitäten auf die vier Cluster (1) Sport- und Spielgruppen, (2) Gemeinnützige Vereine, (3) Musik, Kunst, Kultur und (4) Garten, Wald, Natur geachtet. Eine erste Kontaktaufnahme, in der um einen telefonischen Interviewtermin mit einem/r PrimärvertreterIn der jeweiligen Freizeitaktivität (beispielsweise Leitung, Vorstand) gebeten wurde, erfolgte per Telefon oder E-Mail, woran sich entweder unmittelbar oder nach Absprache die Möglichkeit zur Interviewerhebung anschloss. Die Erfahrungen der InterviewerInnen waren in Bezug auf dieses Vorgehen teils unterschiedlich. Während einige GesprächspartnerInnen sich unmittelbar am Telefon bereit erklärten, ein Interview durchzuführen, gab es andere, die nach der Erklärung des Anliegens durch den/die InterviewIn sofort auflegten und fortan auch per E-Mail nicht mehr zu erreichen waren. Im Folgenden sind schlaglichtartig einige Erfahrungen der Interviewerhebung im Handlungsfeld Freizeit dargelegt, die die Spannbreite der Reaktionen verdeutlichen. Eine Interviewperson antwortete zunächst sehr freundlich auf meine E-Mailanfrage. Am Telefon wirkte sie jedoch sehr verunsichert und wies mehrmals darauf hin, dass sie zu manchen Fragen keine Auskunft geben könne, da sie dazu nicht befugt sei. Trotzdem gelang es, alle relevanten Informationen zu erheben (I-6953). Hieran zeigt sich, inwiefern Interviews mit prinzipiell aufgeschlossenen Personen zur Herausforderung werden können und verdeutlicht, wie wichtig eine dezidierte Vorbereitung auf die Interviews ist, um auf Ängste und Unsicherheiten der Interviewpersonen sensibel reagieren und diese auffangen zu können. Ein Beispiel für sehr engagierte und aufgeschlossene Interview- 
personen ist die folgende Schilderung: Das Interview dauerte fast eine Stunde. Die Interviewperson erklärte mir sehr ausführlich, inwiefern auch Menschen mit Behinderung Spaß an der angebotenen Freizeitaktivität finden würden. Auch berichtete sie von Erfahrungen, die sie bereits bei öffentlichen Angeboten beobachtet hatte und erzählte mir ausführlich über die zukünftige Planung von inklusiven Veranstaltungen (I-5637). Insgesamt war die Resonanz auf die Interviewanfragen sehr positiv und Unsicherheiten, die in einigen Interviews anfangs noch gegeben waren, legten sich im Laufe des Gesprächs, was wieder für die kleinteilige und sensible Vorbereitung der Interviewerhebung spricht.

\subsection{Gesamteinschätzung}

Im Handlungsfeld Freizeit wurden durchschnittlich 5,6 \% der Interviewpersonen respektive der Freizeitgruppen, die sie vertreten, als voll ablehnend kategorisiert, wogegen durchschnittlich $29,2 \%$ als teilweise ablehnend eingeschätzt wurden. Diesen beiden (eher) ablehnenden Typen stehen durchschnittlich 38,1 \% (noch) zurückhaltende sowie durchschnittlich 27,2 \% offene Interviewpersonen gegenüber. Ein deutlich vom Durchschnitt abweichender Wert findet sich in Schneverdingen, wo 7,1 \% der untersuchten Freizeitgruppen als offen kategorisiert wurden. Korrespondierend dazu ist dort auch der Anteil der als voll ablehnend typisierten Freizeitgruppen am höchsten (11,9 \%). Im Vergleich zum Handlungsfeld Arbeit zeigt sich, dass eine deutlich höhere Bereitschaft besteht, Menschen mit unterschiedlichen Unterstützungsbedarfen einen Zugang zur jeweiligen Freizeitaktivität zu eröffnen (siehe Kapitel 16.2).

Tabelle 27: Kategorisierung entlang der vier Typen im Handlungsfeld Freizeit

\begin{tabular}{|l|c|c|c|c|}
\hline \multirow{2}{*}{} & \multicolumn{4}{|c|}{ Gesamteinschätzung } \\
\cline { 2 - 5 } & voll ablehnend & $\begin{array}{l}\text { teilweise } \\
\text { ablehnend }\end{array}$ & $\begin{array}{l}\text { (noch) zurück- } \\
\text { haltend }\end{array}$ & offen \\
\hline Erlangen & $4,2 \%$ & $39,6 \%$ & $31,3 \%$ & $25 \%$ \\
\hline Rostock & $8,3 \%$ & $31,7 \%$ & $25 \%$ & $35 \%$ \\
\hline Schneverdingen & $11,9 \%$ & $21,4 \%$ & $59,5 \%$ & $7,1 \%$ \\
\hline
\end{tabular}




\begin{tabular}{|l|c|c|c|c|}
\hline $\begin{array}{l}\text { Schwäbisch } \\
\text { Gmünd }\end{array}$ & $3,4 \%$ & $33,9 \%$ & $32,2 \%$ & $30,5 \%$ \\
\hline VG Nieder-OIm & $0 \%$ & $19,2 \%$ & $42,3 \%$ & $38,5 \%$ \\
\hline$\varnothing$ & $5,6 \%$ & $29,2 \%$ & $38,1 \%$ & $27,2 \%$ \\
\hline
\end{tabular}

\subsection{Beispielinterviews}

Anhand zweier Interviews, die im Folgenden exemplarisch zusammengefasst sind, wird ein Einblick in das erhobene Material gegeben. Zudem wird so ein Stück weit transparent gemacht, wie bei der Kategorisierung der Interviews in die einzelnen Typen vorgegangen wurde respektive die Typen aus dem Material herausgebildet wurden. Das erste abgebildete Interview wurde mit einem/einer PrimärvertreterIn eines Gospelchores geführt.

Tabelle 28: Beispielinterview im Handlungsfeld Freizeit (1)

\begin{tabular}{|l|l|}
\hline Freizeitaktivität & Gospelchor \\
\hline Code & 5200 \\
\hline Kosten & 0 -120€ pro Jahr \\
\hline Nähe zum ÖPNV & 5 -10 Minuten Fußweg \\
\hline Faktor `Auto، & nicht notwendig \\
\hline Barrierefreiheit des Ortes & nicht barrierefrei \\
\hline Barrierefreiheit der Tätigkeit & $\begin{array}{l}\text { eingeschränkt barrierefrei für Menschen } \\
\text { mit Unterstützungsbedarfen in den } \\
\text { Bereichen Mobilität, Sehen oder Lesen/Ver- } \\
\text { stehen; nicht barrierefrei für Menschen mit } \\
\text { Unterstützungsbedarf im Bereich Hören }\end{array}$ \\
\hline Teilnahme von Menschen mit Behinderung & nein \\
\hline $\begin{array}{l}\text { Gründe dafür, dass keine Menschen mit } \\
\text { Behinderung an der Freizeitaktivität } \\
\text { teilnehmen }\end{array}$ & $\begin{array}{l}\text { »Wir sind ein sehr leistungsorientierter } \\
\text { Chor, da muss man sehr gut singen können. } \\
\text { Und das kann ich mir bei Menschen mit } \\
\text { Behinderung eher nicht vorstellen. « }\end{array}$ \\
\hline
\end{tabular}




\begin{tabular}{|c|c|}
\hline Möglichkeit der Teilnahme & eher nicht \\
\hline $\begin{array}{l}\text { Unterstützungsmöglichkeiten durch anwe- } \\
\text { sende Teilnehmende der Freizeitaktivität }\end{array}$ & weiß nicht \\
\hline $\begin{array}{l}\text { Möglichkeit des Mitbringens einer Begleit- } \\
\text { person }\end{array}$ & weiß nicht \\
\hline Teilnahme von Menschen mit Demenz & nein \\
\hline $\begin{array}{l}\text { Gründe dafür, dass keine Menschen mit } \\
\text { Demenz an der Freizeitaktivität teil- } \\
\text { nehmen }\end{array}$ & $\begin{array}{l}\text { - »Unsere Mitglieder sind zu jung." } \\
\text { - »Wenn die Texte vergessen werden, geht } \\
\text { es nicht mehr, da die Auftritte auswendig } \\
\text { stattfinden.» }\end{array}$ \\
\hline Möglichkeit der Teilnahme & $\begin{array}{l}\text { „Die Teilnahme von Menschen mit Demenz } \\
\text { geht glaube ich nicht. Eine Melodie zu } \\
\text { singen geht noch, aber Text zu lernen, ist } \\
\text { schwierig." }\end{array}$ \\
\hline $\begin{array}{l}\text { Unterstützungsmöglichkeiten durch anwe- } \\
\text { sende Teilnehmende der Freizeitaktivität }\end{array}$ & weiß nicht \\
\hline $\begin{array}{l}\text { Möglichkeit des Mitbringens einer Begleit- } \\
\text { person }\end{array}$ & weiß nicht \\
\hline $\begin{array}{l}\text { Teilnahme von Menschen mit Fluchtmigra- } \\
\text { tionshintergrund }\end{array}$ & nein \\
\hline $\begin{array}{l}\text { Gründe dafür, dass keine Menschen mit } \\
\text { Fluchtmigrationshintergrund an der Frei- } \\
\text { zeitaktivität teilnehmen }\end{array}$ & $\begin{array}{l}\text { - »Der Chor ist nicht für Menschen auf der } \\
\text { Durchreise. Aufgrund des großen Reper- } \\
\text { toires ist Beständigkeit notwendig." } \\
\text { - »Die Lieder haben Bezug zu christlichen } \\
\text { Themen. " } \\
\text { - »Früher hatten wir mal ein spezifisches } \\
\text { Angebot in einer Flüchtlingsunterkunft. } \\
\text { Menschen mit Fluchtmigrationshintergrund } \\
\text { haben ein anderes Verständnis von Pünkt- } \\
\text { lichkeit und kamen alle später. Und wer } \\
\text { nicht pünktlich kommt, fliegt. « } \\
\text { - »Die Sopran- und Altstimmen sind voll.» }\end{array}$ \\
\hline Möglichkeit der Teilnahme & »eher nein« \\
\hline
\end{tabular}




\begin{tabular}{|l|l|}
\hline $\begin{array}{l}\text { Unterstützungsmöglichkeiten durch anwe- } \\
\text { sende Teilnehmende der Freizeitaktivität }\end{array}$ & weiß nicht \\
\hline $\begin{array}{l}\text { Möglichkeit des Mitbringens einer Begleit- } \\
\text { person }\end{array}$ & weiß nicht \\
\hline $\begin{array}{l}\text { Ist Inklusion in Ihrer Freizeitaktivität } \\
\text { möglich? }\end{array}$ & $\begin{array}{l}\text { "Ja, wenn die Menschen singen können, } \\
\text { dann ja. « }\end{array}$ \\
\hline $\begin{array}{l}\text { mögliche Schwierigkeiten bei der Um- } \\
\text { setzung von Inklusion }\end{array}$ & "Die Menschen müssen singen können." \\
\hline $\begin{array}{l}\text { Maßnahmen/Vorhaben in Bezug auf } \\
\text { Inklusion }\end{array}$ & $\begin{array}{l}\text { "Es gab mal Gespräche, aber die Fahrstuhl- } \\
\text { nicht finanzierbar. « (2000 Euro pro Jahr) }\end{array}$ \\
\hline $\begin{array}{l}\text { (Veränderungs-)Wünsche an Politik und } \\
\text { Gesellschaft }\end{array}$ & $\begin{array}{l}\text { - Ich sehe die Schule als einen Anfang, } \\
\text { dort gibt es andere personelle Voraus- } \\
\text { setzungen. « } \\
- \text { »Das Umdenken muss in der ganzen } \\
\text { Gesellschaft geschehen, in den Köpfen der } \\
\text { Menschen überhaupt.» }\end{array}$ \\
\hline Sonstiges Erwähnenswertes & $\begin{array}{l}\text { Singen und Leistung stehen im Vorder- } \\
\text { grund }\end{array}$ \\
\hline vosamteinschätzung ablehnend \\
\hline
\end{tabular}

In Bezug auf das Interview mit dem/der PrimärvertreterIn können unterschiedliche Aspekte diskutiert werden. Beispielsweise ist es so, dass der Ort, an dem der Gospelchor probt, zwar ohne größere Einschränkungen erreicht werden kann (die nächste Haltestelle des öffentlichen Personennahverkehrs ist 5 bis 10 Minuten Fußweg entfernt, ein Auto ist nicht notwendig), der Ort selbst allerdings in keiner der untersuchten Dimensionen barrierefrei ist. So können Menschen mit Unterstützungsbedarfen aus den Bereichen Mobilität, Sehen, Hören und/oder Lesen/Verstehen zwar den Ort erreichen, sich aber dennoch nicht einschränkungslos und ohne fremde Hilfe am Ort selbst bewegen. Veränderungen werden im Kontext Mobilität gedacht, gelten aber als nicht umsetzbar, da die Kosten für Wartung und Unterhalt eines Aufzugs $\mathrm{zu}$ hoch seien. Teilhabebarrieren bleiben so bestehen. Die voll ablehnende Haltung des Primärvertreters/der Primärvertreterin zeigt sich unter anderem darin, dass kaum Möglichkeiten eingeräumt werden, Unterstützungen 
zu leisten. Der Gospelchor ist eher leistungsorientiert, was vor allem daraus hervorgeht, dass eine Teilnahme an die Bedingung geknüpft wird, auf hohem Niveau singen zu können. Es besteht also eher nicht die Möglichkeit, die Handlungspraxen oder strukturellen Rahmenbedingungen der Chorprobe $\mathrm{zu}$ verändern (beispielsweise Verlegung an einen barrierefreien Ort, Aufbringen der Kosten für den Aufzug), sodass eine Teilnahme für jene Personen ermöglicht würde, die bislang ausgeschlossen sind. In Bezug auf Menschen mit Behinderung, Menschen mit Demenz und Menschen mit Fluchtmigrationshintergrund werden negative Pauschalisierungen deutlich, die auf eine ablehnende Haltung der Interviewperson verweisen. So werden Menschen mit Demenz als nicht (mehr) dazu in der Lage konstruiert, Texte zu lernen, während Menschen mit Fluchtmigrationshintergrund pauschal als unpünktlich und unzuverlässig bezeichnet werden. Hinzu kommt, dass der Bereich Freizeit respektive die eigene Person nicht in erster Linie als dafür >zuständig، erachtet werden, sich dafür zu engagieren, Menschen, die von Ausschluss bedroht oder betroffen sind, Teilhabemöglichkeiten zu eröffnen. Inklusion wird zwar nicht grundsätzlich abgelehnt, aber die unmittelbare Betroffenheit oder Beteiligung der eigenen Person wird ausgeschlossen. Die Konstruktionen von negativer Andersartigkeit sowie die Zurückweisung von Personen, die unter Umständen einen je bestimmten Unterstützungsbedarf haben, sind schließlich maßgeblich mitentscheidend dafür, dass die Interviewperson als voll ablehnend typisiert wurde. Das folgende Interview mit einem/einer PrimärvertreterIn eines Selbstverteidigungsclubs bildet dagegen eine deutlich offenere Haltung ab.

Tabelle 29: Beispielinterview im Handlungsfeld Freizeit (2)

\begin{tabular}{|l|l|}
\hline Freizeitaktivität & Selbstverteidigungsclub \\
\hline Code & 7504 \\
\hline Kosten & $0-120 €$ pro Jahr \\
\hline Nähe zum ÖPNV & 5-10 Minuten Fußweg \\
\hline Faktor >Autor & nicht notwendig \\
\hline
\end{tabular}




\begin{tabular}{|c|c|}
\hline Barrierefreiheit des Ortes & $\begin{array}{l}\text { nicht barrierefrei im Bereich `Mobilität‘ } \\
\text { eingeschränkt barrierefrei im Bereich } \\
\text { 'Sehen` }\end{array}$ \\
\hline Barrierefreiheit der Tätigkeit & barrierefrei \\
\hline Teilnahme von Menschen mit Behinderung & $\begin{array}{l}\text { ja (8 Personen) } \\
\text { Teilnahme von Menschen mit Unterstüt- } \\
\text { zungsbedarf im Bereich >Hören< und im } \\
\text { Bereich >Lesen/Verstehenく }\end{array}$ \\
\hline Form der Teilnahme & bedingte Teilnahme \\
\hline Erfahrungen im Miteinander & "keine Probleme» \\
\hline Teilnahme von Menschen mit Demenz & nein \\
\hline $\begin{array}{l}\text { Gründe dafür, dass keine Menschen mit } \\
\text { Demenz an der Freizeitaktivität teil- } \\
\text { nehmen }\end{array}$ & »weiß ich nicht» \\
\hline Möglichkeit der Teilnahme & ja \\
\hline $\begin{array}{l}\text { Unterstützungsmöglichkeiten durch anwe- } \\
\text { sende Teilnehmende der Freizeitaktivität }\end{array}$ & ja \\
\hline $\begin{array}{l}\text { Möglichkeit des Mitbringens einer Begleit- } \\
\text { person }\end{array}$ & nein \\
\hline $\begin{array}{l}\text { Teilnahme von Menschen mit Fluchtmigra- } \\
\text { tionshintergrund }\end{array}$ & ja \\
\hline Form der Teilnahme & volle Teilnahme \\
\hline Erfahrungen im Miteinander & $\begin{array}{l}\text { - »keine Probleme« } \\
\text { - »Viele unterschiedliche Nationen (40) sind } \\
\text { im Verein vertreten.» }\end{array}$ \\
\hline $\begin{array}{l}\text { Ist Inklusion in Ihrer Freizeitaktivität } \\
\text { möglich? }\end{array}$ & ja \\
\hline $\begin{array}{l}\text { mögliche Schwierigkeiten bei der Um- } \\
\text { setzung von Inklusion }\end{array}$ & »Keine, wir leben Inklusion." \\
\hline $\begin{array}{l}\text { Maßnahmen/Vorhaben in Bezug auf } \\
\text { Inklusion }\end{array}$ & $\begin{array}{l}\text { »Wenn jemand teilnehmen möchte, wird } \\
\text { alles daran gesetzt, es zu ermöglichen.» }\end{array}$ \\
\hline
\end{tabular}




\begin{tabular}{|l|l|}
\hline $\begin{array}{l}\text { (Veränderungs-)Wünsche an Politik und } \\
\text { Gesellschaft }\end{array}$ & $\begin{array}{l}\text { »Die Zusammenarbeit mit der Stadt klappt } \\
\text { sehr gut.» }\end{array}$ \\
\hline Persönlicher Eindruck Interviewerln & sehr aufgeschlossen, wirkt engagiert \\
\hline Gesamteinschätzung & offen \\
\hline
\end{tabular}

Der Selbstverteidigungsclub ist fünf bis zehn Minuten von einer Haltestelle des öffentlichen Personennahverkehrs entfernt und es ist kein Auto notwendig, um ihn zu erreichen. Insofern entstehen bezüglich des Zugangs zum Ort keine Barrieren, allerdings kann eine Teilhabe durch die Ausgestaltung des Ortes selbst behindert werden, der nicht in allen untersuchten Dimensionen (uneingeschränkt) barrierefrei ist. Hierbei kommt allerdings die Aussage der Interviewperson zum Tragen: »Wenn jemand teilnehmen möchte, wird alles daran gesetzt, es zu ermöglichen«, woraus folgt, dass Barrieren als überwindbar eingeschätzt werden. An der Freizeitaktivität nehmen bereits einige Menschen mit Behinderung sowie Menschen mit Fluchtmigrationshintergrund teil und die Erfahrungen im Miteinander werden als unproblematisch beschrieben. Schwierigkeiten im Kontext Inklusion und Freizeit sieht die Interviewperson nicht, sie stellt im Gegenteil fest: »[W]ir leben Inklusion«. Die qualitativ-inhaltsanalytische Auswertung des Interviews resultierte schließlich in einer Typisierung als offen. PrimärvertreterInnen wie der/die hiesige, die einer Teilhabe von Menschen mit unterschiedlichen Unterstützungsbedarfen offen gegenüberstehen, können von gelingenden Teilhabeerfahrungen berichten und dadurch im jeweiligen Sozialraum zu einer Art MultiplikatorIn werden. Zudem zeigt sich hier ganz konkret, inwiefern gemeinsame Praxen, Austausch und Berührungspunkte zwischen Menschen mit (möglicherweise) diversen Unterstützungsbedarfen zu einer offeneren Haltung führen.

\subsection{Einzelaspekte}

Im Folgenden werden einzelne Analyseergebnisse dargelegt und diskutiert. Dabei wird immer Bezug auf die vier Typen genommen und die Ergebnisse in Relation zu diesen betrachtet. 


\subsubsection{Verteilung der Freizeitgruppen nach Tätigkeit}

Die untersuchten Freizeitaktivitäten verteilen sich auf vier übergeordnete Cluster: (1) Sport- und Spielgruppen, (2) Gemeinnützige Vereine, (3) Musik, Kunst, Kultur sowie (4) Garten, Wald, Natur. Die Clusterbildung beruht auf Ergebnissen der Studie »Inklusion. Zur Dekonstruktion von Diskursteilhabebarrieren im Kontext von Freizeit und Behinderung « und ist daran orientiert (Trescher 2015b, S. 51ff). Ziel der Erhebung war es, die zu untersuchenden Freizeitvereine und -gruppen möglichst gleichmäßig auf die vier Cluster zu verteilen, was im Gros gelang. Ein Blick auf das Verhältnis von Cluster und Typ zeigt, dass Freizeitaktivitäten aus dem Cluster Gemeinnützige Vereine am aufgeschlossensten sind (35,1 \% wurden als offen typisiert), wogegen Sport- und Spielgruppen deutlich zurückhaltender beziehungsweise ablehnender sind (18,3\% der diesbezüglichen Aktivitäten wurden als offen kategorisiert). Hier besteht also noch einiges Potenzial, Teilhabebeschränkungen für Menschen mit Behinderung, Menschen mit Fluchtmigrationshintergrund oder Menschen mit Demenz abzubauen.

Tabelle 30: Freizeittätigkeit

\begin{tabular}{|l|c|c|c|c|}
\hline & voll ablehnend & $\begin{array}{l}\text { teilweise } \\
\text { ablehnend }\end{array}$ & $\begin{array}{l}\text { (noch) zurück- } \\
\text { haltend }\end{array}$ & offen \\
\hline $\begin{array}{l}\text { Sport- und } \\
\text { Spielgruppen }\end{array}$ & $7,3 \%$ & $45,1 \%$ & $29,3 \%$ & $18,3 \%$ \\
\hline $\begin{array}{l}\text { Gemeinnützige } \\
\text { Vereine }\end{array}$ & $1,8 \%$ & $17,5 \%$ & $45,6 \%$ & $35,1 \%$ \\
\hline $\begin{array}{l}\text { Musik, Kunst, } \\
\text { Kultur }\end{array}$ & $7,8 \%$ & $26,6 \%$ & $35,9 \%$ & $29,7 \%$ \\
\hline $\begin{array}{l}\text { Garten, Wald, } \\
\text { Natur }\end{array}$ & $6,3 \%$ & $25 \%$ & $37,5 \%$ & $31,3 \%$ \\
\hline
\end{tabular}

\subsubsection{Kosten}

Die Kategorisierung der Kosten, die im Rahmen der untersuchten Freizeitaktivitäten anfallen, orientiert sich an Trescher (2015b, S. 81f). Hinter den Kostenschritten (a) o€-120€, (b) $>120 €-240 €$ und (c) $>240 €$ liegt die Idee, monatlich bis maximal zehn Euro (beziehungsweise maximal 20 Euro) für 
Freizeitaktivitäten aufzuwenden, was sich als lebenspraktisch sinnvolle Größe erwies. Die Mehrheit der untersuchten Freizeitaktivitäten (81,2 \%) kostet nach Angaben der interviewten PrimärvertreterInnen jährlich im Durchschnitt zwischen null und 120 Euro. Durchschnittlich 12,2 \% der untersuchten Freizeitaktivitäten bedarf eine jährliche Aufwendung zwischen 120 und 240 Euro, während lediglich durchschnittlich 6,6 \% der untersuchten Freizeitaktivitäten mehr als 240 Euro pro Jahr kosten ${ }^{10}$. Für Personen, die ein geringes oder ungeregeltes Einkommen haben, kann es zur Teilhabebarriere werden, wenn Freizeitaktivitäten teuer sind. In besonderer Weise betrifft dies beispielsweise Menschen, die in sogenannten Werkstätten für Menschen mit Behinderung (WfbM) arbeiten, da dort im Jahr 2017 der durchschnittliche monatliche Verdienst lediglich 213,69€ betrug (BAG WfbM 2019), wodurch kostenintensive Freizeitaktivitäten eher nicht ausgeübt werden können. Zum Zusammenhang zwischen Kosten und Typ der jeweiligen Freizeitaktivität kann gesagt werden, dass Freizeitaktivitäten, die weniger als $120 €$ pro Jahr kosten, am häufigsten als offen kategorisiert wurden (27,9\%). Höhere oder hohe Kosten werden also, neben dem größeren monetären Aufwand, auch dadurch zur Teilhabebarriere, dass entsprechende Interviewpersonen respektive Freizeitaktivitäten einer Teilhabe von Menschen mit unterschiedlichen Unterstützungsbedarfen weniger offen gegenüberstehen.

Tabelle 31: Kosten

\begin{tabular}{|l|c|c|c|c|}
\hline \multicolumn{5}{|c|}{ Durchschnittliche Kosten pro Jahr/Typ } \\
\hline & voll ablehnend & $\begin{array}{l}\text { teilweise ab- } \\
\text { lehnend }\end{array}$ & $\begin{array}{l}\text { (noch) zurück- } \\
\text { haltend }\end{array}$ & offen \\
\hline $0 €-120 €$ & $4,1 \%$ & $30,6 \%$ & $37,4 \%$ & $27,9 \%$ \\
\hline$>120 €-240 €$ & $13,6 \%$ & $40,9 \%$ & $27,3 \%$ & $18,2 \%$ \\
\hline$>240 €$ & $8,3 \%$ & $41,7 \%$ & $41,7 \%$ & $8,3 \%$ \\
\hline
\end{tabular}




\subsubsection{Entfernung zum öffentlichen Personennahverkehr, Abhängigkeit von Automobilität}

Die Entfernung zu Haltestellen des öffentlichen Personennahverkehrs ist in den Sozialräumen recht unterschiedlich verteilt. Dabei fällt auf, dass gerade in den beiden kleineren Sozialräumen Schneverdingen und der Verbandsgemeinde Nieder-Olm der Zugang zum öffentlichen Personennahverkehr weiter entfernt ist als in den größeren Sozialräumen. Durchschnittlich ist es in 61,4 \% der untersuchten Freizeitaktivitäten nicht notwendig, ein Auto zur Verfügung zu haben, um die jeweilige Freizeitaktivität zu erreichen. Davon weichen Schwäbisch Gmünd und insbesondere Schneverdingen ab, da in diesen beiden Sozialräumen der Anteil derjenigen Freizeitaktivitäten sehr hoch ist, in denen die Verfügbarkeit eines Autos Vorteile hätte (39 \% in Schwäbisch Gmünd, 54,1 \% in Schneverdingen) oder sogar zwingend notwendig ist (16,9\% in Schwäbisch Gmünd, $27 \%$ in Schneverdingen $)^{11}$. Erkennbar ist diesbezüglich zudem ein deutlicher Unterschied zwischen Großstadt und Kleinstadt/Gemeinde. Personen, die keinen Führerschein (mehr) haben oder denen kein Auto zur freien Verfügung steht, haben mitunter Schwierigkeiten jene Freizeitaktivitäten zu erreichen, wodurch sie in ihren Teilhabemöglichkeiten eingeschränkt oder von der Unterstützung anderer Personen abhängig gemacht werden. Eine solche Abhängigkeit von Automobilität kann also als Behinderungspraxis wirkmächtig werden. Gleichzeitig zeigt sich, dass die Ausgestaltung der jeweiligen Freizeitaktivität bezüglich ihrer Erreichbarkeit mit öffentlichen oder privaten Verkehrsmitteln keinen Einfluss auf die Typisierung hatte, da kein Zusammenhang zwischen diesen beiden Faktoren und dem jeweiligen Typ festgestellt werden konnte.

\subsubsection{Barrierefreiheit des Ortes und Barrierefreiheit der Tätigkeit}

Werden der Typ und die barrierefreie Ausgestaltung des Ortes, an dem die jeweilige Freizeitaktivität stattfindet, miteinander ins Verhältnis gesetzt, so zeigt sich sehr deutlich, dass, je offener der Typ, desto barrierefreier der Freizeitort in Bezug auf Mobilität, Sehen, Hören sowie Lesen/Verstehen ist. Selbiges gilt für die Barrierefreiheit der Tätigkeit. Barrierefreiheit hat also einen unmittelbaren Einfluss auf die Typisierung der Freizeitaktivität als voll/teilweise ablehnend, (noch) zurückhaltend oder offen, was im Umkehrschluss bedeutet, dass eine größere Sensibilität für Barrierefreiheit bei den 
PrimärvertreterInnen und den Teilnehmenden dazu führen kann, dass sie (a) mehr auf die barrierefreie Ausgestaltung von Ort und Tätigkeit achten und (b) schließlich einer Teilhabe von Menschen mit unterschiedlichen Unterstützungsbedarfen offener gegenüberstehen ${ }^{12}$.

Tabelle 32: Barrierefreiheit des Ortes und Barrierefreiheit der Tätigkeit

\begin{tabular}{|c|c|c|c|c|}
\hline & \multicolumn{4}{|c|}{ Der Ort ist barrierefrei in Bezug auf... } \\
\hline & Mobilität & Sehen & Hören & $\begin{array}{l}\text { Lesen/ } \\
\text { Verstehen }\end{array}$ \\
\hline voll ablehnend & $18,2 \%$ & $0 \%$ & $0 \%$ & $0 \%$ \\
\hline $\begin{array}{l}\text { teilweise ab- } \\
\text { lehnend }\end{array}$ & $42,3 \%$ & $18,2 \%$ & $27,3 \%$ & $40,3 \%$ \\
\hline $\begin{array}{l}\text { (noch) zurück- } \\
\text { haltend }\end{array}$ & $57,1 \%$ & $18,6 \%$ & $34,3 \%$ & $45,7 \%$ \\
\hline \multirow[t]{3}{*}{ offen } & $59 \%$ & $30,4 \%$ & $39,3 \%$ & $61,1 \%$ \\
\hline & \multicolumn{4}{|c|}{ Die Tätigkeit ist barrierefrei in Bezug auf... } \\
\hline & Mobilität & Sehen & Hören & $\begin{array}{l}\text { Lesen/ } \\
\text { Verstehen }\end{array}$ \\
\hline voll ablehnend & $8,3 \%$ & $8,3 \%$ & $8,3 \%$ & $0 \%$ \\
\hline $\begin{array}{l}\text { teilweise ab- } \\
\text { lehnend }\end{array}$ & $31,9 \%$ & $22,7 \%$ & $51,5 \%$ & $31,8 \%$ \\
\hline $\begin{array}{l}\text { (noch) zurück- } \\
\text { haltend }\end{array}$ & $51,9 \%$ & $41 \%$ & $62,2 \%$ & $45,3 \%$ \\
\hline offen & $65,1 \%$ & $55,9 \%$ & $61,9 \%$ & $68,9 \%$ \\
\hline
\end{tabular}

12 Es sei erneut auf die eingeschränkte Cültigkeit dieser Ergebnisse aufgrund subsumtionslogischer Schließungen hingewiesen (Kapitel 16.4.4). 
Zusätzlich sei angemerkt, dass die Barrierefreiheit des Gebäudes beziehungsweise Ortes, an dem die jeweilige untersuchte Freizeitaktivität stattfindet, und die (mögliche) barrierefreie Ausgestaltung der jeweiligen Tätigkeit nicht immer sinnhaft ineinandergreifen. Beispielsweise werden die Gebäude und Orte in Bezug auf Sehen und Hören deutlich seltener als barrierefrei eingeschätzt als die jeweilig darin ausgeübte Tätigkeit. Das bedeutet in der Konsequenz, dass für Menschen mit entsprechenden Unterstützungsbedarfen zwar eine Ausübung der jeweiligen Tätigkeit prinzipiell möglich ist, sie sich am Ort, an dem diese stattfindet, aber nicht unbedingt selbstständig zurechtfinden können. Dies ist durchaus problematisch und führt letzten Endes dazu, dass Teilhabemöglichkeiten aufgrund infrastruktureller Barrieren ungenutzt bleiben.

\subsection{Aktuelle Teilhabe von Menschen mit Behinderung, Menschen mit Fluchtmigrationshintergrund und Menschen mit Demenz}

Für das Handlungsfeld Freizeit sind im Folgenden die Anteile der untersuchten Freizeitaktivitäten dargelegt, in denen Menschen mit und ohne Behinderung, Menschen mit Fluchtmigrationshintergrund oder Menschen mit Demenz gemeinsam ihre Freizeit verbringen. Auch hier ist dies im Kontext Behinderung zusätzlich entlang der vier Beeinträchtigungsdimensionen Mobilität, Sehen, Hören und kognitive Beeinträchtigung dargelegt. Darüber hinaus wurde die Kategorie sandere Beeinträchtigungen` gebildet, unter der alle Personen gefasst werden, deren Unterstützungsbedarf nicht mit einer der anderen Kategorien übereinstimmt. Da es zwischen den fünf untersuchten Sozialräumen keine signifikanten Unterschiede gibt, wird hier auf eine sozialraumspezifische Darstellung der Ergebnisse verzichtet und ausschließlich das Gesamtergebnis abgebildet. 
Tabelle 33: Teilnehmende mit (unterschiedlichen) Beeinträchtigungen, Fluchtmigrationshintergrund oder Demenz

\begin{tabular}{|l|l|l|l|l|l|l|l|l|}
\hline & \multicolumn{7}{|c|}{ Teilnehmende mit... } \\
\cline { 2 - 9 } & \multirow{2}{*}{$\begin{array}{l}\text { Behin- } \\
\text { derung } \\
\text { gesamt }\end{array}$} & Mobilität & Sehen & Hören & kognitiv & andere & $\begin{array}{l}\text { Flucht- } \\
\text { migra- } \\
\text { tions- } \\
\text { hinter- } \\
\text { grund }\end{array}$ & Demenz \\
\cline { 2 - 9 } & $53,6 \%$ & $34,1 \%$ & $15,3 \%$ & $16,1 \%$ & $21,2 \%$ & $3,6 \%$ & $35,1 \%$ & $14 \%$ \\
\hline
\end{tabular}

Im Handlungsfeld Freizeit zeigt sich, dass durchschnittlich circa die Hälfte der untersuchten Freizeitangebote auch von Menschen mit Behinderung besucht wird (53,6\%). In Bezug auf die unterschiedlichen Beeinträchtigungsdimensionen bestehen teils deutliche Unterschiede zwischen dem Anteil der Freizeitangebote, in denen gemeinsame Aktivitäten erfolgen. Auch hier überwiegt der Bereich Mobilität deutlich vor den Bereichen Sehen, Hören und kognitive Beeinträchtigung, denn Menschen mit Mobilitätsbeeinträchtigungen sind in durchschnittlich 34,1 \% der untersuchten Freizeitangebote vertreten. Dem folgen Menschen mit kognitiven Beeinträchtigungen, die in 21,2 \% der untersuchten Freizeitangebote teilhaben. Den geringsten Anteil haben im Handlungsfeld Freizeit, wie auch in Bezug auf das Handlungsfeld Arbeit, Menschen mit Hörbeeinträchtigungen (16,1 \%) und Menschen mit Sehbeeinträchtigungen (15,3\%). Die Kategorie andere Beeinträchtigungen wurde in durchschnittlich 3,6 \% der untersuchten Freizeitaktivitäten gewählt. Es sei auch hier darauf hingewiesen, dass die Werte nicht ohne Weiteres aufsummiert werden können, da Mehrfachnennungen möglich waren. Insgesamt nehmen in durchschnittlich 35,1 \% aller Freizeitangebote, deren PrimärvertreterInnen interviewt wurden, auch Menschen mit Fluchtmigrationshintergrund teil. Das Handlungsfeld Freizeit ist dasjenige, in dem Angaben über eine Teilnahme von Menschen mit Demenz sinnhaft sind und deshalb einen detaillierten Einblick in aktuelle Teilhabepraxen von Menschen mit Demenz ermöglichen. An durchschnittlich 14 \% der untersuchten Freizeitangebote nehmen auch Menschen mit Demenz teil, was mit Blick auf die Differenzkategorien Behinderung (durchschnittlich 53,6 \%) und Fluchtmigration (durchschnittlich 35,1\%) der vergleichsweise geringste Anteil ist. 


\subsection{Form der Teilnahme}

Neben der Frage danach, ob derzeit Menschen mit unterschiedlichen Beeinträchtigungen, Fluchtmigrationshintergrund oder Demenz an der jeweiligen Freizeitaktivität teilnehmen, wurde auch untersucht, wie die Form der Teilnahme ausgestaltet ist. Im Folgenden ist dargelegt, wie groß die Anteile derjenigen untersuchten Freizeitaktivitäten und -gruppen sind, in denen unterschiedliche Personen voll, also ohne Einschränkungen, oder bedingt/ eingeschränkt teilnehmen, sowie, wie groß der Anteil der Sonderteilnahme ist. Da hier ebenfalls keine signifikanten Unterschiede zwischen den einzelnen untersuchten Sozialräumen bestehen, wird synonym zu der vorangegangenen Darstellung ausschließlich das Gesamtergebnis dargelegt.

Tabelle 34: Form der Teilnahme

\begin{tabular}{|l|l|c|}
\hline \multirow{4}{*}{ Menschen mit... } & Form der Teilnahme: & $\varnothing$ \\
\hline \multirow{4}{*}{ Sobilitätsbeeinträchtigung } & voll & $70,7 \%$ \\
\cline { 2 - 3 } & bedingt & $21 \%$ \\
\cline { 2 - 3 } & gesondert & $8,4 \%$ \\
\hline \multirow{5}{*}{ Hörbeeinträchtigung } & voll & $56,7 \%$ \\
\cline { 2 - 3 } & bedingt & $35,7 \%$ \\
\cline { 2 - 3 } & gesondert & $7,6 \%$ \\
\hline \multirow{5}{*}{ kognitiven Beeinträchtigungen } & voll & $76,9 \%$ \\
\cline { 2 - 3 } & bedingt & $20,8 \%$ \\
\cline { 2 - 3 } & gesondert & $2,2 \%$ \\
\hline & voll & $57,4 \%$ \\
\cline { 2 - 3 } & bedingt & $23,1 \%$ \\
\cline { 2 - 3 } & gesondert & $19,5 \%$ \\
\hline
\end{tabular}




\begin{tabular}{|l|l|c|}
\hline Menschen mit... & Form der Teilnahme: & $\varnothing$ \\
\hline \multirow{3}{*}{ Fluchtmigrationshintergrund } & voll & $81,3 \%$ \\
\cline { 2 - 3 } & bedingt & $11,4 \%$ \\
\cline { 2 - 3 } & gesondert & $7,3 \%$ \\
\hline \multirow{3}{*}{ Demenz } & voll & $55 \%$ \\
\cline { 2 - 3 } & bedingt & $16,7 \%$ \\
\cline { 2 - 3 } & gesondert & $28,3 \%$ \\
\hline
\end{tabular}

Im Gros zeigt sich, dass, sobald Menschen mit unterschiedlichen Beeinträchtigungen an Freizeitaktivitäten der Mehrheitsgesellschaft teilnehmen, ihre Teilnahme ohne weitergehende Einschränkungen erfolgt. Sonderteilnahmen finden selten statt und zeigen sich am häufigsten bei der Teilnahme von Menschen mit kognitiven Beeinträchtigungen (durchschnittlich 19,5\%). Diskutiert werden kann ebenfalls, dass auch der Anteil derjenigen Personen, die auf eingeschränkte Art und Weise an der jeweiligen Freizeitaktivität teilnehmen, nicht sehr hoch ist (durchschnittlich zwischen $20,8 \%$ in Bezug auf Menschen mit Hörbeeinträchtigungen und 35,7 \% in Bezug auf Menschen mit Sehbeeinträchtigungen). Dies kann einerseits ein Anzeichen dafür sein, dass die jeweiligen Freizeitgruppen nicht sehr flexibel in ihrer Ausgestaltung sind. Demgegenüber ist es andererseits jedoch auch möglich, dass gerade eine flexible Gestaltung der jeweiligen Freizeitaktivität dazu beiträgt, dass Menschen mit unterschiedlichen Beeinträchtigungen eine volle, uneingeschränkte Teilnahme möglich ist. Menschen mit Fluchtmigrationshintergrund nehmen in durchschnittlich 81,3\% der untersuchten Freizeitgruppen ohne weitere Einschränkungen an der jeweiligen Freizeitaktivität teil. Eine bedingte Teilnahme besteht in durchschnittlich 11,4 \% der Fälle, während durchschnittlich 7,3\% dieser als Sonderteilnahme eingeschätzt wurden. In Bezug auf die Form der Teilnahme von Menschen mit Demenz ist auffällig, dass hier sehr viel häufiger eine Sonderteilnahme stattfindet, als dies in Bezug auf Menschen mit unterschiedlichen Beeinträchtigungen und Menschen mit Fluchtmigrationshintergrund der Fall ist. Durchschnittlich 28,3\% der Teilnehmenden mit Demenz erleben eine Sonderteilnahme. Dies kann ambivalent gelesen werden, so ist eine Sonderteilnahme zwar Ausdruck be- 
sondernder Praxen, gleichzeitig werden dadurch jedoch Teilhabemöglichkeiten eröffnet beziehungsweise weiterhin genutzt.

\subsection{Erfahrungen im Miteinander}

\subsubsection{Erfahrungen im Miteinander mit Teilnehmenden mit Behinderung}

Im Handlungsfeld Freizeit sind die Erfahrungen derjenigen Interviewpersonen, die Freizeitaktivitäten gemeinsam mit Menschen mit Behinderung nachgehen, meist positiv. Eine Interviewperson sagt beispielsweise: »Also, meine Erfahrungen sind da sehr gut. Wir unterstützen uns alle gegenseitig und erleben viel Freude miteinander. Insgesamt herrscht hier eine sehr familiäre Atmosphäre« (6407). Diese wertschätzende Verbindung stellt auch diese Interviewperson fest, die sagt: $» D i e$ Kinder mit Behinderung werden bei uns gut aufgenommen. Ein Kind hat in der Gruppe sein erstes Wort gesprochen und eine große Entwicklung gemacht. Insgesamt haben wir gute Beziehungen zueinander, besonders mit den Eltern, da gemeinsame Ausflüge gemacht werden - das verbindet« (3600). Demgegenüber gibt es immer wieder Interviewpersonen, die weniger positive Erfahrungen gemacht haben. Eine Interviewperson sagt beispielsweise, die Erfahrungen seien gut, allerdings nur »dank der großen Rücksicht der anderen Teilnehmer« (6383). Menschen mit Behinderung werden hier also als Personen konstruiert, die einer Art Sonderbehandlung bedürfen. Zudem wird dadurch eine Grenze zwischen Teilnehmenden mit Behinderung und den »anderen Teilnehmer[n] « gezogen, die annehmbar auch als soziale Grenze wirksam wird, es also nicht unbedingt zu gemeinsamen Praxen und/oder tiefergehenden sozialen Kontakten von Menschen mit und Menschen ohne Behinderung kommt (gegebenenfalls auch über die konkrete Freizeitaktivität hinaus). Diese Manifestation einer sozialen Grenze wird ebenfalls in der Aussage dieser Interviewperson deutlich: »Bei uns im Sportverein sind die nicht auffällig und froh, dass sie sich mit normalen Menschen bewegen können und dürfen. Der Normalsportler bei uns ist auch sehr offen und hilfsbereit" (9611). Die soziale Trennung wird in dieser Darstellung an der Unterscheidung zwischen dem sogenannten »Normalsportler« und SportlerInnen mit Behinderung ganz besonders deutlich. Zudem zeigt sich in der Aussage der Interviewperson, dass Menschen mit Behinderung in gewisser Weise als bemitleidenswert konstruiert werden und so, als sollten sie sich dankbar dafür 
zeigen, dass sie gemeinsamen Aktivitäten mit Menschen ohne Behinderung nachgehen »dürfen«. Die Interviewperson selbst respektive die von ihr vertretene Freizeitaktivität tritt dadurch als eine Art caritativer Fürsprecher für Menschen mit Behinderung auf, was hoch ambivalent ist: Menschen mit Behinderung werden dadurch zwar ausdrücklich adressiert und sind im Verein willkommen. Demgegenüber werden jedoch so gut wie keine Möglichkeiten eingeräumt, den Status `Behinderung abzulegen und in der Rolle als »Normalsportler« an Freizeitaktivitäten teilzunehmen. Andere Interviewpersonen dagegen berichten davon, dass zwar Menschen mit Behinderung an den von ihnen repräsentierten Freizeitaktivitäten teilnehmen, es allerdings zu keinem Kontakt zwischen Menschen mit und Menschen ohne Behinderung käme. Sie sagt: "Man grüßt sich, aber ansonsten gibt es da keinen Kontakt, denn die Behinderten spielen nur mit sich Tennis« (4575). Hieran zeigt sich, inwiefern sich Ausschlusspraxen auch dann vollziehen, wenn vermeintlich alle Voraussetzungen dafür erfüllt sind, gemeinsam Freizeitaktivitäten nachzugehen. Es ist die Frage zu stellen, ob Lebenspraxen bereits dann als sinklusiv bezeichnet werden können, wenn Menschen mit und Menschen ohne Behinderung am selben Ort dieselbe Freizeitaktivität ausüben, oder ob Inklusion nicht viel eher der (tatsächlich) gemeinsamen Praxis bedarf. Eine weitere Herausforderung inklusiver Freizeitaktivitäten besteht darin, dass beiderseits Ängste und Vorbehalte bestehen, sich in gemeinsamen Freizeitaktivitäten zu engagieren. Eine Interviewperson berichtet zwar davon, dass Menschen mit Behinderung an der von ihr vertretenen Freizeitaktivität teilnehmen. Sie sagt allerdings auch: »Man muss sie [gemeint sind Menschen mit Behinderung] überzeugen, dass sie mitmachen sollen und auch dürfen« (3466). Inklusion heißt also auch, Ängste zu überwinden und Wege der Beteiligung zu finden. Schließlich gibt es einige Interviewpersonen, die selbst beeinträchtigt sind und deshalb die Frage nach den Erfahrungen im Miteinander aus der Perspektive eines Menschen mit Behinderung schildern können. Dabei wird teils von Diskriminierungserfahrungen berichtet wird, wie sie beispielsweise diese Interviewperson erlebt hat: »Ich bin selbst das behinderte Mitglied und manchmal ist meine exponierte Rolle im Vorstand schwierig, da körperliche Beeinträchtigung oft mit geistiger Beeinträchtigung gleichgesetzt wird. Teilweise habe ich da schon Angriffe auf meine Person erlebt. Zum Beispiel musste ich mir anhören, so jemand wie ich könne doch nicht den Verein führen« (6298). Menschen mit Behinderung erfahren also unabhängig ihrer Rolle, als Teilnehmende oder als Leitungen, 
dass ihnen andere Personen mit Vorbehalten begegnen, wodurch schließlich ihre Teilhabemöglichkeiten eingeschränkt werden. In Bezug auf die zitierte Interviewperson bleibt zu diskutieren, dass sie ihre eigene Diskriminierung anprangert, allerdings selbst eine Grenze zwischen körperlicher und kognitiver Beeinträchtigung zieht, wodurch sie Menschen mit geistiger Behinderung diskriminiert. Immer wieder, auch an dieser Stelle, kristallisiert sich heraus, inwiefern geistige Behinderung sozusagen als Inklusionsgrenze konstruiert wird, was wiederum dazu führt, dass jene Personen drohen, zu >InklusionsverliererInnen`(Becker 2016b, S. 33; Schäper 2015) zu werden.

\subsubsection{Erfahrungen im Miteinander mit Teilnehmenden mit Fluchtmigrationshintergrund}

Die Interviewpersonen, die in den von ihnen vertretenen Freizeitaktivitäten Teilnehmende mit Fluchtmigrationshintergrund haben, berichten größtenteils von guten Erfahrungen, dass keine Probleme bestehen oder dass es völlig unerheblich sei, ob Teilnehmende fluchtmigriert sind oder nicht. Dies beeinflusse ihre Teilhabe in keiner Weise, wie beispielsweise diese Interviewperson sagt: »Es macht wirklich keinen Unterschied, ob die jetzt geflüchtet sind oder nicht. Ob mit Fluchtmigration oder ohne, bei uns sind alle willkommen« (1513). Eine Erfahrung, die viele Interviewpersonen teilen, betrifft Sprachbarrieren aufseiten der Teilnehmenden mit Fluchtmigrationshintergrund, die die Kommunikation aller Beteiligten behindern könne. Der Umgang mit kommunikativen Schwierigkeiten gestaltet sich teils unterschiedlich. Einige Interviewpersonen nehmen die Sprachbarrieren der Teilnehmenden mit Fluchtmigrationshintergrund als eher hinderlich wahr, während andere Strategien entwickelt haben, diese unkompliziert zu umgehen. Davon berichtet beispielsweise diese Interviewperson: „Bei uns klappt alles super. Es gibt höchstens mal Sprachbarrieren, aber dann spricht man langsam und verständigt sich mit Händen und Füßen« (5331). Von einer solchen Praxis des Aushandelns berichtet auch diese Interviewperson: »Also, bei uns geht's immer recht lustig zu. Zum Beispiel hat einer der Geflüchteten beim Pflanzen einen Baum verkehrt herum in die Erde gesetzt und einer von unseren anderen Teilnehmern hat ihm dann [im Dialekt, der im Sozialraum gesprochen wird] zugerufen, dass er das so nicht machen könne. Da hat der erstmal verdutzt geguckt, aber hat wohl verstanden, was da schiefgelaufen ist, und dann mussten alle lachen " (3105). Menschen mit und ohne Fluchtmigrationshintergrund begegnen sich hier ohne besondere Vorbehalte und 
ohne in besonderer Weise aufeinander Rücksicht nehmend, wodurch, auch wenn unter Umständen teils ein eher >flapsiger Umgangston herrscht, eine angenehme, wertschätzende Atmosphäre geschaffen wird. Darüber hinaus thematisieren einige Interviewpersonen strukturelle Probleme, die die Teilhabe von Menschen mit Fluchtmigrationshintergrund erschweren. Eine Erfahrung dabei sind finanzielle Hürden, da die Zuschüsse beschränkt seien, die einige Interviewpersonen als notwendig erachten, um Menschen mit Fluchtmigrationshintergrund in die von ihnen repräsentierte Freizeitaktivität einzubeziehen. Eine Interviewperson sagt: »Zwischenmenschlich läuft es bei uns sehr gut. Ein Problem ist eher die Finanzierung, weil wir immer extra Gelder beantragen müssen und die bekommen wir nicht zuverlässig« (4609). In Bezug darauf kann problematisiert werden, dass Menschen mit Fluchtmigrationshintergrund so als Belastung konstruiert werden und als Personen, die nicht als gleichwertige Mitglieder an der jeweiligen Freizeitaktivität teilhaben können. Ebenfalls im Zusammenhang mit Kosten wird auf das Strukturproblem aufmerksam gemacht, dass vielen Menschen mit Fluchtmigrationshintergrund nur sehr geringe finanzielle Mittel zur Verfügung stehen und sie aufgrund dessen nur eingeschränkt an Freizeitaktivitäten in ihrem Sozialraum teilhaben können. In diesem Zusammenhang berichtet eine Interviewperson: »Bei uns lief alles gut, aber dann blieben die Zahlungen aus, da es keine staatliche Übernahme der Kosten gab. Und deshalb kommen die meisten jetzt nicht mehr« (6383). Einige Interviewpersonen berichten, sie hätten die Erfahrung gemacht, dass viele der teilnehmenden Menschen mit Fluchtmigrationshintergrund unzuverlässig seien, was Terminabsprachen oder andere Vereinbarungen angehe. Eine Interviewperson sagt beispielsweise diesbezüglich: »Die Teilnehmer waren extrem unzuverlässig, gerade mit den Afrikanern war das sehr schwierig. Dabei ist Pünktlichkeit bei uns das $A$ und $O$, aber durch die andere Lebensweise sind viele von denen nicht regelmäßig und pünktlich erschienen, was uns wirklich vor Schwierigkeiten gestellt hat« (7501). Problematisiert werden kann diesbezüglich, dass solche negativen Erfahrungen zu Vorbehalten gegenüber einer Teilhabe von Menschen mit Fluchtmigrationshintergrund führen, die wiederum, zumindest potenziell, in Ausschluss resultieren. Vermehrt schildern Interviewpersonen die Erfahrung, dass Kinder deutlich positivere Erfahrungen machen bei gemeinsamen Freizeitaktivitäten mit Kindern mit Fluchtmigrationshintergrund als dies aufseiten der Erwachsenen der Fall ist. Eine Interviewperson berichtet beispielsweise: »Die Kinder sind aufge- 
schlossen und haben keine Berührungsängste, sondern eher die Älteren und Erwachsenen « (2698). Eine andere Interviewperson sagt: »Besonders die Eltern verlieren sich in Problemen. Mit den Kindern sind unsere gemeinsamen Unternehmungen immer sehr schön« (1481). Es hat den Anschein, als knüpften Kinder ohne größere Vorbehalte Sozialbeziehungen und seien weniger durch Vorurteile geprägt.

\subsubsection{Erfahrungen im Miteinander mit Teilnehmenden mit Demenz}

Die Interviewpersonen, die gemeinsam mit Menschen mit Demenz an der von ihnen vertretenen Freizeitaktivität teilnehmen, berichten im Gros von positiven Erfahrungen oder sagen, der Umgang sei unproblematisch. Teils berichten die Interviewpersonen von Veränderungen bei Teilnehmenden, die als demenziell erkrankt bezeichnet werden, und bezeichnen diese als »verwirrt, penetrant, nervig" (2915). Diese Interviewperson betont: "Das muss man aushalten können « (2915). Andere Interviewpersonen sehen das ähnlich und berichten aber teils von Bewältigungsstrategien, die sie im Umgang mit Teilnehmenden mit Demenz entwickelt haben. Eine Interviewperson sagt: "Das kann manchmal anstrengend sein. Wir besprechen das dann aber immer gemeinsam und schauen, wer mit der Person zusammengehen kann. Die eine Frau mit Demenz ist seit Anfang an dabei, seit der Gründung des Vereins vor 35 Jahren, und sie möchte unbedingt weiter teilnehmen und das wird ihr dann auch ermöglicht« (8578). Auch eine andere Interviewperson berichtet, dass anfangs für Teilnehmende mit Demenz noch Sonderregelungen gefunden werden, allerdings sei es auch so: »Nach der Krankheit kommen die Leute nicht mehr" (2005). Dieser Aspekt ist problematisch, da der Diagnosestellung oder, sobald sich schwerere Einschränkungen bemerkbar machen, ganz offenbar unweigerlich Ausschlusspraxen folgen. Menschen mit Demenz sind dadurch in der Mehrheitsgesellschaft weniger handlungsmächtig, obwohl sie dieser, davon ist im Regelfall auszugehen, bislang immer angehörten und dort aktiv waren. Menschen mit Demenz drohen dadurch zu vereinsamen, was insbesondere dahingehend bedacht werden muss, dass ältere Menschen (und dazu gehören zumeist Menschen mit Demenz) oftmals dem Risiko stark verminderter Sozialkontakte ausgesetzt sind (siehe dazu u.a. Gronemeyer 2013, S. 40; Schuhmacher 2018, S. 146f; zur Problematik von Demenz und Einsamkeit siehe Trescher 2013, S. 278f). 


\subsection{Begründungsfiguren der Ablehnung}

\subsubsection{Ablehnung von Menschen mit Behinderung}

Die Interviewpersonen nennen unterschiedliche Gründe dafür, dass (bislang) keine Menschen mit Behinderung an den von ihnen repräsentierten Freizeitaktivitäten teilnehmen. Interessant ist, dass hier ganz ähnliche Begründungsfiguren herangezogen werden, wie sie im Handlungsfeld Arbeit festgestellt wurden (siehe Kapitel 16.7).

\section{Mangelnde Leistungsfähigkeit}

Immer wieder kommt es vor, dass Interviewpersonen pauschal annehmen, Menschen mit Behinderung seien aufgrund mangelnder Leistung nicht dazu in der Lage an den jeweiligen Freizeitaktivitäten teilzuhaben. Eine Interviewperson sagt beispielsweise: »Der Sport ist schwer mit Behinderung auszuführen, man braucht ein gutes Körpergefühl« (4928). Menschen mit Behinderung werden dadurch umfassend als Personen angenommen, die ein sschlechtes Körpergefühk haben, weshalb eine Teilhabe grundsätzlich ausgeschlossen wird. Andere Interviewpersonen schließen eine Teilhabe von Menschen mit Behinderung zwar nicht grundsätzlich aus, befürchten allerdings, dass die Gruppendynamik gestört würde, wenn Menschen mit Behinderung teilnehmen, da diese nicht den Leistungsansprüchen genügen. Eine Interviewperson sagt diesbezüglich: »Es muss halt einfach Ballgefühl da sein. Und Menschen mit Behinderung sind nicht in der Lage so zu spielen, dass es Spaß macht«(3000). Zudem werden Menschen mit Behinderung hier als Last konstruiert, die die Freude an der Freizeitaktivität verderben. Insgesamt argumentieren viele Interviewpersonen, Menschen mit Behinderung seien in ihrer körperlichen Leistungsfähigkeit zu eingeschränkt, um - in ihren Augen adäquat - an der jeweiligen Freizeitaktivität teilhaben zu können. Dies findet sich nicht nur in sportlichen, sondern auch in musikalischen Aktivitäten. Ein Beispiel dafür ist die Aussage der folgenden Interviewperson: »Bei unseren Auftritten muss man 45 Minuten am Stück stehen und schunkeln können, das ist sehr anstrengend « (6801). Ganz grundsätzlich stellt sich die Frage, warum Leistung, die von den Interviewpersonen immer wieder thematisiert wird, überhaupt im Handlungsfeld Freizeit eine so große Rolle spielt. Gerade im Freizeitbereich besteht die Möglichkeit zu Praxen, die nicht primär von Leistung und Konkurrenz geprägt sind (wie es im Bereich Arbeit zumeist der Fall ist). Im Kontext Freizeit können sich 
Menschen herrschaftsfrei und gleichberechtigt begegnen (Trescher 2015b, S. 34). Darüber hinaus finden sich immer wieder Aussagen, die als behindertenfeindlich verstanden werden können. Eine Interviewperson sagt beispielsweise: „Warum sollte mit Menschen mit Behinderung Schachspielen funktionieren? Die haben doch echt andere Probleme« (4647). In Aussagen wie diesen werden stark ablehnende Haltungen deutlich. Schließlich gibt es vereinzelt auch diejenigen Interviewpersonen, die eine Teilnahme von Menschen mit Behinderung an der von ihnen repräsentierten Freizeitaktivität kategorial ausschließen. Dies wird größtenteils nicht weiter begründet und äußert sich in Aussagen wie der der folgenden Interviewperson: »Wir wollen keine« (2396).

\section{Keine Anfragen}

Einige Interviewpersonen zeigen sich prinzipiell (mehr oder weniger) offen, Teilhabemöglichkeiten für Menschen mit Behinderung zu bereiten, problematisieren jedoch, dass bislang noch niemand ein entsprechendes Interesse geäußert hätte. Eine Interviewperson sagt diesbezüglich: »Ich kann mir absolut nicht vorstellen, wieso niemand kommt. An sich sind wir da sehr aufgeschlossen« (5017). Dies verweist auf das übergeordnete Strukturproblem, dass es an einer Verbindung von aufgeschlossenen Freizeitaktivitäten und interessierten Menschen mit Behinderung fehlt. Es stellt sich die Frage, wie zwischen diesen beiden Beteiligten vermittelt werden kann, die wechselseitig Interesse haben, jedoch nicht zueinander finden (siehe dazu auch Trescher 2015b, S. 312ff).

\section{Mangelnde Barrierefreiheit}

Vereinzelt benennen die Interviewpersonen mangelnde Barrierefreiheit als Problematik, die eine Teilhabe von Menschen mit Behinderung an der von ihnen repräsentierten Freizeitaktivität erschwert. Hervorgehoben werden muss dabei, dass sich Barrierefreiheit beziehungsweise die Problematisierung mangelnder Barrierefreiheit grundsätzlich immer auf die Örtlichkeit bezieht, an der die Freizeitaktivität stattfindet. Eine gegebenenfalls zu geringe Barrierefreiheit der Tätigkeit an sich wird nicht angesprochen, woraus folgt, dass auch hier der Blick eher vom als sbehindert adressierten Individuum ausgeht, das nicht fähig ist, die je bestimmte Tätigkeit auszuüben. Eine Veränderung der Tätigkeit wird demgegenüber größtenteils nicht als Möglichkeit eingeräumt. Darüber hinaus kann problematisiert werden, dass 
Barrierefreiheit primär als barrierefrei für Menschen mit Mobilitätsbeeinträchtigungen verstanden wird und andere Beeinträchtigungsdimensionen nicht bedacht werden. Dies zeigt erneut, wie sehr Barrierefreiheit nach wie vor über Mobilitätsbeeinträchtigung konstruiert wird, was sich im allgegenwärtigen Symbol für Barrierefreiheit - ein Rollstuhlpiktogramm - widerspiegelt und wechselseitig dadurch reproduziert wird (Trescher 2018c, S. 18).

\section{Verweis auf gesonderte Angebote für Menschen mit Behinderung} Interviewpersonen berichten teils, dass Sonderstrukturen für Menschen mit Behinderung vorhanden sind, in denen diese - exklusiv - Freizeitaktivitäten nachgehen können. Darin sehen sie einen Grund, weshalb keine Menschen mit Behinderung an der von ihnen vertretenen Freizeitaktivität teilnehmen. Eine Interviewperson sagt: »Es gibt doch Sportangebote in den Wohnheimen. Bei uns müsste dann ja ein Betreuer mitkommen« (4003). In Aussagen wie diesen wird deutlich, dass exklusive Betreuungsstrukturen in der Mehrheitsgesellschaft als sinnvolle und positiv bewertete Institutionen verstanden werden. Reproduziert wird dadurch allerdings auch ein Bild von Menschen mit Behinderung als grundsätzlich betreuungsbedürftig (wie in der Aussage der oben zitierten Interviewperson deutlich wird, die davon ausgeht, dass Menschen mit Behinderung nicht ohne Betreuung an der Freizeitaktivität teilhaben können). Dies steht einer selbstermächtigten Freizeitgestaltung in mehrheitsgesellschaftlichen Freizeitpraxen entgegen. Problematisch ist, dass dies mit dazu führt, dass Teilhabemöglichkeiten von Menschen mit Behinderung stark eingeschränkt beziehungsweise gar nicht erst eröffnet werden. Eine andere Interviewperson stellt fest: "Sportvereine sind nicht der erste Ansprechpartner, eher Inklusionsgruppen« (8652). Sie sieht also die Zuständigkeit weder bei sich selbst respektive der von ihr vertretenen Freizeitaktivität noch bei geschlossenen, behindertenspezifischen Angeboten, sondern in sogenannten »Inklusionsgruppen«. Dass diese außerhalb routinemäßiger Freizeitangebote konstruiert werden, ist ein Strukturproblem, das letztlich in Ausschluss resultiert, da sozusagen die Zuständigkeit wechselseitig verschoben wird - vertreten doch beispielsweise MitarbeiterInnen von Behindertenhilfeträgern oftmals den Standpunkt, der Anstoß zu >inklusiver Freizeit müsse von mehrheitsgesellschaftlichen Vereinen und Gruppen ausgehen (Trescher 2015b, S. 234f). 


\section{Vorbehalte und Ängste aufseiten von Menschen mit Behinderung} Ein weiterer Grund dafür, dass keine Menschen mit Behinderung an der von der jeweiligen Interviewperson vertretenen Freizeitaktivität teilnehmen, wird von einigen Personen darin gesehen, dass Menschen mit Behinderung Vorbehalte und Ängste gegenüber einer Teilhabe an mehrheitsgesellschaftlichen Freizeitpraxen haben. Eine Interviewperson sagt: »Menschen mit Behinderungen glauben, dass sie ohnehin nicht am gesellschaftlichen Leben teilhaben können und kommen deshalb nicht" (3108). Eine andere Interviewperson sagt: »Ich könnte mir vorstellen, dass sie [gemeint sind Menschen mit Behinderung] vielleicht Angst haben, hierher zu kommen. Das Leben ist schon schwierig genug für sie, darum sollte man ihnen das Leben so einfach wie möglich machen« (7292). In diesen Äußerungen wird ein paternalistischer Blick auf Menschen mit Behinderung deutlich, der diese als schutz- und betreuungsbedürftig konstruiert und mit dem wiederum Ausschlusspraxen begründet werden, was problematisch ist, da Interviewpersonen, die solche oder ähnliche Begründungen für eine Nicht-Teilnahme von Menschen mit Behinderung äußern, es eher nicht als ihre Aufgabe ansehen, Teilhabemöglichkeiten für Menschen mit Behinderung zu bereiten.

\section{Vorbehalte aufseiten der Teilnehmenden}

Gegenüber angenommenen Vorbehalten aufseiten der potenziell teilnehmenden Menschen mit Behinderung sehen einige Interviewpersonen Vorbehalte aufseiten der Teilnehmenden an der von ihnen vertretenen Freizeitaktivität und ziehen diese als Begründung dafür heran, weshalb keine Menschen mit Behinderung an dieser teilnehmen. Eine Interviewperson äußert sich diesbezüglich wie folgt: »Behinderte würden hier bei uns gegen Wände laufen. Die würden sicher unbewusst gemobbt werden« (8776). Indem die Interviewperson betont, das Mobbing von Menschen mit Behinderung würde »unbewusst« erfolgen, entschuldigt sie ein Stück weit das Verhalten der Teilnehmenden und verschleiert die Ablehnung als nicht vorsätzlich. Dass daraus dennoch massive Teilhabebarrieren hervorgehen, reflektiert die Interviewperson nicht. Interviewpersonen dagegen, die Menschen mit Behinderung als defizitär konstruieren, gehen oftmals davon aus, dass ihnen eine entsprechende Expertise fehlt, diese in die jeweilige Freizeitaktivität miteinzubeziehen. Eine Interviewperson sagt beispielsweise: »Wir machen keine Therapie« (7855). Vorbehalte zeigen sich also auch darin, dass einige Interviewpersonen Menschen mit Behinderung als Objekt je be- 
stimmter Professionen medizinischer und/oder therapeutischer Provenienz verstehen und sich selbst folglich nicht zzuständig، fühlen. Ähnlich protektive Ansichten finden sich in einigen Aussagen von Interviewpersonen, die Menschen mit Behinderung pauschal als schutzbedürftig konstruieren. Eine Interviewperson sagt: »Ich denke, bei uns kann die Aufsichtspflicht nicht garantiert werden. Außerdem finden manche unserer Auftritte auch nachts statt, da ist es für Behinderte ja eher schwierig, mitzumachen « (2707). Diese Interviewperson geht (pauschal) davon aus, dass Menschen mit Behinderung beaufsichtigt werden müssen und keine Termine in den Abend- und Nachtstunden wahrnehmen können, was nicht nur protektiv, sondern auch infantilisierend ist. Menschen mit Behinderung werden wie kleine Kinder adressiert, die nicht auf sich selbst achten können und frühzeitig zu Bett gehen müssen. Demgegenüber kann argumentiert werden, dass die Interviewperson mit der Einschätzung nicht ganz falsch liegt, es sei für Menschen mit Behinderung schwierig, sich abends und nachts an Auftritten zu beteiligen. Denn gerade für institutionalisiert lebende Menschen mit (oftmals geistiger) Behinderung ist es schwierig, am Abend und nachts eine Betreuung zu organisieren (Trescher 2015b, S. 240, 2017f, S. 188). Dies ist ein Strukturproblem, dessen Bearbeitung eher auf der Seite der sogenannten Behindertenhilfe liegt als auf der der Mehrheitsgesellschaft.

\subsubsection{Ablehnung von Menschen mit Fluchtmigrationshintergrund}

Die interviewten VertreterInnen von Freizeitvereinen und -gruppen in den Sozialräumen begründen es ganz unterschiedlich, dass (bislang) keine Menschen mit Fluchtmigrationshintergrund an der von ihnen repräsentierten Freizeitaktivität teilnehmen. Die Bandbreite der Begründungen ist im Folgenden zusammenfassend dargestellt.

\section{(Beiderseitige) Ablehnung}

Ein Teil der Interviewpersonen lehnt es pauschal ab, dass Menschen mit Fluchtmigrationshintergrund an der von ihnen vertretenen Freizeitaktivität teilnehmen. Eine Interviewperson sagt beispielsweise: »Der Verein will das nicht, denn die sehen darin eine Gefahr « (5363). Diffuse Ängste und Vorbehalte spielen dabei sicherlich eine große Rolle, wird die vermeintliche "Gefahr« doch nicht weitergehend ausdifferenziert beziehungsweise näher benannt. Menschen, die derart starke Vorbehalte haben, werden wohl kaum durch (bewusstseins-)bildende Maßnahmen oder das Schaffen von 
Kontaktmöglichkeiten zu Menschen mit Fluchtmigrationshintergrund beeinflusst werden können. Dies trifft unter anderem auch auf diese Interviewperson zu: »Es gab über das Thema große Diskussionen. Insgesamt ist es so, dass die Vereinsmitglieder gegenüber Flüchtlingen noch mehr Vorbehalte haben als gegenüber Behinderten« (6298). Hier werden Menschen mit Fluchtmigrationshintergrund von Menschen mit Behinderung unterschieden, wobei Letztere in gewisser Weise als >das kleinere Übel dargestellt werden. Andere Interviewpersonen zeigen sich erleichtert darüber, dass sie bislang nicht von Menschen mit Fluchtmigrationshintergrund angesprochen wurden, wie beispielsweise diese Interviewperson: »Wir sind davon zum Glück noch nicht betroffen, dass irgendwelche Anfragen von Flüchtlingen kamen« (9783). Demgegenüber äußern einige Interviewpersonen, sie hätten Ablehnung durch Menschen mit Fluchtmigrationshintergrund erfahren. Dies wird zum Teil mit unpopulären Freizeitaktivitäten begründet, an denen Menschen mit Fluchtmigrationshintergrund kein Interesse zeigten, sowie damit, dass Akquiseversuche scheiterten. Eine Interviewperson sagt: »Wir haben wirklich versucht, Werbung zu machen, aber von den Angesprochenen wurde das abgelehnt« (5477). Diese Interviewpersonen zeigen sich prinzipiell offen gegenüber einer Teilnahme von Menschen mit Fluchtmigrationshintergrund, allerdings scheint hier die Vermittlung nicht zu gelingen. Hier besteht ein großes Potenzial, sowohl Freizeitvereine und -gruppen als auch Menschen mit Fluchtmigrationshintergrund aneinander $\mathrm{zu}$ vermitteln beziehungsweise dabei zu unterstützen.

\section{Bislang kein Kontakt}

Eine zentrale Begründung dafür, warum bislang noch keine Menschen mit Fluchtmigrationshintergrund an der von der Interviewperson vertretenen Freizeitaktivität teilnehmen, liegt darin, dass es (a) noch keine Kontaktanfragen seitens Menschen mit Fluchtmigrationshintergrund gab und (b) Kontaktversuche seitens der Freizeitvereine gescheitert sind (wie oben bereits problematisiert wurde). Problematisch wird ein solcher fehlender Kontakt dann, wenn er als nicht gewünscht dargestellt wird. So gehen einige Interviewpersonen davon aus, dass Menschen mit Fluchtmigrationshintergrund ohnehin kein Interesse an der von ihnen vertretenen Freizeitaktivität haben, wie es beispielsweise diese Interviewperson schildert: »Die Flüchtlinge bleiben ja gerne unter sich und kommen nicht so unter Menschen. Da bildet sich eine Parallelgesellschaft mit ganz eigenen Interessen« (3108). Die Folge 
ist, dass Annäherungsversuche gar nicht erst unternommen werden, was schließlich Ausschluss (re-)produziert - und Strukturmerkmale einer von der Interviewperson genannten "Parallelgesellschaft" überhaupt erst hervorbringt (zur Problematisierung dieser Bezeichnung siehe u.a. Hill 2016, S. 61ff; Bukow 2007, S. 50). Es bestätigen sich hier erneut die Annahmen der sogenannten Kontakthypothese (siehe Kapitel 6.2 und Kapitel 23). Demgegenüber gibt es vereinzelt allerdings auch selbstkritische Einschätzungen, in denen (c) begründet wird, dass sich bislang noch nicht genug dafür eingesetzt wurde, Kontakte zu Menschen mit Fluchtmigrationshintergrund zu knüpfen. Eine Interviewperson sagt: »Wir sind nicht offensiv darauf zugegangen « (8578). An dieser Selbstkritik kann angeknüpft werden, indem beispielsweise Unterstützungsmöglichkeiten für PrimärvertreterInnen aus dem Freizeitbereich geschaffen und transparent gemacht werden.

\section{Verweis auf gesonderte Angebote für Menschen mit Fluchtmigrationshintergrund}

Menschen mit Fluchtmigrationshintergrund werden teils als AdressatInnen bestimmter helfender oder sozialer Professionen gesehen, die von dieser Seite gesonderte Freizeitangebote erhalten. Menschen mit Fluchtmigrationshintergrund werden dadurch, ganz ähnlich wie oftmals Menschen mit Behinderung, in gewisser Weise als betreuungsbedürftig hervorgebracht, wodurch Peer-Kontakte als gleichberechtigt und ähnliche Interessen verfolgend von vorneherein eingeschränkt oder sogar verunmöglicht werden.

\section{Menschen mit Fluchtmigrationshintergrund haben keinen Bedarf, einer Freizeitaktivität nachzugehen}

Ein Grund für eine Nicht-Teilhabe, der sehr häufig genannt wird, ist, dass Menschen mit Fluchtmigrationshintergrund keinen Bedarf und auch keine Kapazität hätten, einer Freizeitaktivität nachzugehen, da sie gegenwärtig andere Herausforderungen zu bewältigen hätten. Eine Interviewperson sagt: »Die haben jetzt ja erstmal andere Dinge zu tun, wie zum Beispiel in Deutschland anerkannt $\mathrm{zu}$ werden, $\mathrm{zu}$ wohnen und sich eine Arbeit $\mathrm{zu}$ suchen « (7774). Eine andere Interviewperson sagt: »Na, die haben ja eventuell gerade andere Baustellen« (1106). Dies kann dahingehend problematisiert werden, dass Interviewpersonen, die derart argumentieren, Freizeit ganz offensichtlich nicht als grundständigen Lebensbereich anerkennen, der von jeder Person uneingeschränkt ausgelebt werden darf, sondern als eine Art 
Verdienst und etwas, das erfolgt, sobald alle anderen Lebensbereiche, die eher als Pflicht verstanden werden, sinnhaft gefüllt wurden. Menschen mit Fluchtmigrationshintergrund wird Freizeit nicht zugestanden. Dadurch wird eine Differenz manifestiert zwischen Menschen mit und Menschen ohne Fluchtmigrationshintergrund, die grundsätzlich ist und, in der Darstellung der Interviewpersonen, vorerst nicht überwunden werden kann. Freizeit wird nicht als Möglichkeit betrachtet, Teilhabechancen zu eröffnen, die sich dann auch auf andere Lebensbereiche auswirken könnten, beispielsweise über Kontakte, die in der Freizeit geknüpft wurden, Arbeit oder Wohnung vermittelt zu bekommen. Darüber hinaus kann inklusive Freizeit auch dazu beitragen, Sprachbarrieren abzubauen, da ein informelles Miteinander oftmals das Erlernen einer Sprache erleichtern beziehungsweise unterstützen kann.

\section{Fehlende Lizenzen oder Prüfungen}

Einige Interviewpersonen sehen den Grund für eine Nicht-Teilnahme von Menschen mit Fluchtmigrationshintergrund an der von ihnen repräsentierten Freizeitaktivität darin, dass diese bestimmte Voraussetzungen nicht erfüllen, die für eine Teilnahme unbedingt notwendig sind, wie beispielsweise eine Funklizenz (Amateurfunkverein) oder einen Waffenschein (Schützenverein) zu haben. Eine Unterstützung dabei, diese Lizenzen zu erwerben, bieten die Interviewpersonen nicht an.

\section{Zu hohe Kosten}

Ein weiterer Aspekt, der von einigen Interviewpersonen angesprochen wird, ist, dass die Kosten für die von ihnen vertretene Freizeitaktivität so hoch sind, dass Menschen mit Fluchtmigrationshintergrund sich eine Teilnahme nicht leisten können. Erneut zeigt sich also, ähnlich wie in Bezug auf viele Menschen mit Behinderung, dass ein Leben in Armut oder an der Armutsgrenze zur Teilhabebarriere werden kann. In diesem Zusammenhang kann zudem auf die wechselseitige Bedingtheit von fehlender oder zu niedrig entlohnter Arbeit und einer möglichen Teilnahme an Freizeitaktivitäten aufmerksam gemacht werden, wodurch schließlich eine Teilhabe an Praxen der Mehrheitsgesellschaft unter Umständen weitergehend eingeschränkt wird. 


\section{Sprachbarriere}

Ähnlich wie im Handlungsfeld Arbeit werden im Handlungsfeld Freizeit Sprachbarrieren als Grund dafür gesehen, dass keine Menschen mit Fluchtmigrationshintergrund teilhaben. Dies formulieren auch Interviewpersonen, die Freizeitangebote vertreten, von denen angenommen werden kann, dass die Rolle von Sprache gegebenenfalls gar nicht sehr groß ist, wie beispielsweise bei Ballsportarten oder Outdooraktivitäten. Zu bedenken gegeben werden muss auch, dass statusgleiche Begegnungen, wie sie der Freizeitbereich bietet, dazu beitragen können, Sprache im intersubjektiven Austausch zu erlernen. Dies wird von den Interviewpersonen, die bislang in der von ihnen vertretenen Freizeitaktivität noch keine Teilnehmenden mit Fluchtmigrationshintergrund hatten, nicht als Möglichkeit eingeräumt.

\section{Unüberwindbare kulturelle Differenzen}

Schließlich gehen einige Interviewpersonen davon, dass die kulturellen Differenzen zu groß sind zwischen der von ihnen vertretenen Freizeitaktivität und dem, von dem sie glauben, dass es Menschen mit Fluchtmigrationshintergrund interessiert. Eine Interviewperson, die einen Chor in einem der Sozialräume repräsentiert, sagt beispielsweise: »Sie [gemeint sind Menschen mit Fluchtmigrationshintergrund] singen nicht« (2520). Menschen mit Fluchtmigrationshintergrund werden hier als grundsätzlich sanders konstruiert und ausgehend davon wird angenommen, es sei ohnehin kein Interesse vorhanden. Vorbehalte befürchtet auch jene Interviewperson, die sagt: »Bei uns im Tanzverein ist immer wieder enger Körperkontakt, da gibt es bestimmt kulturelle Differenzen« (7972).

\subsubsection{Ablehnung von Menschen mit Demenz}

Die Interviewpersonen begründen es auf unterschiedliche Art und Weise, dass keine Menschen mit Demenz an der von ihnen repräsentierten Freizeitaktivität teilnehmen. Diese Begründungen werden im Folgenden gebündelt dargelegt und diskutiert.

\section{Ältere Menschen sind nicht Zielgruppe}

Einige Interviewpersonen sehen den Grund für eine Nichtteilnahme von Menschen mit Demenz darin, dass sich die von ihnen vertretene Freizeitaktivität an Kinder und Jugendliche richtet. Diese Begründung scheint nachvollziehbar und bedarf auch keiner weiteren problematisierenden Aus- 
einandersetzung, da es nicht routinemäßig ist, dass SeniorInnen (wie es auf die überwiegende Zahl der Menschen mit Demenz zutrifft) gemeinsam mit Kindern und Jugendlichen Freizeitaktivitäten nachgehen, die sich ausschließlich an Letztere richten. Denkbar wären selbstredend generationenübergreifende Angebote, die es teilweise auch bereits gibt.

\section{Verweis auf gesonderte Angebote für Menschen mit Demenz}

Vereinzelt kommt es vor, dass Interviewpersonen auf Angebote verweisen, die sich explizit an Menschen mit Demenz richten und die somit erste Anlaufstelle für diese Personen seien. Eine Interviewperson sagt beispielsweise: »Bei der AWO wird speziell was für diese Menschen angeboten« (2278). Dies ist eine Aussage, aus der Ausschluss folgen kann, da nicht der Bedarf gesehen wird, Menschen mit Demenz Teilhabe an der eigenen Freizeitaktivität zu ermöglichen. Diese Argumentationslinie findet sich ganz ähnlich in Bezug auf Menschen mit Behinderung (siehe Kapitel 17.8.1). Hier können unter Umständen Bildungsmaßnahmen sinnvoll sein, die PrimärvertreterInnen bestehender Freizeitangebote für eine explizite Ansprache von Menschen mit Demenz sensibilisieren. Denkbar sind darüber hinaus Angebote, in denen Menschen mit und ohne Demenz gemeinsam einer Freizeitaktivität nachgehen und die dadurch eine gewisse Vorreiterrolle einnehmen können. Inwiefern eine Sensibilisierung für das Phänomen Demenz in der Breite notwendig ist, zeigt sich an Aussagen, in denen Menschen mit Demenz abgesprochen wird, überhaupt Interesse und/oder Bedarf an Freizeitaktivitäten $\mathrm{zu}$ haben (eine deutliche Parallele zu entsprechenden Argumentationen in Bezug auf Menschen mit Fluchtmigrationshintergrund ist zu erkennen; siehe Kapitel 17.8.2). Der/die PrimärvertreterIn einer musikalischen Freizeitaktivität sagt: »Für Menschen mit Demenz und Alzheimer ist Musik wahrscheinlich ganz weit weg. Bei denen stehen eher Hilfen zur Bewältigung des Alltags im Fokus und keine Freizeitaktivitäten « (6076). Menschen mit Demenz werden dadurch in gewisser Weise als krank und hilfebedürftig konstruiert. Zudem trägt die Auffassung, Menschen mit Demenz bräuchten Schutz und Hilfe, mit dazu bei, dass sie aus Diskursen der Mehrheitsgesellschaft pauschal ausgeschlossen werden. 


\section{Ängste und Vorbehalte aufseiten von Menschen mit Demenz}

Immer wieder gehen Interviewpersonen davon aus, dass Menschen mit Demenz zu ängstlich sind, um an Freizeitangeboten der Mehrheitsgesellschaft (weiterhin) teilzunehmen. Eine Interviewperson sagt: »Die haben Angst und trauen sich nicht zu kommen« (8649). Eine andere Interviewperson führt diesbezüglich aus: »Viele haben bestimmt kein Interesse mehr oder können es nicht mehr wahrnehmen und bleiben dann einfach Zuhause. Vor allem aus Angst, sich lächerlich zu machen« (4148). Jene Interviewpersonen nehmen die Ängste und Vorbehalte, von denen sie bei Menschen mit Demenz ausgehen, als gegeben hin und sehen sich infolgedessen auch nicht in der Verantwortung, diesen entgegenzusteuern und gegebenenfalls dazu beizutragen, Hemmschwellen abzubauen. Ganz grundsätzlich stellt sich hier die Frage, ob dies überhaupt etwas ist, was von PrimärvertreterInnen geleistet werden kann und, falls nicht, wessen Aufgabe es ist, Ängste und Vorbehalte abzubauen und somit für Menschen mit Demenz Teilhabemöglichkeiten zu eröffnen.

\section{Abhängigkeit von anderen}

Einzelne Interviewpersonen sehen in der eingeschränkten Selbstständigkeit, von der sie bei Menschen mit Demenz ausgehen, einen Grund dafür, dass jene Personen nicht an der von ihnen vertretenen Freizeitaktivität teilnehmen. Sie seien abhängig davon, dass andere Personen sie zur Freizeitaktivität bringen, gegebenenfalls dort begleiten und auch wieder nach Hause zurückbringen. Jedoch verfügen nicht alle Menschen mit Demenz über die dazu notwendigen Ressourcen. Eine Interviewperson sagt: »Wer dement ist, wird sich von sich aus nicht bei uns einfinden« (2081). Hier braucht es also die Unterstützung durch Außenstehende, Menschen mit Demenz den Zugang zu ihren Freizeitaktivitäten aufrechtzuerhalten oder neue Zugänge zu schaffen. Ungeklärt scheint, wer diese Rolle einnehmen kann. In Bezug auf die zitierte Interviewperson erscheint besonders problematisch, dass es sich bei der von ihr vertretenen Freizeitaktivität ausdrücklich um ein generationenübergreifendes Angebot handelt, dem aber offenbar Ideen (oder möglicherweise auch Interesse) dazu fehlt, wie Menschen mit Demenz eingebunden werden können. Andere Interviewpersonen konkretisieren die Abhängigkeit von anderen im Bereich Mobilität. Eine Interviewperson sagt: "Menschen, die im Chor aktiv sind und an Demenz erkranken, hören auf zu singen, weil sie nicht transportiert werden können« (6766). Ausschluss be- 
gründet sich hier also nicht, zumindest nicht primär, in einem vermeintlichen Defizit der Person mit Demenz, sondern in unzureichenden Strukturen, die nicht sicherstellen können, dass die Person die Freizeitaktivität erreicht.

\section{Abschottung durch Angehörige}

Einige Interviewpersonen sehen in der Abschottung durch Angehörige einen Grund dafür, dass Menschen mit Demenz nicht oder nicht mehr an der von ihnen repräsentierten Freizeitaktivität teilnehmen. Eine Interviewperson sagt: »Unsere Erfahrungen mit Mitgliedern mit Demenz ist, dass diese von ihrem privaten Umfeld abgeschottet werden und dann deswegen nicht mehr kommen« (6298). Eine andere vermutet etwas ganz Ähnliches, sie sagt: »Ich gehe mal davon aus, dass Angehörige zu protektiv sind« (1530). Hier zeichnet sich ein dringender Bedarf ab, Angehörige zu sensibilisieren und dahingehend aufzuklären, dass Demenz nicht immer heißen muss, das Haus nicht mehr verlassen zu können (siehe u.a. Brandenburg 2014; Schuhmacher 2018, S. 222ff). Gleichzeitig braucht es umfassende Unterstützungsangebote, die über den Bereich Pflege hinausgehen und eine pädagogische Alltagsbetreuung ermöglichen, insbesondere auch, um Angehörige zu entlasten.

\section{Fachpersonal und Schulungen sind notwendig}

In einigen Fällen überwiegen defizitäre Konstruktionen, in denen Demenz als degenerative, wesensverändernde Krankheit wahrgenommen wird, die besondere Kenntnisse und Fähigkeiten notwendig macht, um Personen zu begleiten, die als erkrankt bezeichnet werden. Infolgedessen stellen einige Interviewpersonen fest, eine Teilhabe von Menschen mit Demenz sei nur dann möglich, wenn entsprechend ausgebildetes Personal vorhanden sei oder die Personen, die die Freizeitaktivität ausrichten, im Hinblick auf den Umgang mit Menschen mit Demenz geschult werden. Eine Interviewperson sagt: "Eine Teilnahme wäre bei uns nicht möglich, weil man da Schulungen und Fachpersonal braucht. Ich selbst hab so meine Erfahrungen mit der Krankheit gemacht und ich habe wirklich großen Respekt davor" (1481). Auch hieran zeigt sich, wie wichtig eine Begleitung aller Personen ist, die (potenziell) zum Sozialraum des jeweiligen Menschen mit Demenz dazugehören, um Ängste und Vorbehalte abzubauen und dort Unterstützung zu ermöglichen, wo Teilhabemöglichkeiten andernfalls deutlich eingeschränkt werden. In Bezug auf die zitierte Interviewperson kann zudem problemati- 
sierend hervorgehoben werden, dass diese ein Freizeitangebot vertritt, das sich explizit an ältere Menschen richtet. Dass Menschen mit Demenz von dort ausgeschlossen werden, zeigt einmal mehr, wie gering ihre Möglichkeiten sind, routinemäßigen Freizeitaktivitäten in der Mehrheitsgesellschaft nachzugehen.

\section{Kein Kontakt}

Sehr häufig berichten Interviewpersonen davon, Menschen mit Demenz suchten keinen Kontakt zu der von ihnen vertretenen Freizeitaktivität, womit erneut auf die eingangs problematisierte Kontakthypothese verwiesen sowie darauf aufmerksam gemacht werden kann, wie wichtig es ist, lebensweltliche Begegnungsmöglichkeiten zwischen Personen $\mathrm{zu}$ schaffen, um (gegenseitige) Vorbehalte abzubauen und schließlich Teilhabemöglichkeiten $\mathrm{zu}$ eröffnen. Eine Interviewperson problematisiert in diesem Zusammenhang die Heimunterbringung von vielen Menschen mit Demenz. Sie sagt: "Meist sind die Menschen ja in Heimen untergebracht, da gibt es dann einfach keinen Kontakt« (2098). Im Rahmen der Kontakthypothese geht es also darum, totale Strukturrahmen, wie sie in stationären Wohnkontexten größtenteils vorliegen (u.a. Trescher 2013, 2017f), infrage zu stellen und offenere Formen der Unterbringung und Betreuung von Menschen mit Demenz zu schaffen.

\section{Mangelnde körperliche und kognitive Leistungsfähigkeit}

Einige Interviewpersonen gehen davon aus, dass Menschen mit Demenz körperlich und/oder kognitiv nicht dazu in der Lage sind, an der von ihnen vertretenen Freizeitaktivität teilzunehmen, und sehen darin den Grund, dass jene Personen nicht teilhaben. Eine Interviewperson sagt beispielsweise: »Bei uns müssen die Lieder auswendig gelernt werden, davon sind Menschen mit Demenz überfordert. Und dann noch die Menschenmengen, das ist einfach zu viel. Menschen mit Demenz können ja auch gar kein Instrument spielen« (6018). Eher auf die körperliche Leistungsfähigkeit bezieht sich diese Interviewperson: »Tennis ist ein aktiver und schneller Sport und Demenz verringert unter anderem die Reaktionsgeschwindigkeit« (3802). An Aussagen wie diesen wird zweierlei deutlich: Zum einen zeigt sich, inwiefern Demenz als Krankheit konstruiert wird, die von umfänglichem Verlust geprägt ist. Zum anderen kristallisiert sich heraus, dass routinemäßige Freizeitaktivitäten nicht auf die diversen Bedarfe unterschiedlicher Perso- 
nen eingestellt sind. Menschen mit Demenz werden in diesem Zusammenhang oftmals als gefährdet oder schutzbedürftig dargestellt, wie im Vorigen bereits diskutiert wurde und sich auch in der Aussage der folgenden Interviewperson zeigt: »Das ist zu gefährlich und deshalb wäre das auch rechtlich gar nicht möglich« (6868). Hier werden rechtliche Begründungen herangezogen, ohne selbst RechtsexpertIn zu sein, weshalb sich erneut zeigt, wie wichtig Aufklärung und Sensibilisierung sind, um keine vorschnellen Urteile zu fällen.

\section{Mehrheitsgesellschaftlicher Ausschluss}

Vereinzelt kommt es vor, dass Interviewpersonen davon ausgehen, Menschen mit Demenz würden pauschal von einer Teilhabe an Praxen der Mehrheitsgesellschaft ausgeschlossen und nähmen deshalb nicht an der von ihnen vertretenen Freizeitaktivität teil. Eine Interviewperson sagt: »Demenz ist nicht hoffähig in unserer Gesellschaft, deshalb tritt auch niemand mit Demenz an unseren Verein heran« (1926). Eine Aussage wie diese offenbart einen kritischen Blick, der Missstände wahrnimmt. Offen bleibt die Frage, wie aus einer solchen, prinzipiell problematisierenden Haltung Praxen der Öffnung entstehen können, die schließlich das Bild von Menschen mit Demenz in der Mehrheitsgesellschaft verändern können, beispielsweise dadurch, dass mehr Menschen mit Demenz an routinemäßigen Freizeitaktivitäten teilhaben.

\section{Selbstkritik: zu wenig Werbung und Angebote für Menschen mit Demenz}

Immer wieder kommt es vor, dass Interviewpersonen durch die Nachfrage, weshalb keine Menschen mit Demenz an der von ihnen vertretenen Freizeitaktivität teilnehmen, nachdenklich werden und schließlich folgern, selbst zu wenig Werbung und Angebote für Menschen mit Demenz zu machen. Eine Interviewperson sagt: »Vielleicht müssen wir unsere Werbung deutlich in diese Richtung schreiben. Das ist eine gute Idee! Aber es wäre auch gut, wenn wir irgendwie merken, dass wir diese Leute nicht erreichen « (9813). Interviewpersonen, die sich derart äußern, scheinen sehr aufgeschlossen, gegebenenfalls Änderungen an ihrer Freizeitaktivität vorzunehmen, um so mehr und andere Menschen zu erreichen. Personen wie die hier zitierte können zu MultiplikatorInnen werden und dabei unterstützen, die Idee von Öffnung in den Sozialraum zu tragen. Zudem zeigt sich hier einmal mehr, wie wichtig es ist, dass Untersuchungen wie die hiesige vorgenommen werden, 
da diese (a) Kenntnisse über Teilhabepraxen im Sozialraum eröffnen und (b) selbst ein Stück weit zur Reflexion der Interviewpersonen beitragen.

\subsection{Möglichkeit der Teilhabe}

Die Interviewpersonen wurden gefragt, inwiefern sie es als möglich einschätzen, dass Menschen mit unterschiedlichen Beeinträchtigungen, Fluchtmigrationshintergrund und/oder Demenz an der von ihnen vertretenen Freizeitaktivität teilhaben können. Die Ergebnisse dessen sind in der nachfolgenden Tabelle übersichtlich dargelegt und werden daran anschließend eingehender betrachtet.

Tabelle 35: Prinzipielle Möglichkeit der Teilhabe an Freizeitaktivitäten für Menschen mit unterschiedlichen Beeinträchtigungen, Fluchtmigrationshintergrund oder Demenz

\begin{tabular}{|l|l|l|l|l|l|c|}
\hline $\begin{array}{l}\text { Ja, es besteht die } \\
\text { prinzipielle Möglich- } \\
\text { keit der Teilhabe von } \\
\text { Menschen mit }\end{array}$ & $\begin{array}{l}\text { Erlan- } \\
\text { gen }\end{array}$ & $\begin{array}{l}\text { Ros- } \\
\text { tock }\end{array}$ & $\begin{array}{l}\text { Schne- } \\
\text { verdin- } \\
\text { gen }\end{array}$ & $\begin{array}{l}\text { Schwä- } \\
\text { bisch } \\
\text { Gmünd }\end{array}$ & $\begin{array}{l}\text { VG } \\
\text { Nieder- } \\
\text { Olm }\end{array}$ & $\varnothing$ \\
\hline $\begin{array}{l}\text { Mobilitätsbeeinträch- } \\
\text { tigung }\end{array}$ & $41,7 \%$ & $47,8 \%$ & $45 \%$ & $32,3 \%$ & $66,7 \%$ & $46,7 \%$ \\
\hline Sehbeeinträchtigung & $29,2 \%$ & $43,5 \%$ & $35 \%$ & $29 \%$ & $66 \%$ & $34,2 \%$ \\
\hline Hörbeeinträchtigung & $33,3 \%$ & $60,9 \%$ & $63,2 \%$ & $71 \%$ & $87,5 \%$ & $63,2 \%$ \\
\hline $\begin{array}{l}\text { kognitiven Beein- } \\
\text { trächtigungen }\end{array}$ & $29,2 \%$ & $56,5 \%$ & $36,8 \%$ & $45,2 \%$ & $87,5 \%$ & $51 \%$ \\
\hline $\begin{array}{l}\text { Fluchtmigrations- } \\
\text { hintergrund }\end{array}$ & $60 \%$ & $63 \%$ & $71,4 \%$ & $89,5 \%$ & $85,7 \%$ & $73,9 \%$ \\
\hline Demenz & $28,6 \%$ & $43,6 \%$ & $48,1 \%$ & $43,6 \%$ & $57,9 \%$ & $44,4 \%$ \\
\hline
\end{tabular}

Im Gegensatz zur korrespondierenden Untersuchung im Handlungsfeld Arbeit können hier keine signifikanten Unterschiede zwischen Großstadt und Kleinstadt/Gemeinde festgestellt werden, weshalb die Ergebnisse sozialraumbezogen dargestellt werden. Gleichzeitig wird daran ein zentrales Ergebnis der Studie erkennbar, denn es kann gesagt werden, dass die 
Teilhabe an Freizeitaktivitäten viel weniger von der Lage des Wohnortes abhängig ist, als dies im Bereich Arbeit der Fall ist (siehe Kapitel 16.8). Zu erkennen ist darüber hinaus, dass die prinzipielle Möglichkeit der Teilhabe an Freizeitaktivitäten für Menschen mit Hörbeeinträchtigungen am höchsten eingeschätzt wird (durchschnittlich 63,2 \%), während für Menschen mit Sehbeeinträchtigung die geringsten Teilhabemöglichkeiten gesehen werden (durchschnittlich 34,2 \%). Zu beachten ist, dass die Ergebnisse in den Sozialräumen sehr unterschiedlich ausfallen, so wird beispielsweise eine Teilhabe für Menschen mit kognitiven Beeinträchtigungen in der Verbandsgemeinde Nieder-Olm (87,5 \%) erheblich häufiger als möglich eingeschätzt als dies beispielsweise in Erlangen der Fall ist (29,2 \%). Auffällig ist beim Blick in die Ergebnisse zudem, dass Menschen mit Fluchtmigrationshintergrund deutlich größere Teilhabemöglichkeiten eingeräumt werden (durchschnittlich $73,9 \%$ ) als Menschen mit unterschiedlichen Beeinträchtigungen (beispielsweise Menschen mit Mobilitätsbeeinträchtigungen: durchschnittlich 46,7\%). Beeinträchtigungen beziehungsweise Behinderung sind also in der Einschätzung der Interviewpersonen eine deutliche Teilhabebarriere. Dass dies auch durch Vorbehalte und mangelnden lebensweltlichen Kontakt zu Menschen mit (unterschiedlichen) Beeinträchtigungen begründet ist, scheint klar und verdeutlicht einmal mehr, inwiefern es wichtig ist, gemeinsame Lebenspraxen von Menschen mit und Menschen ohne Beeinträchtigungen zu unterstützen. Der Anteil der Interviewpersonen, die eine Teilhabe von Menschen mit Demenz als möglich einschätzt, liegt bei durchschnittlich $44,4 \%$. Ein Blick auf die fünf untersuchten Sozialräume zeigt, dass die Einschätzung der Interviewpersonen, eine Teilhabe von Menschen mit Demenz sei möglich, recht unterschiedlich verteilt ist. Während in Erlangen 28,6 \% der Interviewpersonen dies für möglich halten, sind es in der Verbandsgemeinde Nieder-Olm 57,9\%.

\subsection{Möglichkeit, eine Begleitperson mitzubringen}

Im Gros schätzen es die interviewten PrimärvertreterInnen als möglich ein, dass Menschen mit Behinderung, Fluchtmigrationshintergrund oder Demenz eine Begleitperson mitbringen können, wie aus der Tabelle hervorgeht und im Folgenden noch einmal erläutert wird. Da zwischen den Sozialräumen keine signifikanten Unterschiede bestehen, wird das Gesamtergebnis fokussiert. 
Tabelle 36: Möglichkeit, eine Begleitperson mitzubringen

\begin{tabular}{|c|c|c|c|}
\hline \multicolumn{2}{|c|}{ Ja, es besteht die Möglichkeit, eine Begleitperson mitzubringen für Menschen mit... } \\
\hline & Behinderung & $\begin{array}{l}\text { Fluchtmigrations- } \\
\text { hintergrund }\end{array}$ & Demenz \\
\hline$\varnothing$ & $88,6 \%$ & $87,3 \%$ & $80,2 \%$ \\
\hline
\end{tabular}

Die Mehrheit der interviewten PrimärvertreterInnen (durchschnittlich 88,6 \%) hält es für möglich, dass Menschen mit Behinderung eine Begleitperson zur Freizeitaktivität mitbringen. Es besteht also eine gewisse Offenheit dafür, die jeweils vertretene Freizeitaktivität insofern $\mathrm{zu}$ verändern, dass Personen begleitend dabei sind, ohne selbst aktiv teilzunehmen. Hieraus können Teilhabemöglichkeiten erwachsen. Durchschnittlich 87,3 \% der Interviewpersonen gehen davon aus, dass es Teilnehmenden mit Fluchtmigrationshintergrund möglich ist, zu der von ihnen vertretenen Freizeitaktivität eine Begleitperson mitzubringen. Dies unterscheidet sich nur marginal vom entsprechenden Zustimmungswert in Bezug auf Menschen mit Behinderung. Folglich kann auch hieraus eine gewisse Bereitschaft geschlossen werden, die jeweilige Freizeitaktivität Personen mit möglicherweise diversen Bedarfen zu öffnen. In Bezug auf die Teilhabe von Menschen mit Demenz gehen durchschnittlich 80,2 \% der interviewten PrimärvertreterInnen davon aus, dass es möglich sei, eine Begleitperson zu der von ihnen repräsentierten Freizeitaktivität mitzubringen. Im Vergleich zu den Möglichkeiten von Menschen mit Behinderung und Menschen mit Fluchtmigrationshintergrund, eine Begleitperson mitzubringen, zeigt sich, dass die Interviewpersonen hier im Durchschnitt etwas weniger aufgeschlossen sind.

\subsection{Unterstützungsmöglichkeiten durch die anderen Teilnehmenden}

Im Folgenden ist dargelegt, inwiefern die interviewten PrimärvertreterInnen es als möglich einschätzen, dass die Personen, die an der von ihnen repräsentierten Freizeitaktivität teilnehmen, Menschen mit Behinderung, Menschen mit Fluchtmigrationshintergrund oder Menschen mit Demenz darin unterstützen, an der Freizeitaktivität teilzuhaben. In Bezug auf diese Frage hat es sich als sinnvoll erwiesen, die Sozialräume in zwei Gruppen zusammenzuführen und einen Vergleich zwischen Großstadt und Kleinstadt/ 
Gemeinde anzustellen, da signifikante Unterschiede zwischen diesen beiden Gruppen ausgemacht werden konnten.

Tabelle 37: Unterstützungsmöglichkeiten durch die anderen Teilnehmenden

\begin{tabular}{|l|c|c|c|}
\hline $\begin{array}{l}\text { Ja, Unterstützung } \\
\text { ist möglich für } \\
\text { Menschen mit }\end{array}$ & Großstadt & $\begin{array}{l}\text { Kleinstadt/Ge- } \\
\text { meinde }\end{array}$ & $\varnothing$ \\
\hline Behinderung & $48,9 \%$ & $58,3 \%$ & $53,6 \%$ \\
\hline $\begin{array}{l}\text { Fluchtmigrations- } \\
\text { hintergrund }\end{array}$ & $53,9 \%$ & $72,2 \%$ & $63,1 \%$ \\
\hline Demenz & $41,6 \%$ & $56,9 \%$ & $49,2 \%$ \\
\hline
\end{tabular}

Der Vergleich zwischen Großstadt und Kleinstadt/Gemeinde zeigt, dass bei Interviewpersonen aus den kleineren untersuchten Sozialräumen die Bereitschaft deutlich höher ist, Menschen mit Behinderung, Menschen mit Fluchtmigrationshintergrund oder Menschen mit Demenz insofern zu unterstützen, dass ihre Teilnahme an der jeweiligen Freizeitaktivität möglich oder vereinfacht wird. In Bezug auf Menschen mit Behinderung sehen in der Kleinstadt/Gemeinde 58,3\% der Interviewpersonen Unterstützungsmöglichkeiten durch die anderen Teilnehmenden, während es in der Großstadt $48,9 \%$ sind. Bezüglich Menschen mit Fluchtmigrationshintergrund und Menschen mit Demenz ist das Verhältnis ähnlich. Hervorgehoben werden kann darüber hinaus, dass in Bezug auf Menschen mit Fluchtmigrationshintergrund die antizipierten Unterstützungsmöglichkeiten am größten sind (durchschnittlich 63,1 \%) und in Bezug auf Demenz am geringsten (durchschnittlich 49,2\%). Dies liegt möglicherweise daran, dass mit Menschen mit Demenz (ebenso wie mit Menschen mit Behinderung) unter Umständen primär unterstützende Tätigkeiten aus dem Bereich Pflege assoziiert werden, woraus Berührungsängste und schließlich ablehnendere Haltungen resultieren können. 


\subsection{Schwierigkeiten und Handlungsperspektiven im Kontext von Inklusion und Freizeit}

Ebenso wie in Bezug auf die Interviews im Handlungsfeld Arbeit ergeben die Analysen der Interviews mit PrimärvertreterInnen im Handlungsfeld Freizeit unterschiedliche Schwierigkeiten und Handlungsperspektiven, die die Interviewpersonen im Kontext von Inklusion und Freizeit erkennen. Die Ergebnisse sind im Folgenden problemfokussiert zusammengefasst. Auch hier wurde sich für eine sozialraumübergreifende Darstellung entschieden, da die erlebten Schwierigkeiten und möglichen Handlungsperspektiven im Gros sehr ähnlich sind. Anhand einzelner Interviewpassagen werden diese dargelegt und diskutiert.

\subsubsection{Schwierigkeiten im Kontext von Inklusion und Freizeit}

Während einige Personen keine oder kaum Schwierigkeiten darin sehen, das von ihnen repräsentierte Freizeitangebot für eine Teilhabe unterschiedlicher Personen zu öffnen, gibt es einige, die Vorbehalte haben und diese auch als solche benennen. Welche Schwierigkeiten im Kontext Inklusion und Freizeit gesehen werden, wird im Folgenden qualitativ-inhaltsanalytisch zusammengefasst und problemfokussiert diskutiert. Dabei werden einzelne Zitate aus den Interviews exemplarisch zur Illustration herangezogen.

\section{Körperliche und/oder kognitive Minderleistung}

Viele Freizeitaktivitäten werden von den jeweiligen PrimärvertreterInnen als $\mathrm{zu}$ herausfordernd für bestimmte Personen beschrieben, da diese die erforderlichen körperlichen und/oder kognitiven Anforderungen nicht erfüllen können. Menschen mit unterschiedlichen Unterstützungsbedarfen werden dadurch pauschal als >MinderleisterInnen Argument herangezogen wird, jene Personen von der jeweiligen Freizeitaktivität auszuschließen. Diese Argumentation findet sich zum einen bei PrimärvertreterInnen, die ihre Freizeitaktivität als besonders komplex und kognitiv sowie körperlich herausfordernd beschreiben, wie beispielsweise diese Interviewperson: »Tennis erfordert geistig schnelle Reaktionen und eine hohe Kondition, da darf man nicht beeinträchtigt sein« (4575). Zum anderen gibt es einige Interviewpersonen, die die von ihnen vertretene Freizeitaktivität als besonders leistungsorientiert charakterisieren und die Teilnahme sogenannter leistungsschwacher Personen ausschließen, zu denen 
sie fast übereinstimmend Menschen mit Behinderung im Allgemeinen und Menschen mit geistiger Behinderung im Besonderen zählen, jedoch auch andere Beeinträchtigungsdimensionen werden als unpassend eingestuft, wie beispielsweise aus der Aussage dieser Interviewperson hervorgeht: »Die Anforderungen in unserem Leistungschor sind zu hoch. Wir kaufen Dirigenten von extern ein, das ist immer sehr teuer, und die verlangen Leistung. Für Menschen mit Demenz haben die keine Geduld« (6766). Menschen mit Demenz werden hier als langsam konstruiert und so, als sei für sie eine besondere Betreuung notwendig, die die Leitung des Chores nicht erbringen könne. Neben mangelnder Leistungsfähigkeit wird teilweise eine mangelnde Repräsentativität von Personen beklagt, die nicht dem Leistungsniveau entsprechen, das ihre Freizeitaktivität verlangt. Eine Interviewperson sagt: »Geistig Behinderte können nicht bei uns im Orchester mitspielen, immerhin haben die Zuschauer einen gewissen Anspruch. Im Fanfarenzug ist das vielleicht noch möglich, da ist der Anspruch nicht so hoch « (7501). Auch hier zeigt sich, inwiefern in Bezug auf Menschen mit - insbesondere geistiger Behinderung Konstruktionen negativer Andersartigkeit vorherrschen, die in diesem Fall zu pauschalem Ausschluss führen.

\section{Geistige Behinderung als Ausschlusskategorie}

In den Aussagen vieler Interviewpersonen wird deutlich - wie oben bereits anklang -, dass sie teils Menschen mit Behinderung Teilhabemöglichkeiten einräumen, dies in Bezug auf Menschen mit geistiger Behinderung allerdings grundsätzlich ausschließen. Geistige Behinderung wird so als soziale Grenze wirksam, die die Teilhabe an Praxen der Mehrheitsgesellschaft behindert (Trescher 2017a, S. 244ff). Während einige Interviewpersonen diesen Ausschluss sehr pauschal aussprechen, gibt es zum einen einige, die eher behindertenfeindliche Aussagen treffen, wie beispielsweise diese Interviewperson: »Inklusion ist schwierig, bei vielen Dingen funktioniert das einfach nicht. Zum Beispiel ist es einfach so, dass geistige Behinderung nicht mit gesunden Kindern harmoniert « (1279). Zum anderen gibt es Interviewpersonen, die ein differenzierteres Bild zeichnen und vor allem auf den - bislang - mangelnden Kontakt zwischen der Mehrheitsgesellschaft und Menschen mit geistiger Behinderung verweisen. Eine Interviewperson sagt: »Dort, wo Menschen miteinander engen Kontakt aufnehmen, könnte es schwierig oder unangenehm werden. Das ist dann vielleicht frustrierend. Gerade geistige Behinderung ist schwierig, da das vielen Menschen fremd ist« (1467). Hier 
zeichnet sich, trotz aller Vorbehalte, ein gewisses Potenzial ab, Menschen mit geistiger Behinderung die Teilhabe an routinemäßigen Freizeitaktivitäten zu ermöglichen. Notwendig ist dabei in erster Linie, Kontaktmöglichkeiten zu schaffen, um möglicherweise bestehende Berührungsängste abzubauen.

\section{Tätigkeitsbezogene Schwierigkeiten und rechtliche Hürden}

Ähnlich wie im Handlungsfeld Arbeit werden Schwierigkeiten bei der Teilhabe an Freizeitaktivitäten teils in der jeweiligen Tätigkeit selbst gesehen, die unpassend für bestimmte Personen sei und woraus in der Folge Teilhabebeschränkungen errichtet würden. Eine Interviewperson sagt: „Beim Tauchen ist es zum Beispiel so, dass Verlässlichkeit ganz wichtig ist, denn der Tauchpartner muss bei Not aus dem Wasser gebracht werden. Der Schwächere bestimmt den Verlauf des Tauchgangs« (5149). Die Möglichkeit, die Tätigkeit zu verändern, wird von vielen Interviewpersonen nicht eingeräumt oder sie haben keine Idee, inwiefern die jeweilige Tätigkeit verändert werden könnte. Darüber hinaus werden teils auch rechtliche Hürden gesehen, was sich fast ausschließlich darauf bezieht, dass es für die Teilnahme an der je bestimmten Freizeitaktivität einer Prüfung bedarf (zum Beispiel Angelschein, Waffenschein etc.), die, davon gehen einige Interviewpersonen aus, nicht alle potenziellen Teilnehmenden ablegen können oder dürfen. Inwiefern dies tatsächlich zutrifft, ist einzelfallabhängig und hängt beispielsweise vom Status der Betreuung ab (insofern ein solcher vorliegt). Was sich hier zeigt, ist, dass die Interviewpersonen teilweise nur wenig über Beeinträchtigung und daraus folgende rechtliche Hürden wissen. Teilhabe braucht also auch Information und Wissen aufseiten der Personen, die Freizeitaktivitäten repräsentieren und infolgedessen oftmals die ersten Ansprechpersonen sind, wenn Interesse an einer Teilnahme besteht.

\section{Personal hat keine Expertise}

Sehr häufig problematisieren die Interviewpersonen, dass diejenigen, die das jeweilige Freizeitangebot durchführen oder leiten (zum Beispiel TrainerInnen etc.), nicht die notwendige Expertise haben, um Menschen mit unterschiedlichen Unterstützungsbedarfen eine Teilnahme zu ermöglichen. Den jeweiligen Personen fehlten spezielle Schulungen oder Weiterbildungen, die sie dafür qualifizieren, unterschiedliche Personen in das Freizeitangebot einzubinden, geben einige Interviewpersonen zu bedenken. Eine Interview- 
person differenziert diesbezüglich und sieht Schwierigkeiten insbesondere in Bezug auf Menschen mit Demenz und Menschen mit Behinderung. Sie sagt: »Bei Flüchtlingen ist alles top, da gibt es keine Schwierigkeiten. Ein oder zwei Demenzkranke oder Behinderte pro Angebot sind vielleicht auch noch möglich, aber alles, was darüber hinaus geht, nicht. Die Übungsleiter sind nicht ausgebildet und eine separate Betreuung ist unmöglich (8655). Es zeigt sich, dass Menschen mit unterschiedlichen Unterstützungsbedarfen primär über ein antizipiertes Defizit wahrgenommen werden, aus dem heraus besondere Qualifikationen im Umgang notwendig werden. Freizeit bekommt so einen quasi-therapeutischen Charakter verliehen, entlang dessen Behinderung oder Demenz als medizinisches Problem (re-)produziert werden.

\section{Mangelnde Barrierefreiheit}

Einige Interviewpersonen sehen eine Schwierigkeit bei der Teilnahme von Menschen mit unterschiedlichen Unterstützungsbedarfen darin, dass die Örtlichkeiten nicht barrierefrei sind, in denen die Freizeitaktivität stattfindet. Barrierefreiheit wird dabei beinahe ausschließlich in Bezug auf Mobilität gedacht, beispielsweise dann, wenn Treppen zur Freizeitaktivität führen, die nicht per Rampe oder Aufzug umgangen werden können. Eine Interviewperson kommt im Zuge der Reflexion von Barrierefreiheit auf ambivalente Herausforderungen und Ansprüche zu sprechen: »Man müsste dann das Programm in Bezug auf Inklusion anders gestalten. Ein Problem dabei ist, dass sich solche Angebote mit dem beißen, was die Leute kennen und erwarten. Wenn man jetzt zum Beispiel ein Biotop komplett erschließt, damit es auch für Rollstuhlfahrer zugänglich ist, dann hat man ja einen Stadtpark. Die Wege sind manchmal schon für Menschen ohne Behinderung schwer zugänglich, weil sie nicht befestigt sind, und müssten also erstmal für Menschen mit körperlicher Behinderung zugänglich gemacht werden« (2794). Die Argumentation der Interviewperson ist durchaus nachvollziehbar und es wird deutlich, dass Barrierefreiheit respektive die damit oftmals verbundenen Forderungen durchaus ambivalent sind. Eine barrierefreie Natur ist kaum denkbar, dennoch muss reflektiert werden, dass dadurch bestimmte Personen, insbesondere jene, die Unterstützungsbedarf in den Bereichen Mobilität oder Orientierung haben, davon ausgeschlossen werden, naturnahe Freizeitaktivitäten zu verfolgen. Dadurch, dass jene Interviewperson über diese Problematik nachdenkt, zeigt sich ein gewisses Bewusstsein für 
diverse Bedarfe. Eine solche Haltung und ein solches Reflexionsvermögen in der breiten Bevölkerung zu verankern, ist sicherlich hilfreich, um auf Teilhabebarrieren aufmerksam zu machen und schließlich zu ihrem Abbau beizutragen, ist Bewusstsein und Infragestellen doch oftmals der erste Schritt hin zu einem Ermöglichen von Teilhabe.

\section{Sicherheitsbedenken, Gefahren}

Immer wieder kommt es vor, dass Interviewpersonen Teilhabeschwierigkeiten, von in erster Linie Menschen mit Behinderung und Menschen mit Demenz, darin sehen, dass die jeweilige Freizeitaktivität gefährlich sei und die Sicherheit jener Personen bedrohe. Der/die PrimärvertreterIn eines Bogensportclubs sagt: »Generell sehe ich keine Schwierigkeiten, außer, die Menschen sind körperlich zu stark eingeschränkt und haben eine starke geistige Behinderung, sodass die Sicherheit nicht mehr gewährleistet ist« (8311). Ein weiteres Beispiel ist die Aussage des Primärvertreters/der Primärvertreterin eines Turnvereins. Er/sie sagt: »Zum Teil ist es so, dass die Geräte zu gefährlich sind« (9783). Menschen mit Behinderung und Menschen mit Demenz werden dadurch als besonders gefährdet konstruiert und so, als seien sie nicht dazu in der Lage, auf sich selbst zu achten. Erschwert wird dies dadurch, dass die sogenannten Gefahren routinemäßig nicht als solche eingestuft würden, beispielsweise sagt eine Interviewperson, ein bestimmter Kampfsport sei zu gefährlich. Inwiefern diese Gefahr für Menschen mit Unterstützungsbedarfen höher sein soll als für Menschen ohne ebenjene Bedar$\mathrm{fe}$, wird nicht weiter erläutert. Hieran zeigt sich, dass ein Bild von Menschen mit Behinderung oder Demenz als schwach und hilfebedürftig manifestiert wird, als Personen, auf die besondere Rücksicht genommen werden muss. Maßnahmen, die die Teilhabemöglichkeiten von Menschen mit unterschiedlichen Unterstützungsbedarfen erweitern sollen, müssen also vor allem darauf abzielen, jene festgefahrenen Bilder aufzuweichen und durch einen Blick zu ersetzen, der weniger vorurteilsbelastet ist, als es bislang der Fall ist.

Vorurteile und geringe Offenheit (der Mitglieder)

Insgesamt offenbaren die Aussagen der Interviewpersonen viel seltener (mehr oder minder latenten) Rassismus oder Behindertenfeindlichkeit als dies in Bezug auf das Handlungsfeld Arbeit noch der Fall war (siehe Kapitel 16.7 und Kapitel 16.9.1). Dies ist nicht überraschend, spricht dafür doch auch die Verteilung der einzelnen Typen, die im Handlungsfeld Freizeit deutlich 
aufgeschlossener waren als im Handlungsfeld Arbeit (siehe Kapitel 16.2 und Kapitel 17.2). Dennoch äußerten einige Interviewpersonen die Sorge, dass die Mitglieder der Freizeitaktivität sich vorurteilsbehaftet äußern könnten und sich Menschen mit Unterstützungsbedarfen infolgedessen teilweise »dumme Sprüche« (9296) anhören müssten. Ebenfalls problematisiert wurde teilweise, dass aufseiten der potenziellen Teilnehmenden mit Behinderung, Fluchtmigrationshintergrund und/oder Demenz eine zu geringe Offenheit herrsche. Eine Interviewperson gibt zu bedenken: »Man muss sich halt auch trauen, neue Menschen kennenzulernen« (2912). Teilhabe ermöglichen geht in dieser Hinsicht also nicht nur von der Mehrheitsgesellschaft aus, sondern auch von jenen Personen, die bislang Ausschluss erfahren, und kann insofern für diese krisenhaft sein und sie ganz neu herausfordern.

\section{Finanzielle Hürden}

Einige Interviewpersonen problematisieren zu hohe Kosten, die einer Teilhabe von Menschen mit unterschiedlichen Unterstützungsbedarfen im Wege stehen. Dabei wird sich nicht darauf bezogen, dass die jeweilige Freizeitaktivität gegebenenfalls zu teuer für einige Person wäre (was gerade in Bezug auf Menschen, die in einer sogenannten Werkstatt für Menschen mit Behinderung beschäftigt sind, durchaus zur Teilhabebarriere werden kann, da dort, wie oben bereits diskutiert, im Jahr 2017 der durchschnittliche Monatsverdienst lediglich 213,69€ betrug; BAG WfbM 2019), sondern dass dem Verein oder Träger der jeweiligen Freizeitaktivität selbst Kosten entstehen, wenn sie sich (mehr als bislang) für Personen mit diversen Unterstützungsbedarfen öffnen. Eine Interviewperson sagt beispielsweise: »Ein Problem ist halt einfach, dass wir zu wenig Geld haben, um alle möglichen Ansprüche an Barrierefreiheit umzusetzen« (6721). Dies mag oftmals richtig sein, ist aber dennoch Ausdruck für eine gewisse Inflexibilität, Barrieren kreativ und kostensparend abzubauen. Hinzu kommt, dass viele Barrieren nicht physisch manifest sind, sondern viel eher in mangelndem Bewusstsein und fehlender Offenheit aufseiten der Mehrheitsgesellschaft bestehen. Bewusstseinsbildende Maßnahmen können dem entgegenwirken und müssen nicht unbedingt teuer sein. 


\subsubsection{Handlungsperspektiven im Kontext von Inklusion und Freizeit}

Ein Großteil der Interviewpersonen skizziert keine Handlungsperspektiven, die die Teilhabemöglichkeiten von Personen erhöhen sollen, die Ausschluss erfahren. Diese Interviewpersonen begründen dies vor allem darin, dass sie in der von ihnen vertretenen Freizeitaktivität derzeit oder in absehbarer Zukunft keine Maßnahmen ergreifen oder dass sie bislang keine Ideen diesbezüglich entwickelt haben. Diese Einstellung entsteht nicht unbedingt aus einer ablehnenden Haltung heraus, sondern teils aus einer gewissen Befangenheit. Eine Interviewperson sagt beispielsweise: »Bei uns ist erstmal nichts geplant. Ich lasse das auf mich zukommen und reagiere dann angemessen. Wichtig ist ja sowieso ein möglichst unvoreingenommener, menschlicher Umgang (9587). So positiv wie diese Aussage einzuschätzen ist, zeigt sie doch auch, inwiefern Abwarten zur Teilhabebarriere werden kann. Sich selbst nicht als AkteurIn im Kontext Inklusion zu adressieren, kann zu Stillstand führen und dadurch wiederum Barrieren aufrechterhalten, die eine Teilhabe von Menschen mit unterschiedlichen Unterstützungsbedarfen behindern. Einige andere Interviewpersonen benennen dagegen konkretere Maßnahmen und Handlungsvorschläge, die sie dabei unterstützen (würden), Teilhabemöglichkeiten in der von ihnen repräsentierten Freizeitaktivität zu eröffnen. Diese sind im Folgenden problemzentriert zusammengefasst und werden anhand einzelner Aussagen von Interviewpersonen exemplarisch diskutiert.

\section{Begegnungsmöglichkeiten schaffen}

Immer wieder problematisieren Interviewpersonen, dass bislang zu wenig Kontakt zwischen den an der Freizeitaktivität Teilnehmenden und Menschen mit unterschiedlichen Unterstützungsbedarfen, die (bislang) nicht teilnehmen, besteht. In der Konsequenz wird eine Handlungsperspektive im Kontext Inklusion und Freizeit darin gesehen, Begegnungsmöglichkeiten zu schaffen, um so gegebenenfalls Vorbehalte abzubauen. Eine Interviewperson sagt: »Wir wollen mehr Begegnungen schaffen und organisieren, um in Kontakt mit Flüchtlingen zu treten. Außerdem wollen wir einen Sportanleiter einstellen, da wir die Idee haben, dass Sport verbindet« (1644). Eine andere Interviewperson schlägt vor: »Man müsste mehr Plattformen schaffen und mehr Treffpunkte, zum Beispiel Cafés« (6695). Sie sieht Schwierigkeiten bei der Kontaktaufnahme also vor allem darin, dass sich Menschen aus der Mehrheitsgesellschaft und jene, die von Ausschluss bedroht oder betroffen 
sind, im Alltag kaum begegnen. Offen bleibt, inwiefern Personen den $\mathrm{Zu}$ gang zu jenen speziellen Plattformen und Treffpunkten finden. Schließlich sei noch die Aussage dieser Interviewperson herangezogen: »Es sollte nicht nur über technische Sachen geredet werden, wie zum Beispiel Behindertentoiletten, sondern es sollten Begegnungen mit Menschen mit Behinderung geschaffen werden« (5920). Diese Kritik an einer Technisierung trifft den Kern einer zentralen Debatte um Inklusion, in der problematisiert wird, dass Inklusion nicht per Gesetz, Vorgabe oder anderer technisierender Weise sumgesetzt werden kann, sondern dass es sich dabei um einen Prozess handelt, der krisenhaft ist und der zuallererst Menschen betrifft, die Inklusion aushandeln müssen (Trescher 2015b, S. 333f, 2018b, 2017d).

\section{Barrierefreiheit verbessern}

Die Verbesserung barrierefreier Zugänge zu Freizeitaktivitäten und -angeboten ist ein Aspekt, den einige Interviewpersonen als Handlungsperspektive skizzieren. Teilhabemöglichkeiten resultieren in dieser Hinsicht aus einem Abbau von Barrieren, die Teilhabe bislang behindern. Interessant ist, dass viele Interviewpersonen neben physischen Barrieren insbesondere auch informationstechnologische thematisieren. Eine Interviewperson sagt beispielsweise: „Wir sind noch ganz am Anfang. Gerade bauen wir eine Behindertentoilette und überlegen, wie wir unsere Internetseite barrierefreier machen können. Wir planen, dass die Informationen über unsere Angebote in verschiedenen Sprachen gelesen werden können « (9168). Daraus kann gefolgert werden, dass aufseiten der PrimärvertreterInnen teilweise bereits eine gewisse Sensibilität für unterschiedliche Barrieren und Möglichkeiten ihres Abbaus besteht, auf der weiter aufgebaut werden kann. Während einige Interviewpersonen bereits konkrete Veränderungen vornehmen, problematisieren andere, dass zu wenig finanzielle Mittel zur Verfügung stehen, mit denen Umbau- oder andere Maßnahmen finanziert werden könnten. Eine Interviewperson sagt: »Wir brauchen unbedingt Geld für mehr Barrierefreiheit. Unsere Arbeit wird ausschließlich über Fördergelder finanziert und die sind aber oftmals gebunden an bestimmte Ausgaben, weshalb wir davon keine Umbauten oder so etwas bezahlen können« (6721). Es zeigt sich also, dass es, zumindest aus der Perspektive der Menschen vor Ort, einer größeren Flexibilität beim Einsatz von Fördergeldern bedarf. Daran ist darüber hinaus zu erkennen, inwiefern bürokratische Barrieren wirksam wer- 
den, was wiederum möglicherweise das Engagement der PrimärvertreterInnen einschränkt, wodurch letzten Endes Teilhabe behindert wird.

\section{Information und Bewusstseinsbildung}

Immer wieder kommt es vor, dass Interviewpersonen es als notwendig erachten, in der Breite der Bevölkerung mehr für unterschiedliche Unterstützungsbedarfe zu sensibilisieren, um Barrieren abzubauen. Eng verknüpft mit dem Thema Information und Bewusstseinsbildung ist die Forderung einiger Interviewpersonen, sich mehr für Toleranz und Offenheit einzusetzen, um so Diskriminierung und Ausschluss entgegenzutreten. Eine Interviewperson sagt: »Oft fehlt es an Akzeptanz, zum Beispiel gibt es Eltern, die denken, Behinderung sei ansteckend. Deshalb ist Aufklärung wichtig. Das Thema muss in die Mitte der Gesellschaft getragen werden. Eine Möglichkeit dazu wäre zum Beispiel, Lehrgänge anzubieten. Oft scheitert es ja an den kleinkarierten Geistern in Gesellschaft und Politik« (9150). An Aussagen wie diesen wird deutlich, dass Information und Bewusstseinsbildung als eine der zentralen Herausforderungen im Kontext Inklusion und Freizeit erkannt werden. Interviewpersonen, wie die hier zitierte, lassen einen hohen Grad an Reflexion erkennen, an dem es anzuknüpfen gilt, um eine Sensibilisierung der Mehrheitsgesellschaft voranzutreiben.

\section{Mitsprache von Menschen mit unterschiedlichen} Unterstützungsbedarfen

Einige Interviewpersonen sehen eine Handlungsperspektive, um Teilhabebarrieren abzubauen, darin, Menschen mit unterschiedlichen Unterstützungsbedarfen verstärkt in die Planung und Gestaltung von Freizeitaktivitäten miteinzubeziehen. Diese Aufgabe stelle sich, so wird es teils betont, gerade im Kontext Inklusion. Eine Interviewperson sagt beispielsweise: "Betroffene müssen gefragt werden, die wissen doch ganz genau, wo es noch Probleme gibt « (5638). Dies sei, so eine andere Interviewperson, insbesondere bei der Arbeit in Gremien notwendig (2915). Aussagen wie diese zeigen, dass die Expertise von Menschen mit Unterstützungsbedarfen anerkannt werden soll. Sie sollen als ExpertInnen in eigener Sache auftreten. Darin liegt allerdings die Ambivalenz, dass eine solche Expertise untrennbar an den Status sunterstützungsbedürftig` geknüpft ist und sich die Person infolgedessen nicht davon lösen kann. Vielmehr wird sie als Mensch mit Unterstützungsbedarfen reproduziert und ist so - nach wie vor - nicht uneingeschränkt 
Teil der Mehrheitsgesellschaft. Demgegenüber gibt es Interviewpersonen, die Möglichkeiten der Beteiligung primär darin sehen, dass sich sogenannte Betroffene gegen Inklusion (beziehungsweise das, was die jeweiligen Interviewpersonen darunter verstehen) aussprechen können. Eine Interviewperson sagt: »Die Menschen, also die mit geistiger Behinderung und so weiter, die sollten gefragt werden, ob sie Inklusion denn überhaupt wollen« (7491). Hier klingt ein gewisser Zweifel an, ob Inklusion überhaupt im Sinne der Menschen mit unterschiedlichen Unterstützungsbedarfen sei. Wie genau dieser begründet wird, bleibt zumeist unklar. Problematisiert werden kann allerdings, dass dadurch ebenfalls ein Stück weit infrage gestellt wird, ob Menschen mit Unterstützungsbedarfen überhaupt Ausschluss erfahren und ob dies etwas ist, dem entgegengesteuert werden sollte. In Aussagen wie der folgenden zeigt sich zudem, inwiefern ein vermeintlich protektiver Gedanke Ausschluss reproduzieren kann. Eine Interviewperson sagt: „Das mit der Inklusion ist eine schwierige Geschichte, die kann nicht einfach erzwungen werden. Man muss einen Weg dazwischen finden. Nicht jeder Behinderte ist in der Lage, bei Inklusion mitzumachen. Behinderte leiden eventuell darunter« (4647). Zudem zeigt sich hier, inwiefern ein unscharfes Verständnis von Inklusion vorherrscht, das grob damit umfasst wird salle sind bei allem dabei<. Es bedarf also auch einer Sensibilisierung dafür, was Inklusion bedeutet.

\section{Bessere Finanzierungsmöglichkeiten}

Die Frage danach, wie Finanzierungsmöglichkeiten erweitert werden können, treibt einige Interviewpersonen um, wie in den Interviews immer wieder deutlich wurde. Dies betrifft unter anderem die Finanzierung von Personal, Räumlichkeiten oder Werbung. Eine Interviewperson konkretisiert dies und sagt: »Wir brauchen finanzielle Mittel, um mehr Flüchtlinge aufnehmen zu können« (2759). Es wird also immer wieder das Schaffen von Teilhabemöglichkeiten unmittelbar an die Finanzierung je bestimmter Aspekte geknüpft, wobei die Interviewpersonen nicht näher darauf eingehen, wie genau aus (in ihren Augen) besseren Finanzierungsmöglichkeiten Teilhabe für Menschen hervorgehen soll, die bislang ausgeschlossen waren. Inklusion wird in diesem Zusammenhang also auch als Belastung verstanden, der finanziell begegnet werden muss. Festzuhalten bleibt, dass es neben einer entsprechenden finanziellen Ausstattung auch Ideen und Menschen braucht, die diese umsetzen. 


\section{Bürokratieabbau}

Vereinzelt problematisieren Interviewpersonen, dass bürokratische und verwaltungstechnische Vorgaben es ihnen erschweren, Menschen mit diversen Bedarfen den Zugang zu der von ihnen vertretenen Freizeitaktivität zu ermöglichen. Ein Problem sei dabei vor allem mangelnde Flexibilität, wie beispielsweise diese Interviewperson zu bedenken gibt: "Die ganzen Vorgaben, zum Beispiel beim Denkmalschutz oder Brandschutz, sind sehr unflexibel. Man bräuchte mehr Spielräume im Gesetz« (2915). Die Interviewperson bezieht sich hierbei annehmbar auf Bauvorhaben, die nur eingeschränkt umgesetzt werden können, was gerade in Bezug auf Umbauten entlang einer Idee von Barrierefreiheit dazu führen kann, dass bestimmte Personen kategorisch von der Freizeitaktivität ausgeschlossen werden. Eine andere Interviewperson hat konkrete Veränderungsvorschläge: »Man sollte eine Vermittlungsstelle schaffen. Förderanträge zu stellen, ist nämlich sehr kompliziert. Zum Beispiel fände ich eine bessere Unterstützung wichtig, wenn man ein Kulturzentrum eröffnen möchte« (9942). Ein Problem liegt also auch darin, dass bürokratische Vorgaben oft umfangreich und nur schwer verständlich sind. Dies ist gerade bei Freizeitvereinen ein Problem, die größtenteils auf ehrenamtlich Tätige angewiesen sind, die sich selbst in ihrer Freizeit engagieren und nicht unbedingt die notwendige Expertise haben, um bei Antragstellungen oder Ähnlichem mitzuwirken. Bürokratieabbau könnte also hier Ressourcen freisetzen, die dafür genutzt werden können, Teilhabemöglichkeiten für Menschen mit unterschiedlichen Unterstützungsbedarfen zu schaffen.

\section{Erfahrungen im Miteinander erforschen: Gesamtbetrachtung und Diskussion}

Nach der detaillierten Darstellung der Ergebnisse der ersten Sozialraumanalysen in den Handlungsfeldern Arbeit und Freizeit werden in diesem Kapitel zentrale Ergebnisse noch einmal einander gegenübergestellt. Zudem werden, wie in den vorangegangenen Kapiteln ebenfalls, theoretische, methodische und handlungspraktische Ergebnisse und Erfahrungen diskutiert und Anschlussmöglichkeiten abgewogen. 


\section{Gegenüberstellung der Ergebnisse}

In der nachfolgenden Tabelle sind die Ergebnisse der Kategorisierung entlang der vier Typen voll ablehnend, teilweise ablehnend, (noch) zurückhaltend und offen zusammengefasst. Die beiden Handlungsfelder Arbeit und Freizeit sind einander dabei gegenübergestellt.

Tabelle 38: Gegenüberstellung der Typisierungen in den Handlungsfeldern Arbeit und Freizeit

\begin{tabular}{|c|c|c|c|c|c|}
\hline & & \multicolumn{4}{|l|}{ Typ } \\
\hline & & $\begin{array}{l}\text { voll ab- } \\
\text { lehnend }\end{array}$ & $\begin{array}{l}\text { teilweise } \\
\text { ablehnend }\end{array}$ & $\begin{array}{l}\text { (noch) } \\
\text { zurückhal- } \\
\text { tend }\end{array}$ & offen \\
\hline \multirow{6}{*}{ 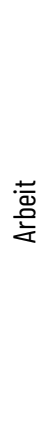 } & Erlangen & $16,2 \%$ & $48,6 \%$ & $24,3 \%$ & $10,8 \%$ \\
\hline & Rostock & $8,2 \%$ & $49 \%$ & $34,7 \%$ & $8,2 \%$ \\
\hline & Schneverdingen & $17,6 \%$ & $76,5 \%$ & $5,9 \%$ & $0 \%$ \\
\hline & $\begin{array}{l}\text { Schwäbisch } \\
\text { Gmünd }\end{array}$ & $19 \%$ & $35,7 \%$ & $21,4 \%$ & $23,8 \%$ \\
\hline & VG Nieder-Olm & $23,1 \%$ & $53,8 \%$ & $7,7 \%$ & $15,4 \%$ \\
\hline & $\varnothing$ & $16,8 \%$ & $52,7 \%$ & $18,8 \%$ & $11,6 \%$ \\
\hline \multirow{6}{*}{$\begin{array}{l}\frac{\sqrt{\mathrm{N}}}{\mathrm{N}} \\
\frac{\mathrm{N}}{\mathrm{W}}\end{array}$} & Erlangen & $4,2 \%$ & $39,6 \%$ & $31,3 \%$ & $25 \%$ \\
\hline & Rostock & $8,3 \%$ & $31,7 \%$ & $25 \%$ & $35 \%$ \\
\hline & Schneverdingen & $11,9 \%$ & $21,4 \%$ & $59,5 \%$ & $7,1 \%$ \\
\hline & $\begin{array}{l}\text { Schwäbisch } \\
\text { Gmünd }\end{array}$ & $3,4 \%$ & $33,9 \%$ & $32,2 \%$ & $30,5 \%$ \\
\hline & VG Nieder-Olm & $0 \%$ & $19,2 \%$ & $42,3 \%$ & $38,5 \%$ \\
\hline & $\varnothing$ & $5,6 \%$ & $29,2 \%$ & $38,1 \%$ & $27,2 \%$ \\
\hline
\end{tabular}


Die Gegenüberstellung der beiden Handlungsfelder Arbeit und Freizeit zeigt noch einmal übersichtlich, was oben bereits mehrfach angesprochen und problematisiert wurde: Die Interviewpersonen im Bereich Arbeit stehen einer Teilhabe von Menschen mit Behinderung und Menschen mit Fluchtmigrationshintergrund deutlich ablehnender gegenüber als dies im Bereich Freizeit der Fall ist, in dem ebenfalls die Teilhabemöglichkeiten von Menschen mit Demenz untersucht wurden. Während im Bereich Arbeit 16,8 \% der Interviewpersonen als voll ablehnend kategorisiert wurden, betrifft dies im Bereich Freizeit lediglich 5,6 \% der Interviewpersonen. Offen gegenüber der Teilhabe von Menschen mit Behinderung und Menschen mit Fluchtmigrationshintergrund (und Menschen mit Demenz) sind korrespondierend dazu im Bereich Arbeit 11,6\% der Interviewpersonen, im Bereich Freizeit dagegen $27,2 \%$. Folglich besteht insbesondere im Bereich Freizeit ein großes Potenzial, Teilhabemöglichkeiten für die Personen zu eröffnen, die bislang ausgeschlossen waren. Korrespondierend dazu zeigt sich, dass im Bereich Freizeit bereits deutlich häufiger Menschen mit Behinderung, Fluchtmigrationshintergrund oder Demenz an den von den interviewten Personen repräsentierten Aktivitäten teilnehmen, als dies im Bereich Arbeit der Fall ist (siehe Kapitel 16.5 und Kapitel 17.5). Es kann also davon ausgegangen werden, dass gemeinsame Praxen zu mehr Offenheit gegenüber je bestimmten Personen führen. Ein weiteres zentrales Ergebnis besteht darin, dass teilweise ein signifikanter Unterschied zwischen großstädtischen und kleinstädtischen Sozialräumen herausgearbeitet wurde, was den Vergleich dieser beiden Gruppen zulässt. Im Konkreten betrifft dies die Frage, inwiefern Interviewpersonen die Teilhabe von Menschen mit Behinderung, Menschen mit Fluchtmigrationshintergrund oder Menschen mit Demenz als möglich einschätzen (Kapitel 16.8 und Kapitel 17.9). Hier zeigt sich, dass im Handlungsfeld Arbeit die Unterscheidung Großstadt und Kleinstadt/Gemeinde mit der Einschätzung der Interviewpersonen bezüglich der Teilhabemöglichkeiten von Menschen mit Behinderung oder Menschen mit Fluchtmigrationshintergrund korreliert: In der Großstadt wird eine Teilhabe jener Personen an Arbeit eher als möglich eingeschätzt als in der Kleinstadt/Gemeinde. Die Lage des je individuellen Wohnortes kann also möglicherweise Einfluss darauf haben, ob es Menschen mit Behinderung oder Menschen mit Fluchtmigrationshintergrund gelingt, einen Arbeitsplatz zu finden beziehungsweise dort als KollegIn anerkannt zu werden. Im Handlungsfeld Freizeit dagegen bildet sich dies nicht ab, dort spielt die Lage des Wohnortes keine 
signifikante Rolle was die Teilhabemöglichkeit an freizeitlichen Aktivitäten angeht ${ }^{13}$. Deutliche Parallelen zwischen den beiden Handlungsfeldern Arbeit und Freizeit zeigt demgegenüber die Kontrastierung der Analysen offener Fragen, die in Schwierigkeiten und Handlungsperspektiven im Kontext von Inklusion und Arbeit beziehungsweise Freizeit zusammengefasst sind (siehe Kapitel 16.9 und Kapitel 17.12). Beispielsweise wird sowohl in Bezug auf Arbeit als auch in Bezug auf Freizeit problematisiert, dass mangelnde Barrierefreiheit oftmals zur Teilhabebarriere würde und die Möglichkeiten einschränke, an der eigenen Arbeitsstelle zu arbeiten oder an der von der Interviewperson vertretenen Freizeitaktivität teilzunehmen. Eine andere Schwierigkeit wird übereinstimmend darin gesehen, dass KollegInnen oder andere Teilnehmende je bestimmten Personen mit Vorurteilen begegnen würden und deshalb befürchten, diese würden nicht akzeptiert. Weiterhin argumentieren sowohl die Interviewpersonen aus dem Bereich Arbeit als auch jene aus dem Bereich Freizeit, die jeweils durch inklusionsorientierte Maßnahmen adressierten Personen seien (kognitiv und/oder körperlich) nicht in ausreichender Weise dazu in der Lage, die jeweilige (Arbeits- oder Freizeit-)Tätigkeit auszuüben. Jedoch ist es so, und darin ist ein zentraler Unterschied zwischen den beiden Handlungsfeldern zu erkennen, dass ausschließlich im Bereich Freizeit problematisiert wird, die Personen, die die jeweilige Freizeitaktivität gestalten (zum Beispiel TrainerInnen, ÜbungsleiterInnen etc.), hätten nicht die notwendigen Kenntnisse, um Personen miteinzubeziehen, die womöglich in der einen oder anderen Weise der Unterstützung bedürfen. Hieran zeigt sich, wie unterschiedlich diese beiden Handlungsfelder ausgestaltet sind und von den jeweiligen RezipientInnen wahrgenommen werden. Während sich im Bereich Freizeit die Interviewpersonen ganz offenbar teilweise selbst dafür verantwortlich sehen, Teilhabemöglichkeiten zu eröffnen oder Aktivitäten für unterschiedliche Teilnehmende zu eröffnen, schreiben im Bereich Arbeit die Interviewpersonen eine solche Verantwortung eher übergeordneten Stellen zu. Dass dies in gewisser Weise womöglich auf die

13 Unterschiede zwischen Croßstadt und Kleinstadt/Gemeinde können im Handlungsfeld Freizeit dagegen bezüglich der Frage dargelegt werden, ob und inwiefern es als möglich eingeschätzt wird, dass Teilnehmende der Freizeitaktivität dazu bereit sind, für Menschen mit Behinderung, Menschen mit Fluchtmigrationshintergrund oder Menschen mit Demenz Unterstützungsleistungen zu erbringen. Interviewpersonen aus der Kleinstadt/ Cemeinde schätzen dies eher als möglich ein als jene aus der Croßstadt (siehe Kapitel 17.11). 
Form der Erhebung zurückzuführen ist - Interviews mit Arbeitnehmenden versus Interviews mit PrimärvertreterInnen, die Verantwortung für die von ihnen repräsentierte Freizeitaktivität haben, - ist klar. Dennoch gibt dies ein Stück weit Aufschluss darüber, wie die Interviewpersonen im Gros die eigene Person im Kontext Inklusion und Arbeit beziehungsweise Freizeit verorten. Ein weiterer, besonders deutlicher Unterschied bezüglich Schwierigkeiten im Kontext Inklusion, der zwischen den Handlungsfeldern Arbeit und Freizeit ausgemacht werden kann, liegt darin, dass im Handlungsfeld Arbeit deutlich offener rassistische und/oder behindertenfeindliche Aussagen getroffen werden, als dies im Handlungsfeld Freizeit der Fall ist. Hier bildet sich qualitativ $a b$, was die obige Typenbildung deutlich zeigt: Interviewpersonen im Bereich Freizeit sind deutlich offener gegenüber der Teilhabe je bestimmter Personen als im Bereich Arbeit und nehmen also auch weniger menschenfeindliche Haltungen ein. Sowohl im Handlungsfeld Arbeit als auch im Handlungsfeld Freizeit zeigen die Analysen, dass die Interviewpersonen teils konkrete Vorschläge haben, wie an ihrem Arbeitsplatz oder bei ihrer Freizeitaktivität Teilhabemöglichkeiten eröffnet werden könnten. Übergreifend wird beispielsweise darauf aufmerksam gemacht, dass durch mehr Barrierefreiheit Menschen mit je bestimmten Unterstützungsbedarfen die Teilhabe an Arbeit und Freizeit ermöglicht werden könnte. Dass dabei ein eher eindimensionales Verständnis von Barrierefreiheit vorherrscht, ist eine Problematik, die theoretisch weiter durchdacht und empirisch untersucht werden muss. Daraus resultierend können, neben der tieferen theoretischen Durchdringung, handlungspraktische Hinweise dafür gegeben werden, wie Personen dafür sensibilisiert werden können, dass Barrierefreiheit durchaus ambivalent ist und es vor allem darauf ankommt, je situativ Barrieren auszuhandeln (siehe Kapitel 5.4 und Kapitel 11.3). Eine weitere Handlungsperspektive, die von einigen Interviewpersonen aus den Bereichen Arbeit und Freizeit formuliert wird, betrifft finanzielle Unterstützung für Unternehmen und Vereine etc., die diesen ermöglichen soll, sich für unterschiedliche Personengruppen zu öffnen. Daneben wird teils übereinstimmend der Abbau bürokratischer Vorgaben gefordert, um die eigenen Strukturen flexibler gestalten oder verwaltungstechnische Hürden überwinden zu können (insbesondere im Bereich Arbeit, wenn es um die Anerkennung von Abschlüssen und einer Arbeitserlaubnis geht). Interessant sind nun die Unterschiede zwischen den beiden Handlungsfeldern, die vor allem an zwei Themen festgemacht werden können. So zeigt sich, dass pri- 
mär im Bereich Freizeit mehr Begegnungsmöglichkeiten zwischen Menschen mit und Menschen ohne je bestimmte Unterstützungsbedarfe gefordert werden. Hier kann bereits eine gewisse Sensibilität und ein Erfahrungswissen darüber festgestellt werden, inwiefern der Abbau von Berührungsängsten und gemeinsame Lebenspraxen $\mathrm{zu}$ mehr Teilhabemöglichkeiten führen können. Die Interviewpersonen im Handlungsfeld Arbeit benennen dies größtenteils (noch) nicht als mögliche Perspektive beziehungsweise Handlungsmöglichkeit im Kontext Inklusion und Arbeit, was darauf hindeutet, dass es in diesem Bereich nach wie vor oftmals an gemeinsamen Erfahrungen mangelt. Die deskriptiv-statistischen Ergebnisse der Studie bestätigen das insofern, dass, wie oben bereits diskutiert, weniger Menschen mit unterschiedlichen Unterstützungsbedarfen am Bereich Arbeit teilhaben, als dies in Bezug auf Freizeitaktivitäten festgestellt werden konnte (siehe Kapitel 16.5 und Kapitel 17.5). Hier deutet sich darüber hinaus ein Bedarf an bewusstseinsbildenden Maßnahmen an. Eine zweite Handlungsperspektive konnte dagegen nahezu ausschließlich bei Interviewpersonen aus dem Handlungsfeld Arbeit festgestellt werden. Es häufen sich bei diesen die Stimmen, die für eine weitergehende Festigung behindertenspezifischer Beschäftigungsmöglichkeiten plädieren. Bemerkenswert ist, dass dies als eine Handlungsmöglichkeit im Kontext Inklusion gedacht wird. Im Handlungsfeld Freizeit findet sich diese Perspektive weniger und wenn, dann primär als Begründung dafür, warum bislang keine Menschen mit Behinderung (oder Fluchtmigrationshintergrund/Demenz) an der jeweiligen Freizeitaktivität teilnehmen. Handlungsperspektiven werden dort nahezu ausschließlich jenseits des Hilfesystems gedacht - was einem allgemeinen Verständnis von Inklusion näherkommt. An diesem Ergebnis zeigt sich, dass die Frage offenbleibt, was die Interviewpersonen im Konkreten unter Inklusion verstehen und, darüber hinaus, welche Bedeutung sie Inklusion im Kontext von Arbeit oder Freizeit zuschreiben.

\section{Theoretische Gesamtbetrachtung und Diskussion}

In einem Großteil der Interviews überwiegen Konstruktionen negativer Andersartigkeit, anhand derer Menschen mit Behinderung, Menschen mit Fluchtmigrationshintergrund und/oder Menschen mit Demenz pauschal als >das andere adressiert und hervorgebracht werden. Sehr deutlich zeigt sich immer wieder, inwiefern mit solchen Konstruktionen Praxen einhergehen, die schließlich in lebenspraktischem Ausschluss resultieren, da Absichten, 
Ideen und Erfahrungen darüber fehlen, gemeinsam zu arbeiten oder die Freizeit zu verbringen. Dies wirft die Frage auf, wie behindernde Praxen infrage gestellt werden können, sodass ihre ausschließenden Funktionen aufgeweicht werden. Es braucht also eine Idee von Dekonstruktion, anhand derer Behinderungspraxen und daraus resultierende Barrieren abgebaut werden können. Einleitend wurde diesbezüglich auf Derrida referiert, der in Begriff und Praxis der Dekonstruktion einführte und diese entscheidend prägte (siehe Kapitel 4.2). In der Praxis der Dekonstruktion wird ein Akt der "Selbstbefreiung des Denkens aus gewohnten Grenzziehungen und Hierarchisierungen [gesehen], insbesondere aus den herkömmlichen Dichotomien [...], die oft genug zur Rechtfertigung des Hegemonieanspruchs einer Kultur, Klasse (class), Rasse (race) oder eines Geschlechts (gender) über das andere missbraucht wurden " (Zapf 2013, S. 123f). Dies kann übertragen werden auf die dichotomen Kategorien sbehindert - nicht behindert<, sfluchtmigriert - nicht fluchtmigriert< und >dement - nicht dement<, mit denen ebenfalls je bestimmte Subjektpositionen einhergehen. Derrida stellt diesen Dichotomien eine Mehrdeutigkeit des Sinns entgegen (Münker und Roesler 2012, S. 141), in der Zuschreibungspraxen »äußerlich und kontingent« (Derrida 2016, S. 275) werden. Forschungsperspektiven eröffnen sich hierbei vor allem in Bezug auf eine weitere Konturierung des Theorems, das das Verhältnis von behindernden und dekonstruktivistischen Praxen ausleuchtet - insbesondere hinsichtlich des Verständnisses von Inklusion als Kritik. In Bezug auf die Konstruktion von Demenz überwiegt (noch deutlicher als in Bezug auf Behinderung) ein medizinischer Blick, der Demenz als Krankheit hervorbringt. Eine solche Konstruktion ist nicht nur bei den Interviewpersonen zu finden, sondern mehrheitsgesellschaftlich - und in den jeweiligen Bezugsdisziplinen - weit verbreitet. Dieser Konstruktion von Demenz als Krankheit kann die der Zuschreibungspraxis gegenübergestellt werden, anhand derer Demenz (ebenso wie Behinderung oder Fluchtmigrationshintergrund) als Praxis verstanden wird, die sich je situativ vollzieht und die so bezeichnete Person auf bestimmte, >demente Subjektpositionen festlegt. Demenz als Zuschreibungspraxis zu verstehen ist kein neuer Gedanke, in vielerlei Hinsicht allerdings einer, der nur von wenigen geteilt wird (u.a. sind hier zu nennen: Gronemeyer 2013; Whitehouse und George 2009; Wißmann und Gronemeyer 2008; Wißmann 2010; Trescher 2013; Trescher und Hauck 2015). Weitere Anschlussmöglichkeiten bezüglich des Ergebnisses, dass Menschen mit Behinderung, Menschen mit Fluchtmigrationshintergrund 
und/oder Menschen mit Demenz im Gros über ein attribuiertes negativ konnotiertes Defizit konstruiert werden, eröffnen sich hinsichtlich der Frage, inwiefern dies gegebenenfalls mit medial und öffentlich transportierten Bildern zusammenhängt, in denen Diversität primär über Differenz und/oder Stereotype vermittelt wird (Eckert 2014, S. 116ff; Reinhardt und Gradinger 2007, S. 102ff; Trescher 2017a, S. 37ff; Scholz 2010; Bosse 2006) ${ }^{14}$. Dabei ist auch die Rolle der Wissenschaft und ihre Beteiligung an der Reproduktion ebenjener Bilder und Haltungen noch nicht erschöpfend untersucht. Weiterhin muss problematisiert werden, dass auch im Rahmen dieser Studie die Kategorien >Behinderung ‘, Fluchtmigrationshintergrund und >Demenz $<$ im Forschungsprozess manifest wurden, um sich ihnen forschungspraktisch überhaupt annähern zu können. Diese manifesten Kategorien gilt es, bei der Diskussion der Ergebnisse nun wieder infrage zu stellen, sodass diese theoretisch dekonstruiert werden können. Weitere theoretische Anschlussmöglichkeiten ergeben sich mit Blick auf die beiden Handlungsfelder Arbeit und Freizeit. Hierbei steht die Frage im Vordergrund, wie Arbeit und Freizeit jeweils theoretisch fundiert werden können - insbesondere mit Blick auf Inklusion. Dazu bedarf es Studien, die jenseits der Untersuchung (behinderten-)spezifischer oder anderer protektiver Beschäftigungs- und Freizeitstrukturen verortet sind und die Themen Inklusion und Arbeit sowie Inklusion und Freizeit nicht aus einer primär bezugswissenschaftlichen Perspektive analysieren (beispielsweise aus Perspektive der Sonder- oder Sozialpädagogik). Forschung zum Thema Inklusion muss insofern bereits selbst inklusiv sein, dass durch sie nicht ausschließende Strukturen bestätigt und so reproduziert werden. Dekonstruktivistische Ansätze können hierbei die Möglichkeit zum Perspektivwechsel eröffnen. In diesem Zusammenhang ist darüber hinaus die Forschung zu Sozialräumen zu betrachten, die neben einer dezidierteren Beschreibung ihres Gegenstandes im Lichte raumtheoretischer Verstehenszugänge (siehe Kapitel 5) ebenfalls in Bezug auf Inklusion geschärft werden kann.

14 Eine problematisierende Auseinandersetzung mit der Darstellung von Behinderung in Flyern und Werbetexten der Aktion Mensch e.V. findet sich in Lingelbach 2010. 


\section{Methodische Gesamtbetrachtung und Diskussion}

Es hat sich gezeigt, dass die für die Sozialraumanalysen entwickelte Methodenkombination zu sinnhaften und breiten Ergebnissen führt. Dabei profitierten Erhebung und Auswertung in besonderer Weise von den Erfahrungen, die beim methodischen Design und der forschungspraktischen Ausgestaltung der Studie »Inklusion. Zur Dekonstruktion von Diskursteilhabebarrieren im Kontext von Freizeit und Behinderung« (Trescher 2015b) gemacht wurden. Zudem bestätigt sich erneut die Güte des Methodensettings, das für diese Studie entwickelt und hier in großen Teilen adaptiert wurde. Dass im Handlungsfeld Arbeit vom ursprünglichen Plan, ArbeitgeberInnen anhand eines Leitfadens $\mathrm{zu}$ interviewen, abgewichen werden musste (siehe Kapitel 16.1), eröffnete durch die persönliche Befragung von ArbeitnehmerInnen vor Ort die Möglichkeit, tieferen Einblick in das Miteinander am Arbeitsplatz zu nehmen, in das Vorgesetzte nicht immer unmittelbaren Einblick nehmen können (selbstredend abhängig davon, wie groß das jeweilige Unternehmen ist). Dadurch konnten Darstellungen und Einschätzungen gemeinsamen Arbeitens generiert werden, die deutlich näher an der Lebenspraxis sind, als dies andernfalls möglich gewesen wäre. Dies bekräftigt noch einmal, wie sinnvoll es ist, Pre-Tests durchzuführen - insbesondere bei einer so großen Studie, wie der hiesigen. Dennoch wäre es sehr interessant gewesen, die Perspektive der ArbeitgeberInnen (ebenfalls) untersuchen zu können, sind diese doch annehmbar diejenigen, die im Unternehmen mit darüber entscheiden, ob und wie Inklusion am Arbeitsplatz thematisiert wird, inwiefern dies gegenüber den MitarbeiterInnen kommuniziert wird und schließlich ganz konkret welche Personen eingestellt werden. In gewisser Weise betrifft diese Leerstelle auch das Handlungsfeld Freizeit, in dem zwar durch die Interviews mit sogenannten PrimärvertreterInnen für den Gegenstand und die Untersuchung zentrale Informationen und kurze Geschichten erhoben werden konnten, es aber dennoch interessant wäre, konkrete Erfahrungen zu sammeln von Teilnehmenden mit und ohne Behinderung, Fluchtmigrationshintergrund oder Demenz sowie von denjenigen, die die Freizeitangebote gestalten (insofern sie nicht selbst der/die PrimärvertreterIn sind und somit die Interviewperson waren). Es bleiben also einige Forschungsfragen offen, die möglicherweise in anschließenden oder weiteren Untersuchungen beforscht werden könnten. In Bezug auf die Auswertung des Materialkorpus kann nach Abschluss der Analysen festgestellt werden, dass sich das mehrdimensionale Auswertungsdesign im Gros bewährt hat. Insbesondere 
die Verknüpfung quantitativer und qualitativer Auswertungsverfahren hat sich als besonders gewinnbringend erwiesen, Ergebnisse zu generieren, die einen breiten Einblick in sowohl deskriptiv-statistische Verteilungen und Zusammenhänge als auch lebenspraktische Erfahrungen ermöglichen. Hervorzuheben ist dabei das Verfahren der Typenbildung, das sich als sinnvolle Methode erwiesen hat, um einen breiten und vielgestaltigen Datensatz vergleichbar zu machen und auf einen gemeinsamen Gegenstand zu beziehen. Das hier angewendete Verfahren der induktiven Kategorienbildung erlaubt, offen an das Material heranzutreten, was durch eine vorgängige Kategorienbildung nicht möglich gewesen wäre und die Breite der Ergebnisse sicherlich geschmälert hätte. Eine Problematik muss diesbezüglich allerdings hervorgehoben werden und zwar zeigt sich, dass kaum ein Bezug zwischen den Typen und der jeweiligen Ausgestaltung hergestellt werden kann. Denn es ist beispielsweise so, dass die Angaben einer Interviewperson zu ihrem Arbeitsort, der als barrierefrei in allen untersuchten Dimensionen verzeichnet wurde, nicht zwingend zur Folge haben, dass diese als aufgeschlossener kategorisiert wurde. Es ist also kaum möglich, die Typen auf die jeweiligen Aussagen zu beziehen (zum Beispiel Barrierefreiheit des Ortes, Vorhandensein eines sogenannten Behindertenparkplatzes etc.). Es zeigt sich, dass Teilhabemöglichkeiten von der je konkreten Tätigkeit abhängig sind und kaum verallgemeinerbare Aussagen getroffen werden können (beispielsweise in der Form, handwerklich ausgerichtete Betriebe seien besonders aufgeschlossen oder Ähnliches). Das bedeutet in der Konsequenz, dass es kaum sharte< Faktoren gibt, an denen der Grad von Inklusion am Arbeitsplatz oder Inklusion in der Freizeit 'gemessen rieren entstehen je individuell, ebenso wie Teilhabemöglichkeiten, weshalb es individuelle und je situative Herangehensweisen braucht, Barrieren abzubauen - weniger entlang einer statistischen Aussage als entlang einer geteilten Erfahrung. Dadurch wird die Herausforderung, Inklusion in Arbeit und Freizeit zu ermöglichen, komplex und vielschichtig.

\section{Handlungspraktische Gesamtbetrachtung und Diskussion}

Im Anschluss an die Darstellung der Ergebnisse der Sozialraumanalysen stellt sich die Frage, wie mit diesen handlungspraktisch weiter verfahren werden kann. Dabei kann beispielsweise aus einer solchen handlungspraktischen Perspektive heraus problematisiert werden, dass gerade Interviewpersonen des Typs voll ablehnend und des Typs teilweise ablehnend 
insbesondere durch sensibilisierende und bewusstseinsbildende Maßnahmen angesprochen werden sollten. Da bei den meisten dieser Personen die Konstruktion negativer Andersartigkeit von Menschen mit Behinderung, Fluchtmigrationshintergrund und/oder Demenz derart fest im Denken und Handeln verankert ist, muss davon ausgegangen werden, dass es hier längerfristiger Begleitung bedarf, um so möglicherweise ein Umdenken anzustoßen. Hilfreich können dabei auch sogenannte Generationenwechsel in Unternehmen, Einrichtungen, Vereinen etc. sein, da dadurch oftmals gängige Praxen hinterfragt und so für Veränderungen geöffnet werden können. Das größte Veränderungspotenzial - und damit das Potenzial, Teilhabemöglichkeiten für je bestimmte Personen zu eröffnen - zeichnet sich bei der Gruppe des Typs (noch) zurückhaltend ab. Hier steht neben bewusstseinsbildenden Maßnahmen, anhand derer Verantwortliche und Teilnehmende weitergehend informiert und sensibilisiert werden, primär das Schaffen von Begegnungsmöglichkeiten im Vordergrund, um (gegebenenfalls beiderseitig vorhandene) Vorbehalte abzubauen. Dies stützt sich vor allem auf das Ergebnis, dass gemeinsame Erfahrungen im Kontext Arbeit oder Freizeit durchweg positiv sind, woraus gefolgert werden kann, dass dort, wo sich Begegnung vollzieht, diese auch als gelungen und oftmals bereichernd wahrgenommen wird. Interviewpersonen, die dem Typ offen zugeordnet wurden, stehen einer Teilhabe von Menschen mit Behinderung, Menschen mit Fluchtmigrationshintergrund und/oder Menschen mit Demenz offen gegenüber. Hier gilt es nun dahingehend aktiv zu werden, interessierte Personen und Gruppen aneinander zu vermitteln, sodass das Potenzial auch genutzt wird, das Personen und die von ihnen dargestellten Unternehmen, Aktivitäten und Einrichtungen eröffnen. Teilweise haben die Interviewpersonen sehr konkrete Vorstellungen davon, wodurch Teilhabe behindert wird und wie Barrieren abgebaut werden können. In der Konsequenz wird dadurch klar, dass es Möglichkeiten zum Dialog braucht, damit Personen, die Ideen und Handlungsvorschläge haben, mit denen zusammenkommen und sich austauschen können, die im jeweiligen Ort Entscheidungsgewalt und Handlungsmacht haben. Denkbar wäre eine Art Bürgerdialog, den es in der einen oder anderen Form in vielen Städten und Gemeinden bereits gibt und in dem das Thema Teilhabe verstärkt platziert werden könnte. Da viele Interviewpersonen große Unsicherheit darüber bekunden, wie Menschen mit diversen Unterstützungsbedarfen am eigenen Arbeitsplatz oder bei der jeweiligen Freizeitaktivität miteinbezogen werden können, wäre es 
auch sinnvoll, mehr beratende und begleitende Angebote für Menschen aus der Mehrheitsgesellschaft zu etablieren, die unter anderem über finanzielle Unterstützungsmöglichkeiten Auskunft geben. Wichtig erscheint vor dem Hintergrund der Ergebnisse ebenfalls, mehr aufklärende und bewusstseinsbildende Maßnahmen in den Sozialräumen umzusetzen, um dadurch die Mehrheitsgesellschaft aufmerksamer für diverse Unterstützungsbedarfe zu machen und die Offenheit in Bezug auf Inklusion und Arbeit beziehungsweise Freizeit zu vergrößern. 


\section{Einstellung(en) zu Inklusion erforschen}

Ziel des Surveys »Einstellung(en) zu Inklusion« ist es, die Einstellungen der Bevölkerung Deutschlands zum Thema >Inklusion zu untersuchen. Dabei wird entlang der übergeordneten Forschungsfrage vorgegangen:

Tabelle 39: Forschungsleitende Fragestellung des Surveys »Einstellung(en) zu Inklusion«

Welche Einstellung(en) zu Inklusion haben die Menschen in Deutschland?

Im Zuge des Surveys konnten umfangreiche Daten erhoben und profunde Ergebnisse in Bezug auf die Einstellung(en) zu Inklusion in Deutschland generiert werden. Im nachfolgenden Kapitel werden einige dieser Ergebnisse exemplarisch dargelegt. Eine detaillierte Darstellung des Surveys und der dadurch gewonnenen Ergebnisse und Erkenntnisse ist an anderer Stelle veröffentlicht (Trescher et al. 2020b, 2020a).

\section{Zur wissenschaftlichen Relevanz}

Skalen zur Einstellungsmessung zum Thema Inklusion sind nicht nur international, sondern auch im deutschsprachigen Raum weit verbreitet. Argumentative Ausgangslage ist dabei im Regelfall, dass die Einstellung von Personen zu Inklusion mit Blick auf die Realisierung inklusiver Prozesse von entscheidender Bedeutung ist. Haben Personen der jeweils untersuchten Personengruppen eine positivere Einstellung zu Inklusion, so wird davon ausgegangen, dass sich dies günstig auf die Realisierung inklusiver Prozesse auswirkt und umgekehrt (Schwab et al. 2014, S. 21; Schwab und Seifert 
2015, S. 73; Gasterstädt und Urban 2016, S. 55; Seifried und Heyl 2016, S. 23). Interessant ist nun, dass, mit Blick auf den derzeitigen Forschungsstand, beinahe alle Skalen, die sich mit Einstellungsmessung im Kontext Inklusion beschäftigen, einzig den Lebensbereich Schule fokussieren. Die konstruierten Skalen richten sich dabei primär an die Beforschung von Eltern von Kindern mit und ohne Behinderung (Stoiber et al. 1998; Kunz et al. 2010) oder an (angehende) LehrerInnen (Abbott 2006; Bosse et al. 2016; Bosse und Spörer 2014; De Boer et al. 2011; Gebhardt et al. 2011; Hecht et al. 2016; Hellmich et al. 2016; Scholz und Rank 2016; Schwab et al. 2012; Schwab und Seifert 2015; Schwab 2015). Fragestellungen, die im Zuge dessen aufgeworfen werden, drehen sich darum, wie sich die jeweiligen Personen zu Inklusion positionieren. Fraglos stellt der Lebensbereich Schule einen zentralen Aspekt des Lebens eines Menschen dar. Hier werden maßgeblich Weichen für den weiteren Lebensverlauf gestellt und potenzielle zukünftige Lebensräume, innerhalb derer sich die Personen bewegen, erfahren und ausdifferenzieren können, mehr oder weniger (un-)flexibel vordefiniert beziehungsweise festgelegt. Gleichzeitig muss jedoch hervorgehoben werden, dass Schule keinesfalls als einziger relevanter Lebensbereich zu betrachten ist. Als mindestens ebenso bedeutsam erweisen sich, nicht zuletzt aufgrund ihrer lebenslangen Relevanz, weitere Lebensbereiche wie beispielsweise Freizeit, Arbeit und Wohnen. Diese werden im Fachdiskurs häufig ausgeblendet und im Rahmen von Studien nur selten berücksichtigt - gerade in der sonderpädagogischen Forschung (Trescher 2017a, S. 56; Buchner und Koenig 2008, S. 21; Beck und Schuck 2001; Langfeldt und Wember 1994). Dies gilt vor allem dann, sobald die Untersuchungen einen klaren Inklusionsbezug haben. Es reproduziert sich hierdurch ein Blick auf Inklusion, der diese einzig - oder zumindest doch in erster Linie - als schulische Herausforderung fasst. Hiermit einher geht eine sich stetig reproduzierende Kopplung von Inklusion an die Lebensalter Kindheit und Jugend, was ebenfalls zu hinterfragen ist. Dieser einseitigen Fokussierung auf Schule sollte mit dem Survey »Einstellung(en) zu Inklusion« ausdrücklich nicht gefolgt werden. 


\section{Konstruktion des Surveys, Pre-Test, Erhebung}

\subsection{Geistige Behinderung als Differenzkategorie}

Ausgehend von den obigen Überlegungen wurde der letztlich erhobene Survey entworfen. Er deckt vier Lebensbereiche ab: Freizeit, Arbeit, Wohnen und Schule. Mit Blick auf das formulierte Erkenntnisinteresse wird deutlich, dass sich dieses in mehrerlei Hinsicht als sehr komplex darstellt. Einerseits sollen möglichst alle zentralen Bereiche des Lebens abgedeckt werden, andererseits bezieht sich Inklusion - insbesondere in ihrem Verständnis als gesamtgesellschaftliche Herausforderung - auf einen äußerst heterogenen Personenkreis, mit je unterschiedlichen Voraussetzungen und Erfahrungen (beispielsweise Menschen mit Behinderung, Menschen mit Fluchtmigrationshintergrund oder auch Menschen mit Demenz). Um den je konkreten Lebenskontexten der verschiedenen Personen, die als primäre Zielgruppe sogenannter inklusiver Maßnahmen definiert werden können, zumindest in Ansätzen gerecht zu werden beziehungsweise diese im Rahmen der Auswertung zu berücksichtigen, wäre es nötig, für jeden einzelnen Personenkreis, der gegenwärtig in irgendeiner Art und Weise von Ausschluss betroffen ist, je eigene Fragen $\mathrm{zu}$ den jeweiligen Lebensbereichen $\mathrm{zu}$ stellen, um überhaupt so etwas wie eine differenzierte Bearbeitung des Surveys durch die Gesamtbevölkerung zu ermöglichen. In diesem Sinne finden sich beispielsweise verschiedene Studien, die darauf hinweisen, dass die Zugehörigkeit $\mathrm{zu}$ einem bestimmten Personenkreis dazu führen kann, dass sich die Offenheit beziehungsweise Ablehnung von inklusiven Prozessen unterschiedlich darstellt. So konstatieren zum Beispiel Scholz und Rank (2016, S. 6off), dass angehende LehrerInnen inklusiven Prozessen dann aufgeschlossener gegenüberstehen, wenn es sich dabei um Personen mit tendenziell geringeren lebenspraktischen Einschränkungen handelt - etwa Menschen mit Mobilitätsbeeinträchtigungen. Dementgegen positioniert sich die befragte Personengruppe weitaus kritischer, sobald es um Personen geht, denen gemeinhin höhere lebenspraktische Einschränkungen zugeschrieben werden - etwa Menschen mit sogenannten herausfordernden Verhaltensweisen und/ oder >geistiger Behinderung (Scholz und Rank 2016, S. 60ff) ${ }^{1}$. Der Anspruch,

1 Ähnliche Ergebnisse finden sich an anderer Stelle (Forlin et al. 1996; Avramidis und Norwich 2002; Kuhl und Walther 2008; Cebhardt etal. 2011; Lindemann 2016, S. 6ff; Schwab und Seifert 2015, S. 75). 
sowohl der Heterogenität der Lebenswelt (vertreten durch die Differenzierung nach Lebensbereichen) als auch der Heterogenität der von Ausschluss betroffenen Personen gerecht zu werden, würde den Umfang des Surveys um ein Vielfaches ausweiten und damit letztlich, aufgrund des massiv erhöhten Zeitaufwands der Beantwortung, auch den erwarteten Rücklauf beziehungsweise die Anzahl der vollständigen Beantwortungen deutlich schmälern. Damit der breite Lebensfeldbezug aufrechterhalten werden konnte, wurde sich für die Auswahl eines einzigen Personenkreises als Referenzpunkt für die Beantwortung der Fragen entschieden. Die Auswahl fiel dabei auf Menschen, die entlang gesellschaftlicher Klassifikationssysteme als 'geistig behindert gelten. Begründet liegt diese Auswahl darin, dass es oftmals gerade diese Personen sind, denen - wie oben bereits dargestellt im Kontext der Einstellung zu Inklusion verstärkt Ablehnung entgegengebracht werden. Ausdruck dessen ist unter anderem die gelegentlich hervorgebrachte Mahnung, Menschen mit 'geistiger Behinderung`dürften nicht zu sogenannten `InklusionsverliererInnen per 2015), was als Kritik an einer 'Tendenz des Übergehens verstanden werden kann (siehe hierzu auch Graumann 2016, S. 56; Trescher 2017a, S. 262f, 2017f, S. 197ff, 2015b, S. 329ff). Es wurde sich also für einen Personenkreis entschieden, der nicht selten als potenzielle »Grenze von Inklusion bzw. IInkludierbarkeit« (Trescher und Börner 2016, o.S.) gehandelt wird und als besonders von Ausschluss bedroht oder betroffen gilt. Angesichts dessen, dass sich Inklusion (zumindest im hier zugrunde gelegten Verständnis) explizit von Zuschreibungen wie inkludierbar beziehungsweise nicht inkludierbar distanziert, erscheint die Fokussierung eines besonders von Ausschluss gefährdeten Personenkreises bedeutsam, um sich der Frage zu nähern, wie sich Menschen der Gesamtbevölkerung zu Inklusion positionieren.

\subsection{Zur Konstruktion und Testung des Fragebogens}

Ziel des Surveys war, wie oben dargelegt, die Einstellung(en) der Gesamtbevölkerung Deutschlands zu Inklusion zu beforschen. Zentrale Referenzkategorien der Einstellungsforschung waren die Lebensbereiche Wohnen, Arbeit, Freizeit und Schule ${ }^{2}$ sowie die Personengruppe Menschen mit sogenannter

2 Da der Lebensbereich Schule sich in der Auswertung als nicht relevant für die Einstellungsmessung zu Inklusion herausstellte, wird in diesem exemplarischen Einblick in die Ergeb- 
geistiger Behinderung. Forschungspraktisch wurden $\mathrm{zu}$ jedem Lebensbereich Thesen formuliert, $\mathrm{zu}$ denen sich die am Survey teilnehmenden Personen verhalten sollten. Hierfür wurden unipolare Likert-Skalen mit einem Ratingspektrum von 1 bis 7 ( $1=$ Stimme überhaupt nicht $\mathrm{zu} ; 7=$ Stimme voll und ganz $\mathrm{zu}$ ) konstruiert und den Thesen beigefügt. Im Kern wurden die Thesen so gestaltet, dass sie, abgesehen von ihrem je kontextspezifischen Bezug, gleich beziehungsweise (möglichst) ähnlich formuliert waren, um eine spätere Gegenüberstellung der Lebensbereiche zu ermöglichen. Gearbeitet wurde dabei entlang eines festgelegten Kategoriensystems, anhand dessen die Thesen inhaltlich ausgestaltet wurden. Folgende Kategorien wurden berücksichtigt:

Tabelle 40: Itemkategorien (1)

\begin{tabular}{|l|l|}
\hline & Kategorien \\
\hline 1 & Unterstützung \\
\hline 2 & Engagement \\
\hline 3 & Erfordernisse \\
\hline 4 & Adressatlnnenauswirkung \\
\hline 5 & Umsetzung \\
\hline 6 & Geteilte Lebenspraxis \\
\hline 7 & Protektion \\
\hline 8 & Finanzierung \\
\hline 9 & Inklusion als Aufgabe \\
\hline 10 & Ausgrenzung \\
\hline 11 & Veränderungen \\
\hline 12 & Schulungsbedarfe \\
\hline 13 & Überforderung \\
\hline
\end{tabular}

Zusätzlich zu jenen lebensbereichsvergleichenden Thesen wurden darüber hinaus Thesen formuliert, die bestimmte Aspekte der einzelnen Lebensbe- 
reiche aufgreifen. Hierüber sollte der Besonderheit der jeweiligen Lebensbereiche entsprochen und über die Ebene des Vergleichs zwischen den Lebensbereichen hinausgegangen werden. Insgesamt wurden sowohl negative als auch positive Formulierungsvarianten für die Thesen gewählt. In seiner ersten Version umfasste der Survey je Lebensbereich 21 Items. Im Anschluss an seine Konstruktion wurde der Fragebogen im Rahmen zweier Pre-Tests erprobt und jeweils überarbeitet. Während es im ersten Pre-Test $(\mathrm{N}=100)$ vor allem um die Formulierung und Verständlichkeit der im Fragebogen offerierten Thesen ging (Beobachtung beziehungsweise Begleitung der Bearbeitung des Fragebogens mitsamt anschließender offener Feedbackgespräche), wurden im Rahmen des zweiten Pre-Tests $(\mathrm{N}=370)$ stärkere Anpassungen vorgenommen. Grundlage hierfür war eine erste Faktorenanalyse, die durch die Offenlegung der latenten Zusammenhänge unter anderem statistisch fundierte Kürzungspotenziale des Fragebogens aufzeigte. Hierüber war es möglich, den Fragebogen zu verdichten und die Bearbeitungszeit zu senken. Zugleich bestätigte die Faktorenanalyse die bereits im Vorfeld bestehende Annahme, dass sich der Lebensbereich Schule nicht mit den anderen drei Lebensbereichen vergleichen lässt ${ }^{3}$. Im Anschluss an die skizzierten Arbeitsschritte wurde die finale Version des Fragebogens erstellt, wobei sich je Lebensbereich auf 15 Thesen beschränkt wurde. Hinzu kommt ein Abschlussteil, in dem neben personenbezogenen Daten (Alter, Geschlecht, Postleitzahl, höchster Bildungsabschluss, Hauptbeschäftigung) unter anderem auch nach einem lebensgeschichtlichen persönlichen Kontakt zu Menschen mit Behinderung im Allgemeinen sowie Menschen mit geistiger Behinderung im Besonderen gefragt wird, was mit Blick auf die spätere Auswertung der Daten, gerade in Anlehnung an die sogenannte 'Kontakthypothese (Allport 1954; Cloerkes 2007), als relevantes Kriterium für eine offene beziehungsweise ablehnende Haltung gegenüber der gewählten Thematik eingestuft wurde (Trescher 2015b, S. 118f; siehe Kapitel 6.2). Gleiches gilt für die Frage danach, ob bei der jeweils ausfüllenden Person gegebenenfalls selbst eine (geistige)

3 Während die erstgenannten Bereiche bei den vergleichenden Thesen noch eine einheitliche Struktur aufwiesen, wurde diese im Kontext des Lebensbereichs Schule durchbrochen. Es wurde sich deshalb dafür entschieden, sSchule <im Rahmen der Haupterhebung separat zu führen und schwerpunktmäßig über lebensbereichsspezifische Thesen zu berücksichtigen, um der identifizierten Sonderstellung zu entsprechen beziehungsweise den Bereich in seiner Besonderheit zu würdigen. Der Lebensbereich Schule wurde deshalb einer ausführlichen Einzelauswertung unterzogen, deren Ergebnisse hier nicht abgebildet sind. 
Behinderung vorliegt oder nicht. Die Reihenfolge, in der die einzelnen Lebensbereiche durch die Befragten bearbeitet wurden, wurde randomisiert, um eine mögliche Beeinflussung des Antwortverhaltens (Priming-Effekte), die eine festgelegte Abfolge der Lebensbereiche womöglich mit sich bringen könnte, bestmöglich zu verhindern.

\subsection{Haupterhebung}

Die Haupterhebung des Surveys erfolgte anhand eines deutschlandweiten Panels, das repräsentativ die Bevölkerung Deutschlands abbildet. Die Befragung wurde in zwei Wellen durchgeführt, in denen - nach Bereinigung des Datensatzes (unter anderem, um sogenannte Erwünschtheitsantworten herauszufiltern) - eine Anzahl von 3695 Teilnehmenden erreicht werden konnte. Die Stichproben wurden zusätzlich zur Quotierung gewichtet (ausgehend von den Merkmalen Alter, Geschlecht und Religion). Die Stichprobe setzt sich zu 49,85\% aus Frauen und zu 49,82 \% aus Männern zusammen. $0,32 \%$ der Befragten machten hinsichtlich ihres Geschlechts keine Angabe. Das Durchschnittsalter der Teilnehmenden beläuft sich auf 48,81 Jahre (SD 16,045). Befragt wurden Personen zwischen 18 und 95 Jahren. Nach eigenen Angaben haben 11,64 \% der befragten Personen einen Migrationshintergrund. Zum Zeitpunkt der Befragung waren mehr als zwei Drittel der Personen hauptbeschäftigt angestellt ( $43,09 \%$ ) oder im Ruhestand (25,85\%). Etwa die Hälfte $(47,01 \%)$ gab an, regelmäßig Kontakt zu Menschen mit Behinderung (nicht nur geistige Behinderung) zu haben. Von diesen wiederum gaben 67,13\% an, regelmäßig Kontakt zu Menschen mit geistiger Behinderung $\mathrm{zu}$ haben (dies entspricht 31,56\% der gesamten Stichprobe). Nach eigenen Angaben haben 12,29\% der Befragten selbst eine Behinderung. 1,19\% der Teilnehmenden gaben an, selbst eine geistige Behinderung zu haben.

\section{Auswertung: Clusteranalyse}

Die Frage nach der Einstellung der Gesamtbevölkerung zu Inklusion wurde über die Durchführung einer Clusteranalyse operationalisiert, die sich im Wesentlichen dadurch kennzeichnet, dass innerhalb des Datensatzes nach signifikanten Beantwortungsmustern (Clustern) gesucht wird, die in der Folge klassifiziert und beschrieben werden. Die Grundlage für die Clus- 
teranalyse bildete eine weitere Faktorenanalyse, mittels derer die innere Struktur des Datensatzes bestimmt wurde. Diese bestätigte das Ergebnis der gleichförmigen Strukturierung der Lebensbereiche Wohnen, Arbeit und Freizeit, wie sie bereits im Zuge des Pre-Tests festgestellt wurde. Die Analyse offenbarte jeweils für die vergleichenden Items von acht der generierten Kategorien, die die Gegenüberstellung und den Vergleich der verschiedenen Lebensbereiche gewährleisten, eine konsistente Zweifaktorenstruktur. Auf dem ersten Faktor, der sich als >Befürwortung von Inklusion ‘ bestimmen lässt, luden in jedem Lebensbereich jeweils die gleichen fünf Items. Die übrigen drei Items hingegen luden auf dem zweiten Faktor, der sich als >Ablehnung von Inklusion ‘ fassen lässt. Die übrigen vergleichenden Items ließen sich nicht klar zuordnen (unter anderem aufgrund von Doppelladungen) und wurden insofern aus der Analyse ausgeklammert. Dies betraf die Items der Überkategorien »Inklusion als Aufgabe«, »Ausgrenzung«, »Veränderungen«, "Schulungsbedarfe« und "Überforderung«. Für die Clusteranalyse blieben insofern die Items folgender Überkategorien in den Lebensbereichen Wohnen, Arbeit und Freizeit bestehen:

Tabelle 41: Itemkategorien (2)

\begin{tabular}{|l|l|}
\hline & Kategorien \\
\hline 1 & Unterstützung \\
\hline 2 & Engagement \\
\hline 3 & Erfordernisse \\
\hline 4 & Adressatlnnenauswirkung \\
\hline 5 & Umsetzung \\
\hline 6 & Geteilte Lebenspraxis \\
\hline 7 & Protektion \\
\hline 8 & Finanzierung \\
\hline
\end{tabular}


Um die Aussagen der verschiedenen Faktoren im Hinblick auf die Einstellung(en) zu Inklusion miteinander vergleichen zu können, musste die LikertSkala der Items, die auf dem inklusionsablehnenden Faktor luden, umgepolt werden, damit in diesen Fällen - wie auch bei Items des inklusionsbefürwortenden Faktors - ein hoher Zustimmungsgrad der Items einen hohen Zustimmungsgrad zu Inklusion bedeutet und umgekehrt. Beispielhaft veranschaulicht heißt das: Der fiktive Zustimmungsgrad einer Person von $80 \%$ in Bezug auf die inklusionsablehnende Aussage »Wenn an meinem Arbeitsplatz Menschen mit geistiger Behinderung integriert würden, würde ich lieber meinen Arbeitsplatz wechseln « bedeutet, dass die Person eine eher negative Einstellung zu Inklusion hat. Im Zuge der Umpolung werden aus den $80 \%$ nun $20 \%$. Dieser Wert stellt infolgedessen nicht mehr den Zustimmungsgrad zur Aussage dar, sondern, und darin liegt ein zentrales Ergebnis der Analyse, basiert vielmehr auf einer inneren Kohärenz des Datensatzes, die als Zustimmungsgrad zu Inklusion gefasst werden kann. Sind im Folgenden Zustimmungsgrade abgebildet, so stellen diese nicht den Zustimmungsgrad zur Aussage dar, sondern immer den Zustimmungsgrad zu Inklusion. Auf dieser Grundlage wurde anhand des Datensatzes eine Clusteranalyse entlang des Ward-Verfahrens ${ }^{4}$ durchgeführt. Zur Identifizierung der geeignetsten Clusterlösung wurden jeweils pro Clusterlösung eine Einfaktorielle

4 Die Clusteranalyse, die zu den explorativen Verfahren der multivariaten Datenanalyse gehört, fasst unterschiedliche Verfahren zur Gruppenbildung zusammen. Dabei unterscheiden sich die Verfahren hinsichtlich des Proximitätsmaßes und der Wahl des Cruppierungsverfahrens (Backhaus et al. 2018). Das agglomerative Ward-Verfahren gehört zu den hierarchischen Clusterverfahren, die nicht von einer gegebenen Cruppierung der Objekte und einer festgelegten Anzahl an Clustern ausgehen, sondern die zu gruppierenden Objekte schrittweise zu immer größeren Clustern zusammenfassen (Backhaus et al. 2018). Die Anwendung des Verfahrens setzt metrische Merkmale voraus, die hier gegeben sind. Das Ward-Verfahren vereinigt diejenigen Objekte, die die Fehlerquadratsumme in einer Cruppe am wenigsten erhöhen und bildet somit möglichst intern homogene Cluster. Dadurch kann das Ziel erreicht werden, möglichst homogene Gruppen zu identifizieren, die in ihrer Einstellung zu Inklusion gleich oder sehr ähnlich sind. 


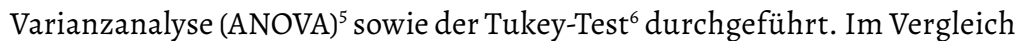
stellte sich die Vier-Cluster-Lösung als beste Clusterlösung heraus, da sich dort alle vier Cluster signifikant $(\mathrm{p}<=.00)$ voneinander unterscheiden sowohl bei der Einfaktoriellen Varianzanalyse als auch beim Tukey-Test. Des Weiteren verweisen die Werte des Etaz bei allen sechs Faktoren auf eine große Effektstärke (nach Cohen 1988, S. 79ff). Dies bedeutet, dass ein großer Prozentsatz (mind. 58,5 \%) der beobachteten Variationen der Zustimmungswerte auf die Clusterzugehörigkeit zurückgeführt werden kann. Die identifizierten Cluster sind damit das Ergebnis statistischer Berechnungen, die in der Folge $\mathrm{zu}$ interpretieren beziehungsweise inhaltlich $\mathrm{zu}$ bestimmen sind. Die vier Cluster lassen sich insofern auch als eine Form der Typisierung verstehen, die entlang des Antwortverhaltens der TeilnehmerInnen erstellt wurde und die statistisch fundiert ist.

5 Die Einfaktorielle Varianzanalyse beschreibt im Wesentlichen ein Verfahren, bei dem über einen Mittelwertvergleich der Frage nachgegangen wird, ob sich signifikante - also statistisch aussagekräftige - Unterschiede zwischen mehreren unabhängigen Cruppen in Bezug auf eine abhängige Variable finden lassen (Diekmann 2016, S. 694ff). Im hiesigen Zusammenhang ging es folglich um die Frage, ob sich in dem verfügbaren Datensatz tatsächlich belastbar unterschiedliche Typen (Cluster) in Bezug auf die »Einstellung zu Inklusion« (abhängige Variable) finden und beschreiben lassen.

6 Mit dem signifikanten Ergebnis der Einfaktoriellen Varianzanalyse wird zunächst lediglich die Aussage getroffen, dass es einen Unterschied zwischen den Cruppen gibt, allerdings nicht, worin dieser liegt. Um dies zu identifizieren, muss ein Post-hoc-Test herangezogen werden. Die paarweisen Vergleiche des Post-hoc-Tests suchen durch sogenannte multiple Mittelwertvergleiche nach signifikanten Unterschieden zwischen den Gruppen. Eine besondere Eigenschaft des Tukey-Tests ist dabei, dass das Fehlerniveau konstant nahe $5 \%$ gehalten wird. Die Anwendung des Tukey-Tests ist zu empfehlen, wenn Varianzhomogenität gegeben und die Cruppengröße gleich ist. Die Varianzhomogenität wurde in der hiesigen Auswertung zuvor mit dem Levene-Test bestätigt. Allerdings handelt es sich bei den generierten Clustern um ungleiche Cruppengrößen. Das Statistikprogramm SPSS berechnet in diesem Fall automatisch den Tukey-Kramer-Test, der speziell für ungleiche Gruppengrößen entwickelt wurde, jedoch in der Interpretation identisch ist. 


\section{Ergebnisse}

\subsection{Zusammenfassung und Charakterisierung der Cluster}

Wie oben dargestellt, konnten insgesamt vier Cluster unterschieden werden. Cluster 1 fasst diejenigen Personen zusammen, die über alle Lebensbereiche hinweg einen hohen Zustimmungsgrad zu Inklusion aufweisen und somit in allen Bereichen - Wohnen, Arbeit und Freizeit - eine positive Einstellung zu Inklusion haben. In Cluster 2 sind Personen gruppiert, die tendenziell einen höheren Zustimmungsgrad zu Inklusion aufweisen. Die Personen in Cluster 3 zeichnen sich durch mittlere Zustimmungsgrade aus. Die unter Cluster 4 subsumierten Personen zeigen in allen Lebensbereichen einen niedrigen Zustimmungsgrad zu Inklusion. Wichtig ist darauf hinzuweisen, dass die hier dargestellten Cluster nicht ohne Weiteres mit den Typen vergleichbar sind, die bei den Sozialraumanalysen im Kontext Arbeit und Freizeit herausgebildet wurden, da (unter anderem) eine unterschiedliche Interpretationsebene eingenommen wird (Einstellung vs. Typisierung), andere Aussagen getroffen werden (Einstellung vs. konkrete lebenspraktische Bezüge), unterschiedliche Designs zugrundeliegen (quantitativ vs. qualitativ) und sich der Einstellungssurvey ausschließlich auf Menschen mit geistiger Behinderung bezieht (siehe Kapitel 24). Dennoch ist bereits an dieser Stelle festzuhalten, dass erstaunliche Parallelen zwischen Einstellungssurvey und Sozialraumanalysen herausgearbeitet werden konnten, was für die Qualität des multiperspektivischen Forschungsdesigns der Gesamtstudie spricht. Ausgehend von den Werten wurde sich für die folgende inhaltliche Bestimmung der Cluster entschieden.

Tabelle 42: Benennung der vier Cluster: offen, eher offen, eher ablehnend, klar ablehnend

\begin{tabular}{|l|l|}
\hline Cluster 1 & offen \\
\hline Cluster 2 & eher offen \\
\hline Cluster 3 & eher ablehnend \\
\hline Cluster 4 & klar ablehnend \\
\hline
\end{tabular}


Nachfolgend werden die vier Cluster näher dargestellt und charakterisiert, anhand derer die Einstellung zu Inklusion der Personen abgebildet werden kann, die an der Befragung teilgenommen haben.

\subsubsection{Cluster 1 - Typ: offen}

In Cluster 1 sind die Personen zusammengefasst, die in allen Faktoren sehr hohe Zustimmungswerte zeigen und somit in allen Bereichen - Wohnen, Arbeit und Freizeit - eine positive Einstellung gegenüber Inklusion aufweisen. Den höchsten Zustimmungswert haben die Personen des Clusters 1 im Bereich Freizeit (mit einem Zustimmungsgrad von insgesamt 90,63\%), doch auch die anderen Bereiche sind durch eine sehr offene, positive Einstellung gegenüber Inklusion gekennzeichnet. So weist beispielsweise die Aussage »Inklusion erfordert, dass Menschen mit geistiger Behinderung (im selben Rahmen wie Menschen ohne geistige Behinderung) entscheiden können, wie, wo und mit wem sie wohnen möchten « mit der niedrigsten Zustimmung gegenüber Inklusion immer noch einen Zustimmungsgrad von 80,30 \% auf. Der durchschnittliche Zustimmungswert aller berücksichtigten Aussagen liegt bei $87,54 \%$.

\section{Personenbeschreibung}

Die Personen aus Cluster 1 sind im Durchschnitt 52,85 Jahre alt und lassen sich hauptsächlich in die Altersgruppe der 55- bis 64-jährigen einordnen (31,92\%). Die Geschlechterverteilung ist eher ausgeglichen, wobei etwas mehr Frauen (52,03 \%) als Männer (47,97\%) vorzufinden sind.

\section{Kontakt zu Menschen mit (geistiger) Behinderung}

In Cluster 1 haben oder hatten 60,00\% der Personen regelmäßig Kontakt zu Menschen mit Behinderung. Zu Menschen mit geistiger Behinderung haben oder hatten 43,37\% der Personen aus Cluster 1 regelmäßig Kontakt.

\section{Politisches Interesse und Zugehörigkeit}

Die Personen in Cluster 1 sind mittelmäßig bis sehr stark am politischen Tagesgeschehen interessiert. Insgesamt sind nur 16,16 \% der Personen wenig bis gar nicht politisch interessiert. Circa die Hälfte der Personen (50,85\%) ist ziemlich stark beziehungsweise stark politisch interessiert. Abgesehen von den Nichtwählenden wählten in Cluster 1 im Zuge der Bundestagwahl 2017 
die meisten Personen die Parteien CDU/CSU (22,69\%), dicht gefolgt von der Partei SPD (19,73\%).

\section{Interesse an Inklusion}

Mit einem Zustimmungsgrad von 66,48 \% geben die Personen aus Cluster 1 an, dass sie sich für das Thema Inklusion interessieren. Des Weiteren zeigt der Zustimmungsgrad von $56,04 \%$ an, dass sich mehr als die Hälfte der Personen in Cluster 1 bereits mit dem Thema Inklusion auseinandergesetzt hat.

Inklusion als (persönliches) Anliegen und die Frage nach einer höheren Steuerlast

Mit einem Zustimmungsgrad von 57,13 \% geben die Personen an, dass ihnen die Umsetzung von Inklusion ein persönliches Anliegen ist. Die Aussage, dass die Personen bereit wären, eine höhere Steuerlast zu tragen, um Inklusion in Deutschland zu ermöglichen, erreicht einen Zustimmungsgrad von $44,87 \%$.

\section{Zusammenfassung}

Zusammenfassend kann gesagt werden, dass die Personen in Cluster 1 politisch interessiert sind, sich bereits mit Inklusion auseinandergesetzt haben und sich sehr für Inklusion interessieren. Wahrscheinlich auch aufgrund des Kontakts mit Menschen mit Behinderung ist Inklusion den Personen in Cluster 1 ein persönliches Anliegen. Personen aus Cluster 1 können somit als offen gegenüber Inklusion bezeichnet werden.

\subsubsection{Cluster 2 - Typ: eher offen}

Cluster 2 sind Personen gruppiert, die tendenziell einen höheren Zustimmungsgrad zu Inklusion bei allen Faktoren aufweisen. Den höchsten $\mathrm{Zu}$ stimmungswert haben die Personen des Clusters 2 im Bereich Freizeit (mit einem Zustimmungsgrad von insgesamt 72,92\%). Auch die anderen Bereiche sind durch eine eher offene, positive Einstellung gegenüber Inklusion gekennzeichnet. Die Zustimmungswerte der einzelnen Aussagen schwanken zwischen $57,64 \%$ und $85,95 \%$, wobei der durchschnittliche Zustimmungswert bei $67,92 \%$ liegt. 


\section{Personenbeschreibung}

Die Personen aus Cluster 2 sind im Durschnitt 48,95 Jahre alt und lassen sich hauptsächlich der Altersgruppe der 55- bis 64-jähringen zuordnen (24,19\%). Die Geschlechterverteilung ist relativ ausgeglichen - es befinden sich etwas mehr Frauen (53,68 \%) als Männer (46,32\%) in Cluster 2.

\section{Kontakt zu Menschen mit (geistiger) Behinderung}

In Cluster 2 haben oder hatten 46,82 \% der Personen regelmäßig Kontakt zu Menschen mit Behinderung. Zu Menschen mit geistiger Behinderung haben oder hatten 30,07 \% der Personen aus Cluster 2 regelmäßig Kontakt.

\section{Politisches Interesse und Zugehörigkeit}

Politisch sind die Personen in Cluster 2 vor allem mittelmäßig bis sehr stark politisch interessiert. Insgesamt sind $22,82 \%$ der Personen weniger stark beziehungsweise überhaupt nicht politisch interessiert. Abgesehen von den Nichtwählenden wählten in Cluster 2 im Zuge der Bundestagswahl 2017 die meisten Personen die Parteien CDU/CSU (24,00\%), gefolgt von der Partei $\operatorname{SPD}(13,52 \%)$.

\section{Interesse an Inklusion}

Mit einem Zustimmungsgrad von 46,23\% geben die Personen aus Cluster 2 an, dass sie sich für das Thema Inklusion interessieren. Des Weiteren zeigt der Zustimmungsgrad von 40,24 \% an, dass sich Personen in Cluster 2 bereits mit Inklusion auseinandergesetzt haben.

\section{Inklusion als (persönliches) Anliegen und die Frage nach der höheren Steuerlast}

Mit einem Zustimmungsgrad von 35,37\% geben die Personen in Cluster 2 an, dass ihnen die Umsetzung von Inklusion ein persönliches Anliegen ist. Die Aussage, dass die Personen bereit wären, eine höhere Steuerlast zu tragen, um Inklusion in Deutschland zu ermöglichen, erreicht einen Zustimmungsgrad von $28,52 \%$.

\section{Zusammenfassung}

Zusammenfassend kann gesagt werden, dass die Personen in Cluster 2 politisch interessiert sind, sich bereits mit Inklusion auseinandergesetzt haben und sich für Inklusion interessieren. Wahrscheinlich auch aufgrund 
des Kontakts mit Menschen mit Behinderung ist Inklusion den Personen in Cluster 2 teilweise ein persönliches Anliegen. Personen aus Cluster 2 können somit als eher offen gegenüber Inklusion bezeichnet werden.

\subsubsection{Cluster 3 - Typ: eher ablehnend}

Die Personen in Cluster 3 zeichnen sich durch mittlere Zustimmungswerte $\mathrm{zu}$ Inklusion aus. Dabei fällt jedoch auf, dass die Zustimmungswerte abhängig von der Formulierung der Aussagen sind und sich je nach positivem, inklusionsbefürwortendem oder negativem, inklusionsablehnendem Aussagetypus unterscheiden. Werden die verschiedenen Aussagetypen in jedem Bereich verglichen, wird deutlich, dass die Personen in Cluster 3 bei Aussagen des negativen Typus einen höheren Zustimmungsgrad in Bezug auf Inklusion aufweisen als bei Aussagen des positiven Typus. Den höchsten Zustimmungswert haben die Personen des Clusters 3 im Bereich Freizeit (mit einem Zustimmungsgrad von insgesamt 51,05 \%) und den niedrigsten Zustimmungswert im Bereich Arbeit (mit einem Zustimmungsgrad von insgesamt 49,64 \%). Insgesamt schwanken die Zustimmungswerte der einzelnen Aussagen zwischen 41,30\% und 62,82 \%, wobei der durchschnittliche Zustimmungswert bei 49,04 \% liegt.

\section{Personenbeschreibung}

Die Personen aus Cluster 3 sind im Durchschnitt 42,97 Jahre alt und lassen sich hautsächlich der Altersgruppe der 18- bis 34-jährigen zuordnen (36,96\%). Die Geschlechterverteilung ist relativ ausgeglichen, wobei in Cluster 3 etwas mehr Männer (55,08 \%) als Frauen (44,92 \%) zu finden sind.

\section{Kontakt zu Menschen mit (geistiger) Behinderung}

In Cluster 3 haben oder hatten 30,34 \% der Personen regelmäßig Kontakt zu Menschen mit Behinderung. Circa die Hälfte davon hat oder hatte regelmäßig Kontakt zu Menschen mit geistiger Behinderung (17,58 \%).

\section{Politisches Interesse und Zugehörigkeit}

Politisch sind die Personen in Cluster 3 sehr unterschiedlich stark interessiert. Der Großteil der Personen ist mittelmäßig stark interessiert (37,05 \%). Fast ein Drittel der Personen ist wenig bis gar nicht politisch interessiert (insgesamt 29,49\%) beziehungsweise ziemlich stark bis sehr stark politisch interessiert (33,46 \%). Abgesehen von den Nichtwählenden wählten in Clus- 
ter 3 im Zuge der Bundestagswahl 2017 die meisten Personen die Partei CDU/ CSU (21,36 \%). Als zweitstärkste vertretene Partei wählten die Personen in Cluster 3 die SPD (11,15\%), dicht gefolgt von der AfD (10,68\%).

Interesse an Inklusion

Mit einem Zustimmungsgrad von 31,57 \% geben die Personen des Clusters 3 an, dass sie sich für das Thema Inklusion interessieren. Des Weiteren zeigt der Zustimmungsgrad von 34,63\% an, dass sich circa ein Drittel der Personen in Cluster 3 bereits mit dem Thema Inklusion auseinandergesetzt hat.

Inklusion als (persönliches) Anliegen und die Frage nach der höheren Steuerlast

Mit einem Zustimmungsgrad von 29,74 \% geben die Personen an, dass ihnen die Umsetzung von Inklusion ein persönliches Anliegen ist. Die Aussage, dass die Personen bereit wären, eine höhere Steuerlast zu tragen, um Inklusion in Deutschland zu ermöglichen, erreicht einen Zustimmungsgrad von $25,30 \%$.

\section{Zusammenfassung}

Zusammenfassend kann gesagt werden, dass die Personen in Cluster 3 politisch interessiert sind, sich bereits mit Inklusion auseinandergesetzt haben und sich teilweise für Inklusion interessieren. Wahrscheinlich auch aufgrund des teilweisen Kontakts mit Menschen mit Behinderung ist Inklusion den Personen in Cluster 3 ein mittelmäßiges persönliches Anliegen. Aufgrund der insgesamt ambivalenten Werte können die Personen aus Cluster 3 somit als eher ablehnend gegenüber Inklusion bezeichnet werden.

\subsubsection{Cluster 4 - Typ: klar ablehnend}

Die unter Cluster 4 subsumierten Personen zeigen bei allen Faktoren einen niedrigen Zustimmungsgrad. Den höchsten Zustimmungsgrad haben die Personen dieses Clusters im Bereich Freizeit (mit einem Zustimmungsgrad von insgesamt $29,33 \%$ ) und den niedrigsten Zustimmungswert im Bereich Arbeit (mit einem Zustimmungswert von insgesamt 22,09 \%). Insgesamt schwanken die Zustimmungswerte der einzelnen Aussagen zwischen 10,94\% und 43,13\%. Der durchschnittliche Zustimmungswert liegt bei $24,93 \%$. 


\section{Personenbeschreibung}

Die Personen aus Cluster 4 sind im Durchschnitt 49,17 Jahre alt und lassen sich hauptsächlich der Altersgruppe der 18- bis 34-jährigen zuordnen $(22,14 \%)$. Es befinden sich mehr Männer (60,31 \%) als Frauen (39,69 \%) in Cluster 4.

Kontakt zu Menschen mit (geistiger) Behinderung

In Cluster 4 haben oder hatten 35,88\% der Personen regelmäßig Kontakt zu Menschen mit Behinderung. Mehr als die Hälfte hiervon hat oder hatte regelmäßig Kontakt zu Menschen mit geistiger Behinderung (22,14\%).

\section{Politisches Interesse und Zugehörigkeit}

Politisch sind die Personen in Cluster 4 vor allem mittelmäßig bis sehr stark politisch interessiert. Insgesamt ist fast ein Fünftel der Personen (19,08 \%) weniger stark beziehungsweise überhaupt nicht politisch interessiert. Mehr als die Hälfte der Personen (57,25 \%) ist ziemlich stark beziehungsweise sehr stark politisch interessiert. Abgesehen von den Nichtwählenden wählten in Cluster 4 im Zuge der Bundestagswahl 2017 die meisten die Partei AfD (29,77 \%). Die zweitstärkste Partei ist die CDU/CSU mit 15,27\%.

\section{Interesse an Inklusion}

Mit einem Zustimmungsgrad von 17,81 \% geben die Personen in Cluster 4 an, dass sie sich für das Thema Inklusion interessieren. Des Weiteren zeigt der Zustimmungsgrad von $39,44 \%$ an, dass sich die Personen in Cluster 4 bereits mit Inklusion auseinandergesetzt haben.

Inklusion als (persönliches) Anliegen und die Frage nach der höheren Steuerlast

Mit einem Zustimmungsgrad von 3,29\% geben die Personen an, dass ihnen die Umsetzung von Inklusion ein persönliches Anliegen ist. Die Aussage, dass die Personen bereit wären, eine höhere Steuerlast zu tragen, um Inklusion in Deutschland zu ermöglichen, erreicht einen Zustimmungsgrad von $1,65 \%$. 


\section{Zusammenfassung}

Zusammenfassend kann gesagt werden, dass die Personen in Cluster 4 zwar politisch interessiert sind und sich bereits mit Inklusion auseinandergesetzt haben, sich aber dennoch in der Breite nicht für Inklusion interessieren und Inklusion ihnen, vielleicht auch aufgrund des fehlenden Kontakts zu Menschen mit Behinderung, kein persönliches Anliegen ist. Personen aus Cluster 4 können somit als klar ablehnend gegenüber Inklusion bezeichnet werden.

\subsection{Deskriptive Statistik der Cluster}

Anhand der Clusteranalyse konnten vier verschiedene Cluster identifiziert werden, die sich signifikant in ihren Einstellungen zu Inklusion unterscheiden.

Tabelle 43: Aufteilung der Gesamtgruppe in die jeweiligen Cluster

\begin{tabular}{|l|c|c|c|c|c|}
\hline & $\begin{array}{l}\text { Cluster 1: } \\
\text { offen }\end{array}$ & $\begin{array}{l}\text { Cluster 2: } \\
\text { eher offen }\end{array}$ & $\begin{array}{l}\text { Cluster 3: } \\
\text { eher } \\
\text { ablehnend }\end{array}$ & $\begin{array}{l}\text { Cluster 4: } \\
\text { klar } \\
\text { ablehnend }\end{array}$ & Gesamt \\
\hline $\begin{array}{l}\text { Häufigkeit } \\
\text { (N) }\end{array}$ & 1.485 & 1.021 & 1.058 & 131 & 3.695 \\
\hline Prozent & $40,19 \%$ & $27,63 \%$ & $28,63 \%$ & $3,55 \%$ & $100 \%$ \\
\hline
\end{tabular}

Cluster 1 umfasst die größte Gruppe mit 1.485 Personen und beinhaltet somit 40,19\% der Gesamtgruppe. Cluster 2 (1.021 Personen) und Cluster 3 (1.058 Personen) unterscheiden sich hinsichtlich ihrer Gruppengröße marginal - beide Cluster bündeln mehr als ein Viertel der Gesamtgruppe (Cluster 2: 27,63\% und Cluster 3: 28,63\%). Cluster 4 fasst insgesamt 131 Personen und ist mit Abstand das kleinste Cluster, das 3,55\% der Gesamtgruppe ausmacht. Dass lediglich ein solch geringer Anteil Inklusion ablehnend gegenübersteht, kann zunächst einmal als positives Ergebnis betrachtet werden: Inklusion scheint etwas zu sein, dem in der Breite eher keine konstitutive Ablehnung entgegengebracht wird. Die höheren Werte in den Clustern 2 und 3 zeigen jedoch, dass nicht von einer rein positiven Einstellung der Gesamtbevölkerung zu Inklusion gesprochen werden kann. Dem vergleichsweise hohen Anteil, der sich >offen` gegenüber Inklusion positioniert, steht ein noch grö- 
ßerer Anteil gegenüber, der sich weder vollständig befürwortend noch vollständig ablehnend zu Inklusion verhält. Es scheinen, dies legen die Ergebnisse nahe, gerade jene beiden Lager zu sein, die prägend für die Einstellung der Gesamtbevölkerung Deutschlands zu Inklusion sind. Darüber hinaus können diese Ergebnisse dahingehend interpretiert werden, dass Inklusion oder Teilhabe in gewisser Hinsicht als moralische Idee weit verbreitet sind, dies aber nicht unbedingt zu veränderten Handlungspraxen führt.

\subsubsection{Häufigkeitsverteilungen der personenbezogenen Daten innerhalb der Cluster}

Nachdem die verschiedenen Cluster hinsichtlich ihrer Zustimmungsgrade zu Inklusion untersucht wurden, wurde überprüft, ob sich die Cluster bezüglich ihrer demografischen Daten signifikant unterscheiden. Dabei wurde analysiert, ob Eigenschaften herausgearbeitet werden können, die einer bestimmten Einstellungsgruppe zuzuordnen sind und als Einflussfaktor für die Einstellung(en) zu Inklusion identifiziert werden können. Forschungspraktisch wurden zunächst die Häufigkeitsverteilungen der verschiedenen demografischen Faktoren pro Cluster betrachtet und eine demografische Clusterbeschreibung erstellt. Anschließend wurde anhand einer Einfaktoriellen Varianzanalyse (ANOVA) - zwischen den Clustern und den jeweiligen demografischen Daten - überprüft, ob signifikante Unterschiede zwischen den Clustern bestehen, in Bezug auf den jeweiligen demografischen Faktor. Ein Post-hoc-Test konnte daraufhin Aufschluss darüber geben, zwischen welchen Clustern genau ein signifikanter Unterschied besteht. Im Zuge der Durchführung jener Arbeitsschritte wurde deutlich, dass signifikante Unterschiede zwischen den Clustern und den demografischen Daten Alter, Altersgruppe, Geschlecht sowie Kontakt zu Menschen mit Behinderung, Kontakt $z u$ Menschen mit geistiger Behinderung, Politisches Interesse und Abstimmverhalten während der Bundestagswahlen 2017 bestehen. Die demografischen Daten Migrationshintergrund, Familienstand, Glaube, Selbst eine (geistige) Behinderung, Höchster Bildungsabschluss, Wohnort (Großstadt ja/nein) wurden ebenfalls untersucht, allerdings sind hier nur vereinzelt knappe signifikante Unterschiede oder teils keine Signifikanzen zwischen den Clustern zu verzeichnen. Die signifikanten Zusammenhänge werden im Folgenden dargelegt. 


\section{Alter}

Personen aus Cluster 1 (offen), die den höchsten Zustimmungsgrad zu Inklusion aufweisen, sind mit durchschnittlich 52,85 Jahren am ältesten. Die Personen aus Cluster 2 (eher offen) sind durchschnittlich 48,95 Jahre alt, wogegen die Personen aus Cluster 3 (eher ablehnend) und Cluster 4 (klar ablehnend) durchschnittlich 42,97 beziehungsweise 49,17 Jahre alt sind. Es kann also davon ausgegangen werden, dass Personen, die Inklusion befürworten, eher älter sind als diejenigen, die Inklusion ablehnen.

\section{Altersgruppe}

Die Altersverteilung innerhalb der einzelnen Cluster gestaltet sich wie folgt:

Tabelle 44: Häufigkeitsverteilung der Variablen >Altersgruppe< innerhalb der Cluster

\begin{tabular}{|c|c|c|c|c|c|}
\hline & & $\begin{array}{l}\text { Cluster 1: } \\
\text { offen }\end{array}$ & $\begin{array}{l}\text { Cluster 2: } \\
\text { eher offen }\end{array}$ & $\begin{array}{l}\text { Cluster 3: } \\
\text { eher } \\
\text { ablehnend }\end{array}$ & $\begin{array}{l}\text { Cluster 4: } \\
\text { klar } \\
\text { ablehnend }\end{array}$ \\
\hline Altersgruppe & $\begin{array}{l}18-34 \\
\text { Jahre }\end{array}$ & $14,21 \%$ & $23,51 \%$ & $36,96 \%$ & $22,14 \%$ \\
\cline { 2 - 7 } & $\begin{array}{l}35-44 \\
\text { Jahre }\end{array}$ & $12,79 \%$ & $13,03 \%$ & $16,82 \%$ & $17,56 \%$ \\
\cline { 2 - 7 } & $\begin{array}{l}45-54 \\
\text { Jahre }\end{array}$ & $18,32 \%$ & $19,69 \%$ & $18,62 \%$ & $20,61 \%$ \\
\cline { 2 - 7 } & $\begin{array}{l}55-64 \\
\text { Jahre }\end{array}$ & $31,92 \%$ & $24,19 \%$ & $17,67 \%$ & $19,85 \%$ \\
\cline { 2 - 6 } & $65+$ & $22,76 \%$ & $19,59 \%$ & $9,92 \%$ & $19,85 \%$ \\
\hline
\end{tabular}

In Cluster 1 sind mit Abstand die meisten Personen zwischen 55 und 64 Jahre alt (31,92 \%). Die zweitgrößte Altersgruppe ist über 65 Jahre alt. Insgesamt sind in Cluster 1 mehr als die Hälfte der Personen über 55 Jahre alt (54,68 \%). In Cluster 3 hingegen sind mit Abstand die meisten Personen zwischen 18 und 34 Jahre alt $(36,96 \%)$ und mehr als die Hälfte der Personen $(53,78 \%)$ unter 45 Jahre alt. Die wenigsten Personen sind über 65 Jahre alt (9,92 \%). Sowohl in Cluster 2 als auch in Cluster 4 unterscheidet sich die prozentuale Häufigkeit der Altersgruppen nicht so deutlich, wie in Cluster 1 und Cluster 3. In Cluster 4 unterscheidet sich die Häufigkeitsverteilung der Altersgruppen 
höchstens um 4,58 \%. Es fällt auf, dass sowohl in Cluster 1 als auch in Cluster 2 die meisten Personen zwischen 55 und 64 Jahre alt sind - beide Cluster sind zudem diejenigen mit den höchsten Zustimmungsgraden zu Inklusion. Jüngere Personen sind in diesen beiden eher inklusionsbejahenden Clustern weniger vertreten, was noch einmal bestätigt, dass die Zustimmung zu Inklusion mit zunehmendem Alter steigt.

\section{Geschlecht}

In der folgenden Tabelle kann abgelesen werden, wie das Verhältnis von männlichen und weiblichen Personen in den jeweiligen Clustern verteilt ist.

Tabelle 45: Häufigkeitsverteilung der Variablen >Geschlecht innerhalb der Cluster

\begin{tabular}{|l|l|l|l|l|l|}
\hline & & $\begin{array}{l}\text { Cluster 1: } \\
\text { offen }\end{array}$ & $\begin{array}{l}\text { Cluster 2: } \\
\text { eher offen }\end{array}$ & $\begin{array}{l}\text { Cluster 3: } \\
\text { eher } \\
\text { ablehnend }\end{array}$ & $\begin{array}{l}\text { Cluster 4: } \\
\text { klar } \\
\text { ablehnend }\end{array}$ \\
\hline Geschlecht & Männlich & $47,97 \%$ & $46,32 \%$ & $55,08 \%$ & $60,31 \%$ \\
\cline { 2 - 6 } & Weiblich & $52,03 \%$ & $53,68 \%$ & $44,92 \%$ & $39,69 \%$ \\
\hline
\end{tabular}

Es fällt auf, dass bei den Personen in Cluster 1 und Cluster 2, die eine (eher) offene Einstellung zu Inklusion haben, prozentual der Anteil an Frauen höher ist als der der Männer. Bei Personen in Cluster 3 und Cluster 4, die eine (eher/klar) ablehnende Einstellung zu Inklusion aufweisen, ist dies der umgekehrte Fall - hier ist der Anteil der Männer höher als der der Frauen. Es kann also gesagt werden, dass Frauen gegenüber Inklusion offener eingestellt sind als Männer.

Kontakt zu Menschen mit Behinderung

Inwiefern der lebenspraktisch erlebte Kontakt zu Menschen mit Behinderung in Zusammenhang mit der Einstellung zu Inklusion steht, kann aus der folgenden Tabelle gelesen werden. 
Tabelle 46: Häufigkeitsverteilung der Variablen >Kontakt zu Menschen mit Behinderung< innerhalb der Cluster

\begin{tabular}{|l|l|l|l|l|l|}
\hline & $\begin{array}{l}\text { Cluster 1: } \\
\text { offen }\end{array}$ & $\begin{array}{l}\text { Cluster 2: } \\
\text { eher offen }\end{array}$ & $\begin{array}{l}\text { Cluster 3: } \\
\text { eher } \\
\text { ablehnend }\end{array}$ & $\begin{array}{l}\text { Cluster 4: } \\
\text { klar } \\
\text { ablehnend }\end{array}$ \\
\hline $\begin{array}{l}\text { Kontakt zu } \\
\text { Menschen } \\
\text { mit Behinde- } \\
\text { rung }\end{array}$ & Ja & $60,00 \%$ & $46,82 \%$ & $30,34 \%$ & $35,88 \%$ \\
\hline
\end{tabular}

Während in Cluster 1 - das Cluster mit den höchsten Zustimmungsgraden $\mathrm{zu}$ Inklusion - 60,00 \% der Personen regelmäßig Kontakt zu Menschen mit Behinderung haben oder hatten, betraf dies in Cluster 4 - das Cluster mit den niedrigsten Zustimmungsgraden zu Inklusion - lediglich 35,88 \% der Personen. Cluster 1 ist das einzige Cluster, in dem die Mehrheit der Personen Kontakt zu Menschen mit Behinderung hat oder hatte. Dies kann als Indiz dafür gewertet werden, dass der lebensweltliche Kontakt zu Menschen mit Behinderung in der Breite eher dazu führt, dass die Einstellung zu Inklusion positiv beeinflusst wird. Dass es sich hierbei jedoch eher um eine (sehr deutliche) Tendenz als um eine Zwangsläufigkeit handelt, wird daran ersichtlich, dass nicht Cluster 4, sondern Cluster 3 mit 30,34 \% den niedrigsten Wert erreicht. Dies kann so gelesen werden, dass der Kontakt zu Menschen mit Behinderung durchaus auch dazu führen kann, dass die Einstellung zu Inklusion eher negativ beeinflusst wird. Das Ergebnis der Auswertung gibt insofern einen Hinweis darauf, dass nicht allein der Kontakt zu Menschen mit Behinderung ausschlaggebend ist, sondern noch weitere Facetten berücksichtigt werden müssen (beispielsweise die Art und Weise des Kontakts oder der jeweils gegebene Unterstützungsbedarf der Person, zu der Kontakt besteht). Dennoch kann gesagt werden, dass sich die Kontakthypothese bestätigt, entlang derer davon ausgegangen wird, dass ein lebenspraktischer Kontakt zu bestimmten Personen die Aufgeschlossenheit ihnen gegenüber erhöht (Trescher 2015b, S. 118f). 


\section{Kontakt zu Menschen mit geistiger Behinderung}

Neben dem Kontakt zu Menschen mit Behinderung wurde nach dem lebenspraktisch erlebten Kontakt zu Menschen mit geistiger Behinderung gefragt. Die folgende Tabelle zeigt die Verteilung entlang der Cluster.

Tabelle 47: Häufigkeitsverteilung der Variablen >Kontakt zu Menschen mit geistiger Behinderung< innerhalb der Cluster

\begin{tabular}{|l|c|c|c|c|c|}
\hline & & $\begin{array}{l}\text { Cluster 1: } \\
\text { offen }\end{array}$ & $\begin{array}{l}\text { Cluster 2: } \\
\text { eher offen }\end{array}$ & $\begin{array}{l}\text { Cluster 3: } \\
\text { eher } \\
\text { ablehnend }\end{array}$ & $\begin{array}{l}\text { Cluster 4: } \\
\text { klar } \\
\text { ablehnend }\end{array}$ \\
\hline $\begin{array}{l}\text { Kontakt zu } \\
\text { Menschen mit } \\
\text { geistiger Be- } \\
\text { hinderung }\end{array}$ & Ja & $43,37 \%$ & $30,07 \%$ & $17,58 \%$ & $22,14 \%$ \\
\hline
\end{tabular}

In Bezug auf den Kontakt zu Menschen mit geistiger Behinderung zeigt sich ein sehr ähnliches Bild, wie es bereits im vorangegangenen Abschnitt beschrieben wurde. Die Personen aus Cluster 1 haben oder hatten mit Abstand am häufigsten (43,37 \%) und die Personen aus Cluster 3 am seltensten (17,58 \%) regelmäßig Kontakt zu Menschen mit geistiger Behinderung - die obige Interpretation der Ergebnisse wäre also auf die hiesigen zu übertragen. Insgesamt hat oder hatte in allen Clustern die Mehrheit der Personen keinen regelmäßigen Kontakt zu Menschen mit geistiger Behinderung, was noch einmal bestätigt, inwiefern Menschen mit geistiger Behinderung nach wie vor von Ausschluss und Ausgrenzung betroffen sind. Deutlich wird aber auch hier, dass der lebenspraktisch erlebte Kontakt zu Menschen mit geistiger Behinderung mit einer offeneren Einstellung zu Inklusion einhergeht (Trescher 2015b, S. 118f; siehe auch Kapitel 6.2).

Politisches Interesse

Des Weiteren wurde untersucht, ob und inwiefern ein Zusammenhang zwischen politischem Interesse und der jeweiligen Einstellung zu Inklusion besteht. Die Ergebnisse dessen sind in der nachfolgenden Tabelle dargelegt. 
Tabelle 48: Häufigkeitsverteilung der Variablen >Politisches Interesse < innerhalb der Cluster

\begin{tabular}{|l|l|c|c|c|c|}
\hline & & $\begin{array}{l}\text { Cluster 1: } \\
\text { offen }\end{array}$ & $\begin{array}{l}\text { Cluster 2: } \\
\text { eher offen }\end{array}$ & $\begin{array}{l}\text { Cluster 3: } \\
\text { eher } \\
\text { ablehnend }\end{array}$ & $\begin{array}{l}\text { Cluster 4: } \\
\text { klar } \\
\text { ablehnend }\end{array}$ \\
\hline $\begin{array}{l}\text { Politi- } \\
\text { sches } \\
\text { Interes- } \\
\text { se }\end{array}$ & $\begin{array}{l}\text { Überhaupt } \\
\text { nicht }\end{array}$ & $4,31 \%$ & $7,44 \%$ & $14,84 \%$ & $6,87 \%$ \\
\cline { 2 - 6 } & $\begin{array}{l}\text { Weniger } \\
\text { stark }\end{array}$ & $11,85 \%$ & $15,38 \%$ & $14,65 \%$ & $12,21 \%$ \\
\cline { 2 - 6 } & Mittelmäßig & $33,00 \%$ & $38,69 \%$ & $37,05 \%$ & $23,66 \%$ \\
\cline { 2 - 6 } & $\begin{array}{l}\text { Ziemlich } \\
\text { stark }\end{array}$ & $33,00 \%$ & $28,31 \%$ & $22,87 \%$ & $33,59 \%$ \\
\cline { 2 - 6 } & Sehr stark & $17,85 \%$ & $10,19 \%$ & $10,59 \%$ & $23,66 \%$ \\
\hline
\end{tabular}

Die Personen aus Cluster 1 und Cluster 4 sind insgesamt am meisten politisch interessiert. Personen aus Cluster 3 sind im Vergleich am politisch uninteressiertesten. Es kann kein Zusammenhang zwischen einem (hohen oder niedrigen) politischen Interesse und der Zustimmung zu beziehungsweise Ablehnung von Inklusion festgestellt werden.

\section{Abstimmungsverhalten in der Bundestagswahl 2017}

Eine weitere Korrelation wurde zwischen dem Abstimmungsverhalten bei der Bundestagswahl im Jahr 2017 und der Einstellung zu Inklusion festgestellt, wie die folgende Tabelle zeigt. Abgebildet ist hier das Abstimmungsverhalten bei der Bundestagswahl 2017 in Relation zur Clusterzuordnung. 
Tabelle 49: Häufigkeitsverteilung der Variablen >Abstimmungsverhalten in der Bundestagswahl 2017 innerhalb der Cluster

\begin{tabular}{|l|l|l|l|l|l|}
\hline \multirow{4}{*}{\begin{tabular}{|l|l|l|} 
Cluster 1: \\
offen
\end{tabular}} & $\begin{array}{l}\text { Cluster 2: } \\
\text { eher offen }\end{array}$ & $\begin{array}{l}\text { Cluster 3: } \\
\text { eher } \\
\text { ablehnend }\end{array}$ & $\begin{array}{l}\text { Cluster 4: } \\
\text { klar } \\
\text { ablehnend }\end{array}$ \\
\hline \multirow{2}{*}{$\begin{array}{l}\text { Abstim- } \\
\text { mungs- } \\
\text { verhalten } \\
\text { in der } \\
\text { Bundes- } \\
\text { tagswahl } \\
\text { 2017 }\end{array}$} & CDU/CSU & $22,69 \%$ & $24,00 \%$ & $21,36 \%$ & $15,27 \%$ \\
\cline { 2 - 6 } & SPD & $19,73 \%$ & $13,52 \%$ & $11,15 \%$ & $6,87 \%$ \\
\cline { 2 - 6 } & Linke & $10,71 \%$ & $5,09 \%$ & $4,73 \%$ & $4,58 \%$ \\
\cline { 2 - 6 } & Grüne & $8,75 \%$ & $6,27 \%$ & $3,50 \%$ & $3,82 \%$ \\
\cline { 2 - 6 } & FDP & $6,13 \%$ & $10,58 \%$ & $6,99 \%$ & $9,92 \%$ \\
\cline { 2 - 6 } & AfD & $6,40 \%$ & $8,91 \%$ & $10,68 \%$ & $29,77 \%$ \\
\cline { 2 - 6 } & Sonstige & $3,77 \%$ & $2,15 \%$ & $5,10 \%$ & $3,82 \%$ \\
\cline { 2 - 6 } & $\begin{array}{l}\text { Nichtwäh- } \\
\text { lende (un- } \\
\text { gültig, nicht } \\
\text { gewählt, } \\
\text { nicht wahl- } \\
\text { berechtigt) }\end{array}$ & $21,82 \%$ & $29,48 \%$ & $36,48 \%$ & $25,95 \%$ \\
\hline
\end{tabular}

Sowohl in Cluster 1 als auch in Cluster 2 und Cluster 3 haben die meisten Personen CDU/CSU gewählt. Im Gegensatz dazu haben die meisten Personen aus Cluster 4 die AfD (mit 29,77 \%) gewählt. In Cluster 3 sind mit 36,48 \% über ein Drittel der Personen Nichtwählende. Des Weiteren fällt auf, dass in Cluster 1 die drittstärkste Partei Die Linke ist, die in den anderen Clustern prozentual deutlich weniger gewählt wurde. Mit 10,71 \% befinden sich in Cluster 1 mehr als doppelt so viele Personen, die Die Linke gewählt haben, als bei allen anderen Clustern. Wird das Cluster mit den höchsten Zustimmungsgraden zu Inklusion - Cluster 1 - und das Cluster mit den niedrigsten Zustimmungsgraden zu Inklusion - Cluster 4 - betrachtet, so scheint es einen Zusammenhang zwischen der politischen Orientierung und der Einstellung zu Inklusion zu geben. Es deutet darauf hin, dass Personen, die eine vergleichsweise (eher) linke politische Orientierung aufweisen, eine positi- 
vere Einstellung zu Inklusion haben als Personen, die sich (eher) politisch rechts orientieren.

\subsection{2 Übergeordnete Aussagen}

Wie oben bereits beschrieben, sollte im Rahmen des Surveys zusätzlich zu den lebensbereichsbezogenen Items zu vier übergeordneten Aussagen Stellung bezogen werden, die sich auf Inklusion im Allgemeinen beziehen. Auch in Bezug auf jene übergeordneten Aussagen wurde anhand der Einfaktoriellen Varianzanalyse (ANOVA) überprüft, ob ein signifikanter Unterschied zwischen den Clustern besteht und welche Effektstärke das Antwortverhalten der verschiedenen Aussagen auf die Variationen der Cluster hat. Dies ist in den nachfolgenden Übersichten dargestellt und wird daraufhin noch einmal ausgeführt.

Tabelle 50: Zustimmungsgrade der übergeordneten Aussage "Ich interessiere mich für das Thema Inklusion.«

\begin{tabular}{|l|l|l|l|l|l|}
\hline & $\begin{array}{l}\text { Cluster 1: } \\
\text { offen }\end{array}$ & $\begin{array}{l}\text { Cluster 2: } \\
\text { eher offen }\end{array}$ & $\begin{array}{l}\text { Cluster 3: } \\
\text { eher } \\
\text { ablehnend }\end{array}$ & $\begin{array}{l}\text { Cluster 4: } \\
\text { klar } \\
\text { ablehnend }\end{array}$ & Insgesamt \\
\hline $\begin{array}{l}\text { 1. Ich interessiere } \\
\text { mich für das } \\
\text { Thema Inklusion. }\end{array}$ & $66,84 \%$ & $46,23 \%$ & $31,57 \%$ & $17,81 \%$ & $49,31 \%$ \\
\hline
\end{tabular}

Cluster 1 hat den höchsten Zustimmungsgrad mit 66,84\%. In Cluster 4, das diejenigen Personen subsumiert, die die ablehnendste(n) Einstellung(en) zu Inklusion haben, wird der geringste Zustimmungsgrad erreicht (17,81 \%). Deutlich wird an diesen Zustimmungsgraden, dass ein Interesse an Inklusion zwar überwiegend, jedoch nicht immer und formelhaft zu einer offenen Einstellung zu Inklusion führt beziehungsweise damit einhergeht. Es gibt durchaus Personen, die ein gewisses Interesse an Inklusion haben, allerdings dieser eher oder klar ablehnend gegenüber eingestellt sind. Es findet sich hierin eine Überschneidung zu dem bereits dargelegten Ergebnis bezüglich der Auswirkungen eines lebensweltlichen Kontakts zu Menschen mit Behinderung. Handlungspraktisch können daraus zwei Schlüsse gezogen werden: Zum einen erscheint es sinnvoll, in der Gesamtbevölkerung das Interesse an Inklusion zu wecken oder zu erhöhen. Zum anderen bedarf es 
einer breiteren Aufklärung, die dazu beiträgt, Irrtümer und Vorurteile, die Inklusion betreffen, abzubauen.

Tabelle 51: Zustimmungsgrade der übergeordneten Aussage »Ich habe mich bereits mit dem Thema Inklusion auseinandergesetzt."

\begin{tabular}{|l|l|l|l|l|l|}
\hline & $\begin{array}{l}\text { Cluster 1: } \\
\text { offen }\end{array}$ & $\begin{array}{l}\text { Cluster 2: } \\
\text { eher offen }\end{array}$ & $\begin{array}{l}\text { Cluster 3: } \\
\text { eher } \\
\text { ablehnend }\end{array}$ & $\begin{array}{l}\text { Cluster 4: } \\
\text { klar } \\
\text { ablehnend }\end{array}$ & Insgesamt \\
\hline $\begin{array}{l}\text { 2. Ich habe } \\
\text { mich bereits } \\
\text { mit dem Thema } \\
\text { Inklusion ausei- } \\
\text { nandergesetzt. }\end{array}$ & $56,04 \%$ & $40,24 \%$ & $34,63 \%$ & $39,44 \%$ & $44,95 \%$ \\
\hline
\end{tabular}

Auch hier ist, wie bei der ersten übergeordneten Aussage, der Zustimmungsgrad in Cluster 1 am höchsten mit 56,04 \%. Cluster 3 umfasst die Personen, die bei dieser Aussage den niedrigsten Zustimmungsgrad mit 34,63\% aufweisen. Ähnlich wie bezüglich der obigen Aussage wird allerdings deutlich, dass sich die Personen über alle Cluster hinweg, bereits mit Inklusion befasst haben (zumindest bis zu einem gewissen Grad). Inklusion ist also ganz offenbar als Thema in der Mitte der Gesellschaft angekommen und es wird sich damit auseinandergesetzt - unabhängig davon, ob die eigene Einstellung zu Inklusion eher offen ist oder nicht.

Tabelle 52: Zustimmungsgrade der übergeordneten Aussage »Die Umsetzung von Inklusion ist mir ein persönliches Anliegen.«

\begin{tabular}{|l|l|l|l|l|l|}
\hline & $\begin{array}{l}\text { Cluster 1: } \\
\text { offen }\end{array}$ & $\begin{array}{l}\text { Cluster 2: } \\
\text { eher offen }\end{array}$ & $\begin{array}{l}\text { Cluster 3: } \\
\text { eher } \\
\text { ablehnend }\end{array}$ & $\begin{array}{l}\text { Cluster 4: } \\
\text { klar } \\
\text { ablehnend }\end{array}$ & $\begin{array}{l}\text { Insge- } \\
\text { samt }\end{array}$ \\
\hline $\begin{array}{l}\text { 3. Die Umsetzung } \\
\text { von Inklusion ist } \\
\text { mir ein persön- } \\
\text { liches Anliegen. }\end{array}$ & $57,13 \%$ & $35,37 \%$ & $29,74 \%$ & $3,82 \%$ & $41,38 \%$ \\
\hline
\end{tabular}


Cluster 1 hat erneut den höchsten Zustimmungsgrad mit 57,13 \%. Der Zustimmungsgrad von Cluster 4 grenzt sich deutlich von den Zustimmungsgraden der anderen Cluster ab und ist mit 3,82\% im Vergleich mit Abstand am geringsten. Die Effektstärke von 21,67 \% zeigt, dass der Zustimmungsgrad dieser Aussage einen großen Effekt auf die Clusterzugehörigkeit hat. Die Frage danach, ob Inklusion als persönliches Anliegen betrachtet wird oder nicht, kann insofern als sehr zentral für die Frage nach der Einstellung $\mathrm{zu}$ Inklusion betrachtet werden. Ebenso wie in Bezug auf die vorangegangenen Aussagen wird deutlich, dass die Zustimmung zu dieser Aussage sinkt, je geringer die Zustimmung zu Inklusion ist. Folglich ist in Cluster 4 (klar ablehnend), wie bereits hervorgehoben, der geringste Zustimmungswert zu verzeichnen. Interessant ist zudem, dass die Zustimmung ganz offenbar dann sinkt, wenn es um die konkrete Umsetzung von Inklusion geht und nicht, wie zuvor, um Interesse oder thematische Beschäftigung. Inklusion scheint in der Gesamtbevölkerung also vor allem als Idee oder moralisch hohes Gut wahrgenommen zu werden.

Tabelle 53: Zustimmungsgrade der übergeordneten Aussage "Ich wäre bereit, eine höhere Steuerlast zu tragen, um Inklusion in Deutschland möglich zu machen."

\begin{tabular}{|l|l|l|l|l|l|}
\hline & $\begin{array}{l}\text { Cluster 1: } \\
\text { offen }\end{array}$ & $\begin{array}{l}\text { Cluster 2: } \\
\text { eher offen }\end{array}$ & $\begin{array}{l}\text { Cluster 3: } \\
\text { eher } \\
\text { ablehnend }\end{array}$ & $\begin{array}{l}\text { Cluster 4: } \\
\text { klar } \\
\text { ablehnend }\end{array}$ & Insgesamt \\
\hline $\begin{array}{l}\text { 4. Ich wäre } \\
\text { bereit, eine } \\
\text { höhere } \\
\text { Steuerlast zu } \\
\text { tragen, um } \\
\text { Inklusion in } \\
\text { Deutschland } \\
\text { möglich zu } \\
\text { machen. }\end{array}$ & $44,87 \%$ & $28,52 \%$ & $25,30 \%$ & $1,65 \%$ & $33,22 \%$ \\
\hline
\end{tabular}

Ein weiteres Mal umfasst Cluster 1 die Personen mit dem höchsten Zustimmungsgrad (44,87\%). Wie bereits bei der vorangegangenen Aussage deutlich wurde, unterscheidet sich Cluster 4 deutlich von den anderen Clustern und weist mit 1,65\% einen sehr niedrigen Zustimmungsgrad auf. Insgesamt handelt es sich bei dieser Aussage um diejenige, bei der die geringste $\mathrm{Zu}$ - 
stimmung zu verzeichnen ist. Daran kann, ähnlich wie in Bezug auf die vorangegangene Aussage zur Umsetzung von Inklusion, diskutiert werden, dass die Zustimmung generell abzunehmen scheint, wenn es um konkrete Veränderungen geht - hier eine mögliche finanzielle Belastung. Deutlich wird erneut, dass Inklusion als moralische Idee zwar oftmals positiv bewertet wird, mögliche handlungspraktische Konsequenzen allerdings eher abgelehnt werden. Dies ist insofern problematisch, dass sich Inklusion über Veränderungen vollzieht - Inklusion ist Kritik (siehe Kapitel 4.2) - und an Grenzen stößt, wenn die Gesamtbevölkerung Umwälzungen und Neuem eher skeptisch gegenübersteht.

\section{Gesamtbetrachtung und Diskussion}

Im Anschluss an die Darstellung der Ergebnisse des Surveys »Einstellung(en) zu Inklusion«, werden diese hier, gleich den vorangegangenen Kapiteln, noch einmal zusammenfassend diskutiert sowie theoretische, methodische und handlungspraktische Anschlussfragen reflektiert.

\section{Theoretische Gesamtbetrachtung und Diskussion}

Die Ergebnisse des Surveys »Einstellung(en) zu Inklusion« zeigen, dass die Zustimmung zu Inklusion im Alter zunimmt und Frauen etwas offener eingestellt sind als Männer. Faktoren wie Glaube, urbane oder rurale Lebensorte, Migrationshintergrund oder Familienstand haben demgegenüber keinen Einfluss darauf, wie positiv oder negativ Personen zu Inklusion eingestellt sind. Viel deutlicher kommt dagegen zum Tragen, ob Personen bereits Kontakt zu Menschen mit (geistiger) Behinderung haben oder hatten. Insofern bestätigt sich die eingangs benannte Kontakthypothese, laut der der lebenspraktische Kontakt zu Menschen mit (geistiger) Behinderung dazu führt, dass Personen gegenüber diesen respektive ihrer mehrheitsgesellschaftlichen Teilhabe positiver eingestellt sind. Bezugnehmend auf Allport (1954), der die sogenannte Kontakthypothese maßgeblich in den Fachdiskurs einführte, konkretisiert Cloerkes (2007) dessen Annahmen dahingehend, dass nicht primär die Häufigkeit des Kontakts zwischen unterschiedlichen Personen/Gruppen ausschlaggebend sei, sondern vielmehr die Intensität (Cloerkes 2007, S. 147; siehe auch Cordes und Silter 2016, S. 238ff; Schattenmann 2016, S. 140ff). Dabei ist es wichtig zu betonen, dass Vorbehalte ebenso wie Offen- 
heit durch regelmäßigen Kontakt mit bestimmten Personen oder Gruppen jeweils verstärkt werden können, das bedeutet: »Eine ursprüngliche Einstellung tendiert dazu, sich bei Kontakt mit dem Einstellungsobjekt zum Extrem hin zu verstärken. Eine primär negative Einstellung kann durch Kontakt noch unterstrichen werden. Eine primär positive Einstellung wird hingegen durch Kontakterfahrungen weiter bestärkt« (Cloerkes 2007, S. 147). In diesem Zusammenhang ist es darüber hinaus bedeutsam, dass Personen, die unterschiedlichen anderen Personen oder Gruppen umfassend ablehnend gegenüberstehen, oftmals auch nicht dadurch in ihrer Einstellung verändert werden können, indem ihnen, mit dem Ziel, Vorbehalte und Ängste abzubauen, umfassende Informationen zur jeweiligen Person/Gruppe zur Verfügung gestellt werden. Vielmehr könnte hierbei ein sogenannter Bumerangeffekt entstehen, der Vorbehalte eher noch verstärkt (Cloerkes 2007, S. 140f; siehe auch Allport 1954, S. 263). Welche handlungspraktischen Implikationen damit einhergehen, wird im entsprechenden unteren Kapitel diskutiert. In theoretischer Hinsicht bleibt zu bemerken, dass die Kontakthypothese zwar breit Anwendung findet und auf sie häufig referiert wird, sie dennoch theoretisch eindimensional bleibt. Hier ist eine weitergehende und vertiefende theoretische Fundierung denkbar. Zwei weitere Fragen werden mit Blick auf den Survey und seine Ergebnisse aufgegriffen. Es zeigt sich, dass einige Personen in Deutschland gegenüber Inklusion sehr ablehnend eingestellt sind. Die Frage, die daraus folgt, ist, was es für die Konstitution von Gesellschaft bedeutet, wenn bestimmte Personen - hier Menschen mit geistiger Behinderung - kategorisch abgelehnt werden (auch wenn dies nicht übergreifend der Fall ist, gleichwohl auf einen geringen Anteil der EinwohnerInnen zutrifft). Dies ist letztlich eine demokratietheoretische Frage, die im Kern darauf abzielt, wie demokratisch eine Gesellschaft ist, wenn nur ausgewählte Personen als dem `demos dazugehörig konstruiert werden. Es ist zu klären: »[W]ho has rights worthy of protection and whose living conditions compose the intrinsic aim of governing; who is normak enough, competent enough, and human enough, to be a citizen? (Altermark 2018, S. 5; siehe auch Forst 1994, S. 215). Ein weiterer Aspekt, der hervorgehoben wird, betrifft die im Survey formulierte These zum Thema Unterstützung, die sinngemäß darauf abzielt, ob die befragten Personen sich vorstellen könnten, Menschen mit geistiger Behinderung dabei zu unterstützen, mehrheitsgesellschaftliche Teilhabemöglichkeiten eröffnet zu bekommen und diese zu nutzen. Möglicherweise problematisch ist hierbei nun, dass mit unterstützendem Han- 
deln immer eine gewisse Asymmetrie verbunden ist und zwar zu Ungunsten der Person, die unterstützt wird. Sie wird im Akt des Unterstützens als hilfebedürftig und abhängig hervorgebracht. Es stellt sich also die Frage nach der theoretischen Fassung von Inklusion, denn es muss näher darauf eingegangen werden, wie Inklusion als Kritik mit - grundsätzlich eher behindernden - Unterstützungspraxen zusammengedacht werden kann (siehe Kapitel 26). Denn es ist unklar, wie diese (auch handlungspraktisch ganz konkrete) Ambivalenz, dass Unterstützung Teilhabe ermöglichen kann, gleichzeitig allerdings behindernd wirksam wird, theoretisch aufgelöst werden kann. Eine weitergehende theoretische Auseinandersetzung mit dieser Ambivalenz könnte beispielsweise dekonstruktivistische Lesarten heranziehen.

\section{Methodische Gesamtbetrachtung und Diskussion}

Das methodische Setting des Surveys hat es erfolgreich ermöglicht, Einstellungen $\mathrm{zu}$ Inklusion $\mathrm{zu}$ untersuchen. Es konnte eine repräsentative Stichprobe generiert werden, die die Übertragbarkeit der Ergebnisse sicherstellt. Hervorgehoben werden kann dabei die Einzigartigkeit des Surveys, denn bislang wurden Einstellungen zu Inklusion in Deutschland - zumindest bezüglich der Teilhabemöglichkeiten von Menschen mit geistiger Behinderung - nicht derart umfassend untersucht. Indem die Ergebnisse des Einstellungssurveys mit denen der Sozialraumanalysen in den Handlungsfeldern Arbeit und Freizeit quergelesen werden, können unterschiedliche Perspektiven auf ähnliche Phänomene eingenommen werden. Beispielsweise konnte in den Sozialraumanalysen teilweise ein signifikanter Unterschied zwischen groß- und kleinstädtischen Sozialräumen ausgemacht werden (siehe Kapitel 18), der sich in dieser Weise im Einstellungssurvey nicht zeigte. Im Vergleich der Ergebnisse können sich diese jedoch gegenseitig ergänzen, infrage stellen und mehrperspektivisch erweitern. Ähnliches gilt für die unterschiedliche Fokussierung der Personengruppen, die als von Ausschluss bedroht oder betroffen angenommen werden (Menschen mit geistiger Behinderung im Einstellungssurvey vs. Menschen mit unterschiedlichen Beeinträchtigungen, Menschen mit Fluchtmigrationshintergrund und Menschen mit Demenz in den Sozialraumanalysen). Wichtig ist zudem zu bedenken, dass Einstellung nicht unmittelbar mit Inklusion verknüpft werden können Einstellung ist nicht gleichzusetzen mit Handeln (siehe Kapitel 35). Ein weiterer Faktor, der nicht berücksichtigt werden konnte, betrifft mögliches Unwissen der befragten Personen. Dadurch, dass im Gros nur wenig Kontakt 
besteht zwischen Menschen mit geistiger Behinderung und Menschen ohne (geistige) Behinderung, kann es in der Einschätzung der abgefragten Lebensbereiche zu Wissens- und Erfahrungslücken kommen, die das Ergebnis mitbeeinflussen. Unklar ist darüber hinaus, wie stark die Ausprägung der hier untersuchten Einstellung gegenüber anderen Einstellungen ist. Es ist beispielsweise denkbar, dass Personen zwar offen gegenüber Menschen mit geistiger Behinderung und (zum Beispiel) Menschen mit Fluchtmigrationshintergrund eingestellt sind, sich jedoch, sobald es um konkrete Handlungsoptionen geht, immer eher für Menschen mit Fluchtmigrationshintergrund engagieren würden. Einstellungen, die als ähnlich eingeschätzt werden, können also in der Handlungspraxis anders gewichtet werden. Methodische Anschlussmöglichkeiten wären nun beispielsweise, vertiefende, qualitative Interviews zu führen, die die Personen adressieren, die Inklusion (eher) ablehnend oder (eher) offen gegenüberstehen, um zu erfahren, wie sie Ablehnung oder Offenheit begründen, was ihre lebenspraktischen und biographischen Erfahrungen sind und welche Zukunftswünsche sie haben. Insbesondere latente Sinnzusammenhänge sind dabei zu berücksichtigen, $\mathrm{zu}$ denen im hiesigen Design kein Zugang eröffnet wurde (siehe dazu die Diskussion in Kapitel 24).

\section{Handlungspraktische Gesamtbetrachtung und Diskussion}

In handlungspraktischer Hinsicht ist ebenfalls der Bezug auf die sogenannte Kontakthypothese relevant, die sich im Survey insofern bestätigte, dass die Offenheit in Bezug auf Inklusion mit dem lebenspraktischen Kontakt mit Menschen mit (geistiger) Behinderung korrelierte (siehe Kapitel 22). Es kann also davon ausgegangen werden, dass durch beispielsweise (bewusstseins-) bildende Maßnahmen und/oder das Schaffen von Kontaktmöglichkeiten die Offenheit der Mehrheitsgesellschaft gegenüber Inklusion erhöht werden könnte. Mit Blick auf solche Maßnahmen ist zweierlei festzuhalten: Adressiert werden sollten dabei nicht jene Personen, die Menschen mit diversen Bedarfen grundsätzlich ablehnend gegenüberstehen, sondern eher jene, die ihnen, wenngleich teils sehr geringe, Teilhabemöglichkeiten einräumen

7 An dieser Stelle kann auf die Ebene 3 der Forschung zum hier untersuchten Projekt verwiesen werden, die in Einzelfallstudien (auch) latente Sinnzusammenhänge fokussiert (Leitung: Prof. Dr. Dieter Katzenbach, Goethe-Universität Frankfurt). Zur Ausgestaltung dieser Forschungsebene siehe Kapitel 8.3. 
(dies betrifft insbesondere Personen aus den Clustern 2 und 3). Letztere können durch das Herstellen von Kontakt sehr gut erreicht werden, denn »[b]ei positiver Grundeinstellung wirkt Kontakt als Verstärker" (Cloerkes 1982, S. 567). Die Verantwortung dafür, Kontaktmöglichkeiten zu schaffen, liegt insbesondere auf (sozial-)politischer Seite, deren Aufgabe es ist, »dafür Sorge zu tragen, daß die öffentlichen Akteure vor Ort, also LehrerInnen, SozialarbeiterInnen, Sozialpädagoglnnen, ErzieherInnen, die optimalen Kontaktbedingungen herstellen können" (Dollase 2001, S. 21; siehe auch Allport 1954, S. 489). Kontaktmöglichkeiten tatsächlich nutzen muss allerdings jede Person selbst (Dollase 2001, S. 21). Darüber hinaus bleibt in handlungspraktischer Hinsicht vor allem die Frage nach der Umsetzung von Inklusion, denn, auch wenn einige Personen Inklusion gegenüber (eher) offen eingestellt sind, so ist dies nicht gleichbedeutend damit, dass Menschen mit bestimmten Unterstützungsbedarfen dadurch Teilhabemöglichkeiten eröffnet werden. Aus offener Einstellung folgt nicht automatisch Inklusion. Insofern muss darüber nachgedacht werden, wie (a) mehr Berührungspunkte und Kontaktmöglichkeiten zwischen EinwohnerInnen von Städten und Gemeinden geschaffen werden können (siehe auch Trescher et al. 2020b, 2020a). Dazu braucht es ein Wissen darüber, wer die EinwohnerInnen des jeweiligen sogenannten Sozialraums sind, wie sie erreicht werden können und welche Bedarfe sie haben, und es müssen überhaupt erst Orte geschaffen werden, an denen sich Menschen treffen können und wollen. In diesem Zusammenhang ist es darüber hinaus wichtig, Institutionalisierungspraxen von insbesondere Menschen mit Behinderung infrage zu stellen (siehe Kapitel 39). Denn eine eher abgeschottete Lebensweise (beispielsweise ein Leben im stationär betreuten Wohnen in einer Wohneinrichtung) erschwert Kontakte zu Menschen außerhalb des Strukturrahmens der Behindertenhilfe, da es nur sehr wenige gemeinsame Orte gibt, an denen sich Menschen mit und Menschen ohne Behinderung begegnen könnten. Handlungspraktisch könnte also die Öffnung von (primär behindertenspezifischen) Betreuungsstrukturen angeregt werden, um Strukturrahmen zu dekonstruieren, die nach wie vor eine totale Wirkmächtigkeit haben können (Goffman 1973; Trescher 2017f; Täubig 2009). Weiterhin braucht es Ideen, wie (b) zu mehr Sensibilität im Umgang und einem Bewusstsein für diverse Bedarfe, Barrieren und Möglichkeiten ihres Abbaus beigetragen werden kann. Denkbar sind hier vor allem Aufklärungs- und Bildungsangebote, die insbesondere Raum dafür geben, problematisierende Fragen zu stellen, und die andere (gege- 
benenfalls ablehnendere) Meinungen nicht unreflektiert und kategorisch abwehren, sondern in einen tatsächlichen Austausch treten, in dem sich alle Beteiligten wahr- und ernstgenommen fühlen. Ein weiterer handlungspraktischer Aspekt ist schließlich (c), die EinwohnerInnen eines Sozialraums vermehrt darüber aufzuklären, was Inklusion bedeutet (oder bedeuten kann). Dazu gehört in erster Linie, Inklusion nicht einseitig als Konzept zu verstehen, das angewendet werden kann - Inklusion ist nichts, das entlang der Vorgaben eines Handbuchs umgesetzt werden könnte (siehe Kapitel 27). Vielmehr gilt es, die Einsicht zu wecken, dass Inklusion eine Praxis ist, die sich im Miteinander vollzieht und die Behinderungspraxen gegenläufig ist.

\section{Methodische Gegenüberstellung des Surveys "Einstellung(en) zu Inklusion« und der Sozialraumanalysen in den Handlungsfeldern Arbeit und Freizeit}

Der Survey »Einstellung(en) zu Inklusion« und die Sozialraumanalysen in den Handlungsfeldern Arbeit und Freizeit fokussieren unterschiedliche Untersuchungsebenen, die je eigene Ergebnisse hervorbringen. Der große Gewinn dieses Vorgehens ist, dass sich die Ergebnisse im mehrdimensionalen Forschungsdesign der Studie ergänzen und so eine breite Beforschung von Inklusion und Sozialraum ermöglichen ${ }^{8}$. Im Folgenden werden daher noch einmal Parallelen zwischen diesen beiden Teilstudien gezogen, Unterschiede herausgestellt und auf ebenjenes Ineinandergreifen mehrperspektivischer Forschung im Design der Gesamtstudie eingegangen.

\subsection{Interpretationsebene: Einstellung vs. Typisierung}

Werden der Survey »Einstellung(en) zu Inklusion « und die Sozialraumanalysen im Kontext Arbeit und Freizeit betrachtet, so zeigt sich, dass, auch wenn beide Untersuchungen intentionale Beantwortungen auf Fragen zu ihrem

8 In diesem Zusammenhang soll noch einmal darauf hingewiesen werden, inwiefern ein so komplexes, exploratives Studiendesign wie das hiesige von Vorerfahrungen aus vorangegangener Forschung profitiert. Insbesondere ist hierbei die Studie »Inklusion. Zur Dekonstruktion von Diskursteilhabebarrieren im Kontext von Freizeit und Behinderung« (Trescher 2015b) hervorzuheben. 
Gegenstand machen, sie sich bezüglich der jeweils Anwendung findenden Interpretationsebene voneinander unterscheiden. Während im Kontext des Einstellungssurveys die Interpretation durch die antwortende Person erfolgt, die ihre Meinung einer Zahl auf einer Skala zuordnet, erfolgt dies in den Sozialraumanalysen durch die interpretierenden ForscherInnen, die anhand der Verfahren der Qualitativen Inhaltsanalyse Kategorien aus dem gesamten Material bilden. Folglich ist das Vorgehen bei der Kategorienbeziehungsweise Clusterbildung verschieden. In den Sozialraumanalysen wurden die Kategorien induktiv aus dem Material, also dem Antwortverhalten der Interviewpersonen, herausgebildet und es wurden gewisse Regeln der Zuordnung aufgestellt. Äußerst sich eine Interviewperson beispielsweise eindeutig negativ gegenüber Menschen mit Behinderung, kann sie nicht dem Typ >offen zugeordnet werden, auch wenn ihr Antwortverhalten ansonsten diesem Typ entspricht. Hieran zeigt sich erneut die Stärke des qualitativen Moments der Typisierung (siehe Kapitel 15). In der Einstellungsforschung dagegen wurden die Cluster respektive die zugrundeliegenden Kategorien zwar auch induktiv gebildet, allerdings nicht nach der Qualität der Aussage, sondern dahingehend, wo es (valide) Parallelen beziehungsweise Abweichungen im Antwortverhalten gab. Dies ist einer der Gründe dafür, weshalb die Cluster, die die Einstellungen der befragten Personen zu Inklusion zusammenfassen, nicht mit den Typen zusammenhängen, die im Kontext der Untersuchung von Teilhabe an Arbeit und Freizeit in den fünf Sozialräumen herausgebildet wurden.

\subsection{Legitimation: Testung und Forschungstradition vs. Komplexität und Würdigung des Einzelfalls}

Eine weitere Unterscheidung der beiden Teilstudien ist entlang der Legitimation der Forschungsmethodik möglich. Der Survey versucht, Einstellungen offenzulegen und nutzt dabei ein komplexes System an Fragen. Er legitimiert sich dabei durch die mehr oder weniger lange Tradition der Einstellungsforschung in den Sozialwissenschaften sowie dadurch, dass jenes Fragensystem vorab breit getestet und adaptiert wurde (siehe Kapitel 20). Wichtig ist, dass die Einstellung aus diesem komplexen, getesteten Fragesystem generiert wird und nicht etwa unmittelbar nach ihr gefragt wird (es wurde also nicht etwa gefragt: "Was halten Sie von Inklusion?«). Die Sozialraumanalysen dagegen legitimieren sich dadurch, dass Einzeldaten 
beziehungsweise Interviews (und damit immer auch Einzelpersonen) in ihrer Komplexität gewürdigt werden. Dies bedeutet, dass aus den zahlreichen Einzelfällen induktiv Kategorien gebildet werden, die jene Komplexität fassen und ihr gerecht werden. Die Konsequenz daraus ist, dass durch das methodische Vorgehen im Survey Einstellungen herausgearbeitet und in die Breite gehende Forschungsergebnisse generiert werden können. Allerdings ist dadurch kein Zugang zum Einzelfall mehr möglich. Dies ist bei den Sozialraumanalysen zwar noch eher gegeben (das Material ist zum Beispiel deutlich vielschichtiger und wird umfangreicher ausgewertet), allerdings kann auch hier nicht jedem Interview beziehungsweise Einzelfall in seiner vollen Komplexität gerecht werden (siehe hierzu ausführlicher Kapitel 24.8).

\subsection{Gegenstand: Hypothetische vs. tatsächliche Lebenspraxis}

Ein bedeutsamer Unterschied zwischen beiden Untersuchungen, insbesondere hinsichtlich der Bewertung der Ergebnisse, liegt im je unterschiedlichen Gegenstand der beiden Untersuchungen. Während der Gegenstand des Surveys der eigene Bezug der befragten Personen zu einer eher hypothetischen Lebenspraxis ist, geht es in den Sozialraumanalysen um die tatsächliche, persönliche Lebenspraxis, die die jeweilige Interviewperson ganz unmittelbar betrifft. Der Survey lässt also noch eine gewisse Distanz zu den Fragen zu, während die Sozialraumanalysen ein mögliches Miteinander im persönlichen Arbeits- und Freizeitleben der Interviewpersonen fokussieren, was es schwieriger macht, sich diesen zu entziehen und die Lebenspraxen zu abstrahieren. Diese Unterscheidung zwischen hypothetischer und tatsächlicher Lebenspraxis ist mit ein Grund dafür, dass Einstellungen nicht unmittelbar und uneingeschränkt in Handlungen übertragen werden können (siehe Kapitel 35).

\section{4 Übertragbarkeit: Deutschlandweit, bevölkerungsrepräsentativ vs. sozialraumbezogen, modellhaft}

Während es sich bei der Einstellungsforschung um ein deutschlandweites, bevölkerungsrepräsentatives Setting handelt, bilden die Sozialraumanalysen Erfahrungen und Meinungen von Personen ab, die in den fünf untersuchten Sozialräumen leben. Die Ergebnisse des Einstellungssurveys sind insofern ein Stück weit als valider zu betrachten und eher übertragbar. Die 
Ergebnisse der sozialraumbezogenen Untersuchungen dagegen können nicht ohne Weiteres und uneingeschränkt auf andere Sozialräume übertragen werden. Dennoch eröffnen sie Verstehenszugänge dahingehend, worin Barrieren und Handlungsperspektiven von Inklusion im Kontext Sozialraum liegen und sind dadurch nutzbar für AkteurInnen anderer, nicht untersuchter Sozialräume. Gerade die qualitativen Elemente der Sozialraumanalysen eröffnen also einen Einblick darin, inwiefern gewisse Praxen und Bedingtheiten so oder so ähnlich auch in anderen Sozialräumen gegeben sein können, die im Prozess der Sozialraumentwicklung möglicherweise zu berücksichtigen sind.

\subsection{Datengenerierung: Online-Fragebogenerhebung vs. persönliches Interview}

Einstellungssurvey und Sozialraumanalysen generierten ihre Daten auf unterschiedliche Art und Weise, wodurch diese voneinander different sind. Es können (und sollen) deshalb nicht unmittelbar vergleichbare Aussagen getroffen werden. Die Daten der quantitativen Erhebung im Einstellungssurvey können in mancher Hinsicht als zuverlässiger betrachtet werden, da die befragten Personen den Fragebogen online, anonym und unabhängig einer interviewenden Person bearbeiten konnten und so möglicherweise ehrlicher und offener antworteten. In den Leitfadeninterviews der Sozialraumanalysen, die persönlich vor Ort (im Kontext Arbeit) und telefonisch (im Kontext Freizeit) erfolgten, wurde den Interviewpersonen selbstredend ebenfalls Anonymität zugesichert und im Auswertungsprozess unbedingt eingehalten. Dennoch kann es sein, dass Interviewpersonen im direkten Gespräch unter Umständen etwas verschlossener sind und/oder Antworten beschönigen. Gleichzeitig ist auch das Gegenteil denkbar, dass nämlich Interviewpersonen im persönlichen Gespräch offener sind, wenn sie (mehr oder minder) frei über das gefragte Thema erzählen können. Wichtig ist zudem, dass im persönlichen Gespräch der Gegenstand des Interviews erklärt werden kann, sollte die jeweilige Interviewperson Rückfragen haben. Allerdings konnte in den Sozialraumanalysen nicht die Perspektive derjenigen Personen mitaufgenommen werden, die zwar über Inklusion reden wollen beziehungsweise ein Interesse daran und eine Meinung dazu haben, jedoch über die Interviewerhebung nicht erreicht wurden. Im Einstellungssurvey dagegen konnte auch die Perspektive jener Personen (potenziell) berücksichtigt wer- 
den, da der Fragebogen in ein Panel eingebettet war, das die Bevölkerung Deutschlands repräsentativ abbildet, was, wie oben bereits hervorgehoben, ein zentraler Gewinn und (bezogen auf das Thema Einstellung zu Inklusion) Alleinstellungsmerkmal dieses Surveys ist. Deutlich wird in jedem Fall, dass sich die Erhebungsformen - und die daraus gewonnenen Daten - unterscheiden und nicht unmittelbar miteinander verglichen werden können.

\subsection{Differenzkategorie: Geistige Behinderung vs. Behinderung, Fluchtmigration und Demenz}

Ein weiterer Unterschied liegt darin, dass im Kontext der Einstellungsforschung ausschließlich nach Teilhabemöglichkeiten von Menschen mit geistiger Behinderung gefragt wurde (siehe Kapitel 20.1), während bei den Sozialraumanalysen Menschen mit Behinderung (Beeinträchtigungen im Kontext Mobilität, Sehen, Hören und kognitiv), Menschen mit Fluchtmigrationshintergrund und, im Kontext Freizeit, Menschen mit Demenz im Fokus standen (siehe Kapitel 14.2). In beiden Verfahren wurden Ergebnisse hervorgebracht, die für sich gesehen wichtige Erkenntnisse rund um das Thema Sozialraum und Inklusion liefern - die aber nicht unmittelbar aufeinander bezogen werden können.

\subsection{Gemeinsamkeit: Ergebnisse zeigen ähnliche Problematiken auf}

Obwohl sich die beiden Teilstudien hinsichtlich einiger Punkte voneinander unterscheiden, können Gemeinsamkeiten sowie teils ähnliche Problematiken festgestellt werden, die die Ergebnisse des Einstellungssurveys und der Sozialraumanalysen offenlegen. So gibt es beispielsweise in inhaltlicher Hinsicht einige ähnliche Verteilungstendenzen. Bei beiden Untersuchungen wurde im Handlungsfeld Arbeit die geringste Teilhabe(-möglichkeit) von Menschen mit Behinderung und/oder Menschen mit Fluchtmigrationshintergrund sowie die geringste Zustimmung zu Inklusion ausgemacht. Inklusion steht im Kontext Arbeit also vor besonderen Herausforderungen, Barrieren abzubauen, zu Sensibilität und Bewusstsein beizutragen und letztlich (ausschließende) Handlungspraxen zu verändern, welche die Herausforde- 
rungen anderer Lebensbereiche (insbesondere Freizeit) übersteigen'. Die mehrperspektivische Untersuchung von Barrieren und Teilhabemöglichkeiten im Sozialraum sichert also auch solche Ergebnisse. Anzumerken ist hierbei, dass sich diese Einschätzung bei den Sozialraumanalysen auf die Bereitschaft bezieht, Teilhabemöglichkeiten in Bezug auf alle abgefragten Differenzkategorien zu eröffnen - Behinderung, Fluchtmigrationshintergrund und Demenz. Allerdings hat sich in vielen Fällen gezeigt, dass zum Beispiel die Offenheit gegenüber Menschen mit Mobilitätsbeeinträchtigungen deutlich höher ist als beispielsweise gegenüber Menschen mit geistiger Behinderung (im Einzelfall jedoch auch umgekehrt). Teilweise ist es also möglich, detailliertere und tiefergehende Erkenntnisse aus den Interviews zu gewinnen, was für die Gesamtstudie eine zusätzliche Ergänzung und Bereicherung ist. Eine weitere Einsicht, die aus den Ergebnissen abgeleitet werden kann, ist, dass die Größe respektive Diversität der Gruppe, nach der als potenzielle AdressatInnen von Inklusion gefragt wird, die Haltung zu Inklusion mitbeeinflusst. So kann vermutet werden, dass, je größer die Gruppe derer ist, die miteinbezogen werden sollen und nach denen in Interviews oder Surveys gefragt wird, desto höher die Ablehnung gegenüber dieser Gruppe beziehungsweise Inklusion im Allgemeinen ist.

\subsection{Desiderat: Latente Sinnzusammenhänge erforschen}

Ein Desiderat, das sich nicht nur aus den Untersuchungen bezüglich des Einstellungssurveys und der Sozialraumanalysen ergibt, sondern das die gesamte Studie durchzieht, ist, dass im hier entwickelten Methodensetting keine latenten Sinnstrukturen offengelegt werden. Eine Analyse latenter Sinnstrukturen »bezieht sich auf die durch Regeln erzeugten objektiven Bedeutungen einer Sequenz von sinntragenden Elementen einer Ausdrucksgestalt, in der alle nur denkbaren konkreten (Lebens-)Äußerungen von Lebenspraxis-Formen verkörpert sein können" (Oevermann 2001, S. 39). Gegenstand einer solchen Analyse ist immer der Einzelfall in seiner Komplexität, wodurch die vielschichtigen (gegebenenfalls ambivalenten) Bedingungsstrukturen des Gegenstandes anerkannt und gewürdigt werden. Es kann somit ein Zugang eröffnet werden, zu den Erfahrungen, Geschichten,

9 Die Potenziale von Inklusion im Kontext Freizeit sind vielfältig dargelegt (Trescher 2015b; Trescher et al. 2020a, 2020b; Markowetz; Markowetz 2006). 
Ängsten, Hoffnungen oder anderen Latenzen, die mit intentionalen Äußerungen verknüpft sind beziehungsweise diese mithervorbringen und die andernfalls verschlossen blieben. Leerstellen in der Forschung betreffen also Einzelfallstudien, die je konkrete (Lebens-)Geschichten in den Fokus nehmen und latente Sinnstrukturen herausarbeiten ${ }^{10}$. Insbesondere Fragen von Teilhabe und Ausschluss können anhand solcher Verfahren in den Blick genommen werden, zeigen doch auch die Ergebnisse dieser Studie, dass sich Behinderungspraxen vielfältig, individuell und je situativ vollziehen, weshalb es folglich Forschungsansätze bedarf, die diese Komplexität erfassen können. Gerade bei der Beforschung der Lebenssituation vulnerabler Gruppen beziehungsweise Personen, die von Ausschluss bedroht oder betroffen sind, verspricht die Erforschung latenter Sinnzusammenhänge einen wichtigen Erkenntnisgewinn. Dies liegt insbesondere daran, dass jene Forschungsverfahren »die Sache selbst zum Sprechen bringen, indem sie sich an sie anschmiegen und durch dieses unvoreingenommene, radikale Sicheinlassen auf die jeweilige Besonderheit des Gegenstandes hindurch zum zugleich klärenden wie kritisch überwindenden, allgemeinen Begreifen der gesellschaftlichen Wirklichkeit gelangen« (Oevermann 1983, S. 234). Eine Analyse latenter Sinnstrukturen kann beispielsweise auf Ebene der Sozialraumanalysen in die Studie implementiert werden. Die Interviews in den Sozialraumanalysen bereiten am ehesten den Boden dafür, ein Verstehen latenter Sinnzusammenhänge in der Studie zu ergänzen, da dort nach konkreten Praxen gefragt wird - Was heißt es ganz konkret für die Interviewperson, Menschen mit unterschiedlichen Unterstützungsbedarfen in ihrem Arbeits- oder Freizeitleben zu haben? Dabei können biographische Bezüge eine Rolle spielen, wie etwa die Frage, ob jemand gewissen Personen eher ablehnend gegenüber eingestellt ist, da diese womöglich Erinnerungen an krisenhafte Erfahrungen wecken. Beispielsweise wäre es denkbar, dass eine Person Schwierigkeiten hat, im Rahmen einer Freizeitaktivität in Kontakt mit einer Teilnehmerin mit Demenz zu treten, da diese sie an ihre mittlerweile verstorbene, zu Lebzeiten jedoch schwer an Demenz erkrankte Mutter erinnert, die sie gepflegt hat. Auch die ethnographischen Sozialraumbegehungen können um eine Analyseebene der Latenz erweitert werden. Am Bei-

10 Latente Sinnzusammenhänge können rekonstruktiv-sequenzanalytisch herausgearbeitet werden (u.a. Oevermann 2000, 2002). Konkrete methodische Settings zu unterschiedlichen Gegenständen finden sich in (Trescher 2013, 2015b, 2017a, 2017f, 2018a). 
spiel des sogenannten Rollstuhlkuriers in Schwäbisch Gmünd (siehe Kapitel 13.1.4) können die Fragen aufgeworfen werden, was genau es für ihn bedeutet, dass er jetzt in gewisser Weise am Sozialraum teilhat, wie ihm andere Personen begegnen und warum oder wie er andere Personen sowie seine KundInnen wahrnimmt respektive selbst wahrgenommen wird. Ein Verstehen dieser und weiterer Fragen ermöglicht, die komplexen Bedingungsgefüge nachzuzeichnen, die Lebenspraxis in ihrer Einzigartigkeit hervorbringen. 



\section{VII.Zusammenfassung - Inklusion, Aneignung, Sozialraumentwicklung}

Abschließend werden in diesem Kapitel zentrale Ergebnisse zusammengeführt und weiterführende Anschlussmöglichkeiten diskutiert, wobei immer wieder Rückbezug zu den Fragen gesucht wird, inwiefern Raum und Inklusion miteinander in Beziehung stehen und wie Sozialräume verändert werden können, sodass Personen sich diese als teilhabend aneignen können, die bislang primär an Barrieren stoßen.

\section{Aneignung von Raum}

Raum wird in Aneignungspraxen hervorgebracht, in denen die betreffenden Personen wiederum je bestimmte Subjektpositionen einnehmen. Ausgehend von diesen Positionen kann sich das Subjekt Raum in je bestimmter Art und Weise aneignen - beispielsweise als individuellen Handlungsraum oder als Raum, in dem es primär an Barrieren stößt. Aneignung vollzieht sich also in der Wechselwirkung zwischen hervorbringendem Subjekt und hervorgebrachtem Raum, der wiederum Subjektpositionen bereitet und damit das Subjekt in je bestimmter Weise hervorbringt (Hüllemann et al. 2016, S. 11). Inklusion im Kontext der Aneignung von Raum bedeutet folglich, (a) Teilhabebarrieren abzubauen, sodass Personen Zugang zu Orten haben, an denen sie Raum hervorbringen können, (b) die räumlichen Aneignungsmöglichkeiten von Orten so zu erweitern, dass Ausschluss vermieden wird und (c) Personen als handlungsmächtig zu subjektivieren, sich Raum in je bestimmter, von ihnen gewünschter Weise anzueignen. Notwendig ist darüber hinaus, dass sich die jeweilige Person sozial und emotional zugehörig beziehungsweise verbunden fühlt, denn Teilhabe kann nicht ausschließlich 
durch das vorgängige Bereiten von Zugängen, Normalisierungspraxen und Subjektpositionen ermöglicht werden (siehe Kapitel 4.1). Vielmehr bedarf es zusätzlich der inneren Beteiligung der jeweiligen Subjekte, was zeigt, dass sich Teilhabe insbesondere im sozialen Miteinander vollzieht (siehe Kapitel 5.5). Raum wird gelebt. Handlungspraktisch bedeutet dies, dass Inklusion nicht auf den Bau von Aufzügen oder die Ausgestaltung eines Ortes als sbarrierefrei enggeführt werden kann. Dadurch werden zwar grundlegende Barrieren verringert, es braucht jedoch darüber hinaus insbesondere die intersubjektive Aushandlung von (Wissen um) Barrieren und die Vergabe von Sprechrollen, um sich Raum als teilhabend aneignen zu können. Die Ergebnisse der Gesamtstudie, die in den empirischen Teilen ausführlich dargelegt wurden, zeigen, inwiefern sich Aneignungspraxen von Raum vollziehen und sich Personen Raum als (mehr oder weniger) teilhabend aneignen. Dabei wird immer wieder deutlich, dass bestimmte Personen Ausschluss erfahren, der dazu führt, dass sie sich Raum primär als ,Territorien der Anderen aneignen können (Trescher und Hauck 2017). Jene Ausschlusspraxen vollziehen sich teilweise durch Hindernisse und Barrieren, die physisch wahrnehmbar sind, wie beispielsweise Treppen, die nicht umgangen werden können, oder Informationen, die ausschließlich in schwerer Schriftsprache dargeboten werden - gerade in den ethnographischen Sozialraumbegehungen konnten derartige Barrieren zahlreich dokumentiert werden (siehe Kapitel 13). Eine Aneignung von Raum als weniger oder kaum teilhabend kann darüber hinaus durch Barrieren mithervorgebracht werden, die sich eher latent vollziehen. Dazu gehören beispielsweise ablehnende Haltungen in der Mehrheitsgesellschaft, die bestimmte Personen kategorisch ausgrenzen (ihnen also keine Sprechrolle im jeweiligen Diskurs gewähren; siehe Kapitel 4.1), sowie die Angst, beispielsweise nicht den Erwartungen und Ansprüchen der Gegenüber gerecht zu werden, oder auch mangelnde gemeinsame Erfahrungen, aus denen eine gewisse Zurückhaltung oder Berührungsangst resultiert. Teilhabebarrieren dieser Art zeichnen sich insbesondere im Zuge der Sozialraumanalysen in den Handlungsfeldern Arbeit und Freizeit ab (siehe Kapitel 16 und Kapitel 17). Exemplarisch kann dies an Aussagen von interviewten ArbeitnehmerInnen verdeutlicht werden, die sich vereinzelt sehr ablehnend gegenüber einer Teilhabe von Menschen mit (geistiger) Behinderung und/oder Menschen mit Fluchtmigrationshintergrund äußern (siehe Kapitel 16.9.1). So sagt beispielsweise eine Interviewperson:»Inklusion ist im Gastrobereich schwierig, gerade für Flüchtlinge, wegen dem Kunden- 
kontakt. Die machen einfach mehr Fehler« (4279). Ablehnende Haltungen können zur Teilhabebarriere werden, denn die darin adressierten Personen werden größtenteils nicht als ssprechend im jeweiligen Diskurs anerkannt. Eine Aneignung von Raum als teilhabend ist folglich sehr schwierig. Eine handlungspraktische Folgerung aus diesen Problematisierungen ist, das mehrheitsgesellschaftliche Bewusstsein für Barrieren zu sensibilisieren, insbesondere dahingehend, dass diese vielfältig, je situativ und einzelne Personen betreffend entstehen können, was jedoch nicht ihre Wirkmächtigkeit einschränkt. Um latenten Barrieren zu begegnen, die insbesondere in Ängsten, Skepsis oder je bestimmten (ablehnenden) Haltungen bestehen, gilt es, Begegnungsmöglichkeiten zu schaffen und dadurch gegebenenfalls (beiderseitig) vorhandene Vorbehalte abzubauen. Diese und weitere handlungspraktische Hinweise werden in einigen der nachfolgenden Kapitel erläutert.

\section{Unscharfe Inklusionsverständnisse}

Inklusion ist zwar sowohl begrifflich als auch in der Handlungspraxis weit verbreitet, dennoch bleibt das Begriffsverständnis in Theorie und Praxis häufig unscharf, was vor allem daran liegt, dass Inklusion von den jeweiligen AkteurInnen je unterschiedlich mit Inhalt gefüllt wird. Folglich scheint »ein Konsens darüber, was denn unter >Inklusion` zu verstehen ist, derzeit nicht absehbar" (Ackermann 2013, S. 171; siehe auch Cramer und Harant 2014, S. 642ff; Dangl 2014, S. 258ff; Dannenbeck 2012, S. 55; Trescher 2015b, S. 332). Vielmehr werden Verständnisse, von dem, was Inklusion ist beziehungsweise sein soll, und insbesondere von dem, wie sie >umgesetzt $\measuredangle$ werden soll, zugrunde gelegt, die teilweise sogar eher widerstreitend sind. Insofern ist ein diffuses Begriffsverständnis von Inklusion vor allem dadurch problematisch, dass sie eben gerade nicht zum Problem gemacht und als Begriff vorausgesetzt wird, dabei allerdings in gewisser Art und Weise Leerformel bleibt. Ebenfalls problematisch ist, dass Inklusion teilweise als »ideologische Semantik« (Herz 2015, S. 64) oder idealisierter "Mythos « (Schäper 2015, S. 80) Einzug in den (pädagogischen) Sprachgebrauch hält. Dannenbeck (2012) äußerst sogar die Vermutung, es würde versucht, durch »unscharfe Verwendungen des Inklusionsbegriffs von dessen kritischen Potenzial abzulenken « (Dannenbeck 2012, S. 55). Es braucht also eine dezidierte Begriffsbildung im 
Kontext Inklusion. In theoretischer Hinsicht zielt Begriffsbildung darauf ab, Ambivalenzen, »Brüche und Widersprüche in sich abzubilden« (Pongratz 2010, S. 15), um dadurch »einer dynamischen, spannungsvoll aufgeladenen, ja, von Konflikten regelrecht zerrissenen Gegenwart zum Ausdruck [zu] verhelfen« (Pongratz 2010, S. 15). Inwiefern es wichtig ist, sich mit Inklusion als Begriff und Praxis zu beschäftigen, zeigen auch die Ergebnisse der Sozialraumanalysen in den Handlungsfeldern Arbeit und Freizeit (siehe Kapitel 16 und Kapitel 17), kam es doch beispielsweise teilweise vor, dass sich Interviewpersonen dafür ausgesprochen haben, behindertenspezifische Strukturen (zum Beispiel sogenannte Werkstätten für Menschen mit Behinderung) zu festigen - und stellen dies als eine Handlungsperspektive im Kontext Inklusion dar. Dies wirft ganz offensichtlich die Frage nach dem dabei vorliegenden Verständnis von Inklusion auf, das sich entgegen verbreiteter Verständnisse von Öffnung eher in der Festigung binärer Strukturen begründet, die sozusagen eine Trennung bbehinderter und snicht behinderter Sphären fortschreibt. Offen bleibt darüber hinaus teilweise, ob überhaupt und welche Bedeutung Inklusion im Kontext Arbeit oder Freizeit zugeschrieben wird. Hier deuten sich Anknüpfungspunkte weiterführender Forschungsmöglichkeiten an, die detailliert untersuchen, wie Personen aus der Mehrheitsbevölkerung (und darüber hinaus) Inklusion verstehen. In handlungspraktischer Hinsicht kann ausgehend von diesen Ergebnissen diskutiert werden, dass es für Personen, die es sich zum Ziel gesetzt haben, Inklusion in ihrem Ort oder einem bestimmten Sozialraum voranzubringen, notwendig ist, ein Verständnis von Inklusion zu konkretisieren, das dem Vorhaben zugrunde gelegt werden kann. Es bietet sich hierbei an, die Nähe zum oben entfalteten Inklusionsverständnis zu suchen, das Inklusion als kritische Praxis versteht, die Behinderungspraxen genau gegenläufig ist (siehe Kapitel 4.2; siehe Trescher 2018b). Inklusion als Kritik analysiert Ambivalenzen von Teilhabe und Ausschluss und stellt ausgehend davon eingeschriebene Zusammenhänge und Praxen infrage, die dazu führen, dass bestimmte Personen je situativ behindert werden. Inklusion vollzieht sich in diesem Verständnis also als Prozess der Dekonstruktion von Diskursteilhabebarrieren (Hartmann 2001, S. 80f; siehe auch Engelmann 1990, S. 27). Der Vorteil eines Verständnisses von Inklusion als Kritik ist, dass dieses nahezu losgelöst von vorgängigen Behinderungskategorien entfaltet werden kann, wodurch vermieden wird, >Restgruppen $\mathrm{zu}$ produzieren, deren Belange nicht berücksichtigt werden. Darauf aufbauend ist es möglich, Projekte 
und/oder Maßnahmen zu entwickeln und umzusetzen, die entlang dieses (oder zumindest eines je bestimmten) Inklusionsverständnisses erfolgen. Dadurch können Maßnahmen entlang einer gemeinsamen Grundlage oder Ausrichtung geplant werden, was dem Vorhaben Stringenz und damit womöglich größere Wirksamkeit und Nachhaltigkeit verleiht. Im Kontext eines solchen Vorhabens ist darüber hinaus bedeutsam, das Thema Inklusion den jeweiligen AdressatInnen von Projekten oder Maßnahmen inhaltlich zugänglich zu machen, da, wie die Ergebnisse der Sozialraumanalysen zeigen, nicht davon ausgegangen werden kann, dass ein Verständnis von Inklusion vorliegt - insbesondere kein gemeinsames. Insofern ist es auch eine Aufgabe von Bewusstseinsbildung, ein Verständnis von Inklusion zu entwickeln, ausgehend dessen Lebenspraxis reflektiert werden kann, worin wiederum ein erster Schritt zu etwaigen Veränderungen liegt. Trotz eines solchen anvisierten gemeinsamen Verständnisses ist es wichtig, für Positionen offen zu sein, die dem nicht entsprechen, da nur so der Begriff weiterentwickelt werden kann. Dies ist gerade vor dem Hintergrund relevant, dass eine solche Begriffsbildung nicht mit dem Ziel verfolgt wird, trennscharfe Definitionen $\mathrm{zu}$ entwickeln, sondern (in handlungspraktischer Hinsicht) ein gemeinsames Verständnis auf den Gegenstand zugrunde zu legen, der kontingent ist und somit im Zuge des Vorhabens verändert werden kann. Es kann also festgehalten werden, dass es sowohl aus theoretischer als auch handlungspraktischer Perspektive notwendig ist, sich mit Inklusion und dem Verständnis dieser auseinanderzusetzen - worin sich wiederum Inklusion als kritische Praxis vollziehen kann.

\section{IUmsetzunge von Inklusion}

Eine Frage, die von unterschiedlicher Seite immer wieder gestellt wird, ist die nach der >Umsetzung von Inklusion. Diese Frage wird, neben zahlreichen anderen, auch vonseiten der deutschen Bundesregierung gestellt, die das Programm "gemeinsam einfach machen« aufgelegt hat, im Rahmen dessen die UN-Konvention über die Rechte von Menschen mit Behinderungen in Deutschland sumgesetzt werden soll (einfachmachen 2019; siehe Kapitel 6). In Bezug auf dieses Vorhaben ist zu hinterfragen, inwiefern eine Umsetzung von Inklusion überhaupt möglich ist, schließlich ist Inklusion weniger als eine Art Mechanismus zu verstehen, der technisch umgesetzt werden 
kann, sondern vielmehr als eine Praxis, die krisenhaft ist und sich in der gemeinsamen Aushandlung vollzieht. Forderungen nach und Programme zur Umsetzung von Inklusion sind ambivalent, da sie zwar auf Bedarfe reagieren, oftmals auch Teilhabemöglichkeiten eröffnen, gleichzeitig jedoch Ausschlusskategorien reproduzieren. Dadurch, dass bestimmte Personen durch sogenannte Inklusionsmaßnahmen als Zielgruppe adressiert werden, werden sie als $>$ das andere hervorgebracht und kontinuierlich in diesem Status bestätigt. Maßnahmen, die auf Inklusion abzielen, können so zwar die Teilhabemöglichkeiten bestimmter Personen erweitern, es ist ihnen allerdings dennoch nur eingeschränkt möglich, den Status `AdressatIn von Inklusion< abzulegen, woraus wiederum besondernde Praxen erwachsen können, die möglicherweise in Ausschluss resultieren. Die theoretische Bearbeitung dieser Ambivalenz kann in erster Linie darüber erfolgen, dass Kategorien infrage gestellt und vom Subjekt gelöst werden, um so ihre subjektivierende Wirkmächtigkeit zu mildern. Ziel einer solchen Dekonstruktion ist, dass Personen diverse Subjektpositionen einnehmen können, nicht nur jene, die ihnen mehrheitsgesellschaftlich zugewiesen werden (wenngleich teilweise wohlmeinend, beispielsweise durch die sogenannte Behindertenhilfe). Subjekte können dabei auch selbst die Subjektpositionen infrage stellen, die sie in ihren Teilhabemöglichkeiten einschränken. Sie können »subversiv und kreativ« (Villa 2008, S. 153) mit Diskursgrenzen umgehen. Um diese Möglichkeiten aufzudecken, bedarf es einer Analyse der »dynamischen Aneignungsprozesse[...]« (Motakef 2014, S. 395), anhand derer die Diskursteilhabemöglichkeiten der jeweiligen Person reguliert werden und sie in je bestimmten Subjektpositionen anerkennen. In handlungspraktischer Hinsicht ist denkbar, sogenannte Inklusionsmaßnahmen breiter aufzustellen, um so die Ambivalenz behindernder Praxen aufzulösen, die unweigerlich damit einhergehen, bestimmte Personengruppen zu adressieren. Es kann also darüber nachgedacht werden, die Orientierung an je bestimmten Zielgruppen aufzugeben und (stattdessen) die Gesamtgesellschaft in den Blick zu nehmen. Dabei ist jedoch klar, dass dies handlungspraktisch schwierig ist, denn es ist ja bereits so, dass viele Aktivitäten grundsätzlich allen offenstehen, aber nicht von Personen mit je bestimmten Unterstützungsbedarfen genutzt werden. Die Gründe dafür sind vielfältig und liegen unter anderem in Berührungsängsten, unklaren Regelungen was die gegebenenfalls notwendige Assistenz betrifft oder auch mangelnden Erfahrungen. Wenn also bestimmte Zielgruppen im Besonderen adressiert werden, braucht es unbe- 
dingt Reflexion, um sich bewusst zu machen, dass dadurch Behinderung als Praxis reproduziert wird. Es kann, um zur Ausgangsfrage zurückzukehren, gesagt werden, dass sich Inklusion wohl kaum >umsetzen< lässt, es jedoch notwendig und wichtig ist, ebenjene Diskussion zu führen, da somit ein Thema in die öffentliche Aufmerksamkeit gerückt wird, das bislang oftmals eher randständig behandelt wurde.

\section{Inklusionsprojekte}

Städte und Kommunen, die sich mit Inklusion befassen und diesbezüglich Projekte ins Leben gerufen haben, sind zahlreich. Inklusionskonzepte, -leitlinien, -pläne und einiges mehr werden in großer Zahl entworfen, unter anderem hat jedes Bundesland in Deutschland einen eigenen Aktionsplan Inklusion erstellt (siehe dazu die Ausführungen in Kapitel 5). Selbstredend ist es positiv, dass dem Thema breite Aufmerksamkeit gewidmet wird und sicherlich konnten entsprechende Projekte bereits den ein oder anderen Erfolg verzeichnen, der sich primär darin ausdrückt, dass Personen, die zuvor von Ausschluss bedroht oder betroffen waren, nun eventuell erweiterte Teilhabemöglichkeiten haben. Die Ergebnisse der hiesigen Studie, in der ein konkretes >Inklusionsprojekt $<$ untersucht wurde (siehe Kapitel 7 und Kapitel 8), legen diesbezüglich allerdings auch Problematiken offen, die verschieden ausgestaltet sind. Beispielsweise stellt es sich an mehreren Stellen (unter anderem bezüglich des Vorhabens, über die Kontakte der Projektverantwortlichen einen Survey breit im Sozialraum zu streuen; siehe Kapitel 9.3) als problematisch heraus, dass das Projekt in den jeweiligen Sozialräumen noch nicht sehr bekannt ist, wobei dies auch in der bisher eher kurzen Projektdauer begründet sein kann. Ebenfalls ist zu fragen, weshalb detaillierte und geprüfte Informationen zum jeweiligen Sozialraum erst dann hinzugezogen werden, wenn das Projekt bereits seit einiger Zeit angelaufen ist (siehe Kapitel 12), wodurch nur sehr verzögert auf zum Beispiel Strukturprobleme reagiert beziehungsweise Maßnahmen kaum noch verändert werden können. Problematisch kann gegebenenfalls auch sein, dass es teilweise nur erschwert gelingt, verlässliche Informationen über die Anzahl von Personen mit bestimmten (für eine solche Form der Erhebung pauschalisierten) Unterstützungsbedarfen zu generieren, wodurch auf Bedarfe, die womöglich vorhanden sind, nicht immer passgenau eingegangen werden kann. Selbi- 
ges gilt für Personengruppen, die keine starke Selbst- oder Fremdvertretung haben, welche sich für ihre Interessen einsetzen könnte, und die dadurch in gewisser Weise ein Stück weit unsichtbar bleiben. Es wird also klar, dass ein Projekt, das Inklusion zum Thema hat, bestenfalls eine dezidierte Analyse des Status quo zum Ausgangspunkt nimmt, um aufbauend darauf gemeinsam mit PartnerInnen aus unterschiedlichen Kreisen Maßnahmen zu planen. Bei einer solchen Analyse sollte im Fokus stehen, Barrieren offenzulegen, da diese, »bevor sie abgebaut werden können, wahrgenommen und verstanden werden [müssen] « (Tervooren und Weber 2012a, S. 11). Zusätzlich herausfordernd ist, dass sogenannte Inklusionsprojekte teils einseitig durch Personen, Einrichtungen und Träger verantwortet und durchgeführt werden, die 'klassischen Hilfesystemen angehören, was im Kern die Frage aufwirft, ob überhaupt Strukturveränderungen durch eine solche Reproduktion von Strukturen möglich sind (siehe Kapitel 29 und Kapitel 39). Eine zentrale Problematik dieser einseitigen Fokussierung ist, dass AdressatInnen von Inklusionsprojekten, die durch Hilfesysteme organisiert werden, oftmals primär über ihren Status ‘behindert` und/oder shilfebedürftig wahrgenommen werden. Einzelnen Maßnahmen in einem solchen Projekt ist es infolgedessen kaum möglich, Zuschreibungspraxen und Kategorisierungen aufzuweichen. Vielmehr wird oftmals dazu beigetragen, diese in ihrer Wirkmächtigkeit zu reproduzieren. Inklusionsprojekte vollziehen sich dann als Behinderungspraxen, was im Widerspruch zu ihrem annehmbar ursprünglichen Ansinnen steht. Problematisch ist zudem, dass durch ein solches Vorgehen, eine Dichotomie zwischen rbehindert und snicht behindert beziehungsweise shilfebedürftig` und snicht hilfebedürftig` weitergehend verfestigt wird. Auch dadurch kann die öffentliche Wahrnehmung von Personen, die von Ausschluss bedroht oder betroffen und aufgrund dessen oftmals primäre AdressatInnen sogenannter Inklusionsmaßnahmen sind, von einem paternalistischen Blick geprägt sein, der das Gefälle zwischen diesen und der Mehrheitsgesellschaft eher verschärft und nicht angleicht. Eine große Herausforderung bei der inhaltlichen Gestaltung von Maßnahmen ist in diesem Zusammenhang ebenfalls, ein Gleichgewicht zu finden zwischen der Adressierung Einzelner (oder Gruppen) und der Adressierung der Gesamtbevölkerung. Während mit einer Adressierung von Einzelpersonen oder Gruppen oftmals zwangsläufig irgendeine Form der Pauschalisierung von Einzelmerkmalen einhergeht (beispielsweise als sbehindert), die infolgedessen reproduziert zu werden drohen, besteht bei der Adressierung der 
Gesamtbevölkerung das Problem, dass bestimmte Personen möglicherweise (nach wie vor) nicht von entsprechenden Maßnahmen berührt werden, da ihre Teilhabemöglichkeiten an Praxen der Mehrheitsgesellschaft zu gering sind. Neben der Frage nach der Adressierung stellt sich die Frage nach geeigneten Themen und Inhalten von Maßnahmen und inwiefern diese begründet auszuwählen sind. Ein Schwerpunkt kann diesbezüglich darauf liegen, die sozialräumliche Perspektive zu stärken und deshalb Maßnahmen zu entwickeln, die die Aneignungsmöglichkeiten von Raum in den Vordergrund stellen, sodass sich Personen Raum als teilhabend aneignen können, die bislang primär Ausschluss erfuhren. Dabei gilt es, an zuvor identifizierten Barrieren anzusetzen und gemeinsam Wege zu finden, wie diese überwunden werden können. Inklusionsprojekte, die diese Fragen in den Vordergrund stellen und zudem die Interessen ihrer NetzwerkpartnerInnen, die gegebenenfalls ambivalent sein können (siehe Kapitel 29), reflektieren, sind auf einem guten Weg, Teilhabebarrieren abbauen zu können.

\section{AkteurInnen und kommunale Netzwerke im Kontext von Inklusion}

Im Kontext von Projekten, die sich an Inklusion ausrichten beziehungsweise diese zum Gegenstand haben, stellt sich neben der Frage nach der inhaltlichen Ausgestaltung auch jene nach den AkteurInnen. Es geht also darum, wer in welcher Weise an einem solchen Projekt beteiligt wird und sich beteiligen kann. Eine zentrale Rolle spielen dabei die Personen, die ein sogenanntes Inklusionsprojekt initiieren, leiten und letzten Endes auch durchführen. Diesbezüglich sollte abgewogen werden, welcher fachliche und berufsbiographische Hintergrund bei diesen Personen hilfreich ist, sodass sie für die Leitung eines solchen Projektes geeignet sind. Beispielsweise eröffnet eine berufsbiographische Verortung in Handlungsfeldern des Hilfesystems zwar ein Wissen über Barrieren, Ausschlusspraxen und sbehinderte Biographien`, gleichzeitig ist es jedoch denkbar, dass die Nähe zum Hilfesystem dessen Strukturen eher reproduziert denn infrage stellt, was wiederum als Behinderungspraxis wirksam werden kann. Neben der Überlegung, welche Personen das Projekt leiten, ist darüber nachzudenken, inwiefern unterstützende Personen oder Institutionen und Einrichtungen eingebunden werden können. Jene Unterstützenden bilden eine Art Netzwerk, das im 
Sozialraum verzweigt ist. Die Aufgaben, die dieses Netzwerk erfüllen kann, sind dabei vielfältig. Als MultiplikatorInnen tragen die Personen dieses Netzwerks die Idee des Projekts und einzelne Maßnahmen in den jeweiligen Sozialraum und unterstützen so dabei, das Thema Inklusion vermehrt ins allgemeine Bewusstsein zu rücken sowie die Bekanntheit des Projekts zu vergrößern. Insofern die jeweiligen NetzwerkpartnerInnen selbst je bestimmte Personen(-Gruppen) adressieren, können diese dem Projekt einen Zugang zu unterschiedlichen AdressatInnenkreisen eröffnen, die möglicherweise andernfalls nicht erreicht würden. Daneben hat eine Einbindung von NetzwerkpartnerInnen das Potenzial, dass diese sich sowohl fachlich als auch finanziell an der Ausgestaltung des Projekts beziehungsweise einzelner Maßnahmen beteiligen, was das Vorhaben, Inklusion im Kontext Sozialraum zu denken, weiterhin stärkt. Beim Aufbau eines Netzwerks stehen die AkteurInnen vor der Herausforderung, die möglicherweise unterschiedlichen Zielsetzungen, Anliegen und Interessen potenzieller NetzwerkpartnerInnen miteinander zu vereinbaren. Es bedarf folglich einer Kultur des problemorientierten Austauschs, der eine erfolgreiche Zusammenarbeit unterstützt. An dieser Stelle ist erneut hervorzuheben, dass es sinnvoll ist, beim Aufbau des Netzwerks nicht ausschließlich auf althergebrachte Hilfestrukturen zu bauen, da diese oftmals selbst als Teilhabebarriere wirksam werden (zum Beispiel bezüglich der Unterbringung von Menschen mit geistiger und/oder komplexer Behinderung in eher geschlossenen Wohneinrichtungen; Trescher 2017f, 2018a). Sollten Hilfesysteme respektive ihre VertreterInnen bei Inklusionsprojekten und -netzwerken beteiligt werden, so sind ihre Rolle sowie die ihr inhärenten quasi-automatischen Adressierungen permanent zu reflektieren, was letzten Endes auch als Möglichkeit gesehen werden kann, die Strukturen der Hilfesysteme selbst zu verändern. Veränderung bedeutet dabei immer Krise und es ist eine Herausforderung, diese nicht als Belastung, sondern als Chance zu begreifen ${ }^{1}$. Diese Einsicht gilt es, AkteurInnen im Kontext Inklusion zu vermitteln. Diesbezüglich kristallisierte sich im Zuge der Beforschung des konkreten Inklusionsprojektes, deren Ergebnisse hier dargelegt sind, immer mehr heraus, wie sehr die ProtagonistInnen in der Praxis von einer wissenschaftlichen Perspektive profitieren, die bei der Aushandlung von Krisen unterstützt, indem sie unter

1 Hier wird implizit einem Verständnis von Krise und Routine gefolgt, wie es Oevermann (u.a. 1996) eingeführt hat. 
anderem einen fundierten inhaltlichen Zugang zum Thema Inklusion sowie differenzierte Verstehenszugänge zu einer mitunter ambivalenten Handlungspraxis eröffnet.

\section{Inklusion zwischen Recht und Gerechtigkeit}

Eng verknüpft mit der Ambivalenz von Standards für Barrierefreiheit ist die Frage danach, ob und gegebenenfalls inwiefern gesetzliche Verankerungen von Inklusion Teilhabemöglichkeiten erweitern können. Dabei spielt die UNKonvention über die Rechte von Menschen mit Behinderungen eine besondere Rolle, denn mit ihr ist ein solches Gesetz festgelegt worden, mit dem die Teilhabe von Personen mit unterschiedlichen Unterstützungsbedarfen gesichert werden soll - auch Deutschland sieht sich dieser seit ihrer Ratifizierung verpflichtet. Dreierlei kann in Bezug auf die UN-Konvention über die Rechte von Menschen mit Behinderungen problematisiert werden. (1) Die Konvention ist idealtypisch formuliert, ihr Auslegungsspielraum ist recht groß und sie ist (auch aufgrund dessen) kaum einklagbar. (2) Unscharf bleibt zudem oftmals ihr menschenrechtlicher Bezug (siehe dazu Kapitel 40). Auch daraus folgt, dass die Konvention zwar eine wichtige, letztlich aber kaum bindende Größe ist. Menschenrechtlich basierte Begründungsfiguren erscheinen zwar auf den ersten Blick als 'gut‘ und 'moralisch richtig - schließlich wird kaum jemand in einer demokratischen Gesellschaft Menschenrechte infrage stellen oder (öffentlich) bestimmten Personengruppen absprechen wollen. Dennoch ist ein solcher (oftmals einseitiger) Bezug nicht unproblematisch, denn Inklusion als Menschenrecht kann nicht unabhängig von Behinderung als manifestes, personengebundenes Faktum gedacht werden. Indem Teilhabe für Menschen mit Behinderung aus einer menschenrechtlichen Perspektive gefordert wird, wird der Status >behindert manifestiert. Dadurch wird die Dichotomie sbehindert - snicht behindert< reproduziert, was wiederum einem Verständnis von Inklusion als Kritik, entlang dessen ebenjene vorgängigen Kategorisierungen aufgebrochen werden sollen (siehe Kapitel 4.2), zuwiderläuft. (3) Schließlich kann problematisiert werden, dass, wenngleich die UN-Konvention sicherlich ein wichtiger Meilenstein dafür ist, Menschen mit Behinderung Teilhabemöglichkeiten an Praxen der Mehrheitsgesellschaft zu eröffnen, Gesetzesänderungen nur dann Wirksamkeit zeigen, wenn es Personen gibt, die sich für die Umsetzung und Einhaltung 
des Gesetzes engagieren (Weisser 2010, S. 8). Die gesetzliche Manifestation von Inklusion wird »scheitern [...], wenn Inklusion nicht zugleich zu einem durch eine hinreichende Zahl von Menschen verkörperten und gelebten Wert wird« (Dederich 2013b, S. 34). Dies berührt Fragen bezüglich des Verhältnisses von Recht und Gerechtigkeit, denn nicht alles, was rechtlich abgesichert ist, gilt in jedem Diskurs als gerecht (und umgekehrt) ${ }^{2}$. "Das Recht ist nicht die Gerechtigkeit. Das Recht ist das Element der Berechnung; es ist nur (ge)recht, daß es ein Recht gibt, die Gerechtigkeit indes ist unberechenbar: sie erfordert, daß man mit dem Unberechenbaren rechnet (Derrida 2017, S. 33f). Dabei ist gerade in Bezug auf Inklusion "Vorsicht angebracht, denn die Institutionalisierung von bestimmten Rechtsnormen alleine schafft keine Gerechtigkeit, so sehr eine solche Institutionalisierung auch zu begrüßen sein mag« (Dederich 2013b, S. 22). Es muss darüber hinaus diskutiert werden, inwiefern mit Barrieren und Diskriminierungen umgegangen werden soll, die durch das je bestimmte Gesetz nicht berücksichtigt werden - entsprechen diese dann dem Recht, auch wenn sie je subjektiv als ungerecht empfunden werden? Zudem haben Personengruppen, die durch gesetzliche Regelungen nicht miteinbezogen werden, aber dennoch ausgeschlossen und/oder diskriminiert werden, kaum eine Handhabe, sich gegen diesen Ausschluss und diese Diskriminierung zu wehren, zumindest nicht anhand eines einklagbaren Rechts. Sicherlich profitieren zahlreiche Menschen von gesetzlichen Änderungen, die Ausschluss ahnden und Teilhabe einklagbar machen (genannt werden kann hier beispielsweise das Wahlrecht, das im Jahr 2019 insofern geändert wurde, dass Menschen, die unter umfassender Betreuung stehen, nicht mehr pauschal verweigert wird, zu wählen und gewählt zu werden; BGBl. I). Dennoch ist zu problematisieren, dass eine solche Praxis »darauf hinaus[läuft], davon nicht berührtes Elend gerechtfertigt erscheinen zu lassen « (Weisser 2010, S. 9). So sinnvoll gesetzliche Änderungen in vielen Fällen sein können, so wichtig ist es doch, zu gesamtgesellschaftlicher Bewusstseinsbildung beizutragen, um ungerechte Verhältnisse in der intersubjektiven Aushandlung angehen zu können. Dazu braucht es Praxen des Infragestellens, des Aufrüttelns und Störens - es braucht Dekonstruktion.

2 Zum Verhältnis von Gerechtigkeit und Politik siehe Forst (1994, S. 215ff, 2005, S. 24ff) sowie Kapitel 31. 


\section{Inklusion, Gerechtigkeit und Politik}

Die Beschäftigung mit Gerechtigkeit (Kapitel 30) berührt nahezu unausweichlich die Fragen, inwiefern Personen Gerechtigkeit erfahren, aus welchen Gründen sie möglicherweise sungerecht behandelt werden und wie >gerechte Verhältnisse geschaffen werden können. Fragen von Gerechtigkeit betreffen insofern immer auch Herrschaftsverhältnisse beziehungsweise Fragen von Macht und Ermächtigung - Forst bezeichnet sogar »die Frage der Macht als erste Frage der Gerechtigkeit« (2005, S. 31). Gerechtigkeit und Macht konstituieren sich dabei entlang der Frage nach der Produktion von Gütern ebenso wie entlang der Frage nach der Verteilung dieser (Forst 2005, S. 28). Beide werden jedoch dominiert von der Frage danach, »wer über die Produktion und Verteilung bestimmt (Forst 2005, S. 28f). Darin liege, so Forst, »der ursprüngliche, politische Sinn der Gerechtigkeit« (2005, S. 29). Folglich berührt die Auseinandersetzung mit Gerechtigkeit immer auch die Politik, die regulierendes Organ der Verteilung ist (siehe Forst 2005, S. 29), unter anderem indem ihr auferlegt ist, gerechte Gesetze zu erlassen (Rawls 2017, S. 257). Die BürgerInnen eines Staates sollen bei der Formulierung und dem Erlass ebensolcher Gesetze beteiligt werden, wodurch der »Grundsatz der (gleichen) Teilnahme« (Rawls 2017, S. 251) eingehalten wird. Eine Beteiligung von BürgerInnen an politischen Entscheidungsprozessen beziehungsweise an der Konstitution von Politik selbst bedarf einen uneingeschränkten Zugang zu Informationen, um »beurteilen [zu] können, wie sich Vorschläge auf ihr Wohl auswirken, und welche Pläne ihrer Vorstellung vom öffentlichen Wohl entsprechen« (Rawls 2017, S. 255). Dazu gehört ebenfalls, selbst Vorschläge zu machen und gehört zu werden (Rawls 2017, S. 255) - im Diskurs um politische Entscheidungsfindung also eine Sprechposition einnehmen zu können. Dies macht deutlich, dass Inklusion und Politik untrennbar miteinander verknüpft sind und es kann bestätigt werden, dass die Forderung nach Inklusion ein »im Kern politische[r] Anspruch «(Dederich 2013a, S. 1) ist. Auch die Ergebnisse der Sozialraumanalysen in den Handlungsfeldern Arbeit und Freizeit zeigen einen Zusammenhang zwischen Inklusion und Politik auf, wobei dieser primär darin besteht, dass die Verantwortung, >Inklusion umzusetzen<, teilweise in erster Linie aufseiten der Politik gesehen wird, beispielsweise bezüglich Gesetzesänderungen oder (finanzieller) Unterstützungsmöglichkeiten (siehe Kapitel 16.9.2 und Kapitel 17.12.2). Dies ist gerade vor dem Hintergrund relevant, dass Inklusion häufig vonseiten der Politik als Handlungsauftrag an Einrichtungen und pädago- 
gisch Handelnde herangetragen wird, oftmals jedoch ohne ausreichende Expertise und/oder Unterstützung bereitzustellen. Daraus folgt, dass auch auf politischer Ebene gehandelt und eine Antwort auf die Frage gefunden werden muss, wie Menschen, die von Ausschluss bedroht oder betroffen sind, Teilhabe an den Praxen der Mehrheitsgesellschaft ermöglicht wird (siehe Dederich 2013a, S. 1). Dies ist eine in mehrerlei Hinsicht komplexe Aufgabe, die die Interessen und Ansprüche von (mindestens) drei Beteiligten miteinander aushandeln muss, denn es sind nicht nur die Politik respektive politisch Handelnde betroffen, sondern auch die Mehrheitsgesellschaft sowie die Personen, denen Teilhabe ermöglicht werden soll. Hinzu kommt, dass diese drei Gruppen nicht trennscharf voneinander abgegrenzt werden können. Auf Ebene der Politik beziehungsweise politisch Handelnder geht es im Kontext dessen, Teilhabemöglichkeiten zu eröffnen, primär darum, politische Instrumente zu nutzen und/oder zur Verfügung zur stellen, die die Rahmenbedingungen zu uneingeschränkter Teilhabe bereiten. Dazu gehört beispielsweise, Gesetze auf den Weg zu bringen, (auch strukturelle) Zuständigkeiten zu schaffen oder Expertise zu generieren und zugänglich zu machen ${ }^{3}$. Die Mehrheitsgesellschaft ist primär bezüglich ihrer Haltungen und Einstellungen zu Inklusion gefordert, denn es ist klar, dass Inklusion nichts ist, das dieser rübergestülpt werden könnte. Es bedarf vielmehr einer (inneren) Bereitschaft, Ausschlusspraxen als solche zu erkennen und sich an ihrem Abbau zu beteiligen. Hinsichtlich der Personen, für die Teilhabemöglichkeiten eröffnet werden sollen, kann gesagt werden, dass diese primär der Sichtbarkeit im gesamtgesellschaftlichen Diskurs bedürfen, um auf ihren (je situativen) Ausschluss aufmerksam zu machen und sie schließlich dabei zu unterstützen, Teilhabebarrieren zu überwinden. Insgesamt wird an diesen Ausführungen klar, dass Inklusion im Kontext Politik eine primär handlungspraktische Frage ist. Es geht also erneut um ihre Umsetzung. Dies ist auch vor dem Hintergrund relevant, dass sich Politik, nach Hannah Arendt (2017), »vor allem im Miteinander-Reden und Miteinander-Handeln der Bürgerinnen und Bürger [vollzieht]« (Oeftering 2015, S. 67). Deutlich wird zudem, dass unterschiedliche AkteurInnen beteiligt sind, von

3 Behrendt (2017) schlägt drei Dimensionen inklusionspolitischen Handelns vor: Eine formelle Inklusionspolitik solle rechtliche Teilhabebeschränkungen aufheben, während sich eine informelle Inklusionspolitik in einem nicht-ausschließenden Miteinander aller Beteiligten vollziehe (Behrendt 2017, S. 71ff). Strukturelle Inklusionspolitik schließlich kulminiere darin, Barrierefreiheit umfänglich zu verwirklichen (Behrendt 2017, S. 73). Zur Diskussion der Ambivalenzen von Barrierefreiheit siehe Kapitel 11.3 und Kapitel 13.2. 
denen hier drei exemplarisch herausgegriffen wurden, da sie zentral erscheinen (die Liste ließe sich allerdings beliebig erweitern, beispielsweise um unterschiedliche Institutionen des Hilfesystems, die ihrerseits ambivalente Interessen haben können, Angehörige etc.). Inklusion als Praxis zwischen diesen unterschiedlichen AkteurInnen ist eine große Herausforderung, denn es gilt, ambivalente - oder zumindest potenziell schwierig zu vereinbarende - Interessen und Ansprüche auszuhandeln ${ }^{4}$. Schließlich stellt sich bei der Betrachtung des Verhältnisses von Inklusion und Politik die Frage, wie auf politischer Ebene Veränderungen angestoßen werden können, die nach Einschätzung sowohl einiger FachwissenschaftlerInnen als auch Personen aus der Mehrheitsbevölkerung in der Verantwortlichkeit (zumindest auch) der Politik liegen. In einem ersten Schritt ist es dazu sicherlich notwendig, überhaupt erst auf Ausschlussmechanismen und Barrieren aufmerksam zu machen, sodass die Lebenssituation von Menschen, die von Ausschluss bedroht oder betroffen sind, sichtbarer wird. Dies ist vor allem Aufgabe der Bezugswissenschaften, die dem bereits in vielfältiger Weise nachkommen. Nichtsdestotrotz könnte die Forschung zur Lebenssituation marginalisierter Personen(-Gruppen) noch intensiviert werden. Einer solchen Sichtbarmachung sollte eine Subjektivierung bislang ausgeschlossener Personen als >sprechend im je für sie bezugsrelevanten Diskurs folgen, sodass diese Teilhabemöglichkeiten nutzen können. Um eine Sprechrolle einnehmen zu können, bedarf es teilweise sicherlich einer gewissen Selbstermächtigung der AdressatInnen, sodass diese darin gestärkt werden, für sich einzutreten und sich zu beteiligen (Oeftering 2015, S. 65). Gerade in Anbetracht sogenannter institutionalisierter Biographien (siehe Trescher 2017a; Theunissen 2002, S. 167) ist dies eine wichtige Forderung, denn ein Leben in den Strukturen des Hilfesystems kann als behindernde Praxis wirksam werden und sozusagen die Fähigkeit zum `Sprechen respektive das Wissen um je bestimmte Diskurse einschränken ${ }^{5}$. Im Kontext einer solchen Selbstermächtigung muss darüber hinaus das oftmals ambivalente Verhältnis zu stellvertretendem Handeln reflektiert werden, auf das einige Perso-

4 Im Zusammenhang damit muss darauf hingewiesen werden, dass durch die Art der Darstellung nicht der Eindruck erweckt werden soll, die AkteurInnen der hier skizzierten Gruppen hätten jeweils ein übereinstimmendes Handlungsinteresse. Vielmehr ist das Cegenteil der Fall.

5 Mit s Sprechen < wird hier und davor auf eine Subjektivierung als im Diskurs zum Sprechen befähigte Person referiert, was über Verbalsprache hinausgeht und alle möglichen Äußerungen und Gestaltungsformen umfasst (Foucault 2003, S. 27ff; siehe Kapitel 4.1). 
nen im Kontext von Assistenz und Unterstützung angewiesen sind (Trescher 2018a, S. 310ff; Ackermann und Dederich 2011, S. 7ff).

\section{Ambivalenzen von Barrierefreiheit}

Eine weitere Folge des Anspruchs, Inklusion sumsetzen $\mathrm{zu}$ wollen, ist eine oftmals eher technische Herangehensweise, was sich im hiesigen Kontext insbesondere am Umgang mit und der Ausgestaltung von Barrierefreiheit zeigt. Barrierefreiheit ist ein idealtypisches Konzept, das nicht so umfassend erreicht werden kann, wie es der Begriff impliziert - es ist keine vollständige Abwesenheit von Barrieren möglich. Dies liegt unter anderem daran, dass der Abbau von Barrieren neue Barrieren entstehen lassen kann - was für die eine Person eine Barriere darstellt, kann für eine andere Person Unterstützung sein und umgekehrt. Hinzu kommt, dass Barrieren teilweise durchaus ihre Berechtigung haben, da sie wichtige Funktionen erfüllen. In diesem Sinne ist der Begriff Barriere eher als Zuschreibung einer (Diskursteilhabe-) Begrenzung beziehungsweise Beschränkung zu verstehen, die diskursive Zugehörigkeiten reguliert, vor unerwünschten äußeren Zugriffen schützt und Diskurse begrenzt (Trescher 2020a). In ihrer Funktion als Diskursgrenze vollziehen sich Barrieren in drei Formen: als exkludierende Barrieren, normalisierende Barrieren und Barrieren in Bezug auf die Sprechenden, wodurch reguliert wird, wer in welcher Art und Weise Zugang zu welchen Diskursen hat und ob beziehungsweise welche `Sprechrolle der Person dabei zugewiesen wird. Es können manifeste von latenten Barrieren unterschieden werden (siehe Kapitel 4.1). Die vielgestaltigen Ergebnisse zeigen, dass unter Barrierefreiheit zumeist verstanden wird, dass ein Zugang einschränkungslos für Menschen erreichbar ist, die einen Rollstuhl nutzen. Übergreifend ergaben das die Untersuchungen zu Barrierefreiheit und öffentlichen Orten (Kapitel 11), die ethnographischen Sozialraumbegehungen (Kapitel 13) und in gewisser Hinsicht auch die Sozialraumanalysen im Kontext Arbeit und Freizeit, wurde hierbei doch deutlich, dass in der Mehrheitsgesellschaft ein eher eindimensionales und auf Mobilität enggeführtes Verständnis von Barrierefreiheit vorherrscht (siehe Kapitel 16 und Kapitel 17). Eine Folge dessen ist, dass Barrieren primär in Bezug auf Mobilitätseinschränkungen abgebaut werden, beispielsweise durch Rampen oder Aufzüge. Zudem sind Personen der Mehrheitsgesellschaft ganz offensichtlich kaum für Unterstützungsbe- 
darfe jenseits von Mobilität aufmerksam und sensibilisiert ${ }^{6}$. Menschen mit Unterstützungsbedarfen in den Bereichen Sehen, Hören oder Lesen/Verstehen werden oftmals übergangen, wenn es darum geht, Zugänge zu schaffen. Wenig bedacht wird außerdem, dass Barrieren auch durch Diskriminierung entstehen beziehungsweise damit einhergehen können. Dass Barrierefreiheit nicht nur in handlungspraktischer Hinsicht, sondern auch in Bezug auf ihr theoretisches Fundament problematisiert werden kann, wurde oben breit diskutiert (Kapitel 11.3). Erneut hervorgehoben werden hier deshalb die Ambivalenzen, die damit einhergehen, Standards für Barrierefreiheit zu fordern (Trescher 2018c, S. 139f). Es kann zwar in gewisser Weise nachvollzogen werden, weshalb, insbesondere aus handlungspraktischer Perspektive, die Forderung nach Standards für Barrierefreiheit laut wird, schließlich kann es sinnhaft sein, sich an bestimmten Vorgaben orientieren und auf diese berufen zu können - sowohl aus Perspektive derjenigen, die verantwortlich dafür sind, Barrieren abzubauen, als auch derjenigen, die davon profitieren, indem ihnen dadurch Teilhabemöglichkeiten eröffnet werden, die zuvor verschlossen waren. Gleichzeitig wird bei der Forderung nach Standards ein Stück weit ausgeblendet, dass Barrieren mehrdimensional sind, sich individuell und je situativ vollziehen. Durch entsprechende Vorgaben kann der Komplexität von Barrieren kaum begegnet werden und es besteht die Gefahr, einer Überbürokratisierung, die mehr Barrieren errichtet, als durch sie abgebaut werden können. Dazu kommt, dass die Verordnungen zum barrierefreien Bauen in den einzelnen Bundesländern nicht immer übereinstimmend sind. Auch wenn im Gros zwar dieselben Vorschriften gelten, konnten bei einer beispielhaften Analyse zwischen der Bayrischen Bauordnung (BayBO) und der Landesbauordnung Mecklenburg-Vorpommern (LBauO M-V), die für die untersuchten Sozialräume Erlangen und Rostock relevant sind, doch einige Unterschiede festgestellt werden. Beispielsweise sind laut $\$ 50$ Absatz 3 LBauO M-V Ausnahmen von barrierefreiem Bauen im Kontext des Denkmalschutzes möglich, im entsprechenden Art. 48 BayBO ist dies dagegen nicht vorgesehen. Dafür gibt es dort wiederum einen $\mathrm{Zu}$ satz, wonach »[b]auliche Anlagen und Einrichtungen, die überwiegend oder ausschließlich von Menschen mit Behinderung, alten Menschen und Personen mit Kleinkindern genutzt werden [...] in allen der zweckentsprechenden Nutzung dienenden Teilen barrierefrei sein [müssen]« (Art. 48 (3) BayBO). In

6 Dies problematisiert auch Bertelmann 2019. 
der entsprechenden Verordnung in Mecklenburg-Vorpommern ist dieser Zusatz nicht zu finden. Schließlich erscheint ein weiterer Unterschied besonders bedeutsam: Während in der Landesbauordnung Mecklenburg-Vorpommern die Größe der Fläche genau definiert ist, die zur Verfügung stehen muss, um Mobilitätshilfen wie unter anderem Kinderwagen und Rollatoren abzustellen ( $\$ 48$ (2) LBauO M-V), ist in der Bayrischen Bauordnung lediglich festgehalten, dass diese Flächen »ausreichend groß « und "gut zugänglich" sein sollten (Art. 46 (2) BayBO). Unklare und uneinheitliche Bestimmungen wie diese stellen die Relevanz und Sinnhaftigkeit von Vorschriften infrage, denn es ist offensichtlich schwierig, verlässliche und allgemeingültige Regelungen zum barrierefreien Bauen zu schaffen sowie das Wissen darüber bundeslandübergreifend zu verbreiten. Wird der Blick über Deutschland hinaus gerichtet, so zeichnet sich ein dichtes Geäst unterschiedlicher Bestimmungen, Regularien und Durchsetzungsinstrumente ab, wenn es um barrierefreie Zugangs- und Nutzungsmöglichkeiten geht ${ }^{7}$. Die Beschäftigung mit Barrierefreiheit ist also auch in internationaler Hinsicht eine komplexe Herausforderung. Bezüglich der Ambivalenz von Standards im Kontext Barrierefreiheit kommt erschwerend hinzu, dass keinesfalls alle komplexen und unterschiedlichen Bedarfe, die Personen in Bezug auf den Abbau von Barrieren haben können, durch entsprechende Vorgaben abgedeckt werden können. Auch bei einer noch so kleinteiligen Festsetzung von Vorschriften, die Barrierefreiheit regeln, wird es Personen geben, deren individuelle Bedarfe davon unberührt bleiben und die infolgedessen nach wie vor Ausschluss erfahren. Mit entsprechenden Regelungen und Vorgaben droht also einherzugehen, dass Personen als `Ausgeschlossene der Ausgeschlossenen < hervorgebracht werden und dadurch in gewisser Hinsicht, wie oben bereits mehrfach problematisiert, $\mathrm{zu}>$ InklusionsverliererInnen werden können.

7 Einen Überblick gibt beispielsweise Waddell (2006). Hingewiesen werden soll zudem auf die sogenannte Accessibility World Map, die weltweit Länder danach beurteilt, inwiefern Websites im jeweiligen Land >barrierefrei< nutzbar sind, und so miteinander vergleichbar macht (https://www.accessibilityworldmap.org/, zuletzt am 24.02.2020). 


\section{Bewusstseinsbildung und Sensibilisierung}

Ein Ergebnis, das sich an unterschiedlichen Stellen herauskristallisiert, ist, dass Inklusion als Praxis sich vor allem dann vollziehen kann, wenn in der Mehrheitsgesellschaft ein gewisses Bewusstsein darüber vorhanden ist, dass Personen (systematisch) Ausschluss erfahren, beziehungsweise, im Umkehrschluss, dass Teilhabe durch zu geringe Sensibilität für ausschlussproduzierende Barrieren behindert werden kann. Es stellt sich also die Frage, wie zu mehr Bewusstsein und Sensibilität aufseiten der Mehrheitsgesellschaft beigetragen werden kann und zwar, ohne bestimmte Personen als >behindert $\mathrm{zu}$ labeln und in diesem Status zu reproduzieren - was handlungspraktisch ebenso wie theoretisch eine Herausforderung ist, denn es gilt, auf Barrieren aufmerksam zu machen, ohne je bestimmte Personen(-Gruppen) in pauschalisierender Weise hervorzuheben. Während im hiesigen Verständnis Bewusstseinsbildung darauf abzielt, Aufmerksamkeit und Sensibilität für Barrieren zu wecken, die Ausschluss nach sich ziehen, findet es sich immer wieder, dass Bewusstseinsbildung sozusagen >umgewidmet « wird und dazu führt, dass negative Zuschreibungen an bestimmte Personen verbreitet und manifestiert werden. Dies liegt vor allem daran, dass vorgängige Behinderungskategorien als Ausgangspunkt von Bewusstseinsbildung genommen werden. Diese Problematik findet sich teils in eher praxisorientierten Veröffentlichungen, die sicherlich gutmeinend sind und prinzipiell das Eröffnen von Teilhabemöglichkeiten zum Ziel haben, bei denen aber im Kern ein Gefälle und eine Dichotomie zwischen Menschen mit und Menschen ohne je bestimmte Unterstützungsbedarfe reproduziert wird. Dies vollzieht sich unter anderem dadurch, dass beispielsweise Menschen mit Behinderung pauschal als hilfebedürftig und weniger leistungsfähig konstruiert werden (zum Beispiel in Ravenhorst 2013). Beispiele wie diese zeigen, wie wichtig es ist, ein Verständnis von Behinderung in den Fach- und öffentlichen Diskurs einzubringen, das möglichst ohne Zuschreibungen an die jeweilige Person auskommt und Behinderung vielmehr als eine sich - auch räumlich - vollziehende Praxis versteht, die jenseits vorgängiger Behinderungskategorien verortet ist. Insofern wäre ein erster Aspekt, der bezüglich Bewusstseinsbildung und Sensibilisierung eine zentrale Rolle spielt, das gegenwärtig vorherrschende Verständnis von Behinderung in der Mehrheitsgesellschaft aufzuweichen und, statt auf je bestimmte Personen(-Gruppen), den Blick auf Barrieren zu lenken, die Teilhabe behindern. Damit geht einher, auch die Be- 
darfe von Personen zu berücksichtigen, die gegenwärtig nicht als `behindert< benannt werden, die aber dennoch je situativ an Teilhabebarrieren stoßen. In Bezug auf Bewusstseinsbildung braucht es also ebenfalls, das zeigen diese Ausführungen ganz deutlich, einen dekonstruktivistischen Blick, anhand dessen gängige Vorstellungen, Denkweisen und Handlungspraxen infrage gestellt werden können. In handlungspraktischer Hinsicht sind einige Ansätze denkbar, um das Bewusstsein für Barrieren und Ausschlusspraxen zu sensibilisieren - teilweise werden diese in der ein oder anderen Form bereits umgesetzt. Konterpart oder zumindest Ergänzung zu bisherigen Angeboten soll aber eben sein, die Person als AdressatIn zu verrücken und stattdessen Barrieren selbst zu fokussieren. Durch diese Dezentralisierung der Person sollen Kategorisierungen vermieden und schließlich abgebaut werden. In einem ersten Schritt ist es naheliegend, Berührungspunkte zu schaffen zwischen Menschen, die Ausschluss erfahren, und Menschen, die eher uneingeschränkte Teilhabemöglichkeiten haben, um auf Barrieren aufmerksam zu machen. Dies legen die Ergebnisse der Sozialraumanalysen in den Handlungsfeldern Arbeit und Freizeit insbesondere nahe, zeigt sich doch, dass dort, wo sich Personen begegnen und interagieren, zumeist positive Erfahrungen im Miteinander gemacht werden (siehe Kapitel 16.6 und Kapitel 17.7). Darüber hinaus wurde festgestellt, dass die Einstellung zu Inklusion mit dem lebensweltlich erlebten Kontakt zu Menschen mit (geistiger) Behinderung korreliert und zwar insofern, dass ein vermehrter Kontakt sich in einer positiveren Einstellung zu Inklusion niederschlägt (siehe Kapitel 22; siehe auch Trescher 2015b, S. 118f). Handlungspraktische Ansätze, um Begegnungsmöglichkeiten und -räume zu schaffen, gibt es bereits einige. Diese können sinnhaft mit dem zweiten Schwerpunkt von Bewusstseinsbildung und Sensibilisierung verbunden werden, nämlich dem der Information (siehe auch Schattenmann 2016, S. 146f). Solche Informationsangebote sollen weniger je bestimmte Behinderungskategorien und damit gegebenenfalls einhergehende Unterstützungsbedarfe fokussieren, sondern eher über Barrieren aufklären, auf diese aufmerksam machen, verdeutlichen, inwiefern sich diese alläglich vollziehen, und schließlich aufzeigen, welche Möglichkeiten es bereits gibt, um Barrieren abzubauen. Eng verknüpft mit dem Schwerpunkt Information ist jener der Beratung. Beratung kann beispielsweise Führungspersonen mit Personalverantwortung in Unternehmen und Betrieben angeboten werden oder, im Kontext Freizeit, PrimärvertreterInnen, ÜbungsleiterInnen, Ehrenamtlichen etc. Gegenstand eines solchen Be- 
ratungsangebots kann unter anderem sein, wie Arbeitsstätten und -tätigkeiten oder Freizeitaktivitäten barrierearmer gestaltet werden können, sodass Personen einen Zugang dazu finden, die bislang ausgeschlossen waren. Ganz konkret kann zum Beispiel über finanzielle Unterstützungsmöglichkeiten informiert werden, die zumeist in der ein oder anderen Weise eingeworben werden können, jedoch oftmals einer gewissen bürokratischen Expertise bedürfen, um sie zu beantragen. Dabei ist es wichtig, zu reflektieren, dass die meisten Finanzierungsmöglichkeiten über Defizite je bestimmter Personen begründet werden, was wiederum Behinderung als pauschal und umfassend zugeschriebene Kategorie reproduziert. Diese Ambivalenz gilt es, sich bewusst zu machen und zu reflektieren, sodass Handelnde nicht davon überformt werden.

\section{Frage nach öffentlichen Mitteln}

Die Interviewpersonen im Kontext der Sozialraumanalysen zu Teilhabe in den Bereichen Arbeit und Freizeit machen häufig eine zu geringe finanzielle Ausstattung dafür verantwortlich, dass bestimmte Personen an Teilhabebarrieren stoßen. Einige gehen davon aus, dass Inklusion (auch) durch finanzielle Unterstützung ermöglicht werden kann (siehe Kapitel 16.9.2 und Kapitel 17.12.2). Gleichzeitig ist die Zustimmung der Gesamtbevölkerung eher gering, höhere Steuern dafür zu zahlen, dass Teilhabebarrieren abgebaut werden können (siehe Kapitel 22.2.2). Die Frage nach Inklusion und öffentlichen Mitteln ist durchaus ambivalent. Zwar ist es so, dass durch eine höhere beziehungsweise sichergestellte Finanzierung durchaus der einen oder anderen Person mehr Möglichkeiten eröffnet werden, an Praxen der Mehrheitsgesellschaft teilzuhaben (beispielsweise durch die unbürokratische und routinemäßige Übernahme von Kosten für Gebärdensprachdolmetschung, Kinder- oder Angehörigenbetreuung, je bestimmte Hilfsmittel und vieles mehr). Daraus können Teilhabemöglichkeiten erwachsen und werden teilweise bereits genutzt. Beispielweise wurde von einigen PrimärvertreterInnen in den Interviews zum Thema Freizeit berichtet, dass - gerade im Kontext Fluchtmigration - die Teilhabe von je bestimmten Personen(-Gruppen) massiv davon abhängt, wie viel Mittel zur Verfügung stehen, um beispielsweise Angebote zu gestalten, externe Expertise einzukaufen (zum Beispiel Übersetzungsleistungen) oder zusätzliche Räume anzumieten. Eine 
zuverlässige (hohe) Finanzierung bereitet also durchaus erweiterte Teilhabemöglichkeiten. Gleichzeitig ist nicht einfach zu lösen, wohin und in welchem Umfang die Mittel fließen sollen sowie wer ebendarüber entscheiden darf. $\mathrm{Zu}$ bedenken ist zudem, dass auch bei einer breiten Finanzierung von dem, was Inklusion genannt wird, wiederum möglicherweise bestimmte Personen außen vor bleiben, deren Bedarfe in gewisser Weise unsichtbar bleiben, weil es gegebenenfalls eher latente Barrieren sind, an die sie stoßen und die ihre Teilhabe behindern. Die zentrale Herausforderung in Fragen der Finanzierung von beispielsweise sogenannten Inklusionsprojekten ist also, die zur Verfügung stehenden Mittel so einzusetzen, dass durch sie tatsächlich Teilhabebarrieren abgebaut werden. Dadurch würde einem unüberlegten, eher aktionistischen Handeln entgegengewirkt. Im Kontext Finanzierung und sogenannter Sozialraumorientierung kann demgegenüber allerdings auch problematisiert werden, dass in gewisser Weise eine Überformung gemeinschaftlichen Handelns durch staatliche Aufgaben festgestellt werden kann, indem teilweise Aufgaben, die bislang dem Hilfesystem und seinen Institutionen zufielen, an nicht-professionelle AkteurInnen vor Ort übertragen werden (beispielsweise in einer Art Nachbarschaftshilfe). Durch diese »Zivilisierung des Sozialen (Dahme und Wohlfahrt 2011, S. 149) werden zudem die Kosten auf die Gesamtgesellschaft abgewälzt, welche aufgewendet werden müssen, um Teilhabebarrieren abzubauen (siehe auch Eick 2005, S. 110f). Sozialraumorientierung und die damit einhergehenden Praxen können insofern als "Sozialabbau" (Dahme und Wohlfahrt 2011, S. 149) verstanden werden, »was aber von den Protagonisten der neuen Sozialstaatlichkeit als Gewinn verbucht wird« (Dahme und Wohlfahrt 2011, S. 149) - bei dem jedoch letztlich der Staat der Gewinnende ist. Verschärft wird diese Problematik dadurch, dass mit >Inklusion eine »normative Leitidee formuliert« (Dahme und Wohlfahrt 2011, S. 151) wird, die die Restrukturierung des Hilfesystems entlang ökonomischer Interessen verschleiert (Dahme und Wohlfahrt 2011, S. 151f). Dabei wird (bewusst) außer Acht gelassen, dass auch soziale Arbeit vor Ort mit nicht unerheblichen Kosten verbunden ist. Die Folge ist, »dass sich der Staat aus seiner sozialpolitischen Verantwortung zurückzieht - und dass bisher sozialrechtlich geschützte und gesicherte Hilfen unter Verweis auf die dafür jetzt verantwortliche >Community« abgeschafft werden« (Dahme und Wohlfahrt 2011, S. 152). Eine solche Neoliberalisierung kommunaler Sozial- und Wirtschaftspolitik kann »zu (neuen) Ausgrenzungsprozessen« (Eick 2005, S. 113) führen. Bezüglich der Finanzierung von teilhabeorien- 
tierten Maßnahmen kommt darüber hinaus die Frage auf, welcher Wert Inklusion (und allem, was damit zusammenhängt) in einer Gesellschaft beigemessen wird. Dies kumuliert in der Frage danach, ob und inwiefern eine Volkswirtschaft dazu bereit ist, finanzielle Mittel aufzuwenden, um denjenigen Teilhabe zu ermöglichen, die bislang (je situativ) ausgeschlossen werden. Diese Fragen von Verteilung werfen erneut die Frage nach dem Verhältnis von Inklusion und Politik auf, die in Kapitel 31 bereits eingehender diskutiert wurde, auf das an dieser Stelle verwiesen werden soll.

\section{Einstellung und Handeln}

Ein wichtiges Ergebnis des Surveys »Einstellung(en) zu Inklusion« (Kapitel VI) ist, dass ein großer Teil der Bevölkerung Deutschlands gegenüber Inklusion (eher) offen eingestellt ist. Von besonderer Bedeutung ist dabei, ob die befragte Person gegenwärtig oder in der Vergangenheit Kontakt zu Menschen mit (geistiger) Behinderung hat oder hatte. Denn, entlang der sogenannten Kontakthypothese, wurde festgestellt beziehungsweise bestätigt, dass lebenspraktischer Kontakt zwischen Menschen mit und Menschen ohne Behinderung zu einer offeneren Einstellung bei Letzteren führt (Allport 1954; Cloerkes 2007; Cordes und Silter 2016; Trescher 2015b, S. 118f). Problematisiert wurde diesbezüglich, dass eine offene Einstellung zwar begrüßenswert ist und sicherlich einen wichtigen Ausgangspunkt für anschließende inklusionsorientierte Maßnahmen oder Ähnliches bietet, aus dieser allerdings nicht grundsätzlich ein entsprechendes Handeln resultiert. Einstellung ist nicht gleichbedeutend mit Handlungspraxis. Bei einer Befragung, wie sie im hiesigen Rahmen durchgeführt wurde, werden Ergebnisse generiert, die die subjektive Einschätzung einer Person abbilden - ihre Einstellung -, weshalb beispielsweise keine Aussagen darüber getroffen werden können, wie Personen in einer konkreten Situation tatsächlich handeln. Gleichzeitig ist eine Einstellung Grundvoraussetzung dafür, ob und dass eine Person überhaupt (inklusiv) handelt. Es ist möglich, dass handlungspraktisch auch durch Personen, die eine eher offene Einstellung zu Inklusion haben, Teilhabebarrieren errichtet werden. In gewisser Weise zeichnet sich dies am Ergebnis ab, dass die Bereitschaft bei allen Typen - offen, eher offen, eher ablehnend, klar ablehnend - eher gering ist, höhere Steuern zu zahlen, um Inklusion zu ermöglichen (siehe Kapitel 22.2.2). Eine weitere Problema- 
tik, die mit einem Survey einhergeht, der Einstellungen untersucht, ist, dass sich beim untersuchten Thema - in diesem Falle Inklusion - auf das im Feld vorhandene Wissen gestützt werden muss. Auch wenn untersucht wurde, ob und in welcher Ausprägung sich die Befragten bereits mit Inklusion beschäftigten, so bleibt doch ihr jeweiliges Inklusionsverständnis unklar. Dies wiederum kann potenzielles Handeln im Kontext Inklusion beeinflussen, da Barrieren auch durch Uninformiertheit errichtet werden können und zwar auch durch Personen mit (eher) offener Einstellung zu Inklusion. Anknüpfend daran stellt sich die Frage, wie eine offene Einstellung zu Inklusion in ein Handeln überführt werden kann, das dazu beiträgt, Teilhabebarrieren abzubauen. Bezüglich der Vorhersage des Verhältnisses von Einstellung und Verhalten wird vor allem in der Sozialpsychologie breit geforscht (Haddock und Maio 2014). Für den hiesigen Gegenstand ist allerdings von größerer Bedeutung, ob und inwiefern offene Einstellungen in entsprechenden Verhaltensweisen resultieren können - und ob dies überhaupt etwas ist, das eingefordert werden kann und soll. Es kann schließlich nicht von Personen verlangt werden, sich beispielsweise nicht-ausschließend zu verhalten oder im Kontext Inklusion zu engagieren. Dies ist jeder Person selbst überlassen und es sollte eher nicht darüber geurteilt werden, wie sich eine Person zu (in diesem Fall) Inklusion verhält. Zudem sind Werturteile kontingente Kategorien - was im Kontext Inklusion respektive der Betreuung von Menschen mit Behinderung deutlich gezeigt werden kann, hat sich diese im Laufe der vergangenen hundert Jahre doch grundlegend gewandelt. Es ist eine Aushandlungspraxis, ihre unscharfen Grenzen (Reckwitz 2008b) zu justieren. Dazu bedarf es sicherlich Bewusstseinsbildung und Sensibilisierung, um Einstellungen (und möglicherweise auch Handlungen) zu verändern (siehe Kapitel 33). Die Frage, die sich nun stellt, ist, weshalb es dennoch wichtig ist, Einstellungen zu erforschen. Ein Grund dafür ist, dass durch einen Einblick in die Einstellungen der breiten Bevölkerung zu einem bestimmten Thema das gegenwärtige gesellschaftliche Klima beschrieben werden kann, was Auskunft darüber gibt, wie und an welchen Stellen Veränderungen angestoßen werden können, beispielsweise um das Ziel zu erreichen, Teilhabebarrieren abzubauen. Insofern sind Einstellungen und Handlungen zwar nicht als übereinstimmend und/oder unmittelbar aufeinander bezogen zu betrachten, sie sind jedoch verknüpft und verweisen (zumindest nachgeordnet) aufeinander. In methodischer Hinsicht ist eine mögliche Erweiterung der Forschung darin zu sehen, über Einstellungen von Personen hinaus Ein- 
blick in den Vollzug latenter Barrieren, Vorbehalte oder Nicht-Wissen zu nehmen (siehe Kapitel 24).

\section{Infrastrukturelle Herausforderungen}

Die Ergebnisse der vielgestaltigen Studie machen in mehrerlei Hinsicht darauf aufmerksam, dass Teilhabebarrieren durch eine schwache Infrastruktur im jeweiligen Sozialraum entstehen können, wobei oftmals die Größe und Lage des jeweiligen Ortes eine Rolle spielen. Dies trifft insbesondere auf die Ausgestaltung des öffentlichen Personennahverkehrs zu, allerdings auch auf die Möglichkeiten, Freizeit zu verbringen, einer Arbeit nachzugehen oder eine Wohnform zu finden, die jenseits klassischer Modelle strukturiert ist. Bereits die Strukturdatenrecherche gab erste Hinweise auf Diskrepanzen zwischen eher urbanen und ruralen Gegenden im Kontext der Möglichkeiten, an routinemäßigen Lebenspraxen teilzuhaben. Ein wichtiger Unterschied liegt im Vergleich der fünf Sozialräume darin, dass die unterstützenden Angebote der Behindertenhilfe in den kleineren Sozialräumen weniger breit aufgestellt sind, wodurch längere Anfahrtswege und geringere Auswahlmöglichkeiten in Kauf genommen werden müssen (Kratz et al. 2016, S. 11; siehe Kapitel 12.5). Das Argument, die Behindertenhilfe sei ohnehin keine inklusive Struktur, ist völlig richtig. Dennoch ermöglicht sie in vielerlei Hinsicht Teilhabe, unter anderem dadurch, dass Assistenzen zur Verfügung gestellt werden ${ }^{8}$. Eine weitere infrastrukturelle Differenz, die die Strukturdatenrecherche aufzeigte, betrifft den ungleich verteilten Wohlstand in den fünf untersuchten Sozialräumen, der sich unter anderem in einer ungleichen Arbeitslosenquote und differierendem durchschnittlichen Gehalt ausdrückt (wobei nicht außer Acht gelassen werden darf, dass die Lebenshaltungskosten ebenfalls unterschiedlich sind und das durchschnittliche Gehalt in Relation zu diesen betrachtet werden muss, was die Prekarität bestimmter Regionen zumindest ein Stück weit entschärft). Die ethnographischen Sozialraumbegehungen machen insbesondere auf Differenzen bezüglich der Ausgestaltung des öffentlichen Personennahverkehrs auf-

8 Auf andere Sozialhilfeträger jenseits sogenannter Behinderung trifft dies ebenfalls zu. Diese wurden allerdings nicht so breit untersucht wie die der sogenannten Behindertenhilfe, weshalb letztgenannte bei den hiesigen Ausführungen im Vordergrund steht. 
merksam, der in den kleineren der fünf untersuchten Sozialräume deutlich schwächer aufgestellt ist als in den größeren (siehe Kapitel 13.1). Dies ist nicht ungewöhnlich, muss aber dahingehend problematisiert werden, dass so die Abhängigkeit von individueller, selbstverantwortlicher (Auto-)Mobilität deutlich steigt. Eine weitere infrastrukturelle Herausforderung, die ebenfalls auf die Differenz von Stand und Land heruntergebrochen werden kann, betrifft die Möglichkeit, im jeweiligen Ort Freizeitaktivitäten nachzugehen oder kulturelle Veranstaltungen zu besuchen. Hier sind in eher ländlich gelegenen Sozialräumen die Angebotsstrukturen deutlich geringer ausgeprägt (dies problematisiert auch Schädler 2011, S. 186f). Die Sozialraumanalysen im Kontext Arbeit und Freizeit decken ebenfalls Differenzen zwischen großstädtischen und kleinstädtischen Sozialräumen auf, die darin liegen, dass die Möglichkeit zur Teilhabe im Bereich Arbeit signifikant von der Lage des jeweiligen Sozialraums abhängt (Teilhabe wird in Großstädten eher als möglich eingeschätzt), was im Bereich Freizeit jedoch nicht der Fall ist (siehe Kapitel 16.8 und Kapitel 17.9). Weitere Differenzen zwischen Großstadt und Kleinstadt/Gemeinde wurden sowohl bei der Reflexion der Erhebung sichtbar als auch bezüglich der Offenheit der Interviewpersonen gegenüber einer Teilnahme von Menschen mit unterschiedlichen Unterstützungsbedarfen. Die Erhebungserfahrungen zeigen insbesondere bei den Interviews zum Thema Arbeit, für die die Interviewenden in den Sozialräumen vor Ort ArbeitnehmerInnen interviewten, dass die angesprochenen Personen in den beiden kleineren Sozialräumen als ablehnender gegenüber einer Teilnahme am Interview wahrgenommen wurden als in den größeren Sozialräumen, sobald sie über das Thema informiert wurden. In gewisser Weise spiegeln die Ergebnisse diesen subjektiven Eindruck wider, denn es konnte gezeigt werden, dass die interviewten ArbeitnehmerInnen und PrimärvertreterInnen im Kontext Freizeit in den beiden kleineren Sozialräumen Schneverdingen und der Verbandsgemeinde Nieder-Olm einer Teilhabe von Menschen mit Behinderung, Menschen mit Fluchtmigrationshintergrund oder Menschen mit Demenz im Gros etwas ablehnender gegenüberstehen als die Interviewten in den größeren Sozialräumen, wobei dies im Bereich Arbeit deutlicher ausgeprägt ist als im Bereich Freizeit (siehe Kapitel 16.2 und Kapitel 17.2). Die repräsentative Untersuchung von Einstellungen der Bevölkerung Deutschlands zu Inklusion kann dieses Ergebnis nicht bestätigten, was jedoch insbesondere an den hohen Ansprüchen bezüglich des zugrunde gelegten Korrelationsgrades liegt. Eine Frage, die aus diesem in vielfältiger 
Hinsicht dargelegten Ergebnis erwächst, ist, inwiefern in ländlichen Regionen (a) Infrastruktur verbessert und (b) die Haltung von EinwohnerInnen zur Teilhabe von Menschen mit diversen Unterstützungsbedarfen an mehrheitsgesellschaftlichen Praxen hin zu mehr Offenheit verändert werden können. Denn gerade »[f] ür Menschen mit Behinderungen bergen die strukturellen Nachteile des ländlichen Raums hohe Diskriminierungsrisiken und oft zusätzliche Einschränkungen ihrer Teilhabemöglichkeiten, die nicht durch informelle Ressourcen oder andere spezifisch ländliche Sozialraumqualitäten ausgeglichen werden können« (Schädler 2011, S. 189). Deshalb gilt es, gleichzeitig die Vorteile stark zu machen, die das Leben in eher ländlichen Regionen bietet. Unter anderem ist es durchaus möglich, einen Gewinn daraus zu ziehen, dass die Wege kurz sein können - gerade in bürokratischer Hinsicht - oder dass womöglich der nachbarschaftliche Austausch stärker etabliert ist. Dies sind Faktoren, die prinzipiell dabei unterstützen können, Teilhabebarrieren abzubauen.

\section{Arbeit und Leistung}

Die Sozialraumanalysen im Handlungsfeld Arbeit zeigen, dass häufige Begründungen dafür, weshalb Menschen mit Behinderung oder Menschen mit Fluchtmigrationshintergrund eingeschränkte Teilhabemöglichkeiten haben, darin liegen, diese seien nicht ausreichend qualifiziert, körperlich und/oder kognitiv nicht dazu in der Lage, die erforderlichen Arbeitstätigkeiten auszuführen oder zu gering motiviert beziehungsweise unzuverlässig (siehe Kapitel 16.7). Jene Personen werden insofern als zu weniger Leistung befähigt oder gewillt hervorgebracht. Arbeit wird von einigen Interviewpersonen (und weit darüber hinaus) also oftmals über eine Idee von Leistung verstanden. Um das Verhältnis zwischen Arbeit und Leistung näher zu untersuchen, wird sich im Folgenden den (durchaus differenten) Begriffsverständnissen von Arbeit angenähert. In den Sozialwissenschaften finden sich zahlreiche Lesarten, wie >Arbeit $<$ begrifflich und inhaltlich gefasst werden kann (Voß 2010). Eine Möglichkeit, die Pluralität an Begriffen zu fassen, ist, Arbeit in der Ambivalenz zwischen Belastung und Selbstverwirklichung

9 Weitere Hinweise zu Teilhabe im Kontext Behinderung und Migration im ländlichen Raum gibt Schön (2013). 
zu definieren. "Arbeit belastet das menschliche Leben und bereichert es zugleich, ja sie wird oft als Grundlage für eine erhoffte Befreiung aus Mühsal und Elend, wenn nicht gar als Feld der schöpferischen Selbstentfaltung des Menschen gesehen« (Voß 2010, S. 27). Im Kontext von Selbstverwirklichung wird Arbeit zur Quelle, aus der Identität geschöpft werden kann und die ermöglicht, diverse Subjektpositionen einzunehmen (beispielsweise die Subjektposition, sich über die eigene Arbeit positiv darzustellen oder der eigenen Arbeit einen sozialen und/oder emotionalen Wert zu verleihen). Eine ambivalente Folge dessen ist, dass das Subjekt in gewisser Weise eine "Ökonomisierung des Selbst« (Bröckling 2019, S. 284) betreibt und so zum »unternehmerischen Selbst«(Bröckling 2019, S. 46) wird, das aufgespannt ist zwischen Selbstermächtigung und Selbstausbeutung (Kleemann und Voß 2010, S. 435). Auch die Frage nach dem Verhältnis von Arbeit und Subjekt ist mittlerweile weitergehend ausdifferenziert (Kleemann und Voß 2010). In mancherlei Hinsicht kann diesbezüglich nach wie vor dem Marx zugeschriebenen Diktum gefolgt werden: »[n]ur der Arbeitende ist Subjekt« (Voß 2010, S. 42). Dies verdeutlicht, inwiefern das Ausüben einer Arbeitstätigkeit Subjektpositionen begründet und inwiefern im Umkehrschluss der Ausschluss von Arbeit gleichbedeutend ist mit dem Ausschluss von je bestimmten Subjektpositionen. Der Ausschluss von Arbeit betrifft die jeweilige Person also unmittelbar und umfänglich, kann sie doch beispielsweise nicht die Subjektposition sarbeitende Person einnehmen und ist infolgedessen von je bestimmten Diskursen ausgeschlossen, beispielsweise regelmäßiges Gehalt, Urlaubsanspruch, mitunter eigenständiges Anmieten einer Wohnung sowie darüber hinaus, als Person angesehen zu werden, die Leistung für die Gesamtgesellschaft erbringt. Die Frage ist nun, wie vor diesem Hintergrund das Verhältnis von Arbeit und Leistung ausgelotet werden kann. Ein verbreitetes Leistungsverständnis geht davon aus, »Leistung müsste etwas Nützliches hervorbringen" (Distelhorst 2014, S. 13). In diesem Kontext können die Äußerungen der Interviewpersonen gesehen werden, die Menschen mit Behinderung und/oder Menschen mit Fluchtmigrationshintergrund die Teilhabe an Arbeit absprechen, da diese, in ihren Augen, nicht ausreichend dazu in der Lage seien, die Arbeitstätigkeit auszuführen beziehungsweise sie so auszuführen, dass sie einen gesamtgesellschaftlichen Nutzen hat. Dem wohnt ein utilitaristisches Arbeitsverständnis inne, das unmittelbar mit Anstrengung, Engagement und dem Erreichen von Zielvorgaben verknüpft ist. "Wer etwas erschafft, das für niemanden von Nutzen ist, hat 
nichts geleistet« (Distelhorst 2014, S. 13). Der Leistungsgedanke ist also Instrument dafür, sich von anderen abzugrenzen und kann ein gewisses Konkurrenzverhältnis begründen. Über Leistung wird »die Figur des Anderen« (Distelhorst 2014, S. 13) eingeführt und gefestigt, nämlich desjenigen, der die jeweiligen Erfordernisse nicht erfüllen kann. In dieser Hinsicht werden Menschen mit Behinderung und/oder Menschen mit Fluchtmigrationshintergrund einmal mehr als >das defizitäre andere hervorgebracht, die sich grundsätzlich von der Mehrheitsgesellschaft unterscheiden. Wer nicht als in ausreichendem Maße leistungsfähig konstruiert wird, wird nicht als Teil der Mehrheitsgesellschaft anerkannt. Dabei kommt unweigerlich die Frage nach Gerechtigkeit auf - die immer auch eine Frage von Politik und Moral ist (siehe Kapitel 31 und Kapitel 40). Es ist zu reflektieren, ob oder inwiefern es als 'gerecht gelten kann, dass bestimmte Personen (oftmals pauschal) von der Teilhabe an Arbeit mehr oder minder umfassend ausgeschlossen werden - und damit in vielerlei Hinsicht von der Teilhabe an Praxen der Mehrheitsgesellschaft. Infolgedessen wird auch das Verständnis von Gesellschaft durch diese Fragen berührt, denn einmal mehr ist zu diskutieren, ob oder inwiefern der Ausschluss bestimmter Personen aus gesamtgesellschaftlichen Praxen die Konstitution von Gesellschaft beeinflusst. Indem bestimmte Personen in ihrem Status als BürgerIn eingeschränkt werden, vollzieht sich in gewisser Weise ein "undoing of citizenship « (Altermark 2018, S. 4), das nicht nur die jeweilige Person als BürgerIn, sondern auch Gesellschaft respektive ihre je bestimmte Hervorbringung infrage stellt (siehe dazu auch Kapitel 23). Im Kontext Gerechtigkeit ist zudem die Frage bedeutsam, wie Menschen hervorgebracht werden, wenn ihr Dasein an den Wert oder Mehrwert geknüpft wird, den sie (vermeintlich nicht) erbringen - und was alles als Arbeit anerkannt wird (Voß 2010). Wird das Verhältnis von Arbeit und Leistung aus handlungspraktischer Perspektive betrachtet, so ist klar, dass es für bestimmte Arbeitstätigkeiten körperlicher oder kognitiver Voraussetzungen bedarf, ohne die diese nicht ausgeübt werden können. Dennoch ist es schlicht nicht zutreffend, Menschen mit Behinderung und/oder Menschen mit Fluchtmigrationshintergrund pauschal als Minderleistende zu titulieren. Den Interviewpersonen fehlt es offensichtlich an Erfahrungen mit KollegInnen mit Behinderung und/oder KollegInnen mit Fluchtmigrationshintergrund. Es scheint, dass das Wissen um alternative Tätigkeiten oder Assistenzmodelle $\mathrm{zu}$ wenig verbreitet ist, um Arbeit jenseits des mehrheitsgesellschaftlichen Verständnisses denken zu können. Hier braucht es mögli- 
cherweise Informationen für ArbeitgeberInnen ebenso wie ArbeitnehmerInnen, aber auch Sensibilisierungspraxen auf Ebene der Gesamtgesellschaft.

\section{Freizeit und Kontakt}

Die Ergebnisse zeigen an vielen Stellen, inwiefern Meinungen, Erfahrungen und Einstellungen von Personen, die als Angehörige der Mehrheitsgesellschaft beschrieben werden, durch den lebenspraktischen Kontakt mit Menschen beeinflusst werden können, die (je situativ) von Ausschluss bedroht oder betroffen sind. Diese Beeinflussung ist im Gros positiv, weshalb gesagt werden kann, dass regelmäßiger Kontakt zwischen Personen dazu führen kann, gegenseitige Vorbehalte und Ängste abzubauen, wodurch wiederum Barrieren dekonstruiert werden, die zuvor die Teilhabemöglichkeiten bestimmter Personen behinderten. Im Bereich Freizeit wirkt sich dies besonders deutlich aus, was bei der Diskussion der Ergebnisse eingehend entfaltet wurde (siehe Kapitel 17.7 und Kapitel 17.12.2), insbesondere in Bezug auf die sogenannte Kontakthypothese. Einmal mehr wird also bestätigt, dass Freizeit aufgrund ihrer eher herrschaftsfreien Strukturierung (zumindest im Vergleich zum größtenteils eher hierarchisch strukturierten Arbeitsbereich; Trescher 2015b, S. 32f) dazu geeignet ist, statusgleiche Begegnungen und Handlungspraxen zu ermöglichen (Trescher 2015b, S. 33). Wobei allerdings nicht unterschlagen werden darf, dass Freizeit ebenso statusgenerierende Wirkmächtigkeit haben kann und oftmals hat (Trescher 2015b, S. 30 ${ }^{10}$. Nichtsdestotrotz überwiegt heutzutage ein positives Freizeitverständnis, das Freizeit als eine Zeit versteht, »in der man für etwas frei ist« (Opaschowski 2014, S. 129; Trescher 2015b, S. 28f). Freizeit wird aus wertrationaler Sicht betrachtet und mit einem je bestimmten Sinn verknüpft (Opaschowski 2014, S. 129), was bedeutet, dass Personen in ihrer Freizeit versuchen, diese freie Zeit sinnhaft zu füllen. In ihrer Funktion der Vergemeinschaftung (Trescher 2015b, S. 31) kann Freizeit dazu beitragen, Teilhabebarrieren abzubauen und

10 Damit geht einher, dass »[v]iele Situationen und Tätigkeiten [...] am Leistungsgedanken orientiert [sind], am Streben nach Erfolg, nach guten bzw. im Vergleich mit anderen, besseren Ergebnissen « (Lüdtke 2001, S. 18). Freizeit wird folglich in gewisser Weise messbar, was dadurch weitergehend verfestigt wird, dass sie sich immer mehr an Qualitätskriterien orientiert (Opaschowski 2008, S. 33). Freizeit kommt in dieser Hinsicht ein »Moment der Instrumentalisierung« (Lüdtke 2001, S. 18) zu. 
Einsamkeit oder Isolation zu überwinden (Opaschowski 2008, S. 223ff). Die besondere Bedeutung von Freizeit zeichnet sich also auf mehreren Ebenen ab. Anknüpfend daran braucht es nun Ideen, wie das ganz offensichtlich vorhandene Inklusionspotenzial des Freizeitbereichs genutzt werden kann. Wie im vorigen bereits mehrfach betont, ist es wichtig, Begegnungsräume zu schaffen, sodass sich Menschen mit und Menschen ohne je bestimmte Ausschlusserfahrungen treffen und kennenlernen können, wodurch gegenseitig möglicherweise vorhandene Vorbehalte abgebaut werden und schließlich Personen in Freizeitsituationen zueinanderkommen können, die sich anderweitig unter Umständen nicht kennengelernt hätten - obwohl eine große Bereitschaft besteht (siehe Kapitel 17.2; Trescher 2015b, S. 118f). Weiterhin ist an informierende und aufklärende Angebote zu denken, die langfristig dazu führen können, Freizeitaktivitäten so zu verändern, dass gegebenenfalls vorhandene Teilhabebarrieren abgebaut werden, die zum Teil darin liegen, dass (a) ein Wissen darüber fehlt, dass Freizeitvereine und -gruppen oftmals Interesse daran haben, Menschen mit unterschiedlichen Unterstützungsbedarfen miteinzubeziehen, und dass (b) zu wenig Kenntnis darüber verbreitet ist, welche Unterstützungsmöglichkeiten es bereits gibt und wie diese genutzt werden können (beispielsweise Freizeitassistenzen oder Ähnliches). Ein weiterer Aspekt ist, die Wirkmächtigkeit des Hilfesystems infrage zu stellen, mit dem oftmals ausschließende Praxen einhergehen beziehungsweise von diesem produziert werden. Am Beispiel von Menschen mit (geistiger) Behinderung kann gezeigt werden, inwiefern es möglich ist, nahezu das gesamte Leben unter dem mehr oder weniger einschließenden Protektorat des Hilfesystems zu verbringen (siehe u.a. Trescher 2017f, 2017a; Altermark 2018, S. 3ff), was wiederum in Ausschluss resultiert und sich folglich in mangelnden Teilhabemöglichkeiten an routinemäßigen Lebenspraxen äußert. Die besondere Bedeutung deinstitutionalisierender Praxen wird im nachfolgenden Kapitel eingehender erläutert.

\section{Die Rolle des Hilfesystems}

Das Hilfesystem und die ihm inhärenten Strukturen und Praxen sind ambivalent, da sie zwar Teilhabe ermöglichen können, beispielsweise durch die Bereitstellung von (Mitteln oder Expertise für) Assistenzen oder Ähnliches, jedoch gleichzeitig oftmals in behindernder Weise wirkmächtig werden 
(Schäfers und Wansing 2016; Beck 2016). Solche Behinderungspraxen vollziehen sich insbesondere dadurch, dass Personen, die von den Strukturen des Hilfesystems mehr oder weniger stark abhängig sind, kaum andere Subjektpositionen einnehmen können, als die einer passiven, hilfeempfangenden Person. In der Folge ist es ihnen nur erschwert möglich, Zugang zu mehrheitsgesellschaftlichen Diskursen zu finden und/oder in diesen eine Sprechrolle einzunehmen, durch die sie an der Gestaltung (und damit Hervorbringung) des jeweiligen Diskurses mitwirken können. Die Strukturen des Hilfesystems wurden hier nicht eingehend und unmittelbar untersucht, dennoch zeigt sich an einigen Stellen die Wirkmächtigkeit von Versorgungsinstitutionen sowie die Wahrnehmung dieser in der Mehrheitsgesellschaft. Beispielsweise ist es so, dass einige Personen, die im Rahmen der Sozialraumanalysen in den Bereichen Arbeit und Freizeit interviewt wurden (siehe Kapitel 16 und Kapitel 17), die Bedeutung des Hilfesystems und der ihm eigenen Einrichtungen hervorheben, da diese für die Betreuung und Versorgung von Menschen mit je bestimmten Unterstützungsbedarfen notwendig seien. Weiterhin pochen einige der Interviewpersonen in diesem Zusammenhang darauf, Institutionen und Einrichtungen nicht infrage zu stellen, sondern in ihrem Fortbestand zu bestätigen. Dies bildet sich teils auch in den Ergebnissen des Surveys »Einstellung(en) zu Inklusion« ab, in dem ein Großteil der Befragten angibt, dass segregative Wohn-, Arbeits-, Bildungsund Freizeitangebote bestehen bleiben sollen (Trescher et al. 2020b, 2020a), wobei im hier konkreten Fall ausschließlich nach den Teilhabemöglichkeiten von Menschen mit geistiger Behinderung gefragt wurde (siehe Kapitel 20.1). Eine Frage, die daraus folgt, ist, wie diese Ergebnisse weitergedacht werden können. Ein Ansatzpunkt ist beispielsweise, das Bild zu dekonstruieren, das nach wie vor oftmals von Menschen mit je bestimmten Unterstützungsbedarfen in der Mehrheitsgesellschaft vorherrscht und diese häufig primär als defizitär und/oder nahezu umfassend hilfebedürftig darstellt. Ziel dessen ist, jene Personen weg vom Image des passiven Hilfeempfangs zu bewegen und hin zum aktiv handelnden Gesellschaftsmitglied. Dazu bedarf es der Eröffnung von Zugängen und Subjektpositionen, in denen ebenjene Rolle eingenommen und ausgestaltet werden kann. Darüber hinaus scheint die öffentliche Wahrnehmung von Institutionen und Einrichtungen, die durch das Hilfesystem finanziert werden, eher positiv zu sein, wenngleich immer wieder (auch medial) auf Missstände aufmerksam gemacht wird. Jener überwiegend positive Eindruck hängt womöglich damit zusammen, dass 
diese Strukturen als gegeben hin- und nicht veränderbar angenommen werden - schließlich, so wird annehmbar argumentiert, erfüllen sie einen bestimmten Zweck. Es bedarf also, gerade von wissenschaftlicher Seite, einer breiteren Untersuchung von Unterbringungs- und Betreuungsstrukturen, die durch das Hilfesystem hervorgebracht und finanziert werden, sodass diese fundiert problematisiert und, das darf nicht unterschlagen werden, gelingende Handlungspraxen identifiziert und herausgestellt werden können. Neben einem solchen Zugang gilt es, Verstehenszugänge zu eröffnen, die dabei unterstützen, die Ambivalenzen des Hilfesystems zu reflektieren. Neben der Fachdisziplin und Selbstvertretungsgruppen ist diesbezüglich ausdrücklich die Mehrheitsgesellschaft zu adressieren, sodass diese für Strukturprobleme und Ambivalenzen im Versorgungs- und Hilfesystem sensibilisiert werden. Dabei bleibt die Frage offen, wie die Breite der Bevölkerung erreicht werden kann, sodass Sensibilisierungsmaßnahmen überhaupt ihre AdressatInnen finden. Schließlich bedarf es sicherlich infrastruktureller Veränderungen, die die häufig einengenden und unflexiblen Strukturrahmen des Hilfesystems aufweichen. Eine besondere Rolle spielt dabei der Sozialraum, denn, wenn (insbesondere Komplex-)Einrichtungen sich »ins regionale Umfeld öffnen oder gar dezentralisiert werden, rückt dabei die Region als zu gestaltender, inklusiv wirkender Rahmen stärker in den Vordergrund (Kratz et al. 2016, S. 12). Dadurch könnten ganz konkret beispielsweise mehr Möglichkeiten geschaffen werden, als Mensch mit je bestimmtem Unterstützungsbedarf in Innenstadtnähe zu leben und nicht auf Randbezirke verwiesen zu sein, in denen die Mieten günstiger sind und/oder sozialer Wohnungsbau angesiedelt ist. Dazu gehört auch, sich seine Wohnform selbst wählen zu dürfen und nicht, beispielsweise aus Kostengründen, in stationär betreute Wohneinrichtungen vermittelt $\mathrm{zu}$ werden ${ }^{11}$. Dies ist insbesondere im Kontext Behinderung eine weit verbreitete Problematik. Allerdings ist das Leben vieler Menschen im Alter und/oder Menschen mit Demenz davon ebenso betroffen wie die Situation von Menschen mit Fluchtmigrationshintergrund, die größtenteils sehr geringe Wahlmöglichkeiten

11 Dabei handelt es sich um eine Problematik, die im Zuge des neuen sogenannten Bundesteilhabegesetzes (BTHG) sowie des Reha- und Intensivpflegestärkungsgesetz (RISC) möglicherweise noch verschärft wird (u.a. Rohrmann 2019, S. 10f; König und Wolf 2017, S. 7; Die Fachverbände für Menschen mit Behinderung 2019; AbilityWatch 2019). 
bezüglich Wohnort und Wohnform haben $\left(\$ 56\right.$ AsylG $^{12}$; $\$ 61$ AufenthG ${ }^{13}$; Täubig 2009, S. 23).

\section{Inklusion, Moral und Menschenrecht}

Ausgangspunkt der Auslotung des Verhältnisses von Inklusion, Moral und Menschenrecht ist, dass Inklusion in einigen Lesarten als moralische Idee verstanden wird. Dies geschieht beispielsweise insofern, sie als "normative Basis politischer Ansprüche von Menschen in modernen Gesellschaften« (Rösner 2010, S. 126) zu verstehen. Moral ist ein kontingenter Begriff, denn, was als moralisch gilt beziehungsweise empfunden wird, ist vielgestaltig und sozio-kulturell-historisch variabel (Hoerster 2008, S. 12). Gleichzeitig begründet sich Moral in (je sozio-kulturell-historisch einzigartiger) Allgemeingültigkeit und »universale[r] Zustimmung« (Hoerster 2008, S. 13). Das, was je als moralisch gilt, muss inhaltlich dargelegt werden, was bedeutet, dass keine Leerformeln herangezogen werden dürfen - dies stellt eine größere Herausforderung dar (Williams 2003, S. 84) ${ }^{14}$. Moralnormen erheben, wie beschrieben, den Anspruch der Allgemeingültigkeit. Dieser Anspruch wird dadurch erfüllt, dass die Zustimmung zur Moralnorm für alle Menschen subjektiv nachvollziehbar und aufgrund dessen auch »intersubjektiv begründet « (Hoerster 2008, S. 59) ist. Übertragen auf >Inklusion« kann also gesagt werden, dass diese (als moralische Größe) genau dann allgemein gültig ist, wenn alle Menschen ihr zustimmen. Dies ist jedoch, wie vielfältig gezeigt werden konnte (siehe Kapitel 22), nicht der Fall ${ }^{15}$. Dadurch wird das argumentative Gerüst, auf dem Inklusion als moralische Verpflichtung teils aufgebaut wird, geschwächt. Die Frage, ob die Allgemeingültigkeit moralischer Normen auch Menschen mit (Schwerst-)Behinderung umfasst, wird

12 Asylgesetz.

13 Gesetz über den Aufenthalt, die Erwerbstätigkeit und die Integration von Ausländern im Bundesgebiet.

14 Beispielsweise ist >das Wohl der Menschen eine solche Formel, die zwar oftmals als Moralnorm herangezogen wird, nach der gehandelt werden soll, die allerdings nur schwierig mit allgemeingültigem Inhalt gefüllt werden kann, da diese Formel teils sehr unterschiedlich interpretiert wird (Williams 2003, S. 91f).

15 Wobei angemerkt werden muss, dass im Kontext der hiesigen Studie keine Moralurteile, sondern Einstellungen, Haltungen und Erfahrungen untersucht wurden. 
von Hoerster (2008) bejaht und anhand zweier Aspekte begründet. Zum einen sei eine intersubjektive Geltung auch hier gegeben, da jeder Mensch dem Risiko ausgesetzt sei, selbst, beispielsweise durch einen Unfall, behindert zu werden (Hoerster 2008, S. 72). Die Begründung liegt hier im Interesse jeder Person, »dass solche Moralnormen soziale Geltung erlangen, die nicht nur meinen gegenwärtigen, sondern auch meinen potentiell künftigen Interessen dienen « (Hoerster 2008, S. 72). Zum anderen wird die Allgemeingültigkeit von Moralnormen für beziehungsweise gegenüber Menschen mit (Schwerst-)Behinderung in einem gewissen Mitgefühl gesehen, das jenen Personen entgegengebracht wird und aus dem resultiert, sich ihnen gegenüber moralisch zu verhalten (Hoerster 2008, S. 72f; siehe auch Horster 2012, S. 29f). Dass dies Ausdruck eines gewissen Paternalismus ist, ist potenziell problematisch, kann dies doch $\mathrm{zu}$ bevormundenden und/oder herablassenden Handlungspraxen gegenüber Menschen mit Behinderung führen. Demgegenüber gibt es Ansätze, entlang derer Menschen mit (Schwerst-)Behinderung aufgrund mangelnder Fähigkeit zur Reziprozität nicht als »Mitglied der moralischen Gemeinschaft« (Horster 2012, S. 26) gelten können. Ihre Aufnahme könne ausschließlich über das Solidarprinzip begründet werden, denn die sogenannte moralische Gemeinschaft sei eine »Solidargemeinschaft « (Horster 2012, S. 27). Auch hier zeichnen sich paternalistische Charakteristika ab und darüber hinaus drohen Handlungsmöglichkeiten für Menschen mit Behinderung, wenn nicht ausgeschlossen, so doch zumindest deutlich eingeschränkt zu werden. Eine Möglichkeit, jenen Paternalismus zu überwinden, entwirft Graumann (2011) mit ihrem Konzept der assistierten Freiheit, bei der sie, im Rückbezug auf die UN-Konvention über die Rechte von Menschen mit Behinderungen und Kant, »Wohlfahrtsrechte mit einem konsequenten Paternalismusverbot« (Graumann 2011, S. 200) verknüpft. Die Verantwortung für kollektive Solidarität verortet sie aufseiten des Staates und der Politik (Graumann 2011, S. 245). Sie verbindet dabei Moral mit Menschenrecht und versteht Menschenrechte »als moralische Rechte [...], die mit moralischen Pflichten verbunden sind, die sich primär an Politik und Staat richten« (Graumann 2011, S. 245) ${ }^{16}$. Dadurch ist es möglich, Menschen mit (schwerer) Behinderung als Menschenrechtssubjekte $\mathrm{zu}$

16 Diese Engführung von Moral und Menschenrecht ist zwar nicht unumstritten, wird jedoch oftmals vorgenommen. Zur Diskussion des Verhältnisses von Moral und Menschenrecht siehe ausführlich Graumann (2011, S. 198ff). 
konstituieren (Graumann 2011, S. 134ff). Die Reflexion von Menschenrechten vor dem Hintergrund von (schwerer) Behinderung hat zur Folge, dass diese »neu interpretiert werden [müssen] (Graumann 2011, S. 11), wodurch das Verständnis von Menschenrecht - und schließlich von Inklusion - weiterentwickelt werden kann. Auch Staat und Politik sind von veränderten menschenrechtlichen Perspektiven, wie sie mit der UN-Konvention über die Rechte von Menschen mit Behinderungen einhergehen, nicht unberührt, was sich in "politischen Zielkonflikten« (Herz 2015, S. 64) zeigen kann, die unter anderem darin bestehen, dass herkömmliche Praxen hinterfragt werden müssen und zwar deutlich über die Lebenssituation von Menschen mit Behinderung hinaus (beispielsweise bezüglich der Verknüpfung von Armut und Bildungsbiographie; Herz 2015, S. 64). Inklusion als menschenrechtliche Forderung findet sich im bezugswissenschaftlichen Diskurs häufig, jedoch fehlt dabei zumeist eine so fundierte Herleitung, wie sie Graumann (2011) geleistet hat (siehe u.a. Schulze 2011; Flieger und Schönwiese 2011) ${ }^{17}$. Ein menschenrechtlich begründetes Verständnis von Inklusion wird darüber hinaus auch von Verbänden oder Vertretungen der Behindertenhilfe als Slogan ausgerufen, um mehr Rechte und Möglichkeiten gesellschaftlicher Teilhabe für Personen zu fordern, die von Ausschluss bedroht oder betroffen sind. Dabei geht es oftmals auch darum, mehr oder eine andere Form der Anerkennung für die jeweils vertretenen Personenkreise zu erreichen. Dadurch, dass Inklusion als menschenrechtlich beziehungsweise moralisch begründete Forderung teilweise als eine Art unanfechtbare Zielvorstellung verstanden wird, ist es kaum möglich, diese zu hinterfragen und sich kritisch mit ihr zu beschäftigen. Ein Wandel von einer gewissen »Inklusionsideologie« (Rösner 2010, S. 135) hin zu Inklusion als Kritik ist allerdings notwendig, damit sich Inklusion im Sinne des Aufbrechens und Veränderns tradierter Praxen vollziehen kann. Dies ist eine Problematik, vor der auch Modellprojekte stehen, die sich Inklusion verschrieben haben.

17 Ein Überblick und eine kritische Einordnung zum Verhältnis von Inklusion und menschenrechtlichem Anspruch findet sich darüber hinaus in Dederich (2013a). 


\section{Schlussbetrachtung}

Am Ende des Buches angelangt werden die umfangreiche Studie reüssiert und offene Fragen beziehungsweise Forschungsdesiderate, die aus den Ergebnissen hervorgehen, noch einmal gebündelt dargelegt. In der Studie werden fünf Sozialräume zum Gegenstand gemacht, die umfänglich untersucht wurden, was sich in der Breite der Ergebnisse widerspiegelt, die in den übergeordneten Kapiteln »Sozialräume beschreiben und erfahren« (Kapitel IV), "Erfahrungen im Miteinander erforschen " (Kapitel V) und »Einstellung(en) zu Inklusion erforschen " (Kapitel VI) dargelegt sind. Dabei folgt der Analyse struktureller sozialräumlicher Gegebenheiten (Kapitel 11 und Kapitel 12) eine eingehende ethnographische Untersuchung, die das je individuelle Erleben in den fünf untersuchten Sozialräumen in den Mittelpunkt stellt (Kapitel 13). Dem schließt sich die Darstellung der Ergebnisse an, die im Kontext der Untersuchung von Teilhabe in den Handlungsfeldern Arbeit und Freizeit generiert wurden und die einen Einblick in das (oftmals gelingende) Miteinander geben sowie Kenntnisse über Barrieren vermitteln, die nach wie vor Teilhabemöglichkeiten behindern (Kapitel 16 und Kapitel 17). Die Ergebnisse des deutschlandweiten Surveys, in dem Einstellungen zu Inklusion untersucht wurden, erweitern den Fokus vom je konkreten Sozialraum auf die Gesamtgesellschaft und zeigen, dass, trotz teilweise (mehr oder weniger ausgeprägter) Ablehnung, Inklusion als grundsätzlich wichtiges und unterstützenswertes Thema verstanden wird (Kapitel 22). Gute Forschung wirft mehr Fragen auf, als sie Antworten gibt - dies ist auch bei der vorliegenden Studie der Fall. Wie oben immer wieder herausgearbeitet folgen aus den Ergebnissen zahlreiche theoretische und empirische Anknüpfungsmöglichkeiten sowie handlungspraktische Implikationen, von denen an dieser Stelle ausgewählte erneut aufgegriffen und dargelegt werden. In theoretischer Hinsicht will die Studie einen Beitrag dazu leisten, das Verständnis um Raum und 
seine Aneignung respektive Hervorbringung ein Stück zu erweitern. Anschlussmöglichkeiten werden hier insbesondere darin gesehen, ein diskurstheoretisch hergeleitetes Verständnis von Raum(-Aneignung) weiter zu vertiefen sowie die praxeologische Perspektive auf Raum zu erweitern (siehe dazu Everts und Schäfer 2019, S. 10ff). Auch das Thema Sozialraumentwicklung spielt in diesem Zusammenhang eine bedeutsame Rolle. Hierbei ist ein zentraler Gedanke, Inklusion vom Sozialraum aus zu denken und nicht, wie es bislang oftmals der Fall ist, nahezu ausschließlich über das Individuum. Dabei ist es notwendig, auch auf Ebene von Sozialraum und Inklusion individuelle Verständnisse von Behinderung zu überwinden, die eindimensional an der jeweiligen Person festgemacht werden und untrennbar mit dieser verknüpft sind, um stattdessen Behinderung eher als behindernde Praxis zu verstehen, die sich je situativ vollzieht (siehe Kapitel 4.1). In diesem Zusammenhang ist die Debatte um Barrierefreiheit zu reflektieren, wird diese doch ebenfalls oftmals an Personen festgemacht, denen ein je bestimmtes Defizit attribuiert wird. Die mehrdimensionale Untersuchung von Barrierefreiheit im Rahmen dieser Studie macht darauf aufmerksam, wie ambivalent Barrierefreiheit und der Umgang damit sind (siehe Kapitel 5.4, Kapitel 11.3 und Kapitel 13.2). Eine daran anknüpfende sozial- ebenso wie kulturwissenschaftliche Betrachtung dieses ambivalenten Gefüges kann beispielsweise die Frage fokussieren, inwiefern >Barriere〈 und `Freiheit< begrifflich gefasst werden können sowie welche Implikationen für das Konstrukt damit einhergehen. Bei der Betrachtung von Barrierefreiheit wurde insbesondere die Konzeption von und der Umgang mit sogenannter Leichter Sprache problematisiert (unter anderem in Kapitel 11.3), deren Ambivalenzen ebenfalls einer eingehenderen theoretischen (ebenso wie empirischen) Untersuchung bedürfen. Dabei steht beispielsweise die Frage im Vordergrund, wie das Verhältnis von Teilhabeermöglichung und Behinderungspraxis, in dem Leichte Sprache unweigerlich aufgespannt ist, theoretisch gefasst und - zumindest als analytische Größe - aufgelöst werden kann. Von großem Interesse ist ebenfalls die Frage nach Standards Leichter Sprache, mit denen immer die Homogenisierung einer äußerst heterogenen Gruppe einhergeht, was dieses Unterfangen bereits im Kern als scheiternd anlegt. Problematisch ist zudem, dass dadurch sozusagen eine Restgruppe von Personen geschaffen wird, die nach wie vor Ausschluss erfahren. Diese Ambivalenz gilt es folglich ebenfalls theoretisch zu beleuchten, beispielsweise im Kontext moraltheoretischer Verstehenszugänge. Auch in dieser Studie wurde das Konstrukt Behinde- 
rung als Praxis - Inklusion als Kritik als theoretische Grundlegung herangezogen (siehe Kapitel 4). Erweitert wurde es insofern, dass nicht zuletzt die räumliche Ebene, die jenem Theorem innewohnt, deutlich (gemacht) wurde. Behinderung, Inklusion und Raumaneignung sind Praxen, die in ihrem Vollzug konstituiert werden. Auch wenn Inklusion als ein Ausgangspunkt der empirischen Fragestellungen in der Studie nachvollziehbarerweise sehr präsent ist, kamen dennoch weiterführende Fragen auf, die nicht unbedingt in dieser Weise antizipiert wurden. Umfasst werden diese von der Problematik, dass Begriffsverständnisse rund um Inklusion vielfältig, allerdings im Gros unscharf sind sowie einer theoretischen Fundierung weitestgehend entbehren. Theoretisch begründeter Gegenentwurf dazu ist das hier zugrunde gelegte Verständnis von Inklusion als Kritik, ausgehend von dem Inklusion als ein Prozess verstanden wird, in dem Barrieren dekonstruiert werden, die eine Diskursteilhabe je situativ einschränken oder erschweren. Aus der überwiegenden Begriffsdiffusität folgt also das theoretische Desiderat einer grundlegenden Begriffsbildung von Inklusion, wie oben bereits ausführlich problematisiert wurde (siehe Kapitel 26). Im Kontext dessen ist zudem eine vertiefte Beleuchtung des Verhältnisses von Inklusion und Gerechtigkeit interessant sowie eine moraltheoretische Auseinandersetzung. In methodischer Hinsicht zeigt sich, dass anhand des entwickelten Forschungsdesigns fünf Sozialräume umfassend und mehrdimensional untersucht werden konnten. Als besonders wertvoll und gewinnbringend hat sich dabei das eigens entwickelte mehrdimensionale Forschungsdesign erwiesen, dessen komplexes methodisches Zusammenspiel ermöglichte, breite Ergebnisse zu generieren. Es wurden sowohl strukturelle, deskriptiv-statistisch vergleichbare als auch tiefergehende qualitative Ergebnisse generiert, die auf mehreren Ebenen Einblicke in die Sozialräume, ihre Ausgestaltung und die sich in ihnen vollziehende Lebenspraxis ermöglichen. Folglich kann das Design als Vorbild für ähnliche Forschungsvorhaben dienen (möglicherweise nach entsprechenden Adaptionen). Auch auf Methodenebene wurden Desiderate ausgemacht, die in anschließenden Projekten sukzessive gefüllt werden können - beziehungsweise kann zumindest damit begonnen werden. An erster Stelle ist hierbei das Desiderat der Erforschung latenter Sinnzusammenhänge zu nennen, die im Rahmen dieser Studie nicht miteinbezogen wurde (siehe Kapitel 24). An dieser Stelle eröffnen sich zahlreiche Anschlussmöglichkeiten, die vertiefende Fragestellungen verfolgen, um schließlich latente Barrieren und Bedingungsstrukturen offenzulegen. Ein 
weiteres Beispiel für methodische ebenso wie inhaltliche Vertiefungen der hiesigen Studie ist eine breite Untersuchung von Inklusionsverständnissen, die unterschiedliche Personen ebenso wie Institutionen miteinschließt. Unter anderem wäre es interessant zu untersuchen, welches Inklusionsverständnis gemeinnützige Vereine haben, die sogenannte Inklusionsprojekte anstoßen und tragen. Aus den Ergebnissen der umfassenden Untersuchung der fünf Sozialräume, die jeweils Inklusionsprojekte initiiert haben, kann in vielfältiger Hinsicht Mehrwert für die Handlungspraxis abgeleitet werden. Eine solche sozialraumorientierte Praxisentwicklung kann am Paradigma der Dekonstruktion festgemacht werden beziehungsweise vollzieht sich im Modus der Dekonstruktion: Inklusion heißt, Diskursteilhabebarrieren zu dekonstruieren. Dazu ist die Handlungspraxis unweigerlich auf einen wissenschaftlichen Blick angewiesen, der Strukturprobleme und Ambivalenzen offenlegt und damit überhaupt erst der kritischen - dekonstruktiven Auseinandersetzung zugänglich macht. Inklusion ist ein krisenhafter Prozess, der sich vollziehen muss, der die Beteiligten herausfordert und der unbedingt der Aushandlung bedarf. Inklusion ist nichts, was einfach >umgesetzt werden kann (siehe Kapitel 27), sie kann nicht als eine Art idealisierte Zielvorstellung realisiert werden, sondern entsteht im Miteinander - das braucht oftmals vor allem Zeit. Zudem ist es wichtig, Berührungspunkte und Begegnungsorte zwischen allen Menschen im Sozialraum zu schaffen, denn ein zentrales Ergebnis der Studie ist, dass gemeinsame Aktivitäten zu überwiegend positiven Erfahrungen und einer offeneren Einstellung gegenüber Inklusion führen (Kapitel 16.6, Kapitel 17.7 und Kapitel 22). Von besonderer Bedeutung ist dabei, nicht einfach bestehende Strukturen zu nutzen, die zudem oftmals primär dem Hilfesystem wie beispielsweise der Behindertenhilfe entstammen, sondern neue Netzwerke zu knüpfen und dabei auch auf die Kreativität und das Engagement der Menschen im Sozialraum $\mathrm{zu}$ vertrauen, die viel zu diesem Prozess beitragen können beziehungsweise ihn in gewisser Weise überhaupt erst konstituieren. Ein weiterer Gestaltungshinweis, der Personen und Gruppen gegeben werden kann, die sich in ihrem Sozialraum mit Inklusion beschäftigen und entsprechend ausgerichtete Projekte anstoßen wollen, ist, zu Bewusstseinsbildung beizutragen, um den Blick für diverse Bedarfe zu öffnen. Der Sozialraum sollte dabei in der Breite erreicht werden, was die Frage aufwirft, welche Personen adressiert werden sollen. So ist zum Beispiel zu entscheiden, ob in einem ersten Schritt Personen angesprochen werden sollen, die Inklusion ohnehin positiv gegen- 
überstehen, oder Personen, die eher ablehnend sind. Auch die Form der Adressierung muss bedacht werden. Dabei ist es wichtig, sich gerade bei zielgruppenspezifischen Maßnahmen und Angeboten der Ambivalenz bewusst zu sein, die mit einer Fokussierung bestimmter Personen als >Ausgeschlossene einhergeht. Auch wenn es Ziel ist, jenen Personen Teilhabemöglichkeiten zu eröffnen und Barrieren abzubauen, die Teilhabe behindern, so darf nicht aus den Augen verloren werden, dass sogenannten Inklusionsmaßnahmen ein Moment der Besonderung innewohnt, wodurch diese selbst zur Behinderungspraxis werden können (siehe auch Herzog 2013). Eine Reflexion dieser Ambivalenz ist unerlässlich für inklusionsbezogene Vorhaben nicht nur in der Handlungspraxis, sondern in allen anderen Kontexten ebenfalls, auch und insbesondere in der Wissenschaft. 



\section{Dank}

Eine umfängliche Beforschung von Sozialräumen kann nicht ohne die Unterstützung zahlreicher Personen realisiert werden, denen an dieser Stelle ausdrücklich gedankt werden soll. An erster Stelle sprechen wir allen Personen, die sich so bereitwillig haben beforschen lassen, unseren herzlichen Dank aus! Im Zusammenhang damit danken wir zudem allen unseren PartnerInnen in der Praxis, die uns bei der Vermittlung von Interviewpersonen oder dem Verteilen von Erhebungsinstrumenten unterstützt haben. Im Konkreten sei hier allen KoordinatorInnen des Projekts »Kommune Inklusiv« in den Sozialräumen Erlangen, Rostock, Schneverdingen, Schwäbisch Gmünd und der Verbandsgemeinde Nieder-Olm gedankt. Zudem danken wir unseren MitarbeiterInnen und KollegInnen, Michael Börner, Anna Lamby und Silvia Rügner, die auch an diesem Projekt in der ein oder anderen Weise mitgearbeitet haben. Michael Börner und Anna Lamby danken wir dabei insbesondere für die Unterstützung bei der Auswertung des Surveys »Einstellung(en) zu Inklusion«. In Bezug auf diesen sind wir darüber hinaus Prof. Dr. Michael Fingerle zu Dank verpflichtet, der dem gesamten Team während der Auswertung des Surveys bei Fragen zur Seite stand. Silvia Rügner war uns im gesamten Prozess - von Interviewerhebung und Recherche bis hin zu Literaturverwaltung und Korrekturlesen - die wichtigste Stütze, weshalb wir ihr an dieser Stelle noch einmal ganz besonders danken. Allen (ehemaligen) studentischen Hilfskräften des Arbeitsbereichs gebührt ebenfalls großer Dank, denn ohne ihren unermüdlichen und zuverlässigen Einsatz bei Erhebung, (Literatur-)Recherche, Datenverwaltung und vielem mehr hätte dieses Buch nicht in diesem knapp bemessenen Zeitrahmen entstehen können. Sarah Kühn, Peter Nothbaum, Iris Pfeuffer, Johanna Printz, Stefanie Schneider, Benjamin Schramm, Leonie Winter: Vielen herzlichen Dank! Ein letztes Dankeschön gilt unserem Kooperationspartner Aktion Mensch e.V., 
in persona Andrea Suda und Carolina Zibell, die aufgrund ihres dezidierten Interesses an der wissenschaftlichen Beforschung ihres Projekts »Kommune Inklusiv« diese Studie erst möglich machten. 


\section{Literaturverzeichnis}

Abbott, Lesley (2006): Northern Ireland headteachers' perceptions of inclusion. In: International Journal of Inclusive Education 10 (6), S. 627-643.

AbilityWatch (2019): Pressemitteilung zum Referentenentwurf des veränderten RISG/GKV-IPREG. Online verfügbar unter https://abilitywatch. de/2019/12/08/pressemitteilung-zum-referentenentwurf-des-veraenderten-risg-gkv-ipreg/, zuletzt geprüft am 14.01.2020.

Ackermann, Karl-Ernst (2013): Geistigbehindertenpädagogik zwischen Disziplin und Profession. In: Karl-Ernst Ackermann, Oliver Musenberg und Judith Riegert (Hg.): Geistigbehindertenpädagogik!? Disziplin - Profession - Inklusion. Oberhausen: Athena, S. 171-184.

Ackermann, Karl-Ernst; Dederich, Markus (2011): Einführung in das Thema. In: Karl-Ernst Ackermann und Markus Dederich (Hg.): An Stelle des Anderen. Ein interdisziplinärer Diskurs über Stellvertretung und Behinderung. Oberhausen: Athena, S. 7-24.

Adeos Media GmbH (2018): Vereine in Rostock (447). Laichingen. Online verfügbar unter www.vereinsverzeichnis.eu/stadt,Rostock,2.html, zuletzt geprüft am 23.08.2018.

Allport, Gordon W. (1954): The Nature Of Prejudice. Cambridge, Mass.: Addison-Wesley Publishing Company, Inc.

Altermark, Niklas (2018): Citizenship Inclusion and Intellectual Disability. Biopolitics Post-Institutionalisation. Abigdon, New York: Routledge.

Arendt, Hannah (2017): Was ist Politik? München, Berlin: Piper.

Avramidis, Elias; Norwich, Brahm (2002): Teachers' attitudes towards integration/inclusion: a review of the literature. In: European Journal of Special Needs Education 17 (2), S. 129-147. 
Backhaus, Klaus; Erichson, Bernd; Plinke, Wulff; Weiber, Rolf (2018): Multivariate Analysemethoden. Eine anwendungsorientierte Einführung. Berlin, Heidelberg: Springer-Verlag Berlin Heidelberg.

BAG WfbM (2019): Durchschnittliche monatliche Arbeitsentgelte 2014-2017. Online verfügbar unter https://www.bagwfbm.de/category/34, zuletzt geprüft am 12.01.2020.

Bänziger, Andreas (2009): Telefonbefragung als intersubjektiver Aushandlungsprozess. Die komplexe Kommunikationsstruktur standardisierter Interviews - theoretische Neukonzeption und praktische Anwendung in der Markt- und Meinungsforschung. Baden-Baden: Nomos.

Bauriedl, Sybille (2008): Räume lesen lernen: Methoden zur Raumanalyse in der Diskursforschung. In: Historical Social Research 33 (1), S. 278-312.

Bayerisches Landesamt für Statistik (2018): Statistik kommunal 2017. Kreisfreie Stadt Erlangen 09 562. Eine Auswahl wichtiger statistischer Daten. Fürth: Bayerisches Landesamt für Statistik.

Beck, Iris (2016): Der Bedarfsbegriff »revisited« - Aspekte der Begründung individueller Ansätze zur Bedarfserhebung und -umsetzung. In: Markus Schäfers und Gudrun Wansing (Hg.): Teilhabebedarfe von Menschen mit Behinderungen. Zwischen Lebenswelt und Hilfesystem. Stuttgart: Kohlhammer, S. 24-45.

Beck, Iris; Schuck, Karl Dieter (2001): Der Forschungsgegenstand über Grenzen und Möglichkeiten der Integration aus Sicht der Heil- und Sonderpädagogik. In: Gerhard Igl und Felix Welti (Hg.): Die Verantwortung des sozialen Rechtsstaates für Personen mit Behinderung und für die Rehabilitation. Interdisziplinäre Tagung des Instituts für Sozialrecht und Sozialpolitik in Europa der Christian-Albrechts-Universität zu Kiel und der LVA Schleswig-Holstein. Wiesbaden: Chmielorz.

Becker, Heinz (2016a): ...inklusive Arbeit! Das Recht auf Teilhabe an der Arbeitswelt auch für Menschen mit hohem Unterstützungsbedarf. Weinheim und Basel: Beltz Juventa.

Becker, Uwe (2016b): Die Inklusionslüge. Behinderung im flexiblen Kapitalismus. Bielefeld: transcript.

Behrendt, Hauke (2017): Was ist soziale Teilhabe? Plädoyer für einen dreidimensionalen Inklusionsbegriff. In: Catrin Misselhorn und Hauke Behrendt (Hg.): Arbeit, Gerechtigkeit und Inklusion. Wege zu gleichberechtigter gesellschaftlicher Teilhabe. Stuttgart: J.B. Metzler Verlag, S. 50-76. 
Ben-Moshe, Liat; Powell, Justin J.W. (2007): Sign of our times? Revis(it)ing the International Symbol of Access. In: Disability \& Society 22 (5), S. 489505.

Benninghaus, Hans (2007): Deskriptive Statistik. Eine Einführung für Sozialwissenschaftler. Wiesbaden: VS.

Berdelmann, Kathrin; Reh, Sabine (2015): Adressierung durch den Raum (Lieblings-)Plätze in der Schule. Eine fotoethnographische Exploration. In: Thomas Alkemeyer, Herbert Kalthoff und Markus Rieger-Ladich (Hg.): Bildungspraxis. Körper · Räume · Objekte. Weilerswist: Velbrück Wissenschaft, S. 183-205.

Bernasconi, Tobias (2007): Barrierefreies Internet für Menschen mit geistiger Behinderung. Eine experimentelle Pilotstudie zu technischen Voraussetzungen und partizipativen Auswirkungen. Oldenburg: BIS-Verlag der Carl von Ossietzky Universität Oldenburg.

Bertelmann, Lena (2019): „Im Moment stellt sich die Frage nicht..." - Zur Rolle der Gemeinde und des Ortsbezirks bei der Planung und Gestaltung eines inklusiven Gemeinwesens. In: Zeitschrift für Inklusion (3).

Beyvers, Eva; Helm, Paula; Henning, Martin; Keckeis, Carmen; Kreknin, Innokentij; Püschel, Florian (2017): Einleitung. In: Eva Beyvers, Paula Helm, Martin Henning, Carmen Keckeis, Innokentij Kreknin und Florian Püschel (Hg.): Räume und Kulturen des Privaten. Wiesbaden: VS, S. 1-18.

BGBl. I: Gesetz zur Änderung des Bundeswahlgesetzes und anderer Gesetze. In: Bundesgesetzblatt. Jahrgang 2019 Teil I Nr. 23. ausgegeben zu Bonn am 27. Juni 2019, S. 834-839.

BIH Bundesarbeitsgemeinschaft der Integrationsämter und Hauptfürsorgestellen (2019): BIH-Jahresbericht 2018 | 2019. Die Arbeit der Integrationsämter und Hauptfürsorgestellen. Wiesbaden: Universum Verlag $\mathrm{GmbH}$.

Bilstein, Johannes (2018): Vom Inneren der Seele bis an das Ende der Welt. Raum als pädagogische Kategorie. In: Edith Glaser, Hans-Christoph Koller, Werner Thole und Salome Krumme (Hg.): Räume für Bildung Räume der Bildung. Beiträge zum 25. Kongress der Deutschen Gesellschaft für Erziehungswissenschaft. Opladen, Berlin \& Toronto: Barbara Budrich, S. 24-39.

Boettner, Johannes (2009): Sozialraumanalyse - soziale Räume vermessen, erkunden, verstehen. In: Brigitta Michel-Schwartze (Hg.): Methodenbuch Soziale Arbeit. Basiswissen für die Praxis. Wiesbaden: VS, S. 259291. 
Bosse, Ingo (2006): Behinderung und Fernsehen. Gleichberechtigte Teilhabe als Leitziel der Berichterstattung. Wiesbaden: DUV.

Bosse, Stefanie; Henke, Thorsten; Jäntsch, Christian; Lambrecht, Jennifer; Vock, Miriam; Spörer, Nadine (2016): Die Entwicklung der Einstellung zum inklusiven Lernen und der Selbstwirksamkeit von Grundschullehrkräften. In: Empirische Sonderpädagogik(1), S. 103-116.

Bosse, Stefanie; Spörer, Nadine (2014): Erfassung der Einstellung und der Selbstwirksamkeit von Lehramtsstudierenden zum inklusiven Unterricht. In: Empirische Sonderpädagogik 6 (4), S. 279-299.

Brandenburg, Hermann (2014): Inklusion von Menschen mit Demenz - Vision oder Illusion? In: Pflege \& Gesellschaft 19 (4), S. 364-371.

Breidenstein, Georg; Hirschauer, Stefan; Kalthoff, Herbert; Nieswand, Boris (2013): Ethnographie. Die Praxis der Feldforschung. Konstanz, München: UVK.

Bröckling, Ulrich (2019): Das unternehmerische Selbst. Soziologie einer Subjektivierungsform. Frankfurt a.M.: Suhrkamp.

Buchner, Tobias; Koenig, Oliver (2008): Methoden und eingenommene Blickwinkel in der sonder- und heilpädagogischen Forschung von 1996-2006. Eine Zeitschriftenanalyse. In: Heilpädagogische Forschung 34 (1), S. 15-34.

Bukow, Wolf-Dietrich (2007): Die Rede von Parallelgesellschaften. Zusammenleben im Zeitalter einer metropolitanen Differenzgesellschaft. In: Wolf-Dietrich Bukow, Claudia Nikodem, Erika Schulze und Erol Yildiz (Hg.): Was heißt hier Parallelgesellschaft? Zum Umgang mit Differenzen. Wiesbaden: VS, S. 29-51.

Bundesagentur für Arbeit (2018a): Brancheneinschätzung Herbst 2017. Arbeitsagenturbezirk Aalen. Online verfügbar unter https://arbeitsmarktmonitor.arbeitsagentur.de/faktencheck/branchen/tabelle/517/ $697 /$ ? $r=\& c=2 \% 2 C_{3} \%_{2} \mathrm{C}_{5} \%_{2} \mathrm{C}_{14} \%_{2} \mathrm{C}_{16} \%_{2} \mathrm{C}_{8} \% 2 \mathrm{C}_{10}$, zuletzt geprüft am 23.08.2018.

Bundesagentur für Arbeit (2018b): Brancheneinschätzung Herbst 2017. Arbeitsagenturbezirk Celle. Online verfügbar unter https://arbeitsmarktmonitor.arbeitsagentur.de/faktencheck/branchen/tabelle/540/0/?r=\&c= $2 \%_{2} \mathrm{C}_{3} \% 2 \mathrm{C}_{5} \%_{2} \mathrm{C}_{14} \%_{2} \mathrm{C}_{16} \% 2 \mathrm{C} 8 \% 2 \mathrm{C} 10$, zuletzt geprüft am 23.08.2018.

Bundesagentur für Arbeit (2018c): Brancheneinschätzung Herbst 2017. Arbeitsagenturbezirk Fürth. Online verfügbar unter https://arbeitsmarktmonitor.arbeitsagentur.de/faktencheck/branchen/tabelle/732/o 
/? $\mathrm{r}=\& \mathrm{c}=2 \% 2 \mathrm{C} 3 \% 2 \mathrm{C} 5 \% 2 \mathrm{C} 14 \% 2 \mathrm{C} 16 \% 2 \mathrm{C} 8 \% 2 \mathrm{C} 10$, zuletzt aktualisiert am 23.08.2018.

Bundesagentur für Arbeit (2018d): Brancheneinschätzung Herbst 2017. Arbeitsagenturbezirk Mainz. Online verfügbar unter https://arbeitsmarktmonitor.arbeitsagentur.de/faktencheck/branchen/tabelle/612/ $697 / ? \mathrm{r}=\& \mathrm{c}=2 \% 2 \mathrm{C}_{3} \% 2 \mathrm{C} 5 \% 2 \mathrm{C} 14 \% 2 \mathrm{C} 16 \% 2 \mathrm{C} 8 \% 2 \mathrm{C} 10$, zuletzt geprüft am 23.08.2018.

Bundesagentur für Arbeit (2018e): Brancheneinschätzung Herbst 2017. Arbeitsagenturbezirk Rostock. Online verfügbar unter https://arbeitsmarktmonitor.arbeitsagentur.de/faktencheck/branchen/tabelle/654 $10 / ? \mathrm{r}=\& \mathrm{c}=2 \% 2 \mathrm{C} 3 \% 2 \mathrm{C} 5 \% 2 \mathrm{C} 14 \% 2 \mathrm{C} 16 \% 2 \mathrm{C} 8 \% 2 \mathrm{C} 10$, zuletzt geprüft am 23.08.2018.

Bundesfachstelle Barrierefreiheit (o.J.): InitiativeSozialraumInklusiv. Mehr Barrierefreiheit in Kommunen und Regionen. Hg. v. Deutsche Rentenversicherung Knappschaft-Bahn-See.

Bundesministerium für Arbeit und Soziales (2019): Konferenzreihe »Inklusiver Sozialraum« gestartet. Berlin.

Butler, Judith (1991): Das Unbehagen der Geschlechter. Frankfurt a.M.: Suhrkamp.

Cloerkes, Günther (1982): Die Kontakthypothese in der Diskussion um eine Verbesserung der gesellschaftlichen Teilhabechancen Behinderter. In: Zeitschrift für Heilpädagogik 33 (8), S. 561-568.

Cloerkes, Günther (2007): Soziologie der Behinderten. Eine Einführung. Heidelberg: Winter.

Cohen, Jacob (1988): Statistical Power Analysis for the Behavioral Sciences. Hillsday, New Jersey: Lawrence Erlbaum Associates.

Cordes, Alja; Silter, Katharina (2016): Inklusion ohne Grenzen - Beeinflussung von Einstellungsbarrieren durch Respekt. In: Tobias Bernasconi und Ursula Böing (Hg.): Schwere Behinderung \& Inklusion. Facetten einer nicht ausgrenzenden Pädagogik. Oberhausen: Athena, S. 235-247.

Cramer, Colin; Harant, Martin (2014): Inklusion - Interdisziplinäre Kritik und Perspektiven von Begriff und Gegenstand. In: Zeitschrift für Erziehungswissenschaft 17 (4), S. 639-659.

Dahme, Heinz-Jürgen; Wohlfahrt, Norbert (2011): Sozialraumorientierung in der Behindertenhilfe: alles inklusive bei niedrigen Kosten? In: Teilhabe 50 (4), S. 148-154. 
Dangl, Oskar (2014): Inklusion als Herausforderung für die Geistigbehindertenpädagogik. In: Zeitschrift für Bildungsforschung 4 (3), S. 257-270.

Dannenbeck, Clemens (2012): Wie kritisch ist der pädagogische Inklusionsdiskurs? Entpolitisierungsrisiko und theoretische Verkürzung. In: Kerstin Rathgeb (Hg.): Disability Studies. Kritische Perspektiven für die Arbeit am Sozialen. Wiesbaden: VS, S. 55-67.

Dannenbeck, Clemens (2013): Inklusionsorientierung als pädagogische Herausforderung. In: Unsere Jugend 65 (11+12), S. 460-466.

De Boer, Anke; Pijl, Sip Jan; Minnaert, Alexander (2011): Regular primary school teachers' attitudes towards inclusive education: a review on the literature. In: International Journal of Inclusive Education 15 (3), S. 331-353.

Dederich, Markus (2012): Ästhetische und ethische Grenzen der Barrierefreiheit. In: Anja Tervooren und Jürgen Weber (Hg.): Wege zur Kultur. Barrieren und Barrierefreiheit in Kultur- und Bildungseinrichtungen. Köln, Weimar, Wien: Böhlau, S. 101-115.

Dederich, Markus (2013a): Ethische Aspekte der Inklusion. Hg. v. Inklusion Lexikon. Universität zu Köln. Online verfügbar unter www.inklusion-lexikon.de/Ethik_Dederich.pdf, zuletzt geprüft am 14.01.2020.

Dederich, Markus (2013b): Recht und Gerechtigkeit. In: Markus Dederich, Heinrich Greving, Christian Mürner und Peter Rödler (Hg.): Behinderung und Gerechtigkeit. Heilpädagogik als Kulturpolitik. Gießen: Psychosozial-Verlag, S. 21-36.

Deinet, Ulrich (2010): Aneignungsraum. In: Christian Reutlinger, Caroline Fritsche und Eva Lingg (Hg.): Raumwissenschaftliche Basics. Eine Einführung für die soziale Arbeit. Wiesbaden: VS, S. 35-44.

Derrida, Jacques (2016): Grammatologie. Frankfurt a.M.: Suhrkamp.

Derrida, Jacques (2017): Gesetzeskraft. Der »mystische Grund der Autorität«. Frankfurt a.M.: Suhrkamp.

Die Fachverbände für Menschen mit Behinderung (2019): Stellungnahme der Fachverbände zum Referentenentwurf der Bundesregierung: Gesetz zur Änderung des Neunten und des Zwölften Buches Sozialgesetzbuch und anderer Rechtsvorschriften. Berlin. Online verfügbar unter https://www. lebenshilfe.de/fileadmin/Redaktion/PDF/Wissen/public/GemeinsameStellungnahmen/20190321_Stellungnahme_KFV_Z_Referentenentwurf BR_BTHG_Aenderungsgesetz.pdf, zuletzt geprüft am 14.01.2020.

Diekmann, Andreas (2016): Empirische Sozialforschung. Grundlagen, Methoden, Anwendungen. Reinbek bei Hamburg: Rowohlt. 
Distelhorst, Lars (2014): Leistung. Das Endstadium der Ideologie. Bielefeld: transcript.

Dollase, Rainer (2001): Fremdenfeindlichkeit verschwindet im Kontakt von Mensch zu Mensch. Zur Reichweite der Kontakthypothese. In: Diskurs 10 (2), S. 16-21.

Ebling, Michael; Lensch, Eckart (2018): Vorwort. In: Landeshauptstadt Mainz (Hg.): Sozialraumanalyse Mainz 2017. Fortschreibung der Analyse der sozialräumlichen Struktur der Landeshauptstadt Mainz. Dezernat für Soziales, Kinder, Jugend, Schule und Gesundheit. Mainz.

Eckert, Martin (2014): Werbung mit Behinderung. Eine umstrittene Kommunikationsstrategie zwischen Provokation und Desensibilisierung. Bielefeld: transcript.

Eick, Volker (2005): »Ordnung wird sein ...«. Quartiersmanagement und lokale Sicherheitspolitik. In: Heinz-Jürgen Dahme und Norbert Wohlfahrt (Hg.): Aktivierende Soziale Arbeit. Theorie - Handlungsfelder - Praxis. Baltmannsweiler: Schneider Verlag Hohengehren, S. 110-122.

Eifler, Stefanie; Kay, Ramona; Pinkas, Sarah (2017): Projekt »Zusammenleben in der Stadt« - Eine Sozialraumanalyse aus der Perspektive der Theorie sozialer Desorganisation. Katholische Universität Eichstätt-Ingolstadt.

einfachmachen (2019): Aktionspläne der Länder. Online verfügbar unter https://www.gemeinsam-einfach-machen.de/GEM/DE/AS/Aktionsplaene/Aktionsplaene_Laender/aktionsplaene_laender_node.html, zuletzt geprüft am 29.02.2020.

Engelmann, Peter (1990): Einführung: Postmoderne und Dekonstruktion. Zwei Stichwörter zur zeitgenössischen Philosophie. In: Peter Engelmann (Hg.): Postmoderne und Dekonstruktion. Texte französischer Philosophen der Gegenwart. Stuttgart: Reclam, S. 5-32.

Engler, Stefanie (1997): Zur Kombination von qualitativen und quantitativen Methoden. In: Barbara Friebertshäuser und Annedore Prengel (Hg.): Handbuch Qualitative Forschungsmethoden in der Erziehungswissenschaft. Weinheim, München: Juventa, S. 118-130.

Everts, Jonathan; Schäfer, Susann (2019): Praktiken und Raum. In: Susann Schäfer und Jonathan Everts (Hg.): Handbuch Praktiken und Raum. Humangeographie nach dem practice turn. Bielefeld: transcript, S. 7-19.

Flieger, Petra; Schönwiese, Volker (2011): Die UN-Konvention über die Rechte von Menschen mit Behinderungen: Eine Herausforderung für die Integrations- und Inklusionsforschung. In: Petra Flieger und Volker Schön- 
wiese (Hg.): Menschenrechte, Integration, Inklusion. Aktuelle Perspektiven aus der Forschung. Bad Heilbrunn: Klinkhardt, S. 27-38.

Forlin, Chris; Douglas, Graham; Hattie, John (1996): Inclusive practices: How accepting are teachers? In: International Journal of Disability, Development and Education 43 (2), S. 119-133.

Forst, Rainer (1994): Kontexte der Gerechtigkeit. Politische Philosophie jenseits von Liberalismus und Kommunitarismus. Frankfurt a.M.: Suhrkamp.

Forst, Rainer (2005): Die erste Frage der Gerechtigkeit. In: Aus Politik und Zeitgeschichte 55 (37), S. 24-31.

Foucault, Michel (1978): Dispositive der Macht. Über Sexualität, Wissen und Wahrheit. Berlin: Merve.

Foucault, Michel (1981): Archäologie des Wissens. Frankfurt a.M.: Suhrkamp. Foucault, Michel (1992): Was ist Kritik? Berlin: Merve.

Foucault, Michel (2003): Die Ordnung des Diskurses. Frankfurt a.M.: Fischer. Foucault, Michel (2005): Subjekt und Macht. In: Daniel Defert und François Ewald (Hg.): Schriften in vier Bänden. Dits et Ecrits. Band 4. Frankfurt a.M.: Suhrkamp, S. 269-294.

Foucault, Michel (2014): Der Wille zum Wissen. Sexualität und Wahrheit 1. Berlin: Suhrkamp.

Foucault, Michel (2018): Von anderen Räumen (1967). In: Jörg Dünne und Stephan Günzel (Hg.): Raumtheorie. Grundlagentexte aus Philosophie und Kulturwissenschaften. Frankfurt a.M.: Suhrkamp, S. 317-329.

Friebertshäuser, Barbara (1997): Interviewtechniken - ein Überblick. In: Barbara Friebertshäuser und Annedore Prengel (Hg.): Handbuch Qualitative Forschungsmethoden in der Erziehungswissenschaft. Weinheim, München: Juventa, S. 371-395.

Friebertshäuser, Barbara; Langer, Antje (2010): Interviewformen und Interviewpraxis. In: Barbara Friebertshäuser, Antje Langer und Annedore Prengel (Hg.): Handbuch Qualitative Forschungsmethoden in der Erziehungswissenschaft. Weinheim, München: Juventa, S. 437-455.

Füller, Henning; Michel, Boris (2012): Einleitung. Raum als Heuristik für die sozialwissenschaftliche Machtanalyse. In: Henning Füller und Boris Michel (Hg.): Die Ordnung der Räume. Geographische Forschung im Anschluss an Michel Foucault. Münster: Westfälisches Dampfboot, S. 7-22. 
Gasterstädt, Julia; Urban, Michael (2016): Einstellung zu Inklusion? Implikationen aus Sicht qualitativer Forschung im Kontext der Entwicklung inklusiver Schulen. In: Empirische Sonderpädagogik 2016 (1), S. 54-66.

Gebhardt, Markus; Schwab, Susanne; Reicher, Hannelore; Ellmeier, Barbara; Gmeiner, Sonja; Rossmann, Peter; Gasteiger Klicpera, Barbara (2011): Einstellungen von LehrerInnen zur schulischen Integration von Kindern mit einem sonderpädagogischen Förderbedarf in Österreich. In: Empirische Sonderpädagogik 2011 (4), S. 275-290.

Geiling, Heiko (2005): Integrations- und Ausgrenzungsprobleme in einer städtischen Großsiedlung. Zur Theorie und Methode der Stadtteilanalyse. In: Marlo Riege und Herbert Schubert (Hg.): Sozialraumanalyse. Grundlagen - Methoden - Praxis. Wiesbaden: VS, S. 223-240.

Gesundheitsberichterstattung des Bundes (2018): Schwerbehinderte Menschen mit Ausweis. Online verfügbar unter www.gbe-bund. de/oowa921-install/servlet/oowa/aw92/WSO100/_XWD_FORMPROC?TARGET $=\& P A G E=$ XWD_1O\&OPINDEX $=1 \&$ HANDLER $=X S$ ROTATE_ADVANCED\&DATACUBE=_XWD_38\&D.000=ACROSS\&D. 0 01=DOWN\&D.624=PAGE\&D.623=PAGE\&D.100=PAGE, zuletzt geprüft am 23.08.2018.

Goffman, Erving (1973): Asyle. Über die soziale Situation psychiatrischer Patienten und anderer Insassen. Frankfurt a.M.: Suhrkamp.

Goffman, Erving (1975): Stigma. Über Techniken der Bewältigung beschädigter Identität. Frankfurt a.M.: Suhrkamp.

Graumann, Sigrid (2011): Assistierte Freiheit. Von einer Behindertenpolitik der Wohltätigkeit zu einer Politik der Menschenrechte. Frankfurt a.M.: Campus.

Graumann, Sigrid (2016): Menschenrechtsethische Überlegungen zum notwendigen Paradigmenwechsel im Selbstverständnis von Sozialpolitik und sozialen Diensten. In: Theresia Degener, Klaus Eberl, Sigrid Graumann, Olaf Maas und Gerhard Schäfer (Hg.): Menschenrecht Inklusion. 10 Jahre UN-Behindertenrechtskonvention - Bestandsaufnahme und Perspektiven zur Umsetzung in Sozialen Diensten und diakonischen Handlungsfeldern. Göttingen: Vandenhoeck \& Ruprecht, S. 52-73.

Groenemeyer, Axel (2014): Soziale Praxis - Institutionen - Diskurse - Erfahrung. Behinderung im Problematisierungsprozess. In: Soziale Probleme 25 (2), S. 150-172. 
Gronemeyer, Reimer (2013): Das 4. Lebensalter. Demenz ist keine Krankheit. München: Pattloch.

Haddock, Goeffrey; Maio, Gregory R. (2014): Einstellungen. In: Jonas Klaus, Wolfgang Stroebe und Miles Hewstone (Hg.): Sozialpsychologie. Berlin, Heidelberg: Springer-Verlag, S. 197-229.

Hansestadt Rostock. Der Oberbürgermeister. Presse- und Informationsstelle (2016): Statistisches Jahrbuch. Hansestadt Rostock. 2016. Hauptamt, Kommunale Statistikstelle. Rostock.

Hartmann, Jutta (2001): Bewegungsräume zwischen Kritischer Theorie und Poststrukturalismus. Eine Pädagogik vielfältiger Lebensweisen als $\mathrm{He}$ rausforderung für die Erziehungswissenschaft. In: Bettina Fritzsche, Jutta Hartmann, Andrea Schmidt und Anja Tervooren (Hg.): Dekonstruktive Pädagogik. Erziehungswissenschaftliche Debatte unter poststrukturalistischen Perspektiven. Wiesbaden: VS, S. 65-84.

Hecht, Petra; Niedermair, Claudia; Feyerer, Ewald (2016): Einstellungen und inklusionsbezogene Selbstwirksamkeitsüberzeugungen von Lehramtsstudierenden und Lehrpersonen im Berufseinstieg - Messverfahren und Befunden aus einem Mixed-Methods-Design. In: Empirische Sonderpädagogik 2016 (1), S. 86-102.

Heitmeyer, Wilhelm (2013): Vorwort. Der Anlass sozialräumlicher Analysen. In: Andreas Grau und Wilhelm Heitmeyer (Hg.): Menschenfeindlichkeit in Städten und Gemeinden. Weinheim und Basel: Beltz Juventa, S. 9-10.

Heitmeyer, Wilhelm; Grau, Andreas (2013): Gruppenbezogene Menschenfeindlichkeit im lokalen Raum und bürgerschaftliches Engagement. In: Andreas Grau und Wilhelm Heitmeyer (Hg.): Menschenfeindlichkeit in Städten und Gemeinden. Weinheim und Basel: Beltz Juventa, S. 11-33.

Helfferich, Cornelia (2014): Leitfaden- und Experteninterviews. In: Nina Baur und Jörg Blasius (Hg.): Handbuch Methoden der empirischen Sozialforschung. Wiesbaden: VS, S. 559-574.

Hellmich, Frank; Görel, Gamze; Schwab, Susanne (2016): Einstellungen und Motivation von Lehramtsstudentinnen und -studenten in Bezug auf den inklusiven Unterricht in der Grundschule - Ein Vergleich zwischen Deutschland und Österreich. In: Empirische Sonderpädagogik 2016 (1), S. 67-85.

Hermes, Gisela; Rohrmann, Eckhard (2006): Einführung. In: Gisela Hermes und Eckhard Rohrmann (Hg.): Nichts über uns - ohne uns! Disability 
Studies als neuer Ansatz emanzipatorischer und interdisziplinärer Forschung über Behinderung. Neu-Ulm: AG SPAK, S. 7-11.

Herz, Birgit (2015): Inklusionssemantik und Risikoverschärfung. In: Sven Kluge, Andrea Liesner und Edgar Weiß (Hg.): Inklusion als Ideologie. Frankfurt a.M.: Lang, S. 59-76.

Herzog, Benno (2013): Ausschluss im (?) Diskurs. Diskursive Exklusion und die neuere soziologische Diskursforschung. In: Forum Qualitative Sozialforschung 14 (2), Art. 19.

Hill, Marc (2016): Nach der Parallelgesellschaft. Neue Perspektiven auf Stadt und Migration. Bielefeld: transcript.

Hoerster, Norbert (2008): Was ist Moral? Eine philosophische Einführung. Stuttgart: Reclam.

Honer, Anne (2010): Lebensweltanalyse in der Ethnographie. In: Uwe Flick, Ernst von Kardorff und Ines Steinke (Hg.): Qualitative Forschung. Ein Handbuch. Reinbek bei Hamburg: Rowohlt, S. 194-204.

Hopf, Christel (2010): Qualitative Interviews. Ein Überblick. In: Uwe Flick, Ernst von Kardorff und Ines Steinke (Hg.): Qualitative Forschung. Ein Handbuch. Reinbek bei Hamburg: Rowohlt, S. 349-360.

Horster, Detlef (2012): Was ist Moral? In: Vera Moser und Detlef Horster (Hg.): Ethik der Behindertenpädagogik. Menschenrechte, Menschenwürde, Behinderung. Eine Grundlegung. Stuttgart: Kohlhammer, S. 23-30.

Hüllemann, Ulrike; Reutlinger, Christian; Deinet, Ulrich (2016): Aneignung als strukturierendes Element des Sozialraums. In: Fabian Kessl und Christian Reutlinger (Hg.): Handbuch Sozialraum. Wiesbaden: Springer Fachmedien, S. 1-18.

iib Institut Innovatives Bauen Dr. Hettenbach GmbH (2018a): WohnpreisMarktmietspiegel für Nieder-Olm. Schwetzingen. Online verfügbar unter https://www.wohnpreis.de/mietspiegel/nieder-olm, zuletzt geprüft am 12.09.2018.

iib Institut Innovatives Bauen Dr. Hettenbach GmbH (2018b): WohnpreisMarktmietspiegel für Schneverdingen. Schwetzingen. Online verfügbar unter https://www.wohnpreis.de/mietspiegel/schneverdingen, zuletzt geprüft am 12.09.2018.

Jahoda, Marie; Lazarsfeld, Paul F.; Zeisel, Hans (2018): Die Arbeitslosen von Marienthal. Ein soziographischer Versuch über die Wirkung langandauernder Arbeitslosigkeit. Frankfurt a.M.: Suhrkamp. 
Karhoff, Brigitte; Riege, Marlo (2005): Dynamik ist eine zentrale Konstante ...«. Annäherung an Wohn- und Lebenswelten in »Stadtteilen mit besonderem Erneuerungsbedarf«. In: Marlo Riege und Herbert Schubert (Hg.): Sozialraumanalyse. Grundlagen - Methoden - Praxis. Wiesbaden: VS, S. 261-281.

Kelle, Udo; Erzberger, Christian (2010): Qualitative und quantitative Methoden: Kein Gegensatz. In: Uwe Flick, Ernst von Kardorff und Ines Steinke (Hg.): Qualitative Forschung. Ein Handbuch. Reinbek bei Hamburg: Rowohlt, S. 299-309.

Kelle, Udo; Kluge, Susann (2010): Vom Einzelfall zum Typus. Fallvergleiche und Fallkontrastierung in der qualitativen Sozialforschung. Wiesbaden: VS.

Kessl, Fabian; Otto, Hans-Uwe; Ziegler, Holger (2005): Der Raum, sein Kapital und seine Nutzer. In: Marlo Riege und Herbert Schubert (Hg.): Sozialraumanalyse. Grundlagen - Methoden - Praxis. Wiesbaden: VS, S. 191205.

Kleemann, Frank; Voß, Günter (2010): Arbeit und Subjekt. In: Fritz Böhle, Günter Voß und Günther Wachtler (Hg.): Handbuch Arbeitssoziologie. Wiesbaden: VS, S. 415-450.

Klinkhammer, Dennis; Niehaus, Mathilde (2015): Betriebliche Inklusion auf dem Ausbildungs- und Arbeitsmarkt. In: Horst Biermann (Hg.): Inklusion im Beruf. Stuttgart: Kohlhammer, S. 180-215.

König, Markus; Wolf, Björn (2017): Steuerung in der Behindertenhilfe. Das Bundesteilhabegesetz und seine Folgen. Berlin: Verlag des Deutschen Vereins für öffentliche und private Fürsorge e.V.

Kratz, Dirk; Lempp, Theresa; Muche, Claudia; Oehme, Andreas (2016): Einführung. Zum wechselseitigen Bezug von Region und Inklusion. In: Dirk Kratz, Theresa Lempp, Claudia Muche und Andreas Oehme (Hg.): Region und Inklusion. Theoretische und praktische Perspektiven. Weinheim, Basel: Beltz Juventa, S. 7-26.

Kuckartz, Udo (2014): Mixed Methods. Methodologie, Forschungsdesigns und Analyseverfahren. Wiesbaden: VS.

Kuckartz, Udo (2016): Qualitative Inhaltsanalyse. Methoden, Praxis, Computerunterstützung. Weinheim und Basel: Beltz Juventa.

Kuckartz, Udo; Ebert, Thomas; Rädiker, Stefan; Stefer, Claus (2009): Evaluation online. Internetgestützte Befragung in der Praxis. Wiesbaden: VS. 
Kuckartz, Udo; Rädiker, Stefan; Ebert, Thomas; Schehl, Julia (2013): Statistik. Eine verständliche Einführung. Wiesbaden:VS.

Kuhl, Jan; Walther, Judith (2008): Die Einstellung von Studenten unterschiedlicher Studiengänge zu Menschen mit geistiger Behinderung. In: Heilpädagogische Forschung 2008 (4), S. 206-219.

Kunz, André; Luder, Reto; Moretti, Marta (2010): Die Messung von Einstellungen zur Integration (EZI). In: Empirische Sonderpädagogik 2 (3), S. 8394.

Kupke, Charlotte; Schlummer, Werner (2010): Kommunikationsbarrieren und ihre Überwindung. Leichte Sprache und Verständlichkeit in Texten für Menschen mit Lernschwierigkeiten. In: Teilhabe 49 (2), S. 67-73.

Kurtenbach, Sebastian (2017): Leben in herausfordernden Wohngebieten. Das Beispiel Köln-Chorweiler. Wiesbaden: Springer Fachmedien.

Kurzenberger, Stephan; Niehoff, Ulrich; Walther, Helmut; Sack, Rudi (2012): Barrierefreiheit für Menschen mit kognitiver Beeinträchtigung - Ergebnisse einer Befragung. In: Teilhabe 51 (3), S. 121-126.

Laclau, Ernesto; Mouffe, Chantal (2006): Hegemonie und radikale Demokratie. Zur Dekonstruktion des Marxismus. Wien: Passagen.

Lamnek, Siegfried (2005): Qualitative Sozialforschung. Weinheim und Basel: Beltz.

Landesamt für Statistik Niedersachsen (2018): Meine Gemeinde, meine Stadt - ausgewählte Daten auf Verwaltungseinheitsebene (VE) - Gebietsstand 01.11.2016. Online verfügbar unter https://www.nls.niedersachsen.de/ gemeinden/G358019.html, zuletzt geprüft am 23.08.2018.

Landeshauptstadt Mainz (Hg.) (2018): Sozialraumanalyse Mainz 2017. Fortschreibung der Analyse der sozialräumlichen Struktur der Landeshauptstadt Mainz. Dezernat für Soziales, Kinder, Jugend, Schule und Gesundheit. Mainz.

Landkreis Emsland (2018): Emsland Statistik. Meppen. Online verfügbar unter https://www.emsland.de/pdf_files/zahlen-und-daten/emslandstatistik-2018-07_2132_15.pdf, zuletzt geprüft am 23.08.2018.

Langfeldt, Hans-Peter; Wember, Franz B. (1994): 30 Jahre heilpädagogische Forschung. Bestandsaufnahme und inhaltsanalytische Reflexionen. In: Heilpädagogische Forschung 20 (4), S. 187-198.

Lazarsfeld, Paul (2005): Die soziografische Methode in der Marienthal-Studie. Auszüge aus »Vorspruch« (1960) und »Einleitung« (1933) der Studie. 
In: Marlo Riege und Herbert Schubert (Hg.): Sozialraumanalyse. Grundlagen - Methoden - Praxis. Wiesbaden: VS, S. 71-78.

Leidner, Rüdiger (2007): Die Begriffe »Barrierefreiheit«, »Zugänglichkeit« und »Nutzbarkeit« im Fokus. In: Patrick S. Föhl, Stefanie Erdrich, Hartmut John und Karin Maaß (Hg.): Das barrierefreie Museum. Theorie und Praxis einer besseren Zugänglichkeit. Ein Handbuch. Bielefeld: transcript, S. 28-33.

Leitner, Gerhard (2009): Weltsprache Englisch. Vom angelsächsischen Dialekt zur globalen Lingua franca. München: Verlag C. H. Beck.

Lindemann, Holger (2016): Konstruktion und empirische Validierung eines Instrumentes zur Erfassung der Einstellungen von Schülerinnen und Schülern gegenüber Peers mit Beeinträchtigung, anderer Herkunft und niedrigem sozioökonomischem Status. In: Empirische Sonderpädagogik 2016 (1), S. 5-21.

Lingelbach, Gabriele (2010): Konstruktionen von »Behinderung« in der Öffentlichkeitsarbeit und Spendenarbeit der Aktion Sorgenkind seit 1964. In: Elsbeth Bösl, Anne Klein und Anne Waldschmidt (Hg.): Disability History. Konstruktionen von Behinderung in der Geschichte. Eine Einführung. Bielefeld: transcript, S. 127-150.

Löw, Martina (2001): Raumsoziologie. Frankfurt a.M.: Suhrkamp.

Lüders, Christian (2010): Beobachten im Feld und Ethnographie. In: Uwe Flick, Ernst von Kardorff und Ines Steinke (Hg.): Qualitative Forschung. Ein Handbuch. Reinbek bei Hamburg: Rowohlt, S. 384-402.

Lüdtke, Hartmut (2001): Freizeitsoziologie. Arbeiten über temporale Muster, Sport, Musik, Bildung und soziale Probleme. Münster, Hamburg, Berlin, London: LIT.

Lueger, Manfred (1989): Die soziale Situation im Interview. In: Österreichische Zeitschrift für Soziologie 14 (3), S. 22-36.

Makarova, Taisiia; Mladenow, Andreas; Strauss, Christine (2016): Barrierefreiheit im Internet und Suchmaschinenranking - eine empirische Untersuchung. In: Heinrich C. Mayr und Martin Pinzger (Hg.): Informatik 2016. Bonn: Gesellschaft für Informatik e.V., S. 1071-1085.

Markowetz, Reinhard: Freizeit behinderter Menschen, S. 307-340.

Markowetz, Reinhard (2006): Freizeit und Behinderung - Inklusion durch Freizeitassistenz. In: Spektrum Freizeit 30 (2), S. 54-72.

Maurer, Alfons (2015): Zur Bedeutung einer sozialraumorientierten Altenpflege. In: Hermann Brandenburg, Helen Güther und Ingo Proft (Hg.): 
Kosten kontra Menschlichkeit. Herausforderungen an eine gute Pflege im Alter. Ostfildern: Grünewald, S. 141-150.

Mayring, Philipp (2010a): Qualitative Inhaltsanalyse. In: Uwe Flick, Ernst von Kardorff und Ines Steinke (Hg.): Qualitative Forschung. Ein Handbuch. Reinbek bei Hamburg: Rowohlt, S. 468-475.

Mayring, Philipp (2010b): Qualitative Inhaltsanalyse. Grundlagen und Techniken. Weinheim, Basel: Beltz.

Meier, Monique (2013): Sozialbericht der Universitätsstadt Marburg - 2013. Hg. v. Magistrat der Universitätsstadt Marburg. Fachbereich »Arbeit, Soziales und Wohnen«. Marburg.

Mills, Sara (2007): Der Diskurs. Tübingen: Francke.

Moser, Vera (2010): Perfektibilität - Verbesonderung - Förderung - Teilhabe/Inklusion. Eine Paradigmengeschichte der Behindertenpädagogik. In: Attila Nóbik und Béla Pukánszky (Hg.): Normalität, Abnormalität und Devianz. Gesellschaftliche Konstruktionsprozesse und ihre Umwälzungen in der Moderne. Frankfurt a.M.: Lang, S. 75-86.

Motakef, Mona (2014): Subjektposition. In: Daniel Wrana, Alexander Ziem, Martin Reisigl, Martin Nonhoff und Johannes Angermuller (Hg.): DiskursNetz. Wörterbuch der interdisziplinären Diskursforschung. Frankfurt a.M.: Suhrkamp, S. 394-395.

Münker, Stefan; Roesler, Alexander (2012): Poststrukturalismus. Stuttgart, Weimar: J.B. Metzler.

Netzwerk Leichte Sprache e.V. (o.J.): Leichte Sprache. Berlin.

Nugel, Martin (2017): Die Erziehungswissenschaft als Raumwissenschaft. Herausforderungen durch den spatial turn. In: Christiane Thompson, Rita Casale und Norbert Ricken (Hg.): Die Sache(n) der Bildung. Paderborn: Schöningh, S. 263-278.

Nuss, Felix (2019): Sozialraumorientierung und Migration. Vom Willen geflüchteter Menschen und der Stärke individueller Lebensgeschichten. In: Birgit Wartenpfuhl (Hg.): Soziale Arbeit und Migration. Konzepte und Lösungen im Vergleich. Wiesbaden: VS, S. 227-244.

Oeftering, Tonio (2015): Hannah Arendts Begriff des Politischen und Inklusion. In: Christoph Dönges, Wolfram Hilpert und Bettina Zurstrassen (Hg.): Didaktik der inklusiven politischen Bildung. Bonn: Bundeszentrale für politische Bildung, S. 60-68.

Oevermann, Ulrich (1983): Zur Sache. Die Bedeutung von Adornos methodologischem Selbstverständnis für die Begründung einer materialen 
soziologischen Strukturanalyse. In: Ludwig von Friedeburg und Jürgen Habermas (Hg.): Adorno-Konferenz. 1983. Frankfurt a.M.: Suhrkamp, S. 234-289.

Oevermann, Ulrich (1996): Theoretische Skizze einer revidierten Theorie professionalisierten Handelns. In: Arno Combe und Werner Helsper (Hg.): Pädagogische Professionalität. Untersuchungen zum Typus pädagogischen Handelns. Frankfurt a.M.: Suhrkamp, S. 70-182.

Oevermann, Ulrich (2000): Die Methode der Fallkonstruktion in der Grundlagenforschung sowie der klinischen und pädagogischen Praxis. In: Klaus Kraimer (Hg.): Die Fallrekonstruktion. Sinnverstehen in der sozialwissenschaftlichen Forschung. Frankfurt a.M.: Suhrkamp, S. 58-156.

Oevermann, Ulrich (2001): Die Struktur sozialer Deutungsmuster - Versuch einer Aktualisierung. In: Sozialer Sinn. Zeitschrift für hermeneutische Sozialforschung 2 (1), S. 35-81.

Oevermann, Ulrich (2002): Klinische Soziologie auf der Basis der Methodologie der objektiven Hermeneutik. Manifest der objektiv hermeneutischen Sozialforschung. Unveröffentlichtes Manuskript. Frankfurt a.M.. Online verfügbar unter www.ihsk.de/publikationen/Ulrich_OevermannManifest_der_objektiv_hermeneutischen_Sozialforschung.pdf, zuletzt geprüft am 29.02.2020.

Oomen-Welke, Ingelore (2015): Leichte Sprache, Einfache Sprache und Deutsch als Zweitsprache. In: Didaktik Deutsch 20 (38), S. 24-32.

Opaschowski, Horst W. (2008): Einführung in die Freizeitwissenschaft. Wiesbaden: VS.

Opaschowski, Horst W. (2014): Freizeit. In: Günter Endruweit, Gisela Trommsdorff und Nicole Burzan (Hg.): Wörterbuch der Soziologie. Konstanz: UVK, S. 128-129.

Pongratz, Ludwig A. (2010): Sackgassen der Bildung. Pädagogik anders denken. Paderborn: Schöningh.

Promberger, Markus (2011): Typenbildung mit quantitativen und qualitativen Daten. Methodische Überlegungen. Institut für Arbeitsmarkt- und Berufsforschung (IAB-Discussion Paper, 12).

Przyborski, Aglaja; Wohlrab-Sahr, Monika (2010): Qualitative Sozialforschung. Ein Arbeitsbuch. München: Oldenbourg.

PWIB Wohnungs-Infobörse GmbH (2018a): Mietspiegel Erlangen 2018. Planegg. Online verfügbar unter https://www.wohnungsboerse.net/mietspiegel-Erlangen/1609, zuletzt geprüft am 23.08.2018. 
PWIB Wohnungs-Infobörse GmbH (2018b): Mietspiegel Rostock 2018. Planegg. Online verfügbar unter https://www.wohnungsboerse.net/mietspiegel-Rostock/4174, zuletzt geprüft am 23.08.2018.

PWIB Wohnungs-Infobörse GmbH (2018c): Mietspiegel Schwäbisch Gmünd 2018. Planegg. Online verfügbar unter https://www.wohnungsboerse.net/mietspiegel-Schwaebisch-Gmuend/697, zuletzt geprüft am 23.08.2018.

Raithel, Jürgen (2008): Quantitative Forschung. Ein Praxiskurs. Wiesbaden: VS.

Rathaus Rostock (2018): Bevölkerungsentwicklung insgesamt (Melderegister). Hg. v. Hanse- und Universitätsstadt Rostock. Der Oberbürgermeister. Online verfügbar unter https://rathaus.rostock.de/de/bevoelkerungsentwicklung_melderegister/254076, zuletzt geprüft am 24.01.2020.

Ravenhorst, Volker (2013): Bewusstseinsbildung als Voraussetzung erfolgreicher Inklusion. In: Stephan A. Böhm, Miriam K. Baumgärtner und David J. G. Dwertmann (Hg.): Berufliche Inklusion von Menschen mit Behinderung. Best Practices aus dem ersten Arbeitsmarkt. Berlin, Heidelberg: Springer-Verlag, S. 65-74.

Rawls, John (2017): Eine Theorie der Gerechtigkeit. Frankfurt a.M.: Suhrkamp.

Reckwitz, Andreas (2003): Grundelemente einer Theorie sozialer Praktiken. Eine sozialtheoretische Perspektive. In: Zeitschrift für Soziologie 32 (4), S. 282-301.

Reckwitz, Andreas (2008a): Subjekt. Bielefeld: transcript.

Reckwitz, Andreas (2008b): Unscharfe Grenzen. Perspektiven der Kultursoziologie. Bielefeld: transcript.

Reh, Gerd (2018): Weiterer Anstieg der Bevölkerung in 2017. Online verfügbar unter www.statistik.rlp.de/no_cache/de/einzelansicht/news/detail/ News/2381/, zuletzt geprüft am 24.01.2020.

Reichstein, Martin F. (2016): Teilhabe an der digitalen Gesellschaft? Über (vorgelagerte) Barrieren bei/in der Nutzung digitaler Medien durch Menschen mit einer sogenannten geistigen Behinderung. In: Teilhabe 55 (2), S. 80-85.

Reinhardt, Jan D.; Gradinger, Felix (2007): Behinderung in der Werbung zwischen Unsichtbarkeit und Provokation. In: Michael Jäckel (Hg.): Am- 
bivalenzen des Konsums und der werblichen Kommunikation. Wiesbaden: VS, S. 91-107.

Riege, Mario (2007): Soziale Arbeit und Sozialraumanalyse. In: Detlef Baum (Hg.): Die Stadt in der Sozialen Arbeit. Ein Handbuch für soziale und planende Berufe. Wiesbaden: VS, S. 376-388.

Riege, Marlo; Schubert, Herbert (2005): Zur Analyse sozialer Räume. Ein interdisziplinärer Integrationsversuch. In: Marlo Riege und Herbert Schubert (Hg.): Sozialraumanalyse. Grundlagen - Methoden - Praxis. Wiesbaden: VS, S. 7-68.

Rohrmann, Albrecht (2019): Das Bundesteilhabegesetz - Ausdruck eines Paradigmenwechsels? In: Archiv für Wissenschaft und Praxis der sozialen Arbeit 50 (1), S. 4-14.

Rohrmann, Albrecht; Schädler, Johannes (2014): Inklusive Gemeinwesen Planen. Abschlussbericht eines Forschungsprojektes im Auftrag des Ministeriums für Arbeit, Integration und Soziales in Nordrhein-Westfalen. Unter Mitarbeit von Matthias Kempf, Eva Konieczny, Marcus Windisch, Lena French und Mario Kaiser. Siegen.

Rohrmann, Albrecht; Schädler, Johannes; Wissel, Timo; Gaida, Mareike (2010): Materialien zur örtlichen Teilhabeplanung für Menschen mit Behinderungen. Siegen.

Rösner, Hans-Uwe (2010): Inklusion allein ist zu wenig! Plädoyer für eine Ethik der Anerkennung. In: Markus Dederich, Heinrich Greving, Christian Mürner und Peter Rödler (Hg.): Inklusion statt Integration? Heilpädagogik als Kulturtechnik. Gießen: Psychosozial-Verlag, S. 126-141.

Rössler, Beate (2001): Der Wert des Privaten. Frankfurt a.M.: Suhrkamp.

Saar, Martin (2007): Beschreiben/Zersetzen: Dekonstruktion als Institutionskritik. In: Andreas Niederberger und Markus Wolf (Hg.): Politische Philosophie und Dekonstruktion. Beiträge zur politischen Theorie im Anschluss an Jacques Derrida. Bielefeld: transcript, S. 165-180.

Saar, Martin (2013): Analytik der Subjektivierung. Umrisse eines Theorieprogramms. In: Andreas Gelhard, Thomas Alkemeyer und Norbert Ricken (Hg.): Techniken der Subjektivierung. München: Fink, S. 17-28.

Schädler, Johannes (2011): Örtliche Teilhabeplanung im ländlichen Raum. In: Dorothea Lampke, Albrecht Rohrmann und Johannes Schädler (Hg.): Örtliche Teilhabeplanung mit und für Menschen mit Behinderungen. Theorie und Praxis. Wiesbaden: VS, S. 183-198. 
Schäfers, Markus; Wansing, Gudrun (2016): Zur Einführung: Teilhabebedarfe - zwischen Lebenswelt und Hilfesystem. In: Markus Schäfers und Gudrun Wansing (Hg.): Teilhabebedarfe von Menschen mit Behinderungen. Zwischen Lebenswelt und Hilfesystem. Stuttgart: Kohlhammer, S. $13-23$.

Schäper, Sabine (2015): Vom Verschwinden der Inklusionsverlierer. Gouvernementalitätstheoretische Einblicke in die unsichtbaren Hinterhöfe eines Diskurses. In: Sven Kluge, Andrea Liesner und Edgar Weiß (Hg.): Inklusion als Ideologie. Frankfurt a.M.: Lang, S. 77-90.

Schattenmann, Eva (2016): Inklusion und Bewusstseinsbildung. Die Notwendigkeit bewusstseinsbildener Maßnahmen zur Verwirklichung von Inklusion in Deutschland. Oberhausen: Athena.

Schneverdingen Touristik (Hg.) (2012): barrierefrei erleben. Schneverdingen. Scholz, Markus (2010): Presse und Behinderung. Eine quantitative und qualitative Untersuchung. Wiesbaden: VS.

Scholz, Markus; Rank, Astrid (2016): Perspektive Inklusion. Inklusionsverständnis und Einstellung zur integrativen Beschulung bei Studierenden des Grundschul- und Förderschullehramts. In: Vierteljahresschrift für Heilpädagogik und ihre Nachbargebiete 85 (1), S. 53-67.

Schön, Elke (2013): Gesellschaftliche Teilhabe von Menschen mit Behinderung und Migrationshintergrund in ländlichen Regionen. Erkenntnisse aus der wissenschaftlichen Begleitung des Modellprojekts »Willkommen«. In: Teilhabe 52 (3), S. 102-108.

Schreiber, Verena (2009): Raumangebot bei Foucault. In: Georg Glasze und Annika Mattissek (Hg.): Handbuch Diskurs und Raum. Theorien und Methoden für die Humangeographie sowie die sozial- und kulturwissenschaftliche Raumforschung. Bielefeld: transcript, S. 199-212.

Schroeder, Joachim (2016): Schulen, Gemeinwesen und Inklusion. Möglichkeiten und Grenzen sozialraumorientierter Schulentwicklung. In: Iris Beck (Hg.): Inklusion im Gemeinwesen. Stuttgart: Kohlhammer, S. 85144.

Schroer, Markus (2008): Raum: Das Ordnen der Dinge. In: Stephan Moebius und Andreas Reckwitz (Hg.): Poststrukturalistische Sozialwissenschaften. Frankfurt a.M.: Suhrkamp, S. 141-157.

Schuhmacher, Birgit (2018): Inklusion für Menschen mit Demenz. Exklusionsrisiken und Teilhabechancen. Wiesbaden:VS. 
Schulze, Marianne (2011): Menschenrechte für alle: Die Konvention über die Rechte von Menschen mit Behinderungen. In: Petra Flieger und Volker Schönwiese (Hg.): Menschenrechte, Integration, Inklusion. Aktuelle Perspektiven aus der Forschung. Bad Heilbrunn: Klinkhardt, S. 11-26.

Schwab, Susanne (2015): Lehrersicht der sozialen Partizipation von Grundschülern - Ergebnisse einer Studie mit dem Lehrerfragebogen zur Erfassung der sozialen Partizipation. In: Vierteljahresschrift für Heilpädagogik und ihre Nachbargebiete 84 (3), S. 234-245.

Schwab, Susanne; Gebhardt, Markus; Ederer-Fick, Elfriede M.; GasteigerKlicpera, Barbara (2012): An examination of public opinion in Austria towards inclusion. Development of the sAttitudes Toward Inclusion Scale - ATIS. In: European Journal of Special Needs Education 27 (3), S. 355-371.

Schwab, Susanne; Seifert, Susanne (2015): Einstellungen von Lehramtsstudierenden und Pädagogikstudierenden zur schulischen Inklusion - Ergebnisse einer quantitativen Untersuchung. In: Zeitschrift für Bildungsforschung 5 (1), S. 73-87.

Schwab, Susanne; Tretter, Tobias; Gebhardt, Markus (2014): Entwicklung und Überprüfung eines fallbasierten Instruments zur Messung der Einstellung zur schulischen Integration. Wie denken Studierende, Berufstätige und Schüler/innen über schulische Integration von Kindern mit sonderpädagogischem Förderbedarf? In: Vierteljahresschrift für Heilpädagogik und ihre Nachbargebiete 83 (1), S. 20-32.

Seggern, Hille von; Tessin, Wulf (2005): Einen Ort begreifen. Der Ernst-August-Platz in Hannover. In: Marlo Riege und Herbert Schubert (Hg.): Sozialraumanalyse. Grundlagen - Methoden - Praxis. Wiesbaden: VS, S. 283-298.

Seifert, Alexander (2016): Technikakzeptanz älterer Menschen am Beispiel der allgemeinen und mobilen Internetnutzung. München: kopaed.

Seifert, Monika (2010): Kundenstudie. Bedarf an Dienstleistungen zur Unterstützung des Wohnens von Menschen mit Behinderung. Abschlussbericht. Berlin: Rhombos-Verlag.

Seifert, Monika (2014): Sozialraumorientierte Arbeit im Schnittfeld von Behinderung und Migration. Ergebnisse einer regionalen Studie. In: Gudrun Wansing und Manuela Westphal (Hg.): Behinderung und Migration. Inklusion, Diversiät, Intersektionalität. Wiesbaden: VS, S. 139-158. 
Seifried, Stefanie; Heyl, Vera (2016): Konstruktion und Validierung eines Einstellungsfragebogens zu Inklusion für Lehrkräfte (EFI-L). In: Empirische Sonderpädagogik 2016 (1), S. 22-35.

Shakespeare, Tom (2010): The Social Model of Disability. In: Lennard J. Davis (Hg.): The Disability Studies Reader. New York, Abigdon: Routledge, S. 266-273.

Silkenbeumer, Mirja (2010): Qualitative und quantitative Methoden. In: Detlef Horster und Wolfgang Jantzen (Hg.): Wissenschaftstheorie. Stuttgart: Kohlhammer, S. 261-274.

Speck, Otto (2001): Kindergarten. In: Georg Antor (Hg.): Handlexikon der Behindertenpädagogik. Schlüsselbegriffe aus Theorie und Praxis. Stuttgart: Kohlhammer, S. 376-378.

Stadt Erlangen, Bürgermeister- und Presseamt, Sachgebiet Statistik und Stadtforschung (Hg.) (2016): Statistisches Jahrbuch 2016. Erlangen. Online verfügbar unter https://www.erlangen.de/Portaldata/1/ Resources/080_stadtverwaltung/dokumente/statistik/13-4_B_Jahrbuch_2016.pdf, zuletzt geprüft am 24.01.2020.

Stadt Erlangen, Oberbürgermeister Dr. Florian Janik (2018): Vereine und Organisationen. Erlangen. Online verfügbar unter https://www.erlangen. de/desktopdefault.aspx/tabid-1371, zuletzt geprüft am 23.08.2018.

Stadt Schneverdingen (2018): Stadtportrait. Schneverdingen. Online verfügbar unter https://www.schneverdingen.de/desktopdefault.aspx/tabid-7223/, zuletzt geprüft am 23.08.2018.

Stadt Schwäbisch Gmünd (2018): Vereine in Schwäbisch Gmünd. Schwäbisch Gmünd. Online verfügbar unter www.schwaebisch-gmuend.de/5619Vereine.html, zuletzt geprüft am 23.08.2018.

Statistik der Bundesagentur für Arbeit (2018a): Heidekreis. Arbeitsmarkt im Überblick - Berichtsmonat Juni 2018 - Heidekreis. Online verfügbar unter https://statistik.arbeitsagentur.de/Navigation/Statistik/Statistiknach-Regionen/Politische-Gebietsstruktur/Niedersachsen/HeidekreisNav.html, zuletzt geprüft am 24.01.2020.

Statistik der Bundesagentur für Arbeit (2018b): Mainz-Bingen. Arbeitsmarkt im Überblick - Berichtsmonat Juni 2018 - Mainz - Bingen. Online verfügbar unter https://statistik.arbeitsagentur.de/Navigation/Statistik/ Statistik-nach-Regionen/Politische-Gebietsstruktur/Rheinland-Pfalz/ Mainz-Bingen-Nav.html, zuletzt geprüft am 24.01.2020. 
Statistik der Bundesagentur für Arbeit (2018c): Ostalbkreis. Arbeitsmarkt im Überblick - Berichtsmonat Juni 2018 - Ostalbkreis. Online verfügbar unter https://statistik.arbeitsagentur.de/Navigation/Statistik/Statistiknach-Regionen/Politische-Gebietsstruktur/Baden-Wuerttemberg/Ostalbkreis-Nav.html, zuletzt geprüft am 24.01.2020.

Statistik der Bundesagentur für Arbeit (2018d): Rostock, Hansestadt. Arbeitsmarkt im Überblick - Berichtsmonat Juni 2018 - Rostock, Hansestadt. Online verfügbar unter https://statistik.arbeitsagentur.de/Navigation/Statistik/Statistik-nach-Regionen/Politische-Gebietsstruktur/ Mecklenburg-Vorpommern/Rostock-Hansestadt-Nav.html, zuletzt geprüft am 24.01.2020.

Statistik der Bundesagentur für Arbeit (2020): Erlangen, Stadt. Arbeitsmarkt im Überblick - Berichtsmonat Juli 2018 - Erlangen, Stadt. Online verfügbar unter https://statistik.arbeitsagentur.de/Navigation/Statistik/ Statistik-nach-Regionen/Politische-Gebietsstruktur/Bayern/ErlangenStadt-Nav.html, zuletzt geprüft am 24.01.2020.

Statistische Ämter des Bundes und der Länder, Deutschland (2018): Regionalatlas Deutschland. Indikatoren des Themenbereichs »Verdienste und Einkommen«. Online verfügbar unter https://www-genesis.destatis.de/ gis/genView?CONTEXT=REGATLASol\&GenMLURL=https\%3A//wwwgenesis.destatis.de/regatlas/AIo16-1.xml, zuletzt geprüft am 24.01.2020.

Statistisches Amt Mecklenburg-Vorpommern (2017): Statistisches Jahrbuch Mecklenburg-Vorpommern 2017. Statistisches Amt Mecklenburg-Vorpommern. Schwerin.

Statistisches Landesamt Baden-Württemberg (2018a): Anteil der Altersjahre an der Bevölkerung. Online verfügbar unter https://www.statistik-bw. de/BevoelkGebiet/Alter/01035810.tab?R=GS136065, zuletzt geprüft am 18.09.2018.

Statistisches Landesamt Baden-Württemberg (2018b): Baden-Württemberg: Erstmals mehr als 11 Millionen Einwohner. Bevölkerung hat sich seit Bestehen des Landes um über 4 Millionen Menschen erhöht. Online verfügbar unter https://www.statistik-bw.de/Presse/Pressemitteilungen/2018100, zuletzt geprüft am 24.01.2020.

Statistisches Landesamt Baden-Württemberg (2018c): Eckdaten zur Bevölkerung. Stuttgart. Online verfügbar unter https://www.statistik-bw.de/ BevoelkGebiet/Bevoelkerung/99025010.tab?R=GS136065, zuletzt geprüft am 23.08.2018. 
Statistisches Landesamt Rheinland-Pfalz (2018): Kommunaldatenprofil. Stand 05/2018. Kommunale Gliederung, Bevölkerung, Kommunalwahlen. Landkreis Mainz-Bingen. Online verfügbar unter https://www.statistik.rlp.de/fileadmin/dokumente/kreisdatenprofil/ergebnisse/KrsModul/20180516_KRS339_Mainz-Bingen_01_KommBevWahl.pdf, zuletzt geprüft am 23.08.2018.

Stefanowitsch, Anatol (2014): Leichte Sprache, komplexe Wirklichkeit. In: Aus Politik und Zeitgeschichte 64 (9-11), S. 11-18.

Stoetzer, Sergej (2014): Aneignung von Orten. Raumbezogene Identifikationsstrategien. Technische Universität Darmstadt, Darmstadt.

Stoiber, Karen Callan; Gettinger, Maribeth; Goetz, Donna (1998): Exploring Factors Influencing Parents' and Early Childhood Practitioners' Beliefs about Inclusion. In: Early Childhood Research Quarterly 13 (1), S. 107-124.

Täubig, Vicki (2009): Totale Institution Asyl. Empirische Befunde zu alltäglichen Lebensführungen in der organisierten Desintegration. Weinheim und München: Juventa.

Tervooren, Anja; Weber, Jürgen (2012a): Einleitung: Barrieren wahrnehmen, verstehen und abbauen. In: Anja Tervooren und Jürgen Weber (Hg.): Wege zur Kultur. Barrieren und Barrierefreiheit in Kultur- und Bildungseinrichtungen. Köln, Weimar, Wien: Böhlau, S. 11-28.

Tervooren, Anja; Weber, Jürgen (Hg.) (2012b): Wege zur Kultur. Barrieren und Barrierefreiheit in Kultur- und Bildungseinrichtungen. Köln, Weimar, Wien: Böhlau.

Tesch-Römer, Clemens; Weber, Constanze; Webel, Henry (2016): Nutzung des Internets durch Menschen in der zweiten Lebenshälfte. Hg. v. Deutsches Zentrum für Altersfragen. Berlin.

Theunissen, Georg (2002): Altenbildung und Behinderung. Impulse für die Arbeit mit Menschen, die als lern- und geistig behindert gelten. Bad Heilbrunn: Klinkhardt.

Theunissen, Georg; Kulig, Wolfram (2011): Empowerment und Sozialraumorientierung in der professionellen Unterstützung von Menschen mit Behinderungen. In: Dorothea Lampke, Albrecht Rohrmann und Johannes Schädler (Hg.): Örtliche Teilhabeplanung mit und für Menschen mit Behinderungen. Theorie und Praxis. Wiesbaden: VS, S. 269-284.

Trescher, Hendrik (2013): Kontexte des Lebens. Lebenssituation demenziell erkrankter Menschen im Heim. Wiesbaden: VS. 
Trescher, Hendrik (2015a): Die Würde des Privaten. Zur Diskussion institutionalisierter Lebensbedingungen von Menschen mit kognitiver Beeinträchtigung. In: Behindertenpädagogik 54 (2), S. 136-153.

Trescher, Hendrik (2015b): Inklusion. Zur Dekonstruktion von Diskursteilhabebarrieren im Kontext von Freizeit und Behinderung. Wiesbaden:VS.

Trescher, Hendrik (2015c): Zielperspektive Inklusion. Freizeit von Menschen mit geistiger Behinderung. In: Schweizerische Zeitschrift für Heilpädagogik 21 (9), S. 39-46.

Trescher, Hendrik (2016a): Freizeit als Fenster zur Inklusion. Konstruktionen von Teilhabe und Ausschluss für erwachsene, institutionalisiert lebende Menschen mit 'geistiger Behinderung،. In: Vierteljahresschrift für Heilpädagogik und ihre Nachbargebiete 85 (2), S. 98-111.

Trescher, Hendrik (2016b): Inklusive Freizeitgestaltung für ältere Menschen mit geistiger Behinderung - ein Strukturproblem. In: Teilhabe 55 (1), S. 35-41.

Trescher, Hendrik (2016c): Wahlrecht von Menschen mit Behinderungen. In: Deutsches Institut für Menschenrechte (Hg.): Die Umsetzung ausgewählter OSZE-Verpflichtungen zu Menschenrechten und Demokratie in Deutschland. Unabhängiger Evaluierungsbericht anlässlich des deutschen OSZE-Vorsitzes 2016, S. 96-108.

Trescher, Hendrik (2017a): Behinderung als Praxis. Biographische Zugänge $\mathrm{zu}$ Lebensentwürfen von Menschen mit »geistiger Behinderung«. Bielefeld: transcript.

Trescher, Hendrik (2017b): Behinderung, Fluchtmigration, Kommunikation. In: Teilhabe 56 (4), S. 150-155.

Trescher, Hendrik (2017c): Disabling practices. In: Cogent Social Sciences 3 (1).

Trescher, Hendrik (2017d): Inclusion as Critique. Deconstructionist Approaches Exemplified through >Care of People with Cognitive Disabilities in Germany. In: International Journal of Social Science Studies 5 (8), S. 33-43.

Trescher, Hendrik (2017e): Von behindernden Praxen zu einer Reformulierung des Behinderungsbegriffs. In: Behindertenpädagogik 56 (3), S. 267282.

Trescher, Hendrik (2017f): Wohnräume als pädagogische Herausforderung. Lebenslagen institutionalisiert lebender Menschen mit Behinderung. Wiesbaden: VS. 
Trescher, Hendrik (2018a): Ambivalenzen pädagogischen Handelns. Reflexionen der Betreuung von Menschen mit >geistiger Behinderungく. Bielefeld: transcript.

Trescher, Hendrik (2018b): Inklusion und Dekonstruktion. Die Praxis der ,Versorgung< von Menschen mit Behinderung in Deutschland zum Gegenstand. In: Zeitschrift für Inklusion 12 (2).

Trescher, Hendrik (2018c): Kognitive Beeinträchtigung und Barrierefreiheit. Eine Pilot-Studie. Bad Heilbrunn: Klinkhardt.

Trescher, Hendrik (2019): Sonderpädagogik als Erfahrungswissenschaft aus poststrukturalistischer Perspektive. In: Markus Dederich, Stephan Ellinger und Désirée Laubenstein (Hg.): Sonderpädagogik als Erfahrungsund Praxiswissenschaft. Geistes-, sozial- und kulturwissenschaftliche Perspektiven. Opladen: Verlag Barbara Budrich, S. 35-50.

Trescher, Hendrik (2020a): Barriere. In: Fabian Kessl und Christian Reutlinger (Hg.): Sozialraum. Eine elementare Einführung. Wiesbaden: VS, im Erscheinen.

Trescher, Hendrik (2020b): Eltern und ihre Kinder mit geistiger Behinderung im Hilfesystem. Wie gouvernementale Praxen Familie hervorbringen. In: Vierteljahresschrift für Heilpädagogik und ihre Nachbargebiete 89 (3), im Erscheinen.

Trescher, Hendrik; Börner, Michael (2016): Repräsentanz und Subjektivität im Kontext geistiger Behinderung. In: Zeitschrift für Inklusion 10 (1).

Trescher, Hendrik; Hauck, Teresa (2015): Demenz und Diskurs. Reproduktion einer modernen Ordnungskategorie vs. Inklusive Perspektiven pädagogischen Handelns. In: Der pädagogische Blick 23 (4), S. 197-208.

Trescher, Hendrik; Hauck, Teresa (2017): Raum und Inklusion. Zu einem relationalen Verhältnis. In: Zeitschrift für Inklusion 11 (4).

Trescher, Hendrik; Hauck, Teresa (2018): »Kommune Inklusiv« - Sozialräume beforschen und begleiten. In: Teilhabe 57 (4), S. 156-162.

Trescher, Hendrik; Hauck, Teresa (2019): Inklusion im relationalen Raum. Ethnographische Sozialraumbegehungen zwischen Teilhabe und Ausschluss. In: Gabi Ricken und Sven Degenhardt (Hg.): Vernetzung, Kooperation, Sozialer Raum. Inklusion als Querschnittaufgabe. Bad Heilbrunn: Julius Klinkhardt, S. 227-231.

Trescher, Hendrik; Hauck, Teresa (2020): Behindernde Räume. Aneignungsund Teilhabepraxen im Sozialraum. In: Gemeinsam leben 28 (2), im Erscheinen. 
Trescher, Hendrik; Hauck, Teresa; Börner, Michael (2017): Auf dem Weg $\mathrm{zu}$ Inklusion? - >Busfahren als Praxis ethnografischer Inklusionsforschung. In: Vierteljahresschrift für Heilpädagogik und ihre Nachbargebiete 86 (3), S. 250-252.

Trescher, Hendrik; Lamby, Anna; Börner, Michael (2020a): Einstellungen zu Inklusion im Kontext "geistiger Behinderung«. Lebensbereiche Freizeit, Arbeit und Wohnen im Vergleich. In: Schweizerische Zeitschrift für Heilpädagogik 26 (2), S. 13-19.

Trescher, Hendrik; Lamby, Anna; Börner, Michael (2020b): Einstellungen zur Inklusion von Menschen mit geistiger Behinderung in Deutschland. Erkenntnisse einer bevölkerungsrepräsentativen Studie. In: Teilhabe 59, im Erscheinen.

Verbandsgemeindeverwaltung Nieder-Olm, Bürgermeister Ralph Spiegler (2018):Vereine und Verbände. Nieder-Olm. Online verfügbar unter https:// www.vg-nieder-olm.de/vg_niederolm/Kultur,\%20Freizeit,\%2oSport/ Vereine\%2ound\%20Verb\%C3\%A4nde/, zuletzt geprüft am 23.08.2018.

Villa, Paula-Irene (2008): (De)Konstruktion und Diskurs-Genealogie: Zur Position und Rezeption von Judith Butler. In: Ruth Becker und Beate Kortendiek (Hg.): Handbuch Frauen- und Geschlechterforschung. Theorie, Methoden, Empirie. Wiesbaden: VS, S. 146-158.

Völkl, Kerstin; Korb, Christoph (2018): Deskriptive Statistik. Eine Einführung für Politikwissenschaftlerinnen und Politikwissenschaftler. Wiesbaden: VS.

Voß, Günter (2010): Was ist Arbeit? In: Fritz Böhle, Günter Voß und Günther Wachtler (Hg.): Handbuch Arbeitssoziologie. Wiesbaden: VS, S. 23-80.

Waddell, Cynthia D. (2006): Worldwide Accessibility Laws and Policies. In: Jim Thatcher, Michael R. Burks, Christian Heilmann, Shawn Lawton Henry, Andrew Kirkpatrick, Patrick H. Lauke et al. (Hg.): Web Accessibility. Web Standards and Regulatory Compliance. New York: Springer-Verlag New York, Chapter 17.

Wansing, Gudrun (2016): Soziale Räume als Ort der Lebensführung. Optionen, Beschränkungen und Befähigungen. In: Iris Beck (Hg.): Inklusion im Gemeinwesen. Stuttgart: Kohlhammer, S. 239-267.

Weisser, Jan (2010): Das provokative Essay: Sozialraumorientierung und Situationen der Behinderung - Über die sozialräumliche Strukturierung von Abhängigkeitsbeziehungen. In: Vierteljahresschrift für Heilpädagogik und ihre Nachbargebiete 79 (1), S. 4-10. 
Wendt, Peter-Ulrich (2017): Lehrbuch Methoden der Sozialen Arbeit. Weinheim und Basel: Beltz Juventa.

Wetz, Franz Josef (2002): Die Würde der Menschen ist antastbar. Eine Provokation. Stuttgart: Klett-Cotta.

Whitehouse, Peter J.; George, Daniel (2009): Mythos Alzheimer. Was Sie schon immer über Alzheimer wissen wollten, Ihnen aber nicht gesagt wurde. Bern: Hans Huber.

Williams, Bernard (2003): Der Begriff der Moral. Eine Einführung in die Ethik. Stuttgart: Reclam.

Wißmann, Peter (2010): Demenz - ein soziales und zivilgesellschaftliches Phänomen. In: Kirsten Aner und Ute Karl (Hg.): Handbuch Soziale Arbeit und Alter. Wiesbaden: VS, S. 339-346.

Wißmann, Peter; Gronemeyer, Reimer (2008): Demenz und Zivilgesellschaft. Eine Streitschrift. Frankfurt a.M.: Mabuse.

Zapf, Hubert (2013): Dekonstruktion. In: Ansgar Nünning (Hg.): Metzler Lexikon Literatur- und Kulturtheorie. Ansätze - Personen - Grundbegriffe. Stuttgart, Weimar: J.B. Metzler, S. 123-124.

Zibell, Carolina (2018): Aktion Mensch: Das Projekt »Kommune Inklusiv«. In: Teilhabe 57 (2), S. 93-94.

Zima, Peter V. (2016): Die Dekonstruktion. Einführung und Kritik. Tübingen: Francke.

Zirfas, Jörg (2001): Identitäten und Dekonstruktionen. Pädagogische Überlegungen im Anschluss an Jacques Derrida. In: Bettina Fritzsche, Jutta Hartmann, Andrea Schmidt und Anja Tervooren (Hg.): Dekonstruktive Pädagogik. Erziehungswissenschaftliche Debatte unter poststrukturalistischen Perspektiven. Wiesbaden: VS, S. 49-64.

Zurstrassen, Bettina (2015): Inklusion durch Leichte Sprache? Eine kritische Einschätzung. In: Christoph Dönges, Wolfram Hilpert und Bettina Zurstrassen (Hg.): Didaktik der inklusiven politischen Bildung. Bonn: Bundeszentrale für politische Bildung, S. 126-138. 


\section{Pädagogik}

Kay Biesel,

Felix Brandhorst,

Regina Rätz,

Hans-Ullrich Krause

Deutschland

schützt

seine Kinder!

Eine Streitschrift zum Kinderschutz

$[$ transcript] $\times+2 \times+2$
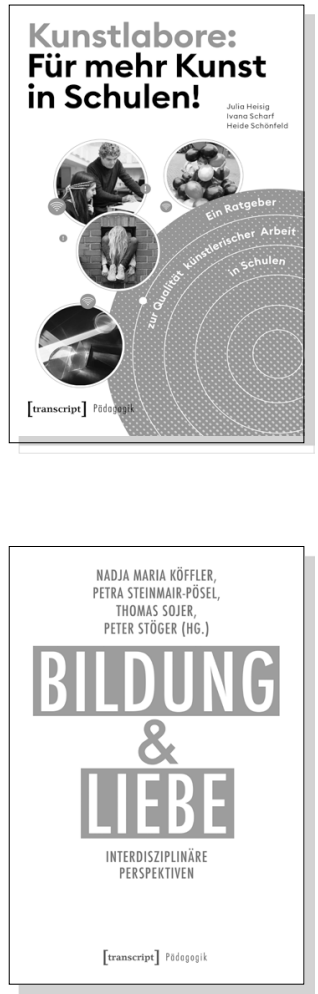

Kay Biesel, Felix Brandhorst, Regina Rätz, Hans-Ullrich Krause Deutschland schützt seine Kinder!

Eine Streitschrift zum Kinderschutz

2019, 242 S., kart., 1 SW-Abbildung

$22,99 €(D E), 978-3-8376-4248-3$

E-Book: 20,99 € (DE), ISBN 978-3-8394-4248-7

EPUB: $20,99 €$ (DE), ISBN 978-3-7328-4248-3

Julia Heisig, Ivana Scharf, Heide Schönfeld

\section{Kunstlabore: Für mehr Kunst in Schulen!}

Ein Ratgeber zur Qualität künstlerischer Arbeit in Schulen

Februar 2020, 216 S., französische Broschur, durchgängig vierfarbig 27,99€ (DE), 978-3-8376-4985-7

E-Book: kostenlos erhältlich als Open-Access-Publikation, ISBN 978-3-8394-4985-1

Nadja Köffler, Petra Steinmair-Pösel, Thomas Sojer, Peter Stöger (Hg.)

\section{Bildung und Liebe}

Interdisziplinäre Perspektiven

2018, 412 S., kart., 11 SW-Abbildungen

39,99€ (DE), 978-3-8376-4359-6

E-Book: 39,99 € (DE), ISBN 978-3-8394-4359-0 


\section{Pädagogik}
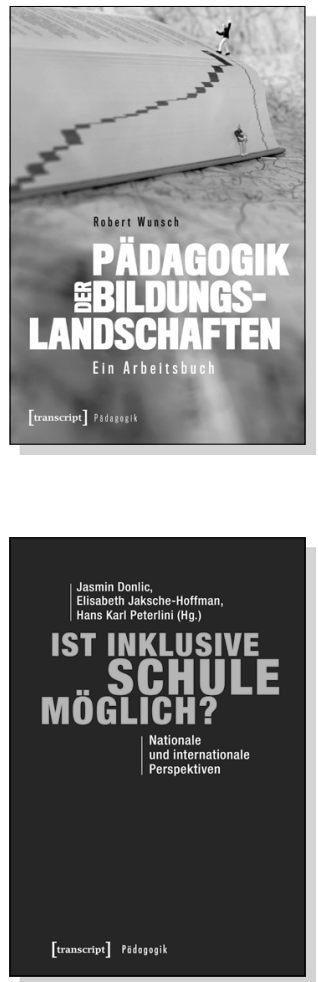

SYBILLE WIESCHOLEK

TEXTIE BHLDUNC

IM ZELTALER DER

DIGITALISIERUNG

VERMITTLUNGSCHANCEN

ZWISCHEN HANDARBE
UND TECHNISIERUNG
Robert Wunsch

\section{Pädagogik der Bildungslandschaften}

Ein Arbeitsbuch

Januar 2020, 210 S., kart., Dispersionsbindung, 1 SW-Abbildung 29,99€ (DE), 978-3-8376-5119-5

E-Book: 26,99 € (DE), ISBN 978-3-8394-5119-9
Jasmin Donlic, Elisabeth Jaksche-Hoffman,

Hans Karl Peterlini (Hg.)

\section{Ist inklusive Schule möglich?}

Nationale und internationale Perspektiven

2019, 312 S., kart., Dispersionsbindung, 11 SW-Abbildungen 29,99 € (DE), 978-3-8376-4312-1

E-Book: $26,99 €(D E)$, ISBN 978-3-8394-4312-5
Sybille Wiescholek

\section{Textile Bildung im Zeitalter der Digitalisierung}

Vermittlungschancen zwischen Handarbeit

und Technisierung

2019, 258 S., kart., Dispersionsbindung, 53 SW-Abbildungen $39,99 €(D E), 978-3-8376-4687-0$

E-Book: 39,99 € (DE), ISBN 978-3-8394-4687-4 
